\title{
HYDROGEOLOGIC DATA FOR THE BLAINE AQUIFER AND ASSOCIATED UNITS IN SOUTHWESTERN OKLAHOMA AND NORTHWESTERN TEXAS
}

By D.L. Runkle, D.L. Bergman, and R.S. Fabian

U.S. GEOLOGICAL SURVEY

Open-File Report 97-50

Prepared in cooperation with the Oklahoma Water Resources Board and the Oklahoma Geological Survey

Oklahoma City, Oklahoma 1997

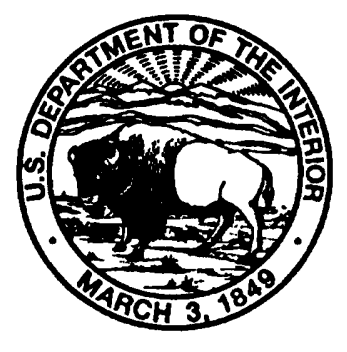




\title{
U.S. DEPARTMENT OF THE INTERIOR
}

BRUCE BABBITT, Secretary

\author{
U.S. GEOLOGICAL SURVEY \\ GORDON P. EATON, Director
}

Any use of trade names in this publleation is for descriptive purposes

only and does not imply endoreoment by the U.S. Covornment.

UNITED STATES GOVERNMENT PRINTING OFFICE: OKLAHOMA CITY 1997

For additional information write to:

District Chief

U.S. Geological Survey

Water Resources Division

202 NW 66th Street, Bullding 7

Oklahoma Clty, OK 73116
Coples of this report can be purchased from:

U.S. Geological Survey

Branch of Information Services

Box 25286

Denver, CO 80225-0286 


\section{CONTENTS}

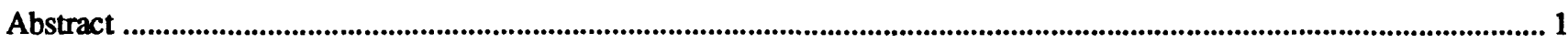

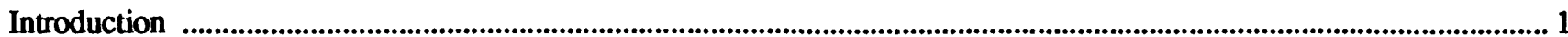

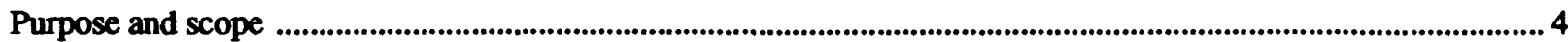

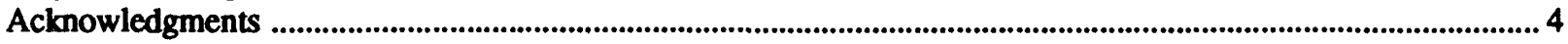

Explanation of tables and methods of data collection ................................................................................................................ 4

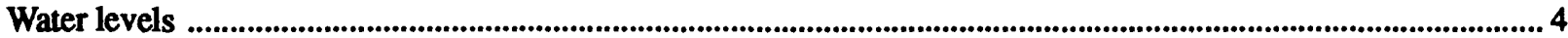

Monthly or periodic water-level measurements .................................................................................................. 4

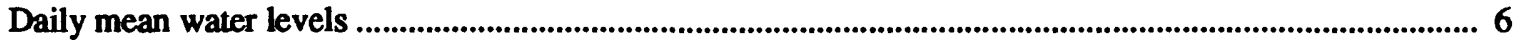

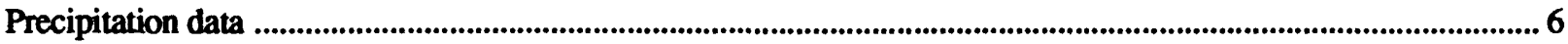

Stream-discharge measurements ............................................................................................................................ 6

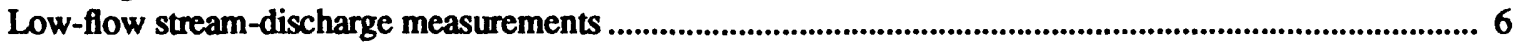

Miscellaneous stream-discharge measurements............................................................................................... 6

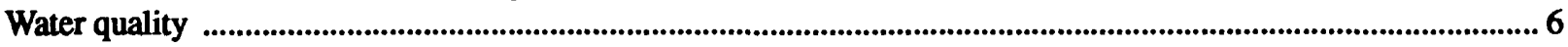

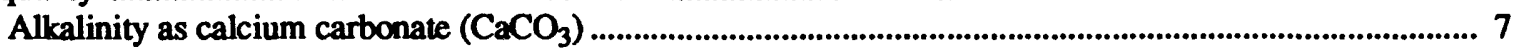

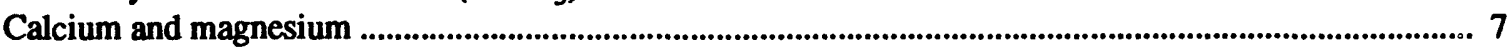

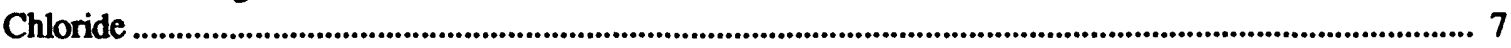

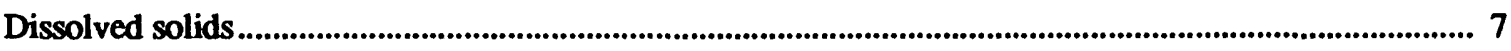

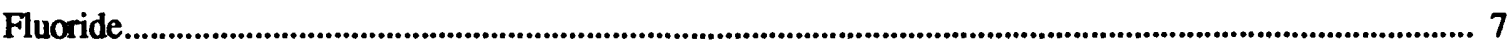

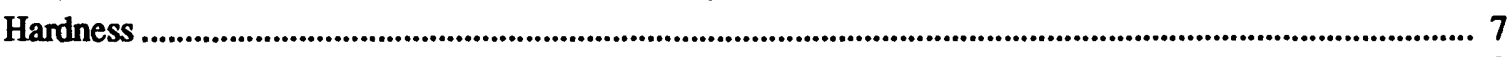

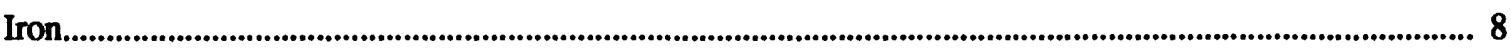

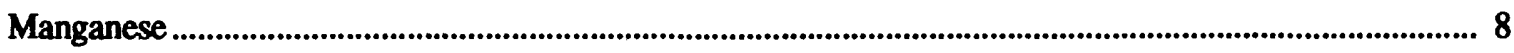

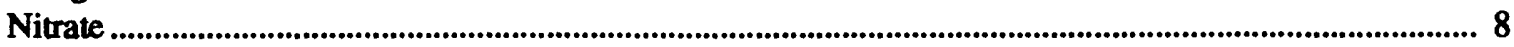

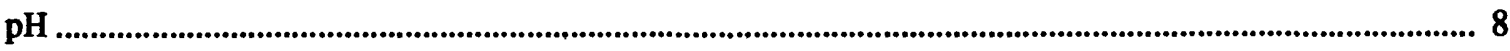

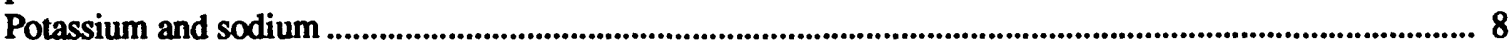

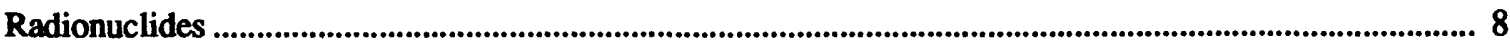

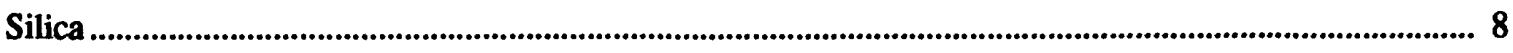

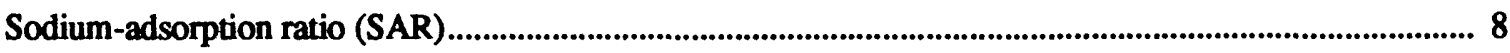

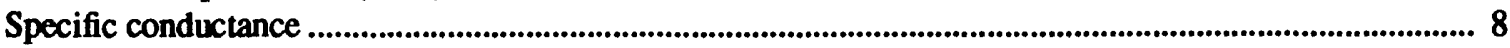

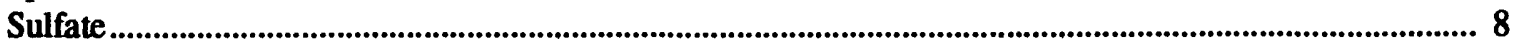

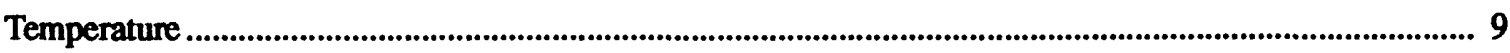

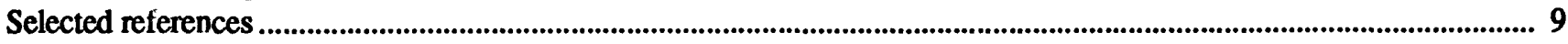

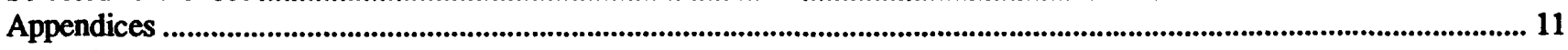

Figures:

3. Map showing location of wells in the water-level network. ............................................................................. 12

4-5 Hydrographs showing:

4. Wells 03N-23W-27 ADAA 1, 02N-26W-05 BCCC 1, and 01S-25W-13 CCAA 1 from June 1948 to March 1988 drilled in the Blaine aquifer................................................................... 78

5. Wells 01N-25W-12 DABA 1, 01N-26W-05 BBCC1, 02N-26W-12 AAAA 1, and 03N-26W-15 BABB 1 from May 1986 to March 1988 drilled in the Blaine aquifer................................. 79

6. Map showing location of wells equipped with water-level recorders in southwestem Oklahoma (index numbers on map refer to sites in table 3). ...................................................................................... 80

7-17 Hydrographs showing:

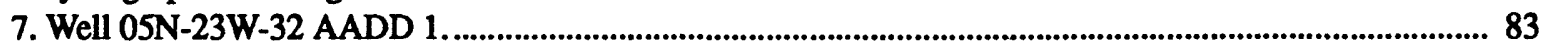

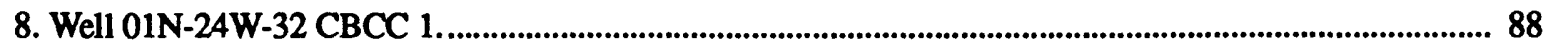

9. Well 02N-24W-21 DDDD 1 (o on hydrograph indicates a point measurement). ...................................... 91

10. Well $02 \mathrm{~N}-24 \mathrm{~W}-21$ DDDD 2 (o on hydrograph indicates a point measurement). ..................................... 94

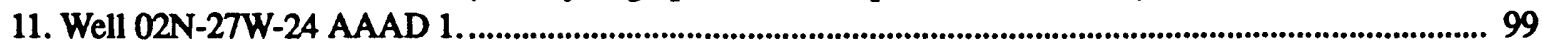

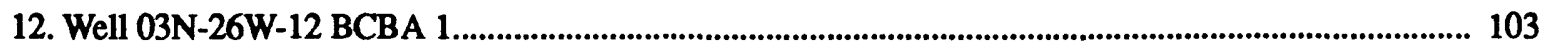


13. Well 03N-26W-33 ABA 1 .

14. Well 04N-25W-30 BBAB 1 .

15. Well 01S-24W-33 BCCC 1

16. Well $01 N-23 W-10$ BBBA 1 .

17. Well 03N-23W-28 DADA 1.

18-29 Maps showing:

18. Location of precipitation gage sites in southwestem Oklahoma (index numbers on map refer to sites in table 4).

19. Location of low-flow stream-discharge measurement sites in southwestern Oklahoma and northwestern Texas (index numbers on map refer to sites in table 5).

20. Location of miscellaneous stream-discharge and field water-quality measurement sites in southwestern Oklahoma (index numbers on map refer to sites in table 6).

21. Location of stream water-quality collection sites sampled during low-flow periods in southwestern Oklahoma and northwestem Texas (index numbers on map refer to sites in table 7a).

22. Location of stream water-quality collection sites sampled during low-flow periods and analyzed for organic chemicals in southwestem Oklahoma and northwestern Texas (index numbers on map refer to sites in table $7 \mathrm{~b}$ ).

23. Location of stream water-quality collection sites sampled during low-flow periods and analyzed for radionuclides in southwestem Oklahoma and northwestern Texas (index numbers on map refer to sites on table 7c).

24. Location of stream water-quality collection sites sampled during low-flow periods, with field water-quality measurements and bromide and chloride analyses in southwestem Oklahoma and northwestern Texas (index numbers on map refer to sites in table 7d).

25. Location of ground-water quality collection sites in southwestern Oklahoma and northwestern Texas (index numbers on map refer to sites in table 8a).

26. Location of ground-water quality collections sites analyzed for organic chemicals in southwestem Oklahoma (index numbers on map refer to sites in table 8b).

27. Location of ground-water quality collection sites analyzed for radionuclides in southwestem Oklahoma (index numbers on map refer to sites in table $8 \mathrm{c}$ ).

28. Location of runoff-water collection sites in southwestem Oklahoma (index numbers on map refer to sites in table 9a)

29. Location of runoff-water collection site analyzed for radionuclides in southwestern Oklahoma (index number on map refers to site in table $9 b$ ).

Tables 2-9b

2. Monthly or periodic water-level measurements in selected wells in southwestern Oklahoma and northwestern Texas.

3. Daily mean water-level measurements in recorder wells in southwestem Oklahoma.

4. Daily precipitation measurements in southwestern Oklahoma .....................................................................130

5. Low-flow stream-discharge measurements in southwestern Oklahoma and northwestern Texas................... 147

6. Miscellaneous stream-discharge measurements and field water-quality properties

in southwestern Oklahoma

7a. Common ion, trace, and nutrient chemical analyses of water collected from streams during low-flow periods in southwestern Oklahoma and northwestern Texas.

7b. Organic chemical analyses of water collected from streams during low-flow periods in southwestem Oklahoma and northwestem Texas

7c. Radionuclide chemical analyses of water collected from streams during low-flow periods in southwestern Oklahoma and northwestem Texas.

7d. Low-flow stream-discharge measurements, field water-quality properties, and bromide and chloride chemical analyses in southwestem Oklahoma and northwestern Texas

8a. Common ion, trace, and nutrient chemical analyses of ground water from selected wells in southwestem Oklahoma and northwestem Texas.

$8 b$. Organic chemical analyses of ground water from selected wells in southwestern Oklahoma. 
8c. Radionuclide chemical analyses of ground water from selected wells in southwestern Oklahoma.

9a. Common ion, trace, and nutrient chemical analyses of runoff water in southwestern Oklahoma.

\section{ILLUSTRATIONS}

Figures 1-2 Maps showing:

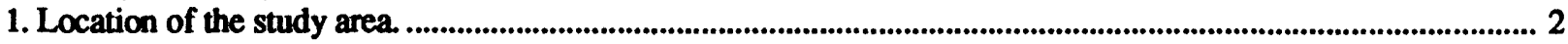

2. Geographic features of the study area. ....................................................................................................................... 3

\section{TABLES}

1. Geologic unit or aquifer names used in this report 5

\section{CONVERSION FACTORS AND VERTICAL DATUM}

\begin{tabular}{rll}
\hline Multiply & By & To obialn \\
\hline cubic foot per second $\left(\mathrm{ft}^{3} / \mathrm{s}\right)$ & 0.02832 & cubic meter per second \\
inch (in) & 25.4 & millimeter \\
foot $(\mathrm{ft})$ & 0.3048 & meter \\
mile $(\mathrm{mi})$ & 1.609 & kilometer \\
square mile $\left(\mathrm{mi}^{2}\right)$ & 2.590 & square kilometer \\
\hline
\end{tabular}

Temperature in degrees Celsius $\left({ }^{\circ} \mathrm{C}\right)$ may be converted to degrees Fahrenheit $\left({ }^{\circ} \mathrm{F}\right)$ as follows:

${ }^{\circ} \mathrm{F}=1.8^{\circ} \mathrm{C}+32$

Sea level: In this report, "sea level" refers to the National Geodetic Vertical Datum of 1929 (NGVD of 1929)--a geodetic datum derived from a general adjustment of the first-order level nets of both the United States and Canada, formerly called Sea Level Datum of 1929. 


\section{Location-numbering System in Oklahoma}

The location-numbering system used in this report is based on the public land-classification system used by the Bureau of Land Management, U.S. Department of the Interior, and the Oklahoma District of the U.S. Geological Survey. The location number is often referred to as the local number or the station name. The location-numbering system illustration follows. The first number indicates the township north or south of a baseline (or standard parallel), the second number indicates the range west of the Indian meridian, and the third number indicates the section in which the well in located. The letters A, B, C, and D designate the northeast, northwest, southwest, and southeast quarters of a section or quarters of any smaller square area section. The first letter of the quarters, reading from left to right, designates the 160-acre quarter, the second letter designates the 40-acre quarter, the third letter designates the 10-acre quarter, and the fourth letter designates the 2.5 -acre quarter. The number following the quarters is a sequential number that allows for a unique local number when more than one site is located in the same smallest quarter subdivision. For example, well $02 \mathrm{~N}-25 \mathrm{~W}-09$ DCBC 1 is located in the SW $1 / 4$, of the NW $1 / 4$, of the SW $1 / 4$, of the SE $1 / 4$, of section 9 , in township 2 north, and range 25 west.

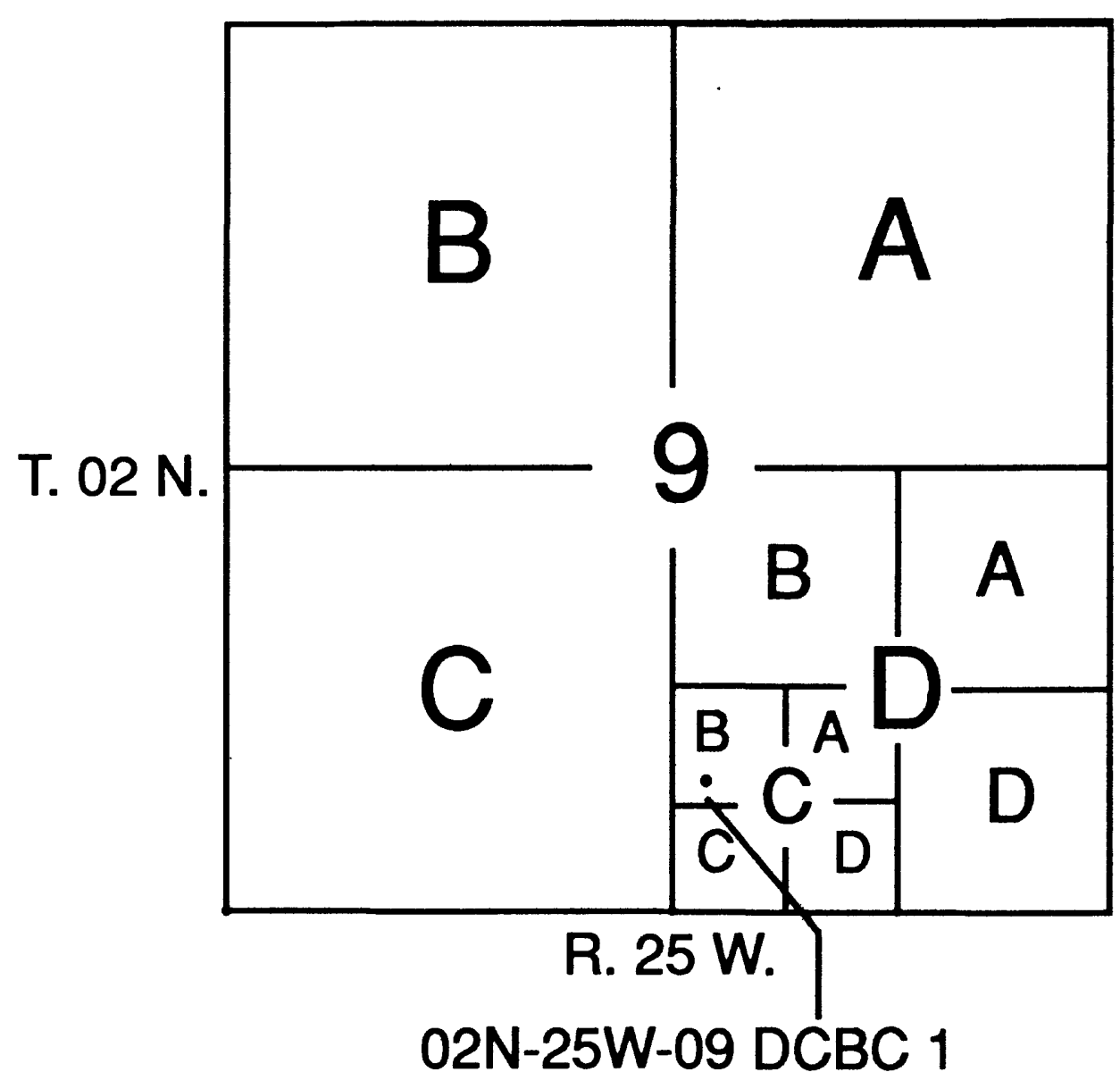


The numbers assigned to wells in Texas in this report conform to the statewide system used by the Texas Water Commission. This system is based on the division of Texas into 1-degree quadrangles bounded by lines of latitude and longitude. Each 1-degree quadrangle is divided into 64 smaller quadrangles, 7 1/2 minutes on a side, each of which is further divided into 9 quadrangles, $21 / 2$ minutes on a side. Each of the 89,1-degree quadrangles in the State has been assigned a 2-digit number for identification. The $71 / 2$ minute quadrangles are numbered from left to right beginning in the upper left-hand comer of the 1-degree quadrangle, and the $21 / 2$ minute quadrangles within each $71 / 2$ minute quadrangle are similarly numbered. Each well in a $21 / 2$ minute quadrangle is assigned a 2-digit number. The well number is determined as follows: the first 2 numbers identify the 1-degree quadrangle, the next 2 numbers identify the $71 / 2$ minute quadrangle, the fifth number identifies the $21 / 2$ minute quadrangle, and the last 2 numbers are sequential numbers to provide a unique well number for the location within the $21 / 2$ minute quadrangle.

The location-numbering system of Oklahoma was extended into Texas for three wells that were located within a mile of the Oklahoma-Texas State line. These three wells are: 03N-27W-23 DD 1 in Childress County, and 02S-24W-05 CADB 1 and 01N-26W-08 BADD 1 in Hardeman County. 


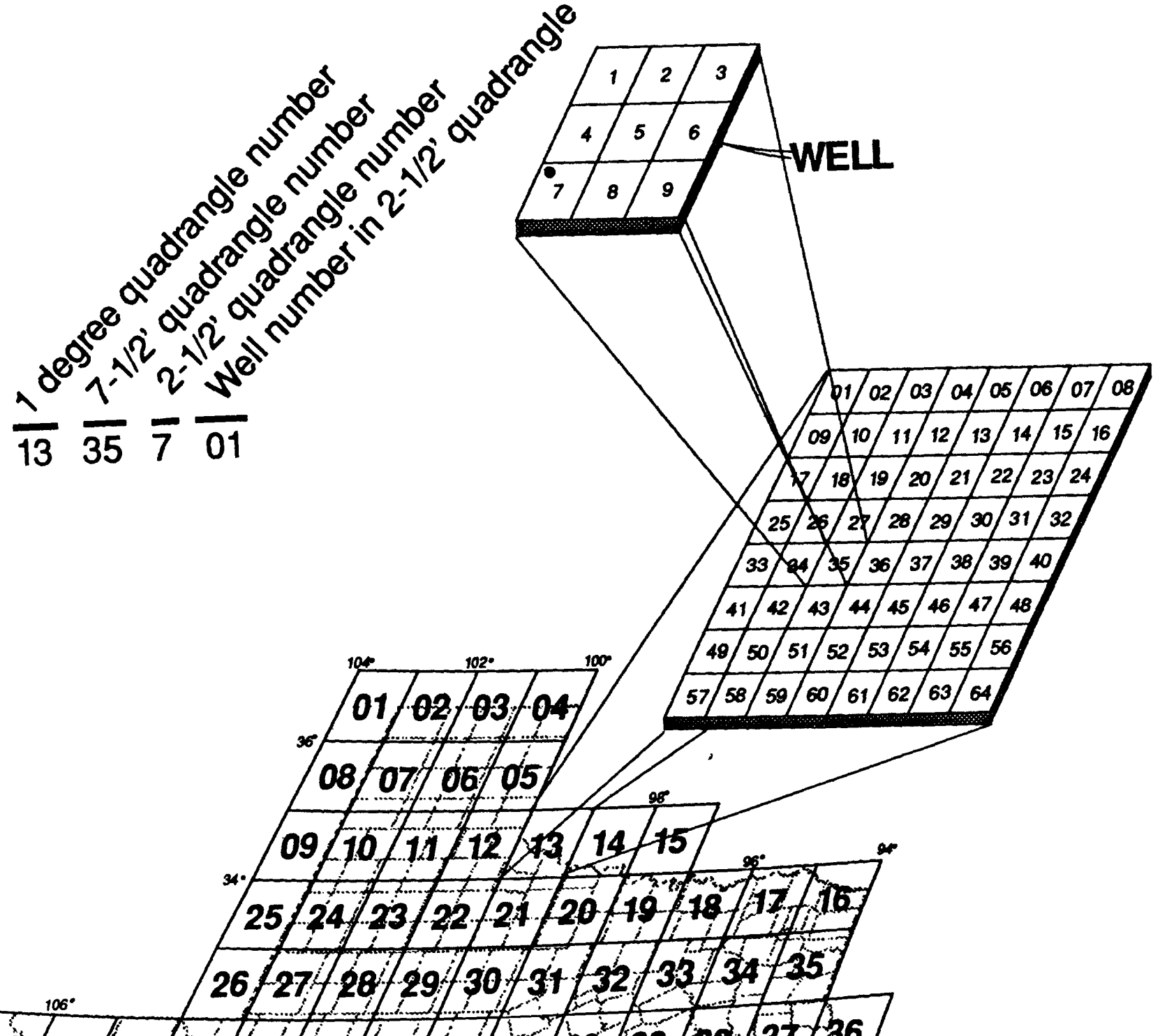

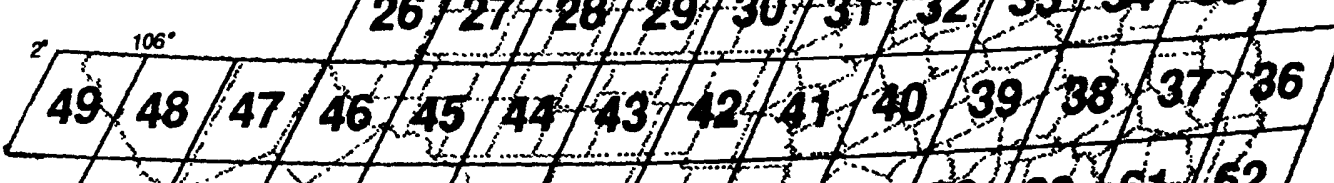

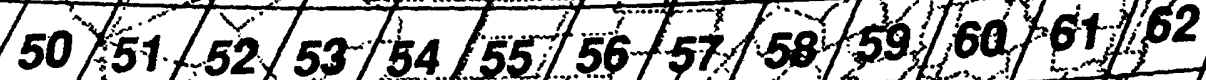

7473.7277 to $69606766.65 \% 63$

${ }_{20} .75$

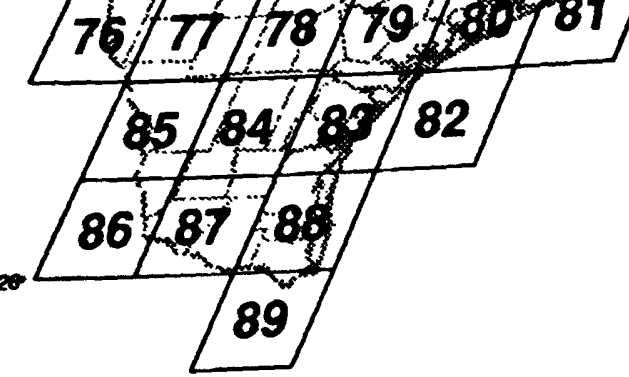




\title{
HYDROGEOLOGIC DATA FOR THE BLAINE AQUIFER AND ASSOCIATED UNITS IN SOUTHWESTERN OKLAHOMA AND NORTHWESTERN TEXAS
}

\author{
By D.L. Runkle, D.L. Bergman, and R.S. Fabian
}

\section{Abstract}

This report is a compilation of hydrogeologic data collected for an areal ground-water investigation of the Blaine aquifer and associated units in southwestern Oklahoma and northwestern Texas. The study area includes parts of Greer, Harmon, and Jackson counties in Oklahoma and parts of Childress, Collingsworth, Hall, Hardeman, and Wilbarger counties in Texas. The Blaine aquifer consists of cavernous gypsum and dolomite beds. Water from the Blaine aquifer supports a local agriculture based mainly on irrigated cotton and wheat. The purpose of the study was to determine the availability, quantity, and quality of ground water from the Blaine aquifer and associated units. This report provides a reference for some of the data that was used as input into a computer ground-water flow model that simulates ground-water flow in the Blaine aquifer. The data in this report consists of: (1) Monthly or periodic water-level measurements in 134 wells; (2) daily mean water-level measurements for 11 wells equipped with water-level recorders; (3) daily total precipitation measurements from five precipitation gages; (4) low-flow stream-discharge measurements for 89 stream sites; (5) miscellaneous stream-discharge measurements at seven stream sites; (6) chemical analyses of surface water from 78 stream sites during lowflow periods; (7) chemical analyses of ground water from 41 wells; and (8) chemical analyses of runoff water collected at five sites.

\section{INTRODUCTION}

The hydrologic data contained in this report were collected for an areal ground-water investigation of the Blaine aquifer and associated units in southwestern Oklahoma and northwestern Texas (fig. 1). The study area includes parts of Greer, Harmon, and Jackson counties in Oklahoma and parts of Childress, Collingsworth, Hall, Hardeman, and Wilbarger counties in Texas (fig. 2).

The Blaine aquifer consists of cavemous gypsum and dolomite beds. Water from the Blaine aquifer supports a local agriculture based mainly on irrigated cotton and wheat. The Blaine aquifer has been artificially recharged since about 1961, although much of the effort was abandoned after 1975; it was restarted in the 1990's as part of a demonstration project by the Oklahoma Water Resources Board. The aquifer was recharged at more than 60 different locations by diverting streamflow and storm runoff into sink holes and wells. Water in the Blaine aquifer is not used for human consumption because of the large dissolved-solids concentrations. The calcium-sulfate type water in the Blaine aquifer is acceptable for irrigation of salt-tolerant crops.

The purpose of the study was to determine the availability, quantity, and quality of ground water from the Blaine aquifer and associated units. Specific objectives were to: (1) Evaluate and map the stratigraphy and structure of all geologic units in the study area adjacent to the Blaine aquifer, (2) evaluate the hydrology of the Blaine aquifer and adjacent units; (3) determine the distribution of major and selected trace and organic chemical constituents in the aquifers; (4) analyze the effects that extensive irrigation development and the artificial recharge program have had on the Blaine and associated aquifers and project the effect they have had on the quantity and quality of water in the aquifers; and (5) determine if opportunities exist for additional artificial recharge.

This was a cooperative study with the Oklahoma Water Resources Board, Oklahoma Geological Survey, and U.S. Geological Survey. The stratigraphic nomenclature used in this report follows that of the Oklahoma Geological Survey. 


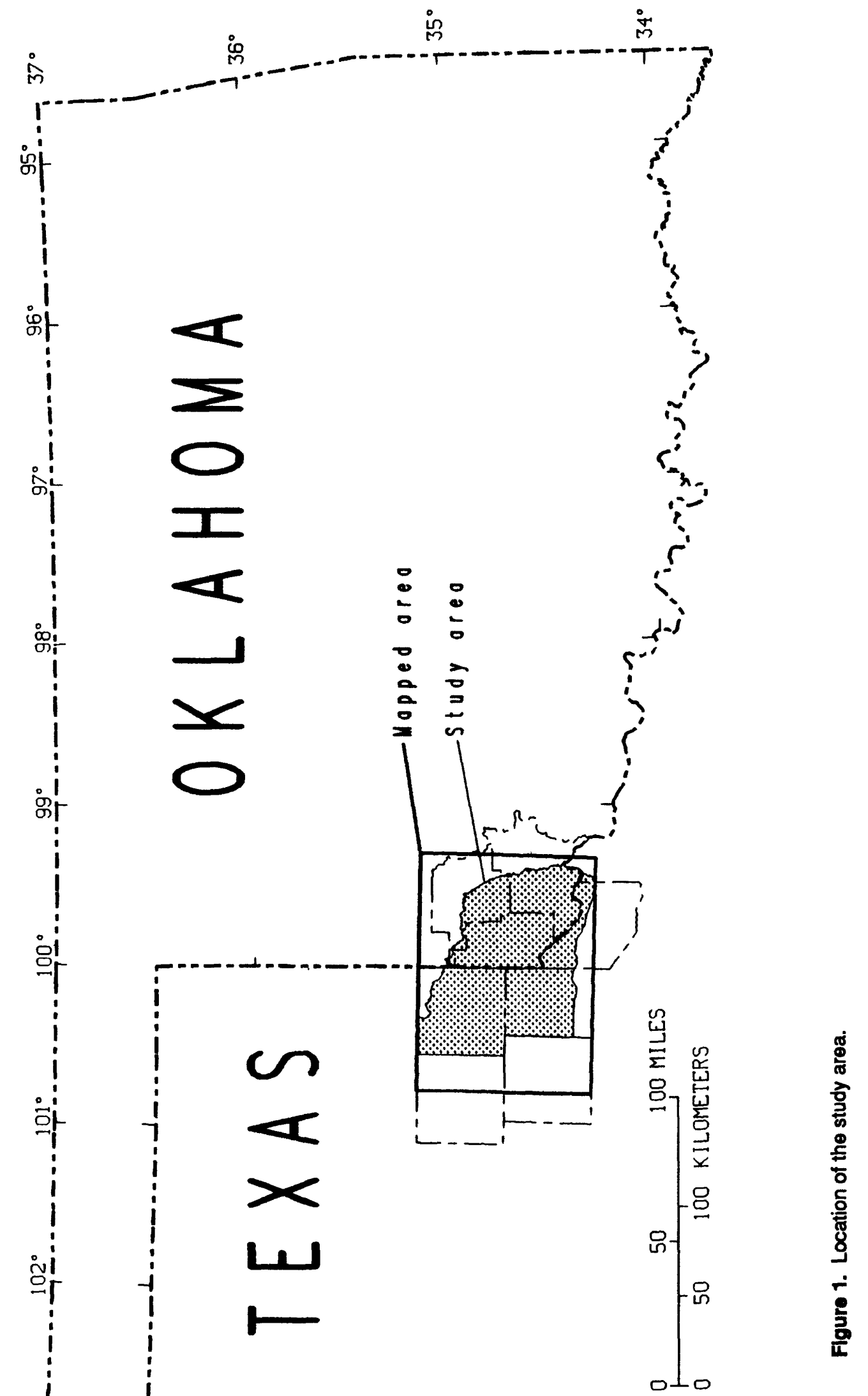




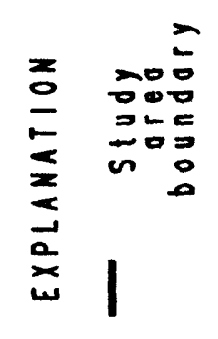

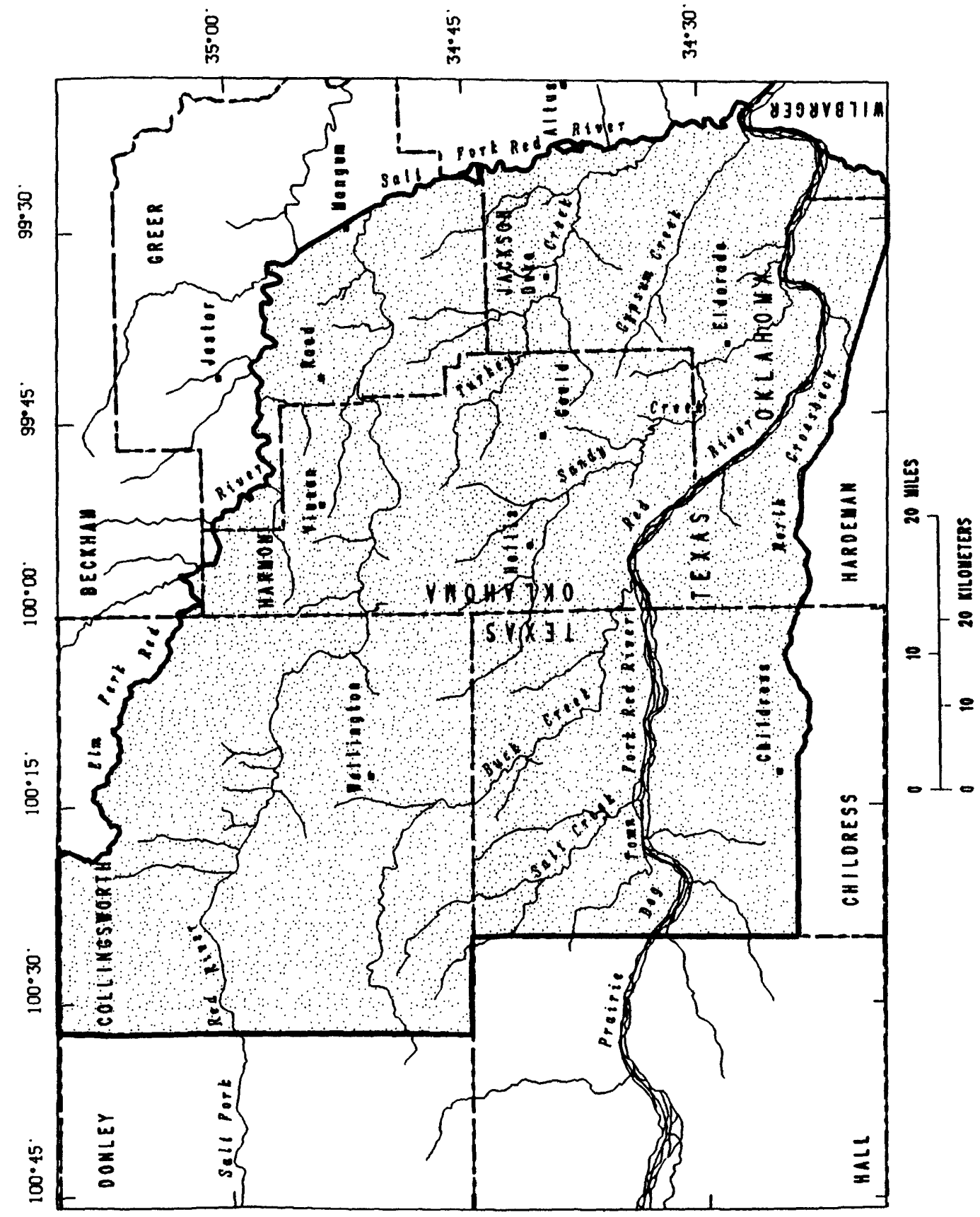

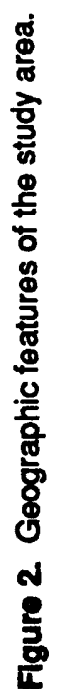


The term Blaine aquifer used in this report refers to the geologic unit, Blaine Gypsum (or Formation) (table 1). The phrase Blaine aquifer and associated units refers to geologic units adjacent to the Blaine aquifer. Other minor aquifers, adjacent to the Blaine aquifer in some areas, include: Red River alluvial aquifer, Salt Fork Red River alluvial aquifer, Red River terrace aquifer, Salt Fork Red River terrace aquifer, Salt Fork Red River terrace and alluvial aquifer and the Whitehorse Group.

\section{Purpose and Scope}

This report is a compilation of the hydrogeologic data collected for the areal ground-water investigation of the Blaine aquifer and associated units in southwestem Oklahoma and northwestem Texas. This report provides a reference for some of the data that was used as input into a computer ground-water model that simulates the ground-water flow in the Blaine aquifer. The results of the ground-water flow model can be found in the report, "Steady-state simulation, Blaine aquifer ground-water flow, southwestern Oklahoma and northwestem Texas" (1995).

\section{Acknowledgments}

The authors would like to extend thanks to the residents and officials of Greer, Harmon, and Jackson counties in Oklahoma and Collingsworth, Childress, Hall, Hardeman, and Wilbarger counties in Texas. Particular recognition is due to Paul Horton for helping arrange contacts for establishing water-quality and water-level networks in the study area. Thanks are due to Charles R. Dyer III and Danny L. Swink, with the Oklahoma Geological Survey, for drilling 23 observation wells and 2 test holes for the project. Thanks also are due to Gary Glover and Dannie Spiser, with the Oklahoma Water Resources Board, for helping process the water samples and measuring water levels in wells. Thanks are extended to the Oklahoma State Department of Health and Oklahoma State Department of Health Radiological Laboratory for analyzing 103 sets of water-quality samples. Also, thanks to the Oklahoma State Department of Transportation, Texas State Department of Transportation, Harmon county commissioners and Jackson county commissioners for allowing the drilling of monitoring wells in the highway and county road rights-of-way.
The authors also would like to acknowledge the assistance of U.S. Geological Survey colleagues, D.L. Adams, L.A. Alf, R.W. Chadd, R.D. Gist, R.L. Goemaat, K.L. Gravitt, G.H. Haff, J.R. Hanlon, J.K. Kurklin, L.D. Mize, L.K. Osbum, L.T. Pham, C.R. Pruitt, D.J. Pruitt, A.R.B. Rooney, and W.B. Simons.

\section{EXPLANATION OF TABLES AND METHODS OF DATA COLLECTION}

The data for this study were collected between 1985 and 1989 but all available water-level data for wells in Oklahoma are listed in tables 2 and 3 and are presented in selected hydrographs. The data in this report consist of: (1) Monthly or periodic water-level measurements in 134 wells; (2) daily mean water-level measurements for 11 wells equipped with water-level recorders; (3) daily total precipitation measurements from five precipitation gages; (4) low-flow stream-discharge measurements for 89 stream sites; (5) miscellaneous stream-discharge measurements at seven stream sites; (6) chemical analyses of surface water from 78 stream sites during low-flow periods; (7) chemical analyses of ground water from 41 ground-water wells; and (8) chemical analyses of runoff water collected at five sites.

The station number is a unique 8- to 15 -digit number for a site. The hydrologic unit code is an 8 digit number for a geographic area representing all or part of a surface-drainage basin of distinct hydrologic features as delineated by the U.S. Geological Survey Office of Water Data Coordination as shown in the State Hydrologic Unit Map of Oklahoma (1976).

\section{Water Levels}

\section{Monthly or Perlodlc Water-level Measurements}

Water levels in 134 wells were measured monthly from May 1986 to March 1988 and are listed in table 2 . Table 2 is organized by state, county, and local well number. The distribution of wells in the monthly water-level network is shown in figure 3. Hydrographs for selected wells in table 2 are shown in figures 4 and 5. Water levels were measured in some wells prior to May 1986. For wells in Oklahoma, all water-level measurements are listed in table 2 . Only water levels measured in wells in Texas from May 
Table 1.--Geologic unit or aquifer names used in this report

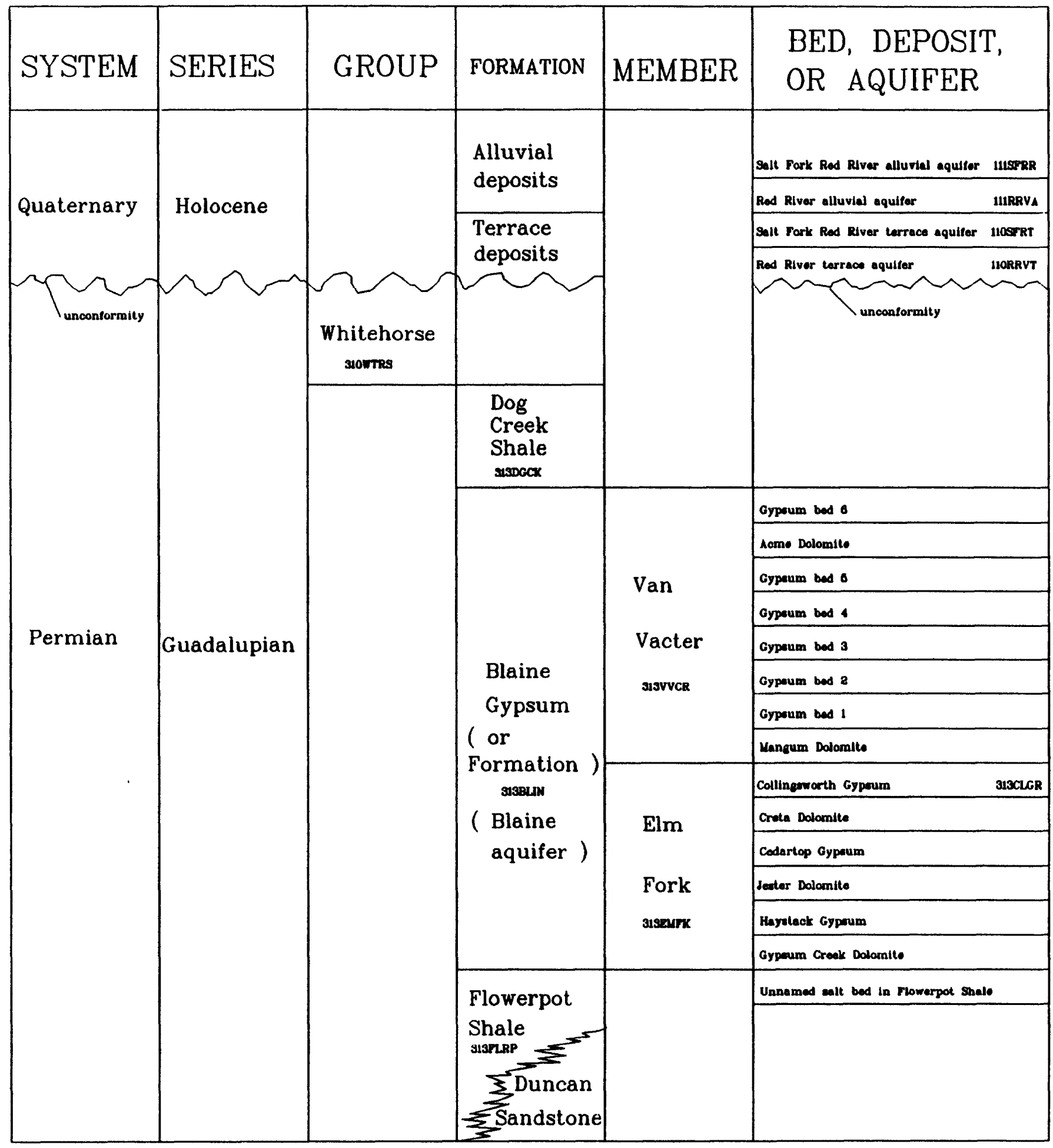


1986 to March 1988 are listed table 2. For a few wells, water levels were measured for a project conducted in the 1950's and then measured for this project in the 1980's.

\section{Dally Mean Water Levels}

Daily mean water levels for 11 wells equipped with digital recorders are listed in table 3 . Table 3 is organized by county and then by local well number. The location of the wells equipped with digital recorders is shown in figure 6. There is a hydrograph (figs. 717) for every recorder well listed in table 3.

\section{Preclpitation Data}

The daily precipitation sums for the five precipitation gages are listed in table 4. The locations of the precipitation gages are shown in figure 18 . The precipitation gages are located adjacent to or within a half mile of five of the recorder wells.

\section{Stream-dlscharge Measurements}

\section{Low-flow Stream-discharge Measurements}

Stream-discharge measurements for 89 stream sites were made during low-flow periods in March 1986, August 1987, and February 1988. Low-flow measurements (also referred to as base-flow measurements or seepage measurements) were made when no precipitation fell within or upstream from the study area for at least 14 days. The low-flow stream-discharge value is a measure of the amount of ground water discharging to the stream. Low-flow discharge measurements are listed in table 5. The locations of the low-flow discharge measurements are shown in figure 19.

\section{Mlscellaneous Stream-dlscharge Measurements}

Miscellaneous stream-discharge measurements for seven sites are listed in table 6 . The locations of the miscellaneous stream-discharge measurement sites are shown in figure 20 .

\section{Water Quallty}

Selected total and dissolved common ion, trace, nutrient, organic or radionuclide chemical analyses, and physical properties of water samples are reported in tables 6, 7a-d, 8a-c, 9a, and 9b. The water samples were analyzed by one or more of the following laboratories: Oklahoma State Department of Health (now Oklahoma Department of Environmental Quality Laboratory), U.S. Geological Survey National Water Quality Laboratory, and Oklahoma State Department of Health Radiological Laboratory (now Oklahoma Department of Environmental Quality Radiochemical Laboratory).

The water samples were collected by either Oklahoma Water Resources Board or U.S. Geological Survey personnel. All stream and runoff samples were collected using a point-sampling method; water was not collected in increments across the stream channel. Ground-water samples from observation wells were collected using a small-diameter squeeze pump. The observation wells were pumped until the water cleared and specific conductance and $\mathrm{pH}$ readings stabilized. Water samples from irrigation, municipal, stock, and domestic wells were collected when specific conductance and $\mathrm{pH}$ readings stabilized after pumping the well sufficiently to evacuate at least 10 well volumes.

Tables 6, 7a-d, 8a-8c, 9a, and 9b have the National Water Information System (NWIS) and Water-Quality Data (QWDATA) parameter (STORET)codes shown in parentheses beneath the chemical constituent. These parameter codes were provided for the project cooperator.

Table 6 lists the field water-quality properties (specific conductance, $\mathrm{pH}$, and air and water temperatures) for the miscellaneous stream-discharge measurements. The locations of the water-quality data collection sites for miscellaneous stream-discharge measurement sites are shown in figure 20. Chemical analyses of water collected from streams during lowflow periods are given in four tables. Table 7a lists the field water-quality properties, common ion, trace, and nutrient chemical analyses, table $7 \mathrm{~b}$ lists the organic chemical analyses, table $7 \mathrm{c}$ lists the radionuclide chemical analyses, and 7d lists the field water-quality properties and bromide and chloride analyses. Data collection sites listed in table 7a are shown in figure 21 , table $7 \mathrm{~b}$ sites are shown in figure 22 , table $7 \mathrm{c}$ sites are shown in figure 23 , and table $7 d$ sites are shown in figure 24. 
Ground-water chemical analyses are listed in three tables. Table 8a lists the field water-quality properties, common ion, trace, and nutrient chemical analyses, table $8 \mathrm{~b}$ lists the organic chemical analyses, and table $8 \mathrm{c}$ lists the radionuclide chemical analyses. Data collection sites listed in table $8 \mathrm{a}$ are shown in figure 25 , table $8 \mathrm{~b}$ sites are shown in figure 26 , and table $8 \mathrm{c}$ sites are shown in figure 27.

Chemical analyses of runoff water are listed in table 9a and 9b. Table 9a lists the field water-quality properties, common ion, trace, and nutrient chemical analyses and table $9 \mathrm{~b}$ lists the radionuclide chemical analyses. Data collection sites listed in table $9 \mathrm{a}$ are shown in figure 28 and table $9 \mathrm{~b}$ sites are shown in figure 29.

Water in the aquifers, except the Salt Fork Red River terrace aquifer, has very large concentrations of dissolved solids, sulfate, and chloride and is unsuitable for human consumption. Drinking-water regulations can be found in the national primary and secondary drinking water regulations (U. S. Environmental Protection Agency, 1989b) and national primary drinking water regulations; radionuclides (U. S. Environmental Protection Agency, 1986).

The following definitions of selected mineral constituents and physical properties are summarized from the U.S. Environmental Protection Agency (1976) and U.S. Public Health Service (1962).

\section{Alkalinity as Calcium Carbonate $\left(\mathrm{CaCO}_{3}\right)$}

Alkalinity is defined as the capacity of a solution to neutralize an acid. In moderate concentrations (200 to 500 milligrams per liter), alkalinity has little effect on most uses of water (Hem, 1985). Bicarbonate $\left(\mathrm{HCO}_{3}{ }^{-}\right)$and carbonate $\left(\mathrm{CO}_{3}{ }^{\circ}\right)$ concentrations were calculated from alkalinity as calcium carbonate $\left(\mathrm{CaCO}_{3}\right)$ concentrations for samples analyzed by the U.S. Geological Survey National Water Quality Laboratory and are shown in table 7a and table 8a. Bicarbonate can be calculated from alkalinity as $\mathrm{CaCO}_{3}$ for samples with a pH between 4.5 and 8.3 by multiplying alkalinity as $\mathrm{CaCO}_{3}$ by 1.22 . The carbonate concentration is assumed to be zero for samples with a pH less than or equal to 8.3.

\section{Cakclum and Magnesium}

Limestone, dolomite, and gypsum are the principal rocks containing calcium and magnesium. Large concentrations in water cause water hardness and form scale. Large concentrations of magnesium in water have a laxative effect (Dunfor and Becker, 1964).

\section{Chioride}

Chloride is dissolved from most rocks. Chloride produces a salty taste in water. Under some conditions it may increase the corrosiveness of water (Dunfor and Becker, 1964).

\section{Dissolved Sollds}

The concentration of dissolved solids is determined from the weight of the dry residue after evaporation from a known quantity of water. Dissolved solids concentrations of 1,000 to 3,000 milligrams per liter are considered slightly saline, 3,000 to 10,000 milligrams per liter are moderately saline, 10,000 to 35,000 are very saline, and more than 35,000 are briny (Hem, 1985).

\section{Fluoride}

Fluoride in ground water is probably derived from solution of fluorite, apatite, and homblende. Optimum concentrations of fluoride are effective in reducing dental carries, especially in adolescents. Excessive concentrations may cause mottling of children's teeth (Dunfor and Becker, 1964).

\section{Hardnese}

Calcium and magnesium are the primary constituents that cause hardness. Hardness is a measure of the soap-consuming properties of water. As hardness increases, a greater amount of soap is required to produce a lather. Water hardness contributes to the formation of scale deposits. The U.S. Geological Survey uses the following classification of water hardness (Dunfor and Becker, 1964).

\begin{tabular}{cc}
\hline $\begin{array}{c}\text { Calclum and megnealum hardnees } \\
\text { as } \mathrm{CaCO}_{3} \text { (milligrams per iticer) }\end{array}$ & $\begin{array}{c}\text { Hardnees } \\
\text { doceription }\end{array}$ \\
\hline $0-60$ & soft \\
$61-120$ & moderately soft \\
$121-180$ & hard \\
more than 180 & very hard \\
\hline
\end{tabular}


Iron

Iron is dissolved from many rocks and soils. The element can cause a reddish-brown stain on plumbing fixtures and fabrics washed in the water and can cause clogging of water mains. The iron criteria are of an esthetic nature (tastes and staining), and do not have a toxicological significance.

\section{Manganese}

Manganese is found in association with salts and iron compounds. The presence of manganese may cause a dark brown or black stain on laundered fabrics or porcelain fixtures. Small concentrations may be objectionable due to taste.

\section{Nitrate}

The occurrence of large nitrate concentrations in shallow ground water has been attributed to leaching in feedlots or to fertilizer from fields where nitrogen compounds have been applied. A large nitrate content is undesirable in drinking water because of its bitter taste. Large nitrate content also is reported to cause methemoglobinemia in infants (Dunfor and Becker, 1964).

\section{pH}

$\mathrm{pH}$ is a mathematical expression indicating hydrogen ion activity. A pH of 7.0 is neutral, $\mathrm{pH}$ less than 7.0 is acidic, and $\mathrm{pH}$ greater than 7.0 is basic or alkaline. The hydrogen ion concentrations affect the corrosiveness of water (Hem, 1985).

\section{Potasslum and Sodlum}

Potassium and sodium are dissolved from igneous and sedimentary rocks. Potassium generally exists in much smaller concentrations than sodium. Mineralized waters containing a large percentage of sodium salts may be unsatisfactory for irrigation. Low-sodium diets are prescribed for certain types of ailments.

\section{Radionuclides}

Radionuclides in drinking water are suspected of increasing the risk of various forms of cancer. Radioactivity is monitored through a screening pro- cess. When gross alpha activity exceeds 5 picocuries per liter the sample is analyzed for radium-226; if the concentration of radium-226 exceeds 3 picocuries per liter, the sample is analyzed for radium-228. The combined radium-226 and radium-228 should not exceed 5 picocuries per liter. Maximum contaminant levels for gross beta are defined in terms of the annual dose rate (millirem per year) from continuous ingestion. The dose rate is calculated on the basis of 2-liter daily intake. Gross beta as Cesium 137 (Cs 137) should not exceed 200 picocuries per liter.

\section{Sillca}

Silica is dissolved from practically all rocks and contributes to the formation of scale in pipes, water heaters, and boilers.

\section{Sodlum-Adsorption Ratio (SAR)}

The sodium-adsorption ratio (SAR) is a measure of the relative concentrations of calcium, magnesium, and sodium ions. SAR is expressed by the equation:

$$
S A R=\sqrt{\frac{\mathrm{Na}^{+}}{\frac{\mathrm{Ca}^{++}+\mathrm{Mg}^{++}}{2}}}
$$

Sodium-adsorption ratio was introduced by the U.S. Salinity Laboratory Staff (1954) and divides water into 16 classes, depending on the SAR and specific conductance. The classifications indicate the usefulness of water for irrigation of various crops grown in different soil types.

\section{Specific Conductance}

Specific conductance is a measure of the ability of water to conduct an electric current. By multiplying specific conductance by a conversion factor of 0.55 to 0.75 , an estimate of total dissolved solids can be approximated (Hem, 1985).

\section{Sulfate}

Sulfate combined with calcium can form scale. Water containing about $\mathbf{5 0 0}$ milligrams per liter of sulfate tastes bitter. Large concentrations of sulfate have a laxative effect (Dunfor and Becker, 1964). 


\section{Temperature}

Water temperature is evaluated for industrial cooling and its effect on the concentrations of dissolved gases and minerals.

\section{SELECTED REFERENCES}

Brown, Eugene, Skougstad, M.W., and Fishman, J.J., 1970, Methods for collection and analysis of water samples for dissolved minerals, and gasses: Techniques of Water-Resources Investigations of the U.S. Geological Survey, book 5, chapter A1, 160 p.

Dunfor, N.C., and Becker, Edith, 1964, Public water supplies of the 100 largest cities in the United States, 1962: U.S. Geological Survey Water-Supply Paper 1812, 364 p.

Havens, J.S., 1977, Reconnaissance of the water resources of the Lawton Quadrangle, southwestem Oklahoma: Oklahoma Geological Survey Hydrologic Atlas 6, 4 sheets, scale 1:250,000.

Hem, J.D., 1985, Study and interpretation of the chemical characteristics of natural water: U.S. Geological Survey Water-Supply Paper 2254, 3d ed., 263 p.

Johnson, K.S., 1967, Stratigraphy of the Blaine Formation and associated strata in southwestern Oklahoma: Urbana, Illinois, University of Illinois, unpublished Ph.D. dissertation, 247 p.

Johnson, K.S., 1986, Hydrogeology and recharge of a gypsum-dolomite karst aquifer in southwestem Oklahoma, U.S.A.: International Symposium of Karst Water Resources, 1985, Ankara, Turkey, International Association of Hydrological Sciences Publication No. 161, p. 343-357.

National Academy of Sciences-National Academy of Enginecring, 1972: U.S. Environmental Protection Agency, Ecological Research Series, Report EPA R3-073-033, March 1973, 594 p.

Oklahoma Water Resources Board, 1966, Ground water in Harmon, Jackson, Greer, and Beckham Counties, Oklahoma: Oklahoma Water Resources Board Publication No. 13, 21 p.

Pettyjohn, W.A., White, Hal, and Dunn, Shari, 1983, Water atlas of Oklahoma: Stillwater, Oklahoma, University Center for Water Research, Oklahoma State University, $72 \mathrm{p}$.

Runkle, D.L., and Johnson, K.S., 1988, Hydrologic study of a gypsum-dolomite karst aquifer in southwestern Oklahoma and adjacent parts of Texas, U.S.A.: Proceedings of the International Association of Hydrogeologists 21st Congress, Karst hydrogeology and karst environment protection, Volume 21, Part 1, p. 400-405.

Runkle, D.L., and McLean, J.S., 1995, Steady-state simulation, Blaine aquifer ground-water flow, southwestern Oklahoma and northwestern Texas: U.S. Geological Survey Open-file Report 94-387, 92 p.

Schoff, S.L., 1948, Ground-water irrigation in the Duke area, Jackson and Greer Counties, Oklahoma: Oklahoma Geological Survey Mineral Report 18, 8 p.

Steele, C.E., and Barclay, J.E., 1965, Ground-water resources of Harmon County and adjacent parts of Greer and Jackson Counties, Oklahoma: Oklahoma Water Resources Board Bulletin 29,96 p.

U.S. Army Corps of Engineers, Arkansas-White-Red Office, 1954, Drainage Area Data, Arkansas, White, and Red River Basins: U.S. Army Corps of Engineers Drainage Area Pamphlet, 221 p.

U.S. Environmental Protection Agency, 1974a, Manual of Methods for Chemical Analysis of Water and Wastes, EPA-625-15-003, Methods Development and Quality Assurance Research Laboratory, National Environmental Research Center, Cincinnati, Ohio, 298 p.

1976, Quality criteria for water: Washington, U.S. Government Printing Office, 256 p.

1986, Advance notice of proposed rule making, National primary drinking water regulations; radionuclides (section 141.50 of part 141) U. S. Federal Register, v. 51, no. 189, September 30, 1986, p. 34, 836-34, 862.

1989b, Proposed rule, National primary and secondary drinking water regulations; (sections 141.50, 141.51, 141.61, and 141.62 or part 141 and 143.3 of part 143) U. S. Federal Register, v. 54, no. 97, May 22, 1989, p.22, 062-22, 160.

U.S. Geological Survey, 1976, Hydrologic Unit Map--1974 State of Oklahoma: U.S. Geological Survey Hydrologic Unit map, 1 sheet, scale 1:500,000.

U.S. Public Health Service, 1962, Public Health Service drinking water standards, 1962: U.S. Public Health Service Publication 956, 61 p.

U.S. Salinity Laboratory Staff, 1954, Diagnosis and improvement of saline and alkali soils: U.S. Department of Agriculture, Agriculture Handbook no. 60, 160 p. 
APPENDICES 


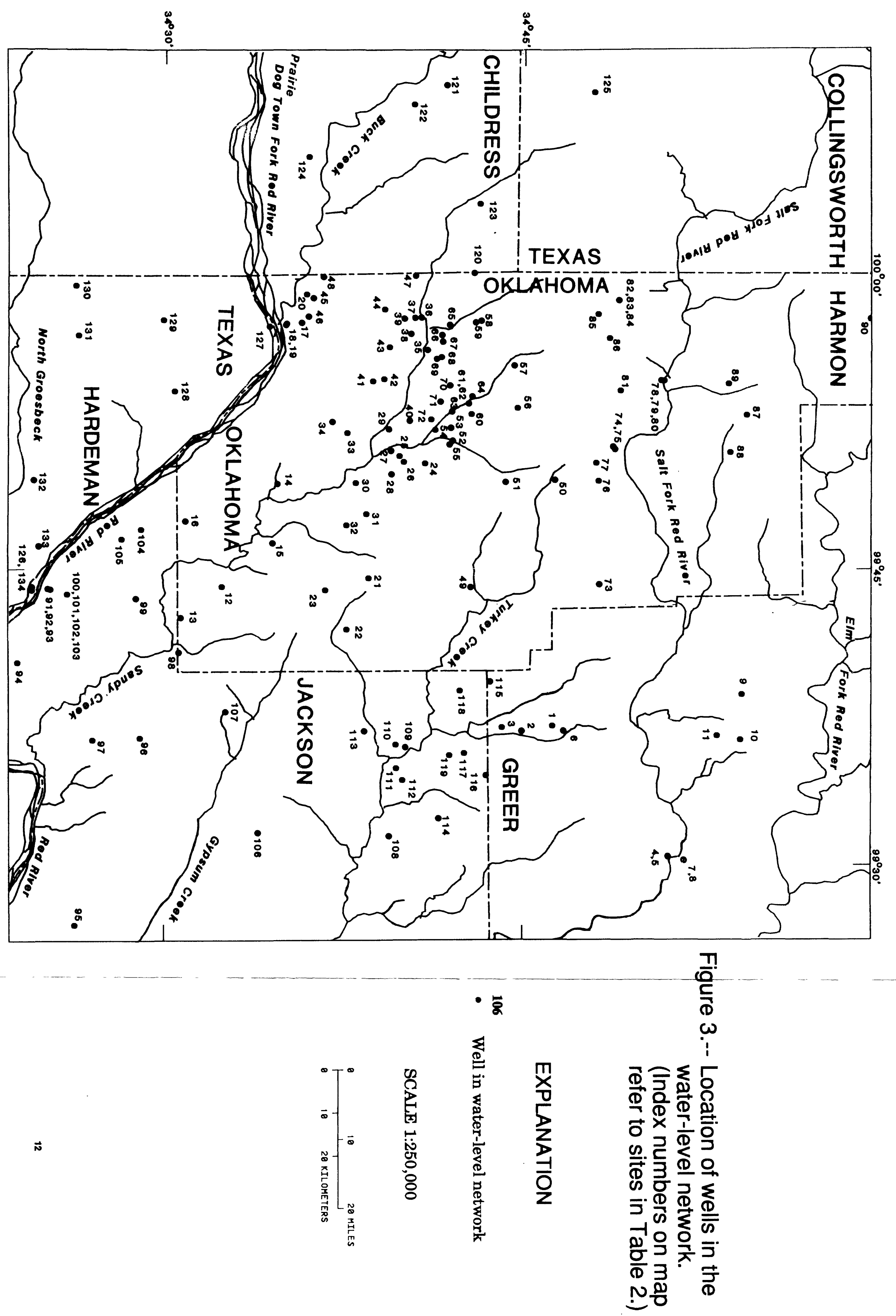


Table 2.-- Nonthly or periodic water-level measurements in selected wells in southwestern Oklahoma and northwestern Texas

[Water levels are in feet below land-surface datum; water levels above land-surface datum are indicated by "+"; index number on location map refers to the number adjacent to the well symbol on figure 6; 116RRVT, Red River terrace aquifer; 110SFRT, Salt Fork Red River terrace aquifer; 110SFTA, Salt Fork Red River terrace and alluvial aquifer; 111RRVA, Red River alluvial aquifer; 111SFRR, Salt Fork Red River alluvial aquifer; 310WTRS, Whitehorse Group; 313BLIN, Blaine Gypsum (or Formation); 313CLGR, Collingsworth Gypsum Bed of the Elm Fork Member; 313DGCK, Dog Creek Shale; 313EMFK, Elm Fork Member of Blaine Gypsum (or Formation); 313FLRP, Flowerpot Shale; 313VVCR, Van Vacter Member of Blaine Gypsum (or Formation); MS, method of measurement and status of site; 0 , obstruction; $P$, well pumping; $R$, well recently pumped; $S$, nearby well pumping; $X$, affected by surface-water site; $Z$, other]

GREER COUNTY, OKLAHOMA

Index number on location map: 1

Station number: 844602099371601 Local number: $63 \mathrm{~N}-23 W-04$ ABBD 1

Location: Lat $34^{\circ} 46^{\prime} 62^{\prime \prime}$, long $99^{\circ} 37^{\prime} 16^{\prime \prime}$, hydrologic unit 11126202

Aquifer: 313BLIN

Altitude of land-surface datum: $1,530 \mathrm{ft}$

Well depth: $20.6 \mathrm{ft}$

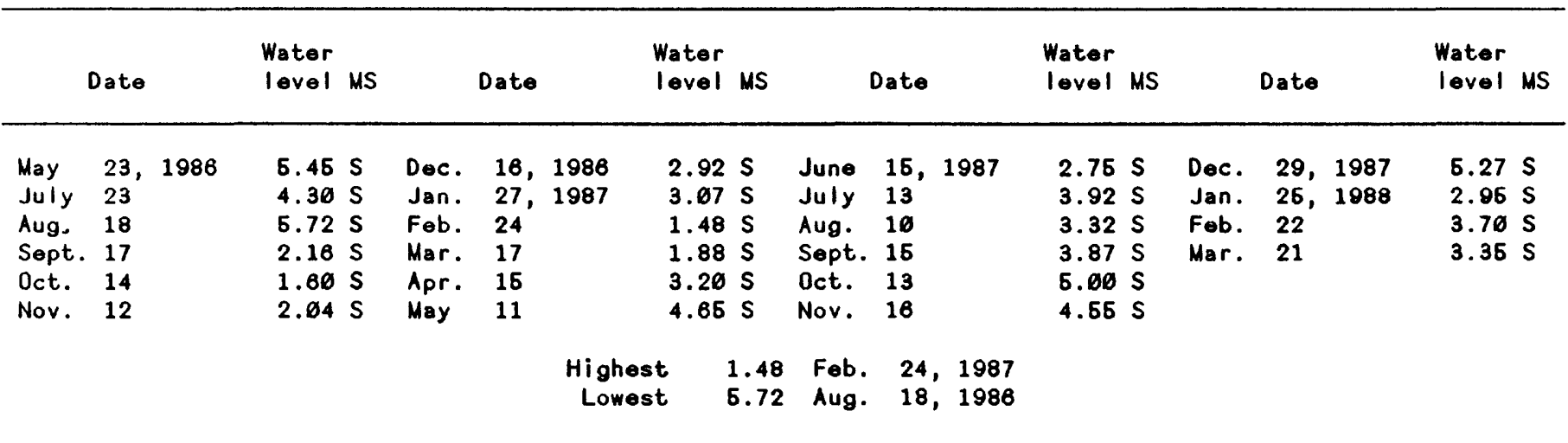

Index number on location map: 2

Station number: 344446099370001 Local number: $63 N-23 W-09$ DAB 1

Location: Lat $34^{\circ} 44^{\prime} 45^{\prime \prime}$, long $99^{\circ} 37^{\prime} 00^{\prime \prime}$, hydrologic unit 11120202

Aquifer: 313BLIN

Altitude of land-surface datum: $1,479.5 \mathrm{ft}$

Well depth: $125 \mathrm{ft}$

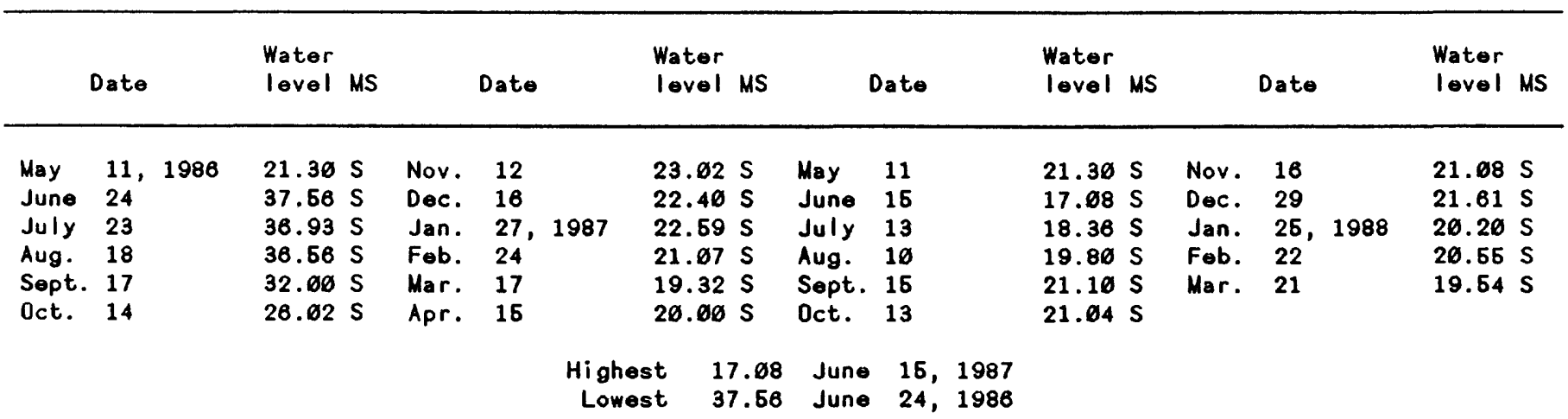


Table 2.-- Monthly or periodic water-level measurements in selected wells in southwestern Oklahoma and northwestern Texas--Continued

GREER COUNTY, OKLAHOMA--CONtinued

Index number on location map: 3

Station number: 344355099371201 Local number: $03 \mathrm{~N}-23 W-16$ DBAA 1

Location: Lat $34^{\circ} 43^{\prime} 65^{\prime \prime}$, long $99^{\circ} 37^{\prime} 12^{\prime \prime}$, hydrologic unit 11120202

Aquifer: 3138 LIN

Altitude of land-surface datum: $1,467 \mathrm{ft}$

Well depth: $95 \mathrm{ft}$

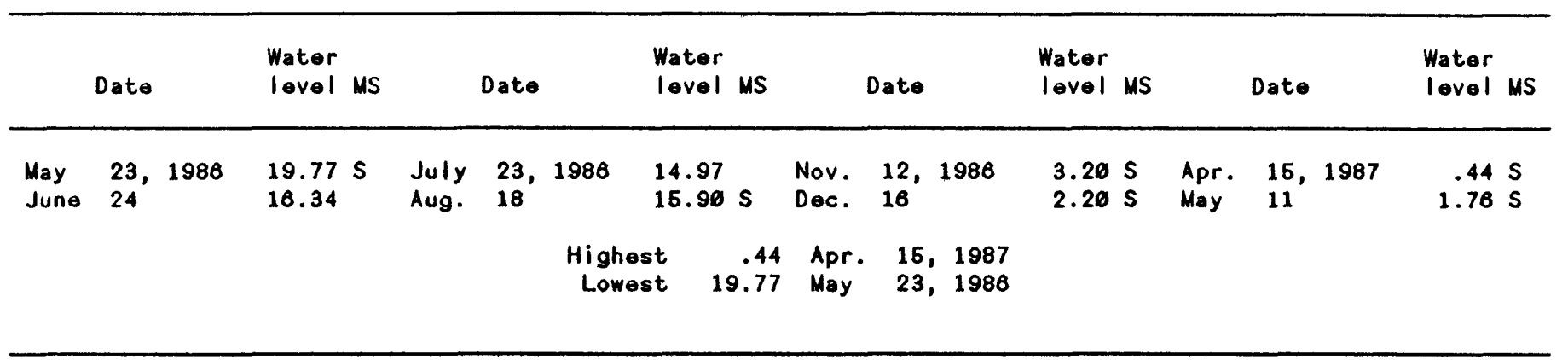

Index number on location map: 4

Station number: 845124099303901 Local number: O4N-22W-64 AAAA 1

Location: Lat $34^{\circ} 51^{\prime} 24^{\prime \prime}$, long $99^{\circ} 3 \sigma^{\prime} 39^{\prime \prime}$, hydrologic unit 11120202

Aquifer: 111SFRR

Altitude of land-surface datum: $1,507 \mathrm{ft}$

Well depth: $53 \mathrm{ft}$

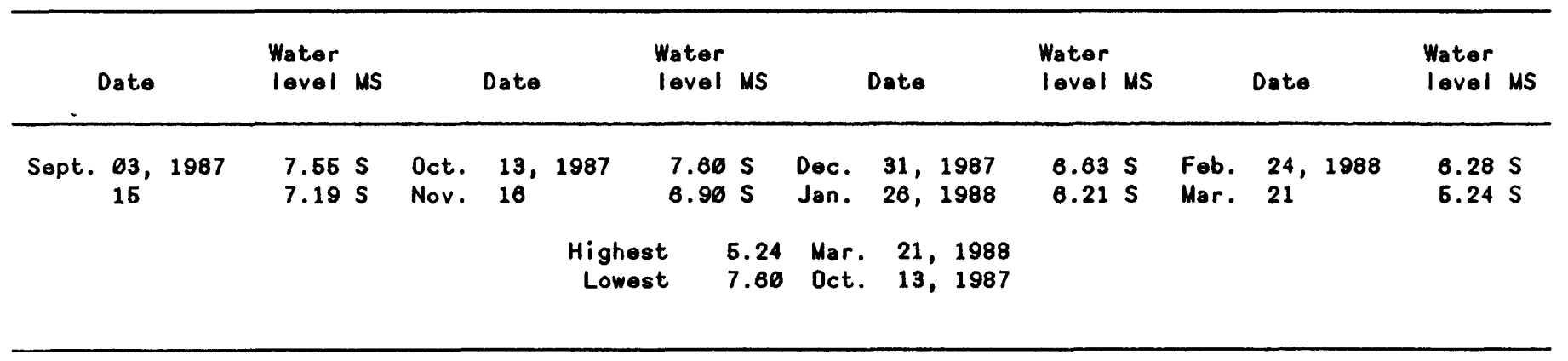

Index number on location map: 6

Station number: 846123099304101 Local number: O4N-22W-04 AAAA 2

Location: Lat $34^{\circ} 51^{\prime} 23^{\prime}$, Iong $99^{\circ} 30^{\prime} 41^{*}$, hydrologic unit 11120202

Aquifar: 111SFRR

Altitude of land-surface datum: $1,509 \mathrm{ft}$

Well depth: $68 \mathrm{ft}$

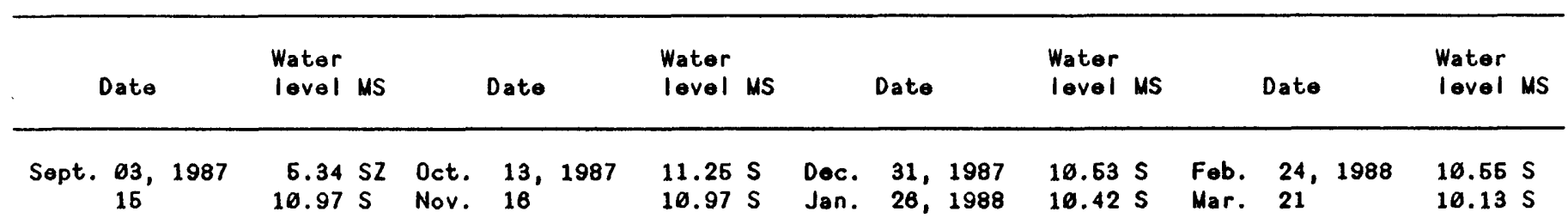

Highest 6.34 Sept. 63, 1987

Lowest 11.26 Oct. 13, 1987 
Table 2.-- Monthly or periodic water-level measurements in selected wells in southwestern Oklahoma and northwestern Texas--Cont inued

GREER COUNTY, OKLAHOMA--Continued

Index number on location map: 6

Station number: 344630699370201 Local number: $4 \mathrm{~N}-23 W-33$ DAB 1

Location: Lat $34^{\circ} 46^{\prime} 36^{\prime \prime}$, long $99^{\circ} 37^{\prime} 62^{\prime \prime}$, hydrologic unit 11120202

Aquifer: $313 V V C R$

Altitude of land-surface datum: $1,650 \mathrm{ft}$

Well depth: $155 \mathrm{ft}$

\begin{tabular}{|c|c|c|c|c|c|c|c|c|c|c|c|c|c|c|c|c|}
\hline \multicolumn{3}{|c|}{ Date } & $\begin{array}{l}\text { Water } \\
\text { level MS }\end{array}$ & \multicolumn{3}{|c|}{ Date } & $\begin{array}{l}\text { Water } \\
\text { level MS }\end{array}$ & \multicolumn{3}{|c|}{ Date } & $\begin{array}{l}\text { Water } \\
\text { level US }\end{array}$ & \multicolumn{3}{|c|}{ Date } & $\begin{array}{l}\text { Water } \\
\text { level }\end{array}$ & MS \\
\hline Apr. & 18 & 1949 & 31.57 & Mar. & 25 , & 1958 & 34.35 & Oct. & 03 & 1962 & 44.40 & Dec. & 10 & 1968 & 44.93 & \\
\hline oct. & $21^{\circ}$ & & 30.93 & Apr. & 21 & & 34.00 & & 16 & & 41.58 & Jan. & 62 , & 1989 & 44.00 & \\
\hline Nov. & 15 & 1950 & 25.43 & May & 20 & & 34.01 & Nor. & 13 & & 38.30 & Fob. & $04^{\circ}$ & & 43.33 & \\
\hline Apr. & 25 & 1951 & 30.61 & June & 24 & & 36.62 & Jan. & 68 & 1963 & 36.03 & Mar. & 11 & & 42.74 & \\
\hline Fob. & 01 & 1952 & 30.88 & July & 29 & & 36.43 & Fob. & $65^{\circ}$ & & 38.19 & Apr. & 69 & & 42.01 & \\
\hline Nov. & 19 & 1953 & 42.62 & Sept. & 23 & & 36.40 & Mar. & 26 & & 35.90 & May & 08 & & 41.27 & \\
\hline Jan. & 26 & 1954 & 42.48 & oct. & 27 & & 36.95 & Apr. & 23 & & 38.00 & June & 18 & & 38.64 & \\
\hline Apr. & 21 & & 48.27 & Nov. & 18 & & 36.24 & May & 20 & & 36.24 & Sept. & 04 & & 47.24 & \\
\hline May & 28 & & 43.62 & Jan. & 09, & 1959 & 48.70 & June & 18 & & 34.95 & Oct. & 07 & & 43.16 & \\
\hline June & 23 & & 42.89 & & 26 & & 40.04 & Nov. & 22 & & 46.11 & Nov. & 18 & & 40.30 & \\
\hline Sept. & 22 & & 61.27 & Fab. & 24 & & 37.90 & Feb. & 10 & 1984 & 43.19 & Dec. & 11 & & 39.82 & \\
\hline Oct. & 26 & & 48.87 & Apr. & 21 & & 42.65 & Jan. & 12 & 1965 & 49.45 & Jan. & 28 & $197 \varnothing$ & 39.50 & \\
\hline Dec. & $D_{1}$ & & 47.55 & May & 19 & & 39.64 & Feb. & 17 & & 48.34 & Fab. & 24 & & 39.88 & \\
\hline Jan. & $\begin{array}{l}\varnothing 3, \\
24\end{array}$ & 1955 & 46.97 & June & $\begin{array}{l}30 \\
22\end{array}$ & & $\begin{array}{l}37.48 \\
47.30\end{array}$ & Apr. & $\begin{array}{l}02 \\
85\end{array}$ & & $\begin{array}{l}48.27 \\
48.24\end{array}$ & Dec. & 29 & 1975 & 52.11 & \\
\hline Feb. & $\varnothing 9$ & & 46.87 & oct. & 20 & & 43.70 & May & 25 & & 49.22 & Jan. & 28, & 1976 & 21.50 & \\
\hline & 22 & & 46.74 & Nov. & 19 & & 42.09 & July & 29 & & 74.11 & Dec. & 67 & & 26.12 & \\
\hline Mar. & 22 & & 51.53 & Dec. & 22 & & 41.40 & Sept. & 29 & & 68.88 & Mar. & 09 , & 1978 & $27.6 \varnothing$ & \\
\hline Apr. & 19 & & 57.60 & Jan. & 16 & 1980 & 40.65 & 0ct. & 12 & & 63.11 & Mar. & 07 & 1979 & 30.68 & \\
\hline May & 26 & & 52.04 & Feb. & 22 & & $39.0 D$ & Nor. & 05 & & 54.25 & Feb. & 28 & 1980 & 37.22 & \\
\hline June & 24 & & 50.12 & Mar. & 21 & & 38.47 & Jan. & 63 & 1966 & 46.46 & Fob. & 19 & 1982 & 33.48 & $s$ \\
\hline Aug. & 24 & & 59.14 & Apr. & 19 & & 39.89 & Apr. & 19 & & 44.18 & Jan. & 26 & 1983 & 38.70 & $S$ \\
\hline Sept. & 28 & & 56.76 & May & 17 & & 37.04 & June & 20 & & 43.79 & Fob. & 02 , & 1984 & 33.39 & $\mathbf{S}$ \\
\hline $0 c t$. & 26 & & 49.10 & June & 21 & & 37.67 & July & 12 & & 65.43 & Jen. & 10 & 1985 & 37.66 & $\mathrm{~s}$ \\
\hline Nov. & 29 & & 46.08 & July & 19 & & 36.05 & Sept. & 13 & & 51.73 & Jan. & 87, & 1986 & 27.72 & $\mathbf{S}$ \\
\hline Dec. & 21 & & 45.05 & Sept. & 13 & & 37.82 & Oct. & 19 & & 46.88 & May & 16 & & 27.48 & \\
\hline Jan. & 31 , & 1956 & 44.22 & Oct. & 24 & & 33.40 & Nov. & 16 & & 43.98 & June & 24 & & 27.76 & \\
\hline Feb. & 21 & & 45.13 & Nov. & 14 & & 31.62 & Dec. & 13 & & 43.36 & July & 23 & & 29.11 & \\
\hline Apr. & 24 & & 45.40 & Dec. & 21 & & 30.64 & Jan. & 17 & 1967 & 43.77 & Sept. & 17 & & 28.06 & \\
\hline May & 22 & & 51.32 & Jan. & 16 & 1961 & 29.90 & Feb. & 16 & & 42.73 & oct. & 14 & & 23.08 & \\
\hline July & 24 & & 52.77 & Feb. & 13 & & 29.60 & Apr. & 13 & & 48.26 & Nov. & 12 & & 18.35 & $s$ \\
\hline Sept. & 18 & & 49.80 & Mar. & 14 & & 29.40 & May & 16 & & 49.73 & Dec. & 16 & & 18.72 & 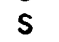 \\
\hline $0 c t$ & 30 & & 54.00 & Apr. & 19 & & 29.20 & June & 15 & & 56.81 & Jan. & 27 , & 1987 & 17.38 & $\mathbf{S}$ \\
\hline Nov. & 27 & & 51.21 & May & 17 & & 30.71 & Sept. & 13 & & 61.46 & Feb. & 24 & & 17.46 & $\mathrm{~s}$ \\
\hline Dec. & 18 & & 50.28 & June & 21 & & 28.29 & $0 \mathrm{ct}$. & 11 & & 54.35 & Mar. & 17 & & 16.38 & $s$ \\
\hline Jan. & 22 & 1957 & 49.16 & July & 12 & & 34.94 & Nov. & 16 & & 51.60 & Apr. & 15 & & 16.50 & 5 \\
\hline Fob. & 28 & & 49.11 & Sept. & 96 & & 36.76 & Dec. & 12 & & 60.84 & May & 11 & & 16.98 & $\mathbf{S}$ \\
\hline Apr. & $\varnothing 4$ & & 48.35 & & 26 & & 34.19 & Jan. & $3 \varnothing$ & 1968 & 49.66 & June & 15 & & 14.75 & $\mathbf{s}$ \\
\hline May & 07 & & 45.53 & Oct. & 24 & & 33.16 & Fob. & 26 & & 48.60 & July & 13 & & 15.32 & $\mathrm{~s}$ \\
\hline & 28 & & 40.89 & Nov. & 14 & & 32.65 & Mar. & 26 & & 47.91 & Sept. & 16 & & 18.73 & 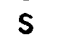 \\
\hline June & 25 & & 35.15 & Dec. & 06 & & 32.01 & Apr. & 26 & & 47.40 & $0 c t$. & 13 & & 17.60 & $s$ \\
\hline Oct. & 01 & & 39.66 & Jan. & 31 & 1962 & 31.93 & June & ø5 & & 46.20 & Nor. & 16 & & 17.10 & $s$ \\
\hline & 29 & & 35.86 & Mar. & 67 & & 31.98 & & 19 & & 44.18 & Dec. & 29 & & 17.35 & $s$ \\
\hline Nov. & 19 & & 34.63 & & 28 & & 40.61 & July & 17 & & 47.88 & Jan. & 25 , & 1988 & 16.95 & $S$ \\
\hline Dec. & 17 & & 34.05 & Apr. & 25 & & 41.25 & Sept. & 19 & & 62.07 & Feb. & 22 & & 16.82 & $\mathbf{s}$ \\
\hline $\begin{array}{l}\text { Jan. } \\
\text { Feb. }\end{array}$ & $\begin{array}{l}28, \\
25\end{array}$ & 1958 & $\begin{array}{l}33.89 \\
33.81\end{array}$ & $\begin{array}{l}\text { May } \\
\text { June }\end{array}$ & $\begin{array}{l}23 \\
27\end{array}$ & & $\begin{array}{l}39.68 \\
35.36\end{array}$ & $\begin{array}{l}\text { Oct. } \\
\text { Nor. }\end{array}$ & $\begin{array}{l}10 \\
14\end{array}$ & & $\begin{array}{l}51.88 \\
51.40\end{array}$ & Mar. & 21 & & 16.55 & $S$ \\
\hline
\end{tabular}


Table 2.--Monthly or periodic water-level measurements in selected wells in southwestern Oklahoma and northwestern Texas--Continued

GREER COUNTY, OKLAHOMA--CONtinUed

Index number on location map: 7

Station number: 346134699362961 Local number: $65 \mathrm{~N}-22 W-34$ DCAB 1

Location: Lat $34^{\circ} 51^{\prime} 34^{\prime \prime}$, long $99^{\circ} 3 \sigma^{\prime} 29^{\prime \prime}$, hydrologic unit 11126202

Aquifer: 111SFRR

Altitude of land-surface datum: $1,506 \mathrm{ft}$

Well depth: $40 \mathrm{ft}$

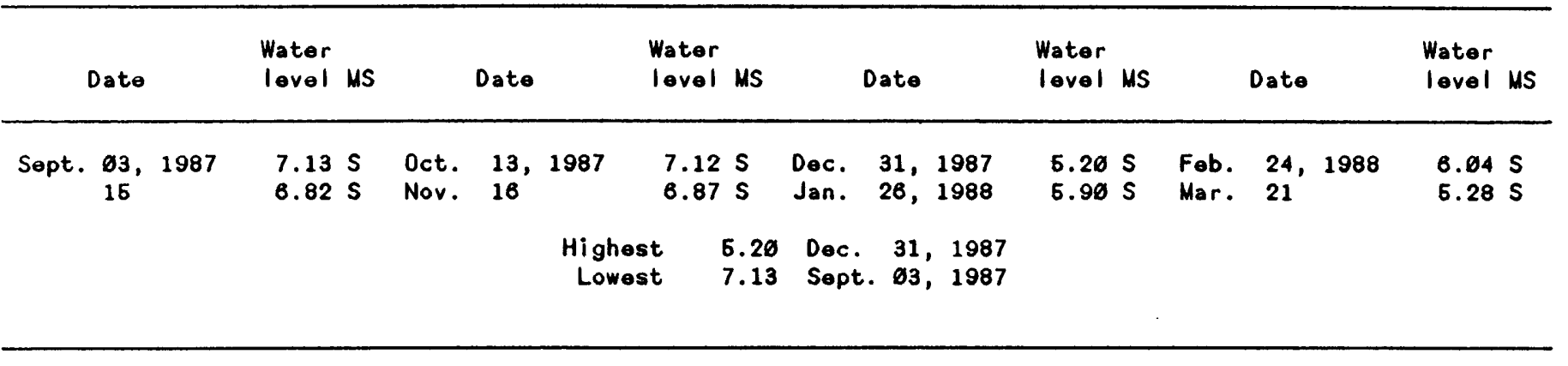

Index number on location map: 8

Station number: $\$ 46132099303001$ Local number: $65 \mathrm{~N}-22 W-34$ DCAC 1

Location: Lat $34^{\circ} 61^{\prime} 32^{\prime \prime}$, long $99^{\circ} 36^{\prime} 39^{\prime \prime}$, hydrologic unit 11120202

Aquifer: 111SFRR

Altitude of land-surface datum: $1604 \mathrm{ft}$

Woll depth: $44 \mathrm{ft}$

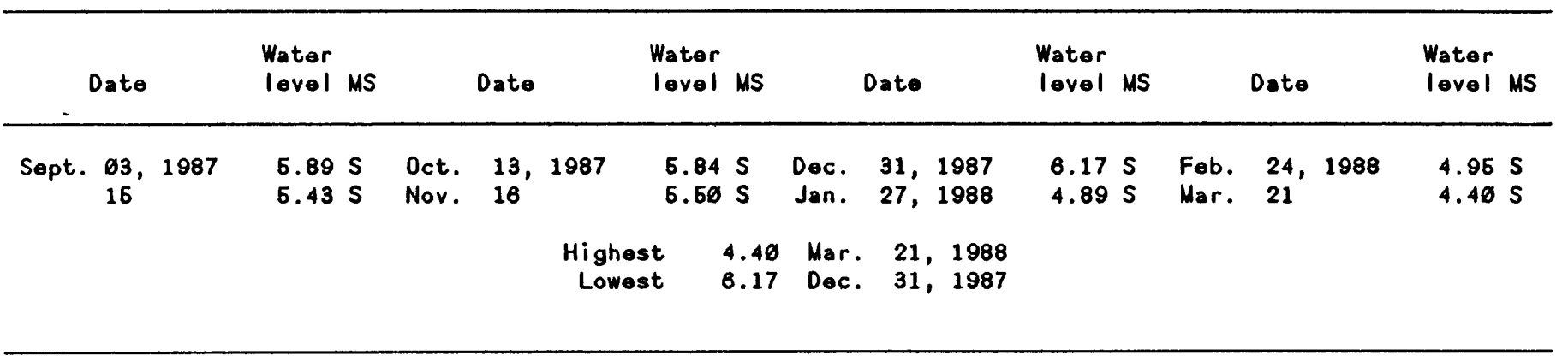

Index number on location map: 9

Station number: $\$ 46368699384601$ Local number: $65 \mathrm{~N}-23 W-2 \varnothing$ ABA 1

Location: Lat $34^{\circ} 63^{\prime} 66^{\prime \prime}$, long $99^{\circ} 38^{\prime} 63^{\prime \prime}$, hydrologic unit 11128282

Aquifer: 313BLIN

Altitude of land-surface datum: $1,735 \mathrm{ft}$

Well depth: $80.6 \mathrm{ft}$

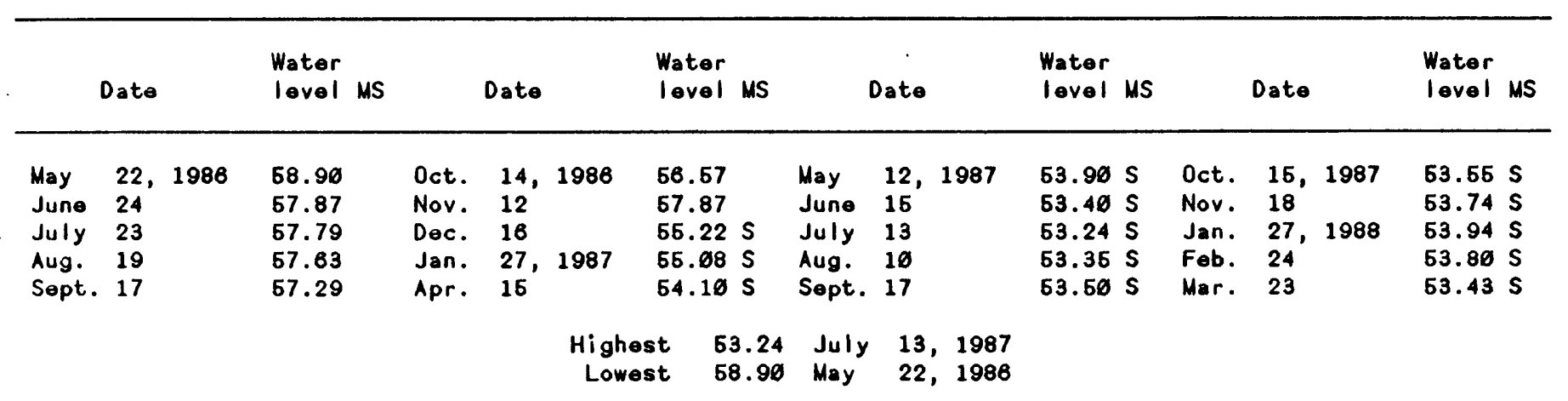


Table 2.--Monthly or periodic water-level measurements in selected wells in southwestern Oklahoma and northwestern Texas--Continued

GREER COUNTY, OKLAHOMA--CONtinuEd

Index number on location map: 10

Station number: 346351099362601 Local number: $65 N-23 W-22$ AADB 1

Location: Lat $34^{\circ} 63^{\prime} 63^{\prime \prime}$, long $99^{\circ} 38^{\prime} 36^{\prime \prime}$, hydrologic unit 11120202

Aquifer: 313BLIN

Altitude of land-surface datum: $1,763.7 \mathrm{ft}$

Well depth: $136 \mathrm{ft}$

\begin{tabular}{|c|c|c|c|c|c|c|c|c|c|c|c|c|c|c|c|c|c|c|c|}
\hline & Date & & $\begin{array}{l}\text { Water } \\
\text { level }\end{array}$ & MS & & Date & & $\begin{array}{l}\text { Water } \\
\text { level }\end{array}$ & MS & & Date & & $\begin{array}{l}\text { Water } \\
\text { lovel }\end{array}$ & MS & & Date & & $\begin{array}{l}\text { Water } \\
\text { level }\end{array}$ & MS \\
\hline $\begin{array}{l}\text { Apr. } \\
\text { Apr. } \\
\text { Nov. } \\
\text { Apr. } \\
\text { Fob. } \\
\text { Mar. } \\
\text { Nov. } \\
\text { Jan. } \\
\text { Mar. } \\
\text { May } \\
\text { June } \\
\text { July } \\
\text { Aug. } \\
\text { Oct. } \\
\text { Dec. } \\
\text { Jan. }\end{array}$ & $\begin{array}{l}16, \\
68, \\
16 \\
15 \\
25, \\
01, \\
21 \\
19, \\
26, \\
25 \\
27 \\
23 \\
29 \\
25 \\
26 \\
02 \\
04,\end{array}$ & $\begin{array}{l}1949 \\
1950 \\
1961 \\
1952 \\
1963 \\
1954\end{array}$ & $\begin{array}{r}91.76 \\
92.80 \\
89.23 \\
89.23 \\
91.78 \\
91.49 \\
91.93 \\
92.13 \\
92.97 \\
95.29 \\
94.40 \\
92.90 \\
98.27 \\
100 . \varnothing 5 \\
96.82 \\
96.4 \varnothing \\
99.71\end{array}$ & $\begin{array}{l}S \\
S \\
S \\
S \\
S \\
S \\
S \\
S \\
S \\
S \\
S \\
S \\
S \\
S \\
S \\
S \\
S\end{array}$ & $\begin{array}{l}\text { Jan. } \\
\text { Fob. } \\
\text { Mar. } \\
\text { Apr. } \\
\text { June } \\
\text { July } \\
\text { Sopt. } \\
\text { Oct. } \\
\text { Nov. } \\
\text { Doc. } \\
\text { Jan. } \\
\text { Jan. } \\
\text { Fob. } \\
\text { Jan. } \\
\text { July } \\
\text { Apr. }\end{array}$ & $\begin{array}{l}25, \\
09 \\
23 \\
23 \\
25 \\
22 \\
27 \\
29 \\
24 \\
29 \\
22 \\
31, \\
22, \\
25, \\
09, \\
19, \\
19,\end{array}$ & $\begin{array}{l}1966 \\
\\
\\
\\
\\
\\
1956 \\
1967 \\
1958 \\
1959 \\
1980 \\
1981\end{array}$ & $\begin{array}{r}103.74 \\
98.86 \\
96.90 \\
96.81 \\
106.10 \\
94.70 \\
94.77 \\
98.90 \\
95.38 \\
94.66 \\
94.16 \\
94.81 \\
108.20 \\
96.98 \\
98.68 \\
92.63 \\
91.19\end{array}$ & $\begin{array}{l}5 \\
S \\
5 \\
5 \\
5 \\
5 \\
S \\
5 \\
5 \\
5 \\
5 \\
5 \\
5 \\
S \\
5 \\
5 \\
S\end{array}$ & $\begin{array}{l}\text { Mar. } \\
\text { Jan. } \\
\text { Fob. } \\
\text { Jan. } \\
\text { Jan. } \\
\text { Jan. } \\
\text { Jan. } \\
\text { Jan. } \\
\text { Jan. } \\
\text { Jan. } \\
\text { May } \\
\text { June } \\
\text { July } \\
\text { Aug. } \\
\text { Sept. } \\
\text { Oct. } \\
\text { Nov. }\end{array}$ & $\begin{array}{l}67, \\
68, \\
10, \\
12, \\
65, \\
19, \\
30, \\
62, \\
27, \\
67, \\
22, \\
24 \\
23 \\
19 \\
17 \\
14 \\
12\end{array}$ & $\begin{array}{l}1962 \\
1983 \\
1964 \\
1986 \\
1966 \\
1967 \\
1968 \\
1969 \\
1970 \\
1976 \\
1986\end{array}$ & $\begin{array}{r}93.76 \\
92.26 \\
100.41 \\
102.42 \\
91.46 \\
94.69 \\
96.34 \\
93.15 \\
93.12 \\
88.4 \varnothing \\
89.98 \\
87.66 \\
86.94 \\
87.35 \\
86.55 \\
83.5 \varnothing \\
80.85\end{array}$ & $\begin{array}{l}S \\
S \\
S \\
S \\
S \\
S \\
S \\
S \\
S \\
S\end{array}$ & $\begin{array}{l}\text { Dec. } \\
\text { Jan. } \\
\text { Fob. } \\
\text { Mar. } \\
\text { Apr. } \\
\text { May } \\
\text { June } \\
\text { July } \\
\text { Aug. } \\
\text { Sept. } \\
\text { Oct. } \\
\text { Nov. } \\
\text { Dec. } \\
\text { Jan. } \\
\text { Feb. } \\
\text { Mar. }\end{array}$ & $\begin{array}{l}16, \\
27, \\
24 \\
18 \\
16 \\
11 \\
16 \\
13 \\
10 \\
17 \\
15 \\
18 \\
30 \\
27 \\
24 \\
23\end{array}$ & $\begin{array}{l}1986 \\
1987\end{array}$ & $\begin{array}{l}79.77 \\
78.10 \\
79.83 \\
78.92 \\
77.85 \\
82.32 \\
75.86 \\
74.32 \\
72.70 \\
75.60 \\
76.23 \\
77.60 \\
78.16 \\
78.90 \\
79.22 \\
78.98\end{array}$ & $\begin{array}{l}S \\
S \\
S \\
S \\
S \\
S \\
S \\
S \\
S \\
S \\
S \\
S \\
S \\
S \\
S \\
S\end{array}$ \\
\hline
\end{tabular}

Highest 72.70 Aug. 10,1887

Lowest 108.20 Jan. 22,1957 
Table 2.--Monthly or periodic water-level measurements in selected wells in southwestern Oklahoma and northwestern Texas--Continued

GREER COUNTY, OKLAHOMA--Continued

Index number on location map: 11

Station number: 345251099363101 Local number: $65 \mathrm{~N}-23 W-27$ ACA 1

Location: Lat $34^{\circ} 52^{\prime} 66^{\prime \prime}$, long $99^{\circ} 36^{\prime} 48^{\prime \prime}$, hydrologic unit 11120202

Aquifer: 313 BLIN

Altitude of land-surface datum: $1,725 \mathrm{ft}$

Well depth: $109 \mathrm{ft}$

Remarks: Previously published as $66 \mathrm{~N}-23 W-27$ ADB

\begin{tabular}{|c|c|c|c|c|c|c|c|c|c|c|c|c|c|c|c|c|c|}
\hline \multicolumn{3}{|c|}{ Date } & \multicolumn{2}{|c|}{$\begin{array}{l}\begin{array}{l}\text { Water } \\
\text { level MS }\end{array} \\
\begin{array}{l}64.82 \mathrm{~S} \\
68.23\end{array}\end{array}$} & \multicolumn{3}{|c|}{ Date } & $\begin{array}{l}\text { Water } \\
\text { level MS }\end{array}$ & \multicolumn{3}{|c|}{ Date } & $\begin{array}{l}\text { Water } \\
\text { level MS }\end{array}$ & \multicolumn{3}{|c|}{ Date } & $\begin{array}{l}\text { Water } \\
\text { level }\end{array}$ & MS \\
\hline $\begin{array}{l}\text { July } \\
\text { June } \\
\text { July } \\
\text { Nov. } \\
\text { Jan. } \\
\text { June } \\
\text { Oct. } \\
\text { Nov. } \\
\text { Dec. } \\
\text { Feb. } \\
\text { Mar. } \\
\text { July } \\
\text { Sept. } \\
\text { Oct. } \\
\text { Nov. } \\
\text { Jan. } \\
\text { Jan. } \\
\text { July } \\
\text { Dec. } \\
\text { Fob. } \\
\text { May } \\
\text { June } \\
\text { Sept. } \\
\text { Oct. } \\
\text { Apr. } \\
\text { June }\end{array}$ & $\begin{array}{l}29, \\
26, \\
24 \\
27 \\
22, \\
25 \\
29 \\
18 \\
16 \\
25, \\
25 \\
36 \\
23 \\
27 \\
18 \\
99, \\
13, \\
19 \\
21 \\
14, \\
17 \\
21 \\
66 \\
26 \\
24 \\
25, \\
27\end{array}$ & $\begin{array}{l}1959 \\
1960 \\
1961\end{array}$ & $\begin{array}{l}64.82 \\
68.23 \\
71.36 \\
68.78 \\
73.53 \\
67.35 \\
69.30 \\
67.74 \\
67.37 \\
66.77 \\
67.69 \\
66.85 \\
69.68 \\
69.46 \\
68.86 \\
68.60 \\
65.60 \\
65.60 \\
66.48 \\
64.65 \\
71.46 \\
65.65 \\
72.17 \\
65.80 \\
65.41 \\
72.26 \\
66.28\end{array}$ & & $\begin{array}{l}\text { Oct. } \\
\text { Nov. } \\
\text { Jan. } \\
\text { Feb. } \\
\text { Mar. } \\
\text { June } \\
\text { Nov. } \\
\text { Jan. } \\
\text { Fob. } \\
\text { Apr. } \\
\text { May } \\
\text { July } \\
\text { Oct. } \\
\text { Nov. } \\
\text { Jan. } \\
\text { Sept. } \\
\text { Oct. } \\
\text { Nov. } \\
\text { Doc. } \\
\text { Jan. } \\
\text { Mar. } \\
\text { Apr. } \\
\text { May } \\
\text { July } \\
\text { Aug. } \\
\text { Sept. }\end{array}$ & $\begin{array}{l}63, \\
17 \\
13 \\
68, \\
65 \\
26 \\
18 \\
23 \\
12, \\
17 \\
19 \\
25 \\
13 \\
12 \\
64 \\
95, \\
14 \\
20 \\
18 \\
14 \\
19, \\
16 \\
13 \\
16 \\
12 \\
15 \\
12\end{array}$ & 1966 & $\begin{array}{l}65.69 \\
65.69 \\
66.29 \\
65.68 \\
66.48 \\
70.79 \\
73.88 \\
70.83 \\
68.63 \\
69.22 \\
65.86 \\
76.15 \\
66.60 \\
66.60 \\
68.68 \\
64.90 \\
66.96 \\
66.35 \\
67.46 \\
66.60 \\
65.86 \\
68.12 \\
68.77 \\
67.61 \\
67.56 \\
74.40 \\
68.46\end{array}$ & $\begin{array}{l}\text { Oct. } \\
\text { Nov. } \\
\text { Dec. } \\
\text { Jan. } \\
\text { Mar. } \\
\text { Apr. } \\
\text { June } \\
\text { July } \\
\text { Sept. } \\
\text { Oct. } \\
\text { Nov. } \\
\text { Jan. } \\
\text { Feb. } \\
\text { Apr. } \\
\text { May } \\
\text { June } \\
\text { Sept. } \\
\text { Oct. } \\
\text { Nov. } \\
\text { Dec. } \\
\text { Jan. } \\
\text { Feb. } \\
\text { Dec. } \\
\text { Jan. } \\
\text { Jan. } \\
\text { Dec. }\end{array}$ & $\begin{array}{l}12, \\
15 \\
12 \\
36, \\
26 \\
25 \\
65 \\
19 \\
17 \\
19 \\
16 \\
13 \\
92, \\
64 \\
68 \\
98 \\
19 \\
64 \\
67 \\
19 \\
11 \\
27, \\
26 \\
29 \\
67, \\
28, \\
68\end{array}$ & $\begin{array}{l}1976 \\
1976\end{array}$ & $\begin{array}{l}67.36 \\
68.65 \\
67.59 \\
67.24 \\
66.73 \\
67.48 \\
65.68 \\
65.36 \\
65.69 \\
68.25 \\
66.93 \\
66.61 \\
65.57 \\
65.62 \\
65.67 \\
65.11 \\
64.76 \\
66.44 \\
66.66 \\
65.73 \\
65.31 \\
64.31 \\
65.16 \\
69.66 \\
62.95 \\
66.56 \\
62.79\end{array}$ & $\begin{array}{l}\text { Mar. } \\
\text { Mar. } \\
\text { Feb. } \\
\text { Feb. } \\
\text { Jan. } \\
\text { Feb. } \\
\text { Jan. } \\
\text { Jan. } \\
\text { May } \\
\text { June } \\
\text { July } \\
\text { Aug. } \\
\text { Sept. } \\
\text { Oct. } \\
\text { Nov. } \\
\text { Dec. } \\
\text { Mar. } \\
\text { May } \\
\text { June } \\
\text { July } \\
\text { Aug. } \\
\text { Sept. } \\
\text { Oct. } \\
\text { Nov. } \\
\text { Jan. } \\
\text { Fob. } \\
\text { Mar. }\end{array}$ & $\begin{array}{l}68, \\
66, \\
28, \\
19, \\
26, \\
92, \\
19 \\
67 \\
16 \\
24 \\
23 \\
19 \\
17 \\
14 \\
12 \\
16 \\
18, \\
11 \\
15 \\
13 \\
16 \\
17 \\
15 \\
18 \\
27, \\
24 \\
23\end{array}$ & $\begin{array}{l}1978 \\
1979 \\
1980 \\
1982 \\
1983 \\
1984 \\
1985 \\
1986\end{array}$ & $\begin{array}{l}64.62 \\
63.60 \\
64.62 \\
64.26 \\
63.34 \\
62.75 \\
67.16 \\
62.21 \\
63.22 \\
61.36 \\
73.99 \\
66.23 \\
59.98 \\
58.72 \\
57.83 \\
57.36 \\
56.38 \\
61.15 \\
56.63 \\
55.76 \\
56.82 \\
56.65 \\
69.78 \\
56.92 \\
56.50 \\
66.82 \\
56.10\end{array}$ & 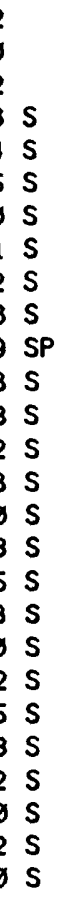 \\
\hline
\end{tabular}


Tablo 2.--Monthly or periodic water-level measurements in selected wells in southwestern Oklahoma and northwestern Texas--Continued

HARMON COUNTY, OKLAHOMA

Index number on location map: 12

Station number: 343214099441401 Local number: $\varnothing 1 \mathrm{~N}-24 W-20$ DDDD 1

Location: Lat $34^{\circ} 32^{\prime} 10^{\prime \prime}$, long $99^{\circ} 44^{\prime} 12^{\prime \prime}$, hydrologic unit 11130101

Aquifer: $313 V V C R$

Altitude of land-surface datum: $1,503 \mathrm{ft}$

Well depth: $97.3 \mathrm{ft}$

Remarks: Well drilled to $140 \mathrm{ft}$ Jan. 15,1953

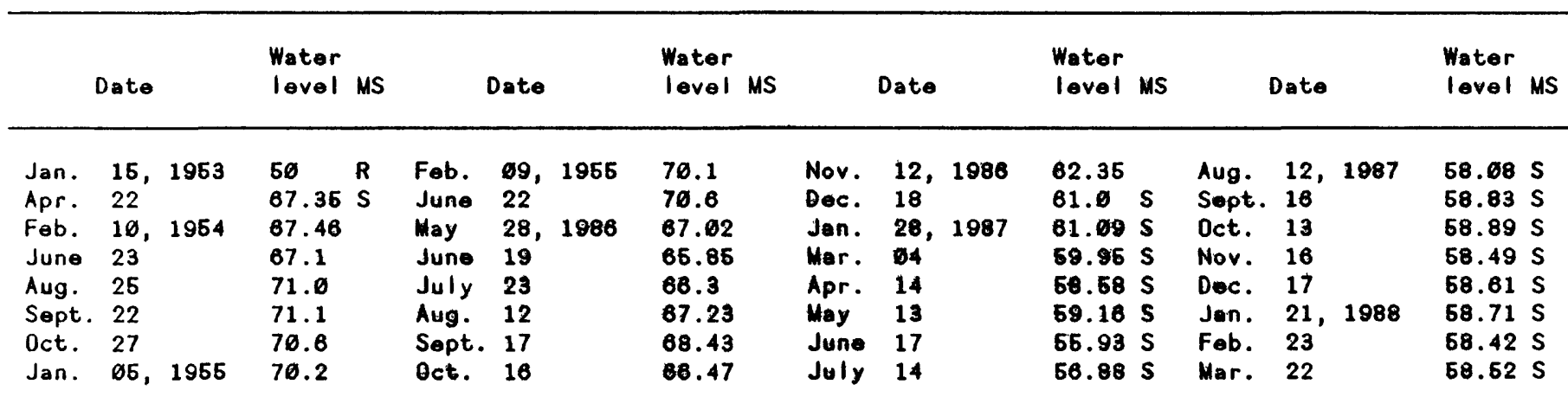

Highest 50 Jan. 16, 1953

Lowest 71.1 Sept. 22, 1954 
Table 2.--Monthly or periodic water-level measurements in selected wells in southwestern Oklahoma and northwestern Texas--Continued

HARMON COUNTY, OKLAHOMA--CONtinued

Index number on location map: 13

Station number: 343020099422001 Local number: $01 \mathrm{~N}-24 W-34$ DCC 1

Location: Lat $34^{\circ} 30^{\prime} 28^{\prime \prime}$, long $99^{\circ} 42^{\prime} 36^{\prime \prime}$, hydrologic unit 11130101

Aquifer: 313VVCR

Altitude of land-surface datum: $1,443.5 \mathrm{ft}$

Well depth: $94 \mathrm{ft}$

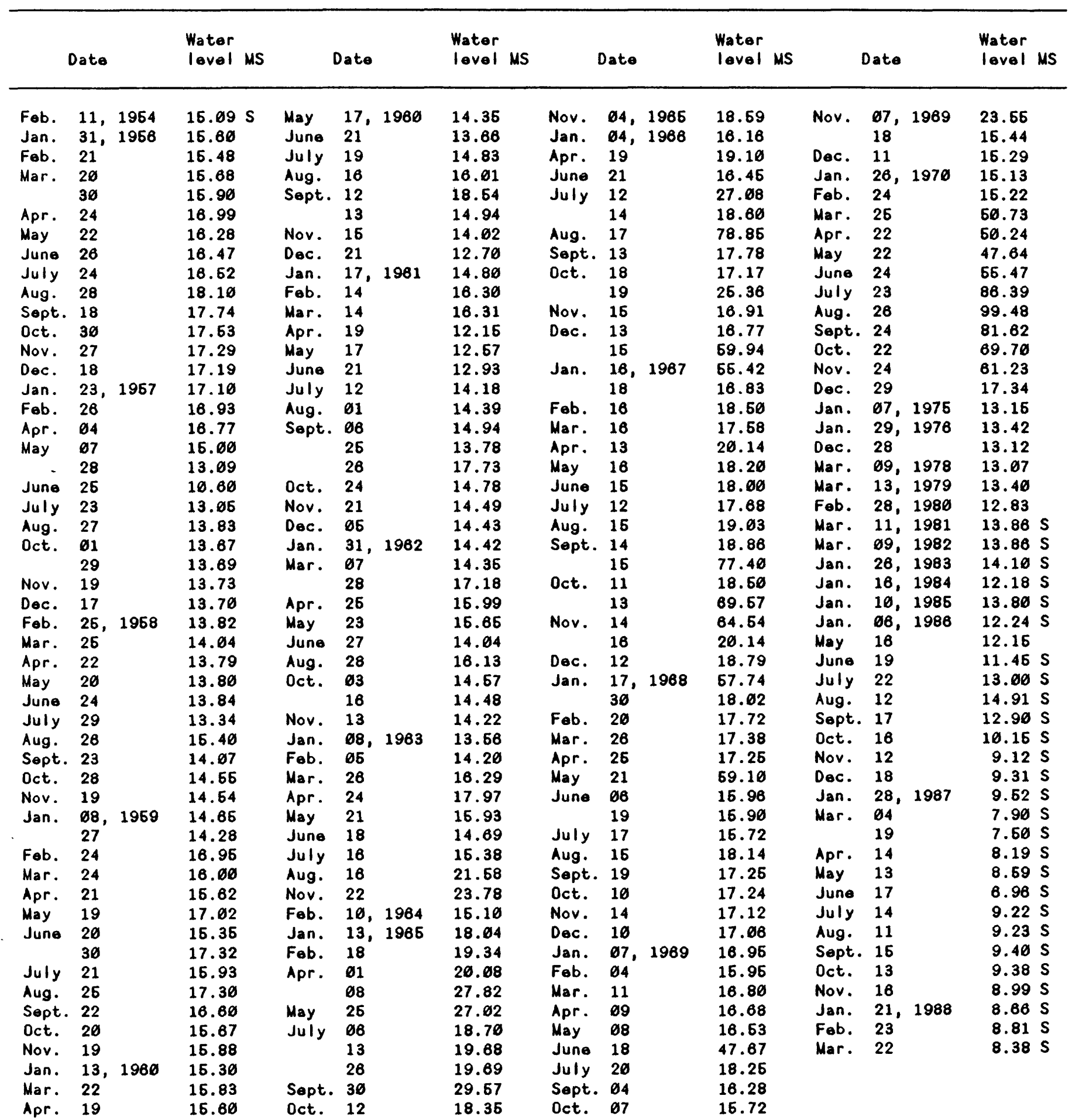


Table 2.-- Monthly or periodic water-level measurements in selected wells in southwestern Oklahoma and northwestern Texas--Continued

HARMON COUNTY, OKLAHOMA--COntinued

Index number on location map: 14

Station number: $\$ 43428699492501$ Local number: $\varnothing 1 \mathrm{~N}-25 \mathrm{~W}-10 \mathrm{BCBC} 1$

Location: Lat $34^{\prime} 34^{\prime} 28^{\prime \prime}$, long $99^{\circ} 49^{\prime} 25^{\prime \prime}$, hydrologic unit 11130101

Aquifer: $313 B L I N$

Altitude of land-surface datum: $1,526 \mathrm{ft}$

Well depth: $120 \mathrm{ft}$

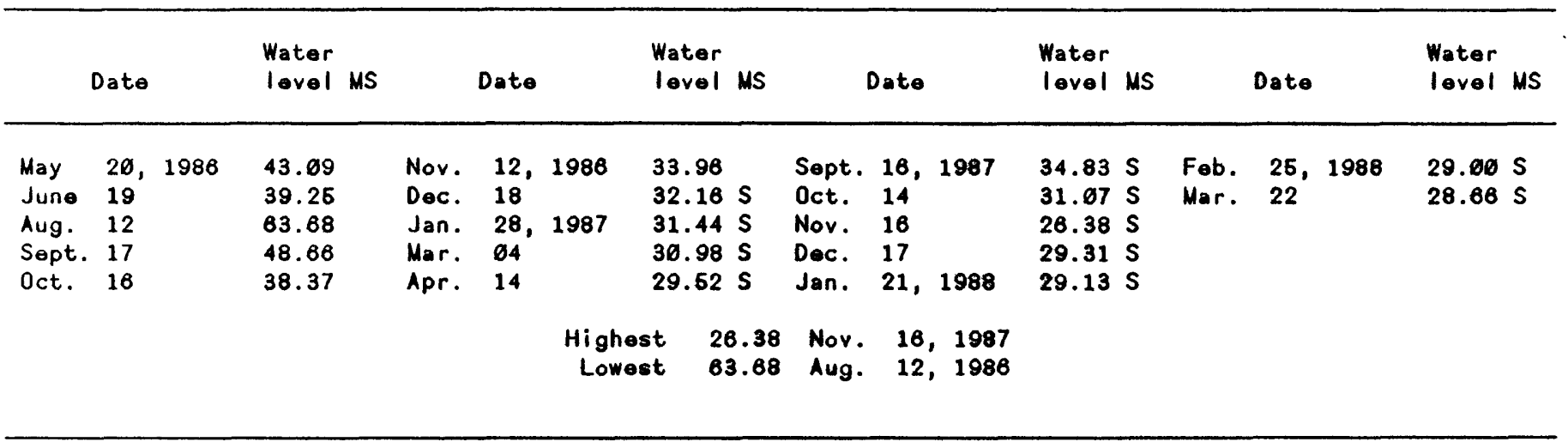

Index number on location map: 16

Station number: 343419099462201 Local number: D1N-25W-12 DABA 1

Location: Lat $34^{\circ} 34^{\prime} 17^{\prime \prime}$, long $99^{\circ} 46^{\prime} 25^{\prime \prime}$, hydrologic unit 11130101

Aquifer: $313 B L I N$

Altitude of land-surface datum: $1,624 \mathrm{ft}$

Well depth: $212 \mathrm{ft}$

Remarks: Previously published as $61 \mathrm{~N}-25 W-12$ DAA

\begin{tabular}{|c|c|c|c|c|c|c|c|c|c|c|c|c|c|c|c|c|c|c|}
\hline & Dato & & $\begin{array}{l}\text { Water } \\
\text { level }\end{array}$ & MS & & Date & & $\begin{array}{l}\text { Water } \\
\text { level }\end{array}$ & MS & & Date & & $\begin{array}{l}\text { Water } \\
\text { level }\end{array}$ & MS & & Date & $\begin{array}{l}\text { Water } \\
\text { level }\end{array}$ & MS \\
\hline $\begin{array}{l}\text { Apr. } \\
\text { Jan. } \\
\text { Jan. } \\
\text { Jan. } \\
\text { Jan. } \\
\text { Dec. } \\
\text { Jan. } \\
\text { Jan. } \\
\text { Dec. } \\
\text { Mar. }\end{array}$ & $\begin{array}{l}19, \\
16, \\
17, \\
07, \\
26, \\
29 \\
20, \\
67, \\
28, \\
69,\end{array}$ & $\begin{array}{l}1986 \\
1967 \\
1968 \\
1969 \\
1970 \\
\\
1972 \\
1976 \\
1976 \\
1978\end{array}$ & $\begin{array}{l}83.38 \\
56.42 \\
67.74 \\
56.38 \\
47.06 \\
61.71 \\
54.94 \\
38.07 \\
36.68 \\
33.92\end{array}$ & $\begin{array}{l}S \\
S \\
S \\
S \\
S \\
S \\
S \\
S\end{array}$ & $\begin{array}{l}\text { Mar. } \\
\text { Fob. } \\
\text { Mar. } \\
\text { Mar. } \\
\text { Jan. } \\
\text { Jan. } \\
\text { Jan. } \\
\text { Jan. } \\
\text { May } \\
\text { June }\end{array}$ & $\begin{array}{l}13, \\
28, \\
11, \\
69, \\
28, \\
16, \\
10, \\
68, \\
16 \\
19\end{array}$ & $\begin{array}{l}1979 \\
1980 \\
1981 \\
1982 \\
1983 \\
1984 \\
1986 \\
1986\end{array}$ & $\begin{array}{l}36.64 \\
36.99 \\
42.22 \\
47.92 \\
51.44 \\
44.92 \\
62.44 \\
36.79 \\
39.82 \\
36.48\end{array}$ & $\begin{array}{l}5 \\
5 \\
5 \\
5 \\
5 \\
5 \\
5 \\
S\end{array}$ & $\begin{array}{l}\text { July } \\
\text { Aug. } \\
\text { Sept. } \\
\text { Oct. } \\
\text { Nov. } \\
\text { Dec. } \\
\text { Jan. } \\
\text { Mar. } \\
\text { Apr. }\end{array}$ & $\begin{array}{l}23, \\
12 \\
17 \\
16 \\
12 \\
18 \\
28, \\
64 \\
19 \\
14\end{array}$ & $\begin{array}{l}1986 \\
1987\end{array}$ & $\begin{array}{l}52.06 \\
70.66 \\
53.61 \\
37.84 \\
36.86 \\
27.86 \\
27.30 \\
26.32 \\
24.78 \\
24.13\end{array}$ & $\begin{array}{l}5 \\
5 \\
S \\
5 \\
S \\
5 \\
5 \\
5 \\
5 \\
S\end{array}$ & $\begin{array}{l}\text { May } \\
\text { June } \\
\text { July } \\
\text { Aug. } \\
\text { Sept. } \\
\text { Oct. } \\
\text { Nov. } \\
\text { Jan. } \\
\text { Fob. } \\
\text { Mar. }\end{array}$ & $\begin{array}{ll}13, & 1987 \\
16 & \\
14 & \\
12 & \\
16 & \\
14 & \\
16 & \\
21, & 1988 \\
26 & \\
22 & \end{array}$ & $\begin{array}{l}29.38 \\
20.24 \\
21.27 \\
36.52 \\
25.78 \\
23.68 \\
22.02 \\
20.92 \\
20.65 \\
19.91\end{array}$ & $\begin{array}{l}5 \\
S \\
5 \\
5 \\
5 \\
5 \\
S \\
25 \\
25 \\
5 \\
S \\
1\end{array}$ \\
\hline
\end{tabular}

Highest 19.91 Mar. 22, 1988

Lowest 70.66 Aug. 12, 1986 
Table 2.--Monthly or periodic water-level measurements in selected wells in southwestern oklahoma and northwestern Texas--Continued

HARMON COUNTY, OKLAHOMA--Continued

Index number on location map: 16

Station number: 843042099474701 Local number: $11 \mathrm{~N}-25 W-35$ DDBA 1

Location: Lat $34^{\circ} 30^{\prime} 38^{\prime \prime}$, long $99^{\circ} 47^{\prime} 30^{\prime \prime}$, hydrologic unit 11130101

Aquifer: $313 V V C R$

Altitude of land-surface datum: $1,485 \mathrm{ft}$

Well depth: $72 \mathrm{ft}$

Remarks: Previously published as $01 \mathrm{~N}-25 \mathrm{~W}-35 \mathrm{DDB}$

\begin{tabular}{|c|c|c|c|c|c|c|c|c|c|c|c|c|c|c|c|}
\hline & Date & & $\begin{array}{l}\text { Water } \\
\text { level }\end{array}$ & MS & & Date & & $\begin{array}{l}\text { Water } \\
\text { level MS }\end{array}$ & & Date & & $\begin{array}{l}\text { Water } \\
\text { level }\end{array}$ & MS & Date & $\begin{array}{l}\text { Water } \\
\text { level MS }\end{array}$ \\
\hline \multirow[t]{2}{*}{$\begin{array}{l}\text { Jan. } \\
\text { Sept. } \\
\text { Dec. } \\
\text { Mar. } \\
\text { Feb. } \\
\text { Mar. } \\
\text { Mar. } \\
\text { Jan. } \\
\text { Jan. }\end{array}$} & $\begin{array}{l}26, \\
63, \\
28, \\
99, \\
28, \\
11, \\
99, \\
26, \\
16,\end{array}$ & $\begin{array}{l}1948 \\
1962 \\
1976 \\
1978 \\
1980 \\
1981 \\
1982 \\
1983 \\
1984\end{array}$ & $\begin{array}{l}16 \\
16.72 \\
17.50 \\
16.60 \\
16.95 \\
18.49 \\
20.80 \\
21.60 \\
18.92\end{array}$ & $\begin{array}{l}R \\
S \\
\\
S \\
S \\
S \\
S\end{array}$ & $\begin{array}{l}\text { Jan. } \\
\text { Jan. } \\
\text { May } \\
\text { June } \\
\text { July } \\
\text { Aug. } \\
\text { Sept. } \\
\text { Oct. } \\
\text { Nov. }\end{array}$ & $\begin{array}{l}10, \\
66, \\
16 \\
19 \\
22 \\
12 \\
17 \\
16 \\
12\end{array}$ & $\begin{array}{l}1986 \\
1988\end{array}$ & $\begin{array}{l}21.67 \mathrm{~S} \\
17.78 \mathrm{~S} \\
17.08 \mathrm{~S} \\
16.62 \mathrm{~S} \\
20.47 \mathrm{~S} \\
24.80 \mathrm{~S} \\
20.99 \mathrm{~S} \\
14.07 \mathrm{~S} \\
11.49 \mathrm{~S}\end{array}$ & $\begin{array}{l}\text { Dec. } \\
\text { Jan. } \\
\text { Mar. } \\
\text { Apr. } \\
\text { May } \\
\text { June } \\
\text { July } \\
\text { Aug. }\end{array}$ & $\begin{array}{l}18, \\
28, \\
64 \\
19 \\
14 \\
13 \\
17 \\
14 \\
11\end{array}$ & $\begin{array}{l}1986 \\
1987\end{array}$ & $\begin{array}{r}11.04 \\
11.38 \\
9.73 \\
9.98 \\
9.16 \\
10.16 \\
7.96 \\
9.98 \\
18.88\end{array}$ & $\begin{array}{l}S \\
S \\
S \\
S \\
S \\
S \\
S \\
S \\
S\end{array}$ & $\begin{array}{lll}\text { Sept. } & 16,1987 \\
\text { Oct. } & 13 & \\
\text { Nov. } & 16 & \\
\text { Jan. } & 21,1988 \\
\text { Fob. } & 23 & \\
\text { Mar. } & 22 & \end{array}$ & $\begin{array}{r}11.69 \mathrm{~S} \\
11.31 \mathrm{~S} \\
11.90 \mathrm{~S} \\
9.98 \mathrm{~S} \\
10.07 \mathrm{~S} \\
9.77 \mathrm{~S}\end{array}$ \\
\hline & & & & & & & $\begin{array}{l}\text { High } \\
\text { Low }\end{array}$ & $\begin{array}{r}7.90 \\
24.80\end{array}$ & $\begin{array}{l}\text { June } \\
\text { Aug. }\end{array}$ & $\begin{array}{l}17, \\
12,\end{array}$ & $\begin{array}{l}1987 \\
, 1988\end{array}$ & & & & \\
\hline
\end{tabular}

Index number on location map: 17

Station number: $\$ 43626099673401$ Local number: $01 \mathrm{~N}-28 W-05$ BACC 1

Location: Lat $34^{\circ} 36^{\prime} 26^{\prime \prime}$, long $99^{\circ} 67^{\prime} 34^{\prime \prime}$, hydrologic unit 11130101

Aquifor: 313BLIN

Altitude of land-surface datum: $1,562 \mathrm{ft}$

Well depth: $81 \mathrm{ft}$

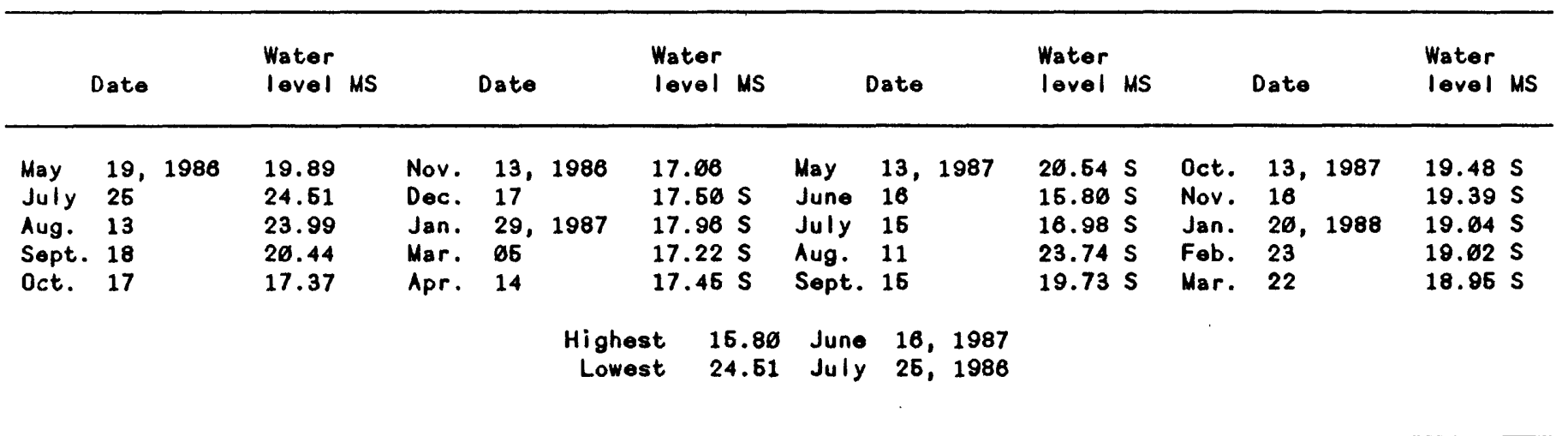

Index number on location map: 18

Station number: $\$ 43449699573101$ Local number: $11 \mathrm{~N}-26 W-05$ CDCD 1

Location: Lat $34^{\circ} 34^{\prime} 49^{\prime \prime}$, long $99^{\circ} 67^{\prime} 31^{\prime \prime}$, hydrologic unit 11130101

Aquifer: 111RRVA

Altitude of land-surface datum: $1,550 \mathrm{ft}$

Well depth: $48 \mathrm{ft}$

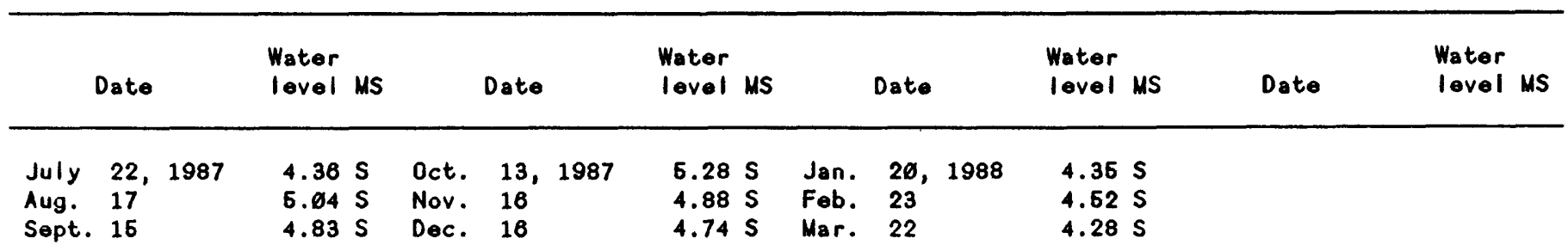


Table 2.--Monthly or periodic water-level measurements in selected wells in southwestern Oklahoma and northwestern Texas--Continued

HARMON COUNTY, OKLAHOMA--CONTInUEd

Index number on location map: 19

Station number: $\$ 43447099572701$ Local number: $01 \mathrm{~N}-26 \mathrm{~W}-08$ BAAB 1

Location: Lat $34^{\circ} 34^{\prime} 47^{\prime \prime}$, long $99^{\circ} 67^{\prime} 27^{\prime \prime}$, hydrologic unit 11130101

Aquifer: 111RRVA

Altitude of land-surface datum: $1,646 \mathrm{ft}$

Well depth: $26 \mathrm{ft}$

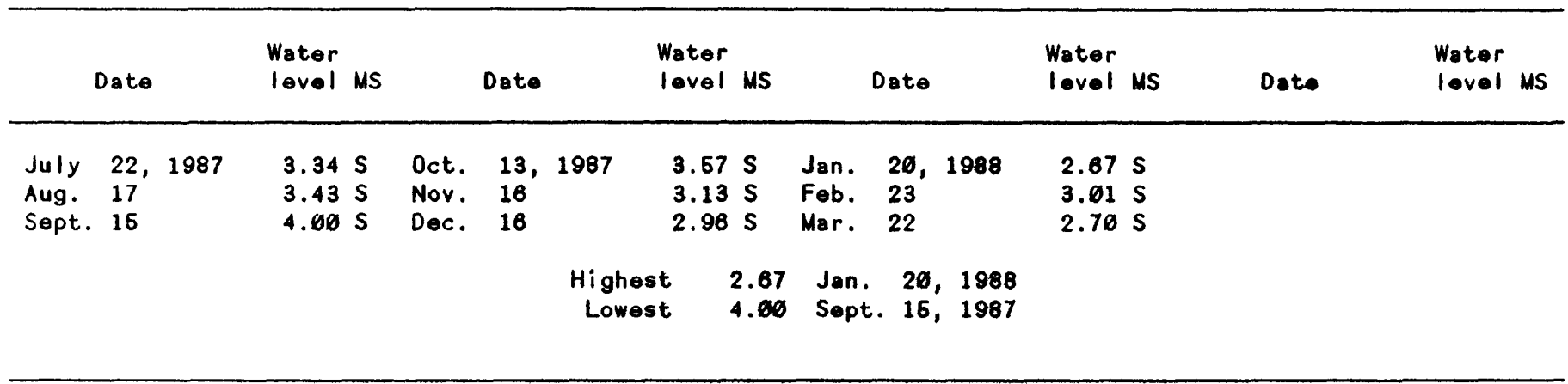

Index number on location map: 20

Station number: 343534699690001 Local number: O1N-27W-61 AAAB 1

Location: Lat $34^{\circ} 35^{\prime} 39^{\prime \prime}$, long $99^{\circ} 59^{\prime} 66^{\prime \prime}$, hydrologic unit 11136101

Aquifer: 313 BLIN

Altitude of land-surface dotum: $1,670 \mathrm{ft}$

Well depth: $70 \mathrm{ft}$

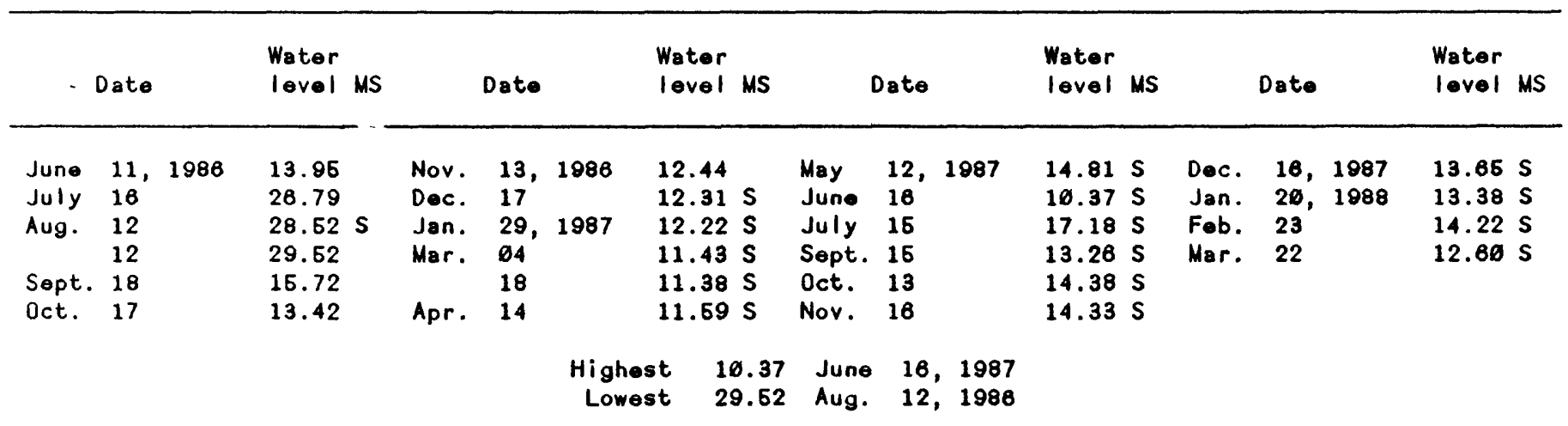

Index number on location map: 21

Station number: 343818099444201 Local number: $62 \mathrm{~N}-24 W-17$ DCCC 1

Location: Lat $34^{\circ} 38^{\prime} 18^{\prime \prime}$, long $99^{\circ} 44^{\prime} 42^{\prime \prime}$, hydrologic unit 11120282

Aquifer: $3130 \mathrm{GCK}$

Altitude of land-surface datum: $1,590 \mathrm{ft}$

Well depth: $35.75 \mathrm{ft}$

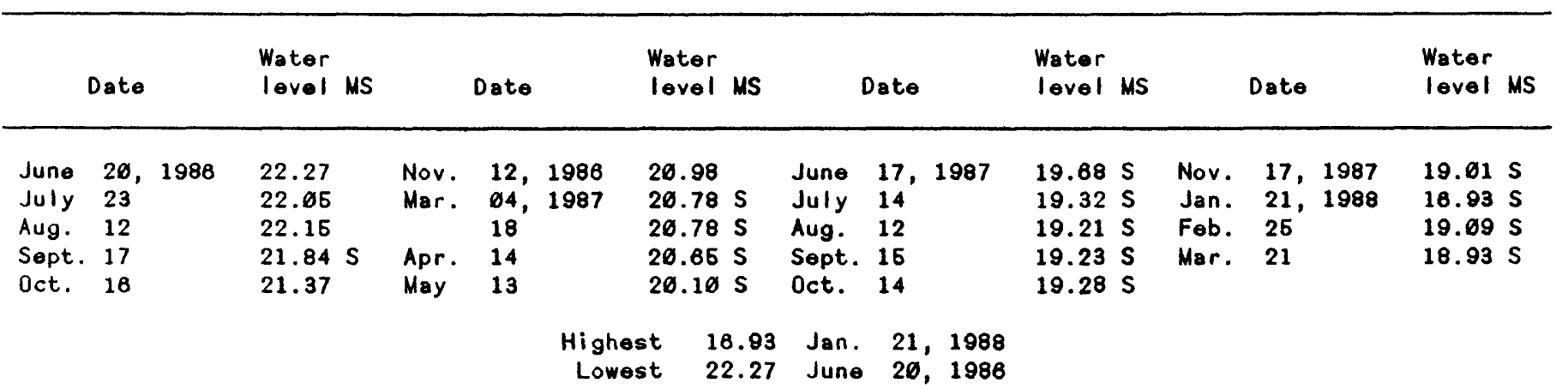


Table 2.--Monthly or periodic water-level measurements in selected wells in southwestern oklahoma and northwestern Texas--Continued

HARMON COUNTY, OKLAHOMA--CONtinued

Index number on location map: 22

Station number: 343728099420201 Local number: $62 \mathrm{~N}-24 W-23$ CCCC 1

Location: Lat $34^{\circ} 37^{\prime} 24^{\prime \prime}$, long $99^{\circ} 42^{\prime} 64^{\prime \prime}$, hydrologic unit 11130101

Aquifer: 313BLIN

Altitude of land-surface datum: $1,531 \mathrm{ft}$

Well depth: $170 \mathrm{ft}$

Remarks: Well depth measured as $148 \mathrm{ft}$ deep, Feb. 27, 1987

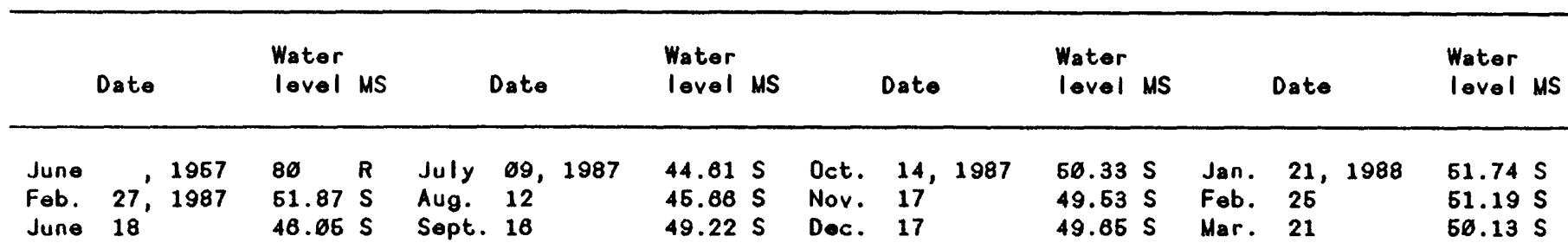

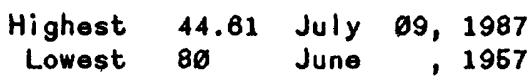

Index number on location map: 23

Station number: 343631099450501 Local number: $02 \mathrm{~N}-24 \mathrm{~W}-32$ ABAB 1

Location: Lat $34^{\circ} 36^{\prime} 29^{\prime \prime}$, long $99^{\circ} 44^{\prime} 64^{\prime \prime}$, hydrologic unit 11120202

Aquifer: 313BLIN

Altitude of land-surface datum: $1,674 \mathrm{ft}$

Well depth: $190 \mathrm{ft}$

Remarks: Original well depth $246 \mathrm{ft}$

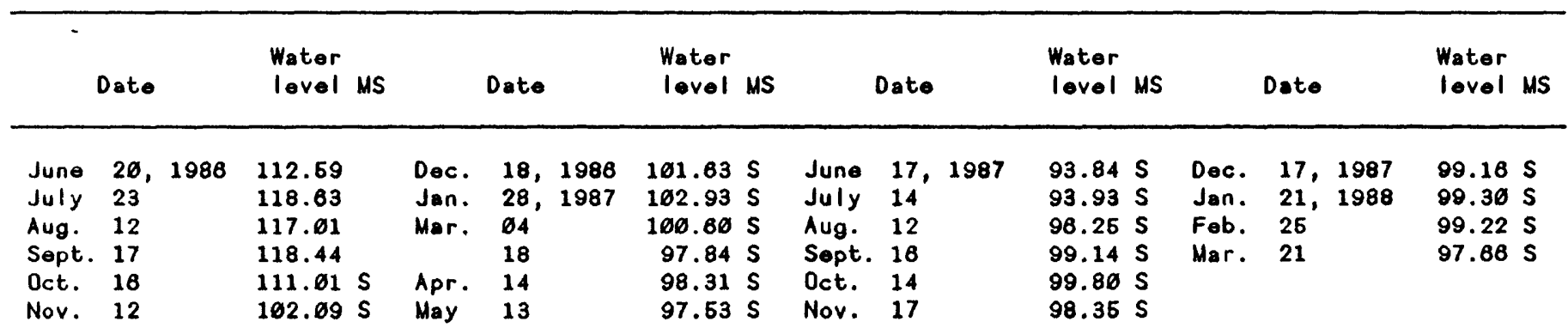

Highest 93.84 June 17,1987

Lowest 118.63 July 23,1986

Index number on location map: 24

Station number: $\$ 44637099503301$ Local number: $62 \mathrm{~N}-25 \mathrm{~W}-\varnothing 5$ AAD 1

Location: Lat $34^{\circ} 40^{\prime} 39^{\prime \prime}$, long $99^{\circ} 50^{\prime} 30^{\prime \prime}$, hydrologic unit 11130161

Aquifer: 313BLIN

Altitude of land-surface datum: $1,602 \mathrm{ft}$

Well depth: $95 \mathrm{ft}$

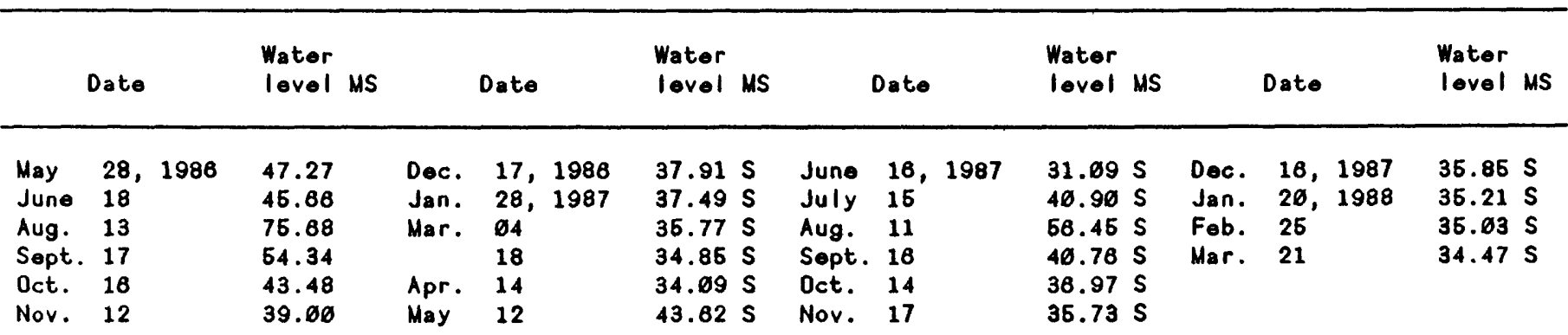


Table 2.--Monthly or periodic water-level measurements in selected wells in southwestern oklahoma and northwestern Texas--Continued

HARMON COUNTY, OKLAHOMA--CONtinued

Index number on location map: 25

Station number: 343934699605201 Local number: $22 N-25 W-\varnothing 8$ ACDC 1

Location: Lat $34^{\circ} 39^{\prime} 34^{\prime \prime}$, long $99^{\circ} 50^{\prime} 62^{\prime \prime}$, hydrologic unit 11130101

Aquifer: $313 B L I N$

Altitude of land-surface datum: $1,583 \mathrm{ft}$

Well depth: $82 \mathrm{ft}$

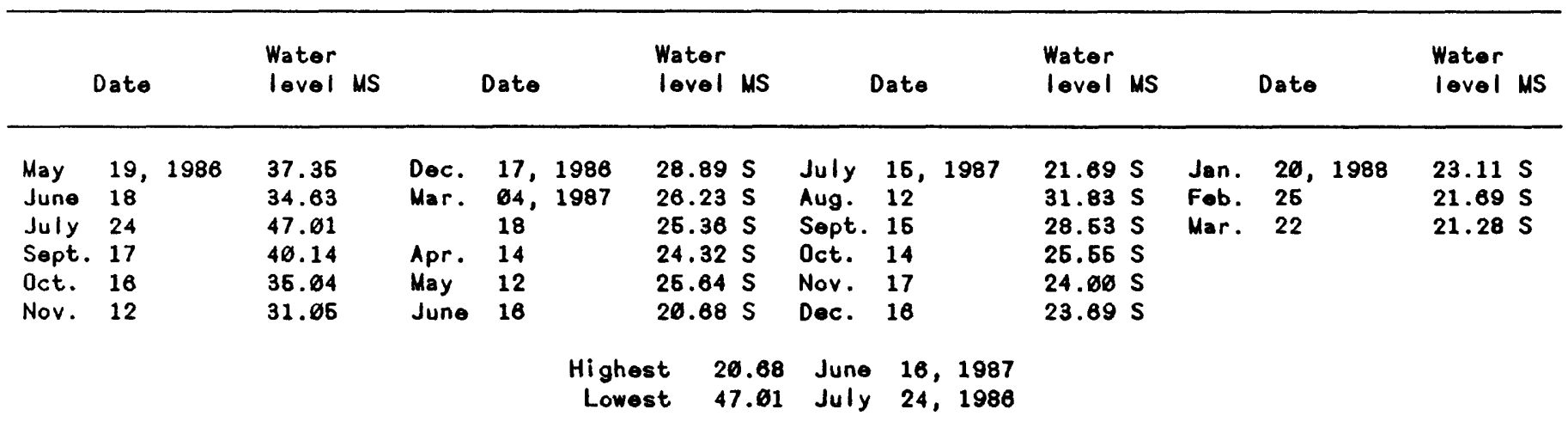

Index number on location map: 28

Station number: 343945099503401 Local number: O2N-26W-08 ADA 1

Location: Lat $34^{\prime} 39^{\prime} 46^{\prime \prime}$, long $99^{\circ} 60^{\prime} 34^{\prime \prime}$, hydrologic unit 11130101

Aquifer: 3138 BIN

Altitude of land-surface datum: 1,692 ft

Well depth: $160 \mathrm{ft}$

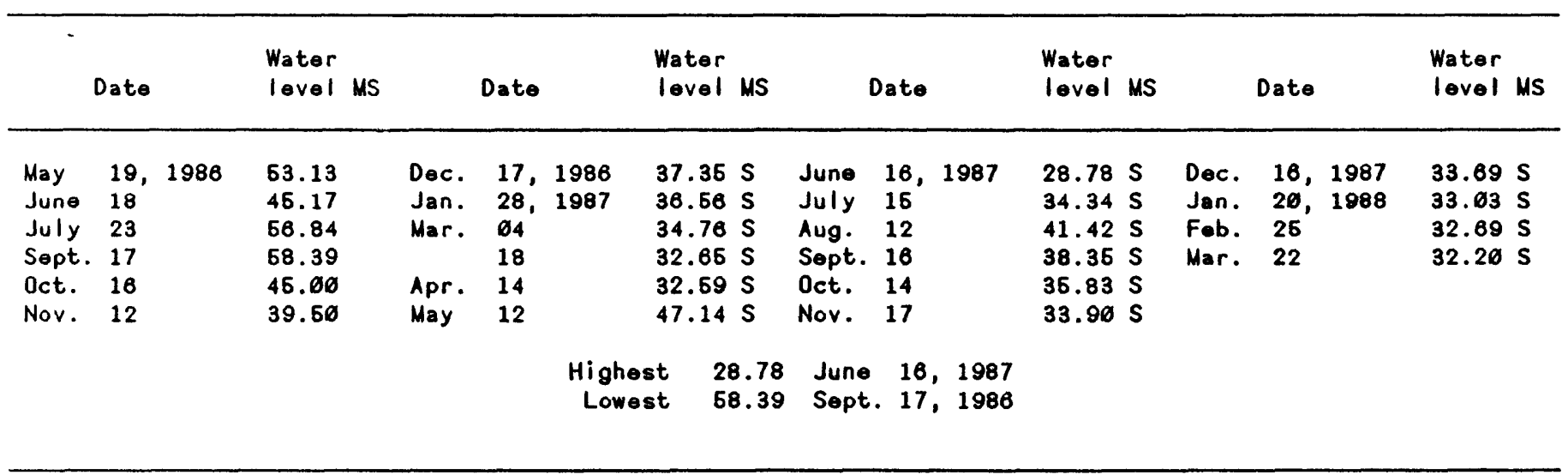

Index number on location map: 27

Station number: 343913099616861 Local number: $62 \mathrm{~N}-26 W-68$ CDCA 1

Location: Lat $34^{\circ} 39^{\prime} 13^{\prime \prime}$, long $99^{\circ} 51^{\prime} 08^{\prime \prime}$, hydrologic unit 11130101

Aquifer: 3138 L.IN

Altitude of land-surface datum: $1,578 \mathrm{ft}$

Well depth: $150 \mathrm{ft}$

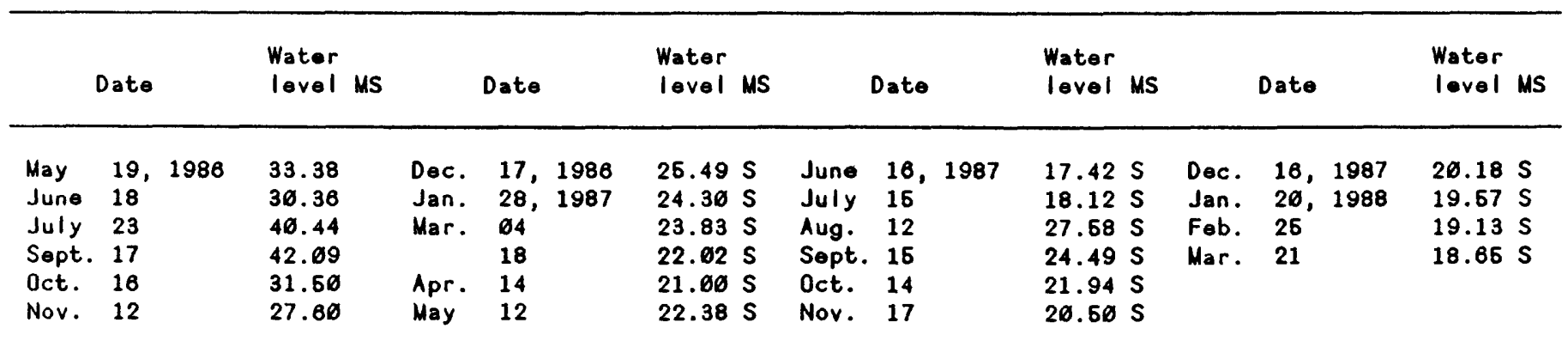


Table 2.--Monthly or periodic water-level measurements in selected wells in southwestern Oklahoma and northwestern Texas--Continued

HARMON COUNTY, OKLAHOMA--CONTInUEd

Index number on location map: 28

Station number: $3439196994956 \varnothing 1$ Local number: $62 \mathrm{~N}-26 \mathrm{~W}-\varnothing 9$ DCBC 1

Location: Lat $34^{\prime} 39^{\prime} 16^{\prime \prime}$, long $99^{\circ} 49^{\prime} 57^{\prime \prime}$, hydrologic unit 11130101

Aquifer: $3138 \mathrm{LIN}$

Altitude of land-surface datum: $1,692 \mathrm{ft}$

Well depth: $200 \mathrm{ft}$

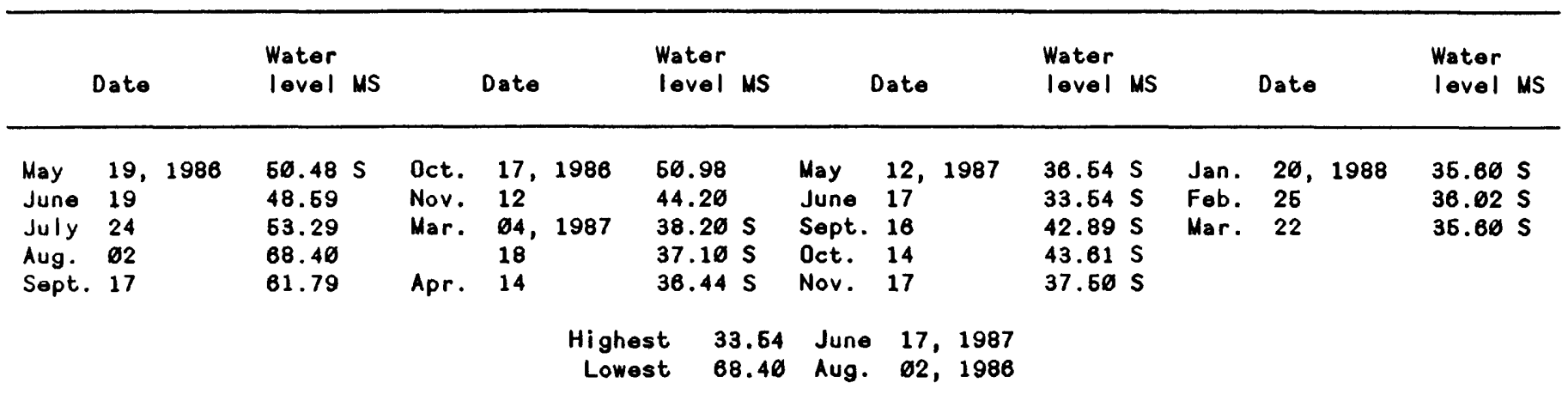

Index number on location map: 29

Station number: $3439080995216 \varnothing 1$ Local number: $02 \mathrm{~N}-26 \mathrm{~W}-18$ BABA 1

Location: Lat $34^{\prime 3} 39^{\prime} 68^{\prime \prime}$, long $99^{\circ} 62^{\prime} 13^{\prime \prime}$, hydrologic unit 11130101

Aquifer: $313 B$ LIN

Altitude of land-surface datum: $1,678 \mathrm{ft}$

Well depth: $84 \mathrm{ft}$

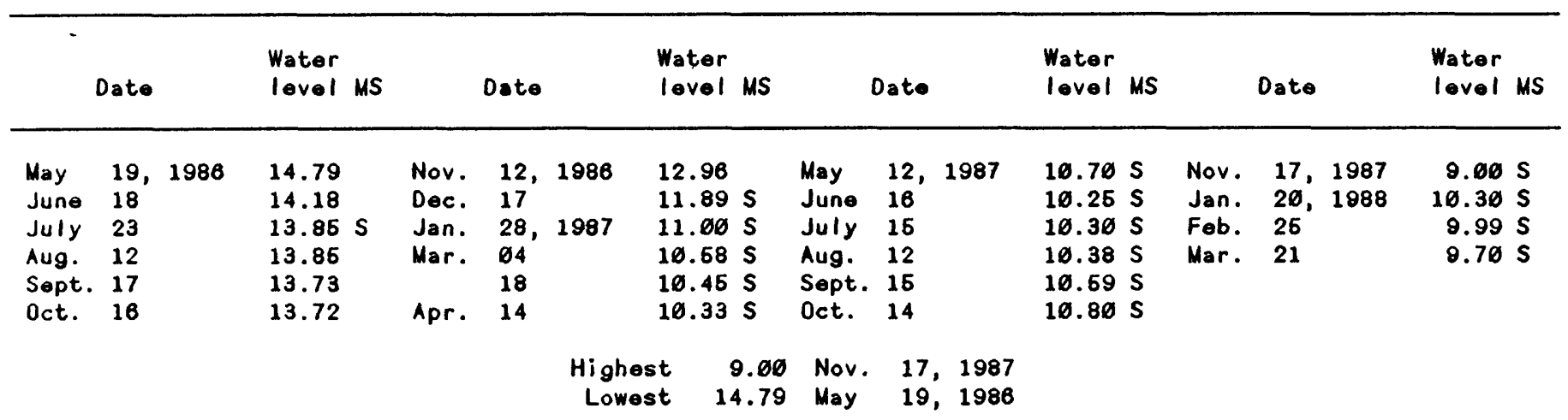


Table 2.--Monthly or periodic water-level measurements in selected wells in southwestern oklahoma and northwestern Texas--Continued

HARMON COUNTY, OKLAHOMA--COntinued

Index number on location map: 30

Station number: 343736097443001 Local number: $02 \mathrm{~N}-25 \mathrm{~W}-21$ DBC 1

Location: Lat $34^{\circ} 37^{\prime} 46^{\prime \prime}$, long $99^{\circ} 49^{\prime} 30^{\prime \prime}$, hydrologic unit 11130101

Aquifer: 313BLIN

Altitude of land-surface datum: $1,566.39 \mathrm{ft}$

Well depth: $112 \mathrm{ft}$

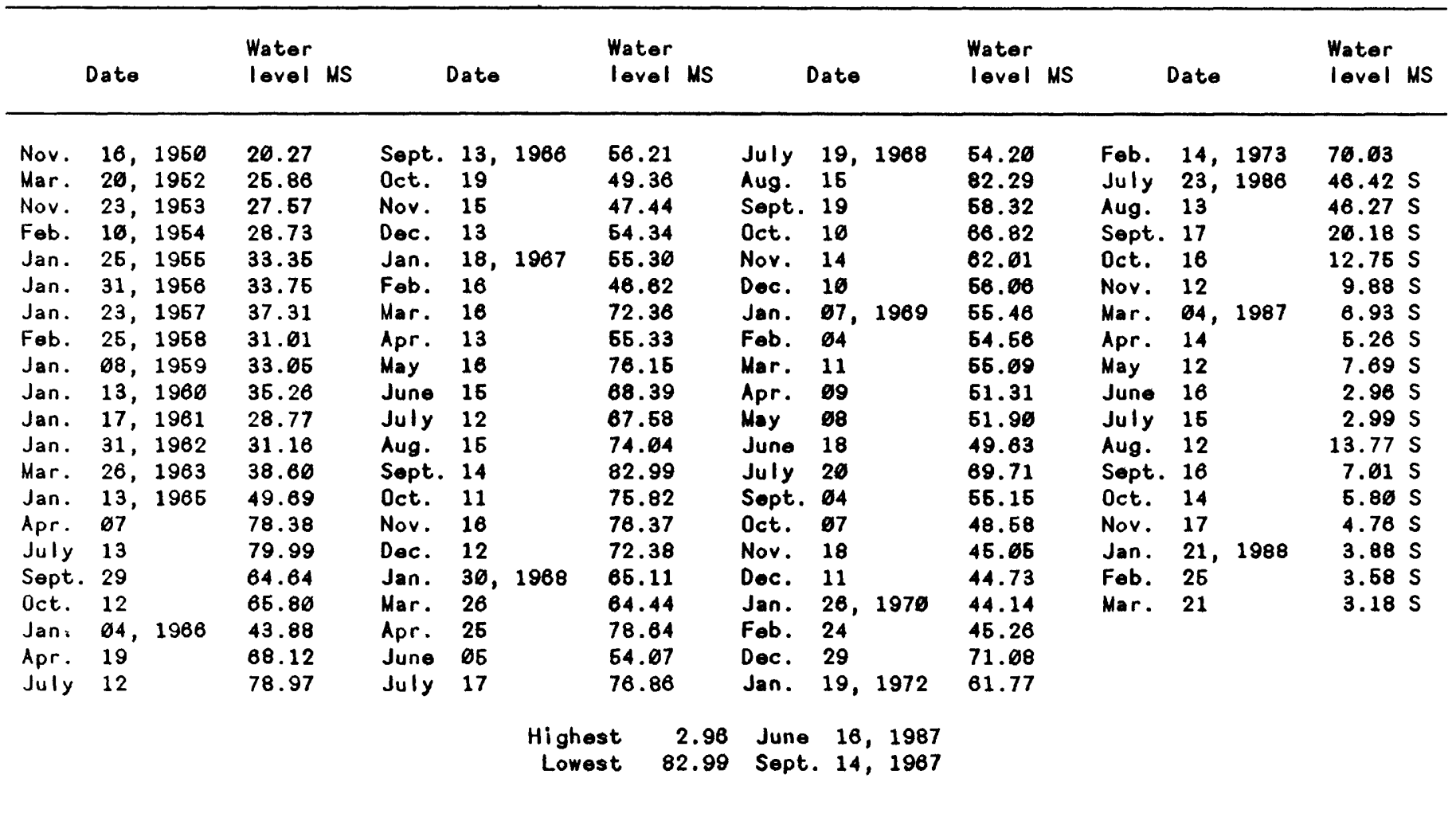

Index number on location map: 31

Station number: 343812099475501 Local number: O2N-25W-23 BAA 1

Location: Lat $34^{\circ} 38^{\prime} 12^{\prime \prime}$, long $99^{\circ} 47^{\prime} 66^{\prime \prime}$, hydrologic unit 11130101

Aquifor: 313 EMFK

Altitude of land-surface datum: $1,565 \mathrm{ft}$

Well depth: $185 \mathrm{ft}$

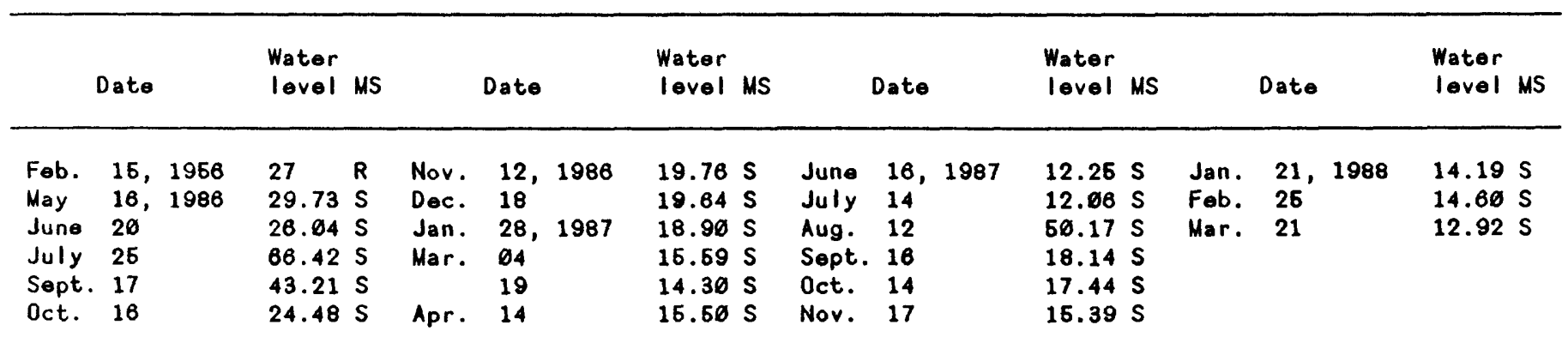

$$
\begin{array}{rrrrr}
\text { Highest } & 12.06 & \text { July } & 14,1987 \\
\text { Lowest } & 66.42 & \text { July } & 26,1986
\end{array}
$$


Table 2.--Monthly or periodic water-level measurements in selected wells in southwestern Oklahoma and northwestern Texas--Continued

\section{HARMON COUNTY, OKLAHOMA--Continued}

Index number on location map: 32

Station number: 843723099472461 Local number: O2N-25W-26 AAAA 1

Location: Lat $34^{\circ} 37^{\prime} 22^{\prime \prime}$, long $99^{\circ} 47^{\prime} 21^{\prime \prime}$, hydrologic unit 11130101

Aquifer: $3138 \mathrm{BLIN}$

Altitude of land-surface datum: $1,545 \mathrm{ft}$

Well depth: $104 \mathrm{ft}$

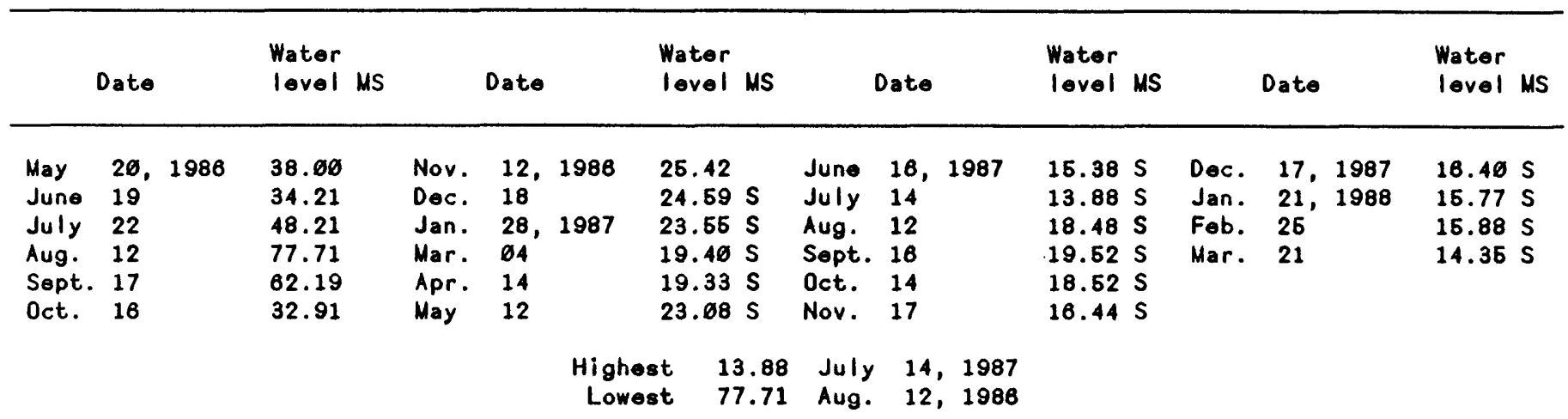

Index number on location map: 33

Station number: $\$ 43720699521201$ Local number: $62 \mathrm{~N}-25 \mathrm{~W}-30$ ABBB 1

Location: Lat $34^{\circ} 37^{\prime} 23^{\prime \prime}$, long $99^{\circ} 52^{\prime} 02^{\prime \prime}$, hydrologic unit 11138101

Aquifer: 313BLIN

Altitude of land-surface datum: $1,801 \mathrm{ft}$

Woll depth: $186 \mathrm{ft}$

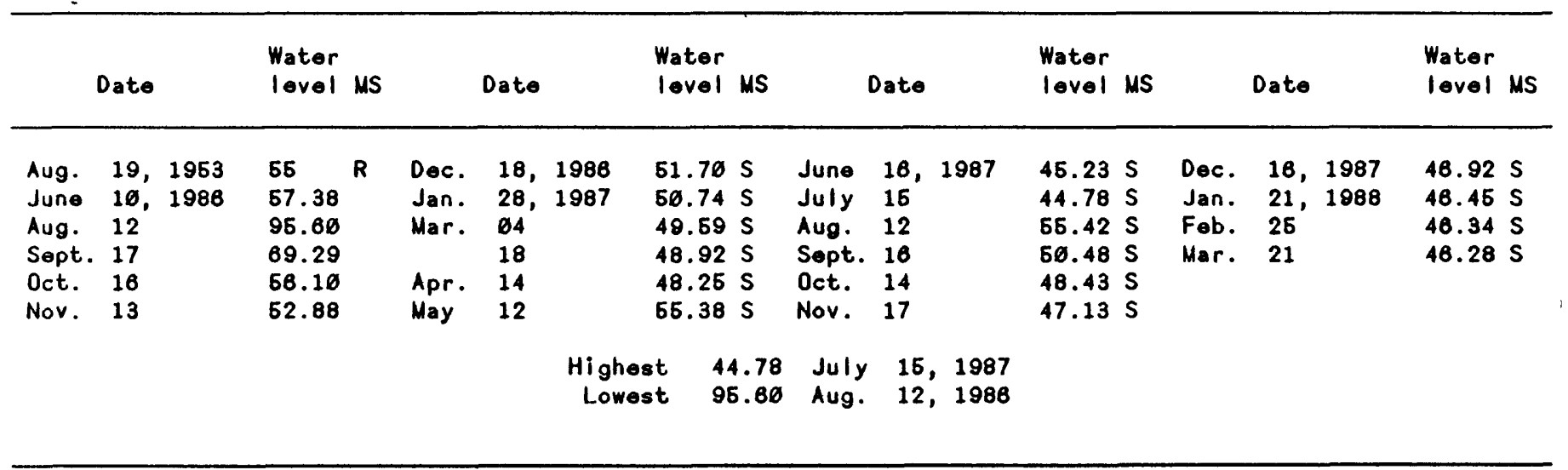

Index number on location map: 34

Station number: $\$ 43720099523001$ Local number: $\varnothing 2 \mathrm{~N}-25 W-30$ CCBB 1

Location: Lat $34^{\circ} 36^{\prime} 45^{\prime \prime}$, long $99^{\circ} 52^{\prime} 34^{\prime \prime}$, hydrologic unit 11130101

Aquifer: 313BLIN

Altitude of land-surface datum: 1,590 ft

Well depth: $175 \mathrm{ft}$

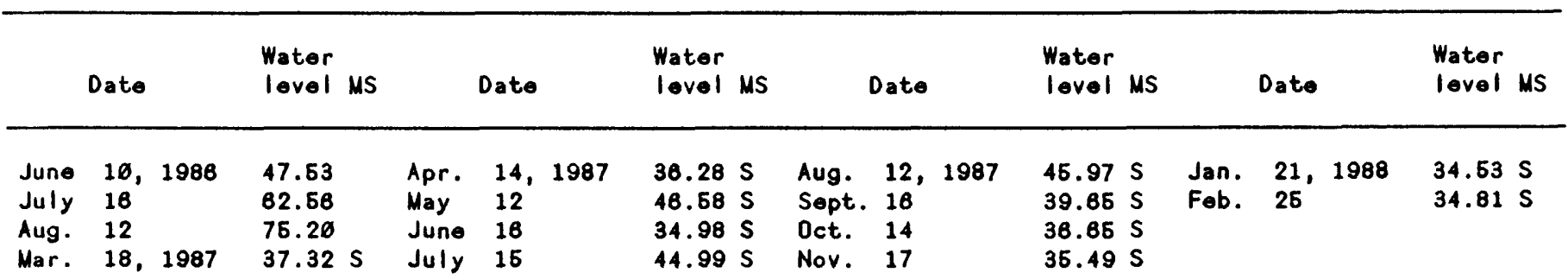


Table 2.--Monthly or periodic water-level measurements in selected wells in southwestern Oklahoma and northwestern Texas--Continued

HARMON COUNTY, OKLAHOMA--CONtinued

Index number on location map: 36

Station number: 344044099661401 Local number: $62 \mathrm{~N}-26 \mathrm{~W}-\varnothing 4$ ABC 1

Location: Lat $34^{\circ} 46^{\prime} 44^{\prime \prime}$, long $99^{\circ} 56^{\prime} 14^{\prime \prime}$, hydrologic unit 11136101

Aquifer: 313EMFK

Altitude of land-surface datum: $1,623 \mathrm{ft}$

Well depth: $220 \mathrm{ft}$

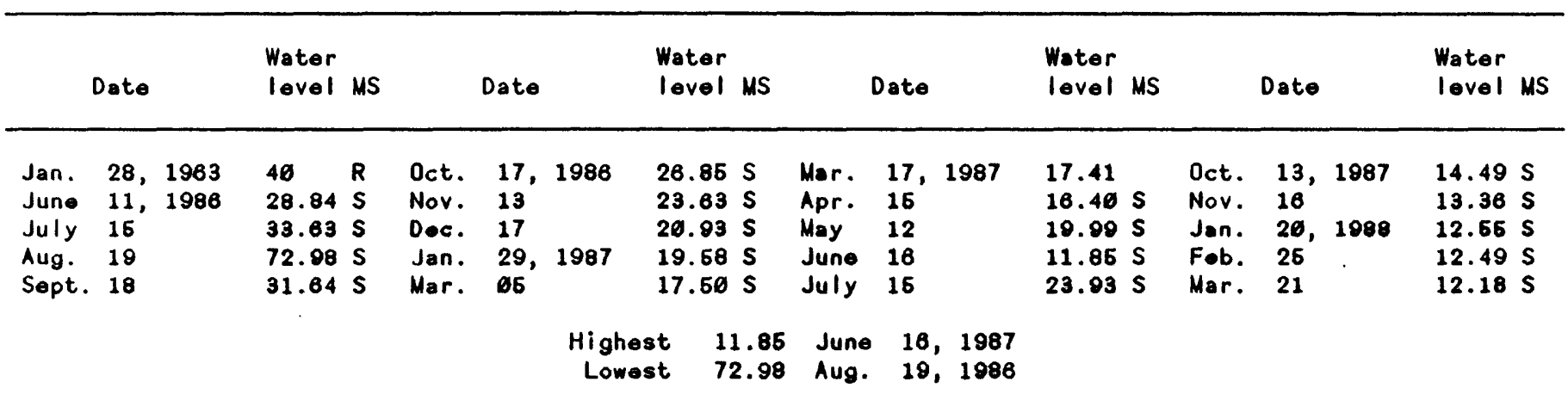


Table 2.--Monthly or periodic water-level measurements in selected wells in southwestern oklahoma and northwestern Texas--Continued

HARMON COUNTY, OKLAHOMA--CONTInUEd

Index number on location map: 36

Station number: $\$ 43612099674701$ Local number: $02 \mathrm{~N}-26 \mathrm{~W}-\varnothing 5$ BCCC 1

Location: Lat $34^{\circ} 40^{\prime} 28^{\prime \prime}$, long $99^{\circ} 57^{\prime} 52^{\prime \prime}$, hydrologic unit 11130101

Aquifor: $3138 \mathrm{LIN}$

Altitude of land-surface datum: $1,641 \mathrm{ft}$

Well depth: $149 \mathrm{ft}$

Remarks: Previously published as 02N-26W-05 BCC

\begin{tabular}{|c|c|c|c|c|c|c|c|c|c|c|c|c|c|c|c|c|}
\hline \multicolumn{3}{|c|}{ Date } & $\begin{array}{l}\text { Water } \\
\text { level MS }\end{array}$ & \multicolumn{3}{|c|}{ Date } & $\begin{array}{l}\text { Water } \\
\text { level MS }\end{array}$ & \multicolumn{3}{|c|}{ Date } & $\begin{array}{l}\text { Water } \\
\text { level MS }\end{array}$ & \multicolumn{3}{|c|}{ Date } & $\begin{array}{l}\text { Water } \\
\text { level }\end{array}$ & MS \\
\hline $\begin{array}{l}\text { Nov. } \\
\text { Apr. } \\
\text { Mar. } \\
\text { Nov. } \\
\text { Jan. } \\
\text { Fob. } \\
\text { Aug. } \\
\text { Oct. } \\
\text { Dec. } \\
\text { Jan. } \\
\text { Fob. } \\
\text { June } \\
\text { Jan. } \\
\text { Fob. } \\
\text { Mar. } \\
\text { Dec. } \\
\text { Jan. } \\
\text { Fob. } \\
\text { Apr. } \\
\text { May } \\
\text { June } \\
\text { July } \\
\text { Oct. } \\
\text { Nov. } \\
\text { Dec. } \\
\text { Fob. } \\
\text { Mar. } \\
\text { Apr. } \\
\text { May } \\
\text { June } \\
\text { July } \\
\text { Jan. } \\
\text { Mar. } \\
\text { June }\end{array}$ & $\begin{array}{l}31, \\
21 \\
20 \\
18 \\
23, \\
28 \\
04 \\
07 \\
28 \\
25 \\
23 \\
01 \\
29 \\
19 \\
17 \\
25, \\
25 \\
22 \\
20 \\
24 \\
29 \\
27, \\
24 \\
30\end{array}$ & $\begin{array}{l}1950 \\
1951 \\
1952 \\
1953 \\
1954\end{array}$ & $\begin{array}{l}32.47 \\
30.49 \\
30.23 \\
52.49 \\
41.65 \\
40.00 \\
37.8 \\
37.8 \\
40.0 \\
39.48 \\
39.25 \\
40.07 \\
49.62 \\
47.34 \\
47.20 \\
47.46 \\
46.83 \\
42.68 \\
37.76 \\
44.04 \\
50.72 \\
36.60 \\
41.86 \\
39.92 \\
38.14 \\
37.20 \\
37.38 \\
37.65 \\
37.63 \\
36.69 \\
37.05 \\
46.70 \\
44.20\end{array}$ & $\begin{array}{l}\text { Oct. } \\
\text { Nov. } \\
\text { Jan. } \\
\text { Mar. } \\
\text { Apr. } \\
\text { May } \\
\text { June } \\
\text { July } \\
\text { Aug. } \\
\text { Nov. } \\
\text { Jan. } \\
\text { Fob. } \\
\text { Mar. } \\
\text { Apr. } \\
\text { May } \\
\text { June } \\
\text { July } \\
\text { Oct. } \\
\text { Nov. } \\
\text { Dec. } \\
\text { Mar. } \\
\text { June } \\
\text { Jan. } \\
\text { Apr. } \\
\text { July } \\
\text { Sept. } \\
\text { Oct. } \\
\text { Jan. } \\
\text { Apr. } \\
\text { July } \\
\text { Sept. } \\
\text { Oct. } \\
\text { Nov. } \\
\text { Dec. } \\
\text { Jan. }\end{array}$ & $\begin{array}{l}20, \\
19 \\
13, \\
22 \\
19 \\
17 \\
21 \\
19 \\
16 \\
15 \\
17, \\
14 \\
14 \\
19 \\
17 \\
21 \\
12 \\
24 \\
14 \\
05 \\
67, \\
28 \\
27 \\
13, \\
67 \\
13 \\
28 \\
12 \\
04, \\
19 \\
12 \\
13 \\
19 \\
16 \\
13 \\
18,\end{array}$ & $\begin{array}{l}1969 \\
1960 \\
1961\end{array}$ & $\begin{array}{r}54.69 \\
49.44 \\
46.54 \\
41.43 \\
52.42 \\
51.74 \\
43.72 \\
41.50 \\
51.90 \\
38.59 \\
34.6 \varnothing \\
32.56 \\
31.76 \\
43.98 \\
43.4 \varnothing \\
35.09 \\
42.34 \\
44.21 \\
41.14 \\
39.05 \\
39.36 \\
53.82 \\
44.68 \\
69.23 \\
121.41 \\
120.88 \\
120.91 \\
106.90 \\
73.93 \\
140.23 \\
143.42 \\
112.05 \\
82.95 \\
79.90 \\
73.17 \\
72.21\end{array}$ & $\begin{array}{l}\text { Feb. } \\
\text { Mar. } \\
\text { Apr. } \\
\text { May } \\
\text { June } \\
\text { July } \\
\text { Aug. } \\
\text { Sept. } \\
\text { Oct. } \\
\text { Nov. } \\
\text { Dec. } \\
\text { Jan. } \\
\text { Feb. } \\
\text { Mar. } \\
\text { Apr. } \\
\text { June } \\
\text { July } \\
\text { Aug. } \\
\text { Sept. } \\
\text { Oct. } \\
\text { Nov. } \\
\text { Dec. } \\
\text { Jan. } \\
\text { Fob. } \\
\text { Mar. } \\
\text { Apr. } \\
\text { June } \\
\text { July } \\
\text { Sept. } \\
\text { Oct. } \\
\text { Nov. } \\
\text { Dec. } \\
\text { Jan. } \\
\text { Fob. }\end{array}$ & $\begin{array}{l}16, \\
16 \\
13 \\
16 \\
16 \\
12 \\
15 \\
14 \\
11 \\
16 \\
12 \\
30, \\
20 \\
26 \\
25 \\
05 \\
19 \\
17 \\
16 \\
19 \\
10 \\
14 \\
10 \\
07, \\
04 \\
11 \\
08 \\
09 \\
18 \\
20 \\
04 \\
07 \\
18 \\
11 \\
26, \\
24\end{array}$ & $197 \varnothing$ & $\begin{array}{r}73.44 \\
83.63 \\
142.42 \\
103.93 \\
87.20 \\
89.78 \\
147.16 \\
121.70 \\
90.60 \\
88.88 \\
82.80 \\
72.95 \\
70.38 \\
67.86 \\
86.93 \\
69.24 \\
67.46 \\
70.68 \\
168.20 \\
82.72 \\
76.43 \\
81.32 \\
71.86 \\
68.63 \\
66.62 \\
65.12 \\
69.83 \\
63.28 \\
63.62 \\
95.67 \\
87.69 \\
72.70 \\
67.98 \\
85.08 \\
61.93 \\
80.36\end{array}$ & $\begin{array}{l}\text { Dec. } \\
\text { Jan. } \\
\text { Jan. } \\
\text { Dec. } \\
\text { Mar. } \\
\text { Mar. } \\
\text { Feb. } \\
\text { Mar. } \\
\text { Mar. } \\
\text { Jan. } \\
\text { Jan. } \\
\text { Jan. } \\
\text { Jan. } \\
\text { May } \\
\text { June } \\
\text { July } \\
\text { Aug. } \\
\text { Sept. } \\
\text { Oct. } \\
\text { Nov. } \\
\text { Dec. } \\
\text { Jan. } \\
\text { Mar. } \\
\\
\text { Apr. } \\
\text { May } \\
\text { June } \\
\text { July } \\
\text { Aug. } \\
\text { Sept. } \\
\text { Oct. } \\
\text { Nov. } \\
\text { Dec. } \\
\text { Jan. } \\
\text { Fob. } \\
\text { Mar. }\end{array}$ & $\begin{array}{l}29, \\
97, \\
29, \\
27 \\
69, \\
13, \\
28, \\
11, \\
99, \\
26, \\
16, \\
16, \\
96, \\
15 \\
18 \\
23 \\
13 \\
18 \\
17 \\
13 \\
17 \\
29, \\
96 \\
17 \\
14 \\
12 \\
16 \\
16 \\
11 \\
16 \\
13 \\
16 \\
16 \\
29, \\
23 \\
21\end{array}$ & $\begin{array}{l}1970 \\
1976 \\
1976 \\
1978 \\
1979 \\
1980 \\
1981 \\
1982 \\
1983 \\
1984 \\
1986 \\
1986\end{array}$ & $\begin{array}{l}93.05 \\
61.90 \\
48.32 \\
48.39 \\
44.84 \\
52.82 \\
57.27 \\
61.04 \\
61.98 \\
58.39 \\
53.80 \\
65.65 \\
51.88 \\
50.02 \\
45.30 \\
53.96 \\
67.20 \\
50.93 \\
44.59 \\
40.45 \\
37.66 \\
35.75 \\
33.76 \\
32.85 \\
32.10 \\
38.81 \\
29.10 \\
33.20 \\
44.94 \\
32.44 \\
31.03 \\
28.99 \\
28.80 \\
27.82 \\
27.42 \\
26.93\end{array}$ & 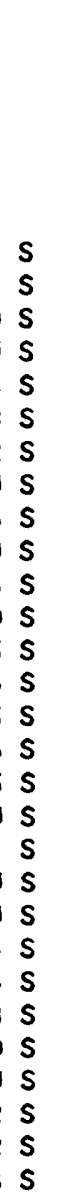 \\
\hline
\end{tabular}

Highest 20.24 Nov. 16,1950

Lowest 147.16 Aug. 16, 1987 
Table 2.--Monthly or periodic water-level measurements in selected wells in southwestern Oklahoma and northwestern Texas--Continued

HARMON COUNTY, OKLAHOMA--Continued

Index number on location map: 37

Station number: $\$ 44612699575161$ Local number: $62 \mathrm{~N}-26 \mathrm{~W}-65$ CCBB 1

Location: Lat $34^{\circ} 40^{\prime} 12^{\prime \prime}$, long $99^{\circ} 57^{\prime} 61^{\prime \prime}$, hydrologic unit 11136101

Aquifer: 313BLIN

Altitude of land-surface datum: $1,638 \mathrm{ft}$

Well depth: $240 \mathrm{ft}$

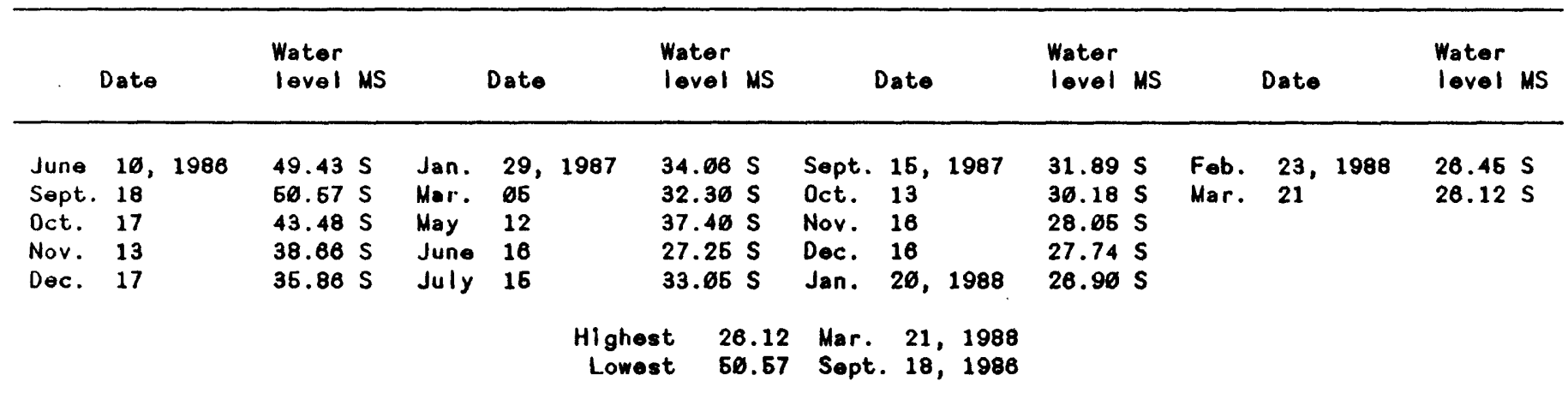

Index number on location map: 38

Station number: 344002099676301 Local number: 62N-26W-65 DDCC 1

Location: Lat $34^{\circ} 40^{\prime} 02^{\prime \prime}$, long $99^{\circ} 57^{\prime} 63^{\prime \prime}$, hydrologic unit 11130101

Aquifer: $3138 \mathrm{LIN}$

Altitude of land-surface datum: $1,830 \mathrm{ft}$

Well depth: $120 \mathrm{ft}$

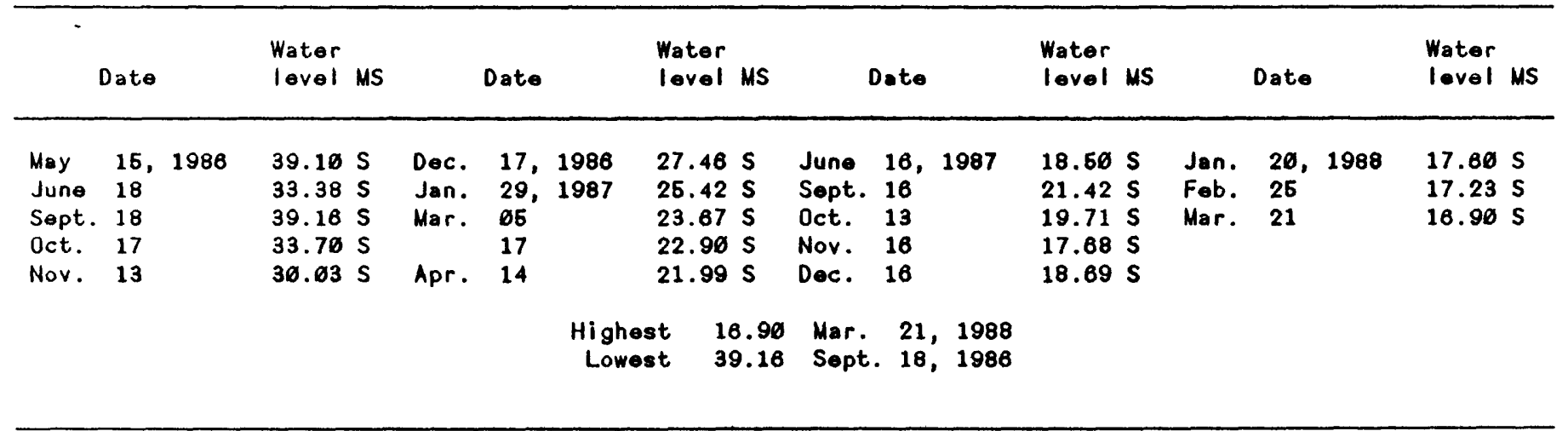

Index number on location map: 39

Station number: 343946099674961 Local number: $62 \mathrm{~N}-26 \mathrm{~W}-68$ BCBB 1

Location: Lat $34^{\circ} 39^{\prime} 46^{\prime \prime}$, long $99^{\circ} 67^{\prime} 49^{\prime \prime}$, hydrologic unit 11130161

Aquifer: 313BLIN

Altitude of land-surface datum: $1,643.8 \mathrm{ft}$

Well depth: $174 \mathrm{ft}$

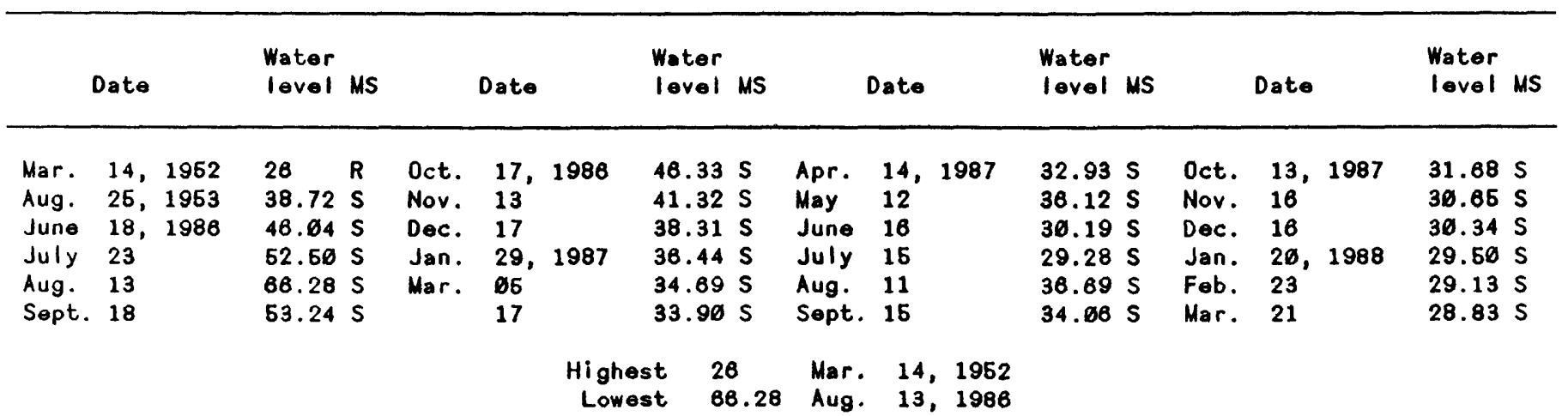


Table 2.-- Monthly or periodic water-level measurements in selected wells in southwestern oklahoma and northwestern Texas--Continued

HARMON COUNTY, OKLAHOMA--CONtinued

Index number on location map: 40

Station number: $344 \varnothing \varnothing \varnothing \varnothing 99523901$ Local number: $02 \mathrm{~N}-28 \mathrm{~W}-12$ AAAA 1

Location: Lat $34^{\circ} 46^{\prime} 60^{\prime \prime}$, I ong $99^{\circ} 52^{\prime} 39^{\prime \prime}$, hydrologic unit 11130101

Aquifer: 3138 LIN

Altitude of land-surface datum: $1,602 \mathrm{ft}$

Well depth: $200 \mathrm{ft}$

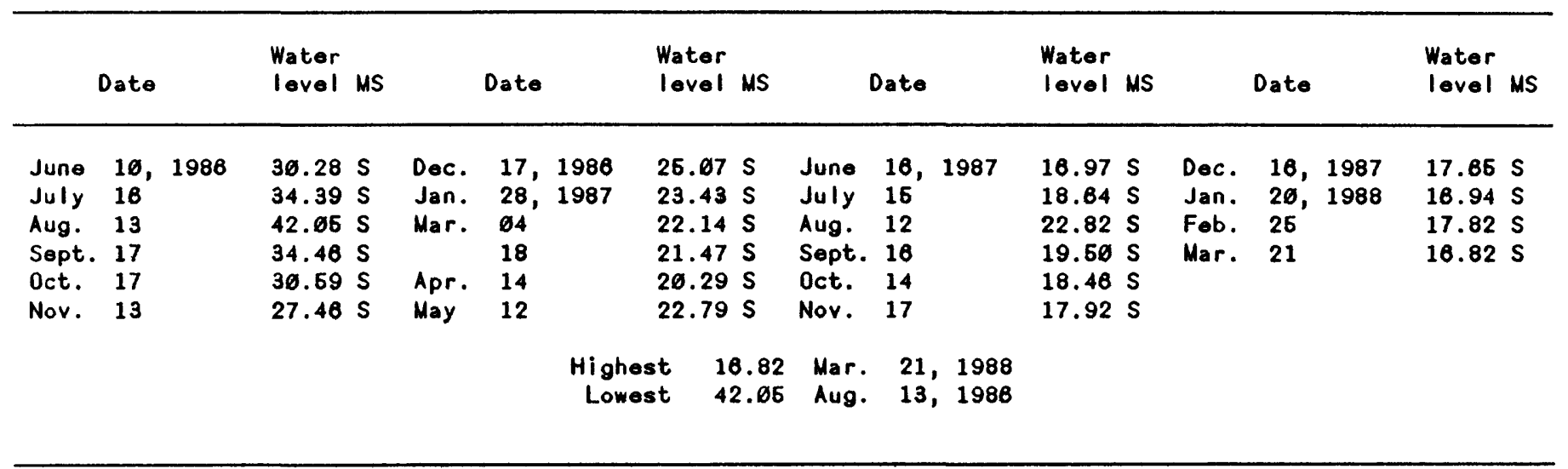

Index number on location map: 41

Station number: $\$ 43847099543801$ Local number: $\$ 2 \mathrm{~N}-26 \mathrm{~W}-14$ CCCC 1

Location: Lat $34^{\circ} 38^{\prime} 27^{\prime \prime}$, long $99^{\circ} 54^{\prime} 38^{\prime \prime}$, hydrologic unit 11130101

Aquifer: $313 B L I N$

Altitude of land-surface datum: $1,643 \mathrm{ft}$

Well depth: $66 \mathrm{ft}$

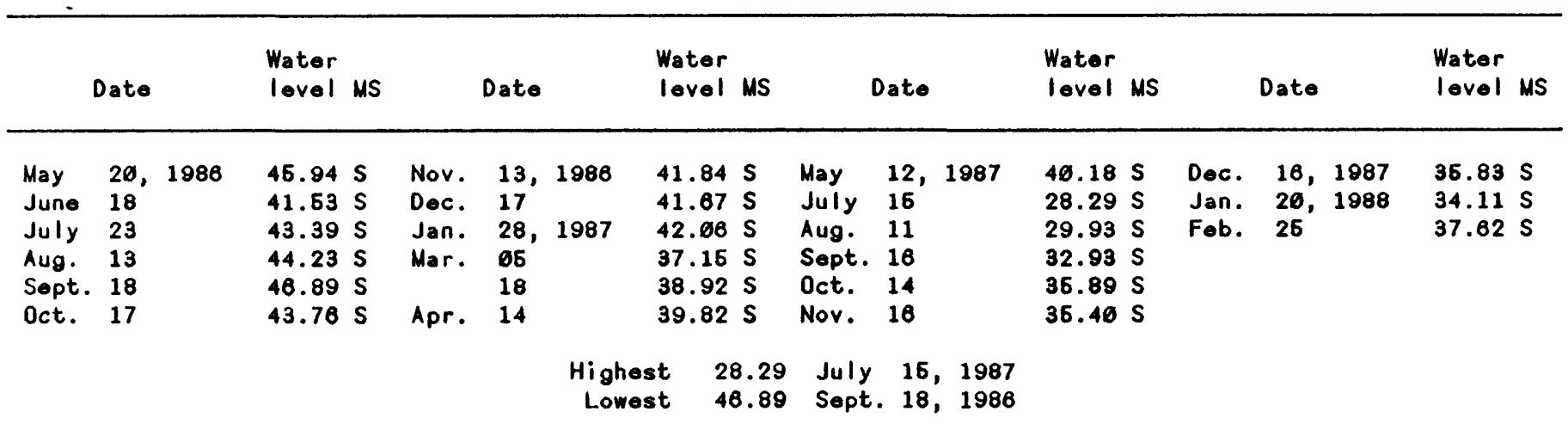


Table 2.--Nonthly or periodic water-level measurements in selected wells in southwestern Oklahoma and northwestern Texas--Continued

HARMON COUNTY, OKLAHOMA--CONtinUEd

Index number on location map: 42

Station number: 343865099644601 Local number: 62N-26W-16 AADD 1

Location: Lat $34^{\circ} 38^{\prime} 55^{\prime \prime}$, long $99^{\circ} 54^{\prime} 45^{\prime \prime}$, hydrologic unit 11136101

Aquifer: 313 BLIN

Altitude of land-surface datum: $1,665 \mathrm{ft}$

Well depth: $130 \mathrm{ft}$

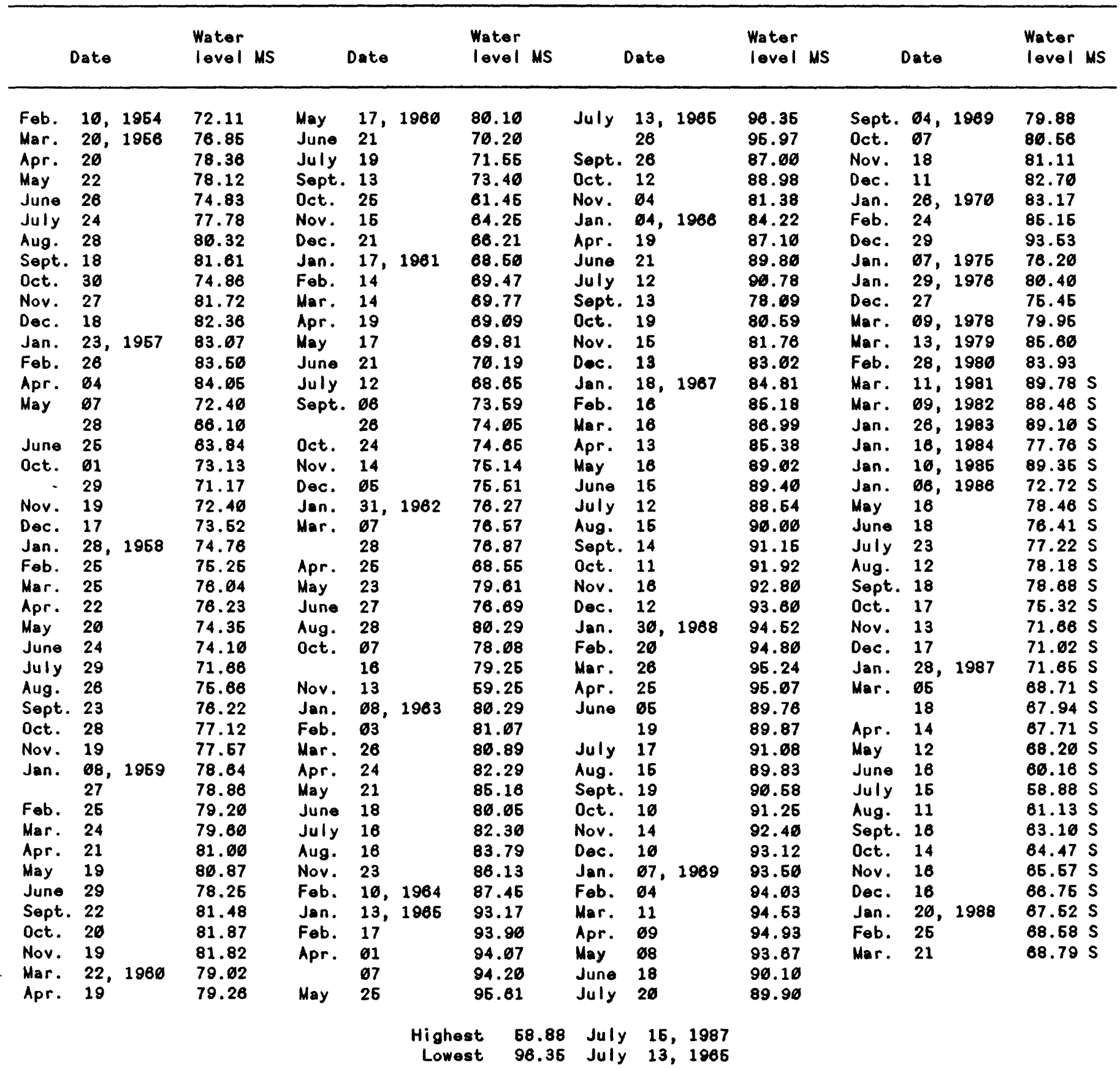


Table 2.-- Monthly or periodic water-level measurements in selected wells in southwestern oklahoma and northwestern Texas--Continued

HARMON COUNTY, OKLAHOMA--Continued

Index number on location map: 43

Station number: 343908999562201 Local number: $02 \mathrm{~N}-26 \mathrm{~W}-16$ BAAB 1

Location: Lat $34^{\circ} 39^{\prime} 98^{\prime \prime}$, long $99^{\circ} 56^{\prime} 22^{\prime \prime}$, hydrologic unit 11130101

Aquifer: $313 B L I N$

Altitude of land-surface datum: $1,648 \mathrm{ft}$

Well depth: $81 \mathrm{ft}$

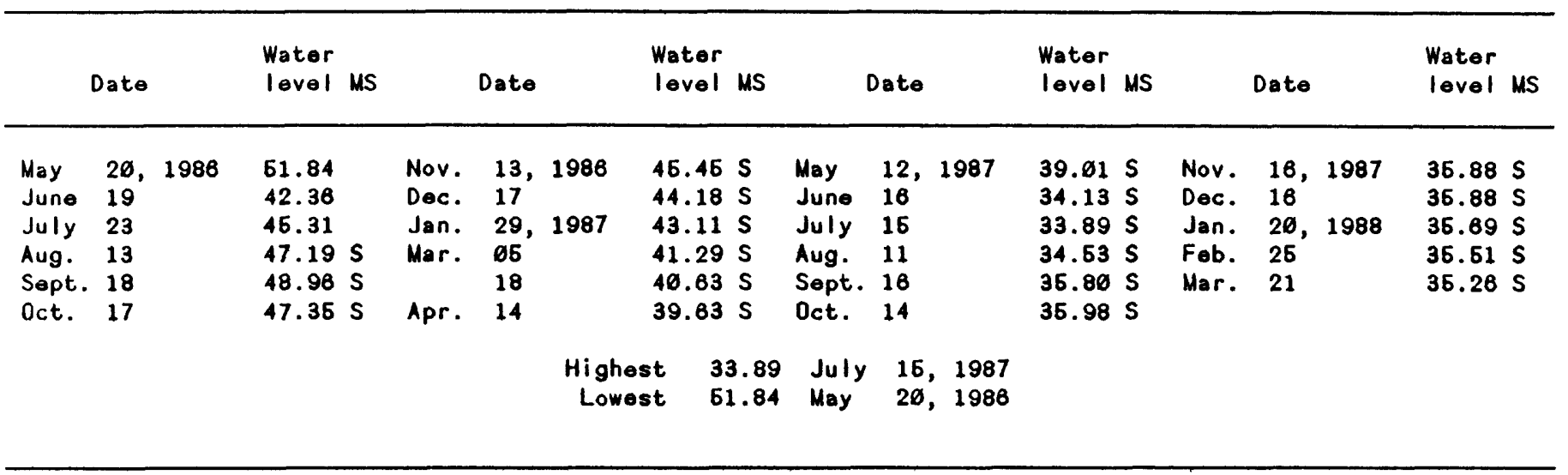

Index number on location map: 44

Station number: 343868099681701 Local number: $62 N-26 W-18$ ACBA 1

Location: Lat $34^{\circ} 38^{\prime} 66^{\prime \prime}$, long $99^{\circ} 68^{\prime} 17^{\prime \prime}$, hydrologic unit 11130101

Aquifer: $313 B L I N$

Altitude of land-surface datum: $1,687 \mathrm{ft}$

Well depth: $145 \mathrm{ft}$

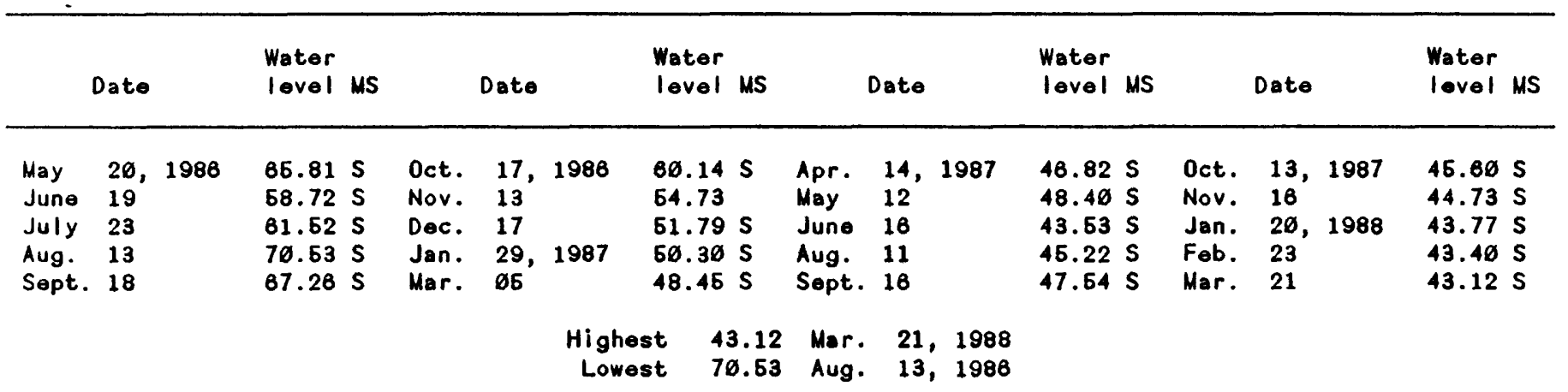


Table 2.--Nonthly or periodic water-level measurements in selected wells in southwestern oklahoma and northwestern Texas--Continued

HARMON COUNTY, OKLAHOMA--CONtinued

Index number on location map: 45

Station number: $\$ 43559899534901$ Local number: $22 \mathrm{~N}-26 \mathrm{~W}-31$ CBCD 1

Location: Lat $34^{\circ} 36^{\prime} 56^{\prime \prime}$, long $99^{\circ} 58^{\prime} 60^{\prime \prime}$, hydrologic unit 11126165

Aquifer: $3138 L I N$

Altitude of land-surface datum: $1,590 \mathrm{ft}$

Well depth: $150 \mathrm{ft}$

Remarks: Previously published as $62 \mathrm{~N}-26 \mathrm{~W}-31 \mathrm{CBC}$

\begin{tabular}{|c|c|c|c|c|c|c|c|c|c|c|c|c|c|c|c|c|}
\hline \multicolumn{3}{|c|}{ Date } & \multirow{2}{*}{$\begin{array}{l}\begin{array}{l}\text { Water } \\
\text { level MS }\end{array} \\
31.51\end{array}$} & \multicolumn{3}{|c|}{ Date } & \multirow{2}{*}{$\begin{array}{l}\begin{array}{l}\text { Water } \\
\text { level MS }\end{array} \\
30.50\end{array}$} & \multicolumn{3}{|c|}{ Date } & \multirow{3}{*}{$\begin{array}{l}\begin{array}{l}\text { Water } \\
\text { level MS }\end{array} \\
34.95 \\
32.29\end{array}$} & \multicolumn{3}{|c|}{ Date } & \multirow[t]{2}{*}{$\begin{array}{l}\text { Water } \\
\text { level }\end{array}$} & \multirow[t]{2}{*}{ MS } \\
\hline Nov. & 16 & 1960 & & July & 29 & 1958 & & May & 21 & 1963 & & Mar. & 11 & 1969 & & \\
\hline Mar. & 20 & 1952 & 33.95 & Aug. & 26 & & 39.18 & June & 18 & & & Apr. & 69 & & 32.63 & \\
\hline Mar. & 20 & 1953 & 34.81 & Sept. & 23 & & 38.22 & Aug. & 16 & & 48.49 & May & 68 & & 32.97 & \\
\hline Oct. & $20^{\circ}$ & & 35.32 & Oct. & 28 & & 35.16 & Nov. & 23 & & 34.63 & June & 18 & & 33.63 & \\
\hline Jan. & 26 & 1954 & 33.82 & Nov. & 19 & & 33.86 & Feb. & 10 & 1984 & 33.30 & Sopt. & 64 & & 36.33 & \\
\hline Mar. & $25^{\circ}$ & & 33.90 & Jan. & $\varnothing 8$, & 1959 & 32.90 & Jan. & 13 & 1965 & 34.33 & oct. & 07 & & 34.27 & \\
\hline Apr. & 21 & & $33.6 \varnothing$ & & 27 & & 33.73 & Fab. & 17 & & 35.62 & Nov. & 18 & & 33.70 & \\
\hline May & 27 & & 32.32 & Feb. & 24 & & 33.50 & Apr. & 01 & & 34.58 & Dec. & 11 & & 33.62 & \\
\hline June & 23 & & 32.48 & Apr. & 21 & & 33.93 & & 67 & & 34.33 & Jan. & 28 & 1970 & 33.14 & \\
\hline oct. & 26 & & 35.73 & May & 19 & & 33.69 & May & 26 & & 35.01 & Føb. & 24 & & 33.68 & \\
\hline Dec. & $\varnothing 2$ & & 35.04 & June & 29 & & 33.44 & June & 13 & & 40.77 & Dec. & 29 & & 36.10 & \\
\hline Jan. & $\varnothing 6$, & 1955 & 44.91 & Sept. & 22 & & 46.45 & July & 26 & & 35.61 & Jan. & 67 , & 1976 & 33.49 & \\
\hline & 25 & & 44.99 & oct. & 20 & & 34.92 & Sept. & 26 & & 36.37 & Jan. & 29 & 1976 & 32.71 & \\
\hline Feb. & 98 & & 34.41 & Nov. & 19 & & 34.62 & Dct. & 12 & & 35.83 & Dec. & $28^{\circ}$ & & 33.17 & \\
\hline & 22 & & 34.98 & Jan. & 13 & 1960 & 33.35 & Nor. & 04 & & 34.71 & Mar. & 09, & 1978 & 33.62 & \\
\hline Mar. & 22 & & 34.42 & Mar. & 22 & & 31.87 & Jan. & 64, & 1986 & 33.80 & Mar. & 13, & 1979 & 34.37 & \\
\hline May & 24 & & 34.65 & Apr. & 19 & & 36.14 & Apr. & 19 & & 37.86 & Fob. & 28 & 1980 & 34.06 & \\
\hline June & 23 & & 27.90 & May & 17 & & 41.20 & June & 21 & & 34.10 & Mar. & 11 , & 1981 & 36.34 & $s$ \\
\hline July & 28 & & 31.29 & June & 21 & & 36.49 & July & 12 & & 38.85 & Mar. & 69, & 1982 & 34.55 & $S$ \\
\hline Sept. & 29 & & 34.64 & July & 19 & & 30.66 & Sept. & 13 & & 34.22 & Jan. & 26 & 1983 & 35.01 & $S$ \\
\hline $0 \mathrm{ct}$. & 25 & & 32.93 & Aug. & 16 & & 39.26 & oct. & 19 & & 31.62 & Jan. & 16 & 1984 & 33.34 & $\mathrm{~S}$ \\
\hline Nov. & 29 & & 32.87 & Sept. & 13 & & 33.72 & Nov. & 15 & & 32.18 & Jan. & 10 & 1986 & 36.23 & $S$ \\
\hline Dec. & 22 & & 32.35 & Nov. & 16 & & 30.45 & Dec. & 13 & & 32.20 & Jan. & 66 , & 1986 & 32.67 & $S$ \\
\hline Jan. & 31 & 1956 & 32.18 & Dec. & 21 & & 30.66 & Jan. & 18 & 1967 & 32.66 & May & 16 & & 33.62 & \\
\hline Mar. & 20 & & 34.74 & Jan. & 17, & 1961 & 30.35 & Feb. & 16 & & 32.51 & June & 19 & & 31.98 & $\mathrm{~S}$ \\
\hline May & 22 & & 39.05 & $F e b$. & $14^{\circ}$ & & 30.33 & Mar. & 16 & & 34.46 & July & 26 & & 49.91 & SR \\
\hline June & 26 & & 32.81 & Mar. & 14 & & 30.34 & Apr. & 13 & & 37.69 & Sept. & 18 & & 34.76 & $S$ \\
\hline July & 23 & & 34.98 & Apr. & 19 & & 31.16 & May & 16 & & 33.09 & Oct. & 17 & & 31.30 & $S$ \\
\hline Sept. & 18 & & 47.00 & May & 17 & & 30.96 & June & 15 & & 32.81 & Nov. & 13 & & 30.08 & \\
\hline oct. & $3 \emptyset$ & & 35.70 & June & 21 & & 30.59 & July & 12 & & 33.71 & Dec. & 17 & & 29.78 & $S$ \\
\hline Nov. & 27 & & 34.89 & July & 12 & & 30.80 & Sept. & 14 & & 35.00 & Jan. & 29 , & 1987 & 31.59 & $S$ \\
\hline Dec. & 18 & & 34.71 & Sept. & 86 & & 33.36 & Oct. & 11 & & 34.68 & Mar. & 65 & & 29.01 & $S$ \\
\hline Jan. & 23 & 1957 & 34.32 & & 26 & & 32.45 & Nov. & 16 & & 34.37 & & 18 & & 28.89 & $\mathbf{S}$ \\
\hline Feb. & 26 & & 32.11 & Oct. & 24 & & 34.98 & Dec. & 12 & & 33.90 & Apr. & 14 & & 29.12 & $\mathbf{S}$ \\
\hline Apr. & 04 & & 33.92 & Nov. & 21 & & 33.62 & Jan. & 30 & 1968 & 33.74 & May & 12 & & 35.69 & $\mathbf{S}$ \\
\hline May & 67 & & 31.78 & Dec. & 65 & & 32.23 & Feb. & 20 & & 34.47 & June & 16 & & 27.79 & $\mathbf{S}$ \\
\hline & 28 & & 30.44 & Jan. & 31 & 1962 & 31.58 & Mar. & 26 & & 33.25 & Juity & 15 & & 35.82 & $S$ \\
\hline June & 25 & & 28.67 & Mar. & 07 & & 31.64 & Apr. & 25 & & 42.38 & Aug. & 11 & & 35.98 & $s$ \\
\hline Oct. & 01 & & 33.82 & & 28 & & 32.34 & June & 85 & & 33.30 & Sept. & 15 & & 32.32 & $S$ \\
\hline & 29 & & 32.82 & May & 23 & & 32.91 & & 19 & & 32.34 & Oct. & 13 & & 31.59 & $S$ \\
\hline Nov. & 19 & & 32.55 & June & 27 & & 31.23 & July & 17 & & 32.62 & Nov. & 16 & & 31.28 & $S$ \\
\hline Dec. & 17 & & 32.30 & Aug. & 28 & & 43.13 & Aug. & 15 & & 43.60 & Dec. & 16 & & 30.27 & $S$ \\
\hline Jan. & 28 & 1958 & 32.20 & oct. & 64 & & 32.45 & Sept. & 19 & & 34.47 & Jan. & 20 & 1988 & 30.90 & $S$ \\
\hline Feb. & 26 & & 32.02 & & 16 & & 32.26 & Oct. & 10 & & 33.29 & Feb. & 23 & & 30.81 & $S$ \\
\hline Mar. & 25 & & 31.83 & Nov. & 13 & & 27.40 & Nov. & 14 & & 33.93 & Mar. & 22 & & 30.49 & $S$ \\
\hline Apr. & 22 & & 31.48 & Jan. & 08, & 1983 & 31.22 & Dec. & 10 & & 33.31 & & & & & \\
\hline May & 20 & & 31.10 & Feb. & 96 & & 31.24 & Jan. & 07, & 1969 & 32.10 & & & & & \\
\hline June & 24 & & 31.70 & Mar. & 26 & & 32.16 & Feb. & 64 & & 33.30 & & & & & \\
\hline & & & & & & Low & $\begin{array}{l}27.40 \\
48.49\end{array}$ & $\begin{array}{l}\text { Nov. } \\
\text { Aug. }\end{array}$ & $\begin{array}{l}13, \\
16,\end{array}$ & $\begin{array}{l}1982 \\
1983\end{array}$ & & & & & & \\
\hline
\end{tabular}


Table 2.-- Nonthly or periodic water-level measurements in selected wells in southwestern Oklahoma and northwestern Texas--Continued

HARMON COUNTY, OKLAHOMA--CONtinued

Index number on location map: 46

Station number: 843547099580101 Local number: 62N-26W-31 DDDA 1

Location: Lat $34^{\circ} 35^{\prime} 44^{\prime \prime}$, long $99^{\circ} 57^{\prime} 54^{\prime \prime}$, hydrologic unit 11130101

Aquifer: 313BLIN

Altitude of land-surface datum: $1,699 \mathrm{ft}$

Well depth: $129 \mathrm{ft}$

\begin{tabular}{|c|c|c|c|c|c|c|c|c|c|c|c|}
\hline & Date & $\begin{array}{l}\text { Water } \\
\text { level MS }\end{array}$ & & Date & & $\begin{array}{l}\text { Water } \\
\text { level MS }\end{array}$ & Date & $\begin{array}{l}\text { Water } \\
\text { level MS }\end{array}$ & & Date & $\begin{array}{l}\text { Water } \\
\text { lovel MS }\end{array}$ \\
\hline \multirow[t]{2}{*}{$\begin{array}{l}\text { June } \\
\text { July }\end{array}$} & $\begin{array}{l}11,1988 \\
17\end{array}$ & $\begin{array}{l}60.03 \\
61.99\end{array}$ & Aug. & $\begin{array}{l}07, \\
12\end{array}$ & 1986 & $\begin{array}{l}70.72 \\
65.33\end{array}$ & $\begin{array}{l}\text { Aug. 19, } 1988 \\
\text { Sept. } 18\end{array}$ & $\begin{array}{l}72.18 \\
61.93\end{array}$ & $\begin{array}{l}\text { Oct. } \\
\text { Nov. }\end{array}$ & $\begin{array}{l}17,1986 \\
13\end{array}$ & $\begin{array}{l}68.91 \\
67.70\end{array}$ \\
\hline & & & & & $\begin{array}{l}\text { High } \\
\text { Low }\end{array}$ & $\begin{array}{l}57.70 \\
72.18\end{array}$ & $\begin{array}{l}\text { Nov. } 13,1986 \\
\text { Aug. } 19,1986\end{array}$ & & & & \\
\hline
\end{tabular}


Table 2.--Monthly or periodic water-level measurements in selected wells in southwestern Oklahoma and northwestern Texas--Continued

HARMON COUNTY, OKLAHOMA--CONtinUEd

Index number on location map: 47

Station number: 344016099694601 Local number: $02 \mathrm{~N}-27 W-01$ CCBB 1

Location: Lat $34^{\circ} 40^{\prime} 13^{\prime \prime}$, long $99^{\circ} 59^{\prime} 68^{\prime \prime}$, hydrologic unit 11130101

Aquifer: $3138 L I N$

Altitude of land-surface datum: $1,660 \mathrm{ft}$

Well depth: $150 \mathrm{ft}$

Remarks: Previously published as $02 \mathrm{~N}-27 \mathrm{~W}-81 \mathrm{CCB}$

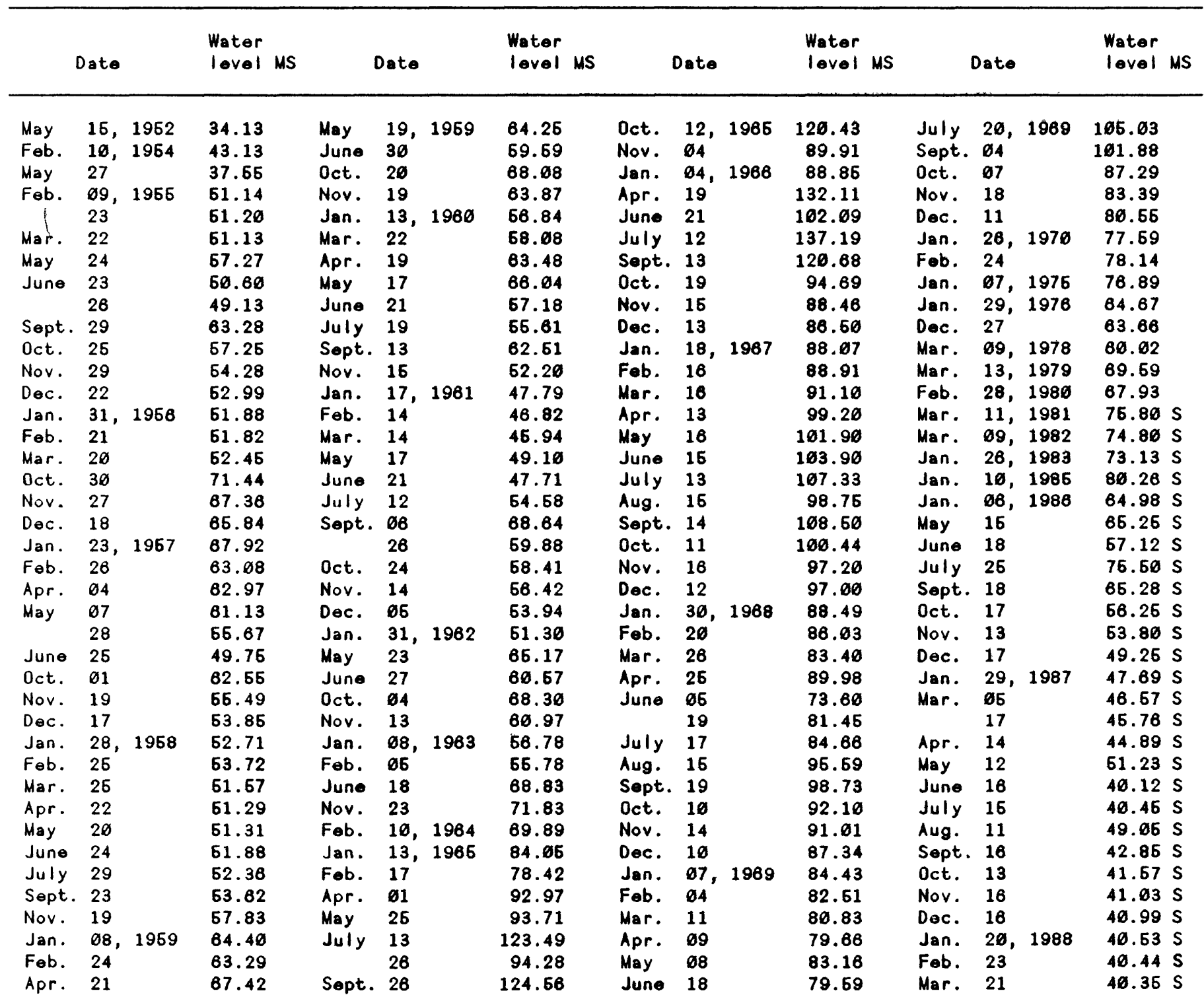

Highest 34.13 May 16,1952

Lowest 137.19 July 12,1966 
Table 2.--Monthly or periodic water-level measurements in selected wells in southwestern Dklahoma and northwestern Texas--Continued

HARMON COUNTY, OKLAHOMA--CONtinUEd

Index number on location map: 48

Station number: $\$ 43636899595561$ Local number: $62 \mathrm{~N}-27 W-36$ BBCD 1

Location: Lat $34^{\circ} 36^{\prime} 21^{\prime \prime}$, long $99^{\circ} 59^{\prime} 63^{\prime \prime}$, hydrologic unit 11120105

Aquifer: $313 B$ LIN

Altitude of land-surface datum: $1,828 \mathrm{ft}$

Well depth: $110 \mathrm{ft}$

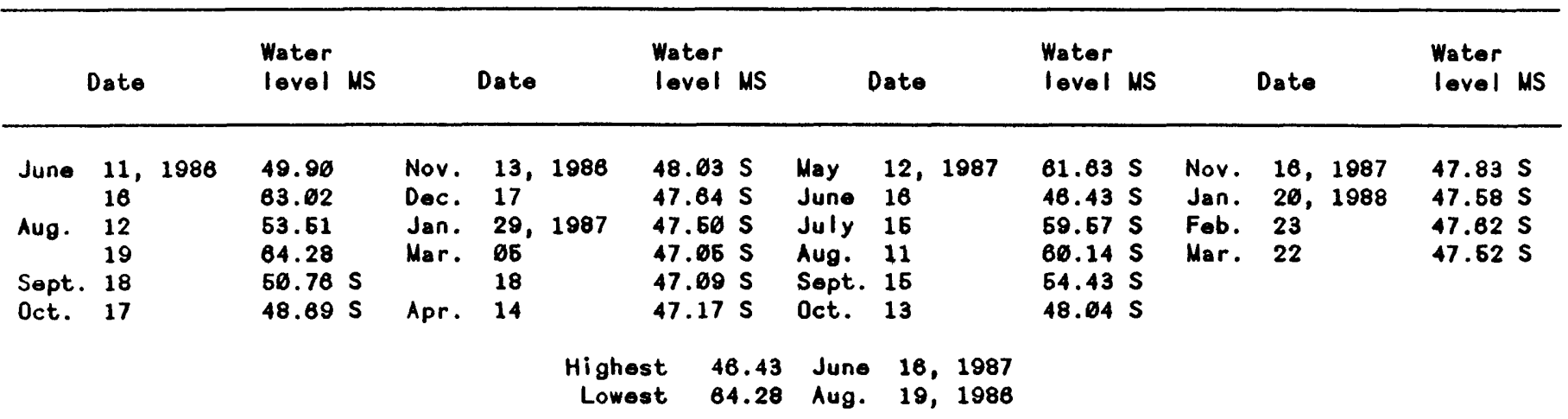


Table 2.--Monthly or periodic water-level measurements in selected wells in southwestern Oklahoma and northwestern Texas--Continued

HARHON COUNTY, OKLAHOMA--Continued

Index number on location map: 49

Station number: $\$ 44220099441601$ Local number: D3N-24W-29 AAA 2

Location: Lat $34^{\circ} 42^{\prime} 36^{\prime \prime}$, long $99^{\circ} 44^{\prime} 16^{\prime \prime}$, hydrologic unit 11120202

Aquifer: $313 B L I N$

Altitude of land-surface datum: $1,526.6 \mathrm{ft}$

Well depth: $169 \mathrm{ft}$

Remarks: Also published as $\oslash 3 N-24 W-29$ AAA 1

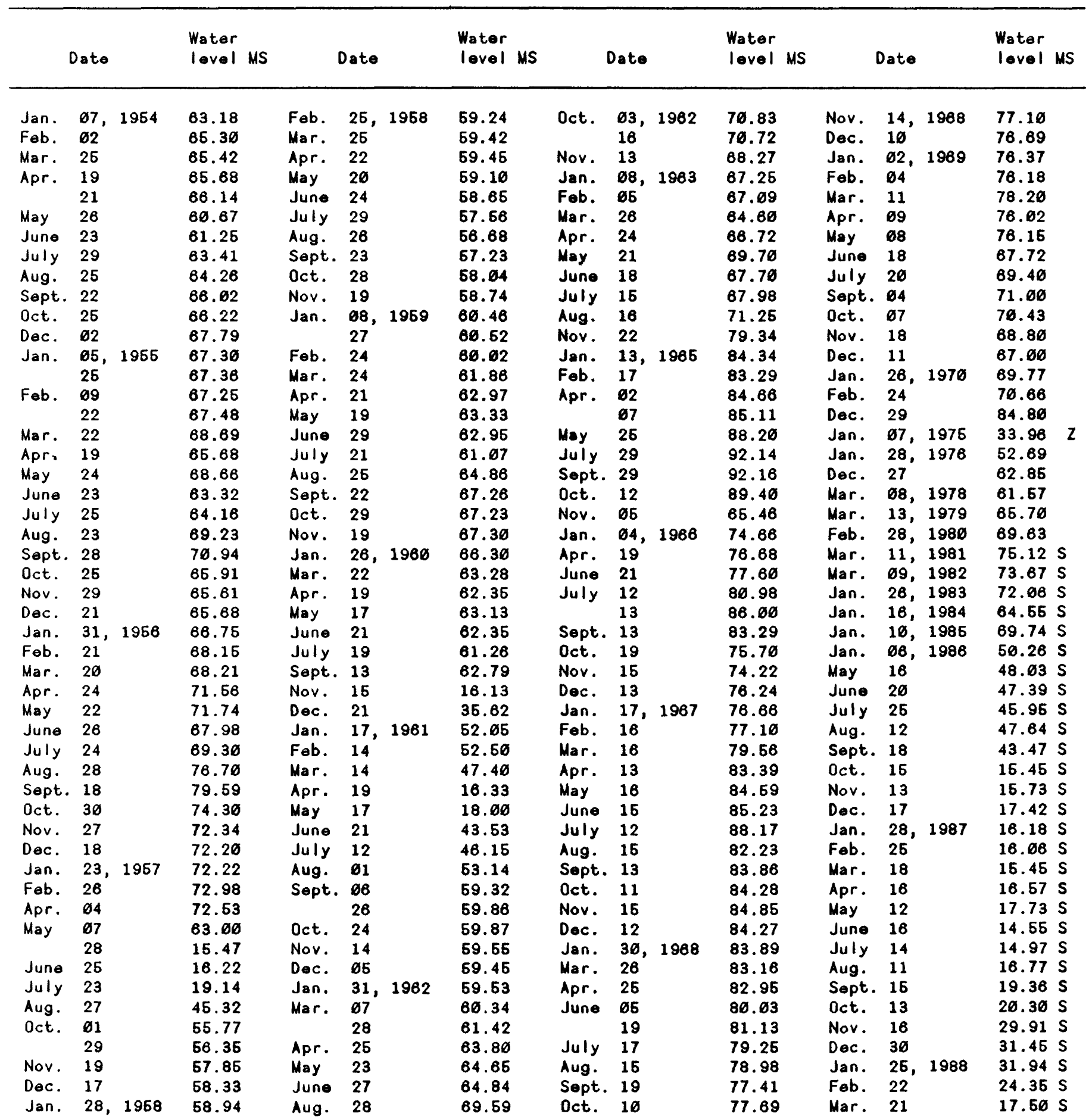


Table 2.--Nonthly or periodic water-level measurements in selected wells in southwestern Oklahoma and northwestern Texas--Continued

HARMON COUNTY, OKLAHOMA---CONtinUed

Index number on location map: 60

Station number: 344868699494201 Local number: $63 \mathrm{~N}-25 W-04$ AABB 1

Location: Lat $34^{\circ} 46^{\prime} 06^{\prime \prime}$, long $99^{\circ} 49^{\prime} 42^{\prime \prime}$, hydrologic unit 11130101

Aquifer: $3130 G C K$

Altitude of land-surface datum: $1,690 \mathrm{ft}$

Well depth: $117 \mathrm{ft}$

\begin{tabular}{|c|c|c|c|c|c|c|c|c|c|c|}
\hline & Date & $\begin{array}{l}\text { Water } \\
\text { level MS }\end{array}$ & & Date & $\begin{array}{l}\text { Water } \\
\text { level MS }\end{array}$ & & Date & $\begin{array}{l}\text { Water } \\
\text { level MS }\end{array}$ & Date & $\begin{array}{l}\text { Water } \\
\text { level MS }\end{array}$ \\
\hline $\begin{array}{l}\text { May } \\
\text { June } \\
\text { July } \\
\text { Oct. } \\
\text { Nov. } \\
\text { Dec. }\end{array}$ & $\begin{array}{ll}22, & 1986 \\
20 & \\
24 & \\
16 & \\
13 & \\
17 & \end{array}$ & $\begin{array}{l}14.44 \mathrm{~S} \\
11.67 \mathrm{~S} \\
12.61 \mathrm{~S} \\
11.62 \mathrm{~S} \\
11.00 \mathrm{~S} \\
10.43 \mathrm{~S}\end{array}$ & $\begin{array}{l}\text { Jan. } \\
\text { Feb. } \\
\text { Mar. } \\
\text { Apr. } \\
\text { May } \\
\text { June }\end{array}$ & $\begin{array}{ll}28, & 1987 \\
25 & \\
18 & \\
16 & \\
12 & \\
18 & \end{array}$ & $\begin{array}{r}10.20 \mathrm{~S} \\
9.02 \mathrm{~S} \\
6.61 \mathrm{~S} \\
6.29 \mathrm{~S} \\
6.96 \mathrm{~S} \\
5.28 \mathrm{~S}\end{array}$ & $\begin{array}{l}\text { July } \\
\text { Aug. } \\
\text { Sept. } \\
\text { Oct. } \\
\text { Nov. } \\
\text { Dec. }\end{array}$ & $\begin{array}{l}14,1987 \\
11 \\
16 \\
14 \\
17 \\
30\end{array}$ & $\begin{array}{r}6.10 \mathrm{~S} \\
8.02 \mathrm{~S} \\
9.50 \mathrm{~S} \\
10.31 \mathrm{~S} \\
9.92 \mathrm{~S} \\
9.14 \mathrm{~S}\end{array}$ & $\begin{array}{ll}\text { Jan. } & 26,1988 \\
\text { Fob. } & 23 \\
\text { Mar. } & 22\end{array}$ & $\begin{array}{r}9.39 \mathrm{~S} \\
9.16 \mathrm{~S} \\
10.10 \mathrm{~S}\end{array}$ \\
\hline
\end{tabular}

Highest 5.28 June 16,1987

Lowest 14.44 May 22, 1988

Index number on location map: 51

Station number: 344402999483201 Local number: $93 \mathrm{~N}-25 W-16$ AOCA 1

Location: Lat $34^{\circ} 44^{\prime} 92^{\prime \prime}$, long $99^{\circ} 49^{\prime} 35^{\prime \prime}$, hydrologic unit 11139101

Aquifer: 313BLIN

Altitude of land-surface datum: $1,882 \mathrm{ft}$

Woll depth: $392 \mathrm{ft}$

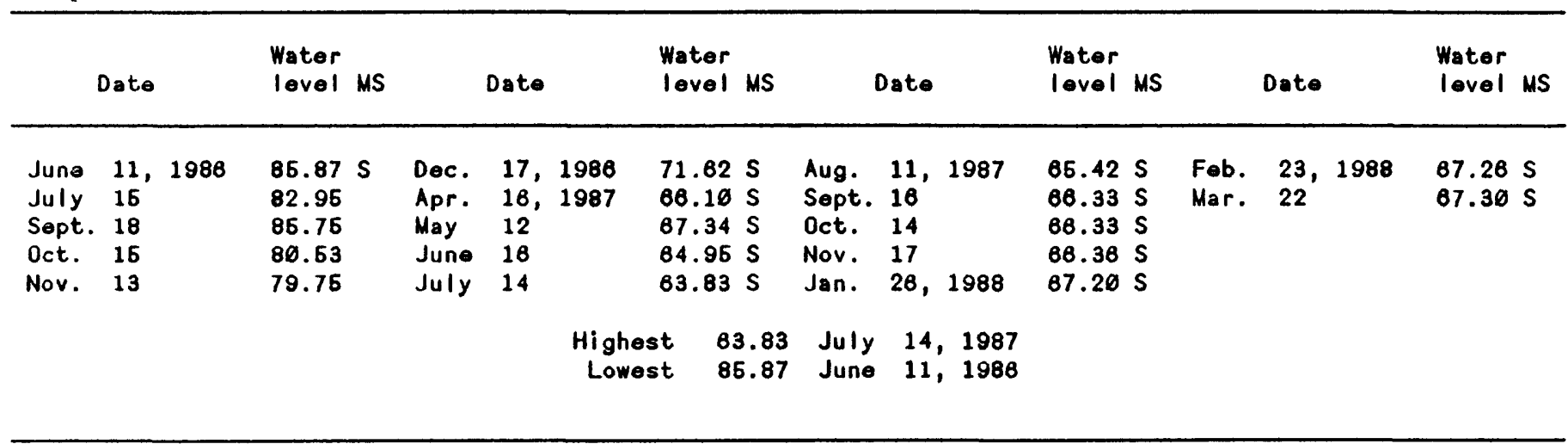

Index number on location map: 62

Station number: $\$ 44148099514001$ Local number: $63 \mathrm{~N}-25 W-30$ DDC 1

Location: Lat $34^{\circ} 41^{\prime} 48^{\prime \prime}$, long $99^{\circ} 51^{\prime} 46^{\prime \prime}$, hydrologic unit 11139101

Aquifer: $313 B L I N$

Altitude of land-surface datum: $1,812 \mathrm{ft}$

Well depth: $150 \mathrm{ft}$

\begin{tabular}{|c|c|c|c|c|c|c|c|c|c|c|c|c|c|}
\hline & Date & $\begin{array}{l}\text { Water } \\
\text { level }\end{array}$ & MS & & Date & & $\begin{array}{l}\text { Water } \\
\text { level MS }\end{array}$ & & Date & $\begin{array}{l}\text { Water } \\
\text { level MS }\end{array}$ & & Date & $\begin{array}{l}\text { Wator } \\
\text { level US }\end{array}$ \\
\hline $\begin{array}{l}\text { June } \\
\text { July } \\
\text { Aug. } \\
\text { Sept. } \\
\text { Oct. } \\
\text { Nov. }\end{array}$ & $\begin{array}{ll}10, & 1986 \\
15 & \\
95 & \\
18 & \\
15 & \\
13 & \end{array}$ & $\begin{array}{l}26.67 \\
26.96 \\
49.68 \\
37.84 \\
28.84 \\
29.42\end{array}$ & $\begin{array}{l}5 \\
5 \\
5\end{array}$ & $\begin{array}{l}\text { Dec. } \\
\text { Jan. } \\
\text { Fob. } \\
\text { Mar. } \\
\text { Apr. } \\
\text { May }\end{array}$ & $\begin{array}{l}17, \\
28, \\
26 \\
18 \\
16 \\
12\end{array}$ & $\begin{array}{l}1986 \\
1987\end{array}$ & $\begin{array}{l}18.06 \mathrm{~S} \\
18.67 \mathrm{~S} \\
14.64 \mathrm{~S} \\
12.80 \mathrm{~S} \\
12.64 \mathrm{~S} \\
15.75 \mathrm{~S}\end{array}$ & $\begin{array}{l}\text { June } \\
\text { July } \\
\text { Sept. } \\
\text { Oct. } \\
\text { Nov. } \\
\text { Dec. }\end{array}$ & $\begin{array}{ll}16, & 1987 \\
14 & \\
16 & \\
14 \\
17 \\
30\end{array}$ & $\begin{array}{r}9.38 \mathrm{~S} \\
11.26 \mathrm{~S} \\
18.17 \mathrm{~S} \\
16.32 \mathrm{~S} \\
16.20 \mathrm{~S} \\
14.25 \mathrm{~S}\end{array}$ & $\begin{array}{l}\text { Jan. } \\
\text { Fob. } \\
\text { Mar. }\end{array}$ & $\begin{array}{ll}26, & 1988 \\
23 & \\
22 & \end{array}$ & $\begin{array}{l}14.20 \mathrm{~S} \\
14.20 \mathrm{~S} \\
13.68 \mathrm{~S}\end{array}$ \\
\hline
\end{tabular}


Tablo 2.--Monthly or periodic water-level measurements in selected wells in southwestern Oklahoma and northwestern Texas--Continued

HARMON COUNTY, OKLAHOMA--Continued

Index number on location map: 63

Station number: 344144099621901 Local number: $03 N-26 W-31$ BABB 1

Location: Lat $34^{\circ} 41^{\prime} 44^{\prime \prime}$, long $99^{\circ} 52^{\prime} 19^{\prime \prime}$, hydrologic unit 11130101

Aquifer: 313BLIN

Altitude of land-surface datum: $1,605 \mathrm{ft}$

Well depth: $78.6 \mathrm{ft}$

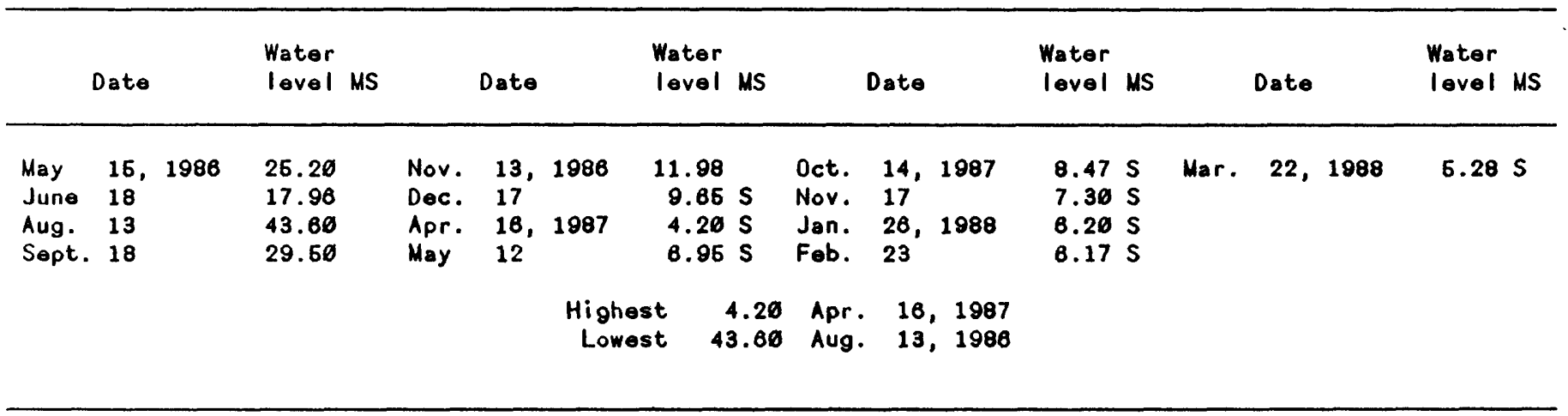

Index number on location map: 64

Station number: 344104699521201 Local number: $03 N-25 W-31$ CDBA 1

Location: Lat $34^{\circ} 41^{\prime} 64^{\prime \prime}$, long $99^{\circ} 62^{\prime} 12^{\prime}$, hydrologic unit 11130101

Aquifer: 313 BLIN

Altitude of land-surface datum: $1,868 \mathrm{ft}$

Woll depth: $180 \mathrm{ft}$

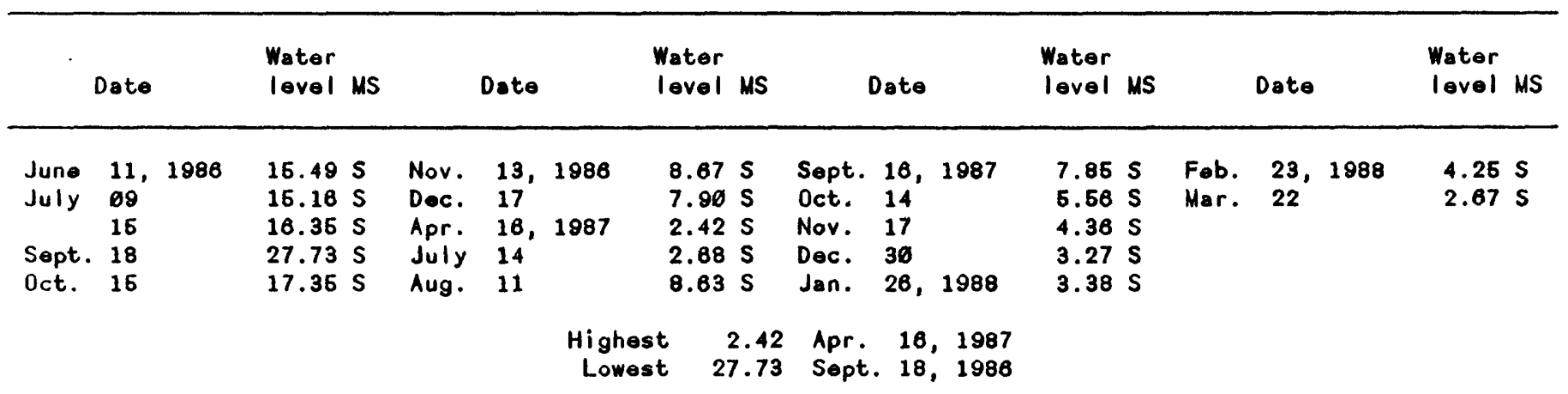


Table 2.--Monthly or periodic water-level measurements in selected wells in southwestern Oklahoma and northwestern Texas-Continued

HARMON COUNTY, OKLAHOMA--Continued

Index number on location map: 65

Station number: $\$ 44146099512501$ Local number: $03 \mathrm{~N}-25 W-32$ BBBD 1

Location: Lat $34^{\circ} 41^{\prime} 40^{\prime \prime}$, long $99^{\circ} 61^{\prime} 28^{\prime \prime}$, hydrologic unit 11130101

Aquifor: 313 BLIN

Altitude of land-surface datum: $1,617 \mathrm{ft}$

Well depth: $160 \mathrm{ft}$

Remarks: Previously published as 03N-25W-32 BBB

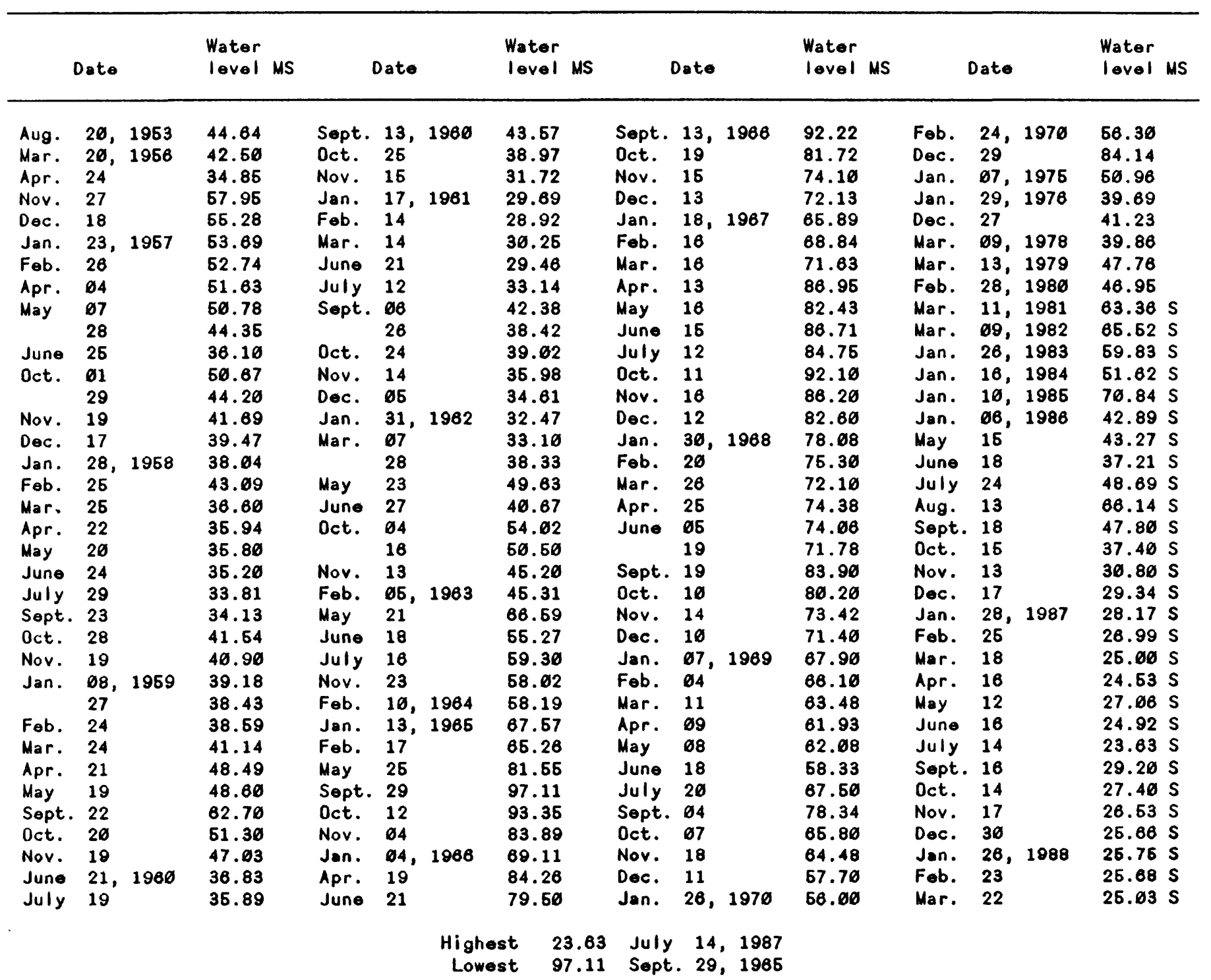


Table 2.--Nonthly or periodic water-level measurements in selected wells in southwestern Oklahoma and northwestern Texas--Continued

HARMON COUNTY, OKLAHOMA--CONtinued

Index number on location map: 66

Station number: 844431699632001 Local number: 63N-28W-12 CDBC 1

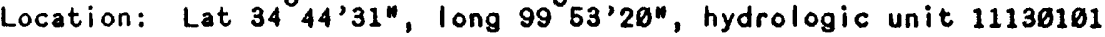

Aquifer: $313 B L I N$

Altitude of land-surface datum: $1,659 \mathrm{ft}$

Well depth: $121.6 \mathrm{ft}$

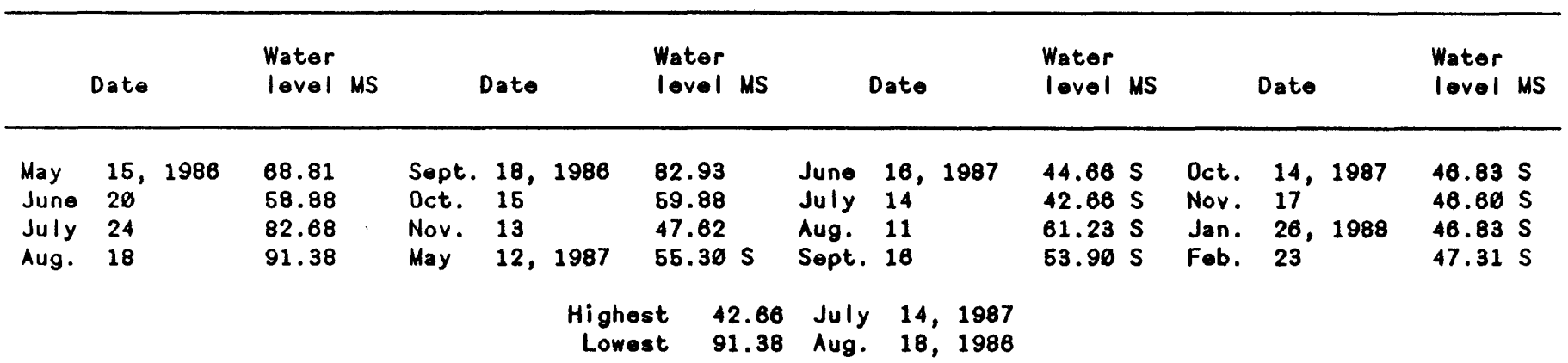

Index number on location map: 57

Station number: 344422099552801 Local number: $63 N-26 W-15$ BABB 1

Location: Lat $34^{\circ} 44^{\prime} 22^{\prime \prime}$, long $99^{\circ} 55^{\prime} 28^{\prime \prime}$, hydrologic unit 11130101

Aquifer: $313 B$ LIN

Altitude of land-surface datum: $1,670 \mathrm{ft}$

Well depth: $214 \mathrm{ft}$

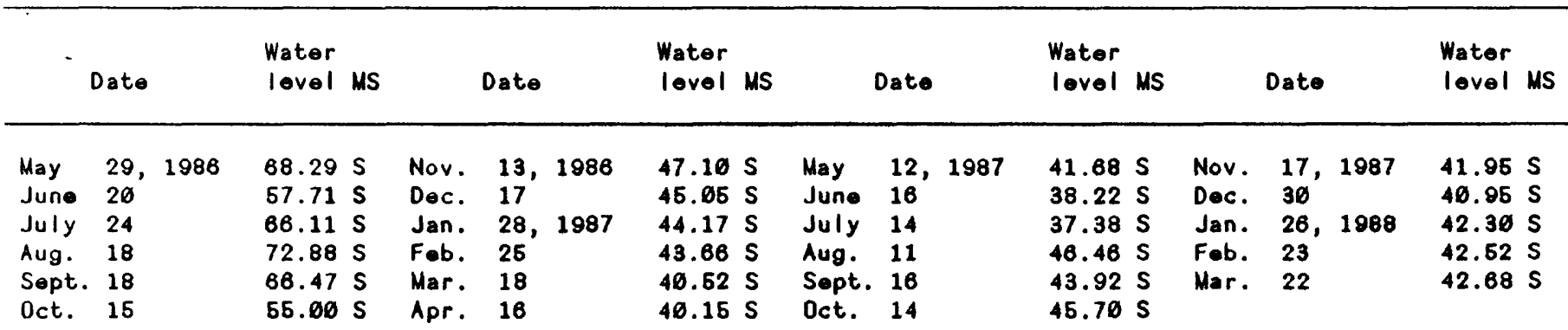

Highest 37.38 July 14,1987

Lowest 72.88 Aug. 18,1986

Index number on location map: 58

Station number: $\$ 44258099674401$ Local number: $63 N-26 W-20$ CBA 1

Location: Lat $34^{\prime 4} 42^{\prime} 58^{\prime \prime}$, long 99 $57^{\prime} 44^{\prime \prime}$, hydrologic unit 11130101

Aquifer: $313 B$ LIN

Altitude of land-surface datum: $1,680 \mathrm{ft}$

Well depth: $220 \mathrm{ft}$

\begin{tabular}{|c|c|c|c|c|c|c|c|c|c|c|c|}
\hline & Date & $\begin{array}{l}\text { Water } \\
\text { level MS }\end{array}$ & & Date & $\begin{array}{l}\text { Water } \\
\text { level MS }\end{array}$ & & Date & $\begin{array}{l}\text { Water } \\
\text { lovel MS }\end{array}$ & & Date & $\begin{array}{l}\text { Water } \\
\text { lovel MS }\end{array}$ \\
\hline $\begin{array}{l}\text { June } \\
\text { July } \\
\text { Nov. }\end{array}$ & $\begin{array}{ll}10, & 1986 \\
15 & \\
13 & \end{array}$ & $\begin{array}{l}65.91 \mathrm{~S} \\
86.68 \mathrm{~S} \\
75.07 \mathrm{~S}\end{array}$ & $\begin{array}{l}\text { Dec. } \\
\text { Mar. } \\
\text { Apr. }\end{array}$ & $\begin{array}{ll}17, & 1986 \\
18, & 1987 \\
16 & \end{array}$ & $\begin{array}{l}73.49 \mathrm{~S} \\
68.15 \mathrm{~S} \\
65.74 \mathrm{~S}\end{array}$ & $\begin{array}{l}\text { May } \\
\text { June } \\
\text { Nov. }\end{array}$ & $\begin{array}{l}12,1987 \\
16 \\
17\end{array}$ & $\begin{array}{l}72.02 \mathrm{~S} \\
64.46 \mathrm{~S} \\
70.94 \mathrm{~S}\end{array}$ & $\begin{array}{l}\text { Jan. } \\
\text { Feb. } \\
\text { Mar. }\end{array}$ & $\begin{array}{ll}26, & 1988 \\
23 & \\
22 & \end{array}$ & $\begin{array}{l}69.91 \mathrm{~S} \\
69.76 \mathrm{~S} \\
69.14 \mathrm{~S}\end{array}$ \\
\hline
\end{tabular}

$\begin{array}{rrrrr}\text { Highest } & 64.46 & \text { June } & 16,1987 \\ \text { Lowest } & 85.68 & \text { July } & 16,1986\end{array}$ 
Table 2.-- Monthly or periodic water-level measurements in selected wells in southwestern Oklahoma and northwestern Texas--Continued

HARMON COUNTY, OKLAHOMA--CONtinued

Index number on location map: 69

Station number: 344244099673901 Local number: $03 \mathrm{~N}-26 \mathrm{~W}-20$ CCDA 1

Location: Lat $34^{\circ} 42^{\prime} 44^{\prime \prime}$, long $99^{\circ} 57^{\prime} 39^{\prime \prime}$, hydrologic unit 11130101

Aquifer: $313 B$ LIN

Altitude of land-surface datum: $1,674 \mathrm{ft}$

Well depth: $141.5 \mathrm{ft}$

\begin{tabular}{|c|c|c|c|c|c|c|c|c|c|c|c|c|c|}
\hline & Date & $\begin{array}{l}\text { Water } \\
\text { level MS }\end{array}$ & & Date & & $\begin{array}{l}\text { Water } \\
\text { level MS }\end{array}$ & & Date & $\begin{array}{l}\text { Water } \\
\text { level MS }\end{array}$ & & Date & & $\begin{array}{l}\text { Water } \\
\text { level MS }\end{array}$ \\
\hline $\begin{array}{l}\text { May } \\
\text { June } \\
\text { July } \\
\text { Aug. } \\
\text { Sept. }\end{array}$ & $\begin{array}{ll}16, & 1986 \\
18 & \\
23 & \\
13 & \\
18 & \end{array}$ & $\begin{array}{l}84.64 \\
77.75 \\
91.35 \\
99.01 \\
87.25\end{array}$ & $\begin{array}{l}\text { Nov. } \\
\text { Dec. } \\
\text { Jan. } \\
\text { Apr. } \\
\text { May }\end{array}$ & $\begin{array}{l}13 \\
17 \\
28 \\
16 \\
11\end{array}$ & $\begin{array}{r}1986 \\
1987\end{array}$ & $\begin{array}{r}28.87 \\
10.74 \mathrm{~S} \\
9.97 \mathrm{~S} \\
62.28 \mathrm{~S} \\
76.60 \mathrm{~S}\end{array}$ & $\begin{array}{l}\text { May } \\
\text { June } \\
\text { July } \\
\text { Aug. } \\
\text { Sept. }\end{array}$ & $\begin{array}{ll}12, & 1987 \\
16 & \\
14 \\
11 \\
16\end{array}$ & $\begin{array}{l}64.13 \mathrm{~S} \\
66.13 \mathrm{~S} \\
68.04 \mathrm{~S} \\
76.60 \mathrm{~S} \\
68.61 \mathrm{~S}\end{array}$ & $\begin{array}{l}\text { Oct. } \\
\text { Nov. } \\
\text { Jan. } \\
\text { Feb. } \\
\text { Mar. }\end{array}$ & $\begin{array}{l}14, \\
17 \\
28 \\
23 \\
22\end{array}$ & $\begin{array}{l}1987 \\
1988\end{array}$ & $\begin{array}{l}65.39 \mathrm{~S} \\
64.38 \mathrm{~S} \\
63.36 \mathrm{~S} \\
63.28 \mathrm{~S} \\
62.65 \mathrm{~S}\end{array}$ \\
\hline
\end{tabular}

Highest 9.97 Jan. 28,1987

Lowest 99.01 Aug. 13,1986

Index number on location map: 60

Station number: 344235099630001 Local number: $\varnothing 3 N-26 W-26$ ABA 1

Location: Lat $34^{\circ} 42^{\prime} 35^{\prime \prime}$, long $99^{\circ} 53^{\prime} \theta \theta^{\prime \prime}$, hydrologic unit 11130101

Aquifer: 313BLIN

Altitude of land-surface datum: $1,640 \mathrm{ft}$

Well depth: $200 \mathrm{ft}$

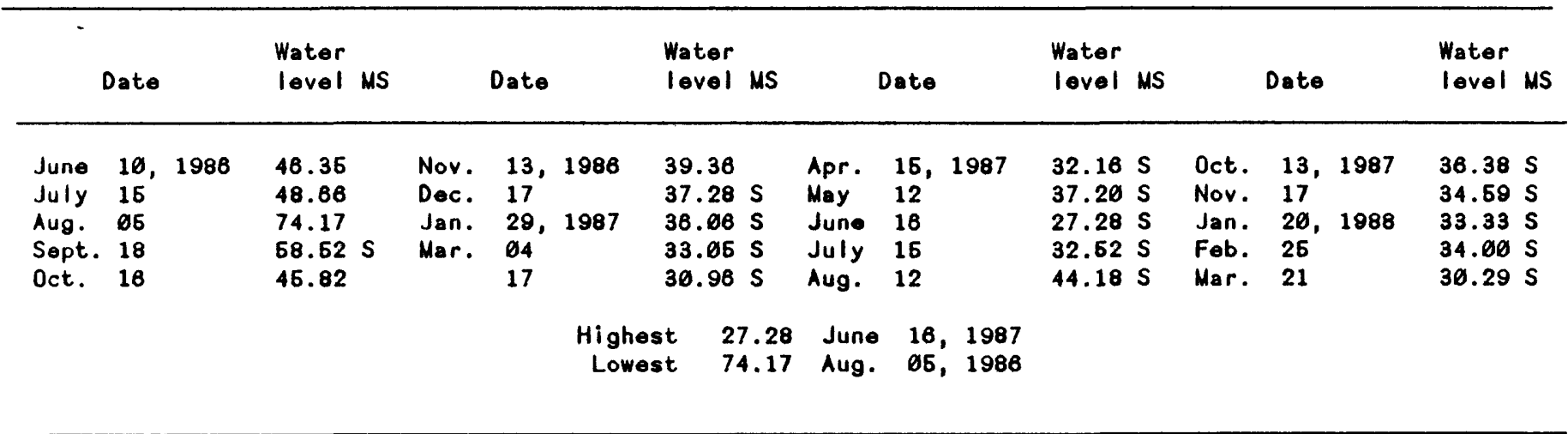

Index number on location map: 61

Station number: 844228099633201 Local number: $03 \mathrm{~N}-26 \mathrm{~W}-25$ BBCD 1

Location: Lat $34^{\circ} 42^{\prime} 28^{\prime \prime}$, long $99^{\circ} 53^{\prime} 32^{\prime \prime}$, hydrologic unit 11130101

Aquifer: 313BLIN

Altitude of land-surface datum: $1,625 \mathrm{ft}$

Well depth: $112.9 \mathrm{ft}$

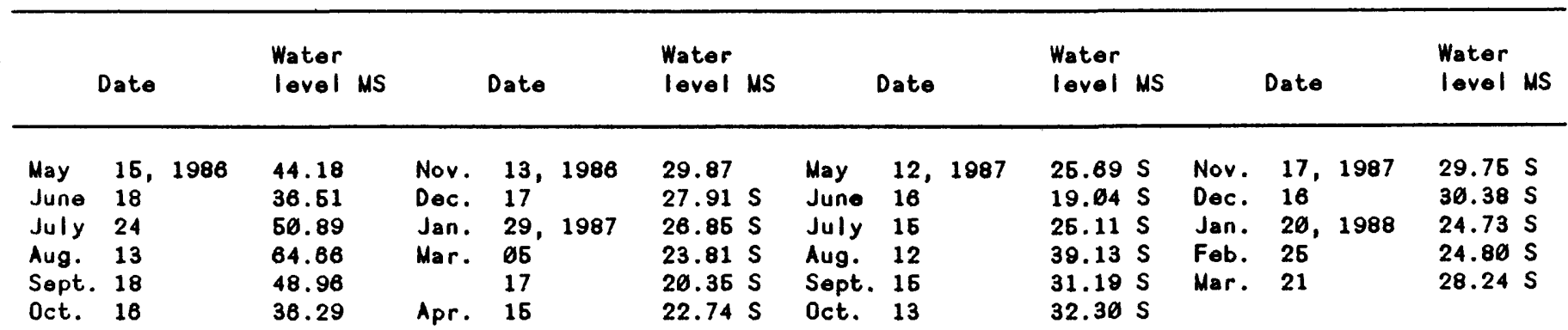


Table 2.-- Monthly or periodic water-level measurements in selected wells in southwestern Oklahoma and northwestern Texas--Continued

HARMON COUNTY, OKLAHOMA--CONtinued

Index number on location map: 62

Station number: $\$ 44228099633202$ Local number: $63 \mathrm{~N}-28 W-26$ BBCD 2

Location: Lat $34^{\circ} 42^{\prime} 28^{\prime \prime}$, long $99^{\circ} 53^{\prime} 32^{\prime \prime}$, hydrologic unit 11130101

Aquifer: $313 B L I N$

Altitude of land-surface datum: $1,625 \mathrm{ft}$

Well depth: $160 \mathrm{ft}$

\begin{tabular}{|c|c|c|c|c|c|c|c|c|c|c|c|c|c|c|c|}
\hline & Date & $\begin{array}{l}\text { Water } \\
\text { level MS }\end{array}$ & & Date & & $\begin{array}{l}\text { Wa } \\
\text { le }\end{array}$ & $\begin{array}{l}\text { or } \\
\text { ol MS }\end{array}$ & & Date & & $\begin{array}{l}\text { Water } \\
\text { level MS }\end{array}$ & & Date & & $\begin{array}{l}\text { Water } \\
\text { level MS }\end{array}$ \\
\hline \multirow[t]{2}{*}{$\begin{array}{l}\text { May } \\
\text { June }\end{array}$} & $\begin{array}{l}15,1986 \\
18\end{array}$ & $\begin{array}{l}40.60 \\
32.92\end{array}$ & $\begin{array}{l}\text { July } \\
\text { Aug. }\end{array}$ & $\begin{array}{l}24, \\
13\end{array}$ & 1986 & \multicolumn{2}{|c|}{$\begin{array}{l}47.30 \\
61.14\end{array}$} & $\begin{array}{l}\text { Sept. } \\
\text { Oct. }\end{array}$ & $\begin{array}{l}18 \\
16\end{array}$ & 1986 & $\begin{array}{l}46.37 \\
32.66\end{array}$ & Nov. & 13 & 1986 & 26.42 \\
\hline & & & & & $\begin{array}{l}\text { High } \\
\text { Low }\end{array}$ & & $\begin{array}{l}26.42 \\
61.14\end{array}$ & $\begin{array}{l}\text { Nov. } \\
\text { Aug. }\end{array}$ & $\begin{array}{l}13, \\
13,\end{array}$ & $\begin{array}{l}1986 \\
1986\end{array}$ & & & & & \\
\hline
\end{tabular}

Index number on location map: 63

Station number: 344146099530801 Local number: $93 \mathrm{~N}-26 \mathrm{~W}-25$ CDDD 1

Location: Lat $34^{\circ} 41^{\prime} 46^{\prime \prime}$, long $99^{\circ} 53^{\prime} 08^{\prime \prime}$, hydrologic unit 11130101

Aquifer: 313 BLIN

Altitude of land-surface datum: $1,820 \mathrm{ft}$

Well depth: $101.9 \mathrm{ft}$

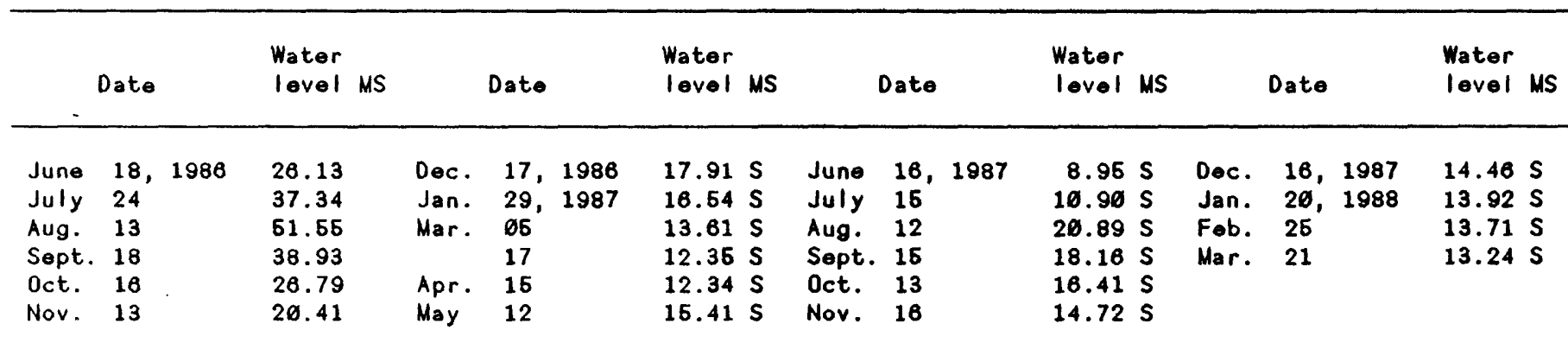

$\begin{array}{rrrr}\text { Highest } & 8.96 & \text { June } 16,1987 \\ \text { Lowest } & 51.65 & \text { Aug. } 13,1986\end{array}$

Index number on location map: 64

Station number: 844234099535001 Local number: ø3N-26W-26 AABB 1

Location: Lat $34^{\circ} 42^{\prime} 37^{\prime \prime}$, long $99^{\circ} 53^{\prime} 64^{\prime \prime}$, hydrologic unit 11130101

Aquifer: $313 B L I N$

Altitude of land-surface datum: $1,640 \mathrm{ft}$

Well depth: $146 \mathrm{ft}$

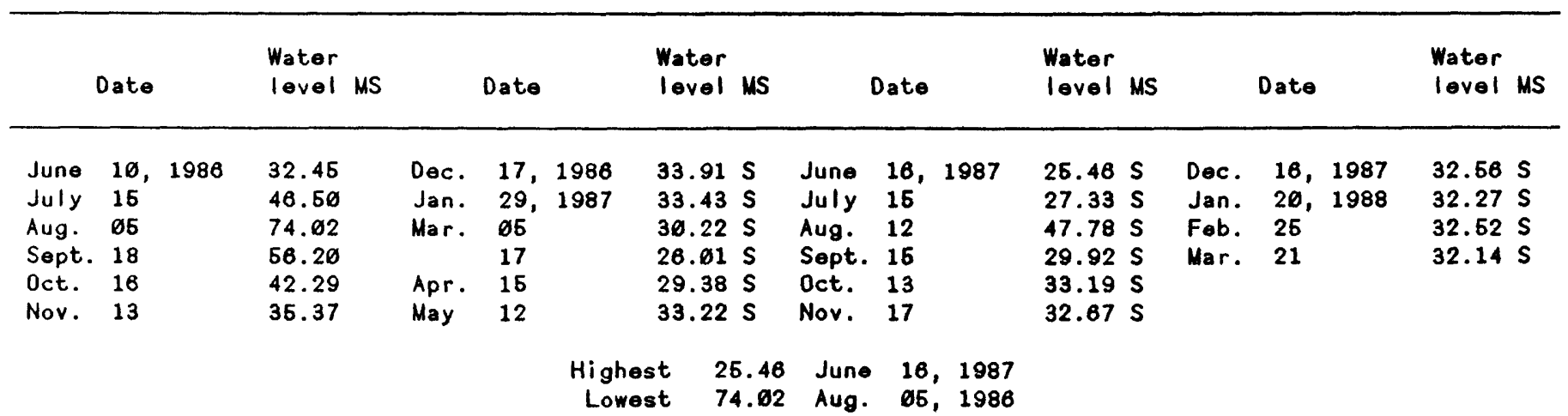


Table 2.--Monthly or periodic water-level measurements in selected wells in southwestern oklahoma and northwestern Texas--Continued

HARMON COUNTY, OKLAHOMA--Continued

Index number on location map: 65

Station number: 344139099573101 Local number: 63N-28W-32 BABD 1

Location: Lat $34^{\circ} 41^{\prime} 39^{\prime \prime}$, long $99^{\circ} 67^{\prime} 31^{\prime \prime}$, hydrologic unit 11130101

Aquifer: 313 BLIN

Altitude of land-surface datum: $1,649 \mathrm{ft}$

Well depth: $68.6 \mathrm{ft}$. Original well depth was $105 \mathrm{ft}$.

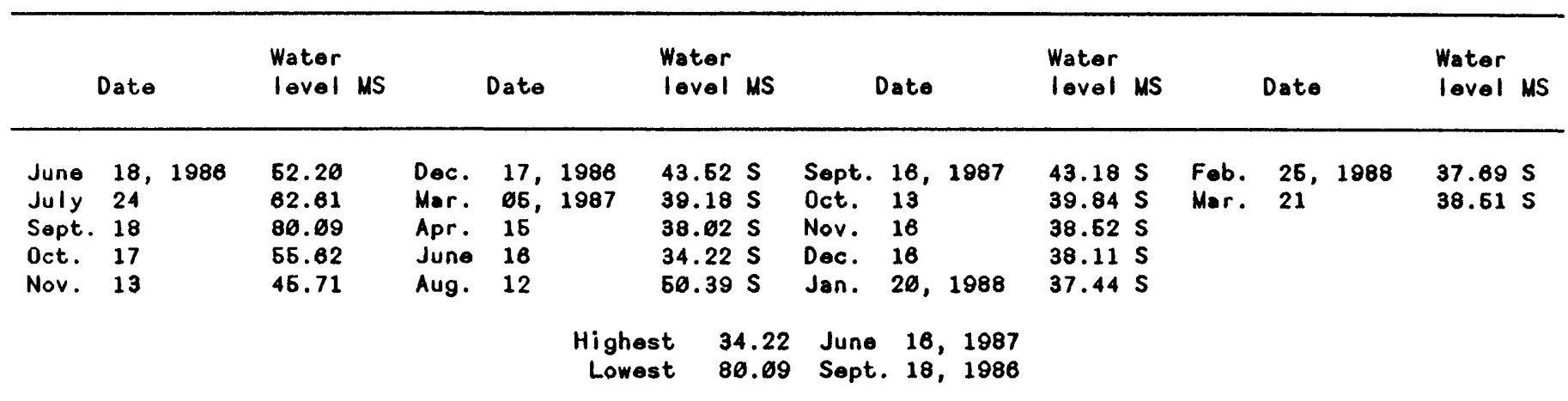

Index number on location map: 66

Station number: 344120099670001 Local number: $03 N-26 W-32$ DABA 1

Location: Lat 34 41'20", long 99 $57^{\prime} 60^{\prime \prime}$, hydrologic unit 11130101

Aquifer: 313BLIN

Altitude of land-surface datum: $1,634 \mathrm{ft}$

Well depth: $280 \mathrm{ft}$

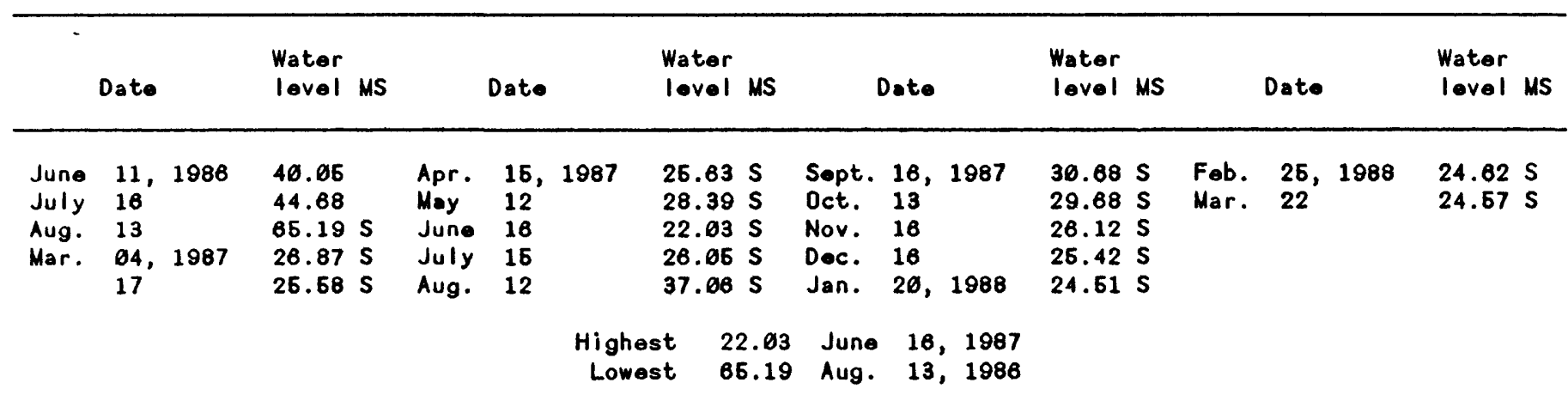

Index number on location map: 67

Station number: 344122099664001 Local number: $03 \mathrm{~N}-26 \mathrm{~W}-33$ BCDC 1

Location: Lat $34^{\circ} 41^{\prime} 22^{\prime \prime}$, long $99^{\circ}$ 68' $46^{\prime \prime}$, hydrologic unit 11130101

Aquifer: 3138 LIN

Altitude of land-surface datum: $1,632 \mathrm{ft}$

Well depth: $119 \mathrm{ft}$

\begin{tabular}{|c|c|c|c|c|c|c|c|c|c|c|}
\hline & Date & $\begin{array}{l}\text { Wator } \\
\text { level US }\end{array}$ & & Date & $\begin{array}{l}\text { Water } \\
\text { level MS }\end{array}$ & & Date & $\begin{array}{l}\text { Water } \\
\text { level MS }\end{array}$ & Date & $\begin{array}{l}\text { Water } \\
\text { lovel MS }\end{array}$ \\
\hline $\begin{array}{l}\text { May } \\
\text { June } \\
\text { July } \\
\text { Aug. } \\
\text { Sept. }\end{array}$ & $\begin{array}{ll}15, & 1988 \\
18 & \\
24 & \\
13 & \\
18 & \end{array}$ & $\begin{array}{l}43.92 \mathrm{~S} \\
37.60 \mathrm{~S} \\
46.79 \mathrm{~S} \\
62.80 \mathrm{~S} \\
46.99 \mathrm{~S}\end{array}$ & $\begin{array}{l}\text { Oct. } \\
\text { Nov. } \\
\text { Dec. } \\
\text { Jan. } \\
\text { Mar. }\end{array}$ & $\begin{array}{l}17,1986 \\
13 \\
17 \\
29,1987 \\
05\end{array}$ & $\begin{array}{l}36.62 S \\
26.66 \mathrm{~S} \\
28.82 \mathrm{~S} \\
27.42 \mathrm{~S} \\
24.72 \mathrm{~S}\end{array}$ & $\begin{array}{l}\text { Apr. } \\
\text { May } \\
\text { June } \\
\text { Oct. } \\
\text { Nov. }\end{array}$ & $\begin{array}{l}16,1987 \\
12 \\
16 \\
13 \\
16\end{array}$ & $\begin{array}{l}22.65 \mathrm{~S} \\
26.19 \mathrm{~S} \\
19.63 \mathrm{~S} \\
26.16 \mathrm{~S} \\
23.76 \mathrm{~S}\end{array}$ & $\begin{array}{ll}\text { Jan. } 20,1988 \\
\text { Fob. } 26 \\
\text { Mar. } 21\end{array}$ & $\begin{array}{l}22.63 \mathrm{~S} \\
22.27 \mathrm{~S} \\
21.79 \mathrm{~S}\end{array}$ \\
\hline
\end{tabular}

Highest 19.53 June 16,1987

Lowest 62.80 Aug. 13, 1986 
Table 2.-- Monthly or periodic water-level measurements in selected wells in southwestern Oklahoma and northwestern Texas--Continued

HARMON COUNTY, OKLAHOMA--CONTInUEd

Index number on location map: 68

Station number: $\$ 44119699655301$ Local number: $63 N-26 W-33$ DAAB 1

Location: Lat $34^{\circ} 41^{\prime} 19^{\prime \prime}$, long $99^{\circ} 55^{\prime} 53^{\prime \prime}$, hydrologic unit 11130101

Aquifer: 313BLIN

Altitude of land-surface datum: $1,630 \mathrm{ft}$

Well depth: $89 \mathrm{ft}$

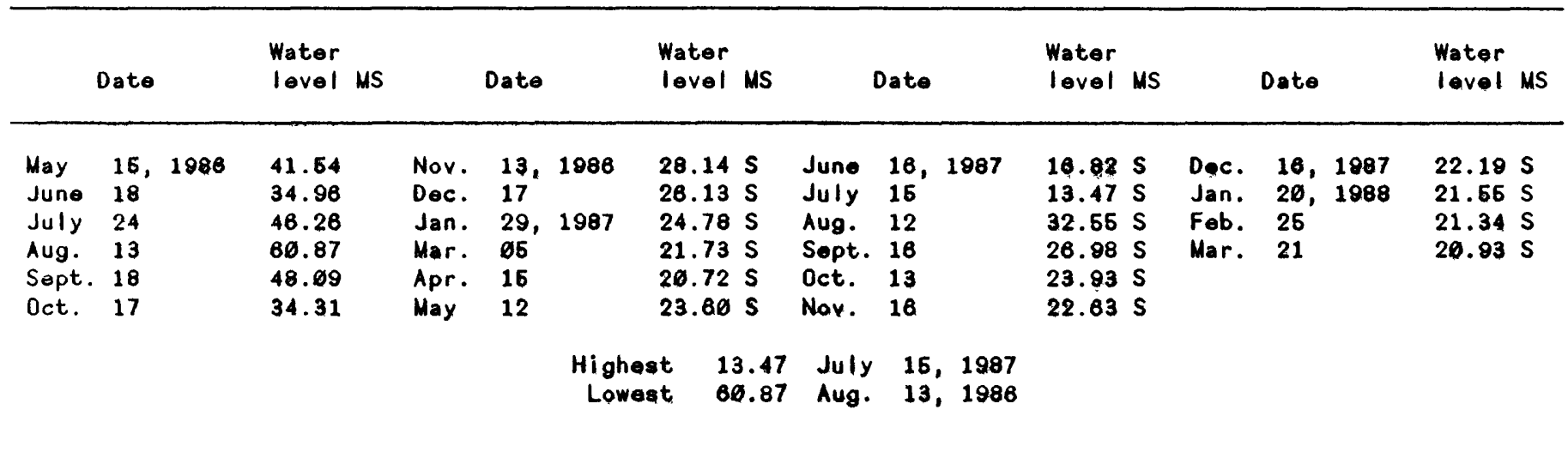

Index number on location map: 69

Station number: $\$ 44118099563001$ Local number: $63 \mathrm{~N}-28 W-33$ DAAD 1

Location: Lat $34^{\circ} 41^{\prime} 07^{\prime \prime}$, long $99^{\circ} 65^{\prime} 47^{\prime \prime}$, hydrologic unit 11130101

Aquifer: 3138 LIN

Altitude of land-surface datum: $1,635 \mathrm{ft}$

Well depth: $200 \mathrm{ft}$

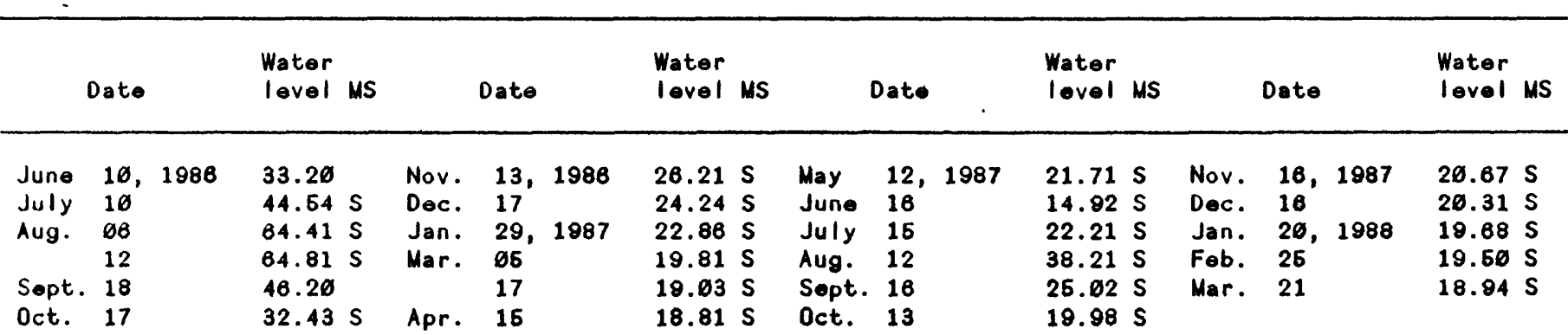

$\begin{array}{rrll}\text { Highest } & 14.92 & \text { June } 16,1987 \\ \text { Lowest } & 64.81 & \text { Aug. } & 12,1986\end{array}$ 
Table 2.-- Monthly or periodic water-level measurements in selected wells in southwestern Oklahoma and northwestern Texas--Continued

HARMON COUNTY, OKLAHOMA--CONTinUed

Index number on location map: 70

Station number: 344141099542701 Local number: $03 \mathrm{~N}-26 \mathrm{~W}-35$ BABC 1

Location: Lat $34^{\circ} 41^{\prime} 41^{\prime \prime}$, long $99^{\circ} 54^{\prime} 27^{\prime \prime}$, hydrologic unit 11130101

Aquifer: $313 B L I N$

Altitude of land-surface datum: $1,838 \mathrm{ft}$

Well depth: $72.2 \mathrm{ft}$. Original well depth was $126 \mathrm{ft}$.

Remarks: Previously published as $03 \mathrm{~N}-26 \mathrm{~W}-36$ BAB

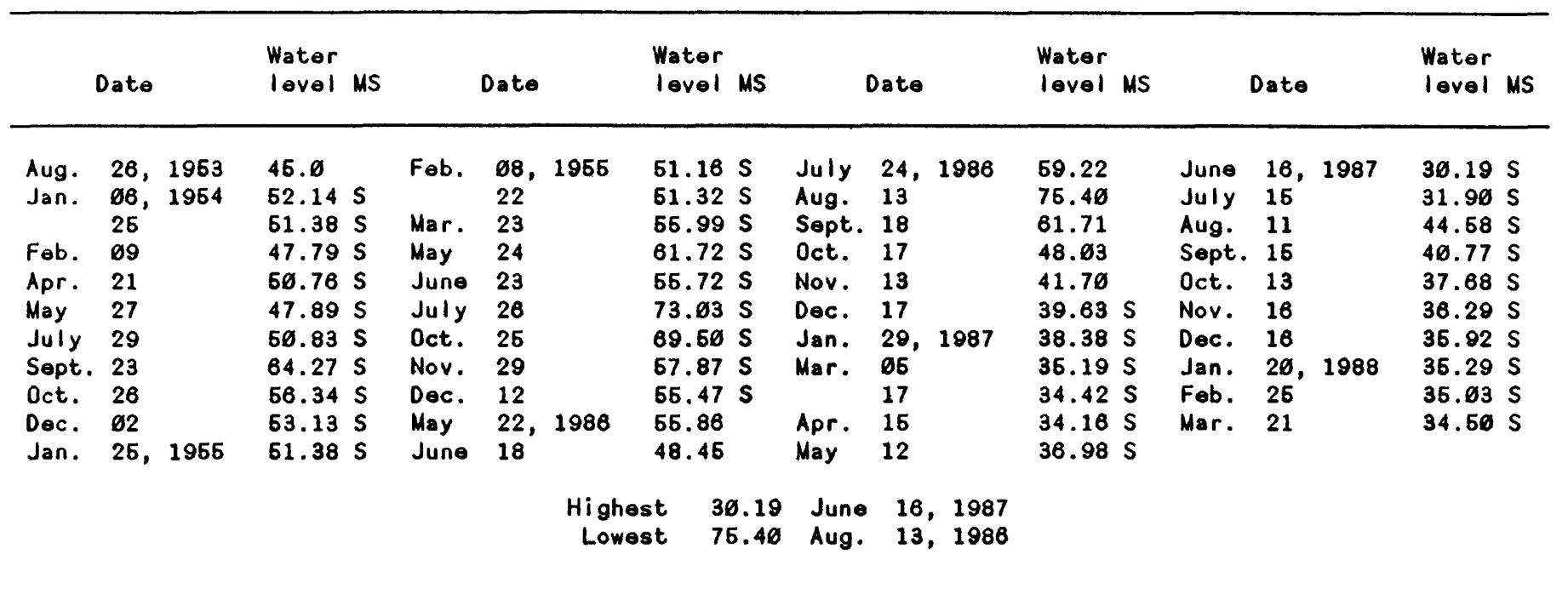

Index number on location map: 71

Station number: 344113099533201 Local number: $93 N-26 W-36$ CBBB 1

Location: Lat $34^{\circ} 41^{\prime} 17^{\prime \prime}$, long $99^{\circ} 63^{\prime} 37^{\prime \prime}$, hydrologic unit 11130101

Aquifer: $313 B L I N$

Altitude of land-surface datum: $1,637 \mathrm{ft}$

Well depth: $72 \mathrm{ft}$. Original well depth was $175 \mathrm{ft}$.

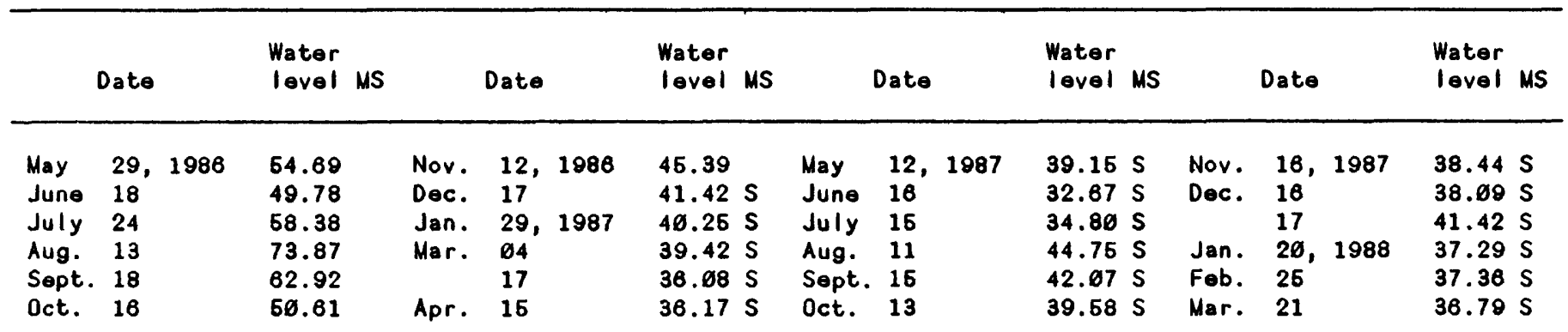

Highest 32.67 June 16,1987

Lowest 73.87 Aug. 13, 1988 
Table 2.--Monthly or periodic water-level measurements in selected wells in southwestern oklahoma and northwestern Texas--Continued

HARMON COUNTY, OKLAHOMA--CONtinUEd

Index number on location map: 72

Station number: $\$ 44658699524601$ Local number: $63 N-28 W-38$ DDDC 1

Location: Lat $34^{\circ} 46^{\prime} 54^{\prime \prime}$, long $99^{\circ} 62^{\prime} 44^{\prime \prime}$, hydrologic unit 11136161

Aquifer: 3138 LIN

Altitude of land-surface datum: $1,619 \mathrm{ft}$

Well depth: $180 \mathrm{ft}$

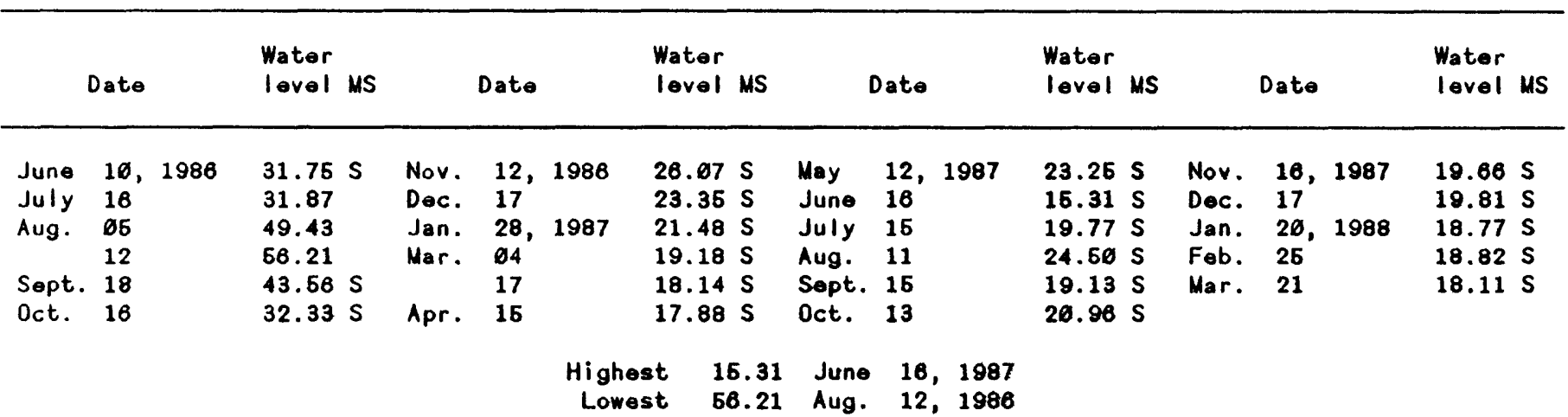

Index number on location map: 73

Station number: $\$ 44758099442601$ Local number: $64 \mathrm{~N}-24 W-20$ DOC 1

Location: Lat $34^{\circ} 47^{\prime} 58^{\prime \prime}$, long $99^{\circ} 44^{\prime} 26^{\prime \prime}$, hydrologic unit 11120202

Aquifer: $3130 \mathrm{GCK}$

Altitude of land-surface datum: $1,700 \mathrm{ft}$

Well depth: $124.6 \mathrm{ft}$

\begin{tabular}{|c|c|c|c|c|c|c|c|c|c|c|c|}
\hline & Date & $\begin{array}{l}\text { Water } \\
\text { level MS }\end{array}$ & & Dato & & $\begin{array}{l}\text { Water } \\
\text { level MS }\end{array}$ & & Date & $\begin{array}{l}\text { Water } \\
\text { level US }\end{array}$ & Date & $\begin{array}{l}\text { Water } \\
\text { level MS }\end{array}$ \\
\hline \multirow[t]{2}{*}{$\begin{array}{l}\text { May } \\
\text { June } \\
\text { July }\end{array}$} & $\begin{array}{ll}29, & 1986 \\
20 & \\
24 & \end{array}$ & $\begin{array}{l}88.98 \\
87.25 \\
86.07\end{array}$ & $\begin{array}{l}\text { Oct. } \\
\text { Nor. } \\
\text { Dec. }\end{array}$ & $\begin{array}{l}15 \\
13 \\
17\end{array}$ & 1986 & $\begin{array}{l}86.48 \mathrm{~S} \\
84.61 \mathrm{~S} \\
84.64 \mathrm{~S}\end{array}$ & $\begin{array}{l}\text { Apr. } \\
\text { May } \\
\text { June }\end{array}$ & $\begin{array}{l}16,1987 \\
12 \\
16\end{array}$ & $\begin{array}{l}77.16 \mathrm{~S} \\
78.27 \mathrm{~S} \\
81.93 \mathrm{~S}\end{array}$ & & \\
\hline & & & & & $\begin{array}{l}\text { High } \\
\text { Low }\end{array}$ & $\begin{array}{l}77.16 \\
88.98\end{array}$ & $\begin{array}{l}\text { Apr. } \\
\text { May }\end{array}$ & $\begin{array}{ll}16, & 1987 \\
29, & 1986\end{array}$ & & & \\
\hline
\end{tabular}

Index number on location map: 74

Station number: 344838699511601 Local number: $64 \mathrm{~N}-25 \mathrm{~W}-2 \varnothing$ BACB 1

Location: Lat $34^{\circ} 48^{\prime} 38^{\prime \prime}$, long $99^{\circ} 61^{\prime} 18^{\prime \prime}$, hydrologic unit 11120202

Aquifer: 110SFRT

Altitude of land-surface datum: $1,750 \mathrm{ft}$

Well depth: $9.8 \mathrm{ft}$

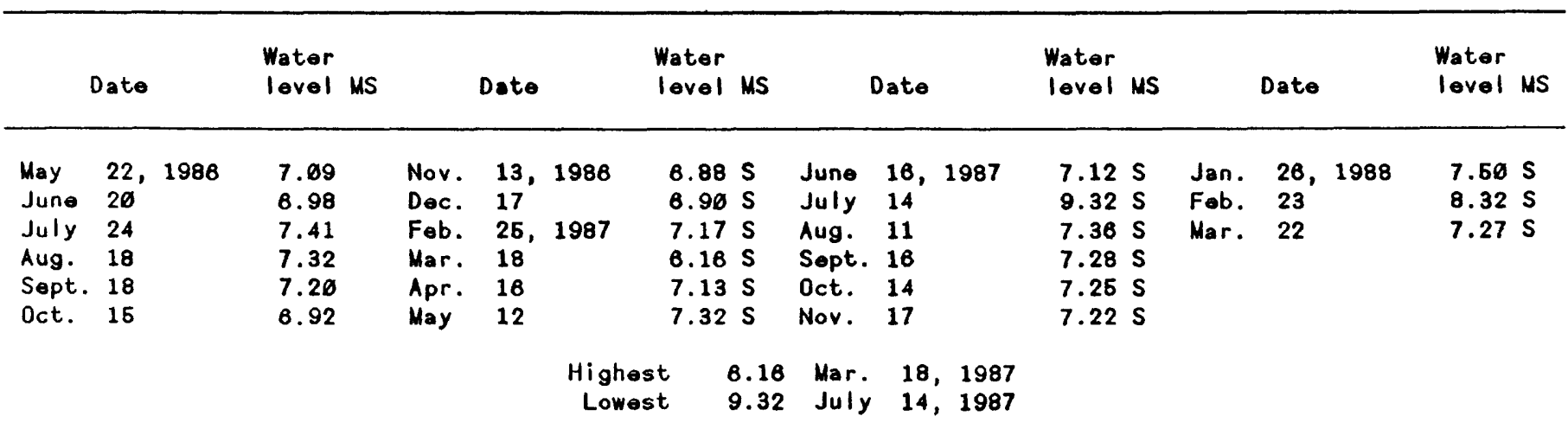


Table 2.--Monthly or periodic water-level measurements in selected wells in southwestern Oklahoma and northwestern Texas--Continued

HARMON COUNTY, OKLAHOMA--CONtinuOd

Index number on location map: 75

Station number: 344831099612401 Local number: $84 \mathrm{~N}-25 \mathrm{~W}-20 \mathrm{BBDC} 1$

Location: Lat $34^{\circ} 48^{\prime} 31^{\prime \prime}$, long $99^{\circ} 51^{\prime} 24^{\prime \prime}$, hydrologic unit 11129202

Aquifer: 11øSFRT

Altitude of land-surface datum: $1,800 \mathrm{ft}$

Well depth: $110 \mathrm{ft}$

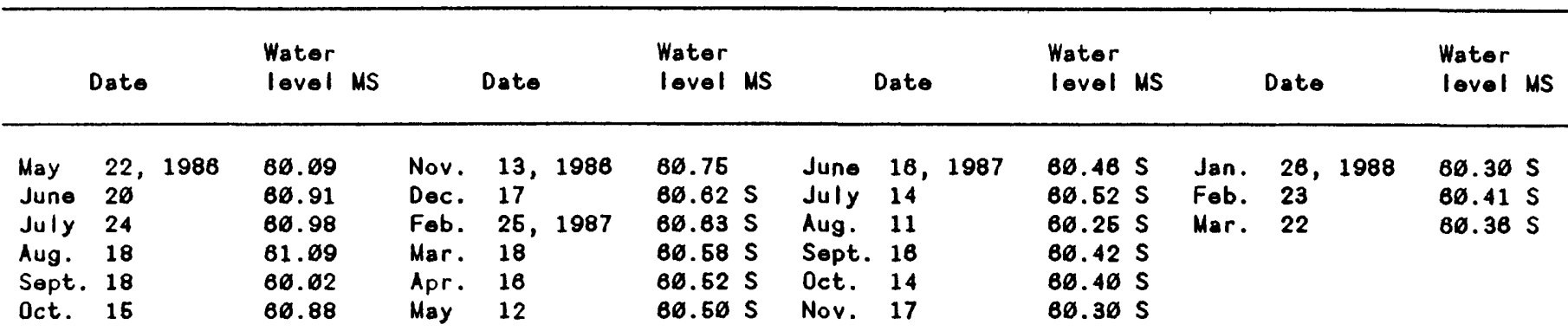

Highest 60.02 Sept. 18,1988

Lowest 61.09 Aug. 18,1986

Index number on location map: 76

Station number: 844756099494001 Local number: $\varnothing 4 \mathrm{~N}-25 W-21$ DDC 1

Location: Lat $34^{\circ} 47^{\prime} 65^{\prime \prime}$, long $99^{\circ} 49^{\prime} 48^{\prime \prime}$, hydrologic unit 11120202

Aquifor: 11øSFRT

Altitude of land-surface datum: $1,829 \mathrm{ft}$

Woll depth: $70 \mathrm{ft}$

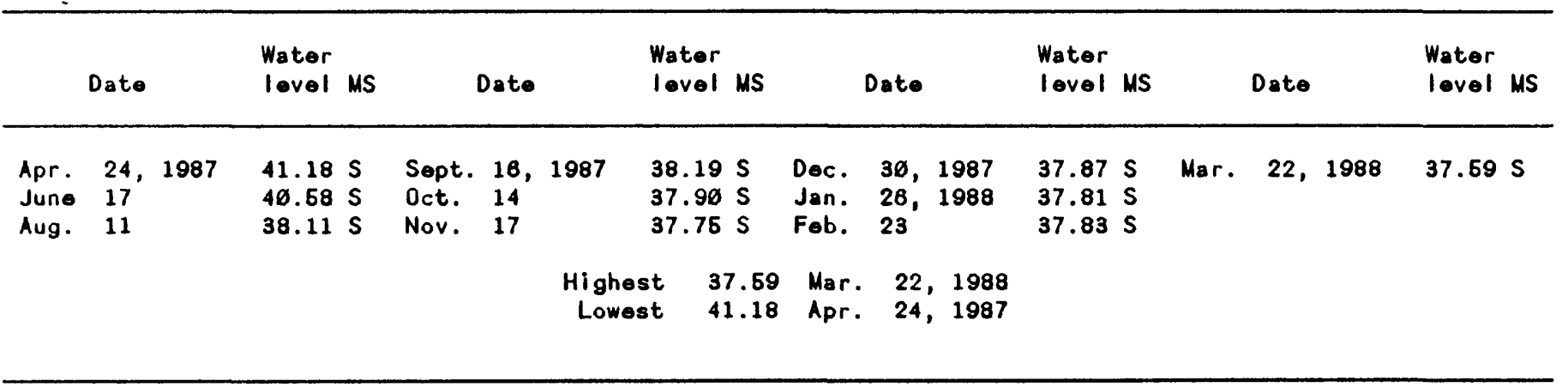

Index number on location map: 77

Station number: $\$ 44749099693692$ Local number: $84 \mathrm{~N}-25 \mathrm{~W}-29$ AAA 2

Location: Lat $34^{\circ} 47^{\prime} 49^{\prime \prime}$, long $99^{\circ} 5 \sigma^{\prime} 36^{\prime \prime}$, hydrologic unit 11120202

Aquifor: 310WTRS

Altitude of land-surface datum: $1,798.4 \mathrm{ft}$

Woll depth: $23 \mathrm{ft}$

\begin{tabular}{|c|c|c|c|c|c|c|c|c|c|c|c|c|}
\hline & Date & & $\begin{array}{l}\text { Wator } \\
\text { level MS }\end{array}$ & & Date & $\begin{array}{l}\text { Wator } \\
\text { lovel }\end{array}$ & MS & & Date & $\begin{array}{l}\text { Water } \\
\text { level MS }\end{array}$ & Date & $\begin{array}{l}\text { Wator } \\
\text { lovel MS }\end{array}$ \\
\hline $\begin{array}{l}\text { Dec. } \\
\text { Apr. } \\
\text { June } \\
\text { Aug. } \\
\text { Sept. } \\
\text { Oct. } \\
\text { Jan. } \\
\text { Feb. }\end{array}$ & $\begin{array}{l}36, \\
21, \\
23 \\
25 \\
22 \\
27 \\
65, \\
69\end{array}$ & $\begin{array}{l}1953 \\
1954 \\
\\
1956\end{array}$ & $\begin{array}{l}18.6 \varnothing \mathrm{S} \\
18.4 \varnothing \\
18.4 \varnothing \\
20.3 \emptyset \\
20.8 \\
19.10 \\
20.6 \varnothing \\
2 \varnothing .4 \varnothing\end{array}$ & $\begin{array}{l}\text { Apr. } \\
\text { June } \\
\text { June } \\
\text { July } \\
\text { Aug. } \\
\text { Sept. } \\
\text { Oct. } \\
\text { Nov. }\end{array}$ & $\begin{array}{ll}26, & 1956 \\
22 & \\
2 \varnothing, & 1986 \\
24 & \\
18 & \\
18 & \\
16 & \\
13 & \end{array}$ & $\begin{array}{l}20.20 \\
19.40 \\
14.68 \\
16.64 \\
15.34 \\
14.50 \\
13.68 \\
12.62\end{array}$ & $\begin{array}{l}\text { S } \\
S \\
S \\
S \\
S \\
S\end{array}$ & $\begin{array}{l}\text { Dec. } \\
\text { Jan. } \\
\text { Fob. } \\
\text { Mar. } \\
\text { Apr. } \\
\text { May } \\
\text { Juno } \\
\text { July }\end{array}$ & $\begin{array}{ll}17, & 1988 \\
28, & 1987 \\
25 & \\
18 & \\
16 & \\
12 & \\
18 & \\
14 & \end{array}$ & $\begin{array}{l}12.69 \mathrm{~S} \\
12.78 \mathrm{~S} \\
12.24 \mathrm{~S} \\
11.63 \mathrm{~S} \\
12.48 \mathrm{~S} \\
12.36 \mathrm{~S} \\
11.89 \mathrm{~S} \\
11.73 \mathrm{~S}\end{array}$ & $\begin{array}{lll}\text { Aug. } & 11, & 1987 \\
\text { Sopt. } & 16 & \\
\text { Oct. } 14 & \\
\text { Nov. } & 17 \\
\text { Dec. } & 3 \emptyset & \\
\text { Jan. } & 26,1988 \\
\text { Fob. } & 23 & \\
\text { Mar. } & 22 & \end{array}$ & $\begin{array}{l}12.50 \mathrm{~S} \\
12.46 \mathrm{~S} \\
11.74 \mathrm{~S} \\
12.66 \mathrm{~S} \\
12.30 \mathrm{~S} \\
12.16 \mathrm{~S} \\
12.61 \mathrm{~S} \\
11.49 \mathrm{~S}\end{array}$ \\
\hline
\end{tabular}


Tablo 2.--Monthly or periodic water-level measurements in selected wells in southwestern Oklahoma and northwestern Texas--Continued

HARMON COUNTY, OKLAHOMA--CONTInUEd

Index number on location map: 78

Station number: $\$ 45039099644401$ Local number: $04 \mathrm{~N}-26 \mathrm{~W}-02$ CCBB 1

Location: Lat $34^{\circ} 50^{\prime} 39^{\prime \prime}$, long $99^{\circ} 54^{\prime} 44^{\prime \prime}$, hydrologic unit 11120202

Aquifer: 111SFRR

Altitude of land-surface datum: $1724 \mathrm{ft}$

Well depth: $22 \mathrm{ft}$

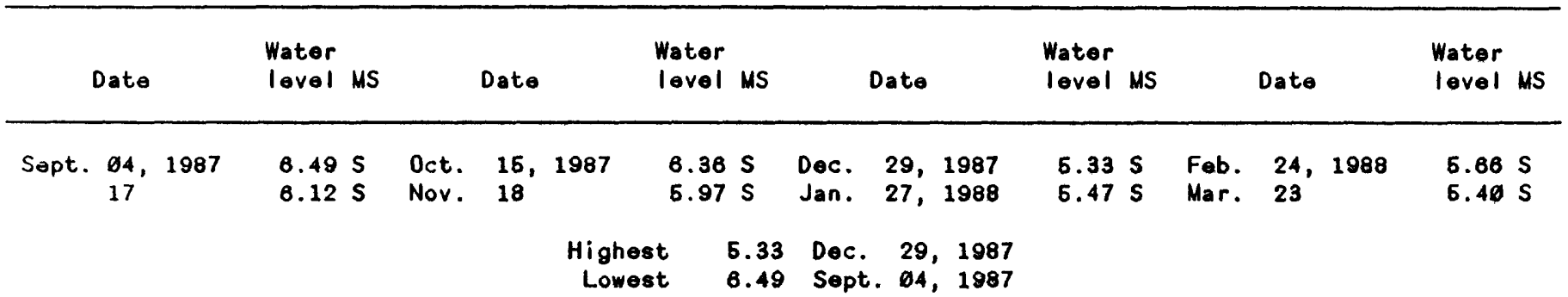

Index number on location map: 79

Station number: $\$ 45037099544401$ Local number: $64 \mathrm{~N}-26 \mathrm{~W}-62$ CCBC 1

Location: Lat $34^{\circ} 50^{\prime} 37^{\prime \prime}$, long $99^{\circ} 54^{\prime} 44^{\prime \prime}$, hydrologic unit 11120202

Aquifer: 111SFRR

Altitude of land-surface datum: $1,727 \mathrm{ft}$

Well depth: $16 \mathrm{ft}$

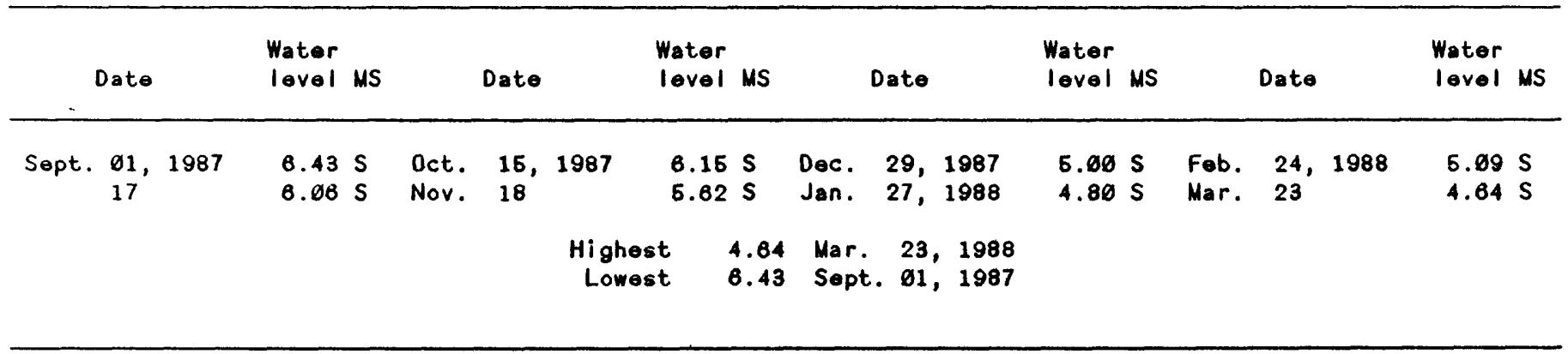

Index number on location map: 80

Station number: $\$ 46031099544501$ Local number: $94 \mathrm{~N}-26 \mathrm{~W}-03$ D0D0 1

Location: Lat $34^{\circ} 50^{\prime} 31^{\prime \prime}$, long $99^{\circ} 54^{\prime} 45^{\prime \prime}$, hydrologic unit 11120202

Aquifer: $313 V V C R$

Altitude of land-surface datum: $1,765 \mathrm{ft}$

Woll depth: $230 \mathrm{ft}$

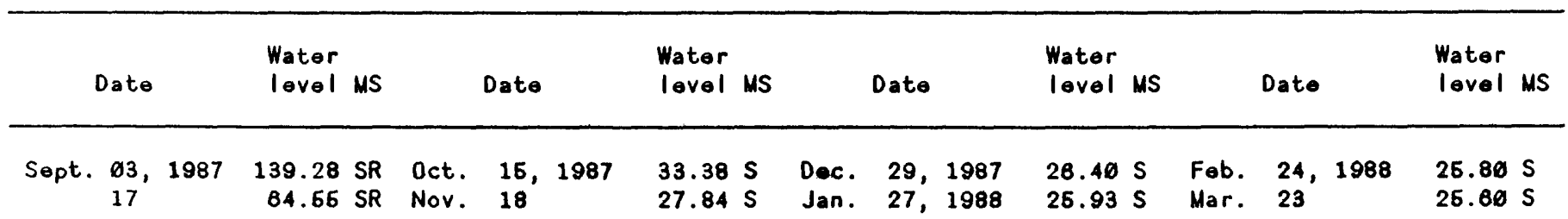

Highest 25.60 Mar. 23, 1988

Lowest 139.28 Sept. 03, 1987 
Table 2.-- Monthly or periodic vater-level measurements in selected wells in southwestern oklahoma and northwestern Texas--Continued

HARMON COUNTY, OKLAHOMA--Continued

Index number on location map: 81

Station number: 844849099541401 Local number: $64 \mathrm{~N}-26 \mathrm{~W}-14$ CDOA 1

Location: Lat $34^{\circ} 48^{\prime} 49^{\prime \prime}$, I ong $89^{\circ} 54^{\prime} 14^{\prime \prime}$, hydrologic unit 11120202

Aquifer: 110SFRT

Altitude of land-surface datum: $1,874 \mathrm{ft}$

Woll depth: $62.2 \mathrm{ft}$

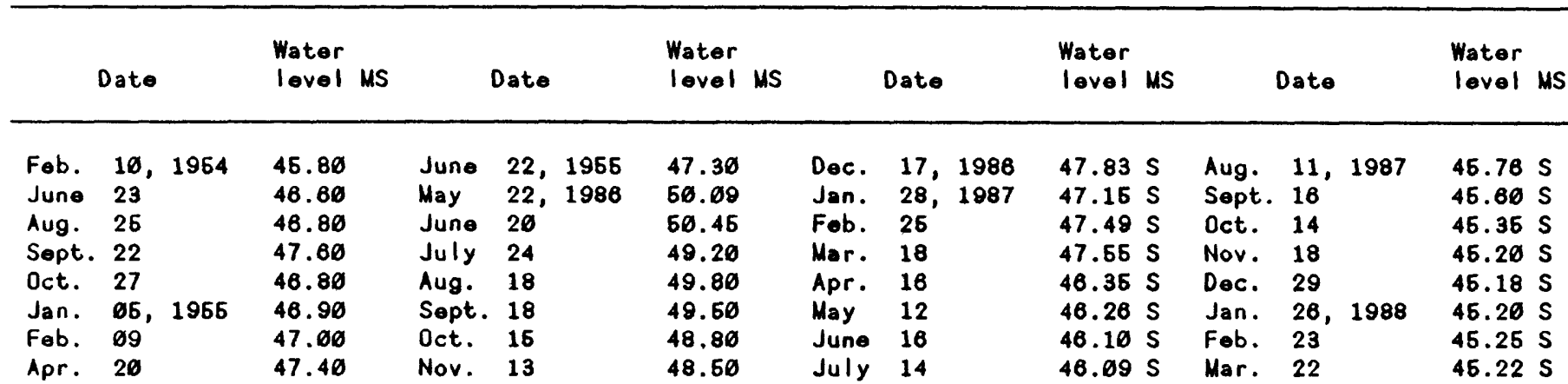

Highest 45.18 Dec. 29,1987

Lowest 60.45 June 20,1986

Index number on location map: 82

Station number: $\$ 44844099684901$ Local number: $04 \mathrm{~N}-26 \mathrm{~W}-19$ BBBA 1

Location: Lat $34^{\circ} 48^{\prime} 44^{\prime \prime}$, Iong $99^{\circ} 58^{\prime} 49^{\prime \prime}$, hydrologic unit 11120202

Aquifer: 310WTRS

Altitude of land-surface datum: $1,930 \mathrm{ft}$

Woll depth: $145 \mathrm{ft}$

\begin{tabular}{|c|c|c|c|c|c|c|c|c|c|c|c|c|c|c|c|c|}
\hline & Date & & $\begin{array}{l}\text { Water } \\
\text { level }\end{array}$ & MS & & Date & & $\begin{array}{l}\text { Wa } \\
\text { lo }\end{array}$ & ier & Date & & $\begin{array}{l}\text { Water } \\
\text { level }\end{array}$ & MS & & Date & $\begin{array}{l}\text { Water } \\
\text { level MS }\end{array}$ \\
\hline \multirow[t]{2}{*}{$\begin{array}{l}\text { July } \\
\text { Aug. } \\
\text { Sopt. }\end{array}$} & $\begin{array}{l}22 \\
17 \\
02\end{array}$ & 1987 & $\begin{array}{l}93.36 \\
32.62 \\
32.21\end{array}$ & $\begin{array}{l}S R \\
S \\
S\end{array}$ & $\begin{array}{l}\text { Sept. } \\
\text { Oct. } \\
\text { Nov. }\end{array}$ & $\begin{array}{l}16 \\
14 \\
17\end{array}$ & 1987 & \multicolumn{2}{|c|}{$\begin{array}{l}32.67 \mathrm{~S} \\
32.48 \mathrm{~S} \\
32.38 \mathrm{~S}\end{array}$} & $\begin{array}{l}\text { Dec. } 29, \\
\text { Jan. } 26, \\
\text { Fob. } 23\end{array}$ & $\begin{array}{l}1987 \\
1988\end{array}$ & $\begin{array}{l}32.36 \mathrm{~S} \\
32.32 \mathrm{~s} \\
32.33 \mathrm{~s}\end{array}$ & $\begin{array}{l}S \\
S \\
S\end{array}$ & Mar. & 22,1988 & $32.23 \mathrm{~S}$ \\
\hline & & & & & & & \multicolumn{2}{|c|}{$\begin{array}{c}\text { Highest } \\
\text { Lowest }\end{array}$} & $\begin{array}{l}32.21 \\
32.62\end{array}$ & $\begin{array}{l}\text { Sept. } 02, \\
\text { Aug. } 17 \text {, }\end{array}$ & $\begin{array}{l}1987 \\
1987\end{array}$ & & & & & \\
\hline
\end{tabular}

Index number on location map: 83

Station number: $\$ 44844099584902$ Local number: $64 \mathrm{~N}-26 \mathrm{~W}-19$ BBBA 2

Location: Lat $34^{\circ} 48^{\prime} 4^{\prime \prime}$, long $99^{\circ} 5^{\prime} 8^{\prime} 49^{\prime \prime}$, hydrologic unit 11120202

Aquifer: 110SFTA

Altitude of land-surface datum: 1,930 ft

Well depth: $70 \mathrm{ft}$

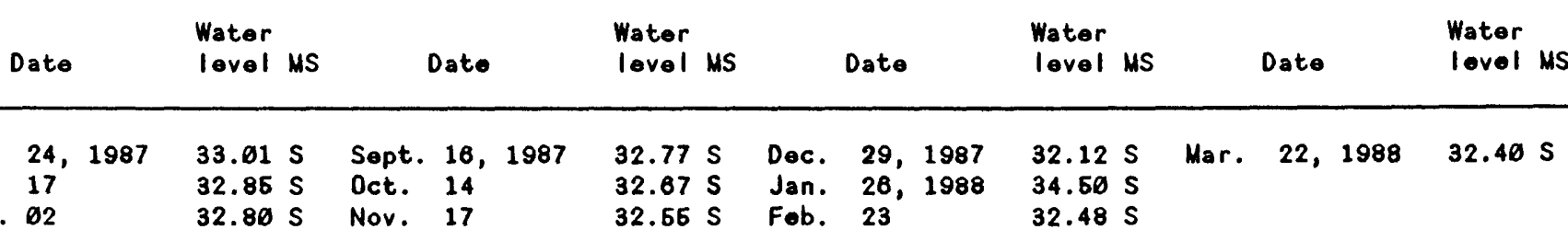

\begin{tabular}{|c|c|c|c|c|c|c|c|c|c|c|c|c|c|c|c|c|c|}
\hline $\begin{array}{l}\text { July } \\
\text { Aug. } \\
\text { Sept. }\end{array}$ & $\begin{array}{l}24, \\
17 \\
92\end{array}$ & 1987 & $\begin{array}{l}33.01 \\
32.85 \\
32.80\end{array}$ & $\begin{array}{l}S \\
S \\
S\end{array}$ & $\begin{array}{l}\text { Sept. } \\
\text { Oct. } \\
\text { Nov. }\end{array}$ & $\begin{array}{l}16 \\
14 \\
17\end{array}$ & 1987 & & $\begin{array}{ll}77 & \mathrm{~S} \\
87 \mathrm{~S} \\
56 \mathrm{~S}\end{array}$ & $\begin{array}{l}\text { Dec. } \\
\text { Jan. } \\
\text { Fob. }\end{array}$ & $\begin{array}{l}29, \\
26, \\
23\end{array}$ & $\begin{array}{l}1987 \\
1988\end{array}$ & $\begin{array}{l}32.12 \mathrm{~S} \\
34.60 \mathrm{~S} \\
32.48 \mathrm{~S}\end{array}$ & Mar. & 22 & 1988 & 32.40 \\
\hline
\end{tabular}


Table 2.-- Monthly or periodic water-level measurements in selected wells in southwestern Oklahoma and northwestern Texas--Continued

HARMON COUNTY, OKLAHOMA--CONtinued

Index number on location map: 84

Station number: $\$ 44844699684963$ Local number: $64 \mathrm{~N}-26 \mathrm{~W}-19$ BBBA 3

Location: Lat $34^{\circ} 48^{\prime} 44^{\prime \prime}$, long $99^{\circ} 58^{\prime} 49^{\prime \prime}$, hydrologic unit 11120202

Aquifer: $3130 G C K$

Altitude of land-surface datum: $1,930 \mathrm{ft}$

Well depth: $278 \mathrm{ft}$

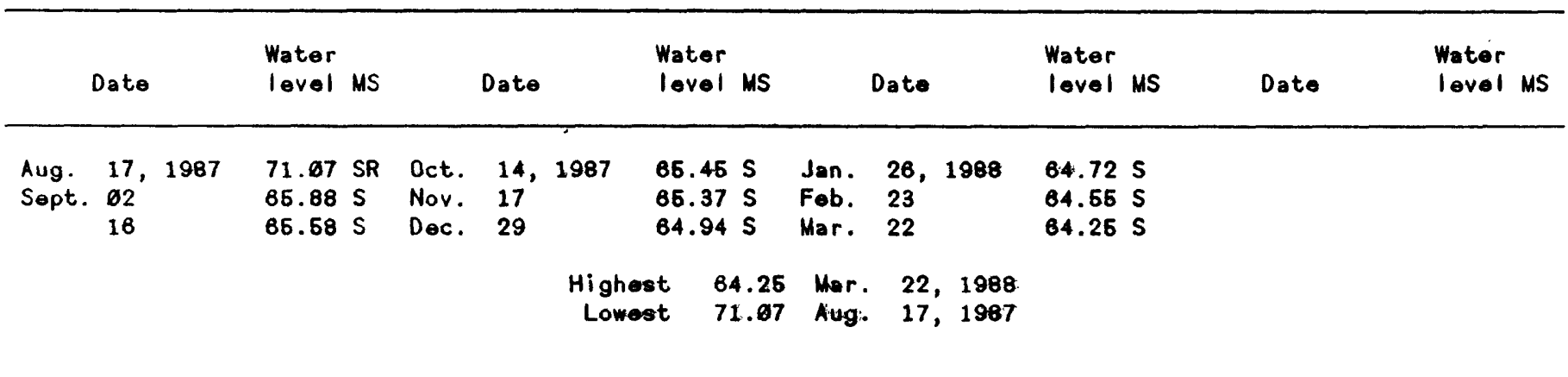

Index number on location map: 85

Station number: 744753699580761 Local number: 64N-26W-19 DDC 1

Location: Lat $34^{\circ} 47^{\prime} 63^{\prime \prime}$, I ong $99^{\circ} 68^{\prime} 97^{\prime \prime}$, hydrologic unit 11130101 .

Aquifer: 11øSFTA

Altitude of land-surface datum: $1,918 \mathrm{ft}$

Well depth: $117 \mathrm{ft}$

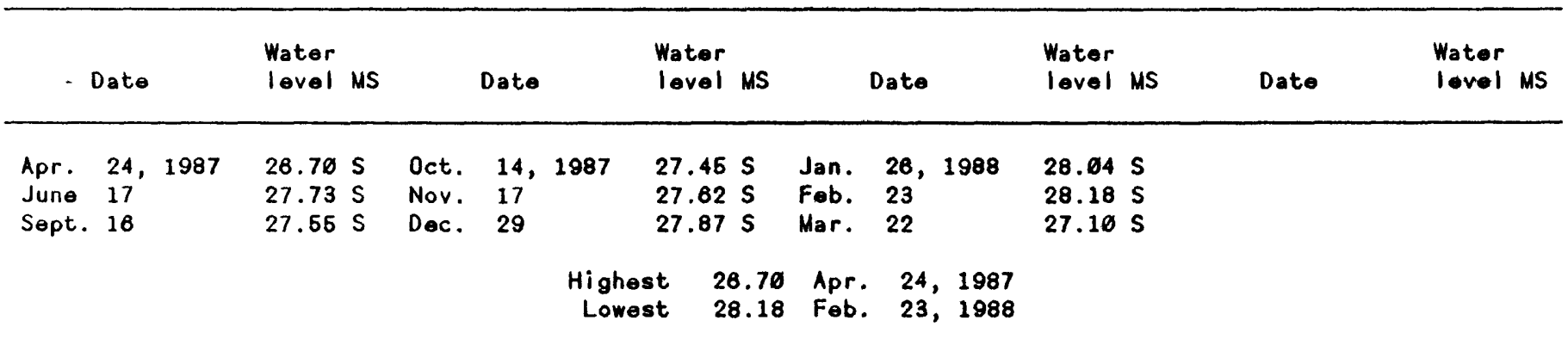

Index number on location map: 86

Station number: $\$ 44821099585401$ Local number: $64 \mathrm{~N}-26 \mathrm{~W}-20$ ADD 1

Location: Lat $34^{\circ} 48^{\prime} 21^{\prime \prime}$, long $99^{\circ} 58^{\prime} 54^{\prime \prime}$, hydrologic unit 11130101

Aquifer: 110SFRT

Altitude of land-surface datum: $1,915 \mathrm{ft}$

Well depth: $94 \mathrm{ft}$

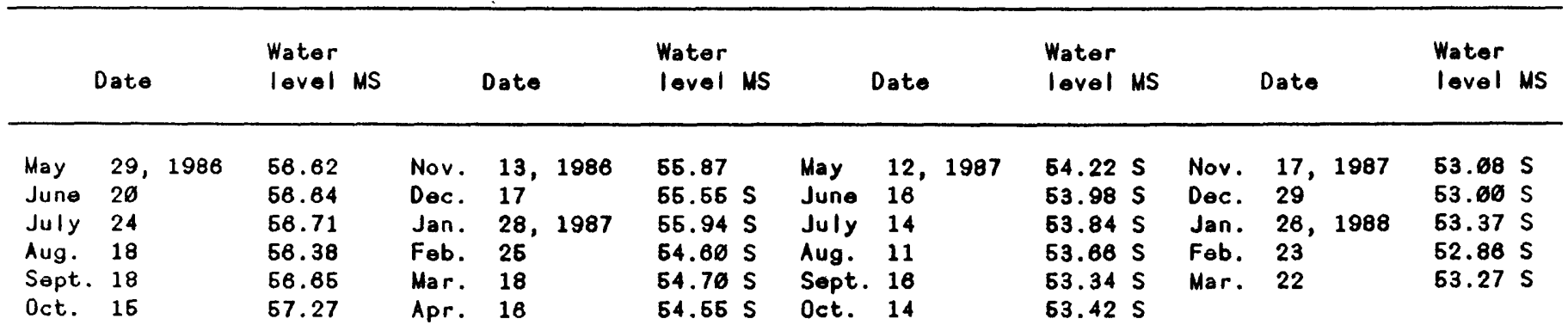

Highest 62.88 Feb. 23,1988

Lowest 57.27 Oct. 15,1986 
Table 2.-- Monthly or periodic water-level measurements in selected wells in southwestern oklahoma and northwestern Texas--Continued

HARMON COUNTY, OKLAHOMA--CONtinUEd

Index number on location map: 87

Station number: 345408099530101 Local number: $65 \mathrm{~N}-25 \mathrm{~W}-18$ CCDA 1

Location: Lat $34^{\circ} 54^{\prime} \theta 6^{\prime \prime}$, long $99^{\circ} 53^{\prime} 02^{\prime \prime}$, hydrologic unit 11120202

Aquifer: 310WTRS

Altitude of land-surface datum: $1,878 \mathrm{ft}$

Well depth: $52.2 \mathrm{ft}$

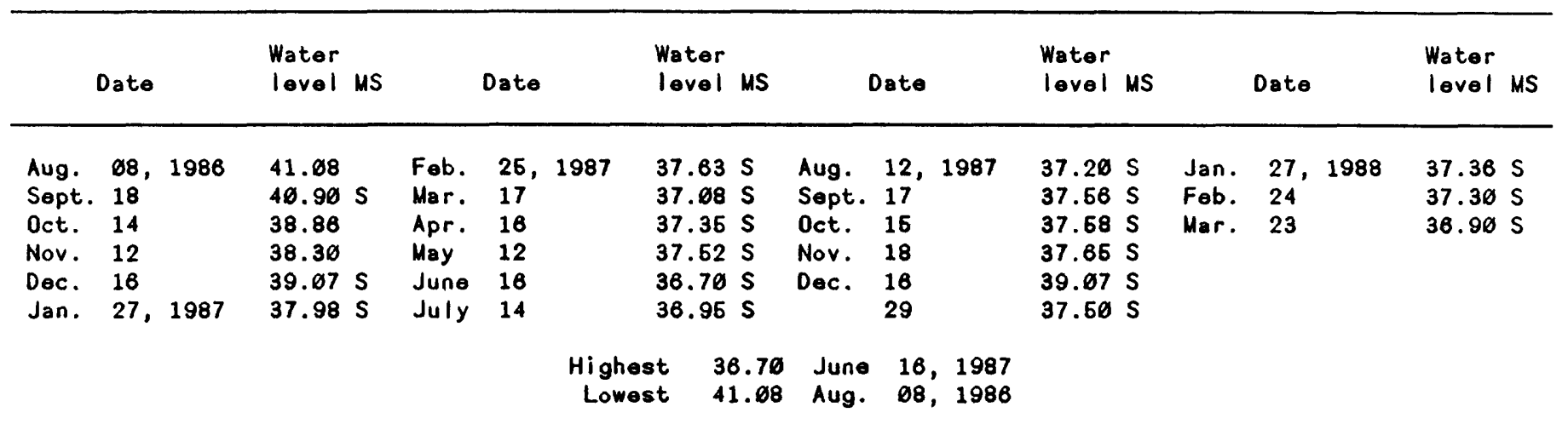

Index number on location map: 88

Station number: 345322099510401 Local number: $05 N-25 W-21$ CBCC 1

Location: Lat $34^{\circ} 53^{\prime} 25^{\prime \prime}$, long $99^{\circ} 51^{\prime} 09^{\prime \prime}$, hydrologic unit 11120202

Aquifer: 310WTRS

Altitude of land-surface datum: $1,662 \mathrm{ft}$

Well depth: $33.1 \mathrm{ft}$

Remarks: Well originally drilled to $42 \mathrm{ft}$.

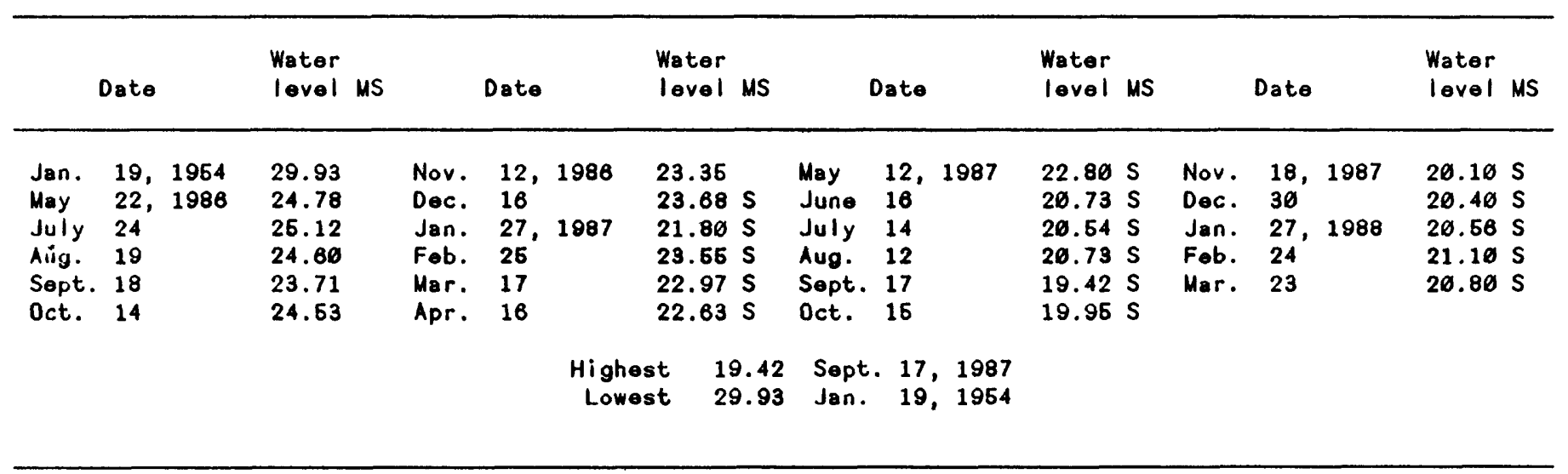

Index number on location map: 89

Station number: $\$ 45320099543601$ Local number: $05 \mathrm{~N}-28 W-23$ DDBB 1

Location: Lat $34^{\circ} 53^{\prime} 20^{\prime \prime}$, long $99^{\circ} 54^{\prime} 36^{\prime \prime}$, hydrologic unit 11120202

Aquifer: 310WTRS

Altitude of land-surface datum: $1,890 \mathrm{ft}$

Well depth: $115 \mathrm{ft}$

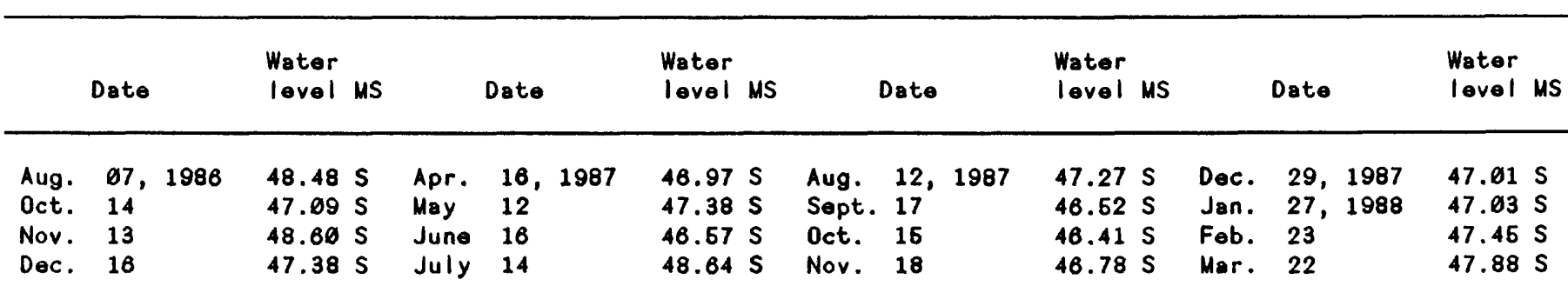


Table 2.- - Monthly or periodic water-level measurements in selected wells in southwestern oklahoma and northwestern Texas-Continued

HARMON COUNTY, OKLAHOMA--CONTinued

Index number on location map: 90

Station number: $\$ 345915099575601$ Local number: $66 \mathrm{~N}-26 \mathrm{~W}-17$ DCCC 1

Location: Lat $34^{\circ} 59^{\prime} 16^{\prime \prime}$, long $99^{\circ} 57^{\prime} 58^{\prime \prime}$, hydrologic unit 11129304

Aquifer: $313 B L I N$

Altitude of land-surface datum: $1,898 \mathrm{ft}$

Woll depth: $69.8 \mathrm{ft}$

Remarks: Well drilled to $116 \mathrm{ft}$ in 1946

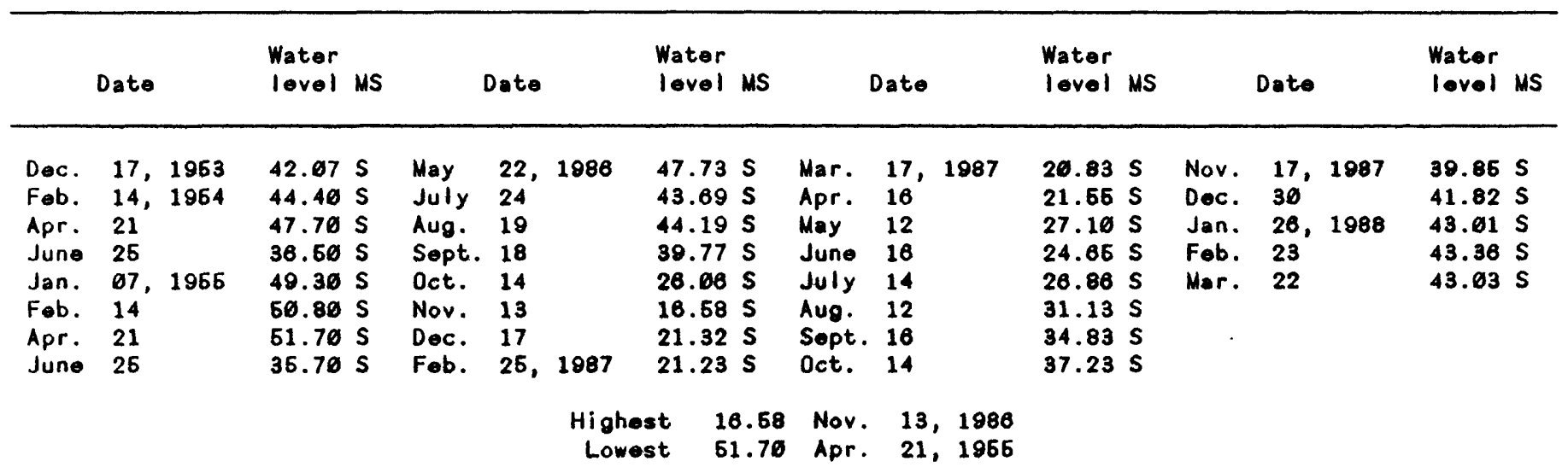


Table 2.--Monthly or periodic water-level measurements in selected wells in southwestern Oklahoma and northwestern Texas--Continued

\section{JACKSON COUNTY, OKLAHOMA}

Index number on location map: 91

Station number: 842500099440301 Local number: 02S-24W-65 ACBC 1

Location: Lat $34^{\circ} 25^{\prime} 00^{\prime \prime}$, long $99^{\circ} 44^{\prime} 03^{\prime \prime}$, hydrologic unit 11130161

Aquifer: 111RRVA

Altitude of land-surface datum: $1,425 \mathrm{ft}$

Hell depth: $47 \mathrm{ft}$

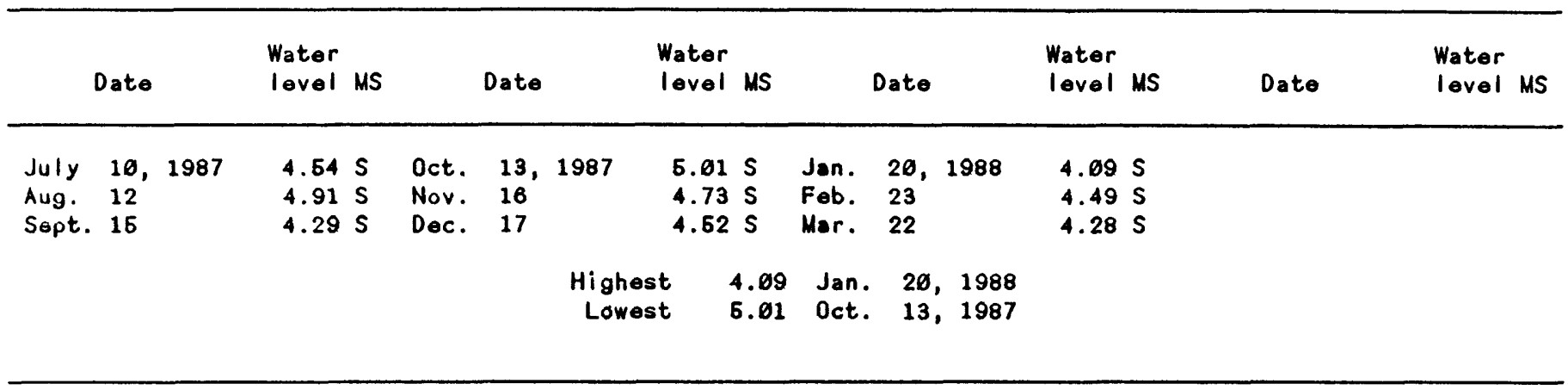

Index number on location map: 92

Station number: $\$ 42500099440302$ Local number: $025-24 W-05$ ACBC 2

Location: Lat $34^{\circ} 25^{\prime} ø \theta^{\prime \prime}$, long $99^{\circ} 44^{\prime} 03^{\prime \prime}$, hydrologic unit 11130101

Aquifer: 313 CLGR

Altitude of land-surface datum: $1,425 \mathrm{ft}$

Well depth: $70 \mathrm{ft}$

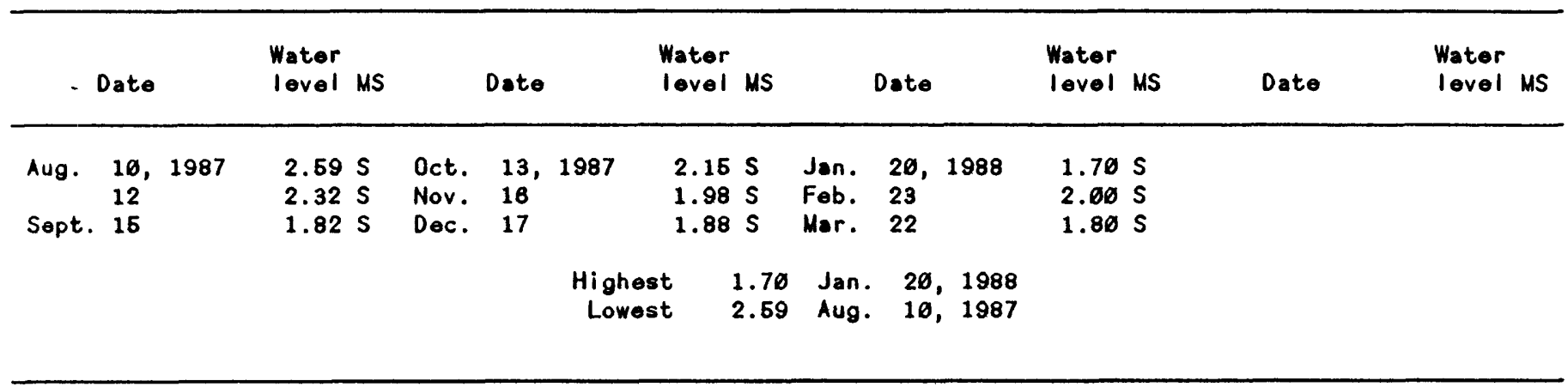

Index number on location map: 93

Station number: $\$ 42464 \varnothing 9944 \varnothing 6 \varnothing 1$ Local number: $025-24 W-\varnothing 5$ BDDD 1

Location: Lat $34^{\circ} 24^{\prime} 64^{\prime \prime}$, long $99^{\circ} 44^{\prime} 05^{\prime \prime}$, hydrologic unit 11130101

Aquifor: 111RRVA

Altitude of land-surface datum: $1,425 \mathrm{ft}$

Well depth: $50 \mathrm{ft}$

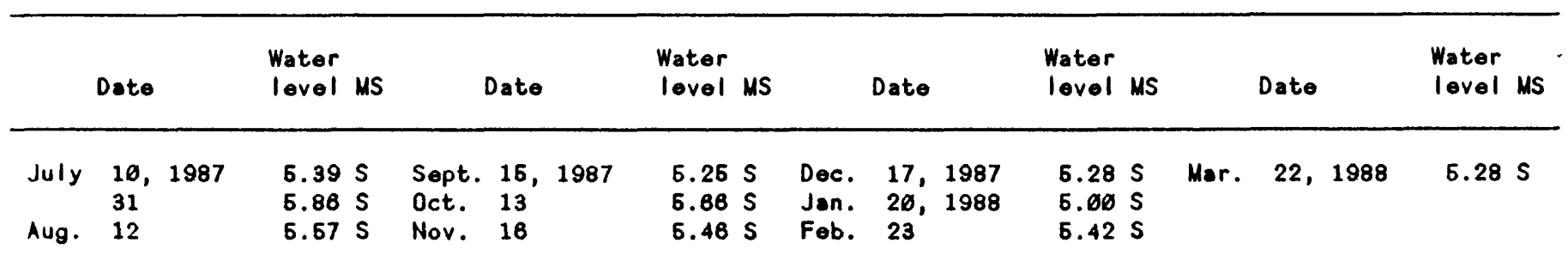

$\begin{array}{rlll}\text { Highest } & 5.00 & \text { Jan. } & 20,1988 \\ \text { Lowest } & 6.86 & \text { July } & 31,1987\end{array}$


Table 2.--Nonthly or periodic water-level measurements in selected wells in southwestern Oklahoma and northwestern Texas--Continued

JACKSON COUNTY, OKLAHOMA--CONTInUEd

Index number on location map: 94

Station number: 342338099392401 Local number: 02S-24W-12 CCCA 1

Location: Lat $34^{\circ} 23^{\prime} 39^{\prime \prime}$, long $99^{\circ} 40^{\prime} 18^{\prime \prime}$, hydrologic unit 11130101

Aquifer: 313 BLIN

Altitude of land-surface datum: $1,479 \mathrm{ft}$

Well depth: $90 \mathrm{ft}$

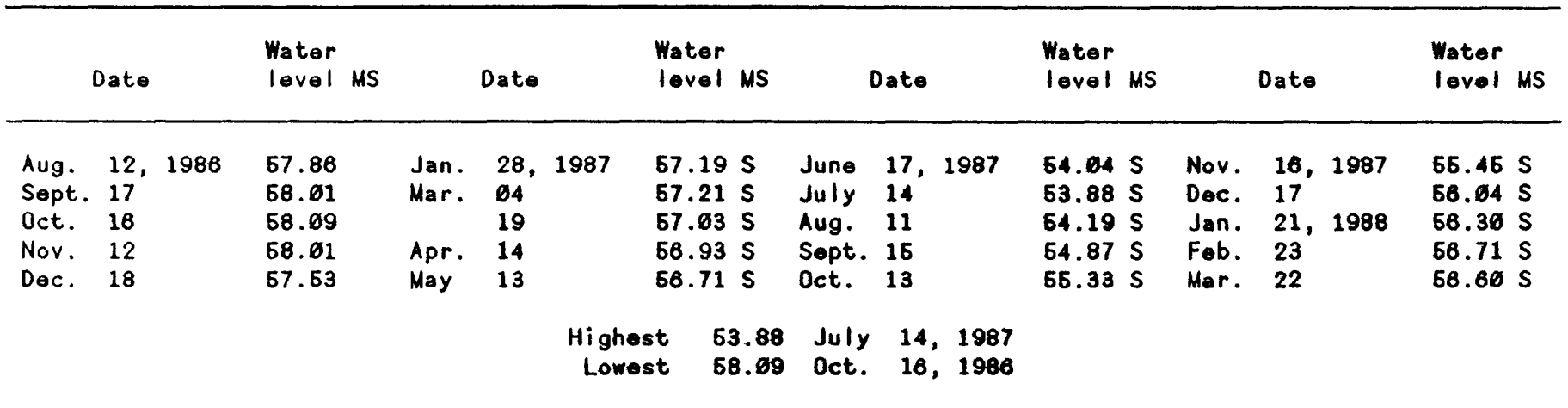

Index number on location map: 95

Station number: 342606099270501 Local number: g1S-22W-36 AABB 1

Location: Lat $34^{\circ} 26^{\prime} 65^{\prime \prime}$, long $99^{\circ} 27^{\prime} 65^{\prime \prime}$, hydrologic unit 11130161

Aquifer: $313 B L I N$

Altitude of land-surface datum: $1,400 \mathrm{ft}$

Well depth: $28.5 \mathrm{ft}$

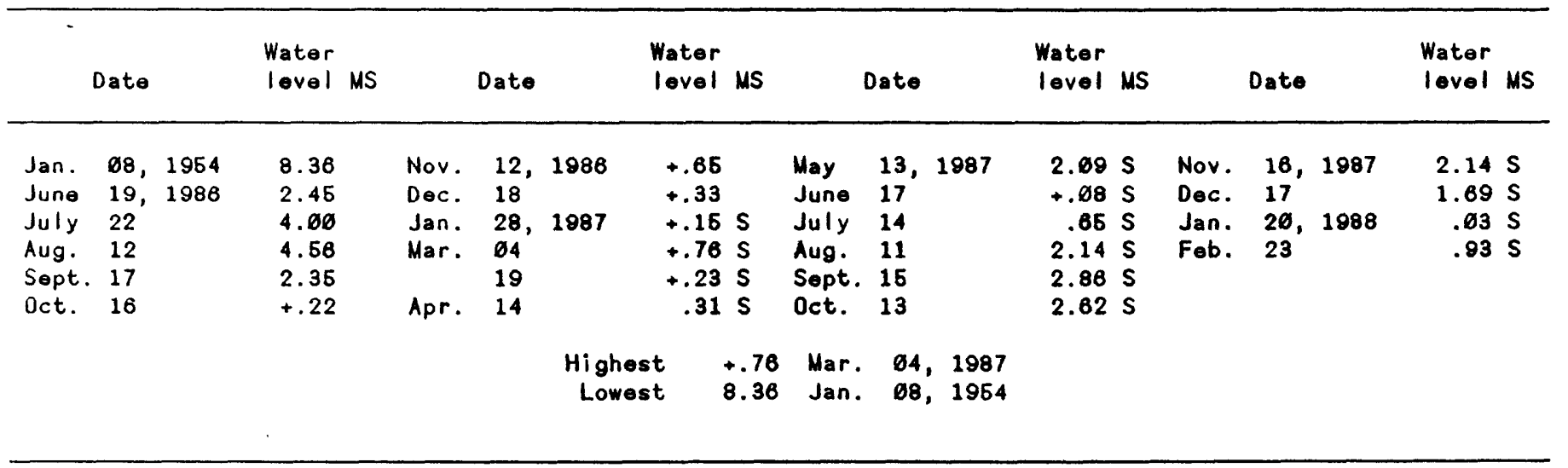

Index number on location map: 98

Station number: $\$ 42848099363361$ Local number: $15-23 W-\varnothing 9$ DCDC 1

Location: Lat $34^{\circ} 28^{\prime} 47^{\prime \prime}$, long $99^{\circ} 36^{\prime} 33^{\prime \prime}$, hydrologic unit 11138101

Aquifer: $313 B L I N$

Altitude of land-surface datum: $1,504 \mathrm{ft}$

Well depth: $103 \mathrm{ft}$

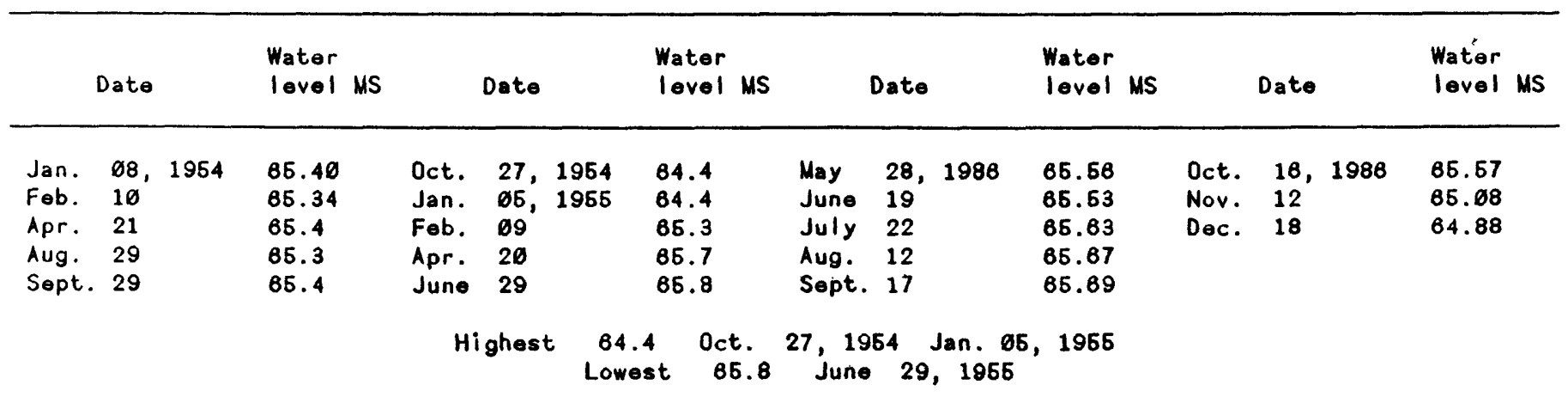


Table 2.--Monthly or periodic water-level measurements in selected wells in southwestern

Oklahoma and northwestern Texas--Continued

JACKSON COUNTY, OKLAHOMA--Continued

Index number on location map: 97

Station number: 342648099362601 Local number: $01 S-23 W-28$ ADBA 1

Location: Lat $34^{\circ} 26^{\prime} 48^{\prime \prime}$, long $99^{\circ} 36^{\prime} 26^{\prime \prime}$, hydrologic unit 11136101

Aquifer: 313BLIN

Altitude of land-surface datum: $1,445 \mathrm{ft}$

Well depth: $39.2 \mathrm{ft}$

Remarks: Well originally drilled to $45 \mathrm{ft}$

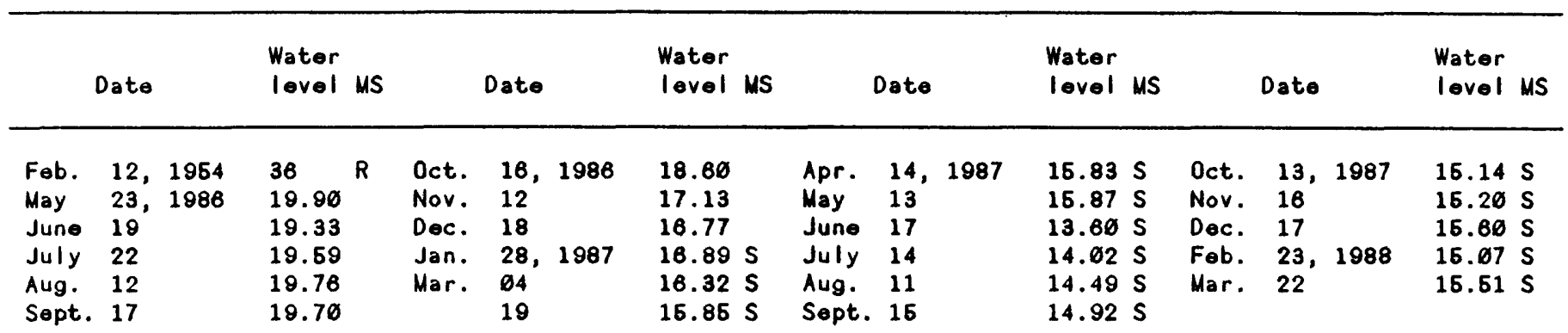

$\begin{array}{clll}\text { Highest } & 13.60 & \text { June } 17,1987 \\ \text { Lowest } & 36 & \text { Feb. } 12,1954\end{array}$

Index number on location map: 98

Station number: $\$ 43020699404601$ Local number: $01 S-24 W-\varnothing 2$ ABB 1

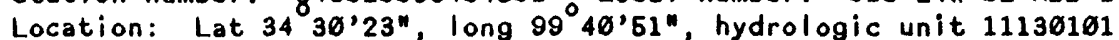

Aquifer: 313BLIN

Altitude of land-surface datum: $1,462 \mathrm{ft}$

Well-depth: $65.5 \mathrm{ft}$

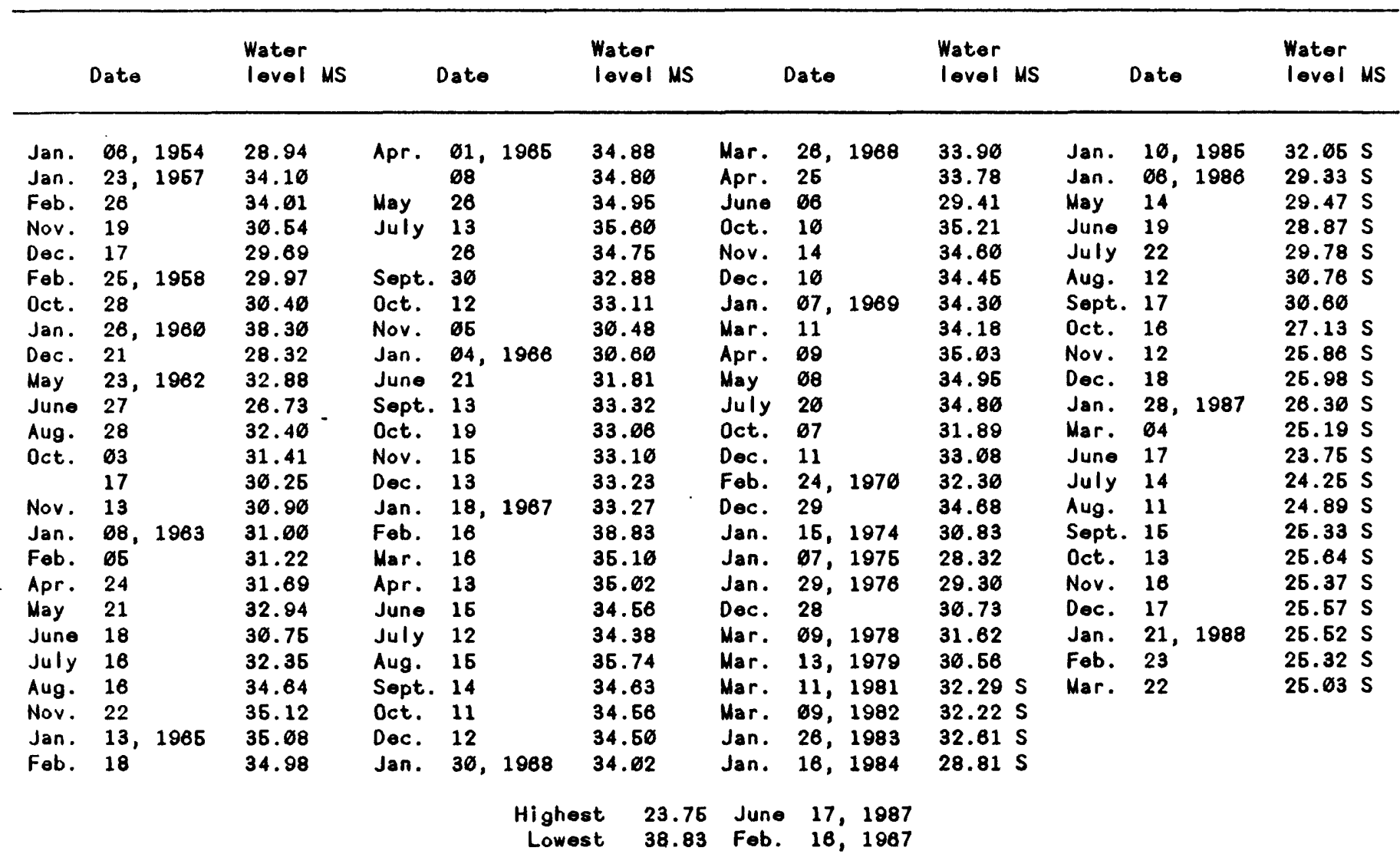


Table 2.-- Monthly or periodic water-level measurements in selected wells in southwestern Oklahoma and northwestern Texas--Continued

JACKSON COUNTY, OKLAHOMA--CONtinued

Index number on location map: 99

Station number: 342836099433601 Local number: 01S-24W-17 AADD 1

Location: Lat $34^{\circ} 28^{\prime} 36^{\prime \prime}$, long $99^{\circ} 43^{\prime} 36^{\prime \prime}$, hydrologic unit 11130101

Aquifer: $313 V V C R$

Altitude of land-surface datum: $1,466 \mathrm{ft}$

Well depth: $75 \mathrm{ft}$

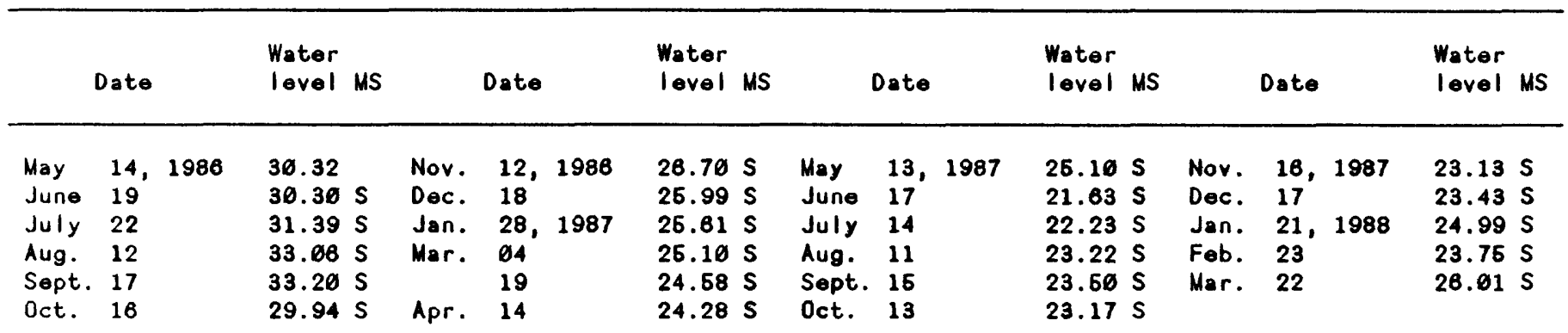

Highest 21.63 June 17,1987

Lowest 33.20 Sept. 17, 1986

Index number on lacation map: 100

Station number: 842642099434801 Local number: $15-24 W-32$ DBAA 1

Location: Lat $34^{\circ} 25^{\prime} 42^{\prime \prime}$, long $99^{\circ} 43^{\prime} 48^{\prime \prime}$, hydrologic unit 11130101

Aquifer: $313 V V C R$

Altitude of land-surface datum: $1,470 \mathrm{ft}$

Well depth: $80 \mathrm{ft}$

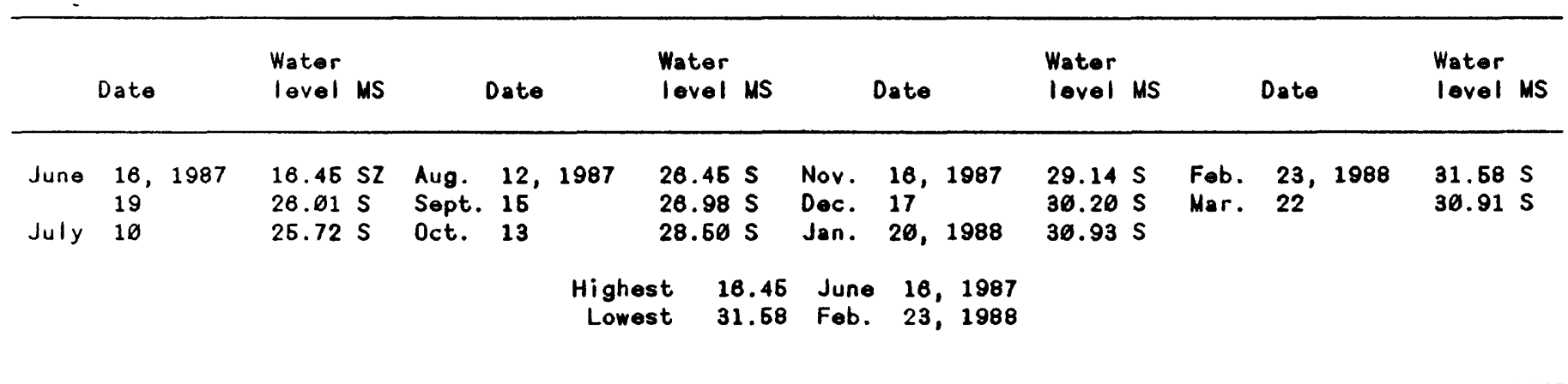

Index number on location map: 101

Station number: 842642099434802 Local number: Ø1S-24W-32 DBAA 2

Location: Lat $34^{\circ} 26^{\prime} 42^{\prime \prime}$, long $99^{\circ} 43^{\prime} 48^{\prime \prime}$, hydrologic unit 11130101

Aquifer: 313 FLRP

Altitude of land-surface datum: $1,470 \mathrm{ft}$

Well depth: $239 \mathrm{ft}$

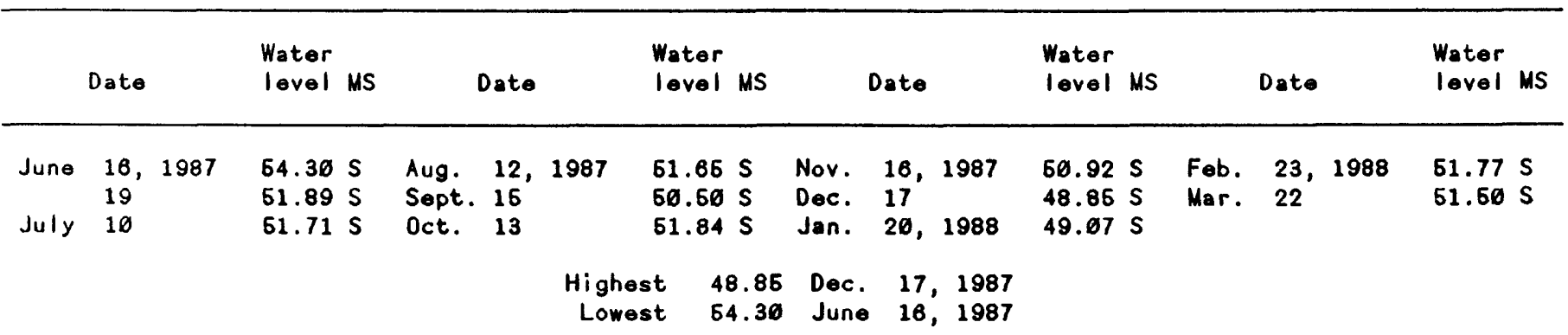


Table 2.-- Monthly or periodic water-level measurements in selected wells in southwestern Oklahoma and northwestern Texas--Continued

JACKSON COUNTY, OKLAHOMA--CONtinued

Index number on location map: 162

Station number: $\$ 42542099434803$ Local number: ø1S-24W-32 DBAA 3

Location: Lat $34^{\circ} 25^{\prime} 42^{\prime \prime}$, long $99^{\circ} 43^{\prime} 48^{\prime \prime}$, hydrologic unit 11136161

Aquifer: $313 E M F K$

Altitude of land-surface datum: $1,470 \mathrm{ft}$

Well depth: $179 \mathrm{ft}$

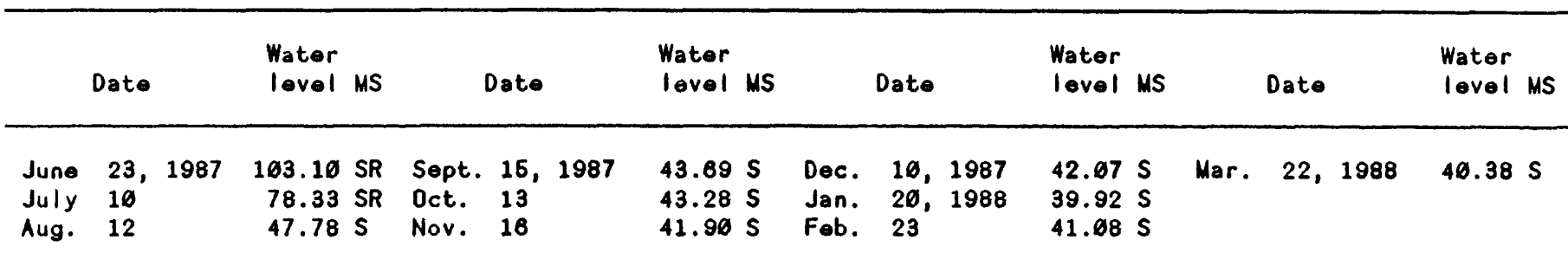

$\begin{array}{rrrrr}\text { Highest } & 39.92 & \text { Jan. } & 20,1988 \\ \text { Lowest } & 78.33 & \text { July } & 10,1987\end{array}$

Index number on location map: 103

Station number: $\$ 42542099434804$ Local number: Ø1S-24W-32 DBAA 4

Location: Lat $34^{\circ} 25^{\prime} 42^{\prime \prime}$, long $99^{\circ} 43^{\prime} 48^{\prime \prime}$, hydrologic unit 11130101

Aquifer: 11øRRVT

Altitude of land-surface datum: $1,470 \mathrm{ft}$

Well depth: $56 \mathrm{ft}$

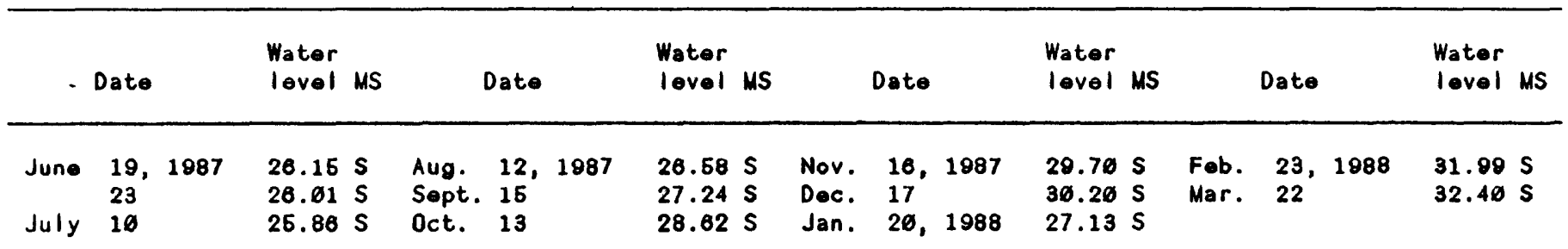

Highest 25.88 July 10,1987

Lowest 32.40 Mar. 22, 1988

Index number on location map: 104

Station number: $\$ 42847099470401$ Local number: $615-25 W-11$ DCDC 1

Location: Lat $34^{\circ} 28^{\prime} 47^{\prime \prime}$, long $99^{\circ} 47^{\prime} 04^{\prime \prime}$, hydrologic unit 11130101

Aquifer: $313 B L I N$

Altitude of land-surface datum: $1,530 \mathrm{ft}$

Well depth: $120 \mathrm{ft}$

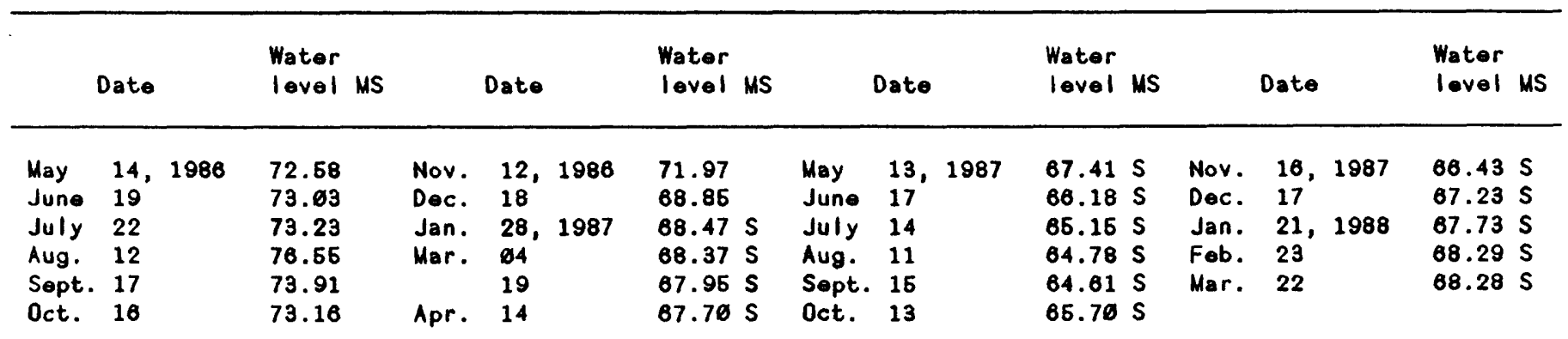

Highest 64.61 Sept. 15, 1987

Lowest 76.55 Aug. 12,1986 
Table 2.-- Nonthly or periodic vater-level measurements in selected wells in southwestern oklahoma and northwestern Texas--Continued

JACKSON COUNTY, OKLAHOMA--COntinued

Index number on location map: 105

Station number: 842744099460701 Local number: $615-25 W-13$ CCCA 1

Location: Lat $34^{\circ} 27^{\prime} 68^{\prime \prime}$, long $99^{\circ} 46^{\prime} 34^{\prime \prime}$, hydrologic unit 11130101

Aquifer: 313BLIN

Altitude of land-surface datum: $1,627 \mathrm{ft}$

Well depth: $130 \mathrm{ft}$

Remarks: Previously published as $015-25 W-13$ CCC

\begin{tabular}{|c|c|c|c|c|c|c|c|c|c|c|c|c|c|c|c|c|}
\hline \multicolumn{3}{|c|}{ Date } & \multirow{2}{*}{$\begin{array}{l}\text { Water } \\
\text { level MS }\end{array}$} & \multicolumn{3}{|c|}{ Date } & $\begin{array}{l}\text { Water } \\
\text { level MS }\end{array}$ & \multicolumn{3}{|c|}{ Date } & \multirow{2}{*}{$\begin{array}{l}\text { Water } \\
\text { level MS }\end{array}$} & \multicolumn{3}{|c|}{ Date } & $\begin{array}{l}\text { Water } \\
\text { level }\end{array}$ & MS \\
\hline Aug. & 12 & 1953 & & June & 24 & 1958 & 71.35 & May & 21 & 1983 & & Mar. & 11 & 1989 & 77.72 & \\
\hline Mar. & 25 & 1964 & 75.67 & July & 29 & & 71.26 & June & 18 & & 74.89 & Apr. & 99 & & 77.75 & \\
\hline Apr. & 20 & & 76.63 & Aug. & 26 & & 72.80 & July & 16 & & 80.90 & May & 68 & & 77.65 & \\
\hline May & 27 & & 76.73 & Sept. & 23 & & 70.91 & Aug. & 16 & & 77.59 & June & 19 & & 77.84 & \\
\hline June & 23 & & 78.64 & 0ct. & 28 & & 70.90 & Nov. & 22 & & 76.80 & Sept. & 94 & & 79.29 & \\
\hline Aug. & 25 & & 78.70 & Nov. & 19 & & 70.23 & Fob. & 10 & 1964 & 87.79 & Oct. & 07 & & 78.50 & \\
\hline Sept. & 23 & & 79.17 & Jan. & 68 & 1959 & 70.62 & Jan. & 13 & 1966 & 78.03 & Nov. & 18 & & 72.92 & \\
\hline oct. & 25 & & 77.03 & & 27 & & 70.79 & Fob. & 18 & & 77.94 & Dec. & 11 & & 77.69 & \\
\hline Dec. & 62 & & 77.20 & Feb. & 24 & & 71.06 & Apr. & 01 & & 85.67 & Jan. & 26 & $197 \varnothing$ & 76.10 & \\
\hline Jan. & 86 & 1956 & 76.49 & Mar. & 24 & & 69.96 & & 68 & & 77.96 & Fob. & 24 & & 77.07 & \\
\hline & 25 & & 76.86 & Apr. & 21 & & 73.36 & May & 26 & & 74.97 & Dec. & 29 & & 77.95 & \\
\hline Feb. & 98 & & 76.19 & May & 19 & & 72.73 & July & 13 & & 78.73 & Jan. & 20 & 1972 & 80.32 & \\
\hline & 22 & & 79.09 & June & 30 & & 72.91 & Sept. & 30 & & 80.86 & Fob. & 14 , & 1973 & 78.63 & \\
\hline Mar. & 22 & & 79.37 & Sept. & 22 & & 74.76 & 0ct. & 12 & & 80.36 & Jan. & 16 & 1974 & 76.18 & \\
\hline Apr. & 20 & & 79.74 & oct. & 20 & & 74.74 & Nov. & 04 & & 79.05 & Dec. & 28 & 1976 & 78.87 & \\
\hline May & 24 & & 78.24 & Nov. & 19 & & 73.84 & Jan. & 64, & 1986 & 77.78 & Mar. & 69 & 1978 & 75.26 & \\
\hline June & 24 & & 78.43 & Jan. & 28 & 1980 & 73.63 & Apr. & 19 & & 78.34 & Mar. & 13 & 1979 & 76.56 & \\
\hline July & 26 & & 76.07 & Mar. & 21 & & 73.67 & June & 21 & & 77.76 & Feb. & 28 , & 1980 & 75.48 & \\
\hline Sept. & 29 & & 76.29 & Apr. & 19 & & 73.62 & Sept. & 13 & & 78.74 & Mer. & 11 & 1981 & 76.72 & $s$ \\
\hline $0 c t$ & 26 & & 75.20 & May & 17 & & 73.86 & Oct. & 19 & & 78.20 & Mar. & 99 & 1982 & 76.78 & $s$ \\
\hline Nov. & 29 & & 73.17 & June & 21 & & 73.76 & Nov. & 15 & & 77.98 & Jan. & 26 & 1983 & 77.14 & $s$ \\
\hline Dec. & 21 & & 72.55 & July & 19 & & 73.38 & Dec. & 13 & & 77.89 & Jan. & 16 & 1984 & 75.65 & $s$ \\
\hline Jan. & 31 , & 1958 & 72.13 & Aug. & 16 & & 76.21 & Jan. & 18 & 1967 & 77.77 & Jan. & 10 & 1986 & 76.10 & $s$ \\
\hline Feb. & 21 & & 73.17 & Sept. & 13 & & 73.74 & Feb. & 16 & & 77.67 & Jan. & $D B$ & 1986 & 73.17 & $s$ \\
\hline Mar. & 20 & & 72.23 & Nov. & 15 & & 72.36 & Mar. & 16 & & 80.91 & May & 14 & & 78.32 & \\
\hline Apr. & 24 & & 75.13 & Dec. & 21 & & 71.45 & Apr. & 13 & & 78.30 & June & 19 & & 74.23 & \\
\hline May & 22 & & 73.65 & Jan. & 17 & 1981 & 71.88 & May & 16 & & 78.48 & July & 22 & & 74.40 & \\
\hline June & 26 & & 72.65 & Feb. & 14 & & 71.06 & June & 15 & & 78.26 & Aug. & 12 & & 76.70 & \\
\hline July & 24 & & 77.02 & Mar. & 14 & & 71.06 & July & 12 & & 78.66 & Sept. & 17 & & 76.17 & \\
\hline Aug. & 28 & & 81.45 & Apr. & 19 & & 70.89 & Aug. & 15 & & 78.30 & oct. & 16 & & 74.79 & \\
\hline Sept. & 18 & & 77.08 & May & 17 & & 70.92 & Sept. & 14 & & 80.30 & Nov. & 12 & & 73.67 & \\
\hline $0 c t$. & 30 & & 76.45 & June & 21 & & 79.42 & Oct. & 11 & & 78.73 & Dec. & 18 & & 72.20 & \\
\hline Nov. & 27 & & 76.10 & July & 12 & & 70.67 & Nov. & 16 & & 79.47 & Jan. & 28 & 1987 & 71.68 & $S$ \\
\hline Dec. & 18 & & 76.14 & Sept. & 86 & & 73.13 & Dec. & 12 & & 79.38 & Mar. & 04 & & 71.13 & $S$ \\
\hline Jan. & 23 & 1957 & 76.16 & & 26 & & 72.38 & Jan. & 30 & 1968 & 79.08 & & 19 & & 70.82 & $S$ \\
\hline Fob. & 26 & & 76.17 & Oct. & 24 & & 72.33 & Feb. & $20^{\circ}$ & & 78.93 & Apr. & 14 & & 70.68 & $S$ \\
\hline Apr. & 04 & & 76.25 & Nov. & 21 & & 71.04 & Mar. & 26 & & 78.85 & May & 13 & & 72.99 & $S$ \\
\hline May & 07 & & 76.37 & Dec. & $\varnothing 5$ & & 72.24 & Apr. & 25 & & 78.73 & June & 17 & & 70.28 & $S$ \\
\hline & 28 & & 75.42 & Jan. & 31 & 1982 & 72.26 & June & 96 & & 78.62 & July & 14 & & 70.46 & $S$ \\
\hline June & 25 & & 72.63 & May & 23 & & 74.87 & & 19 & & 78.60 & Aug. & 11 & & 69.29 & $S$ \\
\hline Oct. & $\theta_{1}$ & & 69.77 & June & 27 & & 74.14 & July & 17 & & 79.60 & Sept. & 16 & & 68.11 & $S$ \\
\hline & 29 & & 69.23 & Aug. & 28 & & 74.08 & Aug. & 15 & & 92.25 & Oct. & 13 & & 68.34 & $S$ \\
\hline Nov. & 19 & & 69.12 & oct. & 93 & & 72.55 & Sept. & 19 & & 88.05 & Nov. & 16 & & 68.62 & $\mathrm{~s}$ \\
\hline Dec. & 17 & & 69.20 & & 17 & & 73.48 & Oct. & 10 & & 87.47 & Dec. & 17 & & 69.17 & $S$ \\
\hline Feb. & 26 & 1958 & 89.87 & Nov. & 13 & & 73.14 & Nov. & 14 & & 84.87 & Jan. & 21 & 1988 & 69.63 & $S$ \\
\hline Mar. & $25^{\circ}$ & & 70.47 & Jan. & 98 & 1963 & 72.90 & Dec. & 10 & & 78.30 & Feb. & $23^{\circ}$ & & 70.01 & $S$ \\
\hline Apr. & 22 & & 70.67 & $F \in b$. & 65 & & 73.20 & Jan. & 87 , & 1989 & 78.00 & Mar. & 22 & & 70.11 & $S$ \\
\hline May & 20 & & 71.23 & Mar. & 26 & & 74.18 & Feb. & 64 & & 77.95 & & & & & \\
\hline & & & & & & Low & $\begin{array}{l}88.11 \\
92.26\end{array}$ & Aug. & $\begin{array}{l}15 \\
16\end{array}$ & $\begin{array}{l}1987 \\
1988\end{array}$ & & & & & & \\
\hline
\end{tabular}


Tablo 2.--Monthly or periodic water-level measurements in selected wells in southwestern Oklahoma and northwestern Texas--Continued

JACKSON COUNTY, OKLAHOMA--Continued

Index number on location map: 186

Station number: $\$ 43359099314701$ Local number: $11 \mathrm{~N}-22 W-\varnothing 8$ DDCD 1

Location: Lat $34^{\circ} 33^{\prime} 44^{*}$, long $99^{\circ} 31^{\prime} 46^{*}$, hydrologic unit 11130101

Aquifer: 313 VVCR

Altitude of land-surface datum: $1,478 \mathrm{ft}$

Well depth: $35 \mathrm{ft}$

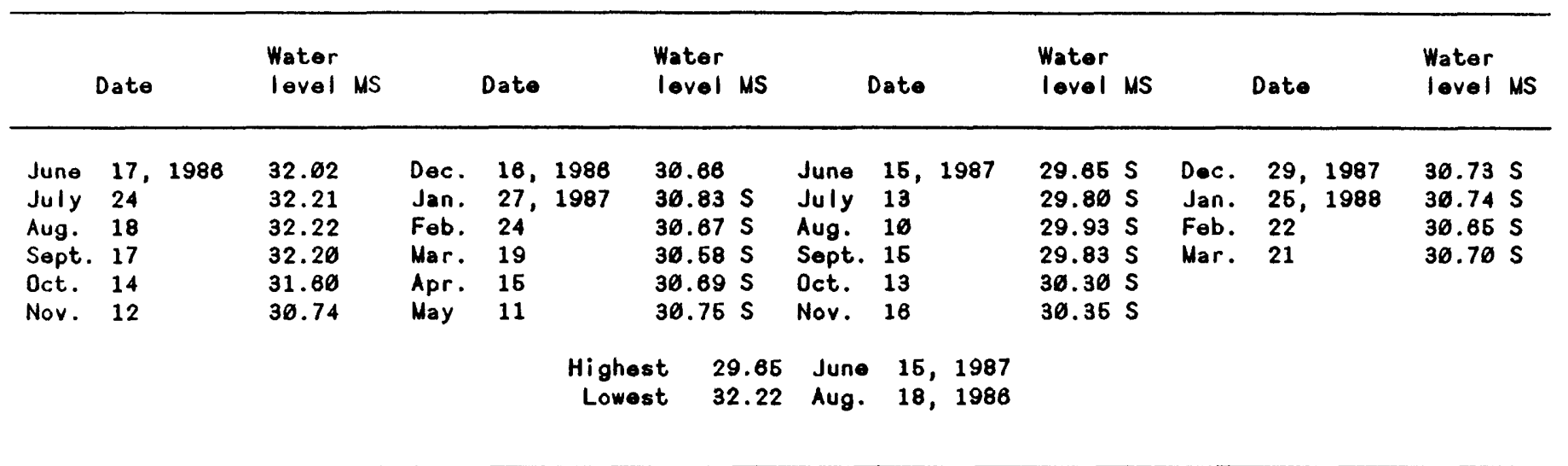

Index number on location map: 107

Station number: $\$ 43221099375701$ Local number: $11 \mathrm{~N}-23 W-20$ DDAA 1

Location: Lat $34^{\circ} 32^{\prime} 21^{\prime \prime}$, long $99^{\circ} 37^{\prime} 63^{\prime \prime}$, hydrologic unit 11130101

Aquifer: $313 V V C R$

Altitude of land-surface datum: $1,440 \mathrm{ft}$

Woll depth: $42.4 \mathrm{ft}$

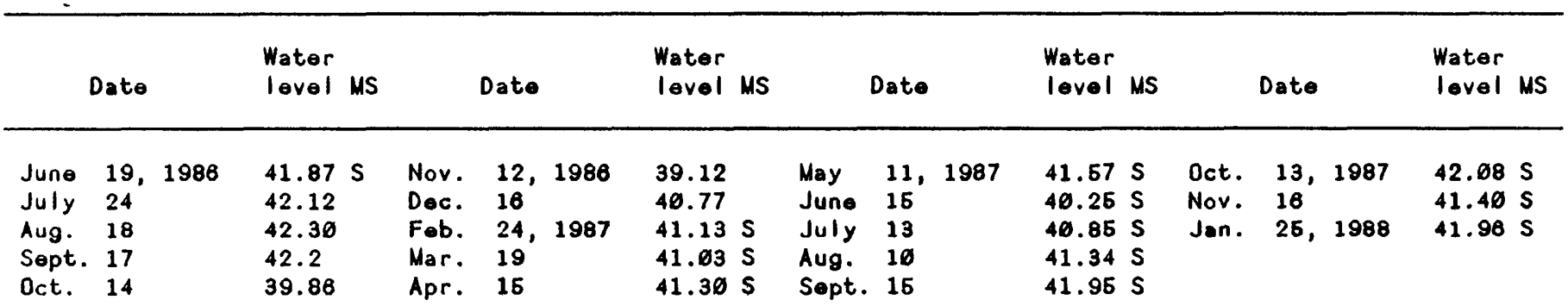

Highest 39.12 Nov. 12, 1986

Lowest 42.36 Aug. 18, 1986 
Table 2.-- Monthly or periodic water-level measurements in selected wells in southwestern Oklahoma and northwestern Texas--Continued

Index number on location map: 188

Station number: 343913099313901 Local number: $02 \mathrm{~N}-22 W-08$ DDA 1

Location: Lat $34^{\circ} 39^{\prime} 13^{\prime \prime}$, long $99^{\circ} 31^{\prime} 39^{\prime \prime}$, hydrologic unit 11120202

Aquifer: $313 B L I N$

Altitude of land-surface datum: $1,417 \mathrm{ft}$

Well depth: $75 \mathrm{ft}$

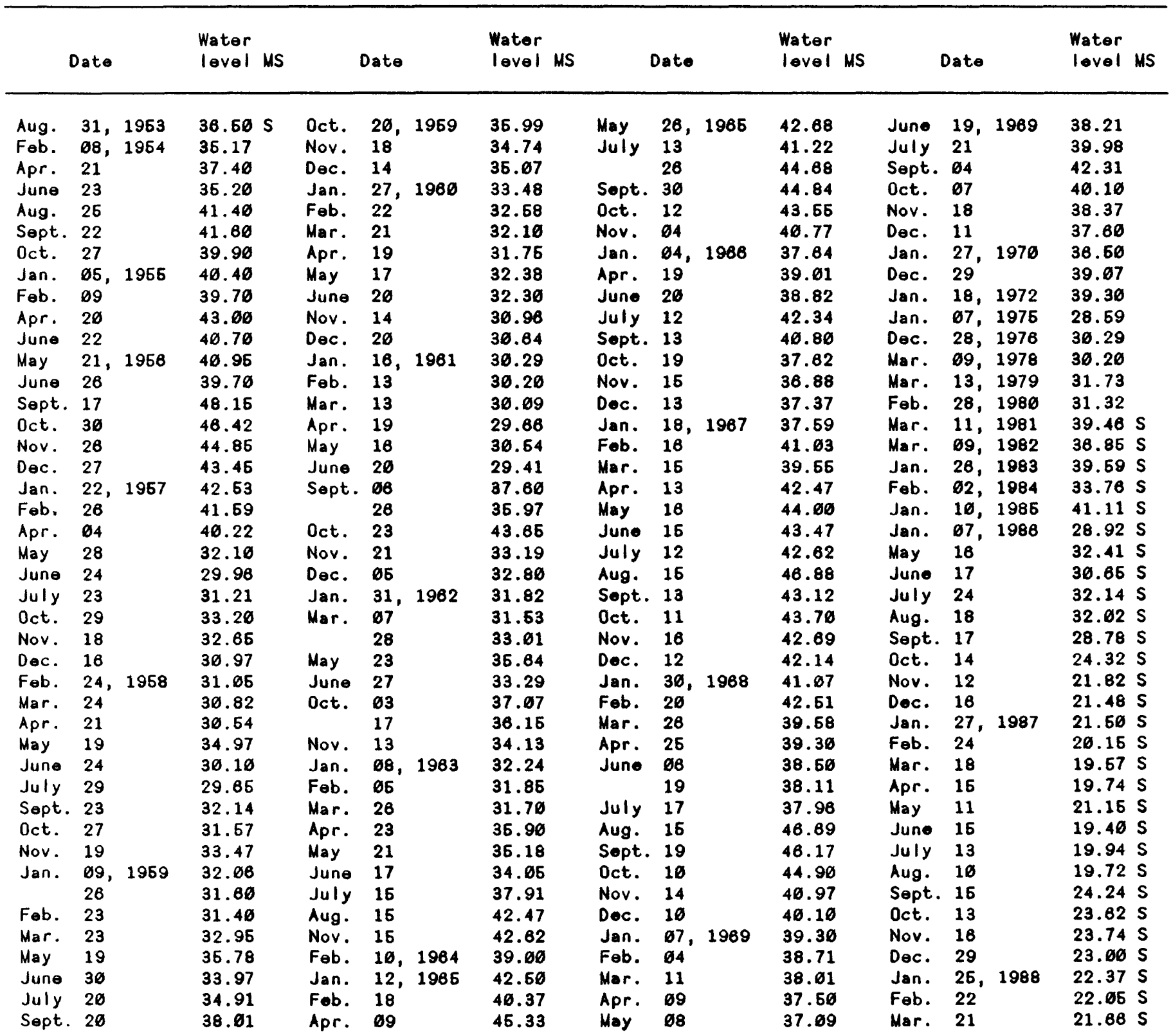

Highest $19.4 \varnothing$ June 15,1987

Lowest 48.15 Sept. 17,1956 
Table 2.-- Monthly or periodic water-level measurements in selected wells in southwestern Oklahoma and northwestern Texas--Continued

JACKSON COUNTY, OKLAHOMA--CONTI nued

Index number on location map: 109

Station number: 343953099360901 Local number: 62N-23W-10 ABDB 1

Location: Lat $34^{\circ} 39^{\prime} 53^{\prime \prime}$, long 99 $36^{\prime} 09^{\prime \prime}$, hydrologic unit 11120202

Aquifer: 3138LIN

Altitude of land-surface datum: $1,431 \mathrm{ft}$

Well depth: $90 \mathrm{ft}$

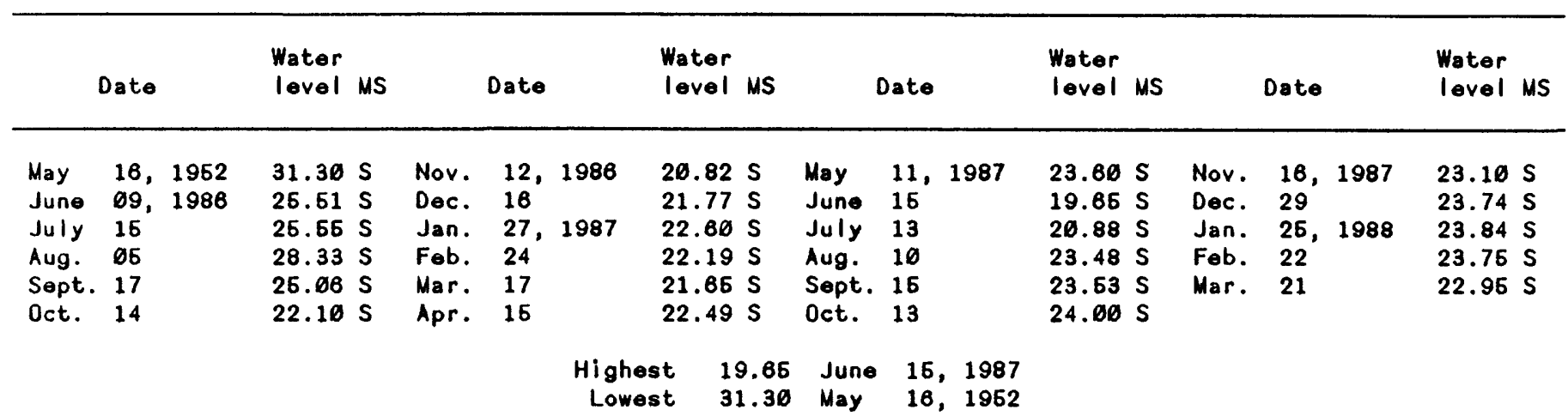

Index number on location map: 110

Station number: 343929099361701 Local number: $62 \mathrm{~N}-23 W-10$ DBBC 1

Location: Lat $34^{\circ} 39^{\prime} 29^{\prime \prime}$, long $99^{\circ} 36^{\prime} 17^{\prime \prime}$, hydrologic unit 11120202

Aquifer: 313 BLIN

Altitude of land-surface datum: $1,422 \mathrm{ft}$

Well depth: $82 \mathrm{ft}$

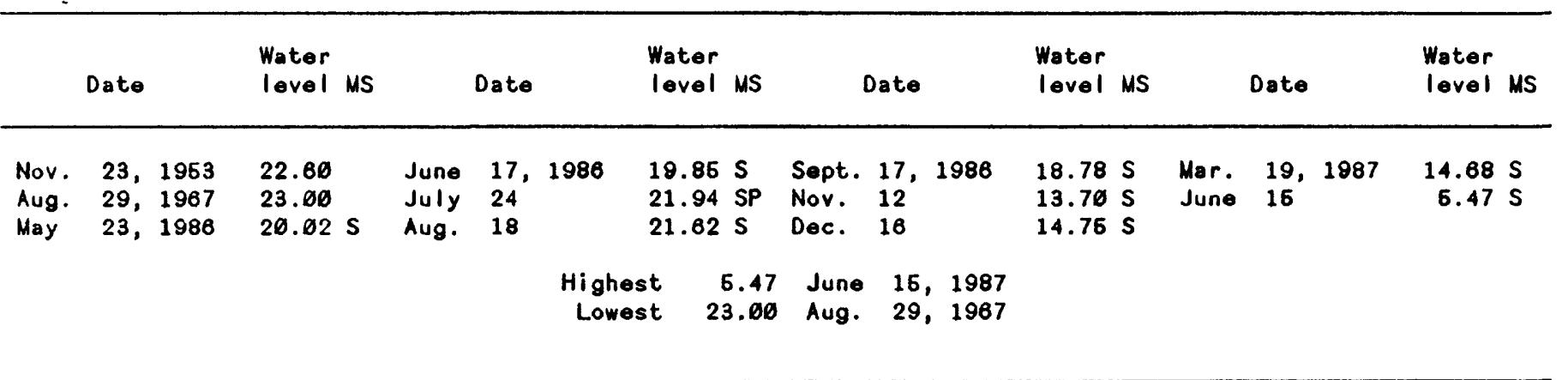

Index number on location map: 111

Station number: $\$ 43930699360601$ Local number: $\$ 2 \mathrm{~N}-23 W-11$ DBAC 1

Location: Lat $34^{\circ} 39^{\prime} 39^{\prime \prime}$, long $99^{\circ} 36^{\prime} 95^{\prime \prime}$, hydrologic unit 11120202

Aquifer: 313 VVCR

Altitude of land-surface datum: $1,413 \mathrm{ft}$

Well depth: $80 \mathrm{ft}$

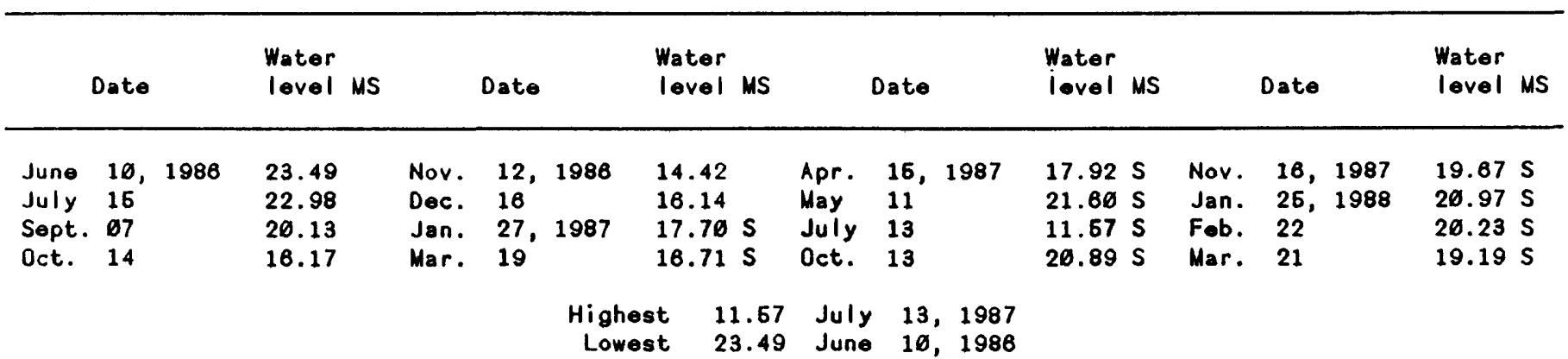


Table 2.-- Nonthly or periodic water-level measurements in selected wells in southwestern oklahoma and northwestern Texas--Continued

JACKSON COUNTY, OKLAHOMA--COntinued

Index number on location map: 112

Station number: 843945099342501 . Local number: $02 \mathrm{~N}-23 \mathrm{~W}-12$ BBDD 1

Location: Lat $34^{\circ} 39^{\prime} 46^{\prime \prime}$, long $99^{\circ} 34^{\prime} 29^{\prime \prime}$, hydrologic unit 11120202

Aquifor: $313 B L I N$

Altitude of land-surface datum: $1,419 \mathrm{ft}$

Well depth: $200 \mathrm{ft}$

Remarks: Previously published as $92 \mathrm{~N}-23 W-12$ B8D.

Well deepened from 98 to $200 \mathrm{ft}$ in April 1953.

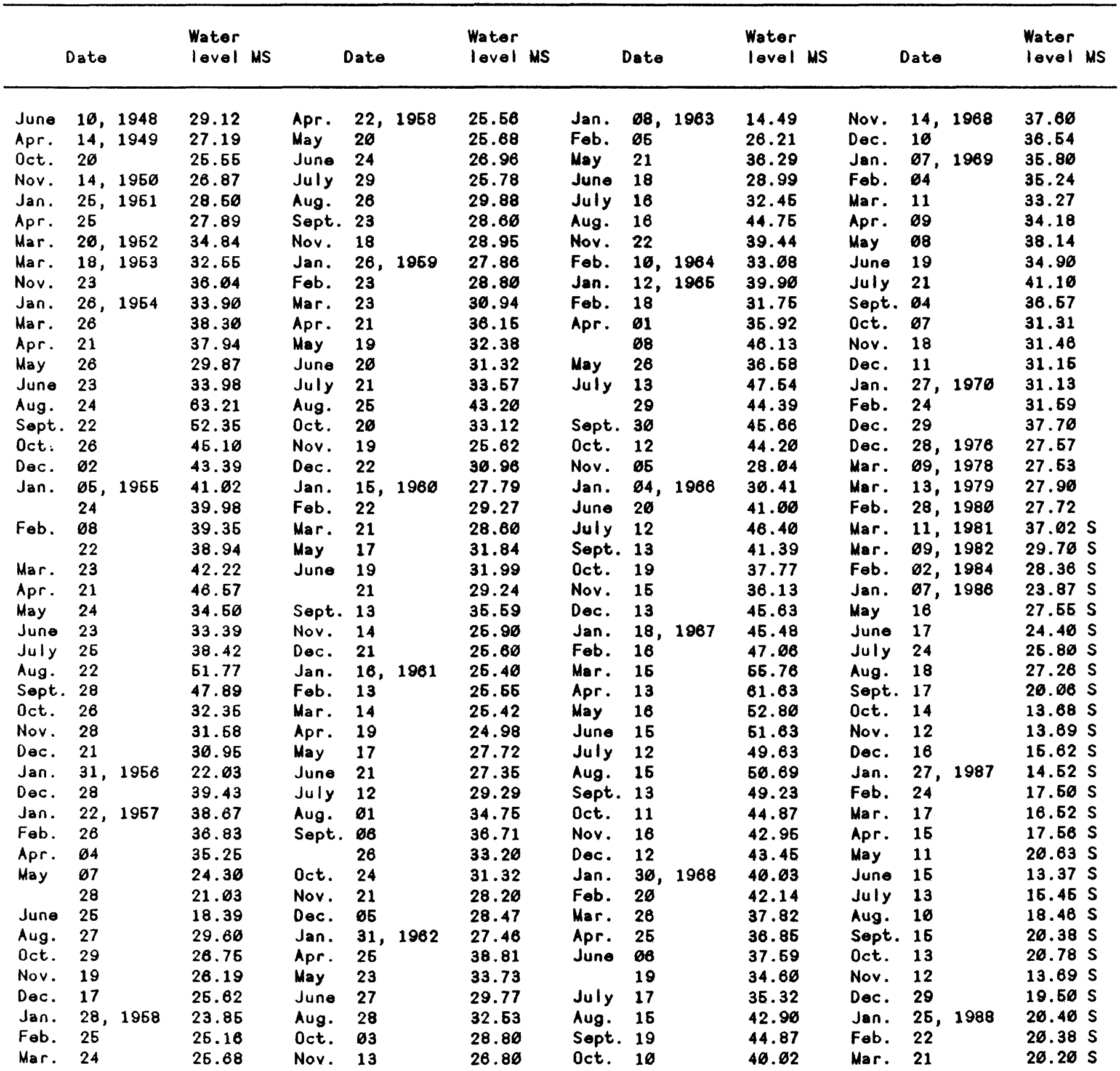

Highest 13.37 June 15,1987

Lowest 63.21 Aug. 24, 1954 
Table 2.--Monthly or periodic water-level measurements in selected wells in southwestern

Oklahoma and northwestern Texas--Continued

JACKSON COUNTY, OKLAHOMA--CONt inued

Index number on location map: 113

Station number: 343810099365701 Local number: $62 N-23 W-21$ AAA 1

Location: Lat $34^{\circ} 38^{\prime} 10^{\prime \prime}$, long $99^{\circ} 36^{\prime} 57^{\prime \prime}$, hydrologic unit 11120202

Aquifer: 313BLIN

Altitude of land-surface datum: $1,428 \mathrm{ft}$

Well depth: $89 \mathrm{ft}$

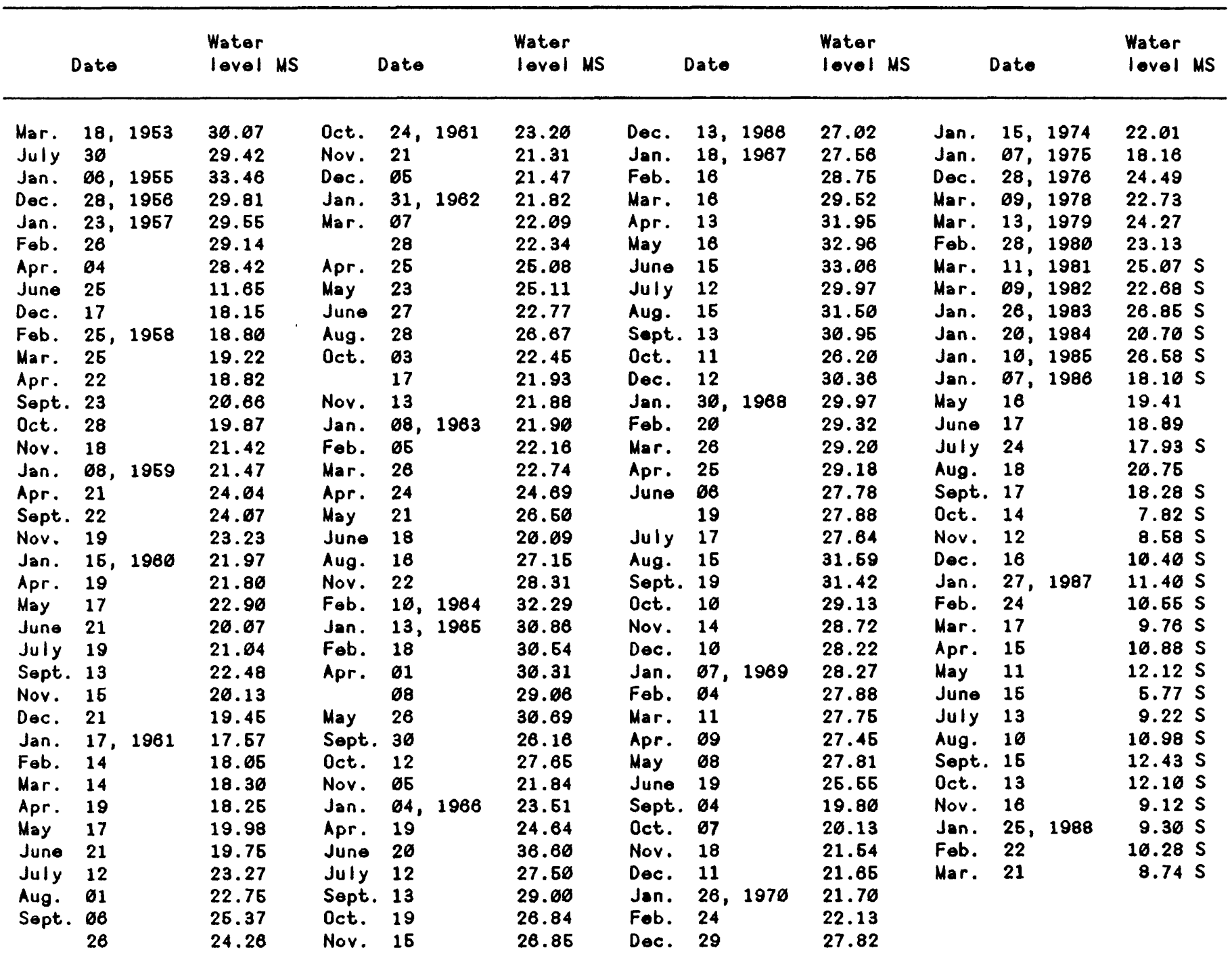


Table 2.--Monthly or periodic water-level measurements in selected wells in southwestern oklahoma and northwestern Texas--Continued

JACKSON COUNTY, OKLAHOMA--Continued

Index number on location map: 114

Station number: $\$ 44114099323701$ Local number: $03 N-22 W-32$ CBAA 1

Location: Lat $34^{\circ} 41^{\prime} 17^{\prime \prime}$, long $99^{\circ} 32^{\prime} 33^{\prime \prime}$, hydrologic unit 11120202

Aquifer: 313BL.IN

Altitude of land-surface datum: $1,432 \mathrm{ft}$

Well depth: $182 \mathrm{ft}$

Remarks: Previously published as $03 \mathrm{~N}-22 \mathrm{~W}-32$ CBB 2

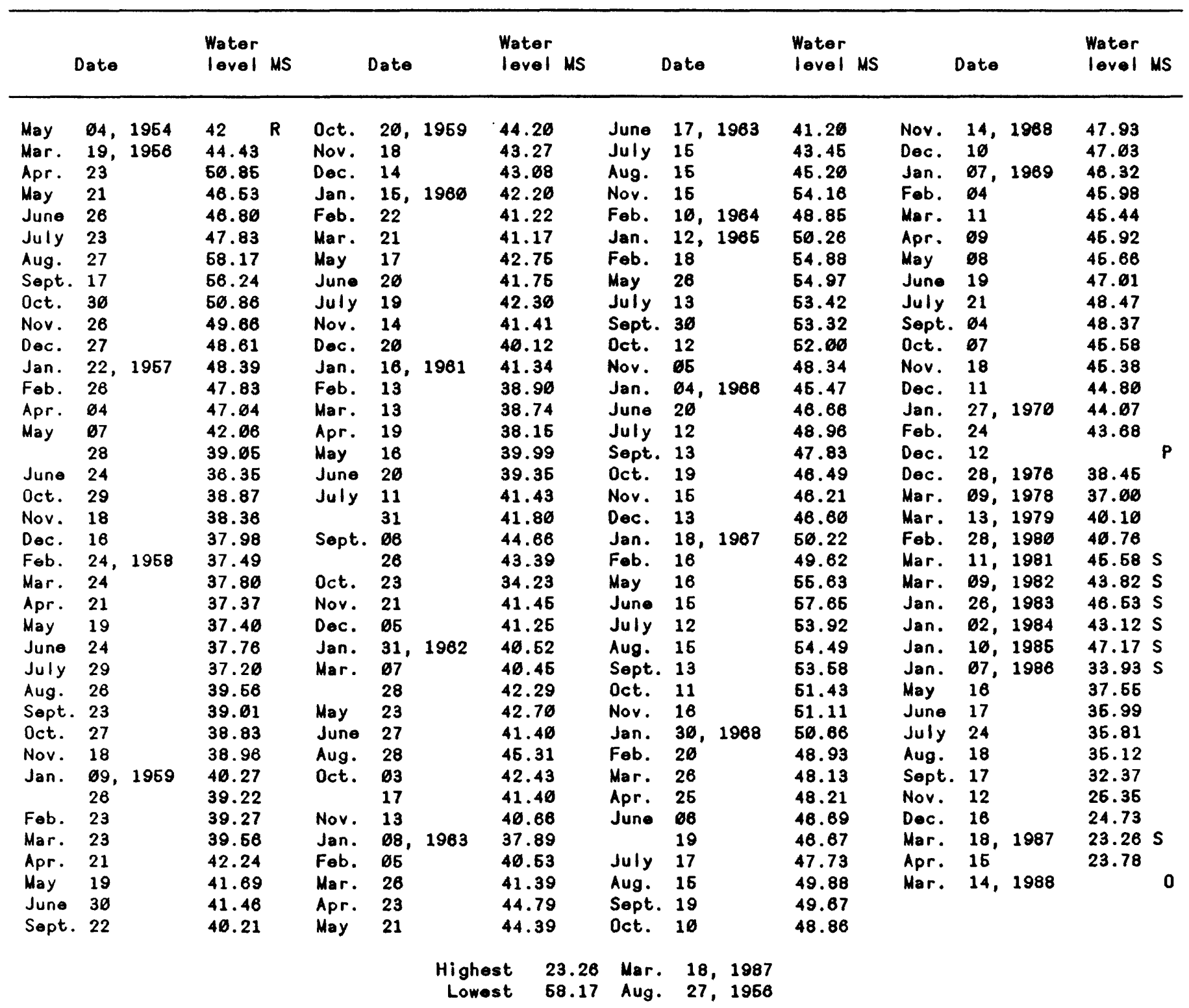


Table 2.--Monthly or periodic water-level measurements in selected wells in southwestern Olclahoma and northwestern Texas--Continued

JACKSON COUNTY, OKLAHOMA-C Continued

Index number on location map: 116

Station number: 844326099396901 Local number: $63 N-23 W-19$ BBBB 1

Location: Lat $34^{\circ} 43^{\prime} 26^{\prime \prime}$, long $99^{\circ} 39^{\prime} 59^{\prime \prime}$, hydrologic unit 11126262

Aquifer: $313 B$ LIN

Altitude of land-surface datum: $1,530.3 \mathrm{ft}$

Well depth: $170 \mathrm{ft}$

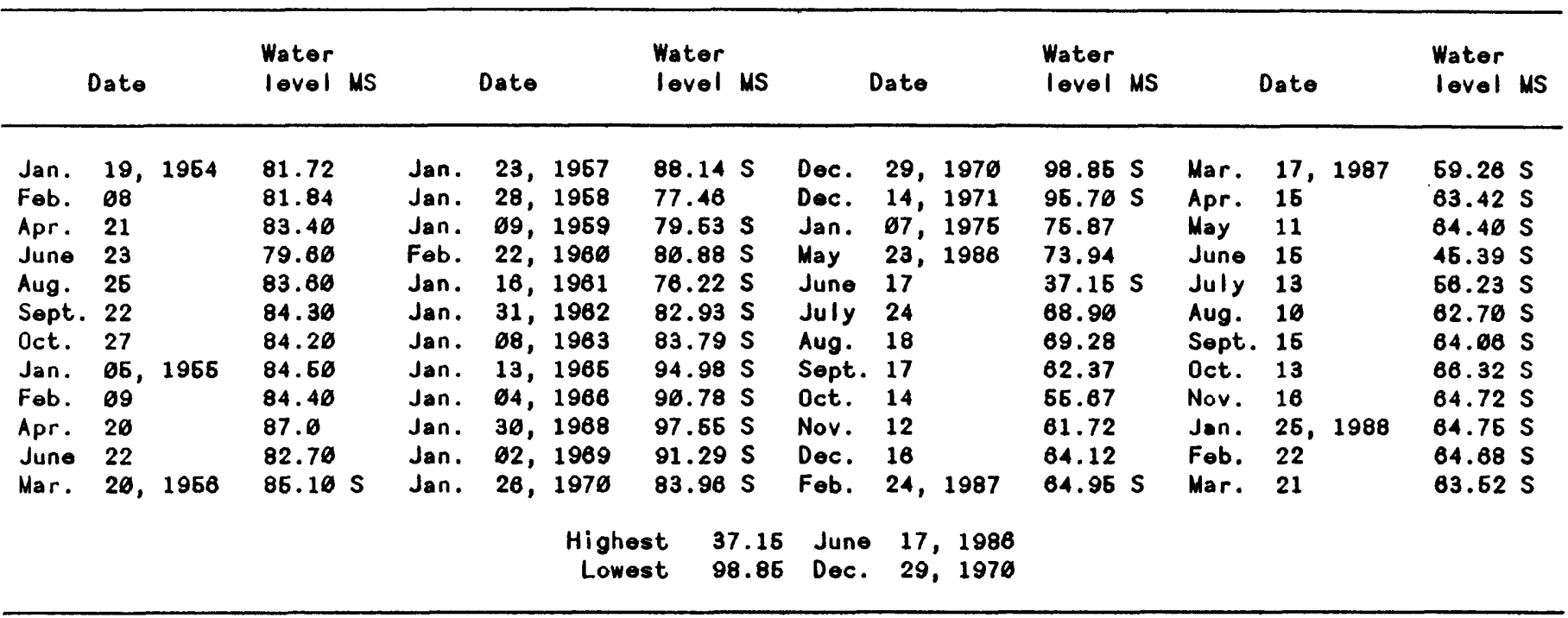

Index number on location map: 116

Station number: 344316099344401 Local number: $63 \mathrm{~N}-23 \mathrm{~W}-24$ BBCC 1

Location: Lat $34^{\circ} 43^{\prime} 16^{\prime \prime}$, long $99^{\circ} 34^{\prime} 46^{\prime \prime}$, hydrologic unit 11120202

Aquifer: $313 B$ LIN

Altitude of land-surface datum: $1,480.6 \mathrm{ft}$

Well depth: $190 \mathrm{ft}$

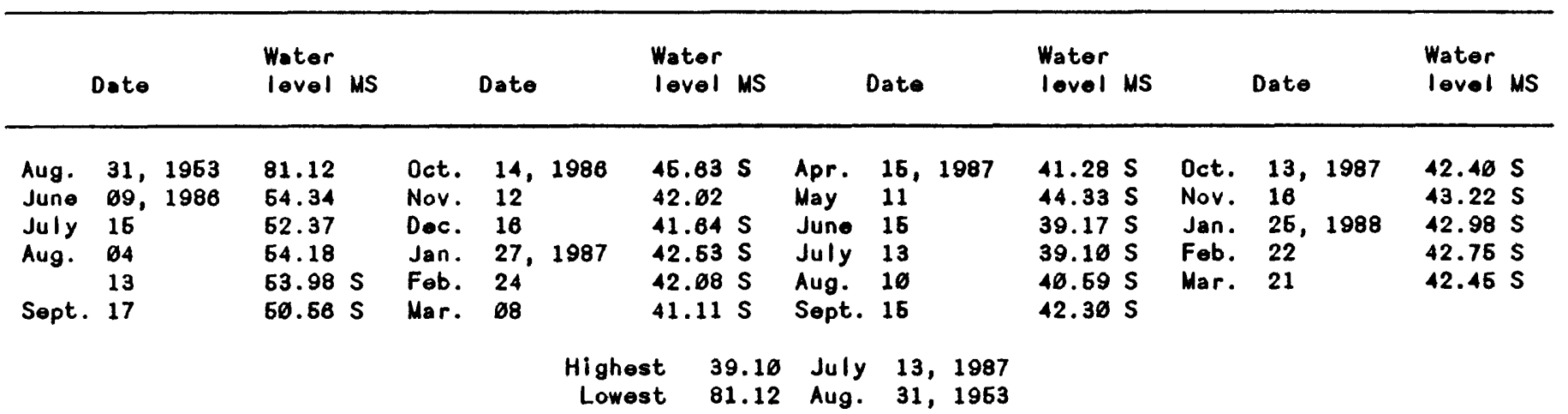


Table 2.--Monthly or periodic water-level measurements in selected wells in southwestern Oklahoma and northwestern Texas--Continued

JACKSON COUNTY, OKLAHOMA--CONtinued

Index number on location map: 117

Station number: 344220099365601 Local number: O3N-23W-27 ADAA 1

Location: Lat $34^{\circ} 42^{\prime} 21^{\prime \prime}$, I ong $99^{\circ} 36^{\prime} 62^{\prime \prime}$, hydrologic unit 11120202

Aquifer: 313BLIN

Altitude of land-surface datum: $1,467.7 \mathrm{ft}$

Well depth: $127 \mathrm{ft}$

Remarks: Previously published $63 \mathrm{~N}-23 W-27$ AAD

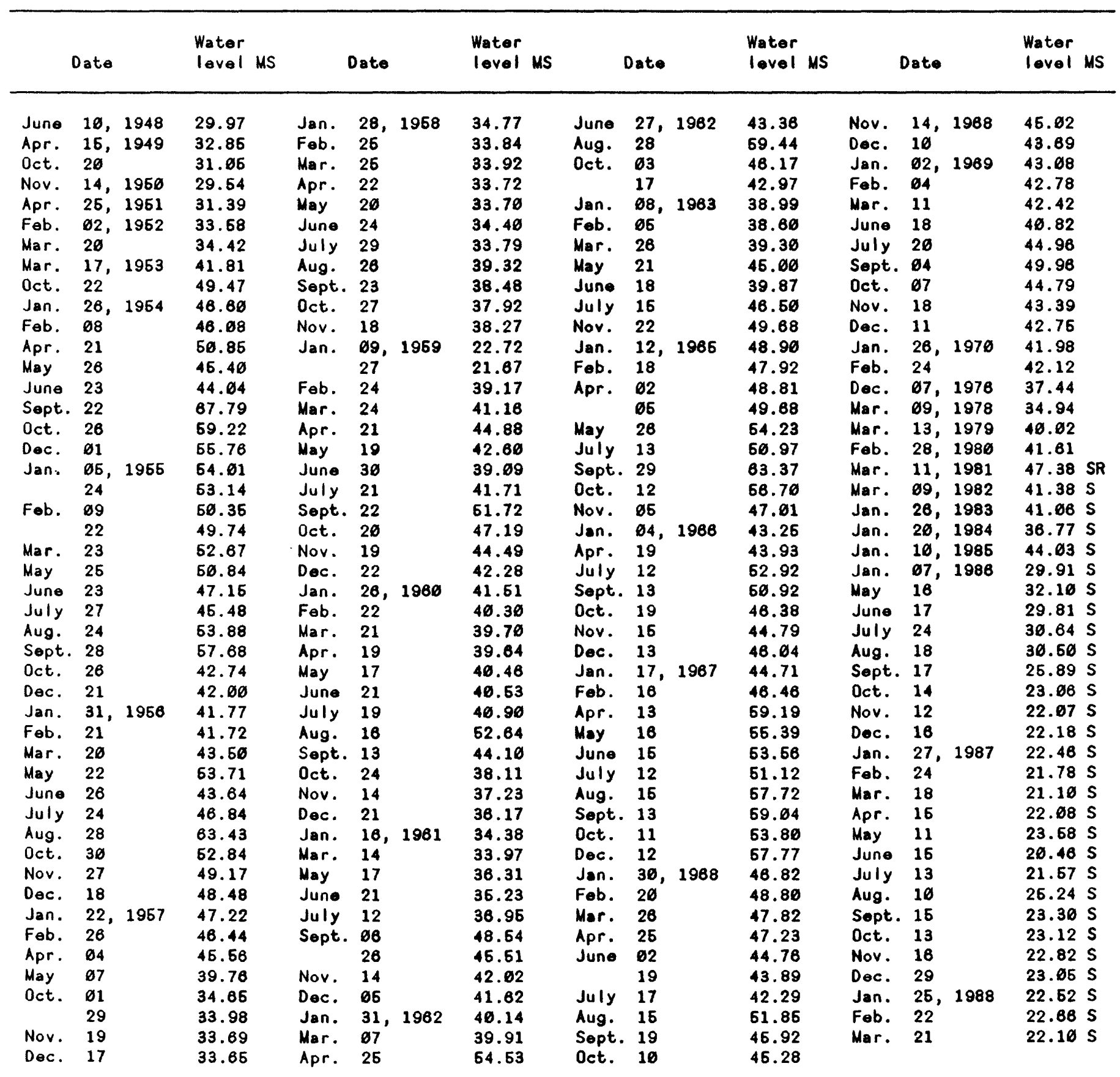

$\begin{array}{ccc}\text { Highest } & 20.46 & \text { June } 16,1987 \\ \text { Lowest } & 67.79 & \text { Sept. } 22,1954\end{array}$ 
Tablo 2.-- Monthly or periodic water-level measurements in selected wells in southwestern Oklahoma and northwestern Texas--Continued

JACKSON COUNTY, OKLAHOMA--CONEInUEd

Index number on location map: 118

Station number: 844032098390201 Local number: $63 N-23 W-30$ DAA 1

Location: Lat $34^{4} 42^{\prime} 09^{\prime \prime}$, long $99^{\circ} 39^{\prime} 01^{\prime \prime}$, hydrologic unit 11120202

Aquifer: 313 BLIN

Altitude of land-surface datum: $1,463.7 \mathrm{ft}$

Well depth: $90 \mathrm{ft}$

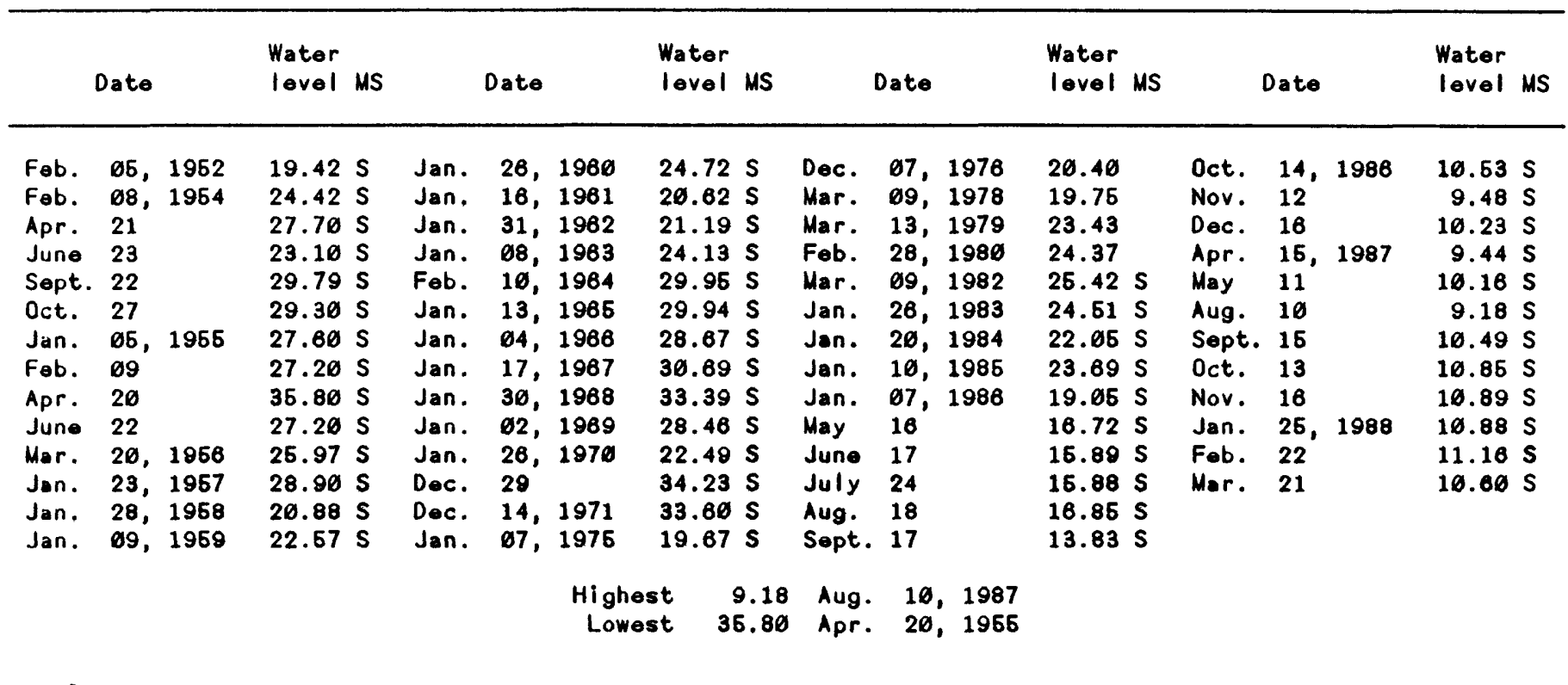

Index number on locetion map: 119

Station number: $\$ 44144099364501$ Local number: $93 \mathrm{~N}-23 W-36$ BBBB 1

Location: Lat $34^{\prime} 41^{\prime} 44^{\prime \prime}$, long $99^{\circ} 36^{\prime} 46^{\prime \prime}$, hydrologic unit 11120202

Aquifer: 313VVCR

Altitude of land-surface datum: $1,438 \mathrm{ft}$

Well depth: $94 \mathrm{ft}$

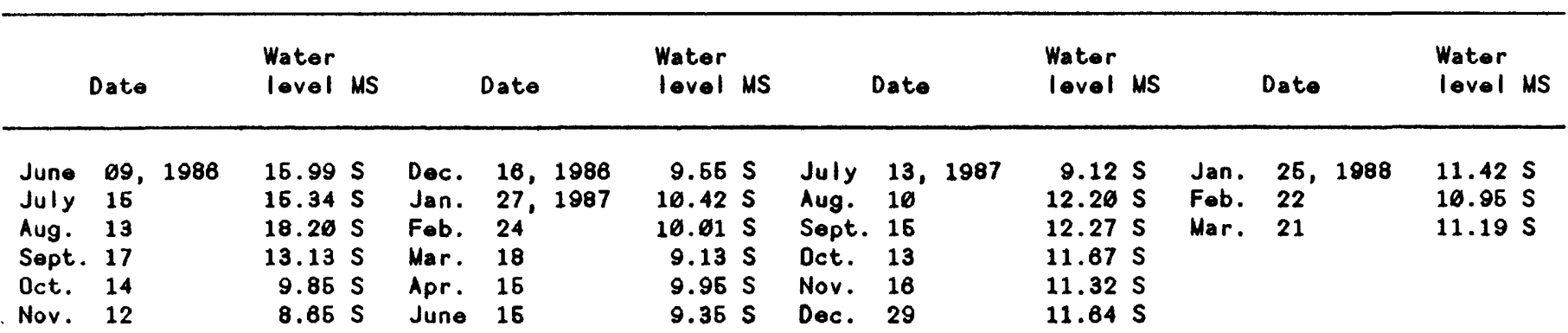

$\begin{array}{rrrr}\text { Highest } & 8.65 & \text { Nov. } 12,1986 \\ \text { Lowest } & 18.20 & \text { Aug. } 13,1986\end{array}$ 
Tablo 2.--Monthly or periodic water-level measurements in selected wells in southwestern okluhoma and northwestern Texas--Continued

CHILDRESS COUNTY, TEXAS

Index number on location map: 120

Station number: 344240100000801 oLocal number: $03 \mathrm{~N}-27 \mathrm{~W}-23$ DD 1

Location: Lat $34^{\circ} 42^{\prime} 40^{\prime \prime}$, long $100^{\circ} 00^{\prime} \theta 8^{\prime \prime}$, hydrologic unit 11130101

Aquifer: $313 B L I N$

Altitude of land-surface datum: $1,697 \mathrm{ft}$

Well depth: $97 \mathrm{ft}$

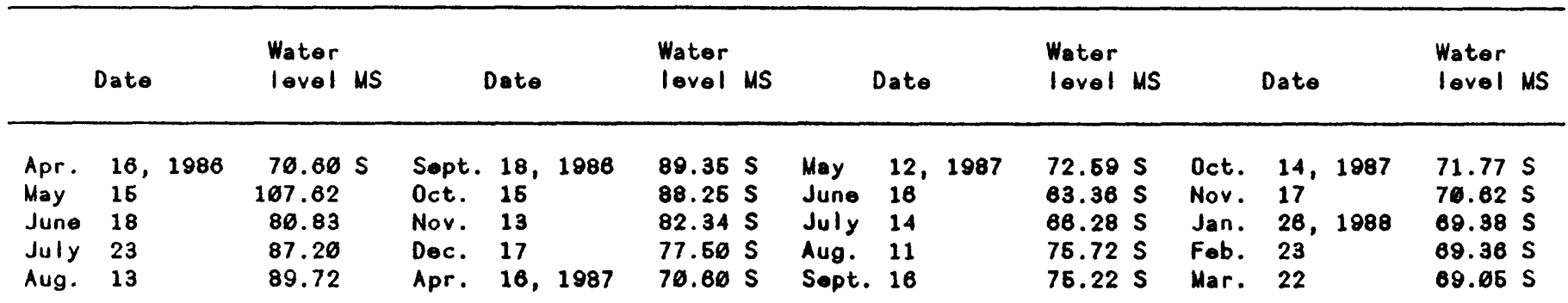

$\begin{array}{rrll}\text { Highest } & 63.36 & \text { June } & 16,1987 \\ \text { Lowest } 107.62 & \text { May } 16,1986\end{array}$

Index number on location map: 121

Station number: 844127100093801 Local number: 12-23-601

Location: Lat $34^{\circ} 41^{\prime} 27^{\prime \prime}$, long 100 $00^{\circ} 38^{\prime \prime}$, hydrologic unit 11120106

Aquifor: $313 B L I N$

Altitude of land-surface datum: $1,762 \mathrm{ft}$

Well depth: $178 \mathrm{ft}$

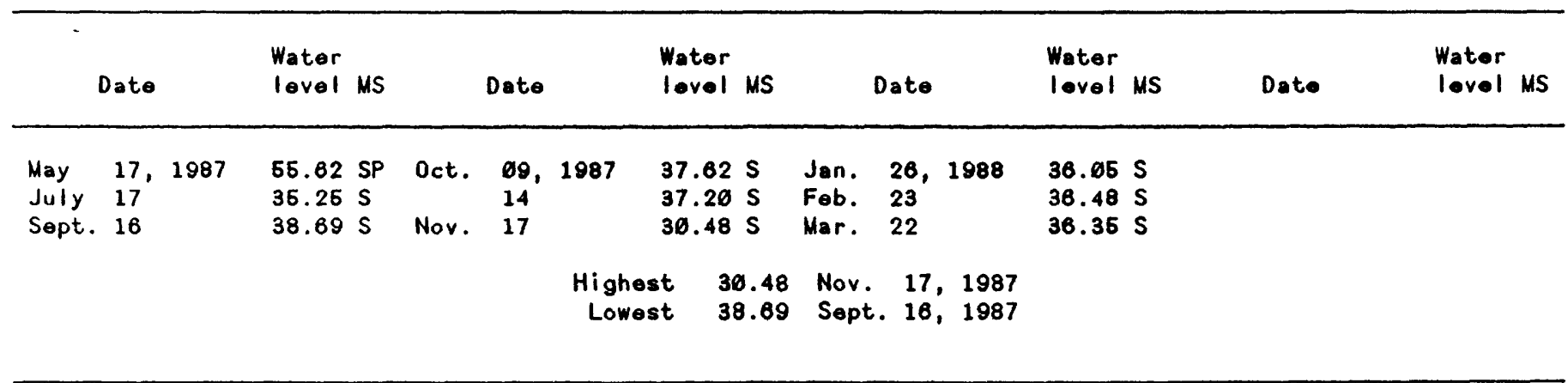

Index number on location map: 122

Station number: 344006100083901 oLocal number: 12-23-603

Location: Lat $34^{\circ} 40^{\prime} 06^{\prime \prime}$, Iong $100^{\circ} 08^{\prime} 39^{\prime \prime}$, hydrologic unit 11120105

Aquifer: $313 B$ LIN

Altitude of land-surface datum: $1,745 \mathrm{ft}$

Well depth: $177 \mathrm{ft}$

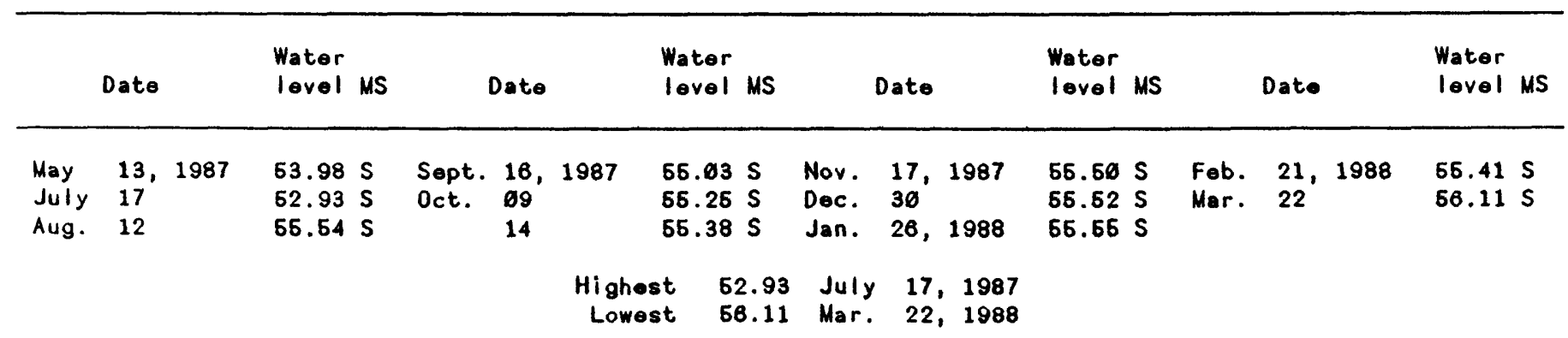


Table 2.--Monthly or periodic water-level measurements in selected wells in southwestern Oklahoma and northwestern Texas--Continued

CHILORESS COUNTY, TEXAS--Continued

Index number on location map: 123

Station number: 844253160033901 Local number: 12-24-207

Location: Lat $34^{\circ} 42^{\prime} 63^{\prime \prime}$, long $100^{\circ} 03^{\prime} 39^{\prime \prime}$, hydrologic unit 11130101

Aquifer: 313 BLIN

Altitude of land-surface datum: $1,732 \mathrm{ft}$

Wall depth: $212 \mathrm{ft}$

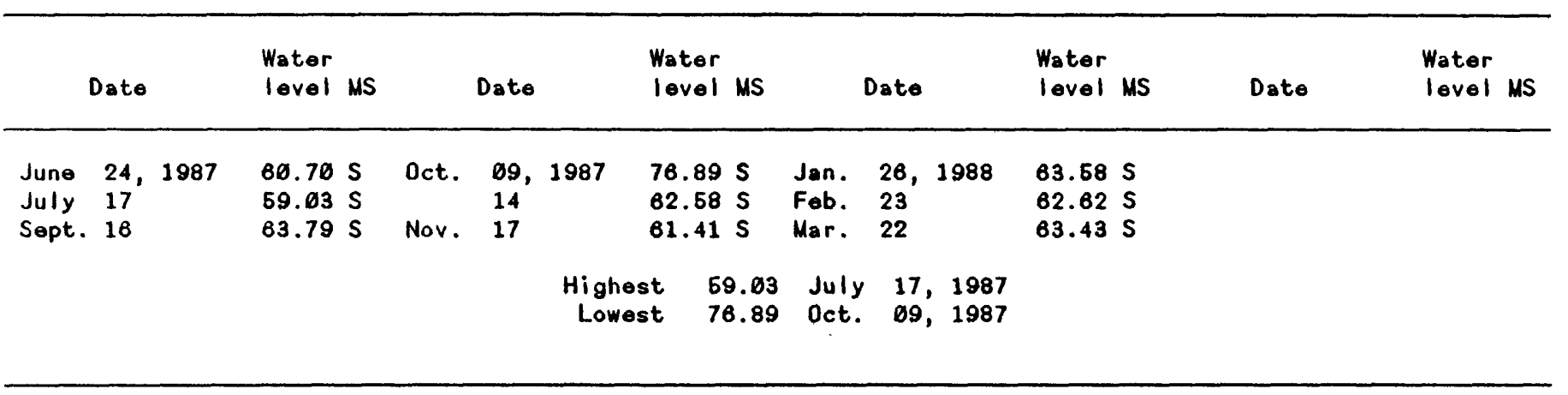

Index number on location map: 124

Station number: 343542100056901 Local number: 12-32-101

Location: Lat $34^{3} 36^{\prime} 42^{*}$, long 180 $86^{\prime} 69^{\prime \prime}$, hydrologic unit 11120105

Aquifer: 313BLIN

Altitude of land-surface datum: 1,702 ft

Well depth: $170 \mathrm{ft}$

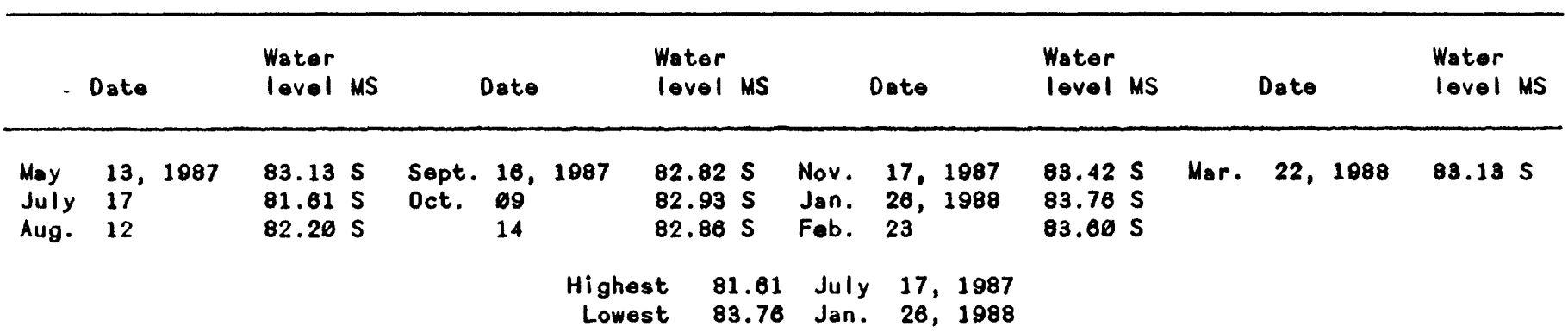


Table 2.-- Monthly or periodic water-level measurements in selected wells in southwestern Oklahoma and northwestern Texas--Continued

\section{COLLINGSWORTH COUNTY, TEXAS}

Index number on location map: 125

Station number: 844739100092001 Local number: 12-15-601

Location: Lat $34^{\circ} 47^{\prime} 39^{\prime \prime}$, long $100^{\circ} 09^{\prime} 20^{\prime \prime}$, hydrologic unit 11120202

Aquifer: 310WTRS

Altitude of land-surface datum: $1,964 \mathrm{ft}$

Well depth: $200 \mathrm{ft}$

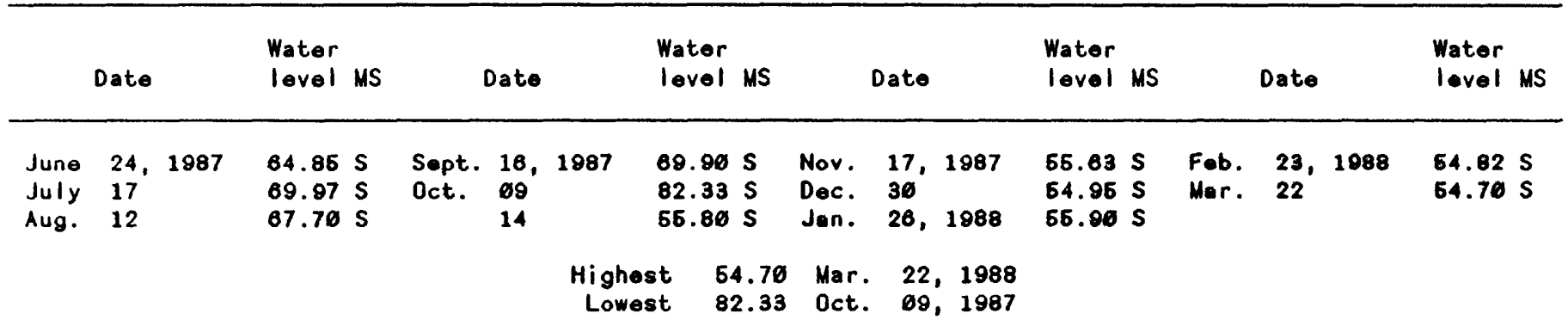


Table 2.--Monthly or periodic water-level measurements in selected wells in southwestern Oklahoma and northwestern Texas--Continued

HARDEMAN COUNTY, TEXAS

Index number on location map: 126

Station number: 342444699441601 Local number: 62S-24W-865 CADB 1

Location: Lat $34^{\circ} 24^{\prime} 44^{\prime \prime}$, long $99^{\circ} 44^{\prime} 10^{\prime \prime}$, hydrologic unit 11130101

Aquifer: 110RRVT

Altitude of land-surface datum: $1,440 \mathrm{ft}$

Well depth: $27 \mathrm{ft}$

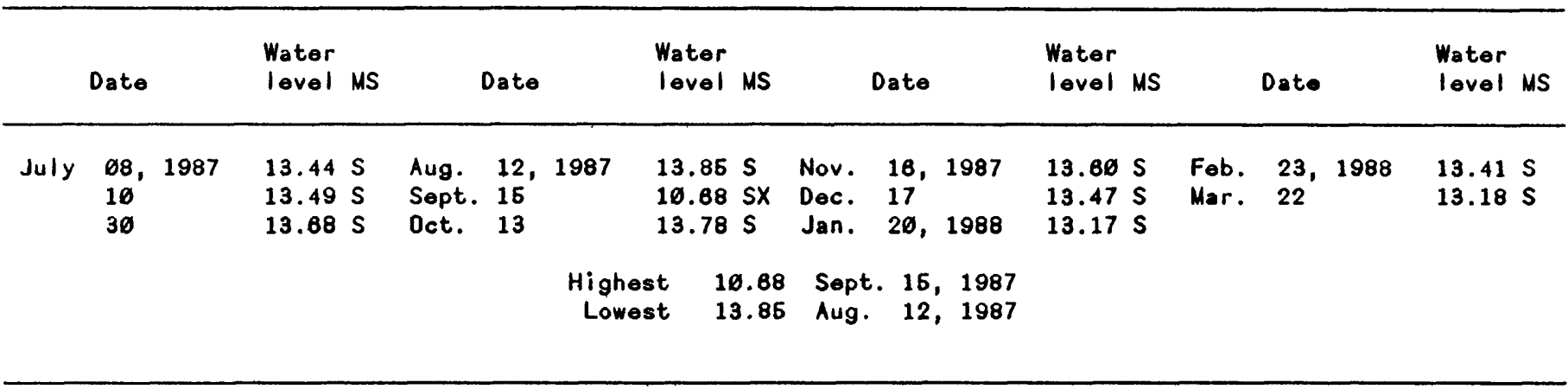

Index number on location map: 127

Station number: $\$ 43436899672281$ Local number: $61 \mathrm{~N}-28 W-68$ BADD 1

Location: Lat $34^{\circ} 34^{\prime} 36^{\prime \prime}$, long $99^{\circ} 67^{\prime} 22^{\prime \prime}$, hydrologic unit 11136101

Aquifer: $313 V V C R$

Altitude of land-surface datum: $1,655 \mathrm{ft}$

Well depth: $70 \mathrm{ft}$

\begin{tabular}{|c|c|c|c|c|c|c|c|c|c|c|c|c|c|c|c|c|}
\hline - & Date & & $\begin{array}{l}\text { Water } \\
\text { level MS }\end{array}$ & MS & & Date & & & ter & & Date & & $\begin{array}{l}\text { Water } \\
\text { level MS }\end{array}$ & & Date & $\begin{array}{l}\text { Water } \\
\text { level MS }\end{array}$ \\
\hline \multirow[t]{2}{*}{$\begin{array}{l}\text { July } \\
\text { Aug. }\end{array}$} & $\begin{array}{l}22, \\
17\end{array}$ & 1987 & $\begin{array}{l}12.65 \mathrm{~S} \\
12.65 \mathrm{~S}\end{array}$ & $\begin{array}{l}S \\
S\end{array}$ & $\begin{array}{l}\text { Sept. } \\
\text { Oct. }\end{array}$ & $\begin{array}{l}16 \\
13\end{array}$ & 1987 & \multicolumn{2}{|c|}{$\begin{array}{l}12.23 \mathrm{~s} \\
12.77 \mathrm{~s}\end{array}$} & $\begin{array}{l}\text { Nov. } \\
\text { Dec. }\end{array}$ & $\begin{array}{l}16 \\
18\end{array}$ & 1987 & $\begin{array}{l}12.64 \mathrm{~S} \\
12.46 \mathrm{~S}\end{array}$ & $\begin{array}{l}\text { Jan. } \\
\text { Feb. }\end{array}$ & $\begin{array}{ll}20, & 1988 \\
23 & \end{array}$ & $\begin{array}{l}12.02 \mathrm{~S} \\
12.40 \mathrm{~S}\end{array}$ \\
\hline & & & & & & & \multicolumn{2}{|c|}{$\begin{array}{l}\text { Highest } \\
\text { Lowest }\end{array}$} & $\begin{array}{l}12.02 \\
12.77\end{array}$ & $\begin{array}{l}\text { Jan. } \\
\text { Oct. }\end{array}$ & $\begin{array}{l}20, \\
. \quad 13,\end{array}$ & $\begin{array}{l}1988 \\
, \quad 1987\end{array}$ & & & & \\
\hline
\end{tabular}

Index number on location map: 128

Station number: 343069699546501 Local number: 13-25-962

Location: Lat $34^{\circ} 30^{\prime} 09^{\prime \prime}$, long $99^{\circ} 54^{\prime} 05^{\prime \prime}$, hydrologic unit 11130101

Aquifer: 313BLIN

Altitude of land-surface datum: $1,598 \mathrm{ft}$

Well depth: $250 \mathrm{ft}$

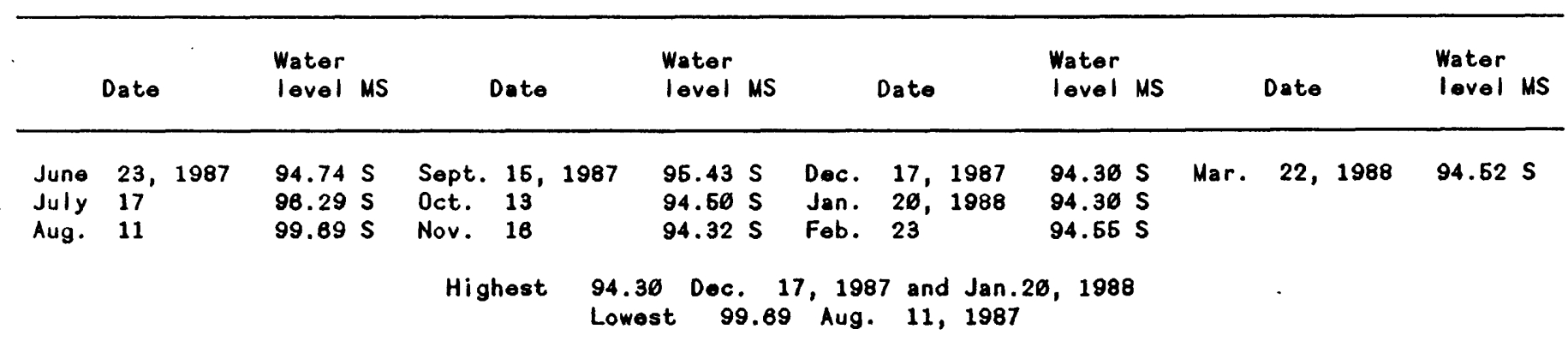


Table 2.-- Monthly or periodic water-level measurements in selected wells in southwestern Oklahoma and northwestern rexas--Continued

HARDEMAN COUNTY, TEXAS--Continued

Index number on location map: 129

Station number: $\$ 42940699574201$ Local number: 13-33-102

Location: Lat $34^{\circ} 29^{\prime} 40^{\prime \prime}$, long $99^{\circ} 57^{\prime} 42^{\prime \prime}$, hydrologic unit 11130161

Aquifer: 313BLIN

Altitude of land-surface datum: $1,781 \mathrm{ft}$

Well depth: $195 \mathrm{ft}$

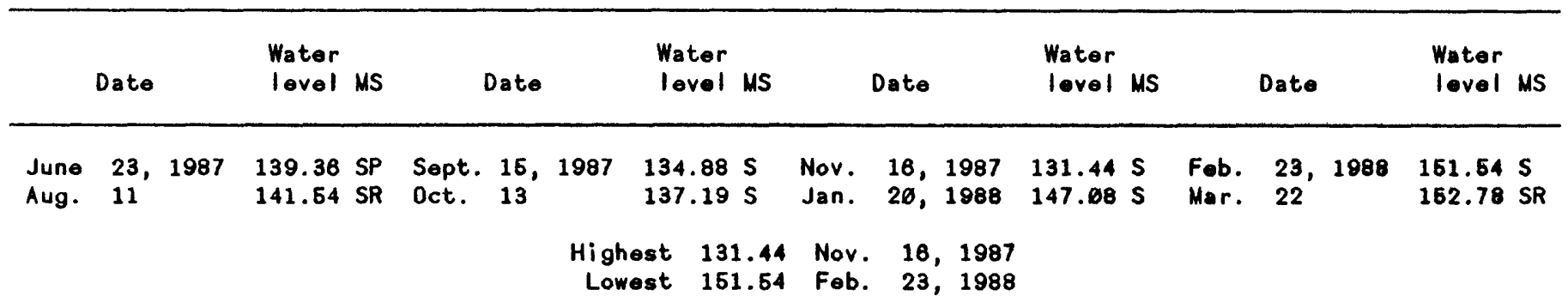

Index number on location map: 130

Station number: 342564699592981 Local number: 13-33-403

Location: Lat $34^{\circ} 28^{\prime} 00^{\prime \prime}$, long $99^{\circ} 69^{\prime} 25^{\prime \prime}$, hydrologic unit 11130101

Aquifer: 313BLIN

Altitude of land-surface datum: $1,677 \mathrm{ft}$

Well depth: $230 \mathrm{ft}$

\begin{tabular}{|c|c|c|c|c|c|c|c|c|c|c|c|c|c|}
\hline \multicolumn{2}{|r|}{ Date } & \multicolumn{2}{|l|}{$\begin{array}{l}\text { Water } \\
\text { level MS }\end{array}$} & \multicolumn{2}{|l|}{ Date } & \multicolumn{2}{|c|}{$\begin{array}{l}\text { Water } \\
\text { level MS }\end{array}$} & \multicolumn{3}{|c|}{ Date } & $\begin{array}{l}\text { Water } \\
\text { level MS }\end{array}$ & \multirow[t]{2}{*}{ Date } & \multirow[t]{2}{*}{$\begin{array}{l}\text { Water } \\
\text { level MS }\end{array}$} \\
\hline $\begin{array}{l}\text { June } \\
\text { July } \\
\text { Aug. }\end{array}$ & $\begin{array}{ll}23, & 1987 \\
17 & \\
11 & \end{array}$ & $\begin{array}{l}79.18 \mathrm{~S} \\
78.53 \mathrm{~S} \\
79.58 \mathrm{~S}\end{array}$ & $\begin{array}{l}\text { Sept. } \\
\text { Oct. } \\
\text { Nov. }\end{array}$ & $\begin{array}{l}15 \\
13 \\
16\end{array}$ & 1987 & $\begin{array}{l}80 \\
80 \\
80\end{array}$ & $\begin{array}{ll}62 & S \\
99 & S \\
97 & S\end{array}$ & $\begin{array}{l}\text { Jan. } \\
\text { Fob. } \\
\text { Mar. }\end{array}$ & $\begin{array}{l}20 \\
23 \\
22\end{array}$ & 1988 & $\begin{array}{l}81.87 \mathrm{~S} \\
82.18 \mathrm{~S} \\
81.85 \mathrm{~S}\end{array}$ & & \\
\hline & & & & & $\begin{array}{l}\text { High } \\
\text { Low }\end{array}$ & & $\begin{array}{l}78.53 \\
82.18\end{array}$ & $\begin{array}{l}\text { July } \\
\text { Fob. }\end{array}$ & $\begin{array}{l}17, \\
23,\end{array}$ & $\begin{array}{l}1987 \\
1988\end{array}$ & & & \\
\hline
\end{tabular}

Index number on location map: 131

Station number: \$42867099585401 Local number: 13-33-501

Location: Lat $34^{\circ} 26^{\prime} 97^{\prime \prime}$, I ong 99 $56^{\prime} 54^{\prime \prime}$, hydrologic unit 11136101

Aquifer: $313 B L I N$

Altitude of land-surface datum: $1,828 \mathrm{ft}$

Well depth: $200 \mathrm{ft}$

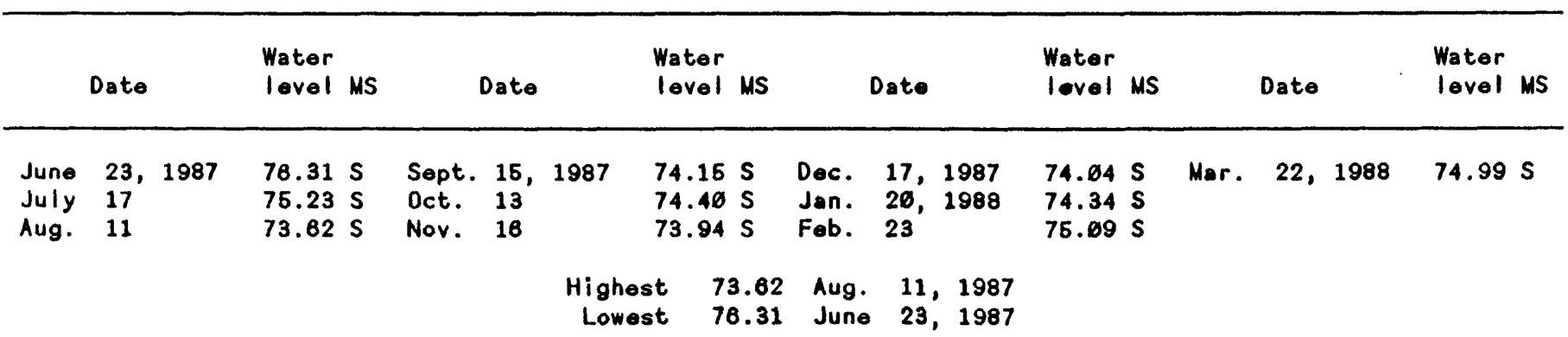


Table 2.--Monthly or periodic water-level measurements in selected wells in southwestern Oklahorna and northwestern Texas--Continued

HARDEMAN COUNTY, TEXAS--Continued

Index number on location map: 132

Station number: $\$ 42417099493601$ Local number: 13-34-802

Location: Lat $34^{\circ} 24^{\prime} 17^{\prime \prime}$, long $99^{\circ} 49^{\prime} 36^{\prime \prime}$, hydrologic unit 11130101

Aquifer: 313BLIN

Altitude of land-surface datum: $1,520 \mathrm{ft}$

Well depth: $110 \mathrm{ft}$

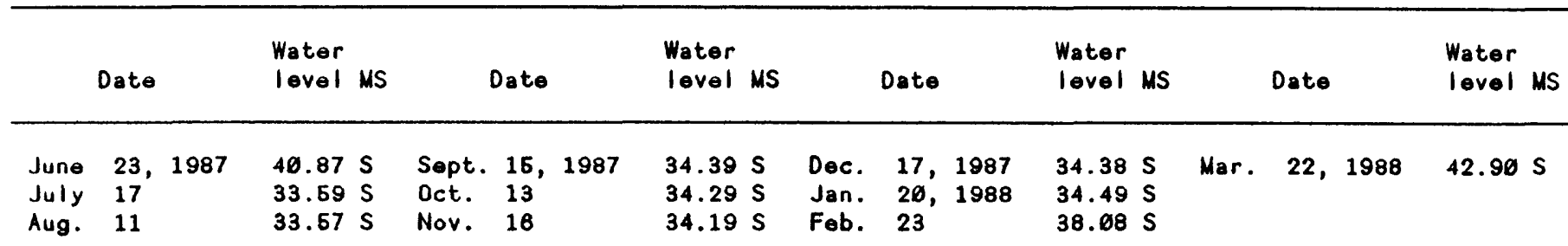

Highest 33.67 Aug. 11, 1987

Lowest 42.90 Mar. 22, 1988

Index number on location map: 133

Station number: 842436099461401 Local number: 13-34-905

Location: Lat $34^{\circ} 24^{\prime} 36^{\prime \prime}$, long $99^{\circ} 46^{\prime} 14^{\prime \prime}$, hydrologic unit 11130101

Aquifer: 3138 LIN

Altitude of land-surface datum: 1,625 ft

Well depth: $137 \mathrm{ft}$

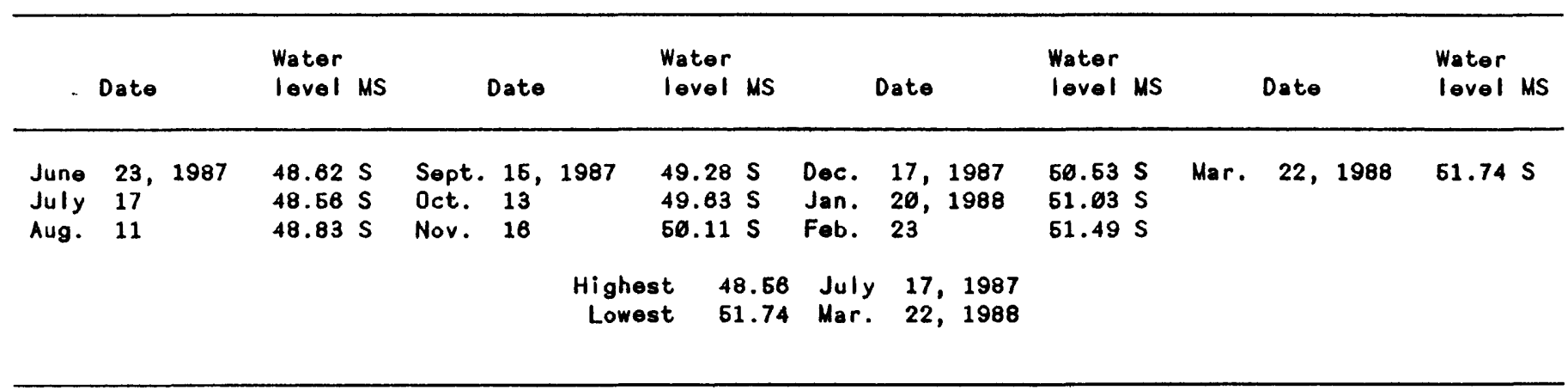

Index number on location map: 134

Station number: 342413099443101 Local number: 13-35-701

Location: Lat $34^{\prime 2} 24^{\prime} 13^{\prime \prime}$, long $99^{\circ} 44^{\prime} 31^{\prime \prime}$, hydrologic unit 11130181

Aquifer: 3138 LIN

Altitude of land-surface datum: $1,490 \mathrm{ft}$

Well depth: $78 \mathrm{ft}$

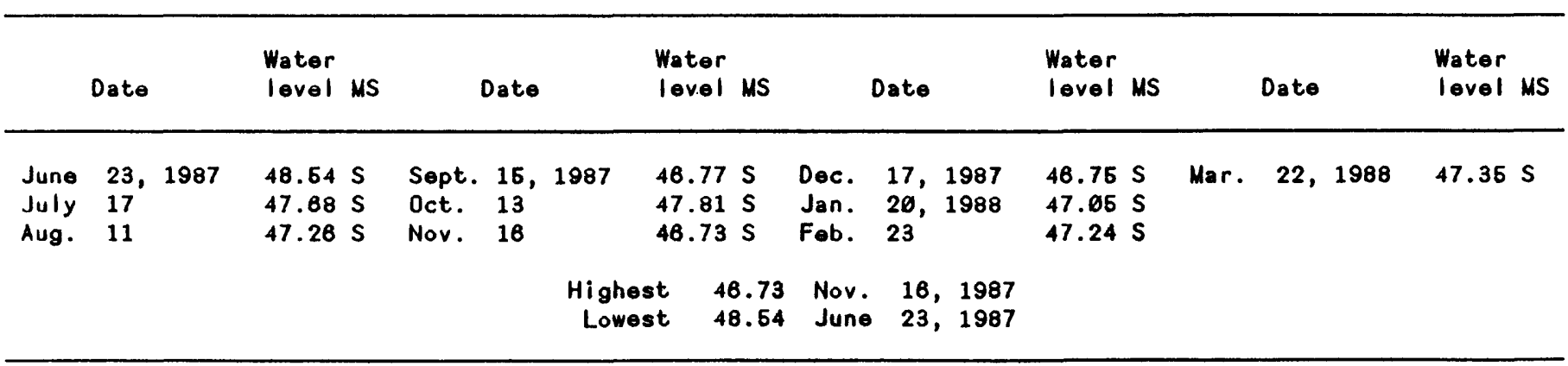




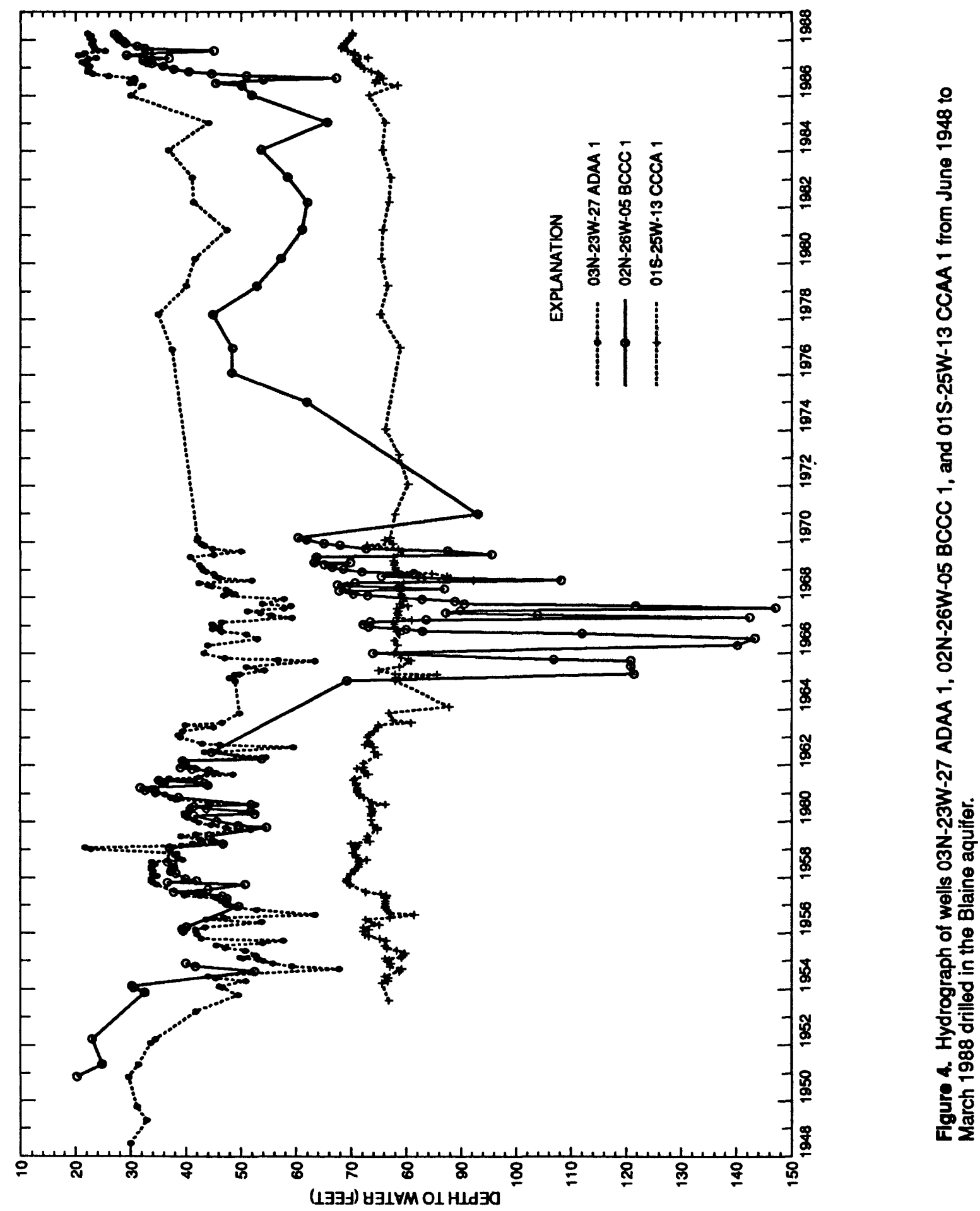




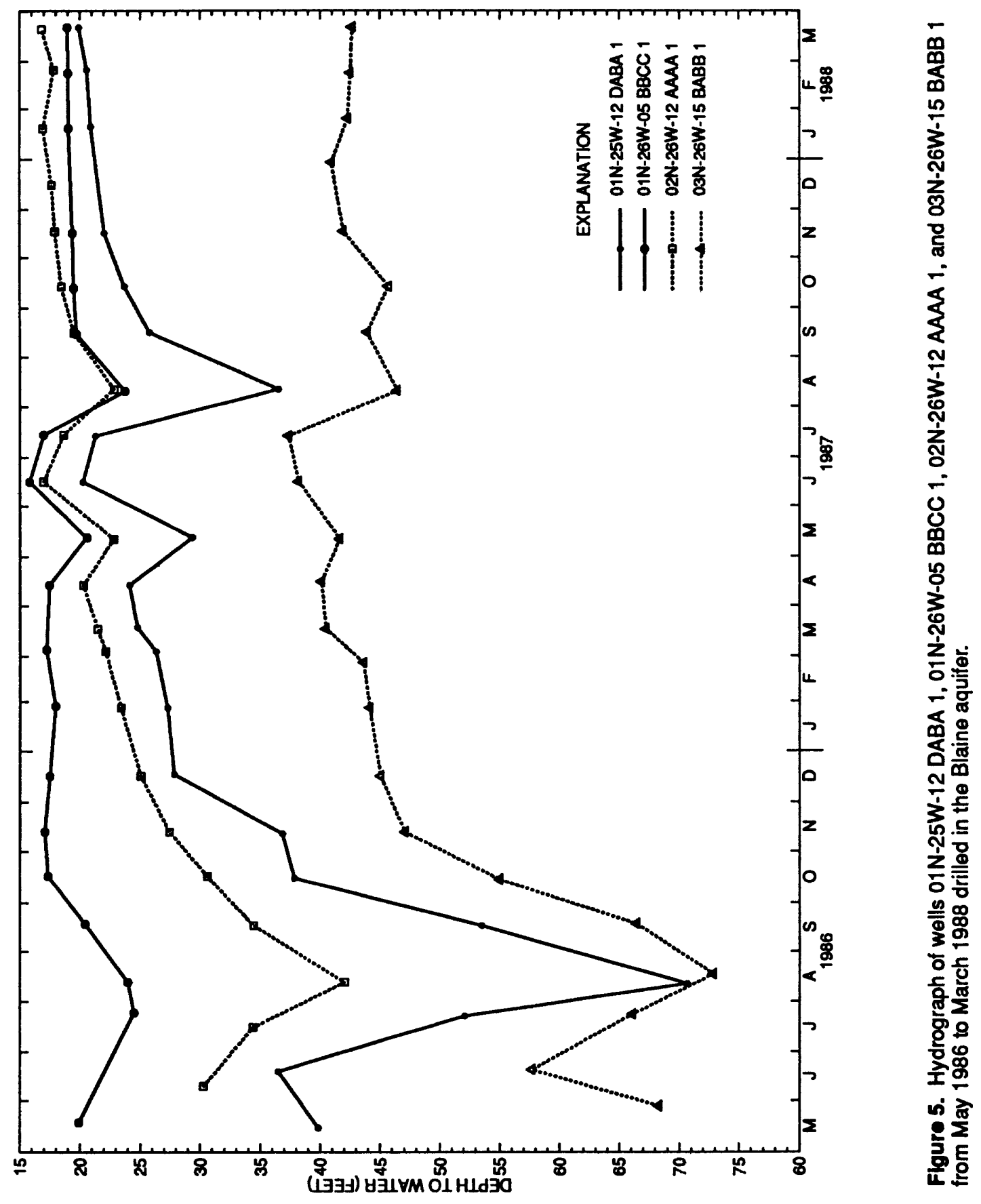




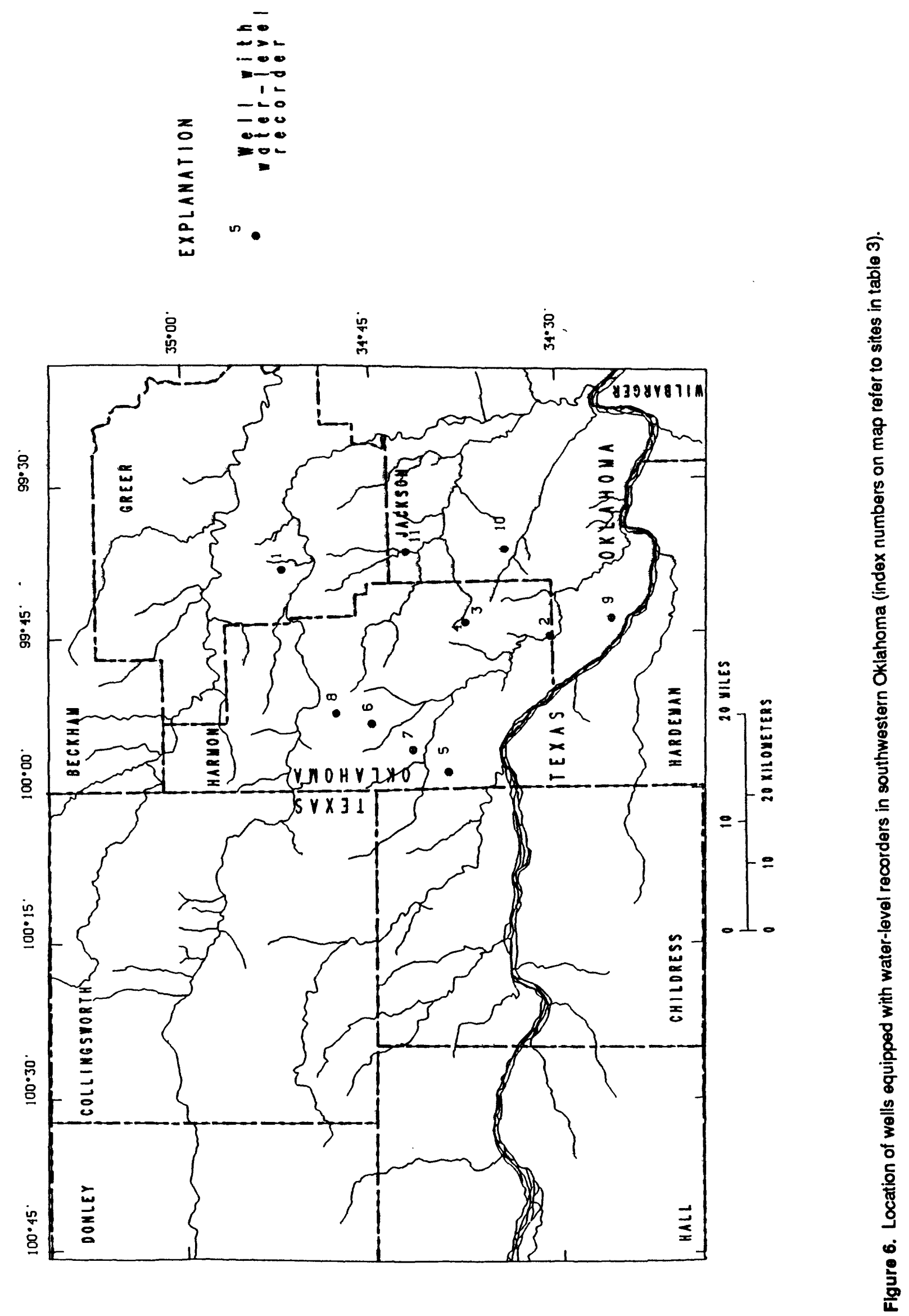


Table 3.--Daily mean water-level measurements in recorder wells in southwestern Oklahoma

[Water levels are in feet below land-surface datum; index number on location map refers to the number adjacent to the well symbol on figure 8; 110RRVT, Red River terrace aquifer; 110SFRT, Salt Fork Red River terrace aquifer; 11øSFTA, Salt Fork Red River terrace and alluvial aquifer; 111RRVA, Red River alluvial aquifer; 111SFRR, Salt Fork Red River alluvial aquifer; 310WTRS, Whitehorse Group; $3138 L I N$, Blaine Gypsum (or Formation); 313CLGR, Collingsworth Gypsum Bed of the EIm Fork Member; $3130 G C K$, Dog Creok Shale; 313EMFK, Elm Fork Member of Blaine Gypsum (or Formation); 313FLRP, Flowerpot Shale; 313VVCR, Van Vactor Member of Blaine Gypsum (or Formation); -- no data]

GREER COUNTY, OKLAHOMA

Index number on location map: 1

Station number: $3452 \varnothing 3 \varnothing 993828 \varnothing 1$ oLCal number: $05 \mathrm{~N}-23 W-32$ AADD 1

Location: Lat $34^{\circ} 62^{\prime} 03^{\prime \prime}$, long $99^{\circ} 38^{\prime} 28^{\prime \prime}$, hydrologic unit 11120202

Aquifer: $313 B$ LIN

Altitude of land-surface datum: $1,705 \mathrm{ft}$

Well depth: $120 \mathrm{ft}$

Water Year, October 1988 to September 1987

Day 0ct. Nov. Dec. Jan. Fob. Mar. Apr. May Juno July Aug. Sept.

\begin{tabular}{|c|c|c|c|c|c|c|c|c|c|c|c|c|}
\hline 1 & -- & -- & -- & 28.19 & 29.12 & 29.91 & 28.64 & 28.63 & 28.06 & 24.16 & 24.60 & 26.98 \\
\hline 2 & -- & -- & -- & 27.97 & 29.22 & 29.87 & 28.76 & 28.67 & 27.97 & 24.10 & 24.62 & 25.98 \\
\hline 3 & -- & -- & -- & 28.25 & 29.32 & 29.90 & 28.84 & 28.71 & 28.08 & 24.09 & 24.67 & 25.99 \\
\hline 4 & -- & -- & -- & 28.25 & 29.38 & 29.88 & 28.65 & 28.88 & 27.98 & 24.62 & 24.76 & 26.02 \\
\hline 6 & -- & -- & -- & 28.16 & 29.68 & 29.69 & 28.66 & 28.97 & 27.78 & 24.06 & 24.78 & 28.08 \\
\hline 6 & -- & -- & -- & 28.25 & $29.6 \emptyset$ & 29.67 & 28.58 & $29 . \varnothing 1$ & 27.63 & 24.09 & 24.78 & 26.13 \\
\hline 7 & -- & -- & -- & 28.44 & 29.64 & 29.49 & 28.67 & 29.01 & 27.62 & 24.09 & 24.83 & 26.19 \\
\hline 8 & -- & -- & -- & 28.38 & 29.69 & 29.36 & 28.63 & 29.80 & 27.44 & 24.12 & 24.86 & 26.29 \\
\hline 9 & -- & -- & -- & 28.35 & 29.59 & 29.43 & 28.42 & 28.94 & 27.34 & 24.11 & 24.95 & 26.28 \\
\hline 10 & -- & -- & -- & 28.51 & 29.49 & 29.43 & 28.47 & 28.92 & 27.18 & 24.10 & 24.97 & 26.31 \\
\hline 11 & - & -- & -- & 28.58 & 29.67 & 29.37 & 28.34 & 28.97 & 26.86 & 24.10 & 24.94 & 28.38 \\
\hline 12 & -- & -- & -- & 28.51 & 29.66 & 29.35 & 28.35 & 29.03 & 26.60 & 24.13 & 24.98 & 26.43 \\
\hline 13 & -- & - & -- & 28.45 & 29.55 & 29.18 & 28.62 & 29.04 & 26.32 & 24.27 & 25.03 & 28.45 \\
\hline 14 & -- & -- & -- & 28.44 & 29.43 & 28.94 & 28.68 & 29.11 & 26.00 & 24.23 & 26.07 & 26.53 \\
\hline 16 & -- & -- & -- & 28.63 & 29.61 & 28.99 & 28.57 & 29.17 & 25.70 & 24.17 & 25.13 & 26.48 \\
\hline 16 & -- & -- & -- & 28.72 & 29.77 & 28.84 & 28.53 & 29.11 & 26.44 & 24.18 & 25.20 & 28.65 \\
\hline 17 & -- & -- & 27.68 & 28.68 & 29.87 & 28.74 & 28.48 & 29.68 & 26.27 & 24.14 & 26.26 & 28.67 \\
\hline 18 & $\ldots$ & -- & 27.73 & 28.66 & 29.89 & 28.86 & 28.46 & 29.10 & 25.15 & 24.24 & 25.32 & 26.77 \\
\hline 19 & -- & -- & 27.65 & 28.80 & 29.92 & 28.89 & 28.62 & 29.16 & 24.91 & 24.31 & 25.37 & 26.79 \\
\hline 20 & -- & -- & 27.75 & 28.94 & 29.96 & 28.81 & 28.70 & 29.15 & 24.76 & 24.37 & 25.42 & 26.82 \\
\hline 21 & -- & -- & 27.89 & 28.82 & 29.87 & 28.83 & 28.83 & 29.30 & 24.64 & 24.39 & 25.46 & 26.90 \\
\hline 22 & - & -- & 27.84 & 28.93 & 29.88 & 28.64 & 28.69 & 29.42 & 24.64 & 24.37 & 25.63 & 26.98 \\
\hline 23 & -- & -- & 27.72 & 28.79 & 29.86 & 28.56 & 28.67 & 29.37 & 24.51 & 24.35 & 26.82 & 28.92 \\
\hline 24 & - & -- & 27.74 & 28.86 & 29.90 & 28.76 & 28.75 & 29.31 & 24.46 & 24.43 & 25.68 & 26.89 \\
\hline 26 & -- & -- & 27.89 & 29.03 & 29.95 & 28.88 & 28.77 & 29.29 & 24.42 & 24.48 & 25.64 & 26.98 \\
\hline 26 & -- & -- & 27.99 & 29.13 & 29.89 & 28.79 & 28.79 & 29.30 & 24.40 & 24.48 & 26.65 & 27.00 \\
\hline 27 & - & - & 28.01 & 29.08 & 29.78 & 28.67 & 28.80 & 29.34 & 24.28 & 24.49 & 25.80 & 27.04 \\
\hline 28 & -- & -- & 28.04 & 28.99 & 29.77 & 28.75 & 28.79 & 29.27 & 24.19 & 24.50 & 25.84 & 27.20 \\
\hline 29 & -- & -- & 28.07 & 29.16 & 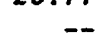 & 28.85 & 28.70 & 28.80 & 24.16 & 24.63 & 26.83 & 27.29 \\
\hline 30 & - & -- & 28.03 & 29.30 & -- & 28.76 & 28.67 & 28.64 & 24.19 & 24.67 & 25.86 & 27.30 \\
\hline 31 & -- & -- & 28.03 & 29.14 & -- & 28.66 & 20.0 & 28.29 & -- & 24.60 & 25.95 & - \\
\hline Mean & - & -- & -- & 28.68 & 29.66 & 29.11 & 28.61 & 29.03 & 25.92 & 24.27 & 25.23 & 28.69 \\
\hline Max. & -- & -- & -- & 29.36 & 29.96 & 29.91 & 28.83 & 29.42 & 28.06 & 24.60 & 26.96 & 27.30 \\
\hline Min. & -- & -- & -- & 27.97 & 29.12 & 28.56 & 28.34 & 28.29 & 24.16 & 24.82 & 24.68 & 25.96 \\
\hline
\end{tabular}


Table 3.--Daily mean water-level measurements in recorder wells in southwestern Oklahoma--Continued

GREER COUNTY, OKLAHOMA--CONtinued

$65 N-23 W-32$ AADD 1--Continued

Water Year, October 1987 to September 1988

\begin{tabular}{|c|c|c|c|c|c|c|c|c|c|c|c|c|}
\hline Day & Oct. & Nov. & Dec. & Jan. & Fob. & Mar. & Apr. & May & June & July & Aug. & Sopt. \\
\hline 1 & 27.24 & 28.58 & 29.69 & 31.60 & 31.99 & -- & 32.99 & -- & -- & 33.86 & 34.61 & -- \\
\hline 2 & 27.39 & 28.57 & 29.70 & 30.95 & 32.06 & -- & 33.63 & -- & -- & 33.89 & 34.64 & -- \\
\hline 3 & 27.49 & 28.59 & 29.83 & 30.90 & 32.06 & -- & 33.67 & -- & -- & 33.89 & 34.67 & -- \\
\hline 4 & 27.34 & 28.67 & 29.86 & 31.69 & 32.19 & -- & 33.04 & $-\infty$ & - & 33.93 & 34.60 & -- \\
\hline 5 & 27.37 & 28.82 & 29.75 & 31.06 & 32.25 & -- & 33.14 & -- & -- & 33.96 & 34.61 & -- \\
\hline 6 & 27.61 & 28.74 & 29.87 & 31.02 & 32.20 & -- & 33.24 & -- & -- & 33.98 & 34.63 & -- \\
\hline 7 & 27.64 & 28.66 & 29.91 & 31.11 & 32.11 & -- & 33.13 & - & -- & 34.00 & 34.64 & -- \\
\hline 8 & 27.43 & 28.84 & 29.98 & 31.11 & 32.13 & -- & 33.89 & -- & -- & 34.03 & 34.87 & -- \\
\hline 9 & 27.62 & 28.98 & 36.06 & 31.18 & 32.21 & 32.84 & 33.23 & -- & -- & 34.02 & 34.70 & -- \\
\hline 10 & 27.82 & 29.06 & 30.01 & 31.20 & 32.23 & 32.76 & 33.27 & - & -- & 34.62 & 34.73 & -- \\
\hline 11 & 27.83 & 28.99 & 30.00 & 31.11 & 32.34 & 32.83 & 33.21 & -- & -- & 34.04 & 34.75 & -- \\
\hline 12 & 27.71 & 28.93 & 30.11 & 31.26 & 32.25 & 32.97 & 33.20 & -- & -- & 34.06 & 34.77 & -- \\
\hline 13 & 27.68 & 28.87 & 30.14 & 31.42 & 32.17 & 33.05 & 33.18 & - & -- & 34.09 & 34.78 & -- \\
\hline 14 & 27.75 & 28.87 & 30.17 & 31.33 & 32.30 & 33.01 & 33.19 & - & - & 34.12 & 34.83 & -- \\
\hline 15 & 27.82 & 28.93 & 30.31 & 31.25 & 32.36 & 32.96 & 33.26 & -- & -- & 34.14 & 34.85 & -- \\
\hline 16 & 27.93 & 29.11 & 30.37 & 31.22 & 32.32 & 32.98 & 33.25 & - & -- & 34.17 & 34.88 & - \\
\hline 17 & 28.00 & 29.19 & 30.34 & 31.32 & 32.46 & 33.04 & 33.17 & - & -- & 34.19 & 34.91 & -- \\
\hline 18 & .27 .89 & 29.39 & 30.27 & 31.26 & 32.44 & 33.08 & 33.22 & -- & -- & 34.19 & 34.91 & -- \\
\hline 19 & 28.02 & 29.39 & 30.22 & 31.36 & 32.48 & 33.02 & 33.22 & -- & -- & 34.24 & 34.82 & -- \\
\hline 20 & 28.22 & 29.37 & 30.49 & 31.65 & 32.56 & 32.96 & 33.17 & -- & -- & 34.31 & 34.94 & -- \\
\hline 21 & 28.16 & 29.21 & 30.44 & 31.57 & 32.55 & 32.95 & 33.14 & -- & -- & 34.32 & 34.97 & -- \\
\hline 22 & 28.06 & 29.22 & 30.47 & 31.64 & 32.56 & 32.93 & -- & -- & -- & 34.28 & 35.01 & -- \\
\hline 23 & 28.15 & 29.37 & 36.37 & 31.51 & 32.73 & 32.95 & -- & -- & -- & 34.28 & 35.06 & -- \\
\hline 24 & 28.29 & 29.42 & 30.58 & 31.68 & 32.69 & 32.95 & -- & -- & -- & 34.34 & 35.09 & -- \\
\hline 26 & 28.24 & 29.63 & 30.64 & 31.72 & 32.76 & 33.06 & -- & -- & -- & 34.38 & 35.68 & -- \\
\hline 26 & 28.28 & 29.65 & 30.69 & 31.75 & 32.76 & 33.11 & -- & -- & -- & 34.39 & 35.09 & -- \\
\hline 27 & 28.46 & 29.67 & 36.67 & 31.79 & 32.83 & 33.61 & -- & -- & -- & 34.41 & 35.12 & -- \\
\hline 28 & 28.38 & 29.57 & 36.83 & 31.78 & 32.76 & 32.94 & -- & -- & -- & 34.43 & 35.21 & -- \\
\hline 29 & 28.34 & 29.60 & 30.89 & 31.86 & -- & 33.11 & -- & -- & -- & 34.47 & 35.23 & -- \\
\hline 30 & 28.41 & 29.66 & 30.70 & 31.78 & -- & 33.67 & -- & -- & -- & 34.60 & 36.21 & -- \\
\hline 31 & 28.51 & -- & 30.88 & 31.88 & -- & 33.02 & -- & -- & -- & 34.60 & -- & -- \\
\hline Mean & 27.90 & 29.11 & 30.27 & 31.37 & -- & -- & -- & -- & -- & 34.18 & -- & -- \\
\hline Max. & 28.61 & 29.66 & 36.89 & 31.88 & -- & -- & -- & -- & -- & 34.60 & -- & -- \\
\hline Min. & 27.24 & 28.67 & 29.69 & 30.96 & -- & -- & -- & -- & -- & 33.86 & -- & -- \\
\hline
\end{tabular}




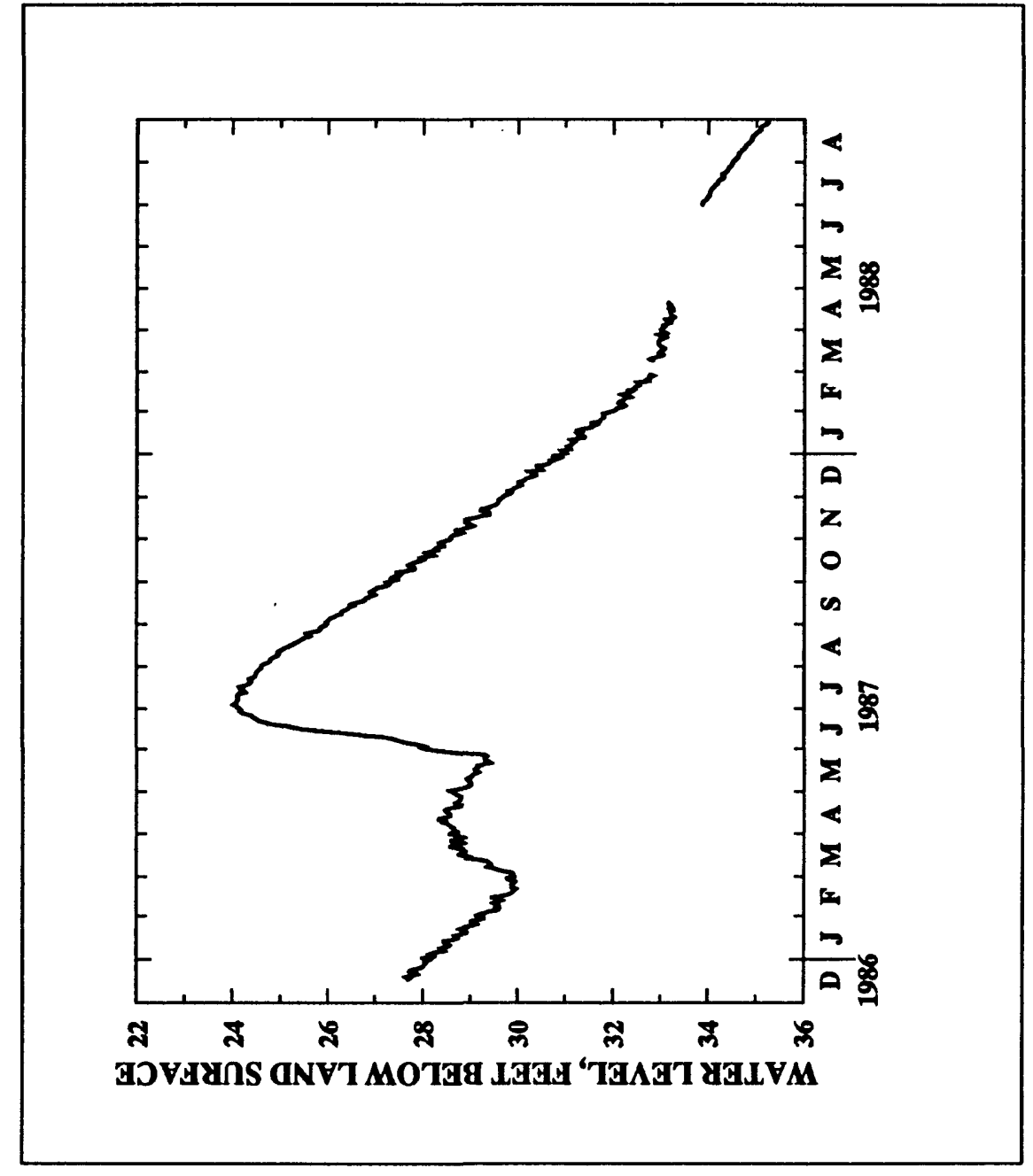

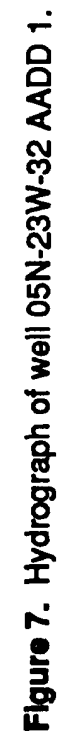


Table 3.--Daily mean water-level measurements in recorder wells in southwestern Oklahoma--Continued

HARMON COUNTY, OKLAHOMA

Index number on location map: 2

Station number: 343641099451001 Local number: $61 \mathrm{~N}-24 W-32$ CBCC 1

Location: Lat $34^{\circ} 36^{\prime} 41^{\prime \prime}$, long $99^{\circ} 46^{\prime} 10^{\prime \prime}$, hydrologic unit 11130101

Aquifer: 313VVCR

Altitude of land-surface datum: $1,483 \mathrm{ft}$

Well depth: $76.6 \mathrm{ft}$

Water Year, October 1986 to September 1986

Day Dct. Nov. Dec. Jan. Fob. Mar. Apr. May Juno July Aug. Sept.

\begin{tabular}{|c|c|c|c|c|c|c|c|c|c|c|c|c|}
\hline 1 & -- & -- & -- & -- & -- & -- & -- & -- & 28.37 & 29.78 & 34.14 & 31.66 \\
\hline 2 & -- & -- & - & -- & - & -- & -- & -- & 28.42 & 29.39 & 33.26 & 31.60 \\
\hline 3 & -- & -- & -- & -- & -- & -- & -- & -- & 28.46 & 29.12 & 32.79 & 31.46 \\
\hline 4 & -- & -- & -- & -- & - & -- & -- & -- & 28.47 & 28.98 & 32.23 & 31.41 \\
\hline 6 & -- & -- & -- & -- & - & -- & -- & -- & 27.73 & 29.09 & 31.89 & 31.28 \\
\hline 6 & -- & -- & -- & -- & -- & -- & -- & -- & 27.22 & 29.63 & 31.68 & 31.18 \\
\hline 7 & -- & -- & -- & -- & -- & -- & $-\infty$ & -- & 27.69 & 30.23 & 31.94 & 31.16 \\
\hline 8 & -- & -- & -- & -- & -- & -- & -- & -- & 27.80 & 31.14 & 32.89 & 31.06 \\
\hline 9 & -- & -- & - & - & - & - & -- & -- & 27.92 & 31.48 & 33.83 & 30.98 \\
\hline 10 & -- & -- & -- & -- & -- & -- & -- & -- & 28.06 & 31.40 & 34.48 & 30.91 \\
\hline 11 & -- & -- & -- & -- & -- & -- & -- & -- & 28.11 & 30.93 & 34.46 & 36.92 \\
\hline 12 & -- & -- & -- & -- & -- & -- & -- & -- & 28.06 & 30.61 & 35.01 & 30.88 \\
\hline 13 & -- & -- & -- & -- & -- & -- & -- & -- & 28.16 & 30.33 & 34.70 & 30.82 \\
\hline 14 & -- & -- & -- & -- & -- & -- & -- & -- & 28.21 & 30.33 & 34.10 & 30.76 \\
\hline 15 & -- & -- & -- & -- & -- & -- & -- & -- & 28.24 & 31.96 & 33.60 & 30.70 \\
\hline 18 & -- & -- & -- & -- & -- & -- & -- & -- & 28.33 & 33.22 & 32.93 & 30.60 \\
\hline 17 & -- & -- & -- & -- & -- & -- & -- & -- & 28.46 & 33.93 & 32.63 & 30.51 \\
\hline 18 & -- & -- & -- & -- & -- & -- & -- & -- & 28.44 & 34.23 & 32.98 & 30.60 \\
\hline 19 & -- & -- & -- & -- & -- & -- & -- & -- & 28.42 & 34.29 & 33.68 & 30.46 \\
\hline 20 & -- & -- & -- & -- & -- & -- & -- & -- & 28.44 & 33.64 & 32.97 & 30.44 \\
\hline 21 & -- & -- & -- & -- & -- & -- & -- & -- & 28.46 & 32.45 & 32.87 & 30.41 \\
\hline 22 & -- & -- & -- & -- & -- & -- & -- & -- & 28.47 & 31.76 & 32.60 & 30.37 \\
\hline 23 & -- & -- & -- & -- & -- & -- & -- & -- & 28.61 & 31.29 & 32.31 & 30.32 \\
\hline 24 & -- & -- & -- & -- & -- & -- & -- & -- & 28.92 & 30.98 & 32.16 & 30.28 \\
\hline 25 & -- & -- & -- & -- & -- & -- & -- & -- & 28.99 & 31.13 & 32.07 & 30.31 \\
\hline 28 & -- & -- & -- & -- & -- & -- & -- & -- & 28.83 & 32.13 & 31.99 & 30.28 \\
\hline 27 & -- & -- & -- & -- & -- & -- & -- & -- & 28.83 & 32.88 & 31.96 & 30.27 \\
\hline 28 & -- & -- & -- & -- & -- & -- & -- & -- & 28.89 & 33.14 & 31.86 & 30.23 \\
\hline 29 & -- & -- & -- & -- & -- & -- & -- & 28.22 & 28.97 & 34.17 & 31.76 & 29.61 \\
\hline 30 & -- & -- & -- & -- & -- & -- & -- & 28.27 & 29.16 & 34.84 & 31.69 & 28.84 \\
\hline 31 & -- & -- & -- & -- & -- & -- & -- & 28.31 & -- & 34.99 & 31.62 & -- \\
\hline Mean & -- & -- & -- & -- & -- & -- & -- & -- & 28.37 & 31.72 & 32.85 & 30.67 \\
\hline Max. & -- & -- & -- & -- & -- & -- & -- & -- & 29.16 & 34.99 & 36.01 & 31.56 \\
\hline Min. & -- & -- & -- & -- & -- & -- & -- & -- & 27.22 & 28.98 & 31.62 & 28.84 \\
\hline
\end{tabular}


Table 3.--Daily mean water-level measurements in recorder wells in southwestern oklahoma--Continued

HARMON COUNTY, OKLAHOMA--CONtinued

$01 \mathrm{~N}-24 \mathrm{~W}-32$ CBCC $1--$ Continued

Water Year, October 1986 to September 1987

\begin{tabular}{|c|c|c|c|c|c|c|c|c|c|c|c|c|}
\hline Day & Oct. & Nov. & Dec. & Jan. & Fab. & Mar. & Apr. & May & June & July & Aug. & Sept. \\
\hline 1 & 28.92 & 26.65 & 26.67 & 26.70 & 26.47 & 25.46 & 28.01 & 26.60 & 24.62 & 25.64 & 27.69 & 27.62 \\
\hline 2 & 28.20 & 26.56 & 26.81 & 26.66 & 26.49 & 25.61 & 26.16 & 26.59 & 24.83 & 26.70 & 27.87 & 27.78 \\
\hline 3 & 27.34 & 26.47 & 26.64 & 26.73 & 26.51 & 25.74 & 26.09 & 26.64 & 25.62 & 25.80 & 28.06 & 27.48 \\
\hline 4 & 26.34 & 26.26 & 26.68 & 26.70 & 26.62 & 25.79 & 26.12 & 26.68 & 26.09 & 25.84 & 28.82 & 27.28 \\
\hline 5 & 26.68 & 26.16 & 26.61 & 26.63 & 26.51 & 25.75 & 26.14 & 26.69 & 26.13 & 25.91 & 28.90 & 27.19 \\
\hline 6 & 26.99 & 26.06 & 28.54 & 26.68 & 26.42 & 26.77 & 26.11 & 26.68 & 26.18 & 26.19 & 27.61 & 27.15 \\
\hline 7 & 27.11 & 26.16 & 26.64 & 26.78 & 26.35 & 26.79 & 26.12 & 26.66 & 26.23 & 27.16 & 27.19 & 27.12 \\
\hline 8 & 27.19 & 26.29 & 26.66 & 26.68 & 26.38 & 25.78 & 28.10 & 26.68 & 25.29 & 27.40 & 27.02 & 27.13 \\
\hline 9 & 27.32 & 26.39 & 26.62 & 26.64 & 26.36 & 25.89 & 26.06 & 26.63 & 25.30 & 27.65 & 26.96 & 27.09 \\
\hline 10 & 27.30 & 26.36 & 26.63 & 26.71 & 26.28 & 25.92 & 26.11 & 26.63 & 25.23 & 27.71 & 26.88 & 27.08 \\
\hline 11 & 27.24 & 26.39 & 26.67 & 26.72 & 26.32 & 26.93 & 26.05 & 26.68 & 24.86 & 27.12 & 26.83 & 27.69 \\
\hline 12 & 26.99 & 26.48 & 26.64 & 26.67 & 26.36 & 25.96 & 26.08 & 26.68 & 24.82 & 28.83 & 27.05 & 27.07 \\
\hline 13 & 26.95 & 26.61 & 26.63 & 26.62 & 26.27 & 25.90 & 26.19 & 26.69 & 25.04 & 28.70 & 27.93 & 27.63 \\
\hline 14 & 27.01 & 26.33 & 26.69 & 26.62 & 26.21 & 25.83 & 26.20 & 26.73 & 26.16 & 26.81 & 29.01 & 27.04 \\
\hline 16 & 27.12 & 26.33 & 26.61 & 26.71 & 26.27 & 25.92 & 26.19 & 26.78 & 26.21 & 27.21 & 27.89 & 26.98 \\
\hline 16 & 27.12 & 26.36 & 26.62 & 26.73 & 26.08 & 25.87 & 26.18 & 26.87 & 25.26 & 27.39 & 27.17 & 27.00 \\
\hline 17 & 27.12 & 26.36 & 26.62 & 26.68 & 26.01 & 25.76 & 26.17 & 26.93 & 25.34 & 26.93 & 26.94 & 27.02 \\
\hline 18 & 27.17 & 26.61 & 26.63 & 26.66 & 26.11 & 26.72 & 26.17 & 27.01 & 25.42 & 26.60 & 26.83 & 27.06 \\
\hline 19 & 27.22 & 26.41 & 28.68 & 26.72 & 26.16 & 25.84 & 26.22 & 27.01 & 26.41 & 28.63 & 26.78 & 27.02 \\
\hline 20 & 27.21 & 26.58 & 26.63 & 26.77 & 26.18 & 25.88 & 26.32 & 26.93 & 25.45 & 26.61 & 26.77 & 27.02 \\
\hline 21 & 28.86 & 26.49 & 26.68 & 26.67 & 26.12 & 26.96 & 26.36 & 26.98 & 25.47 & $26.6 \varnothing$ & 27.16 & 27.02 \\
\hline 22 & 26.98 & 26.44 & 26.64 & 26.71 & 26.14 & 25.88 & 26.29 & 26.98 & 25.49 & 26.71 & 28.43 & 27.82 \\
\hline 23 & 26.68 & 28.69 & 26.67 & 26.61 & 26.12 & 26.86 & 28.31 & 26.69 & 26.65 & 27.07 & 29.56 & 26.97 \\
\hline 24 & 26.87 & 26.66 & 26.68 & 26.64 & 26.14 & 25.99 & 26.37 & 26.33 & 26.69 & 27.16 & 29.66 & 26.94 \\
\hline 26 & 26.08 & 26.46 & 26.66 & 26.71 & 26.16 & 26.04 & 26.38 & 26.00 & 26.62 & 28.29 & 29.24 & 26.96 \\
\hline 26 & 26.21 & 26.58 & 28.68 & 26.73 & 26.68 & 26.08 & 28.39 & 25.71 & 25.65 & 28.50 & 28.67 & 28.95 \\
\hline 27 & 26.27 & 26.68 & 26.67 & 26.66 & 25.81 & 26.92 & 26.42 & 25.71 & 25.64 & 28.10 & 27.94 & 28.95 \\
\hline 28 & 26.33 & 26.56 & 26.67 & 26.68 & 26.19 & 26.07 & 26.62 & 25.02 & 25.65 & 28.74 & 27.62 & 28.88 \\
\hline 29 & 26.44 & 26.60 & 26.68 & 26.66 & - & 26.12 & 28.67 & 23.38 & 25.68 & 28.68 & 27.30 & 26.62 \\
\hline 30 & 26.40 & 26.47 & 26.64 & 26.68 & -- & 26.08 & 28.63 & 23.80 & 25.62 & 28.05 & 27.20 & 26.70 \\
\hline 31 & 26.44 & -- & 26.64 & 26.63 & - & 26.05 & - & 24.38 & -- & 27.69 & 27.18 & - \\
\hline Mean & 26.87 & 26.42 & 26.62 & 26.67 & 26.21 & 26.87 & 26.23 & 26.31 & 25.29 & 27.08 & 27.74 & 27.07 \\
\hline Max. & 28.92 & 26.59 & 26.68 & 26.77 & 26.62 & 26.12 & 26.63 & 27.01 & 25.68 & 28.74 & 29.68 & 27.78 \\
\hline Min. & 25.58 & 28.06 & 26.64 & 26.63 & 25.19 & 25.46 & 26.01 & 23.38 & 24.62 & 25.64 & 26.77 & 26.62 \\
\hline
\end{tabular}


Table 3.--Daily mean water-level measurements in recorder wells in southwestern Oklahoma--Continued

HARMON COUNTY, OKLAHOMA--ContinUed

o1N-24W-32 CBCC 1--Continued

Water Year, October 1987 to September 1988

\begin{tabular}{|c|c|c|c|c|c|c|c|c|c|c|c|c|}
\hline Day & oct. & Nov. & Dec. & Jan. & Fob. & Mar. & Apr. & May & June & July & Aug. & Sept. \\
\hline 1 & 28.70 & 25.70 & 28.63 & 20.72 & 26.43 & 26.46 & 26.29 & 26.09 & 27.13 & 26.91 & -- & 28.94 \\
\hline 2 & 26.80 & 25.99 & 26.61 & 28.64 & 26.46 & 26.30 & 26.33 & 26.12 & 27.03 & 26.89 & -- & 28.89 \\
\hline 3 & 26.84 & 26.11 & 26.68 & 26.60 & 26.44 & 25.86 & 26.36 & 26.22 & 27.04 & 26.86 & -- & 28.83 \\
\hline 4 & $2 \theta .7 \theta$ & 26.22 & 26.06 & 26.69 & 26.62 & 25.96 & 26.33 & 26.26 & 26.98 & $28.8 \theta$ & -- & 28.78 \\
\hline 5 & 26.78 & 28.33 & 26.58 & 26.64 & 26.54 & 26.04 & 26.42 & 26.24 & 26.94 & 26.88 & -- & 28.73 \\
\hline 8 & 26.83 & 26.30 & 28.66 & 28.59 & 26.49 & 26.04 & 26.47 & 26.17 & 27.66 & 26.86 & -- & 28.69 \\
\hline 7 & 26.83 & 26.28 & 26.04 & 20.63 & 26.41 & 20.05 & 26.38 & 26.18 & 27.69 & 26.86 & -- & 28.64 \\
\hline 8 & 26.77 & 26.40 & 26.68 & 26.61 & 26.41 & 26.17 & 26.37 & 26.23 & 27.64 & 26.86 & -- & 28.62 \\
\hline 9 & 26.86 & 28.47 & 26.70 & 26.65 & 26.46 & 28.11 & 28.49 & 26.33 & 27.43 & 26.83 & -- & 28.63 \\
\hline 10 & 26.94 & 26.51 & 26.64 & 26.63 & 26.45 & 26.04 & 26.49 & 26.34 & 27.34 & 26.80 & -- & 28.60 \\
\hline 11 & 26.92 & 20.47 & 26.67 & 26.51 & 28.62 & 26.13 & 26.45 & 26.37 & 27.31 & 26.71 & -- & 28.57 \\
\hline 12 & 26.85 & 26.44 & 26.71 & 26.82 & 26.42 & 26.25 & 26.51 & 28.34 & 27.19 & 26.73 & -- & 28.58 \\
\hline 13 & 26.82 & 26.41 & 26.69 & 26.66 & 26.34 & 26.29 & 26.69 & 26.38 & 27.89 & 26.78 & -- & 28.56 \\
\hline 14 & 26.84 & 26.40 & 26.71 & 26.57 & 26.44 & 28.25 & 26.67 & 26.49 & 27.22 & 26.83 & -- & 28.13 \\
\hline 16 & 26.88 & 26.43 & 26.77 & 26.48 & 26.48 & 26.23 & 26.84 & 26.62 & 27.34 & 27.28 & -- & 28.14 \\
\hline 16 & -26.90 & 26.52 & 26.78 & 26.43 & 26.40 & 26.25 & 27.20 & 26.56 & 27.34 & 28.63 & -- & 28.15 \\
\hline 17 & 26.91 & 26.54 & 26.72 & 26.37 & 26.50 & 26.30 & 26.56 & 26.61 & 27.29 & 28.64 & -- & 28.16 \\
\hline 18 & 26.84 & 26.65 & 26.84 & 26.26 & 26.45 & 26.32 & 25.47 & 26.67 & 27.30 & 28.44 & -- & 27.71 \\
\hline 19 & 26.89 & 26.82 & 26.58 & 26.30 & 26.47 & 26.27 & 25.48 & 26.76 & 27.32 & 28.81 & -- & 26.49 \\
\hline $2 \varnothing$ & 26.98 & 28.60 & 26.71 & 26.40 & 26.61 & 26.23 & 25.76 & 26.68 & 27.41 & 28.94 & -- & 26.18 \\
\hline 21 & 26.92 & 26.50 & 26.63 & 26.38 & 26.49 & 26.24 & 25.77 & 26.68 & 28.25 & 29.36 & -- & 26.23 \\
\hline 22 & 26.85 & 26.51 & 26.86 & 28.41 & 26.42 & 26.24 & 25.88 & 28.64 & 29.00 & 28.69 & -- & 26.58 \\
\hline 23 & 26.87 & 26.58 & 26.68 & 26.29 & 26.69 & 26.27 & 26.00 & 26.64 & 29.81 & 28.79 & -- & 28.68 \\
\hline 24 & 26.73 & 26.69 & 26.69 & 26.43 & 26.57 & 26.26 & 26.97 & 26.66 & 30.02 & 28.59 & -- & 26.66 \\
\hline 26 & 26.62 & 26.83 & 26.66 & 28.41 & 26.64 & 26.33 & 26.03 & 26.71 & 30.15 & 28.22 & -- & 26.77 \\
\hline 26 & $26.7 \varnothing$ & 26.62 & 26.68 & 26.43 & 26.49 & 28.38 & 26.08 & 26.66 & 28.89 & 28.01 & -- & 26.81 \\
\hline 27 & 26.79 & 26.62 & 26.64 & 26.43 & 26.53 & 26.28 & 26.13 & 26.85 & 27.72 & 27.93 & -- & 26.84 \\
\hline 28 & 26.74 & 26.60 & 26.74 & 26.39 & 26.50 & 26.26 & 26.10 & 27.04 & 27.42 & - & -- & 28.87 \\
\hline 29 & 26.73 & 26.61 & 26.71 & 26.34 & 26.56 & 26.41 & 26.67 & 26.97 & 27.03 & -- & -- & 28.98 \\
\hline 30 & 26.76 & 26.82 & 26.56 & 26.31 & -- & 28.34 & 26.15 & 26.84 & 26.93 & -- & -- & 28.97 \\
\hline 31 & 25.73 & -- & 26.68 & 26.37 & -- & 26.31 & -- & 27.05 & -- & -- & -- & -- \\
\hline Mean & 26.79 & 26.44 & 26.67 & 26.49 & 26.48 & 26.22 & 26.25 & 26.52 & 27.67 & -- & -- & 27.78 \\
\hline Max. & 26.98 & 26.65 & 26.78 & 26.72 & 26.59 & 26.46 & 27.20 & 27.05 & 30.15 & -- & -- & 28.94 \\
\hline Min. & 26.73 & 25.70 & 26.56 & 26.25 & 26.34 & 25.85 & 25.47 & 26.09 & 28.93 & -- & -- & 28.18 \\
\hline
\end{tabular}


Table 3.--Daily mean water-level measurements in recorder wells in southwestern oklahoma--Continued

HARMON COUNTY, OKLAHOMA--Continued

01N-24W-32 CBCC 1--Continued

Water Year, October 1988 to September 1989

Day Oct. Nov. Dec. Jan. Feb. Mar. Apr. May June July Aug. Sept.

\begin{tabular}{|c|c|c|c|c|c|c|c|c|c|c|c|c|}
\hline 1 & 26.98 & 27.16 & 27.28 & 27.20 & -- & - & -- & -- & - & -- & - & $\ldots$ \\
\hline 2 & 27.04 & 27.08 & 27.26 & 27.21 & -- & -- & - & -- & -- & -- & -- & - \\
\hline 3 & 27.06 & 27.09 & 27.26 & 27.27 & -- & -- & - & -- & -- & -- & - & $\sim$ \\
\hline 4 & 27.08 & 27.17 & 27.31 & 27.21 & -- & -- & - & -- & -- & -- & -- & - \\
\hline 6 & 27.08 & 27.24 & 27.23 & 27.13 & -- & -- & -- & -- & -- & -- & -- & - \\
\hline 6 & 27.08 & 27.17 & 27.17 & 27.16 & -- & -- & -- & -- & -- & -- & - & - \\
\hline 7 & 27.12 & 27.18 & 27.21 & 27.23 & -- & -- & - & -- & -- & -- & -- & $\ldots$ \\
\hline 8 & 27.06 & 27.21 & 27.30 & 27.31 & -- & -- & - & -- & -- & -- & -- & - \\
\hline 9 & 27.09 & 27.17 & 27.23 & 27.24 & -- & -- & - & -- & -- & -- & -- & -- \\
\hline 10 & 27.10 & 27.26 & 27.20 & 27.20 & -- & -- & - & -- & - & -- & -- & -- \\
\hline 11 & 27.12 & 27.16 & 27.22 & 27.20 & -- & -- & -- & - & -- & -- & -- & - \\
\hline 12 & 27.14 & 27.20 & 27.22 & 27.33 & -- & -- & - & -- & -- & -- & - & -- \\
\hline 13 & 27.13 & 27.22 & 27.17 & 27.30 & -- & -- & - & -- & -- & -- & -- & $\ldots$ \\
\hline 14 & 27.09 & 27.22 & 27.17 & 27.18 & -- & -- & - & -- & -- & -- & - & -- \\
\hline 16 & 27.10 & 27.24 & 27.33 & 27.26 & -- & -- & -- & -- & -- & -- & -- & -- \\
\hline 16 & $\begin{array}{l}27.12 \\
\end{array}$ & 27.32 & 27.27 & 27.28 & -- & -- & -- & -- & -- & -- & -- & -- \\
\hline 17 & 27.11 & 27.28 & 27.25 & 27.20 & -- & -- & - & -- & -- & -- & -- & - \\
\hline 18 & 27.20 & 27.21 & 27.17 & 27.26 & -- & $\ldots$ & -- & $\ldots$ & -- & -- & -- & -- \\
\hline 19 & 27.14 & 27.30 & 27.11 & - & -- & -- & - & -- & -- & -- & - & - \\
\hline 20 & 27.13 & 27.30 & 27.22 & -- & -- & -- & -- & -- & -- & -- & -- & -- \\
\hline 21 & 27.18 & 27.26 & 27.22 & -- & -- & -- & - & -- & -- & -- & -- & - \\
\hline 22 & 27.09 & 27.20 & 27.20 & -- & -- & -- & - & -- & -- & -- & -- & - \\
\hline 23 & 27.16 & 27.16 & 27.16 & -- & -- & -- & - & -- & -- & -- & -- & -- \\
\hline 24 & 27.16 & 27.12 & 27.26 & -- & -- & -- & - & -- & -- & -- & - & - \\
\hline 26 & 27.17 & 27.13 & 27.20 & -- & -- & -- & -- & -- & - & -- & -- & - \\
\hline 26 & 27.17 & 27.16 & 27.14 & -- & -- & -- & -- & -- & -- & -- & - & -- \\
\hline 27 & 27.13 & 27.29 & 27.26 & -- & -- & -- & - & -- & -- & -- & -- & - \\
\hline 28 & 27.24 & 27.22 & 27.28 & -- & -- & -- & - & -- & -- & -- & -- & - \\
\hline 29 & 27.20 & 27.24 & 27.22 & -- & -- & -- & -- & -- & - & - & - & -- \\
\hline 30 & 27.21 & 27.28 & 27.18 & -- & -- & - & -- & -- & -- & -- & -- & -- \\
\hline 31 & 27.20 & - & 27.19 & -- & -- & -- & -- & -- & -- & -- & -- & -- \\
\hline Mean & 27.13 & 27.21 & 27.22 & -- & -- & - & - & -- & - & -- & -- & - \\
\hline Max. & 27.24 & 27.32 & 27.33 & -- & -- & -- & -- & -- & $-\infty$ & -- & -- & - \\
\hline Min. & 26.98 & 27.08 & 27.11 & -- & -- & - & - & -- & -- & - & -- & - \\
\hline
\end{tabular}




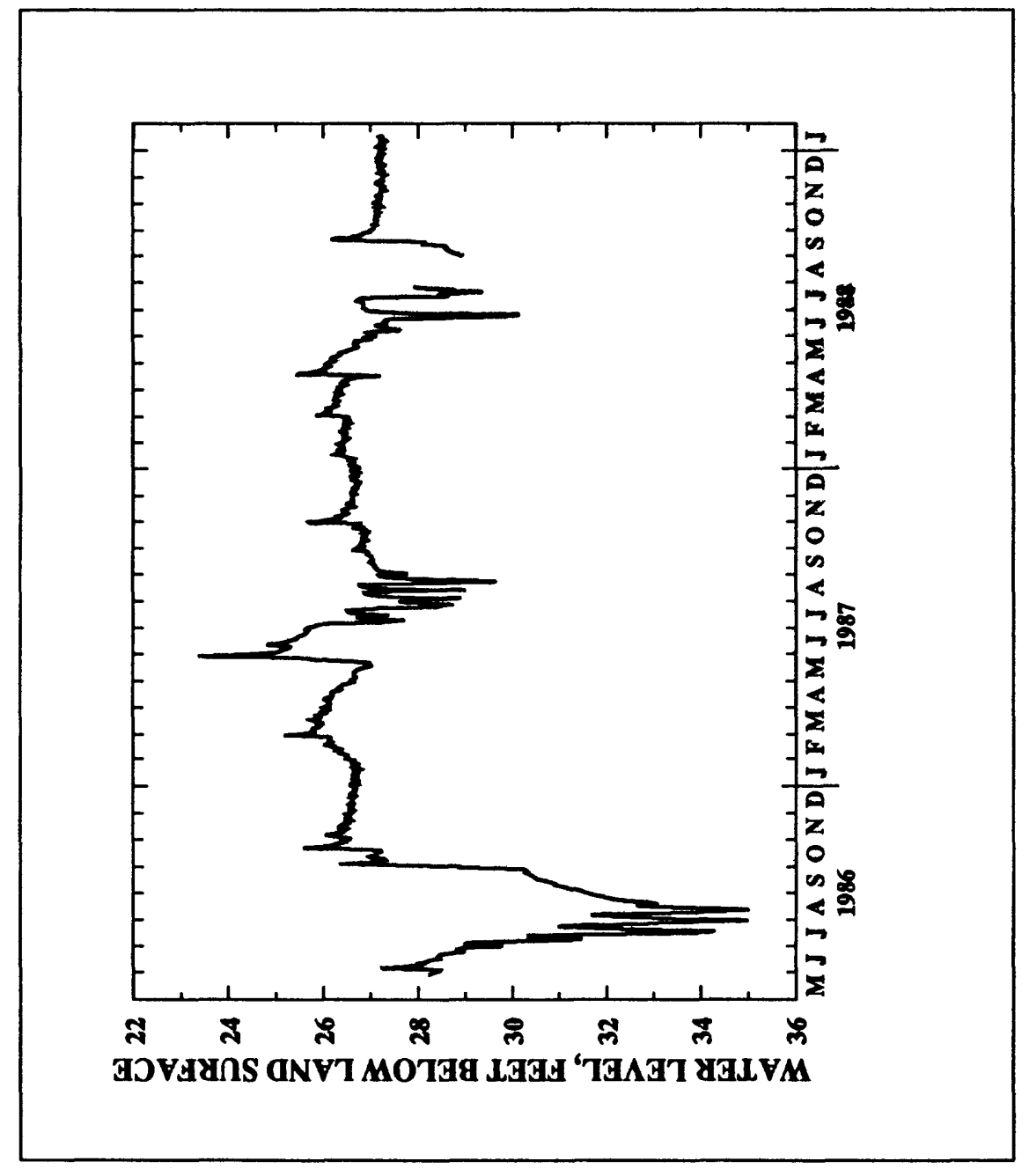

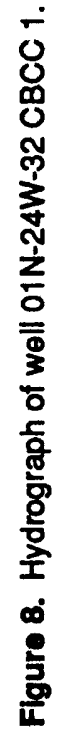


Table 3.--Daily mean water-level measurements in recorder wells in southwestern oklahoma--Continued

HARMON COUNTY, OKLAHOMA--COntinUEd

Index number on location map: 3

Station number: $\$ 43724099434901$ Local number: $02 \mathrm{~N}-24 W-21$ DDDD 1

Location: Lat $34^{\circ} 37^{\prime} 24^{\prime \prime}$, long $99^{\circ} 43^{\prime} 87^{\prime \prime}$, hydrologic unit 11120282

Aquifer: 313 EMFK

Altitude of land-surface datum: $1,625 \mathrm{ft}$

Well depth: $286 \mathrm{ft}$

Water Year, October 1986 to September 1987

Day Oct. Nov. Dec. Jan. Fob. Mar. Apr. May June July Aug. Sept.

\begin{tabular}{|c|c|c|c|c|c|c|c|c|c|c|c|c|c|}
\hline 1 & & - & -- & -- & -- & -- & -- & -- & -- & - & -- & 35.38 & 37.80 \\
\hline 2 & & -- & - & - & -- & -- & -- & -- & -- & -- & -- & 36.49 & 37.85 \\
\hline 3 & & - & - & -- & - & -- & -- & -- & -- & 52.06 & - & 36.67 & 37.86 \\
\hline 4 & & -- & - & -- & -- & -- & -- & -- & -- & -- & $\ldots$ & 35.82 & 37.88 \\
\hline 6 & & -- & - & - & -- & - & -- & -- & -- & -- & -- & 35.88 & 37.94 \\
\hline 6 & & -- & -- & -- & $-\infty$ & -- & -- & -- & -- & -- & $-\infty$ & 35.86 & 37.97 \\
\hline 7 & & -- & $\sim$ & -- & -- & -- & - & $\sim$ & -- & - & -- & 35.80 & 38.08 \\
\hline 8 & & - & -- & -- & -- & -- & -- & -- & -- & -- & - & 35.81 & 38.13 \\
\hline 8 & & -- & -- & -- & -- & -- & -- & -- & -- & -- & 34.01 & 35.87 & 38.10 \\
\hline 10 & & -- & $-\infty$ & -- & -- & -- & -- & - & - & - & $\ldots$ & 35.91 & 38.15 \\
\hline 11 & & -- & -- & - & -- & -- & -- & - & -- & -- & -- & 35.90 & 38.17 \\
\hline 12 & & -- & $-\infty$ & - & -- & -- & -- & -- & -- & 36.79 & - & 36.92 & 38.22 \\
\hline 13 & - & -- & -- & - & -- & -- & -- & -- & -- & -- & -- & 36.08 & 38.26 \\
\hline 14 & & -- & -- & -- & -- & -- & -- & - & - & -- & -- & 36.19 & 38.30 \\
\hline 15 & & -- & - & -- & -- & -- & -- & -- & - & -- & -- & 36.33 & 38.28 \\
\hline 16 & & -- & -- & -- & -- & -- & -- & -- & -- & - & 34.36 & 36.49 & 38.38 \\
\hline 17 & & -- & -- & $\sim$ & -- & -- & -- & - & - & - & 34.31 & 36.60 & 38.39 \\
\hline 18 & & - & - & -- & -- & -- & -- & -- & -- & 34.17 & 34.37 & 36.73 & 38.49 \\
\hline 19 & & - & - & -- & - & -- & -- & -- & - & - & 34.70 & 36.79 & 38.62 \\
\hline 20 & & - & -- & -- & -- & -- & -- & -- & -- & -- & 34.92 & 36.96 & 38.65 \\
\hline 21 & & - & -- & -- & -- & -- & -- & -- & -- & -- & $35 . \varnothing 5$ & 37.08 & 38.60 \\
\hline 22 & & -- & -- & -- & -- & -- & -- & -- & -- & -- & 35.21 & 37.21 & 38.72 \\
\hline 23 & & -- & - & -- & -- & -- & -- & - & -- & -- & -- & 37.34 & 38.71 \\
\hline 24 & & $\ldots$ & -- & -- & -- & -- & -- & -- & -- & -- & -- & 37.41 & 38.67 \\
\hline 26 & & -- & -- & -- & -- & -- & -- & -- & -- & -- & -- & 37.45 & 38.70 \\
\hline 26 & & -- & - & -- & -- & -- & -- & -- & -- & -- & -- & 37.59 & 38.73 \\
\hline 27 & & -- & -- & -- & -- & -- & -- & -- & -- & -- & -- & 37.76 & 38.77 \\
\hline 28 & & -- & -- & -- & -- & - & -- & -- & -- & -- & -- & 37.78 & 38.77 \\
\hline 29 & & -- & -- & -- & -- & -- & -- & -- & - & -- & -- & 37.80 & 38.83 \\
\hline 30 & & -- & -- & -- & -- & - & -- & -- & -- & - & -- & 37.84 & 38.83 \\
\hline 31 & & -- & -- & -- & -- & -- & -- & -- & - & -- & 35.28 & 37.84 & -- \\
\hline Nean & & -- & - & -- & -- & -- & -- & -- & -- & - & -- & 36.60 & 38.38 \\
\hline Max. & & -- & - & -- & - & -- & -- & -- & - & -- & -- & 37.84 & 38.83 \\
\hline Min. & & -- & -- & -- & -- & -- & -- & - & -- & -- & -- & 35.36 & 37.80 \\
\hline
\end{tabular}


Table 3.--Daily mean water-level measurements in recorder wells in southwestern Oklahoma--Continued

HARMON COUNTY, OKLAHOMA--CONTInUed

62N-24W-21 DDDD 1--Continued

Water Year, October 1987 to September 1988

Day Dct. Nov. Dec. Jan. Feb. Mar. Apr. May June July Aug. Sept.

\begin{tabular}{|c|c|c|c|c|c|c|c|c|c|c|c|c|}
\hline 1 & 38.65 & -- & 39.77 & 48.68 & 38.65 & -- & -- & -- & -- & -- & -- & -- \\
\hline 2 & 38.73 & -- & 39.81 & 46.84 & 38.79 & -- & -- & -- & -- & -- & -- & -- \\
\hline 3 & 38.77 & -- & 39.93 & 40.62 & 38.82 & -- & -- & -- & -- & -- & -- & -- \\
\hline 4 & 38.78 & 38.86 & 40.00 & 40.70 & 38.96 & -- & -- & -- & -- & -- & -- & -- \\
\hline 5 & -- & 36.91 & 39.93 & 40.67 & 39.66 & -- & -- & -- & -- & -- & -- & -- \\
\hline 6 & -- & 38.98 & 39.97 & 40.66 & 39.11 & -- & $-\infty$ & $-\dot{-}$ & -- & -- & -- & -- \\
\hline 7 & -- & 37.01 & 40.04 & 46.62 & 38.99 & -- & -- & -- & -- & -- & -- & -- \\
\hline 8 & -- & 37.23 & 40.09 & 40.60 & -- & 37.39 & -- & $-\infty$ & -- & -- & -- & -- \\
\hline 9 & -- & 37.56 & 39.95 & 48.68 & -- & 37.31 & -- & -- & -- & -- & -- & -- \\
\hline 10 & -- & 37.79 & 39.53 & 40.70 & -- & $=-$ & -- & -- & -- & -- & -- & -- \\
\hline 11 & -- & 38.00 & 39.57 & 40.66 & -- & -- & -- & - & -- & -- & -- & -- \\
\hline 12 & -- & 38.09 & 39.74 & 40.67 & -- & -- & -- & -- & -- & -- & -- & -- \\
\hline 13 & -- & 38.69 & 39.81 & 40.89 & -- & -- & -- & -- & -- & -- & -- & - \\
\hline 14 & -- & 38.11 & 39.84 & 46.81 & -- & -- & -- & -- & -- & -- & -- & -- \\
\hline 15 & -- & 38.21 & 39.96 & 40.87 & -- & -- & - & -- & -- & -- & -- & - \\
\hline 16 & -- & 38.43 & 40.68 & 40.46 & -- & -- & -- & -- & -- & -- & -- & -- \\
\hline 17 & -- & 38.60 & 40.09 & 40.37 & -- & -- & -- & -- & -- & -- & -- & -- \\
\hline 18 & -- & 38.90 & 40.04 & 40.16 & -- & -- & -- & -- & -- & -- & -- & -- \\
\hline 19 & -- & 39.06 & 39.91 & 39.84 & -- & -- & -- & -- & -- & -- & -- & -- \\
\hline 20 & -- & 39.14 & 40.16 & 39.86 & -- & -- & -- & -- & -- & -- & -- & -- \\
\hline 21 & -- & 39.67 & 40.16 & 39.61 & -- & -- & -- & -- & -- & -- & -- & -- \\
\hline 22 & -- & 39.08 & 40.18 & 39.54 & -- & -- & -- & -- & -- & -- & -- & -- \\
\hline 23 & -- & 39.20 & 39.99 & -- & -- & -- & -- & -- & -- & -- & -- & -- \\
\hline 24 & -- & 39.29 & 40.20 & -- & -- & -- & -- & -- & -- & -- & -- & -- \\
\hline 25 & -- & 39.43 & 40.30 & -- & -- & -- & -- & -- & -- & -- & -- & -- \\
\hline 26 & -- & 39.61 & 40.36 & -- & -- & -- & -- & -- & -- & -- & -- & -- \\
\hline 27 & -- & 39.66 & 46.37 & -- & -- & -- & -- & -- & -- & -- & -- & -- \\
\hline 28 & -- & 39.69 & 40.49 & 38.29 & -- & -- & -- & -- & -- & -- & -- & -- \\
\hline 29 & -- & 39.63 & 40.63 & 38.37 & -- & -- & -- & -- & -- & -- & -- & -- \\
\hline 30 & -- & 39.68 & 40.43 & 38.36 & -- & -- & -- & -- & -- & -- & -- & -- \\
\hline 31 & -- & -- & 40.60 & 38.47 & -- & -- & -- & -- & -- & -- & -- & -- \\
\hline Mean & -- & -- & 40.08 & -- & -- & -- & -- & -- & -- & -- & -- & -- \\
\hline Max. & -- & -- & 40.63 & -- & -- & -- & -- & -- & -- & -- & -- & -- \\
\hline Min. & -- & -- & 39.63 & -- & -- & -- & -- & -- & -- & -- & -- & -- \\
\hline
\end{tabular}




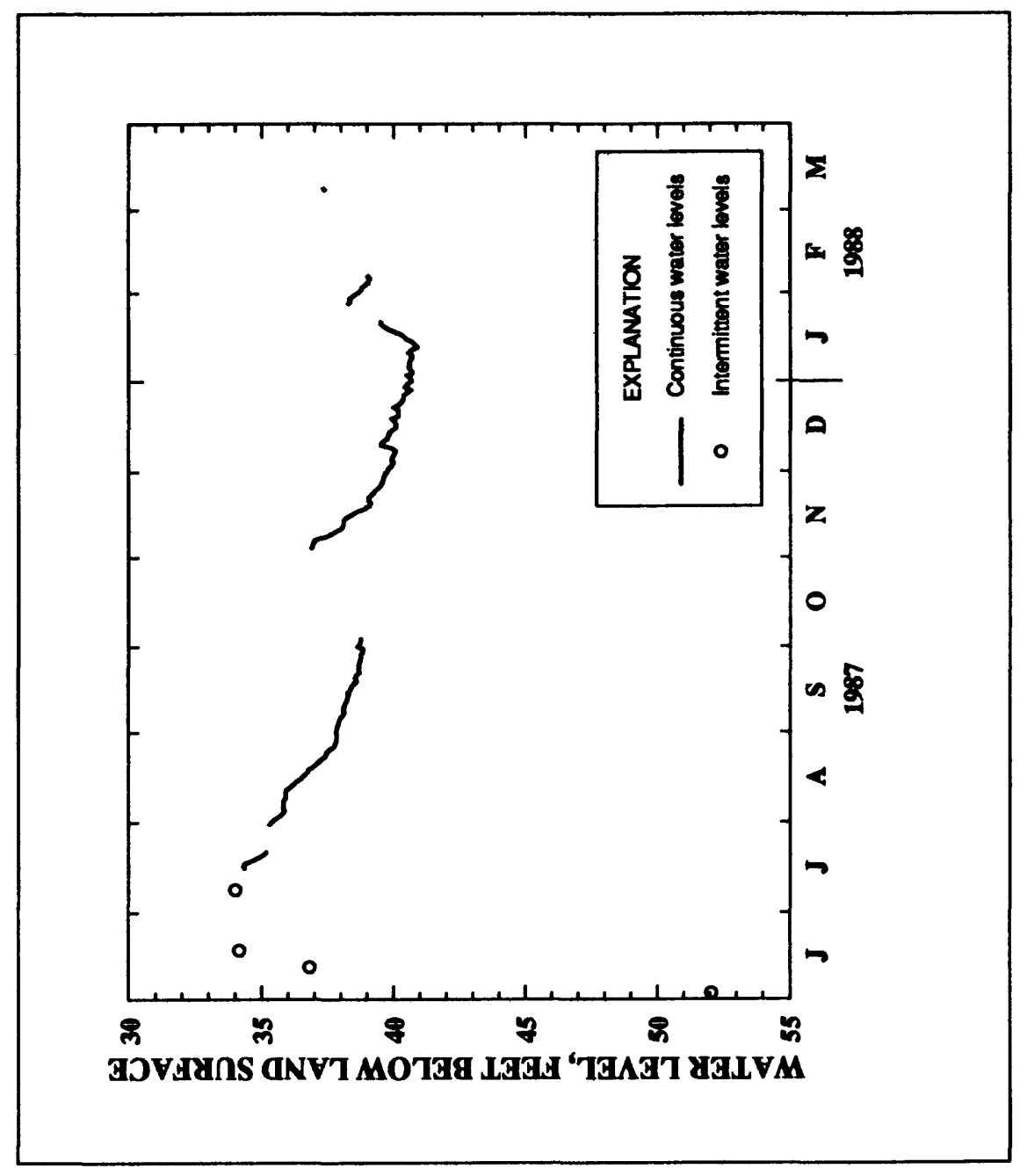

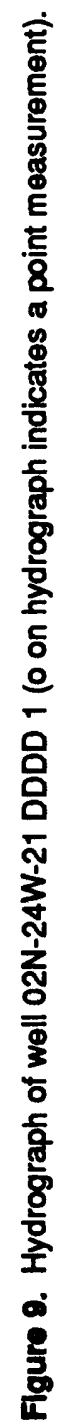


Table 3.--Daily mean water-level measurements in recorder wells in southwestern Oklahoma--Continued

HARMON COUNTY, OKLAHOMA--CONTInUEd

Index number on location map: 4

Station number: 343724099434902 Local number: $62 \mathrm{~N}-24 W-21$ DODD 2

Location: Lat $34^{\circ} 37^{\prime} 24^{\prime \prime}$, long $99^{\circ} 43^{\prime} 07^{\prime \prime}$, hydrologic unit 11120202

Aquifer: $313 V V C R$

Altitude of land-surface datum: $1,525 \mathrm{ft}$

Well depth: $159 \mathrm{ft}$

Woter Year, October 1988 to September 1987

Day Dct. Nov. Dec. Jan. Fob. Mar. Apr. May June July Aug. Sept.

\begin{tabular}{|c|c|c|c|c|c|c|c|c|c|c|c|c|}
\hline 1 & -- & -- & -- & -- & -- & -- & -- & -- & -- & -- & 36.89 & -- \\
\hline 2 & -- & -- & -- & -- & -- & -- & -- & -- & -- & -- & 37.61 & -- \\
\hline 3 & -- & -- & -- & -- & -- & -- & -- & -- & -- & -- & 37.14 & -- \\
\hline 4 & -- & -- & -- & -- & -- & -- & -- & -- & -- & -- & 37.30 & - \\
\hline 5 & -- & -- & -- & -- & -- & -- & -- & -- & -- & -- & -- & -- \\
\hline 6 & -- & -- & -- & -- & -- & -- & -- & -- & -- & -- & -- & -- \\
\hline 7 & -- & -- & -- & -- & -- & -- & -- & -- & -- & -- & -- & -- \\
\hline 8 & -- & -- & -- & -- & -- & -- & -- & -- & -- & -- & -- & -- \\
\hline 9 & -- & -- & -- & -- & -- & -- & -- & -- & 96.62 & 36.20 & -- & - \\
\hline 10 & -- & -- & -- & -- & - & -- & -- & -- & - & -- & -- & -- \\
\hline 11 & -- & -- & -- & -- & -- & -- & -- & -- & -- & -- & -- & - \\
\hline 12 & -- & -- & -- & -- & -- & -- & -- & -- & 37.03 & -- & -- & - \\
\hline 13 & -- & -- & -- & -- & -- & -- & -- & -- & -- & -- & -- & -- \\
\hline 14 & -- & -- & -- & -- & -- & -- & -- & -- & -- & -- & -- & -- \\
\hline 15 & -- & -- & -- & -- & -- & -- & -- & -- & -- & -- & -- & - \\
\hline 16 & -- & -- & -- & -- & -- & -- & -- & -- & -- & 36.89 & -- & -- \\
\hline 17 & -- & -- & -- & -- & -- & -- & -- & -- & -- & 35.85 & -- & -- \\
\hline 18 & -- & -- & -- & -- & -- & -- & -- & -- & 35.21 & 35.92 & -- & -- \\
\hline 19 & -- & -- & -- & -- & -- & -- & -- & -- & -- & 36.00 & -- & -- \\
\hline 20 & -- & -- & -- & -- & -- & -- & -- & -- & -- & 36.08 & -- & -- \\
\hline 21 & -- & -- & -- & -- & -- & -- & -- & -- & -- & 36.13 & -- & -- \\
\hline 22 & -- & -- & -- & -- & -- & -- & -- & -- & -- & 36.16 & -- & -- \\
\hline 23 & -- & -- & -- & -- & -- & -- & -- & -- & -- & 36.13 & -- & -- \\
\hline 24 & -- & -- & -- & -- & -- & -- & -- & -- & -- & 36.15 & -- & -- \\
\hline 25 & -- & -- & -- & -- & -- & -- & -- & -- & -- & 36.26 & -- & - \\
\hline 26 & -- & -- & -- & -- & -- & -- & - & -- & -- & 36.35 & -- & -- \\
\hline 27 & -- & -- & -- & -- & -- & -- & -- & -- & -- & 36.41 & -- & -- \\
\hline 28 & -- & -- & -- & -- & -- & -- & -- & -- & -- & 36.48 & -- & -- \\
\hline 29 & -- & -- & -- & -- & -- & -- & -- & -- & -- & 36.56 & -- & -- \\
\hline 30 & -- & -- & -- & -- & -- & -- & -- & -- & -- & 36.67 & -- & -- \\
\hline 31 & -- & -- & -- & -- & -- & -- & -- & -- & - & 36.81 & -- & -- \\
\hline Mean & -- & -- & -- & -- & -- & -- & -- & -- & -- & -- & -- & - \\
\hline Max. & -- & -- & -- & -- & -- & -- & -- & -- & -- & -- & - & - \\
\hline Min. & -- & -- & -- & -- & -- & -- & -- & -- & -- & -- & -- & -- \\
\hline
\end{tabular}


Table 3.--Daily mean water-level measurements in recorder wells in southwestern Oklahoma--Continued

HARMON COUNTY, OKLAHOMA--CONTINUEd

82N-24W-21 DODD 2--Continued

Water Year, October 1987 to September 1988

Day Oct. Nov. Dec. Jan. Feb. Mar. Apr. May June July Aug. Sept.

\begin{tabular}{|c|c|c|c|c|c|c|c|c|c|c|c|c|c|}
\hline 1 & & -- & -- & 40.40 & -- & 40.78 & -- & -- & -- & -- & -- & -- & -- \\
\hline 2 & & -- & -- & 40.41 & -- & 40.91 & -- & -- & -- & -- & -- & -- & -- \\
\hline 3 & & -- & -- & -- & -- & 40.92 & -- & -- & -- & -- & -- & -- & -- \\
\hline 4 & & -- & 37.94 & -- & -- & 40.97 & -- & -- & -- & -- & -- & -- & -- \\
\hline 5 & & -- & 37.92 & -- & -- & -- & -- & -- & -- & -- & -- & -- & -- \\
\hline 6 & & -- & 37.95 & -- & -- & -- & -- & -- & -- & - & -- & -- & -- \\
\hline 7 & & -- & 37.98 & -- & -- & -- & -- & -- & -- & -- & -- & -- & -- \\
\hline 8 & & -- & 38.19 & -- & -- & -- & 39.73 & -- & -- & -- & -- & -- & -- \\
\hline 9 & & -- & 38.46 & 40.63 & -- & -- & 39.67 & -- & -- & -- & -- & -- & -- \\
\hline 10 & & -- & 38.72 & 40.84 & -- & -- & 39.36 & -- & -- & -- & -- & -- & -- \\
\hline 11 & & -- & 38.85 & 40.82 & -- & -- & 39.38 & -- & -- & -- & -- & -- & -- \\
\hline 12 & & -- & 38.89 & 40.98 & -- & -- & 39.63 & -- & -- & -- & -- & -- & -- \\
\hline 13 & & -- & 38.96 & 41.01 & -- & 46.96 & 39.85 & -- & -- & -- & -- & -- & -- \\
\hline 14 & & -- & 38.92 & 41.01 & -- & 40.92 & 39.97 & -- & -- & -- & -- & -- & -- \\
\hline 16 & & -- & 39.00 & 41.14 & -- & 40.92 & 40.05 & -- & -- & -- & -- & -- & -- \\
\hline 16 & & -- & 39.22 & -- & -- & -- & 40.18 & - & -- & - & -- & -- & -- \\
\hline 17 & & -- & 39.39 & -- & -- & -- & 48.19 & -- & -- & -- & -- & -- & -- \\
\hline 18 & - & -- & 39.68 & -- & -- & -- & 48.19 & -- & -- & -- & -- & -- & -- \\
\hline 19 & & -- & 39.82 & -- & -- & -- & -- & -- & -- & -- & -- & -- & -- \\
\hline 20 & & -- & 39.88 & -- & -- & -- & -- & -- & -- & -- & -- & -- & -- \\
\hline 21 & & -- & 39.79 & -- & -- & -- & -- & -- & -- & -- & -- & -- & -- \\
\hline 22 & & -- & 39.76 & -- & -- & -- & -- & -- & -- & -- & -- & -- & -- \\
\hline 23 & & -- & 39.90 & -- & -- & -- & -- & -- & -- & -- & -- & -- & - \\
\hline 24 & & -- & 39.98 & -- & -- & -- & -- & -- & -- & -- & -- & -- & -- \\
\hline 26 & & -- & 46.14 & -- & -- & -- & -- & -- & -- & -- & -- & -- & -- \\
\hline 28 & & -- & 40.19 & -- & -- & -- & -- & -- & -- & -- & -- & -- & -- \\
\hline 27 & & -- & 40.24 & -- & -- & -- & -- & -- & -- & -- & -- & -- & -- \\
\hline 28 & & -- & 40.24 & -- & 40.67 & -- & -- & -- & -- & -- & -- & -- & -- \\
\hline 29 & & -- & 40.29 & -- & 46.69 & -- & -- & -- & -- & -- & -- & -- & - \\
\hline 30 & & -- & 40.33 & -- & 40.65 & -- & -- & -- & -- & -- & -- & -- & -- \\
\hline 31 & & -- & -- & -- & 40.63 & -- & -- & -- & -- & -- & -- & -- & -- \\
\hline Mean & & -- & -- & -- & -- & -- & -- & -- & -- & -- & -- & -- & -- \\
\hline Max. & & -- & -- & -- & -- & -- & -- & -- & -- & -- & -- & -- & -- \\
\hline Min. & & -- & -- & -- & -- & -- & -- & -- & -- & -- & -- & -- & -- \\
\hline
\end{tabular}




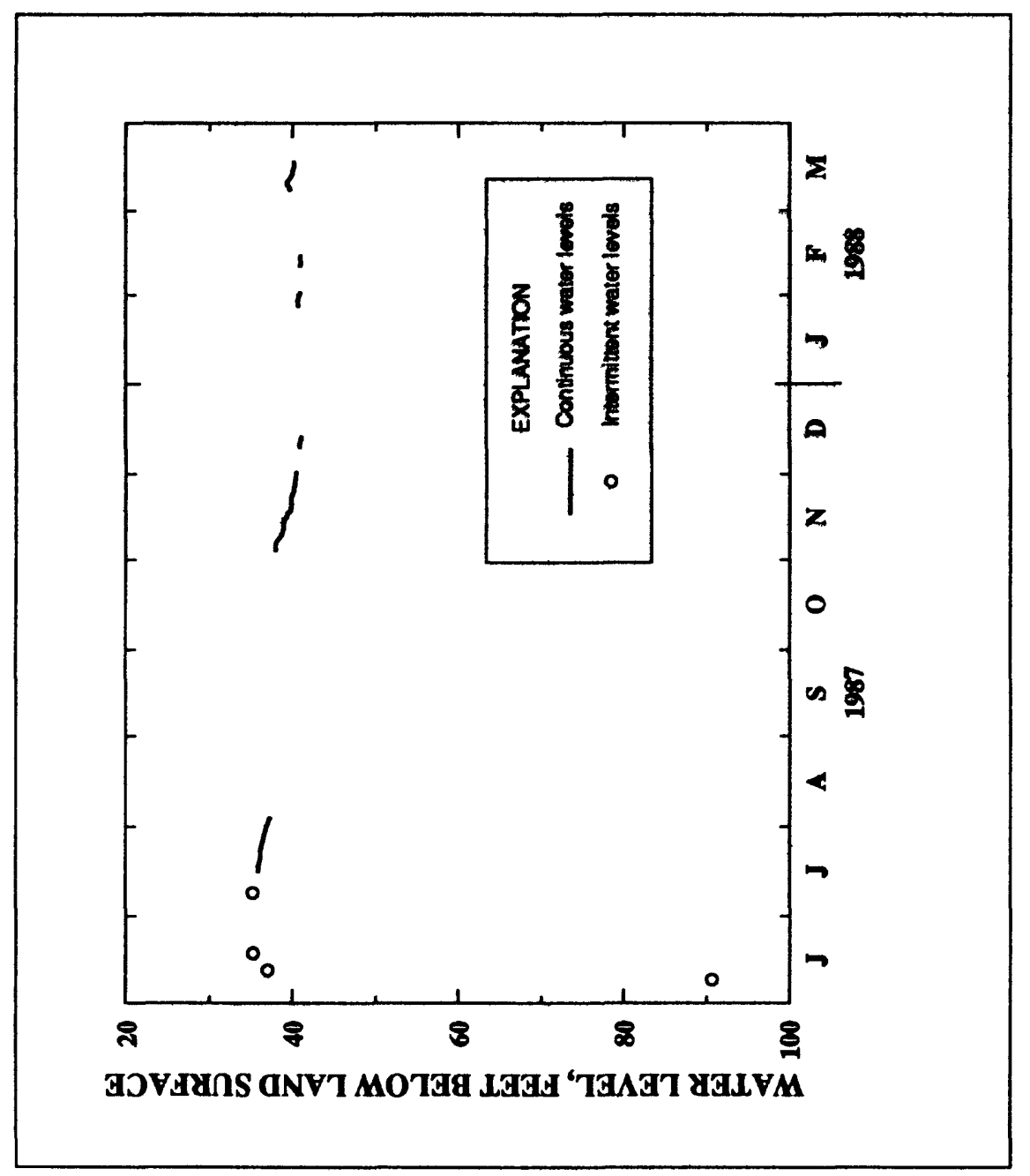

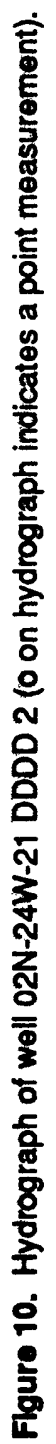


Table 3.--Daily mean water-level measurements in recorder wells in southwestern Oklahoma--Continued

HARMON COUNTY, OKLAHOMA--Continued

Index number on location map: 6

Station number: 343855099581801 Local number: 62N-27W-24 AAAD 1

Location: Lat $34^{\circ} 38^{\prime} 66^{\prime \prime}$, long $99^{\circ} 6^{\prime} 18^{\prime \prime}$, hydrologic unit 11130181

Aquifer: 3138 LIN

Altitude of land-surface datum: $1,715 \mathrm{ft}$

Well depth: $223.6 \mathrm{ft}$

Water Year, October 1985 to September 1986

Day Dct. Nov. Dec. Jan. Fob. Mar. Apr. May June July Aug. Sept.

\begin{tabular}{|c|c|c|c|c|c|c|c|c|c|c|c|c|}
\hline 1 & -- & -- & -- & -- & -- & -- & -- & -- & 98.49 & 86.54 & 90.87 & 97.43 \\
\hline 2 & -- & -- & -- & -- & -- & -- & -- & -- & 98.26 & 86.81 & 91.22 & 97.68 \\
\hline 3 & -- & -- & -- & -- & -- & -- & -- & -- & 98.86 & 86.66 & 91.67 & 97.70 \\
\hline 4 & -- & -- & -- & -- & -- & -- & -- & -- & 97.46 & 86.70 & 91.73 & 97.85 \\
\hline 6 & -- & -- & -- & -- & -- & -- & -- & -- & 94.72 & 86.83 & 91.91 & 97.80 \\
\hline 6 & -- & -- & -- & -- & -- & -- & -- & -- & 92.88 & 86.98 & 92.14 & 97.67 \\
\hline 7 & -- & -- & -- & -- & -- & -- & -- & -- & 91.77 & 87.68 & 92.46 & 97.68 \\
\hline 8 & -- & -- & -- & -- & -- & -- & -- & -- & 90.58 & 87.16 & 92.64 & 97.49 \\
\hline 9 & -- & -- & -- & -- & -- & -- & -- & -- & 89.58 & 87.21 & 92.85 & 97.25 \\
\hline 10 & -- & -- & -- & -- & -- & -- & -- & -- & 88.88 & 87.28 & 93.14 & 97.08 \\
\hline 11 & -- & -- & -- & -- & -- & $-\infty$ & -- & -- & 88.42 & 87.36 & 93.39 & 97.12 \\
\hline 12 & -- & -- & -- & -- & -- & -- & -- & -- & 87.94 & 87.55 & 93.56 & 97.26 \\
\hline 13 & -- & -- & -- & -- & -- & $-\infty$ & -- & -- & 87.72 & 87.81 & 93.77 & 97.21 \\
\hline 14 & -- & -- & -- & -- & -- & -- & -- & -- & 87.54 & 87.95 & 93.94 & 96.95 \\
\hline 16 & -- & -- & -- & -- & -- & -- & -- & -- & 87.38 & 87.97 & 94.12 & 96.11 \\
\hline 16 & -- & -- & -- & -- & -- & -- & -- & -- & 87.32 & 88.10 & 94.30 & 96.48 \\
\hline 17 & -- & -- & -- & -- & -- & -- & -- & -- & 87.34 & 88.26 & 94.68 & 94.95 \\
\hline 18 & -- & -- & -- & -- & -- & -- & -- & -- & 87.20 & 88.41 & 94.86 & 94.66 \\
\hline 19 & -- & -- & -- & -- & -- & -- & -- & -- & 87.10 & 88.49 & 95.00 & 94.24 \\
\hline 20 & -- & -- & -- & -- & -- & -- & -- & -- & 87.09 & 88.70 & 95.23 & 94.02 \\
\hline 21 & -- & -- & -- & -- & -- & -- & -- & -- & 86.93 & 88.86 & 95.62 & 93.81 \\
\hline 22 & -- & -- & -- & -- & -- & -- & -- & -- & 86.85 & 89.04 & 95.67 & 93.62 \\
\hline 23 & -- & -- & -- & -- & -- & -- & -- & -- & 86.77 & 89.23 & 95.87 & 93.43 \\
\hline 24 & -- & -- & -- & -- & -- & -- & -- & -- & 86.63 & 89.42 & 96.04 & 93.27 \\
\hline 26 & -- & -- & -- & -- & -- & -- & -- & -- & 86.62 & 89.49 & 96.26 & 93.35 \\
\hline 26 & -- & -- & -- & -- & -- & -- & -- & -- & 86.45 & 89.68 & 96.46 & 93.39 \\
\hline 27 & -- & -- & -- & -- & -- & $-\infty$ & -- & -- & 88.44 & 89.86 & 96.64 & 93.47 \\
\hline 28 & -- & -- & -- & -- & - & $-\infty$ & -- & -- & 86.34 & 96.67 & 96.88 & 93.49 \\
\hline 29 & -- & -- & -- & -- & -- & -- & -- & 99.78 & 86.38 & 90.27 & 97.01 & 83.48 \\
\hline 30 & -- & -- & -- & -- & -- & -- & -- & 99.33 & 86.40 & 90.48 & 97.12 & 93.63 \\
\hline 31 & -- & -- & -- & -- & -- & -- & -- & 98.86 & -- & 90.68 & 97.27 & -- \\
\hline Mean & -- & -- & -- & -- & - & -- & - & -- & 89.38 & 88.28 & 94.32 & 95.61 \\
\hline Max. & -- & -- & -- & -- & -- & -- & -- & -- & 98.49 & 90.68 & 97.27 & 97.86 \\
\hline Min. & -- & -- & -- & -- & -- & -- & -- & -- & 86.34 & 88.54 & 90.87 & 93.27 \\
\hline
\end{tabular}


Table 3.--Daily mean water-level measurements in recorder wells in southwestern Oklahoma--Continued

HARMON COUNTY, OKLAHOMA--CONTInUEd

$62 N-27 W-24$ AAAD 1--Continued

Water Year, October 1986 to September 1987

Day Oct. Nov. Dec. Jan. Feb. Mar. Apr. May June July Aug. Sept.

\begin{tabular}{|c|c|c|c|c|c|c|c|c|c|c|c|c|}
\hline 1 & 93.68 & 82.16 & 83.00 & 86.80 & 90.64 & 88.38 & 84.13 & 86.70 & 77.90 & 76.98 & 80.64 & 84.61 \\
\hline 2 & 93.55 & 82.18 & 83.03 & 86.80 & 90.65 & 87.81 & 84.50 & 86.73 & 76.68 & 76.07 & 80.66 & 84.68 \\
\hline 3 & 92.39 & 81.97 & 83.26 & 87.25 & 90.85 & 87.35 & 84.36 & 86.93 & 76.14 & 76.22 & 80.79 & 84.67 \\
\hline 4 & 91.38 & 81.77 & 83.36 & 87.25 & 91.01 & 86.87 & 84.55 & 87.12 & 75.78 & 76.28 & 80.98 & 84.77 \\
\hline 5 & 90.49 & 81.70 & 83.47 & 87.28 & 91.62 & 88.24 & 84.60 & 87.32 & 76.62 & 76.61 & 81.11 & 84.87 \\
\hline 6 & 89.66 & 81.47 & 83.63 & 87.45 & 91.68 & 86.78 & 84.56 & 87.49 & 75.37 & 76.78 & 81.18 & 85.00 \\
\hline 7 & 88.87 & 81.35 & 83.60 & 87.66 & 91.64 & 85.45 & 84.82 & 87.67 & 75.32 & 76.84 & 81.33 & 85.13 \\
\hline 8 & 88.22 & 81.41 & 83.64 & 87.68 & 91.85 & 85.04 & 84.64 & 87.69 & 76.34 & 77.12 & 81.46 & 85.30 \\
\hline 9 & 87.82 & 81.63 & 83.92 & 87.83 & 91.95 & 85.84 & 84.67 & 87.63 & 75.35 & 77.27 & 81.63 & 85.37 \\
\hline 10 & 87.33 & 81.42 & 84.06 & 88.12 & 91.89 & 84.86 & 84.84 & 87.71 & 75.28 & 77.43 & 81.74 & 85.49 \\
\hline 11 & 86.95 & 81.46 & 84.04 & 88.16 & 92.01 & 84.60 & 84.71 & 87.82 & 75.14 & 77.64 & 81.81 & 85.63 \\
\hline 12 & 86.87 & 81.51 & 84.26 & 88.18 & 92.18 & 84.51 & 84.80 & 87.86 & 76.01 & 77.71 & 81.92 & 85.74 \\
\hline 13 & 86.53 & 81.67 & 84.43 & 88.20 & 92.13 & 84.31 & 85.16 & 88.07 & 74.85 & 78.11 & 82.06 & 85.83 \\
\hline 14 & 86.34 & 81.33 & 84.47 & 88.32 & 92.01 & 84.05 & 85.33 & 88.24 & 74.69 & 78.24 & 82.18 & 85.98 \\
\hline 15 & 86.29 & 81.25 & 84.57 & 88.73 & 92.06 & 84.64 & 86.22 & 88.38 & 74.58 & 78.31 & 82.30 & 86.01 \\
\hline 16 & 86.12 & 81.25 & 84.69 & 88.95 & 91.64 & 83.89 & 85.23 & 88.40 & 74.50 & 78.43 & 82.44 & 86.13 \\
\hline 17 & 85.97 & 81.27 & 84.87 & 88.98 & 91.38 & 83.72 & 85.28 & 88.47 & 74.50 & 78.53 & 82.69 & 86.30 \\
\hline 18 & 85.84 & 81.63 & 86.01 & 89.07 & 91.07 & 83.87 & 85.36 & 88.61 & 74.58 & 78.72 & 82.72 & 86.48 \\
\hline 19 & 85.98 & 81.44 & 85.02 & 89.15 & 90.90 & 83.82 & 85.65 & 88.79 & 74.53 & 78.92 & 82.86 & 86.57 \\
\hline $2 \emptyset$ & 85.91 & 81.76 & 85.19 & 89.42 & 90.80 & 83.79 & 85.83 & 88.92 & 74.66 & 79.10 & 82.98 & 86.88 \\
\hline 21 & 85.70 & 81.75 & 85.45 & 89.42 & 90.47 & 83.81 & 86.08 & 89.18 & 74.62 & 79.24 & 83.11 & 86.86 \\
\hline 22 & 85.12 & 81.71 & 86.63 & 89.71 & 96.31 & 83.68 & 85.93 & 89.48 & 74.70 & 79.33 & 83.26 & 87.61 \\
\hline 23 & 84.81 & 82.01 & 86.48 & 89.62 & 90.21 & 83.73 & 86.61 & 89.16 & 74.84 & 79.41 & 83.43 & 87.04 \\
\hline 24 & 84.40 & 82.12 & 85.58 & 89.89 & 90.16 & 83.92 & 86.16 & 88.61 & 75.64 & 79.56 & 83.49 & 87.07 \\
\hline 25 & 83.91 & 82.04 & 85.80 & 96.15 & 90.12 & 83.96 & 86.26 & 87.83 & 76.23 & 79.72 & 83.56 & 87.19 \\
\hline 26 & 83.51 & 82.44 & 85.98 & 90.36 & 89.86 & 83.90 & 86.37 & 87.26 & 75.37 & 79.83 & 83.70 & 87.30 \\
\hline 27 & 83.10 & 82.48 & 86.10 & 90.41 & 89.34 & 83.81 & 86.46 & 86.76 & 75.48 & 79.94 & 83.90 & 87.41 \\
\hline 28 & 82.72 & 82.65 & 86.23 & 90.44 & 88.85 & 84.16 & 86.55 & 86.01 & 75.66 & 80.83 & 84.84 & 87.62 \\
\hline 29 & 82.56 & 82.59 & 86.41 & 90.61 & -- & 84.33 & 86.57 & 83.75 & 75.70 & 80.15 & 84.11 & 87.79 \\
\hline $3 \varnothing$ & 82.26 & 82.65 & 86.47 & 90.48 & -- & 84.25 & 86.64 & 82.00 & 75.88 & 80.29 & 84.22 & 87.89 \\
\hline 31 & 82.09 & -- & 86.62 & 90.44 & -- & 84.17 & -- & 80.02 & -- & 80.42 & 84.38 & - \\
\hline Mean & 86.68 & 81.79 & 84.74 & 88.80 & 91.04 & 84.75 & 85.36 & 87.39 & 75.27 & 78.33 & 82.47 & 86.14 \\
\hline Max. & 93.88 & 82.65 & 86.62 & 90.61 & 92.18 & 88.38 & 86.64 & 89.48 & 77.90 & 80.42 & 84.38 & 87.89 \\
\hline & 82.09 & 81.25 & 83.00 & 86.80 & 88.85 & 83.68 & 84.13 & 80.02 & 74.50 & 75.98 & 80.54 & 84.51 \\
\hline
\end{tabular}


Table 3.--Daily mean water-level measurements in recorder wells in southwestern Oklahoma--Continued

HARMON COUNTY, OKLAHOMA--CONtinued

62N-27W-24 AAAD 1--Continued

Water Year, October 1987 to September 1988

Day Dct. Nov. Dec. Jan. Fob. Mar. Apr. May June July Aug. Sept.

\begin{tabular}{|c|c|c|c|c|c|c|c|c|c|c|c|c|}
\hline 1 & 87.84 & 91.84 & 94.66 & 97.09 & 98.66 & 99.71 & 100.84 & 99.75 & 101.05 & 102.49 & 104.24 & 107.06 \\
\hline 2 & 88.19 & 91.90 & 94.67 & 97.07 & 98.75 & 99.79 & 100.94 & 99.81 & 101.04 & 182.54 & 104.34 & 107.13 \\
\hline 3 & 88.46 & 91.98 & 94.86 & 97.01 & 98.72 & 99.97 & 100.86 & 99.95 & 101.12 & 102.56 & 104.42 & 107.20 \\
\hline 4 & 88.43 & 92.12 & 94.96 & 97.25 & 98.87 & 99.92 & 100.88 & 99.92 & 101.17 & 102.81 & 104.50 & 107.29 \\
\hline 6 & 88.63 & 92.37 & 94.87 & 97.32 & 99.01 & 186.03 & 101.03 & 99.92 & 181.23 & 102.67 & 104.59 & 107.33 \\
\hline 6 & 88.78 & 92.34 & 95.00 & 97.32 & 99.04 & 99.96 & 101.06 & 99.89 & 101.26 & 102.71 & 104.67 & 107.38 \\
\hline 7 & 88.96 & 92.30 & 95.10 & 97.39 & 98.94 & 99.98 & 101.00 & 99.82 & 101.24 & 102.75 & 104.75 & 107.41 \\
\hline 8 & 88.87 & 92.66 & 95.19 & 97.35 & 98.96 & 100.17 & 101.03 & 99.83 & 161.23 & 102.81 & 104.84 & 107.46 \\
\hline 9 & 89.12 & 92.80 & 96.35 & 97.44 & 99.06 & 100.01 & 101.25 & 99.93 & 101.38 & 102.82 & 104.93 & 107.67 \\
\hline 10 & 89.44 & 92.99 & 95.34 & 97.61 & 99.09 & 99.90 & 101.29 & 100.00 & 101.54 & 162.85 & 105.03 & 107.62 \\
\hline 11 & 89.68 & 92.98 & 95.34 & 97.41 & 99.22 & 99.98 & 161.20 & 100.08 & 101.56 & 102.88 & 105.11 & 107.68 \\
\hline 12 & 89.54 & 92.96 & 95.51 & 97.69 & 99.06 & 100.17 & 101.14 & 100.06 & 101.56 & 102.92 & 105.20 & 107.77 \\
\hline 13 & 89.56 & 92.91 & 95.58 & 97.80 & 98.97 & 160.28 & 101.13 & 100.06 & 101.65 & 102.96 & 105.31 & 197.86 \\
\hline 14 & 89.69 & 92.92 & 95.65 & 97.71 & 99.12 & 106.24 & 101.16 & 100.10 & 101.74 & 103.03 & 165.42 & 107.87 \\
\hline 16 & 89.82 & 93.01 & 95.86 & 97.64 & 99.19 & 100.25 & 101.32 & 100.10 & 101.80 & 103.08 & 105.50 & 107.90 \\
\hline 16 & 90.02 & 93.27 & 95.99 & 97.65 & 99.13 & 100.36 & 101.33 & 100.16 & 101.80 & 103.17 & 105.69 & 107.95 \\
\hline 17 & 90.19 & 93.41 & 95.99 & 97.71 & 99.35 & 100.60 & 101.01 & 100.19 & 101.80 & 103.23 & 105.68 & 108.02 \\
\hline 18 & 90.14 & 93.72 & 95.92 & 97.66 & 99.32 & 100.65 & 100.58 & 100.28 & 101.85 & 103.25 & 165.75 & 107.74 \\
\hline 19 & 90.33 & 93.83 & 95.83 & 97.87 & 99.32 & 100.46 & 100.40 & 100.32 & 101.93 & 163.34 & 105.83 & 106.33 \\
\hline 20 & 90.65 & 93.86 & 96.15 & 98.19 & 99.42 & 160.39 & 100.21 & 160.32 & 101.99 & 103.48 & 105.92 & 106.13 \\
\hline 21 & 90.68 & 93.72 & 96.16 & 98.09 & 99.44 & 100.38 & 99.98 & 100.46 & 102.04 & 103.63 & 106.05 & 105.98 \\
\hline 22 & 90.63 & 93.74 & 96.21 & 98.19 & 99.43 & 100.46 & 99.97 & 100.64 & 102.08 & 103.53 & 106.18 & 105.78 \\
\hline 23 & 90.78 & 93.94 & 96.08 & 98.10 & 99.63 & 100.50 & 100.000 & 100.66 & 102.13 & 103.67 & 106.31 & 165.63 \\
\hline 24 & 91.00 & 94.04 & 98.32 & 98.39 & 99.86 & 100.50 & 99.90 & 109.67 & 102.22 & 103.66 & 106.42 & 165.65 \\
\hline 26 & 91.03 & 94.22 & 96.46 & 98.41 & 99.63 & 100.69 & 99.90 & 100.69 & 102.30 & 103.76 & 106.46 & 106.46 \\
\hline 28 & 91.13 & 94.30 & 96.66 & 98.47 & 99.62 & 100.72 & 99.91 & 100.73 & 102.32 & 103.82 & 106.51 & 105.36 \\
\hline 27 & 91.40 & 94.38 & 96.57 & 98.63 & 99.69 & 100.68 & 99.88 & 108.79 & 102.35 & 103.88 & 106.60 & 105.27 \\
\hline 28 & 91.38 & 94.42 & 96.79 & 98.51 & 99.68 & 100.69 & 99.82 & 100.85 & 102.37 & 103.94 & 106.78 & 105.18 \\
\hline 29 & 91.38 & 94.47 & 96.90 & 98.61 & 99.84 & 160.92 & 99.74 & 100.89 & 102.36 & 104.03 & 106.87 & 105.26 \\
\hline 30 & 91.51 & 94.57 & 96.70 & 98.49 & -- & 100.84 & 99.75 & 101.07 & 102.40 & 104.12 & 106.90 & 105.20 \\
\hline 31 & 91.69 & -- & 96.90 & 98.49 & -- & 100.79 & - & 101.13 & -- & 104.17 & 166.97 & - \\
\hline $\begin{array}{l}\text { Mean } \\
\text { Max. } \\
\text { Min. }\end{array}$ & $\begin{array}{l}89.96 \\
91.89 \\
87.84\end{array}$ & $\begin{array}{l}93.26 \\
94.67 \\
91.84\end{array}$ & $\begin{array}{l}96.79 \\
96.90 \\
94.66\end{array}$ & $\begin{array}{l}97.81 \\
98.63 \\
97.01\end{array}$ & $\begin{array}{l}99.23 \\
99.84 \\
98.66\end{array}$ & $\begin{array}{r}100.31 \\
100.92 \\
99.71\end{array}$ & $\begin{array}{r}100.62 \\
101.33 \\
99.74\end{array}$ & $\begin{array}{r}100.29 \\
101.13 \\
99.75\end{array}$ & $\begin{array}{l}101.75 \\
102.40 \\
101.04\end{array}$ & $\begin{array}{l}103.23 \\
104.17 \\
102.49\end{array}$ & $\begin{array}{l}105.60 \\
106.97 \\
104.24\end{array}$ & $\begin{array}{l}106.78 \\
108.02 \\
105.18\end{array}$ \\
\hline
\end{tabular}


Table 3.--Daily mean water-level measurements in recorder wells in southwestern Oklahoma--Continued

HARMON COUNTY, OKLAHOMA--CONTInUEd

D2N-27W-24 AAAD 1--Continued

Water Year, October 1988 to September 1989

Day Dct. Nov. Dec. Jan. Fob. Mar. Apr. May June July Aug. Sept.

\begin{tabular}{|c|c|c|c|c|c|c|c|c|c|c|c|c|}
\hline 1 & 105.14 & 106.27 & 107.64 & 108.10 & -- & -- & -- & -- & -- & -- & -- & -- \\
\hline 2 & 105.23 & 106.17 & 107.66 & 108.15 & -- & -- & -- & -- & -- & -- & -- & -- \\
\hline 3 & 105.24 & 166.16 & 107.68 & 168.29 & -- & -- & -- & -- & -- & -- & -- & -- \\
\hline 4 & 105.29 & 168.29 & 107.76 & 108.30 & -- & -- & -- & -- & -- & -- & -- & -- \\
\hline 5 & 105.31 & 166.48 & 107.89 & 108.21 & -- & -- & -- & -- & -- & -- & -- & -- \\
\hline 6 & 105.31 & 166.45 & 107.60 & 108.13 & -- & -- & -- & -- & -- & -- & -- & -- \\
\hline 7 & 165.38 & 166.46 & 107.62 & 108.21 & -- & -- & -- & - & -- & $-\therefore$ & -- & -- \\
\hline 8 & 165.32 & 106.56 & 107.80 & 108.36 & -- & -- & -- & - & - & -- & -- & -- \\
\hline 9 & 105.32 & 106.56 & 107.78 & 168.33 & -- & -- & -- & -- & -- & -- & -- & -- \\
\hline 10 & 105.36 & 106.72 & 107.72 & 108.26 & -- & -- & -- & -- & -- & -- & -- & -- \\
\hline 11 & 105.40 & 106.65 & 107.77 & 108.26 & $-\infty$ & -- & -- & -- & -- & -- & -- & - \\
\hline 12 & 105.47 & 106.72 & 107.81 & 108.49 & -- & -- & - & -- & -- & -- & -- & -- \\
\hline 13 & 105.48 & 108.79 & 167.78 & 100.60 & -- & -- & -- & -- & -- & -- & -- & -- \\
\hline 14 & 165.45 & 106.78 & 107.73 & 198.34 & -- & -- & -- & -- & -- & -- & -- & -- \\
\hline 15 & 165.46 & 106.80 & 107.98 & 108.43 & -- & -- & -- & -- & -- & -- & -- & -- \\
\hline 16 & 105.53 & 107.02 & 167.99 & 168.48 & -- & -- & -- & -- & -- & -- & -- & -- \\
\hline 17 & 105.57 & 107.010 & 107.99 & 108.39 & -- & -- & -- & -- & -- & -- & -- & -- \\
\hline 18 & .106 .73 & 166.95 & 107.91 & 108.60 & -- & -- & -- & -- & -- & -- & -- & -- \\
\hline 19 & 105.72 & 167.08 & 107.79 & -- & -- & -- & -- & -- & -- & -- & -- & -- \\
\hline 20 & 165.71 & 167.23 & 107.91 & -- & -- & -- & -- & -- & -- & -- & -- & -- \\
\hline 21 & 165.83 & 107.25 & 107.95 & -- & -- & -- & -- & -- & -- & -- & -- & -- \\
\hline 22 & 105.75 & 107.22 & 107.96 & -- & -- & -- & -- & -- & -- & -- & -- & -- \\
\hline 23 & 105.86 & 107.18 & 167.93 & -- & -- & -- & -- & -- & -- & -- & -- & -- \\
\hline 24 & 165.91 & 107.14 & 168.04 & -- & -- & -- & -- & -- & -- & -- & -- & -- \\
\hline 25 & 105.96 & 107.16 & 108.01 & -- & -- & -- & -- & -- & -- & -- & -- & -- \\
\hline 28 & 108.04 & 167.18 & 107.95 & -- & -- & -- & -- & -- & -- & -- & -- & -- \\
\hline 27 & 108.01 & 167.43 & 108.08 & -- & -- & -- & -- & -- & -- & -- & -- & -- \\
\hline 28 & 108.23 & 167.44 & 108.17 & -- & -- & -- & -- & -- & -- & -- & -- & -- \\
\hline 29 & 106.23 & 167.48 & 108.11 & -- & -- & -- & -- & -- & -- & -- & -- & - \\
\hline 30 & 108.28 & 107.61 & 108.07 & -- & -- & - & -- & -- & -- & -- & -- & -- \\
\hline 31 & 108.30 & -- & 108.08 & -- & -- & -- & -- & -- & -- & -- & -- & -- \\
\hline Mean & 165.64 & 108.87 & 107.87 & -- & -- & -- & -- & -- & -- & -- & -- & - \\
\hline Max. & 108.30 & 107.61 & 108.17 & -- & -- & - & -- & -- & -- & -- & -- & -- \\
\hline Min. & 105.14 & 106.16 & 107.60 & -- & -- & -- & -- & -- & -- & -- & -- & -- \\
\hline
\end{tabular}




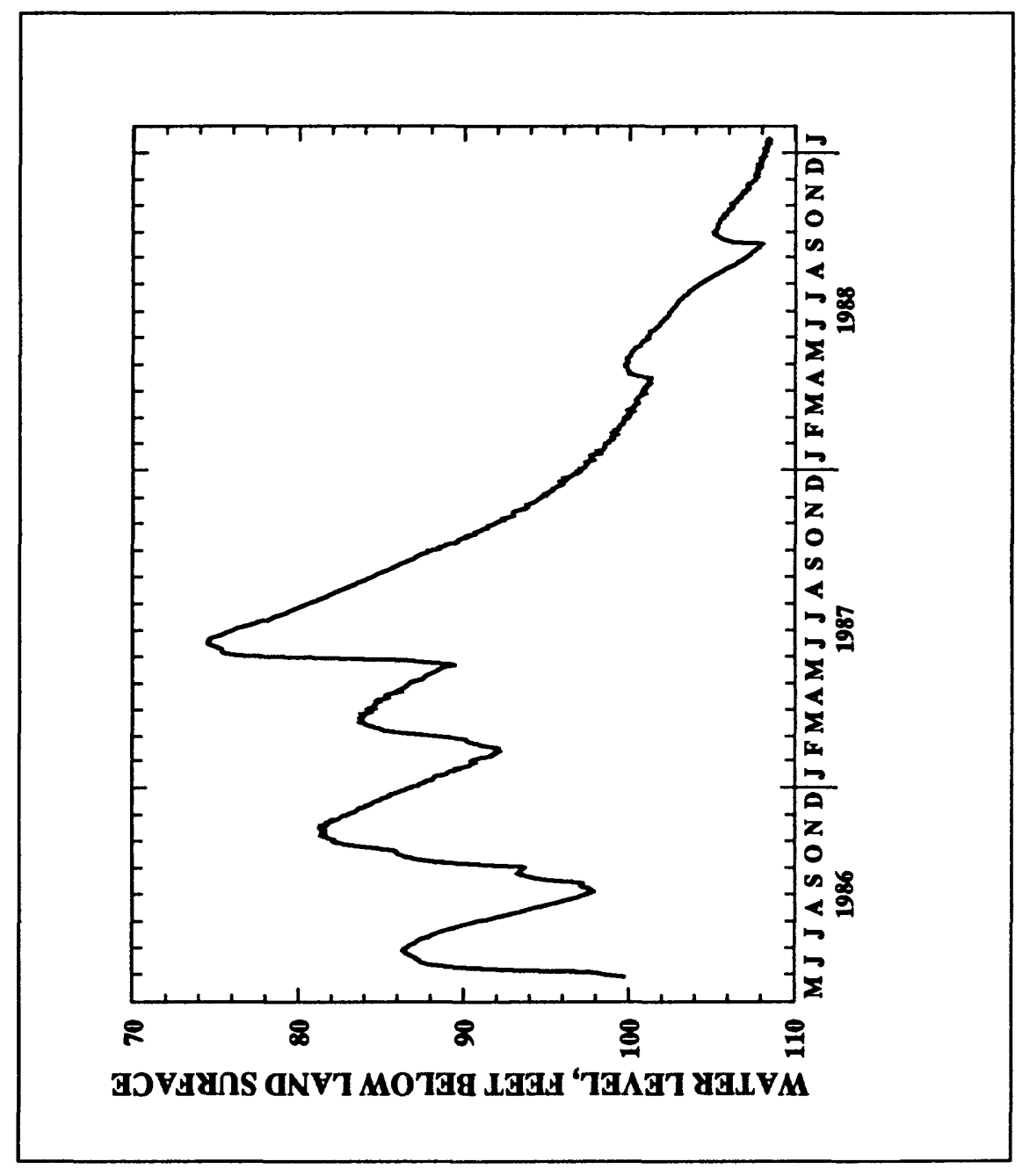

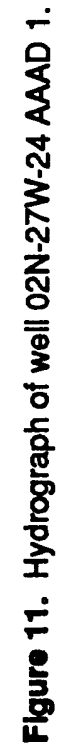


Index number on location map: 6

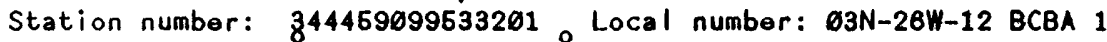

Location: Lat $34^{\circ} 44^{\prime} 59^{\prime \prime}$, long $99^{\circ} 53^{\prime} 32^{\prime \prime}$, hydrologic unit 11130101

Aquifer: 313 BLIN

Altitude of land-surface datum: $1,673 \mathrm{ft}$

Well depth: $275 \mathrm{ft}$

Water Year, October 1985 to September 1986

Day Oct. Nov. Dec. Jan. Feb. Mar. Apr. May June July Aug. Sept.

\begin{tabular}{|c|c|c|c|c|c|c|c|c|c|c|c|c|c|}
\hline 1 & & -- & -- & -- & -- & -- & -- & -- & -- & 61.63 & 56.26 & 75.69 & 77.88 \\
\hline 2 & & -- & -- & -- & -- & -- & -- & -- & -- & 61.70 & 56.29 & 76.42 & 77.29 \\
\hline 3 & & -- & -- & -- & -- & -- & -- & -- & -- & 61.65 & 56.21 & 76.93 & 78.72 \\
\hline 4 & & -- & -- & -- & -- & -- & -- & -- & -- & 60.69 & 56.11 & 77.38 & 76.22 \\
\hline 5 & & -- & -- & -- & -- & -- & -- & -- & -- & 56.26 & 68.07 & 77.90 & 76.67 \\
\hline 6 & & -- & -- & -- & -- & -- & -- & -- & -- & 57.06 & 58.14 & 79.98 & 75.05 \\
\hline 7 & & -- & -- & -- & -- & -- & -- & -- & -- & 57.62 & 56.18 & 80.12 & 74.53 \\
\hline 8 & & -- & -- & -- & -- & -- & -- & -- & -- & 67.96 & 56.20 & 79.66 & 73.91 \\
\hline 9 & & -- & -- & -- & -- & -- & -- & -- & -- & 58.08 & 56.72 & 79.86 & 73.26 \\
\hline 10 & & -- & -- & -- & -- & -- & -- & -- & -- & 65.84 & 67.99 & 79.63 & 72.65 \\
\hline 11 & & -- & -- & -- & -- & -- & -- & -- & -- & 65.53 & 58.45 & 79.36 & 72.25 \\
\hline 12 & & -- & -- & -- & -- & -- & -- & -- & -- & 56.18 & 59.02 & 79.63 & 71.86 \\
\hline 13 & - & -- & -- & -- & -- & -- & - & -- & -- & 56.57 & 59.69 & 80.08 & 71.43 \\
\hline 14 & & -- & -- & -- & -- & -- & -- & -- & -- & 66.75 & 61.08 & 81.14 & 70.72 \\
\hline 15 & & -- & -- & -- & -- & -- & -- & -- & -- & 56.78 & 66.64 & 80.32 & 69.66 \\
\hline 16 & & -- & -- & -- & -- & -- & -- & -- & -- & 56.83 & 68.11 & 79.48 & 69.09 \\
\hline 17 & & -- & -- & -- & -- & - & -- & -- & - & 56.95 & 69.20 & 78.98 & 68.53 \\
\hline 18 & & -- & -- & -- & -- & -- & -- & -- & -- & 56.94 & 70.65 & 78.68 & 68.08 \\
\hline 19 & & -- & -- & -- & -- & -- & -- & -- & -- & 66.74 & 71.96 & 78.22 & 67.64 \\
\hline 20 & & -- & -- & -- & -- & -- & -- & -- & -- & 56.75 & 68.78 & 77.97 & 87.28 \\
\hline 21 & & -- & -- & -- & -- & $-\infty$ & -- & -- & - & 56.79 & 67.47 & 77.93 & 66.88 \\
\hline 22 & & -- & -- & -- & -- & - & -- & -- & -- & 58.83 & 67.60 & 78.14 & 68.60 \\
\hline 23 & & -- & -- & -- & -- & -- & -- & -- & -- & 56.64 & 72.85 & 78.87 & 66.09 \\
\hline 24 & & -- & -- & -- & -- & -- & -- & -- & -- & 58.52 & 75.63 & 79.36 & 65.89 \\
\hline 25 & & -- & -- & -- & -- & -- & -- & -- & -- & 58.45 & 77.46 & 79.70 & 65.42 \\
\hline 26 & & -- & -- & -- & -- & -- & -- & -- & -- & 56.34 & 79.16 & 80.09 & 65.13 \\
\hline 27 & & -- & -- & -- & -- & -- & -- & -- & -- & 56.34 & 75.65 & 80.19 & 64.83 \\
\hline 28 & & -- & -- & -- & -- & -- & -- & -- & -- & 56.32 & 74.57 & 79.81 & 64.46 \\
\hline 29 & & -- & -- & -- & -- & -- & -- & -- & 61.67 & 56.29 & 74.44 & 79.27 & $64.1 \sigma$ \\
\hline 30 & & -- & -- & -- & -- & -- & -- & -- & 61.61 & 56.26 & 74.33 & 78.80 & 63.87 \\
\hline 31 & & -- & -- & -- & -- & -- & -- & -- & 61.62 & -- & 75.20 & 78.37 & -- \\
\hline Mean & & -- & -- & -- & -- & -- & - & - & -- & 57.31 & 65.55 & 78.96 & 70.09 \\
\hline Max. & & -- & -- & -- & -- & -- & -- & -- & -- & 61.70 & 79.16 & 81.14 & 77.88 \\
\hline Min. & & -- & -- & -- & -- & -- & -- & -- & -- & 55.63 & 56.07 & 75.69 & 63.87 \\
\hline
\end{tabular}


Table 3.--Daily mean water-level measurements in recorder wells in southwestern Oklahoma--Continued

HARMON COUNTY, OKLAHOMA--Continued

03N-28W-12 BCBA 1--Continued

\begin{tabular}{|c|c|c|c|c|c|c|c|c|c|c|c|c|}
\hline \multirow[b]{2}{*}{ Day } & \multirow[b]{2}{*}{ oct. } & \multirow[b]{2}{*}{ Nov. } & \multirow[b]{2}{*}{ Dec. } & \multirow{2}{*}{$\begin{array}{l}\text { Water } \\
\text { Jan. }\end{array}$} & \multicolumn{2}{|c|}{ ear, October 1986} & \multicolumn{2}{|c|}{ o September 1987} & \multirow[b]{2}{*}{ June } & \multirow[b]{2}{*}{ July } & \multirow[b]{2}{*}{ Aug. } & \multirow[b]{2}{*}{ Sept. } \\
\hline & & & & & Feb. & Mar. & Apr. & May & & & & \\
\hline 1 & 63.61 & 48.76 & 46.42 & 44.94 & 44.23 & 39.94 & 40.38 & 44.63 & 48.34 & 37.18 & 53.11 & 50.65 \\
\hline 2 & 62.97 & 48.71 & 46.48 & 44.78 & 44.12 & 40.60 & 40.69 & 44.65 & 40.49 & 37.12 & 53.96 & 49.97 \\
\hline 3 & 67.64 & 48.45 & 45.66 & 44.89 & 44.29 & 40.99 & 40.60 & 44.97 & 40.65 & 37.12 & 64.71 & 49.64 \\
\hline 4 & 66.81 & 46.70 & 46.61 & 45.05 & 44.41 & 41.24 & 40.63 & 46.23 & 40.67 & 37.06 & 65.33 & 49.34 \\
\hline 5 & 67.19 & 44.41 & 45.55 & 44.99 & 44.40 & 41.26 & 40.68 & 45.67 & 48.56 & 37.06 & 55.22 & 49.11 \\
\hline 6 & 67.32 & 46.20 & 45.39 & 44.99 & 44.16 & 41.23 & 40.66 & 46.52 & 40.45 & 37.11 & 63.13 & 48.94 \\
\hline 7 & 67.29 & 46.86 & 46.25 & 46.13 & 44.24 & 41.24 & 40.50 & 49.85 & 40.37 & 37.15 & 53.03 & 48.73 \\
\hline 8 & 57.11 & 46.07 & 45.10 & 45.08 & 44.36 & 41.20 & 40.49 & 50.37 & 40.32 & 37.24 & 53.40 & 48.69 \\
\hline 9 & 56.98 & 46.42 & 44.66 & 44.86 & 44.49 & 41.30 & 40.43 & 61.26 & 40.23 & 37.28 & 62.97 & 48.25 \\
\hline 10 & 66.70 & 46.50 & 44.79 & 44.90 & 44.42 & 41.26 & 40.48 & 49.63 & 37.32 & 37.32 & 63.82 & 47.91 \\
\hline 11 & 56.16 & 46.57 & 44.80 & 45.00 & 44.45 & 41.16 & 40.42 & 49.26 & 35.15 & 37.33 & 54.34 & 47.66 \\
\hline 12 & 55.07 & 46.58 & 44.92 & 44.97 & 44.66 & 41.18 & 40.40 & 49.50 & 36.84 & 37.35 & 56.74 & 47.41 \\
\hline 13 & 54.81 & 48.71 & 44.96 & 44.89 & 44.49 & 41.14 & 40.65 & 50.28 & 37.45 & 37.63 & 56.27 & 47.20 \\
\hline 14 & 54.74 & 46.40 & 44.92 & 44.80 & 44.35 & 40.95 & 40.64 & 50.24 & 37.68 & 38.73 & 64.00 & 47.04 \\
\hline 16 & 54.74 & 46.13 & 44.91 & 44.92 & 42.36 & 41.81 & 40.67 & 49.16 & 37.76 & 41.81 & 63.90 & 46.81 \\
\hline 18 & 54.60 & 46.98 & 44.95 & 45.01 & 41.18 & 40.80 & 40.65 & 51.69 & 37.74 & 43.19 & 53.42 & 46.68 \\
\hline 17 & 54.37 & 46.86 & 44.94 & 46.00 & 42.26 & 38.22 & 40.61 & 76.22 & 37.74 & 43.56 & 52.82 & 46.58 \\
\hline 18 & -54.17 & 46.95 & 44.94 & 44.98 & 42.80 & 38.98 & 40.69 & 82.85 & 37.77 & 42.64 & 51.07 & 46.60 \\
\hline 19 & 54.02 & 46.83 & 44.74 & 46.05 & 43.02 & 39.66 & 40.63 & 71.43 & 37.70 & 43.36 & 50.91 & 46.37 \\
\hline 20 & 63.82 & 46.93 & 44.75 & 45.20 & 42.77 & 39.96 & 40.78 & 54.01 & 37.64 & 43.64 & 62.89 & 46.27 \\
\hline 21 & 62.38 & 46.88 & 44.87 & 45.09 & 42.67 & 40.17 & 40.96 & 60.78 & 37.67 & 43.89 & 54.80 & 46.21 \\
\hline 22 & 46.68 & 46.71 & 44.88 & 46.07 & 42.65 & 40.20 & 40.89 & 49.72 & 36.98 & 44.46 & 56.16 & 46.18 \\
\hline 23 & 47.14 & 46.83 & 44.76 & 44.89 & 42.77 & 39.40 & 40.86 & 46.89 & 36.87 & 47.93 & 56.83 & 46.04 \\
\hline 24 & 48.24 & 45.87 & 44.71 & 44.50 & 42.72 & 39.17 & 40.94 & 45.60 & 37.12 & 63.71 & 56.85 & 46.89 \\
\hline 25 & 48.68 & 46.66 & 44.79 & 44.62 & 42.58 & 39.74 & 41.19 & 43.99 & 36.74 & 66.33 & 66.82 & 45.81 \\
\hline 28 & 48.93 & 45.69 & 44.89 & 44.63 & 42.11 & 40.83 & 42.74 & 43.83 & 37.85 & 52.96 & 56.17 & 45.73 \\
\hline 27 & 49.01 & 45.68 & 44.92 & 44.30 & 38.91 & 40.00 & 43.65 & 43.94 & 37.14 & 50.89 & 56.09 & 45.65 \\
\hline 28 & 48.98 & 46.63 & 44.93 & 44.02 & 38.47 & 40.21 & 43.99 & 41.64 & 37.12 & 50.86 & 65.79 & 46.68 \\
\hline 29 & 49.03 & 46.63 & 44.93 & 44.13 & -- & 40.47 & 44.26 & 37.68 & 37.11 & 51.40 & 54.43 & 45.78 \\
\hline 30 & 48.90 & 46.35 & 44.89 & 44.42 & -- & 40.54 & 44.63 & 39.26 & 37.16 & 53.33 & 53.16 & 45.66 \\
\hline 31 & 48.75 & -- & 44.84 & 44.44 & -- & 40.46 & - & 40.03 & -- & 53.20 & 61.65 & - \\
\hline $\begin{array}{l}\text { Mean } \\
\text { Mox. } \\
\text { Min. }\end{array}$ & $\begin{array}{l}53.76 \\
63.61 \\
46.58\end{array}$ & $\begin{array}{l}46.16 \\
48.76 \\
44.41\end{array}$ & $\begin{array}{l}45.00 \\
46.61 \\
44.65\end{array}$ & $\begin{array}{l}44.82 \\
46.20 \\
44.02\end{array}$ & $\begin{array}{l}43.16 \\
44.56 \\
38.47\end{array}$ & $\begin{array}{l}40.44 \\
41.30 \\
38.22\end{array}$ & $\begin{array}{l}41.17 \\
44.63 \\
40.38\end{array}$ & $\begin{array}{l}49.63 \\
82.86 \\
37.56\end{array}$ & $\begin{array}{l}38.19 \\
40.67 \\
35.15\end{array}$ & $\begin{array}{l}43.19 \\
56.33 \\
37.06\end{array}$ & $\begin{array}{l}54.24 \\
56.86 \\
50.91\end{array}$ & $\begin{array}{l}47.27 \\
50.65 \\
46.66\end{array}$ \\
\hline
\end{tabular}


Table 3.--Daily mean water-level measurements in recorder wells in southwestern oklahoma--Continued

HARMON COUNTY, OKLAHOMA--Continued

ø3N-28W-12 BCBA 1--Continued

Water Year, October 1987 to September 1988

Day Dct. Nov. Dec. Jan. Feb. Mar. Apr. May June July Aug. Sept.

\begin{tabular}{|c|c|c|c|c|c|c|c|c|c|c|c|c|}
\hline 1 & 46.54 & 45.26 & 45.36 & 45.26 & 45.45 & 45.84 & 45.66 & -- & -- & -- & -- & -- \\
\hline 2 & 46.56 & 45.23 & 45.32 & 46.28 & 45.54 & 44.84 & 45.68 & -- & -- & -- & -- & -- \\
\hline 3 & 45.62 & 46.21 & 45.38 & 46.18 & 45.63 & 42.25 & 45.69 & - & -- & - ــ & -- & -- \\
\hline 4 & 46.64 & 46.23 & 46.40 & 46.32 & 45.65 & 43.65 & 46.72 & -- & - & -- & -- & -- \\
\hline 5 & 46.46 & 45.35 & 45.27 & 45.34 & 45.72 & 44.25 & 46.84 & $-\infty$ & -- & -- & -- & -- \\
\hline 6 & 45.61 & 45.27 & 46.31 & 45.27 & 45.78 & 44.61 & 46.01 & -- & -- & -- & -- & -- \\
\hline 7 & 45.49 & 45.16 & 45.33 & 45.33 & 45.55 & 44.66 & 47.31 & -- & -- & -- & -- & -- \\
\hline 8 & 46.37 & 46.24 & 45.35 & 46.33 & 45.56 & 44.89 & 65.61 & -- & - & -- & -- & -- \\
\hline 9 & 46.43 & 45.36 & 45.43 & 45.46 & 46.65 & 44.97 & 54.81 & -- & - & -- & -- & -- \\
\hline 10 & 46.58 & 46.44 & 45.39 & 46.42 & 45.61 & 44.90 & 58.00 & $-\infty$ & -- & -- & -- & - \\
\hline 11 & 45.63 & 45.37 & 45.34 & 46.24 & 46.68 & 44.98 & 58.92 & -- & -- & -- & -- & -- \\
\hline 12 & 46.47 & 45.28 & 46.43 & 45.09 & 45.67 & 46.24 & 59.38 & -- & -- & -- & -- & -- \\
\hline 13 & 46.36 & 46.16 & 45.46 & 46.06 & 45.36 & 46.42 & 59.40 & - & -- & -- & -- & -- \\
\hline 14 & 45.32 & 46.08 & 45.43 & 44.98 & 45.37 & 46.45 & 62.04 & -- & $-\infty$ & -- & -- & -- \\
\hline 15 & 46.32 & 45.07 & 46.55 & 44.89 & 45.61 & 46.41 & 63.10 & -- & -- & -- & -- & - \\
\hline 16 & 45.37 & 45.19 & 46.62 & 44.76 & 45.46 & 45.46 & 63.34 & $-\infty$ & -- & -- & -- & -- \\
\hline 17 & 45.41 & 45.24 & 45.68 & 44.87 & 45.68 & 45.46 & 68.82 & -- & -- & -- & $=-$ & - \\
\hline 18 & 45.29 & 45.42 & 46.46 & 44.83 & 45.60 & 45.41 & 49.08 & -- & -- & -- & -- & -- \\
\hline 19 & 45.32 & 46.46 & 44.24 & 44.86 & 45.64 & 45.37 & 48.15 & -- & -- & -- & -- & -- \\
\hline 20 & 45.45 & 46.42 & 44.03 & 46.09 & 46.71 & 46.36 & -- & -- & -- & -- & -- & - \\
\hline 21 & 45.39 & 46.28 & 44.44 & 46.19 & 46.73 & 46.40 & -- & -- & -- & -- & -- & -- \\
\hline 22 & 45.24 & 46.18 & 44.68 & 45.31 & 45.62 & 45.43 & -- & -- & -- & -- & -- & -- \\
\hline 23 & 46.23 & 46.26 & 44.68 & 45.18 & 46.85 & 45.50 & -- & -- & -- & -- & -- & -- \\
\hline 24 & 46.28 & 45.29 & 44.98 & 45.32 & 46.91 & 46.52 & -- & -- & -- & -- & -- & - \\
\hline 25 & 46.24 & 46.37 & 45.04 & 46.41 & 46.80 & 46.66 & -- & -- & -- & -- & -- & - \\
\hline 26 & 46.20 & 46.37 & 45.13 & 45.44 & 46.84 & 46.77 & -- & -- & -- & - & -- & -- \\
\hline 27 & 46.31 & 46.38 & 46.16 & 46.49 & 46.88 & 46.70 & -- & -- & -- & - & -- & - \\
\hline 28 & 45.24 & 46.36 & 46.28 & 46.44 & 46.86 & 45.62 & -- & -- & -- & -- & -- & -- \\
\hline 29 & 45.17 & 46.34 & 45.36 & 46.37 & 45.93 & 45.82 & -- & -- & -- & -- & -- & -- \\
\hline $3 \varnothing$ & 45.19 & 46.36 & 46.68 & 46.30 & -- & 45.82 & -- & -- & -- & -- & -- & -- \\
\hline 31 & 46.22 & -- & 46.10 & 45.34 & - & 46.77 & -- & -- & $-\infty$ & -- & $-\infty$ & -- \\
\hline Mean & 45.38 & 45.29 & 45.18 & 46.21 & 45.65 & 46.17 & - & -- & -- & -- & -- & -- \\
\hline Max. & 45.63 & 46.45 & 46.62 & 45.49 & 46.93 & 46.84 & -- & -- & -- & -- & -- & -- \\
\hline Min. & 46.17 & 46.07 & 44.03 & 44.76 & 45.36 & 42.25 & - & -- & -- & -- & -- & -- \\
\hline
\end{tabular}




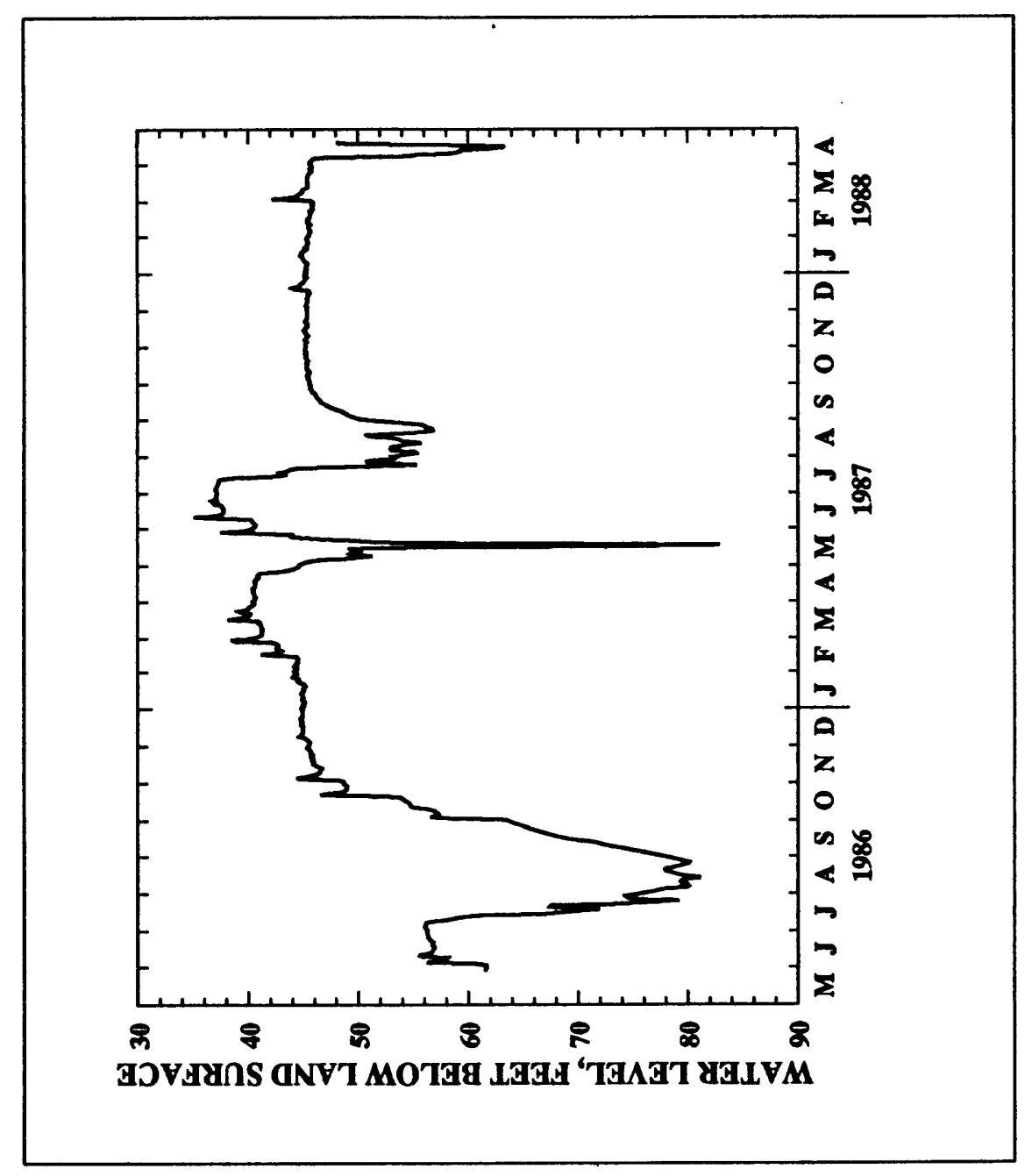


Index number on location map: 7

Station number: 344143099560801 Local number: $63 \mathrm{~N}-26 \mathrm{~W}-33$ ABA 1

Location: Lat $34^{\circ} 41^{\prime} 43^{\prime \prime}$, long $99^{\circ} 66^{\prime} 66^{\prime \prime}$, hydrologic unit 11130101

Aquifer: 313BLIN

Altitude of land-surface datum: $1,640 \mathrm{ft}$

Well depth: $237 \mathrm{ft}$

Water Year, October 1982 to September 1983

Day Oct. Nov. Dec. Jan. Fob. Mar. Apr. May June July Aug. Sept.

\begin{tabular}{|c|c|c|c|c|c|c|c|c|c|c|c|c|c|}
\hline 1 & & -- & -- & -- & -- & -- & -- & -- & -- & -- & 61.80 & 78.52 & 110.62 \\
\hline 2 & & - & -- & -- & -- & -- & -- & -- & -- & -- & 61.61 & 79.17 & 109.22 \\
\hline 3 & & -- & -- & -- & -- & -- & -- & -- & -- & -- & 61.56 & 80.10 & 109.00 \\
\hline 4 & & -- & -- & -- & -- & -- & -- & -- & -- & -- & 81.58 & 82.06 & 110.76 \\
\hline 5 & & -- & - & -- & -- & -- & -- & -- & -- & -- & 61.66 & 84.35 & 110.66 \\
\hline 6 & & -- & -- & -- & -- & -- & - & -- & -- & -- & 61.79 & 86.53 & 111.15 \\
\hline 7 & & -- & -- & -- & -- & -- & - & -- & -- & 64.86 & 62.09 & 90.83 & 106.99 \\
\hline 8 & & -- & -- & -- & -- & -- & - & -- & -- & 04.06 & 62.36 & 93.34 & -- \\
\hline 9 & & -- & -- & -- & -- & -- & -- & -- & - & 64.63 & 62.66 & 94.62 & - \\
\hline 10 & & -- & -- & -- & -- & -- & -- & -- & -- & 63.93 & 63.10 & 96.69 & -- \\
\hline 11 & & -- & - & -- & -- & -- & -- & -- & - & 63.71 & 63.63 & 96.47 & - \\
\hline 12 & & -- & -- & -- & -- & -- & - & -- & -- & 63.39 & 64.96 & 97.07 & -- \\
\hline 13 & . & -- & $\ldots$ & -- & -- & $\ldots$ & - & -- & -- & 63.16 & 66.39 & 97.43 & - \\
\hline 14 & & -- & - & $\ldots$ & -- & - & - & $-\infty$ & -- & 63.07 & 66.73 & 97.98 & -- \\
\hline 16 & & -- & -- & -- & -- & -- & -- & -- & -- & 62.92 & 66.88 & 98.38 & -- \\
\hline 16 & & -- & -- & -- & -- & -- & -- & -- & -- & 62.81 & 67.84 & 98.88 & -- \\
\hline 17 & & -- & -- & -- & -- & - & -- & $-\infty$ & -- & 62.62 & 68.43 & 99.49 & -- \\
\hline 18 & & -- & -- & -- & -- & - & - & -- & - & 62.20 & 69.14 & 167.46 & - \\
\hline 19 & & -- & -- & -- & -- & -- & - & -- & -- & 62.06 & 69.84 & 102.29 & -- \\
\hline 20 & & -- & -- & -- & -- & -- & -- & -- & -- & 61.93 & 70.64 & 162.33 & - \\
\hline 21 & & -- & -- & $-\infty$ & $-\infty$ & -- & - & -- & -- & 61.86 & 71.18 & 102.30 & -- \\
\hline 22 & & -- & - & - & - & -- & -- & -- & -- & 61.78 & 71.62 & 101.09 & - \\
\hline 23 & & -- & -- & -- & -- & -- & -- & -- & -- & 61.71 & 72.66 & 100.88 & -- \\
\hline 24 & & -- & - & -- & -- & -- & - & -- & -- & 61.61 & 73.76 & 102.65 & 100.66 \\
\hline 25 & & -- & -- & -- & -- & -- & -- & -- & -- & 61.53 & 75.36 & 102.92 & - \\
\hline 28 & & -- & -- & -- & -- & -- & - & -- & - & 61.46 & 76.76 & 104.69 & -- \\
\hline 27 & & -- & -- & -- & -- & -- & -- & -- & -- & 61.36 & 76.36 & 165.96 & -- \\
\hline 28 & & -- & -- & -- & -- & -- & -- & -- & -- & 61.29 & 77.16 & 186.66 & -- \\
\hline 29 & & -- & -- & -- & -- & -- & -- & -- & -- & 61.30 & 78.13 & 109.97 & -- \\
\hline $3 \emptyset$ & & -- & - & -- & -- & -- & - & $-\infty$ & - & 61.46 & 77.93 & 110.69 & 98.96 \\
\hline 31 & & -- & -- & -- & -- & -- & -- & $-\infty$ & -- & -- & 78.10 & 111.93 & -- \\
\hline Mean & & -- & - & -- & -- & -- & -- & - & - & -- & 68.46 & 97.49 & -- \\
\hline Max. & & -- & - & -- & -- & -- & -- & -- & -- & -- & 78.13 & 111.93 & - \\
\hline Min. & & -- & -- & -- & -- & -- & -- & -- & -- & -- & 61.66 & 78.62 & - \\
\hline
\end{tabular}


Table 3.--Daily mean water-level measurements in recorder wells in southwestern Dklahoma--Continued

HARMON COUNTY, OKLAHOMA--CONtinued

O3N-26W-33 ABA 1--Continued

Water Year, October 1983 to September 1984

Day Oct. Nov. Dec. Jan. Feb. Mar. Apr. May June July Aug. Sept.

\begin{tabular}{|c|c|c|c|c|c|c|c|c|c|c|c|c|c|}
\hline 1 & & -- & -- & 70.34 & 66.77 & -- & -- & -- & -- & -- & -- & -- & -- \\
\hline 2 & & -- & -- & 70.11 & 86.89 & -- & 69.71 & -- & -- & -- & -- & -- & -- \\
\hline 3 & & -- & -- & 69.95 & 65.52 & -- & -- & -- & -- & -- & -- & -- & -- \\
\hline 4 & & -- & -- & 69.75 & 65.35 & -- & -- & 58.49 & -- & -- & -- & -- & -- \\
\hline 5 & & -- & -- & 69.62 & 65.20 & -- & -- & -- & -- & -- & - & -- & - \\
\hline 6 & & -- & -- & 69.63 & 65.67 & -- & -- & - & -- & -- & -- & -- & -- \\
\hline 7 & & -- & -- & 69.32 & 64.97 & -- & -- & -- & -- & -- & -- & -- & -- \\
\hline 8 & & -- & -- & 69.17 & 64.82 & -- & - & -- & -- & -- & -- & -- & - \\
\hline 9 & & -- & -- & 68.97 & 64.69 & -- & -- & -- & -- & -- & -- & -- & -- \\
\hline 10 & & -- & -- & 68.74 & 64.66 & -- & - & -- & -- & -- & -- & -- & -- \\
\hline 11 & & -- & -- & 68.63 & 64.46 & -- & -- & -- & 69.64 & -- & -- & -- & - \\
\hline 12 & & -- & -- & 68.45 & 64.41 & -- & -- & -- & -- & -- & -- & -- & - \\
\hline 13 & & -- & -- & 68.26 & 64.41 & -- & -- & -- & -- & -- & -- & -- & -- \\
\hline 14 & & -- & -- & 68.10 & 64.40 & -- & -- & -- & -- & -- & -- & -- & -- \\
\hline 15 & & -- & -- & 87.99 & 64.46 & -- & -- & - & -- & $-\infty$ & -- & -- & -- \\
\hline 16 & & -- & -- & 67.92 & 64.40 & -- & -- & -- & -- & -- & - & -- & -- \\
\hline 17 & & -- & 72.63 & 67.78 & 64.41 & -- & -- & -- & -- & -- & -- & -- & -- \\
\hline & - & -- & 70.36 & 67.69 & 64.41 & -- & -- & -- & -- & -- & -- & -- & -- \\
\hline 19 & & -- & 72.27 & 67.50 & 64.41 & -- & -- & -- & -- & -- & -- & -- & -- \\
\hline 20 & & -- & 72.09 & 67.31 & 63.60 & -- & -- & -- & -- & -- & -- & -- & -- \\
\hline 21 & & -- & 71.83 & 67.21 & -- & -- & -- & -- & -- & -- & -- & -- & - \\
\hline 22 & & -- & 71.73 & 67.10 & -- & -- & -- & -- & -- & -- & -- & 98.02 & -- \\
\hline 23 & & -- & 71.59 & 66.95 & -- & -- & -- & -- & -- & -- & -- & -- & - \\
\hline 24 & & -- & 71.47 & 66.93 & -- & -- & -- & -- & - & -- & -- & -- & -- \\
\hline 25 & & -- & 71.26 & 68.74 & -- & -- & -- & -- & 68.84 & -- & -- & -- & -- \\
\hline 26 & & -- & 71.13 & 68.49 & -- & -- & -- & -- & -- & -- & -- & -- & -- \\
\hline 27 & & -- & 76.92 & 66.28 & -- & -- & -- & -- & -- & -- & -- & -- & -- \\
\hline 28 & & -- & 76.78 & 66.24 & -- & -- & -- & -- & -- & -- & -- & -- & - \\
\hline 29 & & -- & 70.62 & 88.21 & -- & -- & -- & -- & -- & -- & -- & -- & -- \\
\hline 30 & & -- & 70.51 & 66.05 & -- & -- & -- & -- & -- & -- & -- & -- & -- \\
\hline 31 & & -- & -- & 65.88 & -- & -- & -- & -- & -- & -- & -- & -- & -- \\
\hline Mean & & -- & -- & 67.97 & -- & -- & -- & -- & -- & -- & -- & -- & -- \\
\hline Max. & & -- & -- & 70.34 & $-\infty$ & -- & -- & -- & -- & -- & -- & -- & -- \\
\hline Min. & & -- & -- & 65.88 & - & -- & -- & -- & -- & -- & -- & -- & - \\
\hline
\end{tabular}


Table 3.--Daily mean water-level measurements in recorder wells in southwestern Oklahoma--Continued

HARMON COUNTY, OKLAHOMA--CONTInUEd

D3N-26W-33 ABA 1--Continued

Water Year, October 1984 to September 1985

Day Dct. Nov. Dec. Jan. Fob. Mar. Apr. May June July Sept.

\begin{tabular}{|c|c|c|c|c|c|c|c|c|c|c|c|c|c|}
\hline 1 & & -- & -- & 83.80 & 79.61 & 76.50 & 74.04 & 71.12 & -- & 69.07 & 68.74 & - & -- \\
\hline 2 & & -- & -- & 83.72 & 79.53 & 76.42 & 73.96 & 71.61 & -- & 69.67 & 68.70 & -- & -- \\
\hline 3 & & -- & -- & 83.60 & 79.39 & 76.32 & 73.81 & 70.88 & -- & 69.07 & 68.67 & -- & -- \\
\hline 4 & & -- & -- & 83.45 & 79.27 & 76.23 & 73.80 & 70.76 & -- & 69.67 & -- & -- & -- \\
\hline 5 & & -- & -- & 83.31 & 79.16 & 76.14 & 73.77 & 70.76 & -- & 69.05 & -- & -- & -- \\
\hline 6 & & -- & 88.60 & 83.23 & 79.02 & 76.07 & 73.68 & 70.70 & -- & 68.99 & -- & -- & -- \\
\hline 7 & & -- & 88.28 & 83.04 & 78.94 & 76.03 & 73.58 & 70.71 & 69.14 & 68.95 & -- & -- & -- \\
\hline 8 & & -- & 88.02 & 82.87 & 78.80 & 75.99 & 73.52 & 70.67 & 69.13 & 68.85 & -- & -- & -- \\
\hline 9 & & -- & 87.78 & 82.74 & 78.67 & 75.76 & 73.47 & 70.56 & 69.11 & 68.81 & -- & -- & -- \\
\hline 10 & & -- & 87.68 & 82.63 & 78.63 & 75.71 & 73.34 & 70.47 & 69.11 & 68.79 & -- & -- & -- \\
\hline 11 & & -- & 87.30 & 82.43 & 78.67 & 75.67 & 73.26 & 70.42 & 69.11 & 68.76 & -- & -- & -- \\
\hline 12 & & -- & 87.08 & 82.27 & 78.48 & 75.66 & 73.19 & 70.45 & 69.11 & 68.76 & -- & -- & -- \\
\hline 13 & & -- & 88.83 & 82.16 & 78.32 & 75.48 & 73.11 & 70.44 & 69.11 & 68.74 & -- & -- & -- \\
\hline 14 & & -- & 86.67 & 82.12 & 78.23 & 75.41 & 73.08 & 70.42 & 69.11 & 68.74 & -- & -- & -- \\
\hline 15 & & -- & 86.44 & 81.95 & 78.10 & 76.29 & 73.01 & 70.36 & 69.11 & 68.74 & -- & -- & -- \\
\hline 16 & & -- & 86.26 & 81.74 & 77.98 & 76.16 & 72.94 & 70.38 & 69.11 & 68.74 & -- & -- & -- \\
\hline 17 & & -- & 86.00 & 81.67 & 77.89 & 76.13 & 72.88 & 70.40 & 69.11 & 68.74 & -- & -- & -- \\
\hline 18 & - & -- & 86.81 & 81.46 & 77.76 & 75.89 & 72.78 & 70.32 & 69.11 & 68.74 & -- & -- & -- \\
\hline 19 & & -- & 86.69 & 81.20 & 77.67 & 74.97 & 72.67 & 70.22 & 69.11 & 68.74 & -- & -- & -- \\
\hline $2 \varnothing$ & & -- & 86.67 & 81.00 & 77.66 & 74.84 & 72.49 & 70.17 & 69.12 & 68.74 & -- & -- & -- \\
\hline 21 & & -- & 86.41 & 80.86 & 77.66 & 74.74 & 72.40 & 70.18 & 69.12 & 68.74 & -- & -- & -- \\
\hline 22 & & -- & 85.25 & 80.77 & 77.46 & 74.66 & 72.29 & 70.24 & 69.12 & 68.74 & -- & -- & -- \\
\hline 23 & & -- & 86.06 & 80.59 & 77.32 & 74.67 & 72.03 & 70.38 & 69.12 & 68.74 & -- & -- & -- \\
\hline 24 & & -- & 84.86 & 80.48 & 77.21 & 74.63 & 71.91 & 70.61 & 69.12 & 68.74 & -- & -- & -- \\
\hline 26 & & -- & 84.64 & 80.42 & 77.14 & 74.40 & 71.81 & 70.64 & 69.12 & 68.74 & -- & -- & - \\
\hline 26 & & - & 84.51 & 80.27 & 77.65 & 74.34 & 71.69 & 70.72 & 69.12 & 68.74 & -- & -- & -- \\
\hline 27 & & -- & 84.44 & 80.12 & 76.93 & 74.29 & 71.54 & 70.33 & 69.10 & 68.74 & -- & -- & -- \\
\hline 28 & & -- & 84.24 & 79.99 & 76.84 & 74.16 & 71.40 & -- & 69.67 & 68.74 & -- & -- & -- \\
\hline 29 & & -- & 84.08 & 79.89 & 76.71 & -- & 71.29 & -- & 69.67 & 68.74 & -- & -- & -- \\
\hline 30 & & -- & 83.97 & 79.81 & 76.63 & -- & 71.24 & -- & 69.67 & 68.74 & -- & -- & -- \\
\hline 31 & & -- & -- & 79.66 & 76.68 & -- & 71.16 & -- & 69.07 & -- & -- & -- & -- \\
\hline Mean & & -- & -- & 81.71 & 78.04 & 76.33 & 72.76 & -- & -- & 68.82 & -- & -- & -- \\
\hline Max. & & -- & -- & 83.80 & 79.61 & 76.50 & 74.04 & -- & -- & 69.07 & -- & -- & -- \\
\hline Min. & & -- & -- & 79.66 & 76.58 & 74.16 & 71.16 & -- & -- & 68.74 & -- & -- & -- \\
\hline
\end{tabular}


Table 3.--Daily mean water-level measurements in recorder wells in southwestern Oklahoma--Continued

HARMON COUNTY, OKLAHOMA--CONTinUEd

63N-26W-33 ABA 1--Continued

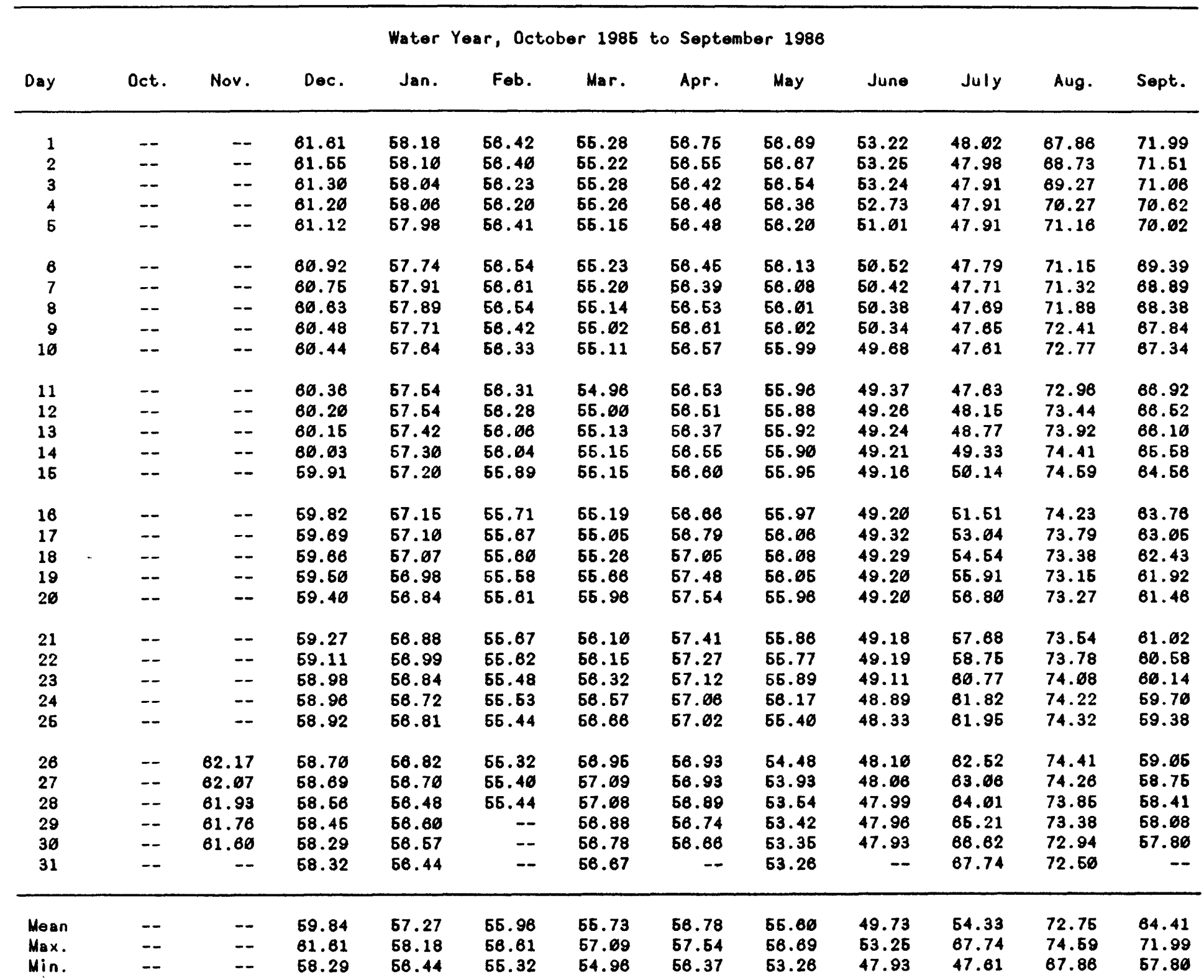


Table 3.--Daily mean water-level measurements in recorder wells in southwestern Oklahoma--Continued

HARMON COUNTY, OKLAHOMA--CONtinued

63N-26W-33 ABA 1--Continued

Water Year, October 1986 to September 1987

\begin{tabular}{|c|c|c|c|c|c|c|c|c|c|c|c|c|}
\hline Day & Oct. & Nov. & Dec. & Jan. & Feb. & Mar. & Apr. & May & June & July & Aug. & Sept. \\
\hline 1 & -- & 45.44 & 42.63 & 41.60 & 40.35 & -- & 36.63 & 36.58 & 35.67 & 32.06 & 47.00 & 48.28 \\
\hline 2 & -- & 45.40 & 42.86 & 41.37 & 46.35 & -- & 36.87 & 36.71 & 36.57 & 32.06 & 47.29 & 47.68 \\
\hline 3 & -- & 46.16 & 42.71 & 41.63 & 40.40 & -- & 36.76 & 37.10 & 35.64 & 32.60 & 46.17 & 47.01 \\
\hline 4 & -- & 44.79 & 42.74 & 41.62 & 40.41 & -- & 36.77 & 37.46 & 35.68 & 31.92 & 46.10 & 46.67 \\
\hline 6 & -- & 43.93 & 42.63 & 41.38 & 40.58 & -- & 36.86 & 37.84 & 36.39 & 31.98 & 46.42 & 46.19 \\
\hline 6 & -- & 43.24 & 42.46 & 41.38 & 40.63 & -- & 36.72 & 38.09 & 36.26 & 32.03 & 46.29 & 45.87 \\
\hline 7 & -- & 43.26 & 42.37 & 41.62 & - & -- & 36.69 & 38.36 & 35.14 & 32.09 & 47.46 & 45.64 \\
\hline 8 & -- & 43.26 & 42.32 & 41.44 & -- & -- & 36.64 & 38.44 & 36.07 & 32.37 & 46.90 & 45.24 \\
\hline 9 & - & 43.28 & 42.31 & 41.34 & -- & -- & 38.61 & 38.71 & 34.98 & 32.62 & 47.56 & 44.89 \\
\hline 10 & -- & 43.28 & 42.29 & 41.41 & -- & -- & 36.64 & 39.65 & 34.21 & 32.98 & 47.09 & 44.60 \\
\hline 11 & -- & 43.48 & 42.14 & 41.43 & -- & 37.47 & 36.39 & 39.36 & 33.34 & 33.32 & 47.62 & 44.34 \\
\hline 12 & -- & 43.83 & 42.20 & 41.33 & -- & 37.60 & 36.37 & 39.59 & 33.18 & 33.68 & 48.47 & 44.07 \\
\hline 13 & -- & 43.92 & 42.15 & 41.22 & -- & 37.37 & 36.64 & 40.69 & 33.63 & 33.64 & 49.45 & 43.77 \\
\hline 14 & -- & 43.77 & 42.05 & 41.13 & -- & 37.14 & 36.69 & 40.88 & 32.89 & 33.89 & 49.76 & 43.63 \\
\hline 16 & -- & 43.76 & 42.02 & 41.25 & -- & 37.21 & 36.67 & 41.36 & 32.81 & 34.69 & 49.62 & 43.19 \\
\hline 16 & -- & 43.68 & 42.01 & 41.30 & -- & 37.66 & 36.62 & 41.49 & 32.75 & 36.44 & 50.42 & 42.95 \\
\hline 17 & -- & 43.63 & 41.98 & 41.22 & -- & 36.81 & 36.46 & 41.97 & 32.76 & 36.69 & 49.29 & 42.80 \\
\hline 18 & -- & 43.60 & 41.98 & 41.15 & -- & 36.89 & 36.40 & 42.56 & 32.78 & 36.12 & 48.76 & 42.68 \\
\hline 19 & -- & 43.08 & 41.82 & 41.20 & -- & 36.91 & 36.43 & 43.50 & 32.67 & 36.58 & 49.06 & 42.50 \\
\hline 20 & -- & 43.24 & 41.81 & 41.33 & -- & 36.86 & 36.62 & 43.47 & 32.62 & 36.99 & 60.19 & 42.31 \\
\hline 21 & -- & 43.11 & 41.86 & 41.18 & -- & 36.90 & 36.78 & 43.21 & 32.60 & 37.66 & 61.91 & 42.20 \\
\hline 22 & -- & 42.92 & 41.80 & 41.23 & -- & 36.73 & 36.64 & 43.68 & 32.66 & 38.28 & 51.75 & 42.08 \\
\hline 23 & -- & 43.08 & 41.64 & 41.00 & -- & 36.63 & 36.61 & 42.28 & 32.58 & 38.66 & 61.78 & 41.84 \\
\hline 24 & -- & 43.05 & 41.58 & 40.93 & -- & 36.76 & 36.70 & 41.24 & 32.60 & 38.83 & 61.90 & 41.61 \\
\hline 25 & -- & 42.82 & 41.64 & 41.01 & -- & 36.84 & 36.71 & 49.23 & 32.60 & 39.65 & 63.28 & 41.47 \\
\hline 26 & - & 42.97 & 41.78 & 41.02 & -- & 36.79 & 36.71 & 39.44 & 32.60 & 41.39 & 63.26 & 41.34 \\
\hline 27 & -- & 42.93 & 41.67 & 40.84 & -- & 36.68 & 36.71 & 39.66 & 32.51 & 43.42 & 52.64 & 41.20 \\
\hline 28 & -- & 42.84 & 41.66 & 48.63 & -- & 36.78 & 36.71 & 38.19 & 32.42 & 44.47 & 51.83 & 41.17 \\
\hline 29 & -- & 42.70 & 41.64 & 40.63 & -- & 36.91 & 36.61 & 36.68 & $32.4 \varnothing$ & 45.29 & 62.08 & 41.12 \\
\hline 30 & 45.42 & 42.64 & 41.56 & 40.63 & -- & 36.86 & 38.67 & 38.19 & 32.19 & 46.40 & 50.22 & 41.00 \\
\hline 31 & 46.36 & -- & 41.50 & 40.44 & -- & 36.76 & - & 36.90 & - & -- & 49.03 & - \\
\hline Mean & -- & 43.63 & 42.05 & 41.17 & -- & -- & 36.62 & 39.61 & 33.65 & -- & 49.37 & 43.63 \\
\hline Max. & - & 46.44 & 42.74 & 41.60 & $-\infty$ & -- & 36.87 & 43.50 & 36.67 & -- & 63.28 & 48.28 \\
\hline Min. & -- & 42.64 & 41.50 & 40.44 & -- & -- & 36.37 & 35.90 & 32.19 & -- & 46.10 & 41.00 \\
\hline
\end{tabular}


Table 3.--Daily mean water-level measurements in recorder wells in southwestern Oklahoma--Continued

HARMON COUNTY, OKLAHOMA--CONtinUEd

D3N-26W-33 ABA 1--Continued

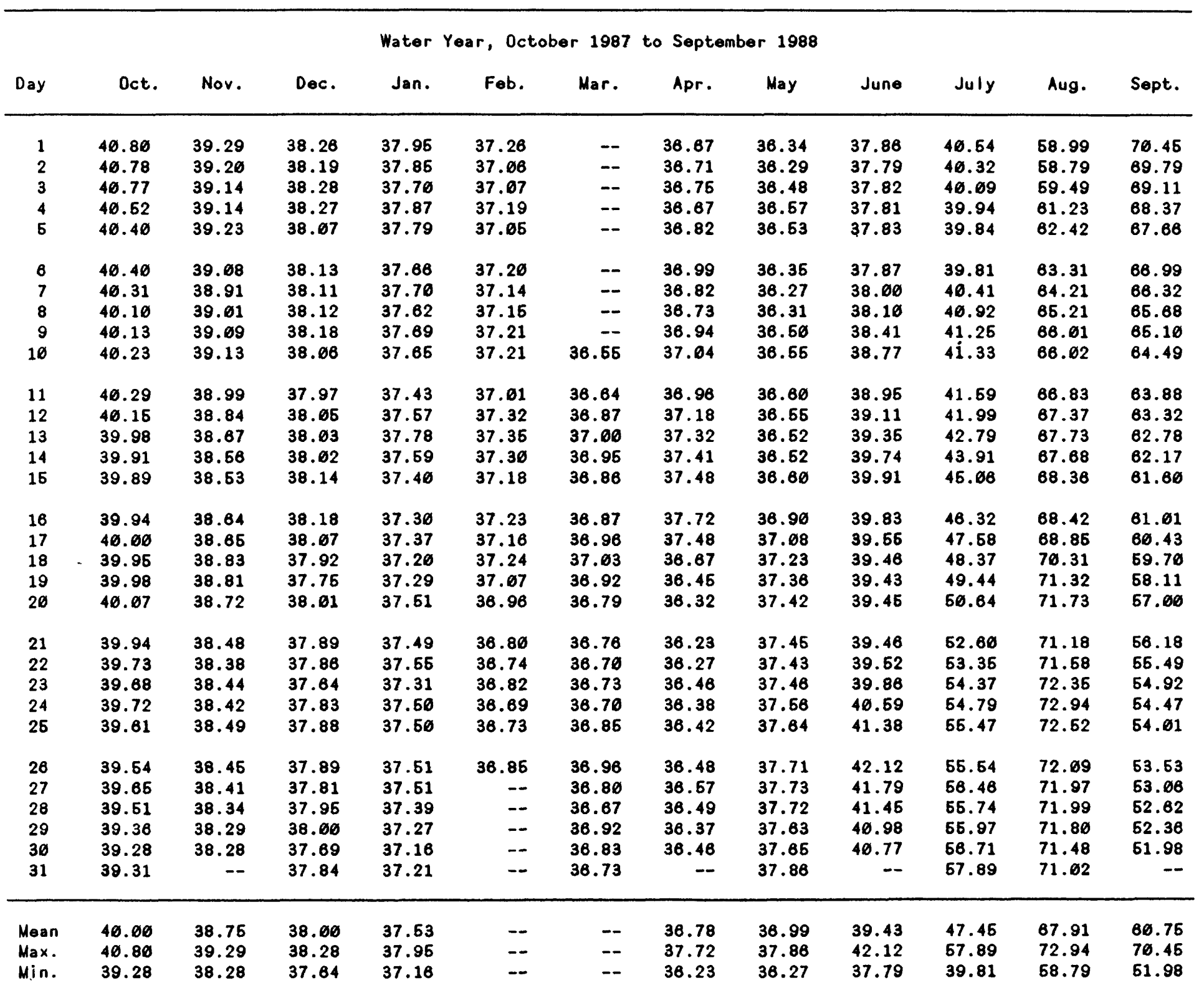


Table 3.--Daily mean water-level measurements in recorder wells in southwestern Oklahoma--Continued

HARMON COUNTY, OKLAHOMA--CONTinued

63N-26W-33 ABA 1--Continued

Water Year, October 1988 to September 1989

Day Dct. Nov. Dec. Jan. Fob. Mar. Apr. May June July Aug. Sept.

\begin{tabular}{|c|c|c|c|c|c|c|c|c|c|c|c|c|}
\hline 1 & 61.68 & 46.33 & 44.78 & 43.20 & -- & -- & -- & -- & -- & -- & -- & -- \\
\hline 2 & 61.36 & 46.04 & 44.68 & 43.19 & -- & -- & -- & -- & -- & -- & -- & -- \\
\hline 3 & 51.05 & 45.89 & 44.68 & 43.28 & - & -- & -- & -- & -- & -- & -- & -- \\
\hline 4 & 50.82 & 46.88 & 44.86 & 43.18 & -- & -- & -- & -- & -- & -- & -- & -- \\
\hline 5 & 58.61 & 46.95 & 44.64 & 42.94 & -- & -- & -- & -- & -- & -- & -- & -- \\
\hline$\theta$ & 50.20 & 46.77 & 44.48 & 42.93 & -- & -- & -- & -- & -- & -- & -- & -- \\
\hline 7 & 49.99 & 45.66 & 44.47 & 43.00 & -- & -- & -- & -- & -- & -- & -- & -- \\
\hline 8 & 49.83 & 45.68 & 44.81 & 43.19 & -- & -- & -- & -- & -- & -- & -- & -- \\
\hline 9 & 49.37 & 46.61 & 44.43 & 43.69 & -- & -- & -- & -- & -- & -- & -- & -- \\
\hline 10 & 49.16 & 46.63 & 44.25 & 42.84 & -- & -- & -- & -- & -- & -- & -- & -- \\
\hline 11 & 48.97 & 46.41 & 44.23 & 42.87 & -- & -- & -- & -- & -- & -- & -- & -- \\
\hline 12 & 48.82 & 45.45 & 44.19 & 43.11 & -- & -- & -- & -- & -- & -- & -- & -- \\
\hline 13 & 48.60 & 46.58 & 44.02 & 43.10 & -- & -- & -- & -- & -- & -- & -- & -- \\
\hline 14 & 48.31 & 46.38 & 43.91 & 42.82 & -- & -- & -- & -- & -- & -- & -- & -- \\
\hline 15 & 48.09 & 46.26 & 44.19 & 42.90 & $-\infty$ & -- & -- & -- & -- & -- & -- & -- \\
\hline 16 & 47.94 & 46.60 & 44.69 & 42.91 & -- & -- & -- & -- & -- & -- & -- & -- \\
\hline 17 & 47.74 & 45.43 & 43.89 & 42.76 & -- & -- & -- & - & -- & -- & -- & -- \\
\hline 18 & 47.78 & 46.34 & 43.79 & 42.82 & -- & -- & -- & -- & -- & -- & -- & -- \\
\hline 19 & 47.68 & 45.47 & 43.65 & -- & -- & -- & -- & -- & -- & -- & -- & -- \\
\hline 20 & 47.38 & 45.60 & 43.67 & -- & -- & -- & -- & -- & -- & -- & -- & -- \\
\hline 21 & 47.34 & 46.47 & 43.68 & -- & -- & -- & -- & -- & -- & -- & -- & -- \\
\hline 22 & 47.67 & 46.36 & 43.58 & -- & -- & -- & -- & -- & -- & -- & -- & -- \\
\hline 23 & 47.08 & 46.19 & 43.46 & -- & -- & -- & -- & -- & -- & -- & -- & -- \\
\hline 24 & 46.99 & 44.96 & 43.69 & -- & -- & -- & -- & -- & -- & -- & -- & -- \\
\hline 25 & 46.92 & 44.83 & 43.50 & -- & -- & -- & -- & -- & -- & -- & -- & -- \\
\hline 28 & 46.88 & 44.73 & 43.31 & -- & -- & -- & -- & -- & -- & -- & -- & -- \\
\hline 27 & 48.71 & 44.95 & 43.46 & -- & -- & -- & -- & -- & -- & -- & -- & -- \\
\hline 28 & 48.90 & 44.85 & 43.65 & -- & -- & -- & -- & -- & -- & -- & -- & -- \\
\hline 29 & 48.77 & 44.77 & 43.39 & -- & -- & -- & -- & -- & -- & -- & -- & -- \\
\hline 30 & 46.67 & 44.81 & 43.27 & -- & - & -- & -- & -- & -- & -- & -- & -- \\
\hline 31 & 46.53 & -- & 43.22 & -- & -- & -- & -- & -- & -- & -- & -- & -- \\
\hline Mean & 48.41 & 46.42 & 43.98 & -- & -- & -- & -- & -- & -- & -- & -- & -- \\
\hline Max. & 51.68 & 46.33 & 44.86 & -- & -- & -- & -- & -- & -- & -- & -- & -- \\
\hline Min. & 46.63 & 44.73 & 43.22 & -- & -- & -- & -- & -- & -- & -- & -- & -- \\
\hline
\end{tabular}




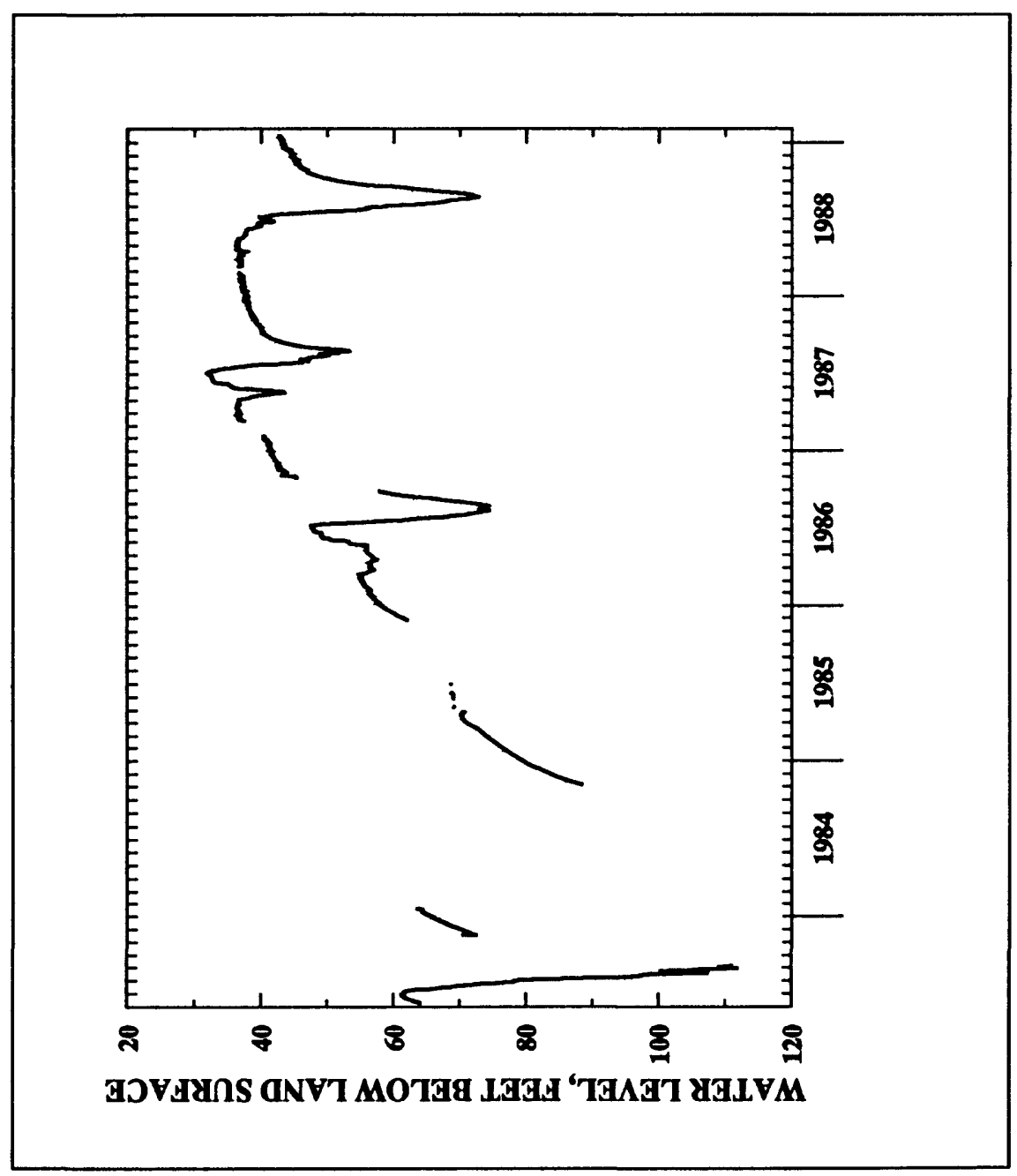


Table 3.--Daily mean water-level measurements in recorder wells in southwestern Oklahoma--Continued

HARMON COUNTY, OKLAHOMA--CONtinued

Index number on location map: 8

Station number: $\$ 44750099522801$ Local number: $94 \mathrm{~N}-25 \mathrm{~W}-30$ BBAB 1

Location: Lat $34^{\circ} 47^{\prime} 50^{\prime \prime}$, long $99^{\circ} 52^{\prime} 26^{\prime \prime}$, hydrologic unit 11130101

Aquifer: 110SFRT

Altitude of land-surface datum: $1,819 \mathrm{ft}$

Well depth: $50 \mathrm{ft}$

Water Year, October 1985 to September 1986

Day Oct. Nov. Dec. Jan. Fob. Mar. Apr. May June July Aug. Sept.

\begin{tabular}{|c|c|c|c|c|c|c|c|c|c|c|c|c|}
\hline 1 & -- & -- & -- & -- & -- & -- & -- & -- & 39.95 & 40.00 & 39.91 & 39.82 \\
\hline 2 & -- & -- & -- & -- & -- & -- & -- & -- & 39.96 & 40.00 & 39.93 & 39.81 \\
\hline 3 & -- & -- & -- & -- & -- & -- & -- & -- & 39.96 & 39.95 & 39.94 & 39.82 \\
\hline 4 & -- & -- & - & -- & -- & -- & -- & -- & 39.96 & 39.92 & 39.90 & 39.83 \\
\hline 5 & -- & -- & -- & -- & -- & -- & -- & -- & 39.96 & 39.93 & 39.88 & 39.81 \\
\hline 6 & -- & -- & -- & -- & -- & -- & -- & -- & 39.95 & 39.98 & 39.89 & 39.82 \\
\hline 7 & -- & -- & -- & -- & -- & -- & -- & -- & 39.97 & 39.98 & 39.89 & 39.85 \\
\hline 8 & -- & -- & -- & -- & -- & -- & -- & -- & 39.96 & 39.96 & 39.90 & 39.80 \\
\hline 9 & -- & -- & -- & -- & -- & -- & -- & -- & 39.96 & 39.84 & 39.88 & 39.77 \\
\hline 10 & -- & -- & -- & -- & -- & -- & -- & -- & 39.98 & 39.92 & 39.92 & 39.76 \\
\hline 11 & -- & -- & -- & -- & -- & -- & -- & -- & 40.01 & 39.92 & 39.91 & 39.82 \\
\hline 12 & -- & -- & -- & -- & -- & -- & -- & -- & 39.99 & 39.93 & 39.88 & 39.82 \\
\hline 13 & -- & -- & -- & -- & -- & -- & -- & -- & 40.00 & 39.97 & 39.86 & 39.81 \\
\hline 14 & -- & -- & -- & -- & -- & -- & -- & -- & 39.99 & 39.97 & 39.87 & 39.79 \\
\hline 15 & -- & -- & -- & -- & -- & -- & -- & -- & 39.97 & 39.85 & 39.87 & 39.79 \\
\hline 16 & -- & -- & -- & -- & -- & -- & -- & -- & 40.00 & 39.94 & 39.86 & 39.78 \\
\hline 17 & -- & -- & -- & -- & -- & -- & -- & -- & 40.63 & 39.96 & 39.88 & 39.76 \\
\hline 18 & -- & -- & -- & -- & -- & -- & -- & -- & 40.00 & 39.96 & 39.89 & 39.77 \\
\hline 19 & -- & -- & -- & -- & -- & -- & -- & -- & 39.98 & 39.94 & 39.86 & 39.77 \\
\hline 20 & -- & -- & -- & -- & -- & -- & -- & -- & 39.99 & 39.96 & 39.86 & 39.77 \\
\hline 21 & -- & -- & -- & -- & -- & -- & -- & -- & 39.99 & 39.94 & 39.88 & 39.78 \\
\hline 22 & -- & -- & -- & -- & -- & -- & -- & -- & 40.01 & 39.95 & 39.86 & 39.74 \\
\hline 23 & -- & -- & -- & -- & -- & -- & -- & -- & 40.00 & 39.93 & 39.86 & 39.72 \\
\hline 24 & -- & -- & -- & -- & -- & -- & -- & -- & 40.00 & 39.91 & 39.85 & 39.71 \\
\hline 25 & -- & -- & -- & -- & -- & -- & -- & -- & 39.99 & 39.91 & 39.85 & 39.75 \\
\hline 28 & -- & -- & -- & -- & -- & -- & -- & -- & 39.99 & 39.91 & 39.85 & 39.75 \\
\hline 27 & -- & -- & -- & -- & -- & -- & -- & -- & 39.99 & 39.90 & 39.86 & 39.75 \\
\hline 28 & -- & -- & -- & -- & -- & -- & -- & -- & 39.97 & 39.91 & 39.87 & 39.73 \\
\hline 29 & -- & -- & -- & -- & -- & -- & -- & -- & 39.96 & 39.91 & 39.83 & 39.72 \\
\hline 30 & -- & -- & -- & -- & -- & -- & -- & 39.96 & 39.96 & 39.90 & 39.83 & 39.74 \\
\hline 31 & -- & -- & -- & -- & -- & -- & -- & 39.94 & -- & 39.92 & 39.82 & -- \\
\hline Mean & -- & -- & -- & -- & -- & -- & -- & -- & 39.98 & 39.94 & 39.88 & 39.78 \\
\hline Max. & -- & -- & -- & -- & -- & -- & -- & -- & 40.03 & 40.00 & 39.94 & 39.85 \\
\hline Min. & -- & -- & -- & -- & -- & -- & -- & -- & 39.95 & 39.90 & 39.82 & 39.71 \\
\hline
\end{tabular}


Table 3.--Daily mean water-level measurements in recorder wells in southwestern oklahoma--Continued

HARMON COUNTY, OKLAHOMA--CONTINUEd

$64 \mathrm{~N}-25 W-30$ BBAB 1--Continued

\begin{tabular}{|c|c|c|c|c|c|c|c|c|c|c|c|c|}
\hline \multirow[b]{2}{*}{ Day } & \multirow[b]{2}{*}{ Oct. } & \multirow[b]{2}{*}{ Nov. } & \multirow[b]{2}{*}{ Dec. } & \multirow{2}{*}{$\begin{array}{l}\text { Water } \\
\text { Jan. }\end{array}$} & \multirow[b]{2}{*}{ Fob. } & \multirow[b]{2}{*}{ Mar. } & \multicolumn{2}{|c|}{ - September 1987} & \multirow[b]{2}{*}{ June } & \multirow[b]{2}{*}{ July } & \multirow[b]{2}{*}{ Aug. } & \multirow[b]{2}{*}{ Sept. } \\
\hline & & & & & & & Apr. & May & & & & \\
\hline 1 & 39.72 & 39.42 & 38.71 & 38.28 & 38.03 & 37.98 & 37.67 & 37.52 & 37.36 & 37.18 & 36.91 & 36.68 \\
\hline 2 & 39.69 & 39.37 & 38.71 & 38.17 & 38.03 & 37.88 & 37.75 & 37.50 & 37.39 & 37.17 & 36.90 & 36.66 \\
\hline 3 & 39.71 & 39.30 & 38.70 & 38.31 & 38.03 & 37.91 & 37.68 & 37.56 & 37.46 & 37.16 & 36.90 & 36.65 \\
\hline 4 & 39.77 & 39.30 & 38.69 & 38.24 & 38.04 & 37.90 & 37.70 & 37.59 & 37.41 & 37.13 & 36.92 & 36.64 \\
\hline 6 & 39.76 & 39.29 & 38.63 & 38.17 & 38.08 & 37.82 & 37.70 & 37.69 & 37.37 & 37.16 & 36.89 & 36.63 \\
\hline 6 & 39.74 & 39.24 & 38.69 & 38.21 & 38.05 & 37.82 & 37.86 & 37.58 & 37.35 & 37.15 & 36.87 & 36.83 \\
\hline 7 & 39.76 & 39.25 & 38.69 & 38.23 & 38.00 & 37.80 & 37.66 & 37.66 & 37.35 & 37.14 & 36.87 & 36.63 \\
\hline 8 & 39.69 & 39.26 & 38.57 & 38.17 & 38.04 & 37.78 & 37.64 & 37.54 & 37.35 & 37.14 & 36.86 & 36.64 \\
\hline 9 & 39.73 & 39.28 & 38.62 & 38.15 & 37.98 & 37.86 & 37.60 & 37.60 & 37.33 & 37.12 & 36.87 & 36.66 \\
\hline 10 & 39.68 & 39.22 & 38.68 & 38.18 & 37.95 & 37.84 & 37.66 & 37.49 & 37.32 & 37.11 & 36.84 & 36.60 \\
\hline $\begin{array}{l}11 \\
12\end{array}$ & $\begin{array}{l}39.69 \\
39.73\end{array}$ & $\begin{array}{l}39.19 \\
39.26\end{array}$ & $\begin{array}{l}38.63 \\
38.56\end{array}$ & $\begin{array}{l}38.16 \\
38.12\end{array}$ & $\begin{array}{l}37.97 \\
37.97\end{array}$ & $\begin{array}{l}37.81 \\
37.80\end{array}$ & $\begin{array}{l}37.68 \\
37.61\end{array}$ & $\begin{array}{l}37.60 \\
37.50\end{array}$ & $\begin{array}{l}37.33 \\
37.33\end{array}$ & $\begin{array}{l}37.10 \\
37.11\end{array}$ & $\begin{array}{l}36.82 \\
36.82\end{array}$ & $\begin{array}{l}36.66 \\
36.66\end{array}$ \\
\hline 13 & 39.66 & 39.19 & 38.62 & 38.08 & 37.90 & 37.73 & 37.66 & 37.49 & 37.31 & 37.14 & 36.82 & 36.57 \\
\hline 14 & 39.67 & 39.08 & 38.49 & 38.08 & 37.86 & 37.71 & 37.65 & 37.61 & 37.30 & 37.09 & 36.80 & 38.68 \\
\hline 16 & 39.68 & 39.67 & 38.49 & 38.13 & 37.97 & 37.76 & 37.63 & 37.60 & 37.29 & 37.06 & 36.80 & 36.64 \\
\hline 16 & 39.64 & 39.08 & 38.47 & 38.11 & 37.97 & 37.70 & 37.61 & 37.46 & 37.28 & 37.05 & 36.81 & 36.56 \\
\hline 17 & 39.62 & 39.83 & 38.46 & 38.06 & 37.98 & 37.76 & 37.58 & 37.45 & 37.29 & 37.03 & 36.80 & 36.67 \\
\hline 18 & -39.62 & 39.10 & 38.46 & 38.65 & 37.86 & 37.76 & 37.68 & 37.46 & 37.30 & 37.06 & 36.79 & 36.58 \\
\hline 19 & 39.62 & 38.98 & 38.41 & 38.08 & 37.95 & 37.76 & 37.68 & 37.45 & 37.25 & 37.06 & 36.78 & 36.55 \\
\hline 20 & 39.69 & 39.08 & 38.43 & 38.10 & 37.95 & 37.72 & 37.68 & 37.43 & 37.25 & 37.05 & 36.78 & 38.66 \\
\hline 21 & 39.64 & 38.97 & 38.43 & 38.02 & 37.88 & 37.73 & 37.67 & 37.48 & 37.26 & 37.03 & 36.77 & 36.57 \\
\hline 22 & 39.62 & 38.94 & 38.39 & 38.06 & 37.89 & 37.65 & 37.69 & 37.50 & 37.26 & 37.01 & 36.77 & 36.56 \\
\hline 23 & 39.64 & 39.00 & 38.34 & 37.97 & 37.86 & 37.70 & 37.60 & 37.46 & 37.28 & 37.06 & 38.78 & 36.61 \\
\hline 24 & 39.61 & 38.93 & 38.34 & 38.01 & 37.88 & 37.76 & 37.62 & 37.41 & -- & 37.01 & 36.72 & 36.49 \\
\hline 26 & 39.49 & 38.88 & 38.36 & 38.03 & 37.88 & 37.78 & 37.61 & 37.41 & 37.25 & 37.00 & 36.71 & 36.60 \\
\hline 26 & 39.48 & 38.89 & 38.36 & 38.03 & 37.84 & 37.73 & 37.60 & 37.40 & 37.24 & 36.98 & 36.73 & 36.49 \\
\hline 27 & 39.46 & 38.79 & 38.33 & 37.97 & 37.82 & 37.66 & 37.60 & 37.41 & 37.21 & 36.96 & 36.75 & 36.48 \\
\hline 28 & 39.44 & 38.75 & 38.31 & 37.97 & 37.86 & 37.76 & 37.68 & 37.41 & 37.20 & 36.95 & 36.73 & 36.53 \\
\hline 29 & 39.46 & 38.76 & 38.31 & 38.13 & -- & 37.77 & 37.54 & 37.42 & 37.20 & 36.95 & 36.76 & 36.62 \\
\hline 30 & 39.39 & 38.69 & 38.26 & 38.12 & -- & 37.72 & 37.54 & 37.48 & 37.21 & 36.94 & 36.76 & 36.49 \\
\hline 31 & 39.39 & -- & 38.27 & 38.01 & -- & 37.70 & -- & 37.38 & -- & 36.93 & 36.71 & -- \\
\hline Mean & 39.61 & 39.08 & 38.48 & 38.11 & 37.95 & 37.77 & 37.63 & 37.48 & -- & 37.06 & 36.80 & 36.67 \\
\hline $\begin{array}{l}\text { Max. } \\
\text { Min. }\end{array}$ & $\begin{array}{l}39.77 \\
39.39\end{array}$ & $\begin{array}{l}39.42 \\
38.69\end{array}$ & $\begin{array}{l}38.71 \\
38.26\end{array}$ & $\begin{array}{l}38.31 \\
37.97\end{array}$ & $\begin{array}{l}38.08 \\
37.82\end{array}$ & $\begin{array}{l}37.91 \\
37.66\end{array}$ & $\begin{array}{l}37.75 \\
37.64\end{array}$ & $\begin{array}{l}37.69 \\
37.38\end{array}$ & -- & $\begin{array}{l}37.18 \\
36.93\end{array}$ & $\begin{array}{l}36.92 \\
36.76\end{array}$ & $\begin{array}{l}36.68 \\
36.48\end{array}$ \\
\hline
\end{tabular}


Table 3.--Daily mean water-level measurements in recorder wells in southwestern Oklahoma--Continued

HARMON COUNTY, OKLAHOMA--CONtinued

$04 \mathrm{~N}-25 \mathrm{~W}-30$ BBAB 1--Continued

Water Year, October 1987 to September 1988

Day Det. Nov. Dec. Jan. Feb. Mar. Apr. May June July Aug. Sept.

\begin{tabular}{|c|c|c|c|c|c|c|c|c|c|c|c|c|}
\hline 1 & 36.46 & 36.35 & 36.25 & 36.36 & 36.36 & 36.39 & 36.64 & 36.72 & 38.95 & 37.10 & 37.26 & 37.35 \\
\hline 2 & 38.63 & 36.32 & 36.24 & 36.28 & 36.36 & 36.38 & 36.68 & 36.74 & 36.93 & 37.10 & 37.22 & 37.37 \\
\hline 3 & 36.61 & 36.32 & 36.29 & 36.27 & 36.36 & 36.47 & 36.68 & 36.82 & 36.97 & 37.08 & 37.24 & 37.37 \\
\hline 4 & 36.42 & 36.35 & 36.26 & 36.36 & 36.43 & 36.46 & 36.66 & 36.84 & 36.96 & 37.11 & 37.24 & 37.36 \\
\hline 6 & 36.46 & 36.37 & 36.20 & 36.30 & 36.46 & 36.60 & 36.67 & 36.81 & 36.97 & 37.13 & 37.24 & 37.34 \\
\hline 6 & 36.46 & 36.30 & 36.26 & 36.27 & 36.37 & 36.44 & 36.69 & 36.73 & 36.96 & 37.12 & 37.24 & 37.36 \\
\hline 7 & 38.44 & 36.28 & 36.23 & 36.30 & 36.34 & 36.43 & 36.69 & 36.72 & 36.92 & 37.13 & 37.23 & 37.32 \\
\hline 8 & 36.39 & 38.38 & 36.27 & 36.28 & 38.33 & 36.61 & 36.69 & 36.76 & 36.91 & 37.14 & 37.25 & 37.34 \\
\hline 9 & 38.47 & 36.37 & 36.26 & 36.31 & 36.37 & 38.45 & 36.71 & 36.86 & 37.62 & 37.11 & 37.26 & 37.39 \\
\hline 10 & 36.61 & 36.38 & 36.22 & 36.29 & 36.38 & 36.37 & 36.71 & 36.86 & 37.04 & 37.11 & 37.26 & 37.38 \\
\hline 11 & 36.47 & 36.32 & 36.24 & 36.19 & 36.41 & 36.46 & 38.65 & 36.88 & 36.89 & 37.11 & 37.25 & 37.36 \\
\hline 12 & 36.39 & 36.28 & 36.26 & 36.35 & 36.34 & 36.64 & 36.65 & 36.84 & 36.97 & 37.12 & 37.26 & 37.41 \\
\hline 13 & 36.38 & 36.26 & 36.25 & 36.35 & 36.26 & 36.68 & 36.63 & 36.83 & 37.01 & 37.14 & 37.27 & 37.41 \\
\hline 14 & 36.39 & 36.24 & 38.25 & 36.28 & 36.37 & 36.62 & 36.65 & 36.80 & 37.08 & 37.15 & 37.29 & 37.38 \\
\hline 15 & 36.39 & 36.27 & 36.30 & 36.21 & 36.36 & 36.61 & 36.69 & 36.80 & 37.04 & 37.16 & 37.28 & 37.38 \\
\hline 16 & 36.43 & 36.31 & 36.31 & 36.23 & 36.32 & 36.53 & 36.67 & 36.86 & 37.01 & 37.17 & 37.29 & 37.40 \\
\hline 17 & 36.40 & 36.31 & 36.26 & 36.26 & 36.41 & 36.69 & 36.61 & 36.83 & 38.98 & 37.16 & 37.29 & 37.41 \\
\hline 18 & .36 .35 & 36.40 & 36.21 & 36.19 & 36.36 & 36.69 & 36.68 & 36.83 & 37.01 & 37.16 & 37.28 & 37.32 \\
\hline 19 & 36.41 & 36.36 & 36.21 & 36.28 & 36.38 & 36.64 & 36.67 & 36.83 & 37.63 & 37.19 & 37.27 & 37.41 \\
\hline 20 & 36.46 & 36.31 & 36.33 & 36.34 & 36.41 & 36.60 & 36.66 & 36.86 & 37.05 & 37.22 & 37.27 & 37.42 \\
\hline 21 & 38.37 & 36.23 & 36.24 & 36.32 & 36.38 & 36.51 & 36.62 & 36.88 & 37.04 & 37.19 & 37.29 & 37.41 \\
\hline 22 & 36.34 & 36.26 & 36.26 & 36.34 & 36.35 & 36.50 & 36.68 & 36.88 & 37.03 & 37.14 & 37.31 & 37.38 \\
\hline 23 & 36.37 & 36.28 & 36.18 & 36.25 & 36.49 & 36.53 & 36.77 & 36.90 & 37.06 & 37.14 & 37.34 & 37.40 \\
\hline 24 & 38.40 & 36.28 & 36.29 & 36.37 & 36.47 & 36.53 & 36.69 & 36.92 & 37.68 & 37.19 & 37.33 & 37.44 \\
\hline 26 & 36.32 & 36.30 & 36.28 & 36.33 & 36.46 & 36.68 & 36.74 & 36.93 & 37.68 & 37.20 & 37.29 & 37.44 \\
\hline 26 & 36.38 & 36.29 & 36.29 & 36.38 & 36.41 & 36.63 & 36.78 & 36.91 & 37.06 & 37.20 & 37.29 & 37.41 \\
\hline 27 & 36.40 & 36.28 & 36.26 & 36.35 & 36.46 & 36.62 & 36.79 & 36.88 & 37.07 & 37.20 & 37.31 & 37.38 \\
\hline 28 & 36.32 & 36.25 & 36.35 & 36.32 & 36.42 & 36.51 & 36.75 & 36.88 & 37.06 & 37.20 & 37.38 & 37.38 \\
\hline 29 & 36.32 & 36.26 & 36.32 & 36.28 & 36.47 & 36.64 & 36.72 & 36.86 & 37.63 & 37.23 & 37.35 & 37.44 \\
\hline 36 & 36.33 & 36.26 & 36.21 & 36.26 & - & 36.57 & 36.78 & 36.89 & 37.05 & 37.23 & 37.31 & 37.40 \\
\hline 31 & 36.36 & -- & 36.33 & 36.31 & -- & 36.66 & -- & 36.93 & -- & 37.20 & 37.31 & - \\
\hline $\begin{array}{l}\text { Moan } \\
\text { Mox. } \\
\text { Min. }\end{array}$ & $\begin{array}{l}38.41 \\
36.63 \\
36.32\end{array}$ & $\begin{array}{l}36.30 \\
36.40 \\
36.23\end{array}$ & $\begin{array}{l}36.26 \\
36.36 \\
36.18\end{array}$ & $\begin{array}{l}36.30 \\
36.38 \\
36.19\end{array}$ & $\begin{array}{l}36.39 \\
36.49 \\
36.26\end{array}$ & $\begin{array}{l}36.51 \\
36.64 \\
36.37\end{array}$ & $\begin{array}{l}36.67 \\
36.78 \\
36.54\end{array}$ & $\begin{array}{l}36.84 \\
36.93 \\
36.72\end{array}$ & $\begin{array}{l}37.01 \\
37.08 \\
36.91\end{array}$ & $\begin{array}{l}37.16 \\
37.23 \\
37.08\end{array}$ & $\begin{array}{l}37.28 \\
37.38 \\
37.20\end{array}$ & $\begin{array}{l}37.38 \\
37.44 \\
37.32\end{array}$ \\
\hline
\end{tabular}


Table 3.--Daily mean water-level measurements in recorder wells in southwestern Oklahoma--Continued

HARMON COUNTY, OKLAHOMA--CONTInUEd

$64 \mathrm{~N}-25 W-30$ BBAB $1--$ Continued

\begin{tabular}{|c|c|c|c|c|c|c|c|c|c|c|c|c|}
\hline \multirow[b]{2}{*}{ Day } & \multirow[b]{2}{*}{ Oct. } & \multirow[b]{2}{*}{ Nov. } & \multirow[b]{2}{*}{ Dec. } & \multirow{2}{*}{$\begin{array}{l}\text { Water } \\
\text { Jan. }\end{array}$} & \multirow{2}{*}{\multicolumn{2}{|c|}{$\begin{array}{l}\text { ar, October } 1988 \text { t } \\
\text { Feb. Mar. }\end{array}$}} & \multicolumn{2}{|c|}{ September 1989} & \multirow[b]{2}{*}{ June } & \multirow[b]{2}{*}{ July } & \multirow[b]{2}{*}{ Aug. } & \multirow[b]{2}{*}{ Sopt. } \\
\hline & & & & & & & Apr. & May & & & & \\
\hline 1 & 37.38 & 37.19 & 37.22 & 37.12 & -- & -- & -- & -- & -- & -- & -- & -- \\
\hline 2 & 37.44 & 37.12 & 37.19 & 37.12 & -- & -- & -- & -- & -- & -- & -- & -- \\
\hline 3 & 37.42 & 37.12 & 37.26 & 37.20 & -- & -- & -- & -- & -- & -- & -- & -- \\
\hline 4 & 37.43 & 37.19 & 37.25 & 37.11 & -- & -- & -- & -- & -- & -- & -- & -- \\
\hline 5 & 37.41 & 37.26 & 37.16 & 37.05 & -- & -- & -- & -- & -- & -- & -- & -- \\
\hline 6 & 37.39 & 37.17 & 37.09 & 37.06 & -- & -- & -- & -- & -- & -- & -- & -- \\
\hline 7 & 37.43 & 37.20 & 37.15 & 37.15 & -- & -- & -- & -- & -- & -- & -- & -- \\
\hline 8 & 37.33 & 37.21 & 37.24 & 37.22 & -- & -- & -- & -- & -- & -- & -- & -- \\
\hline 9 & 37.35 & 37.18 & 37.15 & 37.14 & -- & -- & -- & -- & -- & -- & -- & -- \\
\hline 16 & 37.36 & 37.26 & 37.13 & 37.11 & -- & -- & -- & -- & -- & -- & -- & -- \\
\hline 11 & 37.36 & 37.15 & 37.16 & 37.12 & -- & -- & -- & -- & -- & -- & -- & -- \\
\hline 12 & 37.38 & 37.20 & 37.16 & 37.25 & -- & -- & -- & -- & -- & -- & -- & -- \\
\hline 13 & 37.33 & 37.18 & 37.69 & 37.20 & -- & -- & -- & -- & -- & -- & -- & -- \\
\hline 14 & 37.27 & 37.14 & 37.12 & 37.69 & -- & -- & -- & -- & -- & -- & -- & -- \\
\hline 16 & 37.27 & 37.14 & 37.26 & 37.19 & -- & -- & -- & -- & -- & -- & -- & -- \\
\hline 16 & 37.29 & 37.28 & 37.18 & 37.17 & -- & -- & -- & -- & -- & -- & -- & -- \\
\hline 17 & 37.27 & 37.16 & 37.18 & 37.11 & -- & -- & -- & -- & -- & -- & -- & -- \\
\hline 18 & -37.35 & 37.13 & 37.08 & 37.19 & -- & -- & -- & -- & -- & -- & -- & -- \\
\hline 19 & 37.26 & 37.19 & 37.63 & -- & -- & -- & -- & -- & -- & -- & -- & -- \\
\hline 20 & 37.26 & 37.26 & 37.15 & -- & -- & -- & -- & -- & -- & -- & -- & -- \\
\hline 21 & 37.29 & 37.22 & 37.13 & -- & -- & -- & -- & -- & -- & -- & -- & -- \\
\hline 22 & 37.18 & 37.17 & 37.12 & -- & -- & -- & -- & -- & -- & -- & -- & -- \\
\hline 23 & 37.27 & 37.12 & 37.07 & -- & -- & -- & -- & -- & -- & -- & -- & -- \\
\hline 24 & 37.23 & 37.09 & 37.18 & -- & -- & -- & -- & -- & -- & -- & -- & -- \\
\hline 25 & 37.25 & 37.69 & 37.11 & -- & -- & -- & -- & -- & -- & -- & -- & -- \\
\hline 26 & 37.24 & 37.11 & 37.06 & -- & -- & -- & -- & -- & -- & -- & -- & -- \\
\hline 27 & 37.21 & 37.26 & 37.18 & -- & -- & -- & -- & -- & -- & -- & -- & -- \\
\hline 28 & 37.31 & 37.16 & 37.19 & -- & -- & -- & -- & -- & -- & -- & -- & -- \\
\hline 29 & 37.26 & 37.21 & 37.12 & -- & -- & -- & -- & -- & -- & -- & -- & -- \\
\hline 30 & 37.27 & 37.24 & 37.10 & -- & -- & -- & -- & -- & -- & -- & -- & -- \\
\hline 31 & 37.25 & -- & 37.11 & -- & -- & -- & -- & -- & -- & -- & -- & -- \\
\hline Mean & 37.31 & 37.18 & 37.15 & -- & $-\infty$ & -- & -- & - & -- & -- & -- & - \\
\hline $\operatorname{Max}$. & 37.44 & 37.26 & 37.26 & -- & -- & -- & -- & -- & -- & -- & -- & -- \\
\hline Min. & 37.18 & 37.09 & 37.63 & -- & -- & -- & -- & -- & -- & -- & -- & -- \\
\hline
\end{tabular}




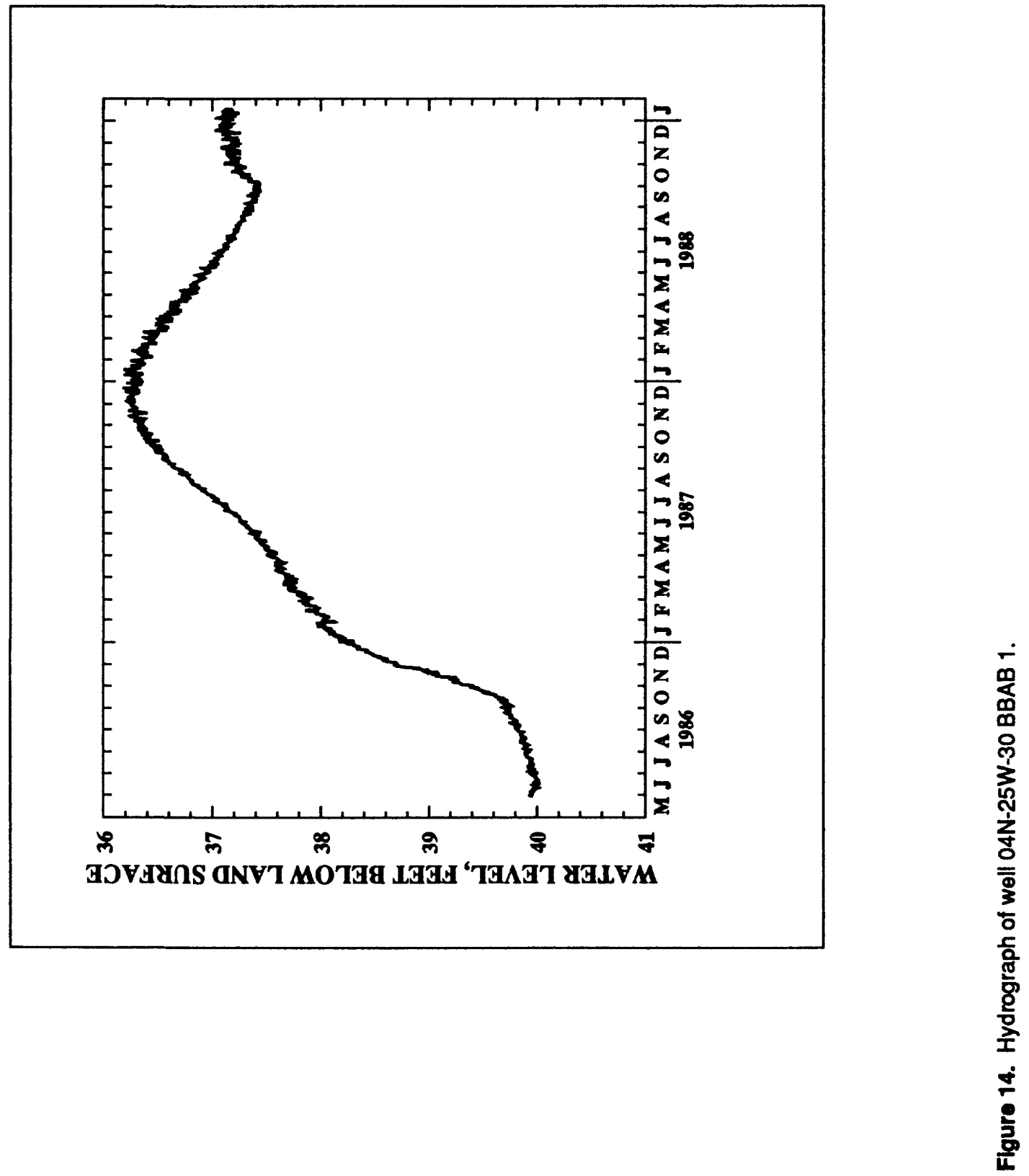


Table 3.--Daily mean water-level measurements in recorder wells in southwestern oklahoma--Continued

JACKSON COUNTY, OKLAHOMA

Index number on location map: 9

Station number: $\$ 42644099433201$ Local number:01S-24W-33 BCCC 1

Location: Lat $34^{\circ} 26^{\prime} 44^{\prime \prime}$, long $99^{\circ} 43^{\prime} 32^{\prime \prime}$, hydrologic unit 11130101

Aquifer: $313 V V C R$

Altitude of land-surface datum: $1,477 \mathrm{ft}$

Woll depth: $74 \mathrm{ft}$

Water Year, October 1986 to September 1987

Day Dct. Nov. Dec. Jan. Feb. Mar. Apr. May June July Aug. Sept.

\begin{tabular}{|c|c|c|c|c|c|c|c|c|c|c|c|c|}
\hline 1 & -- & 38.03 & -- & 37.17 & 37.33 & 36.97 & 37.29 & 37.68 & 32.90 & 33.03 & 33.35 & 34.21 \\
\hline 2 & -- & 38.11 & 37.20 & 37.01 & 37.27 & 36.96 & 37.46 & 37.63 & 32.67 & 32.98 & 33.36 & 34.18 \\
\hline 3 & -- & 38.05 & 37.24 & 37.18 & 37.26 & 37.63 & 37.41 & 37.60 & 32.70 & 32.98 & 33.39 & 34.19 \\
\hline 4 & -- & 38.03 & 37.27 & 37.18 & 37.27 & 37.10 & 37.43 & 37.71 & 32.69 & 32.93 & 33.46 & 34.19 \\
\hline 6 & -- & 37.89 & 37.20 & 37.10 & 37.42 & 37.02 & 37.46 & 37.78 & 32.68 & 32.97 & 33.48 & 34.21 \\
\hline 6 & -- & 37.68 & 37.10 & 37.13 & 37.43 & 36.99 & 37.44 & 37.82 & 32.67 & 33.00 & 33.47 & 34.25 \\
\hline 7 & -- & 37.42 & 37.86 & 37.24 & 37.34 & 36.99 & 37.44 & 37.82 & 32.71 & 33.81 & 33.61 & 34.38 \\
\hline 8 & -- & 37.46 & 37.06 & 37.21 & 37.35 & 36.94 & 37.43 & 37.82 & 32.77 & 33.04 & 33.63 & 34.38 \\
\hline 9 & -- & 37.66 & 37.14 & 37.17 & 37.33 & 37.06 & 37.36 & 37.79 & 32.80 & 33.04 & 33.68 & 34.37 \\
\hline 10 & -- & 37.49 & 37.18 & 37.26 & 37.24 & 37.12 & 37.38 & 37.77 & 32.84 & 33.03 & 33.60 & 34.38 \\
\hline 11 & -- & 37.61 & 37.09 & 37.30 & 37.28 & 37.12 & 37.29 & 37.79 & 32.92 & 33.02 & 33.67 & 34.43 \\
\hline 12 & -- & 37.68 & 37.16 & 37.26 & 37.33 & 37.14 & 37.30 & 37.82 & 32.98 & 33.04 & 33.60 & 34.46 \\
\hline 13 & -- & 37.66 & 37.13 & 37.19 & 37.23 & 37.08 & 37.42 & 37.83 & 33.00 & 33.18 & 33.60 & 34.47 \\
\hline 14 & - & 37.42 & 37.07 & 37.16 & 37.13 & 36.94 & 37.46 & 37.87 & 32.99 & 33.16 & 33.63 & 34.63 \\
\hline 16 & -- & 37.34 & 37.07 & 37.27 & 37.27 & 37.01 & 37.46 & 37.92 & 32.98 & 33.09 & 33.66 & 34.48 \\
\hline 18 & -- & 37.33 & 37.08 & 37.32 & 37.31 & 36.96 & 37.43 & 37.88 & 32.97 & 33.09 & 33.71 & 34.61 \\
\hline 17 & -- & 37.30 & 37.07 & 37.29 & 37.28 & 36.93 & 37.40 & 37.86 & 33.00 & 33.05 & 33.78 & 34.67 \\
\hline 18 & -- & 37.44 & 37.10 & 37.26 & 37.28 & 37.06 & 37.39 & 37.86 & 33.06 & 33.12 & 33.79 & 34.64 \\
\hline 19 & -- & 37.30 & 37.04 & 37.33 & 37.27 & 37.11 & 37.43 & 37.89 & 33.00 & 33.18 & 33.83 & 34.67 \\
\hline 20 & -- & 37.47 & 37.07 & 37.44 & 37.31 & 37.10 & 37.56 & 37.88 & 32.99 & 33.23 & 33.86 & 34.69 \\
\hline 21 & -- & -- & 37.16 & 37.36 & 37.24 & 37.15 & 37.66 & 37.98 & 32.98 & 33.26 & 33.89 & 34.76 \\
\hline 22 & -- & -- & 37.11 & 37.43 & 37.23 & 37.65 & 37.60 & 38.06 & 32.98 & 33.22 & 33.93 & 34.81 \\
\hline 23 & -- & -- & 37.02 & 37.32 & 37.18 & 37.65 & 37.59 & 38.04 & 33.62 & 33.20 & 33.99 & 34.77 \\
\hline 24 & -- & - & 37.00 & 37.33 & 37.20 & 37.20 & 37.66 & 37.92 & 33.05 & 33.26 & 33.97 & 34.73 \\
\hline 25 & -- & -- & 37.07 & 37.44 & 37.24 & 37.29 & 37.67 & 37.76 & 33.07 & 33.30 & 33.93 & 34.76 \\
\hline 28 & -- & -- & 37.13 & 37.61 & 37.19 & 37.29 & 37.69 & 37.49 & 33.10 & 33.29 & 33.98 & 34.77 \\
\hline 27 & -- & -- & 37.13 & 37.47 & 37.10 & 37.17 & 37.70 & 37.27 & 33.05 & 33.29 & 34.69 & 34.79 \\
\hline 28 & 37.76 & -- & 37.13 & 37.46 & 36.96 & 37.32 & 37.70 & 36.69 & 33.00 & 33.29 & 34.13 & 34.89 \\
\hline 29 & 37.85 & $\ldots$ & 37.16 & 37.48 & - & 37.41 & 37.64 & 36.44 & 32.99 & 33.31 & 34.12 & 34.97 \\
\hline 30 & 37.84 & - & 37.10 & 37.64 & -- & 37.40 & 37.61 & 34.23 & 33.04 & 33.34 & 34.12 & 34.99 \\
\hline 31 & 37.87 & -- & 37.08 & 37.39 & -- & 37.35 & - & 33.41 & -- & 33.36 & 34.19 & -- \\
\hline Mean & -- & -- & -- & 37.29 & 37.26 & 37.11 & 37.49 & 37.42 & 32.92 & 33.14 & 33.74 & 34.64 \\
\hline Max. & $\ldots$ & -- & $\ldots$ & 37.64 & 37.43 & 37.41 & 37.70 & 38.06 & 33.16 & 33.36 & 34.19 & 34.99 \\
\hline Min. & - & -- & -- & 37.01 & 36.96 & 36.93 & 37.29 & 33.41 & 32.68 & 32.93 & 33.35 & 34.18 \\
\hline
\end{tabular}


Table 3.--Daily mean water-level measurements in recorder wells in southwestern Oklahoma--Continued

JACKSON COUNTY, OKLAHOMA--CONtinued

015-24W-33 BCCC 1--Continued

Water Year, October 1987 to September 1988

Day Oct. Nov. Dec. Jan. Fob. Mar. Apr. May June July Aug. Sept.

\begin{tabular}{|c|c|c|c|c|c|c|c|c|c|c|c|c|}
\hline 1 & 34.95 & 35.89 & -- & 37.61 & 38.06 & 38.66 & 39.16 & -- & -- & -- & -- & -- \\
\hline 2 & 36.04 & 35.88 & -- & 37.69 & 38.11 & 38.61 & 39.21 & -- & -- & -- & -- & -- \\
\hline 3 & 35.14 & 35.89 & -- & 37.63 & 38.12 & 38.73 & 39.24 & -- & -- & -- & -- & -- \\
\hline 4 & 35.03 & 36.93 & -- & 37.67 & 38.22 & 38.73 & 39.23 & -- & -- & -- & -- & -- \\
\hline 5 & 35.03 & 36.06 & -- & 37.66 & 38.28 & 38.74 & 39.31 & -- & -- & -- & -- & -- \\
\hline 8 & 35.13 & 36.00 & -- & 37.62 & 38.28 & 38.70 & 39.41 & -- & -- & -- & -- & -- \\
\hline 7 & 36.16 & 36.93 & -- & 37.68 & 38.18 & 38.64 & 39.36 & -- & -- & -- & -- & -- \\
\hline 8 & 35.06 & 36.04 & -- & 37.68 & 38.18 & 38.76 & 39.32 & -- & -- & -- & -- & -- \\
\hline 9 & 35.17 & 36.15 & -- & 37.74 & 38.25 & 38.73 & 39.44 & -- & -- & -- & -- & -- \\
\hline 10 & 36.32 & 36.24 & 36.87 & 37.76 & 38.23 & 38.63 & 39.60 & -- & -- & -- & -- & -- \\
\hline 11 & 35.36 & 36.20 & 36.86 & 37.67 & 38.36 & 38.68 & 39.47 & -- & -- & -- & -- & - \\
\hline 12 & 36.28 & 36.15 & 36.96 & 37.73 & 38.28 & 38.82 & 39.48 & -- & -- & - & -- & - \\
\hline 13 & 35.24 & 36.09 & 36.97 & 37.98 & 38.21 & 38.90 & 39.47 & -- & -- & - & -- & -- \\
\hline 14 & 35.27 & 36.05 & 37.00 & 37.84 & 38.26 & 38.90 & 39.48 & -- & -- & -- & -- & -- \\
\hline 15 & 35.32 & 36.07 & 37.11 & 37.76 & 38.34 & 38.87 & 39.54 & -- & $-\infty$ & -- & -- & -- \\
\hline 16 & 35.40 & 36.20 & 37.17 & 37.72 & 38.28 & 38.90 & 39.56 & -- & -- & -- & -- & - \\
\hline 17 & 35.46 & 36.27 & 37.14 & 37.74 & 38.39 & 38.96 & 39.49 & -- & -- & -- & -- & - \\
\hline 18 & 35.39 & 36.42 & 37.09 & 37.64 & 38.39 & 39.63 & 39.63 & -- & -- & -- & -- & -- \\
\hline 19 & 35.46 & 36.43 & 37.03 & 37.66 & 38.42 & 39.00 & 39.49 & -- & -- & -- & -- & -- \\
\hline 20 & 35.61 & 36.46 & 37.23 & 37.80 & 38.47 & 38.95 & -- & -- & -- & -- & -- & -- \\
\hline 21 & 35.60 & -- & 37.20 & 37.82 & 38.49 & 38.96 & -- & -- & -- & -- & -- & -- \\
\hline 22 & 35.52 & -- & 37.22 & 37.89 & 38.41 & 38.97 & -- & -- & -- & -- & -- & -- \\
\hline 23 & 35.56 & -- & 37.12 & 37.78 & 38.60 & 39.00 & -- & -- & - & -- & -- & -- \\
\hline 24 & 35.66 & -- & 37.26 & 37.92 & 38.63 & 39.01 & -- & -- & -- & -- & -- & -- \\
\hline 25 & 35.65 & -- & 37.33 & 37.96 & 38.63 & 39.09 & -- & -- & -- & -- & -- & -- \\
\hline 26 & 36.66 & -- & 37.37 & 37.99 & 38.61 & 39.16 & -- & -- & -- & -- & -- & -- \\
\hline 27 & 36.79 & -- & 37.36 & 38.02 & 38.65 & 39.10 & -- & -- & -- & -- & -- & -- \\
\hline 28 & 36.76 & -- & 37.47 & 37.98 & 38.64 & 39.05 & -- & -- & - & -- & -- & -- \\
\hline 29 & 35.71 & -- & 37.63 & 37.96 & 38.71 & 39.21 & -- & -- & -- & -- & -- & -- \\
\hline 30 & 35.75 & -- & 37.39 & 37.90 & $=$ & 39.19 & -- & -- & -- & -- & -- & -- \\
\hline 31 & 36.83 & -- & 37.61 & 37.96 & -- & 39.16 & -- & -- & -- & -- & -- & -- \\
\hline Mean & 35.40 & -- & -- & 37.78 & 38.37 & 38.90 & -- & -- & -- & -- & -- & - \\
\hline Max. & 35.83 & -- & -- & 38.62 & 38.71 & 39.21 & -- & -- & -- & -- & -- & -- \\
\hline Min. & 34.95 & -- & -- & 37.63 & 38.06 & 38.61 & -- & -- & -- & -- & -- & - \\
\hline
\end{tabular}




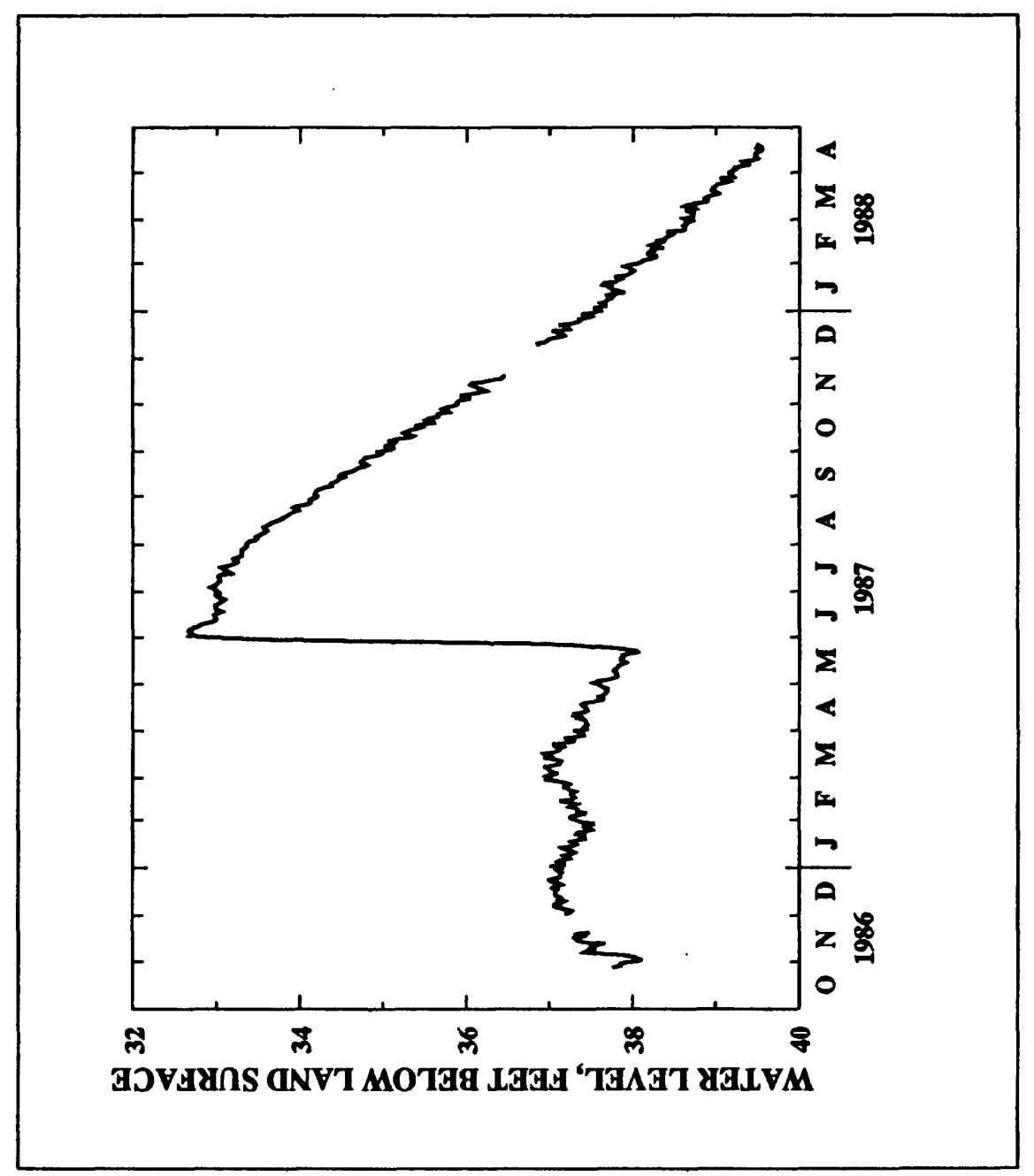

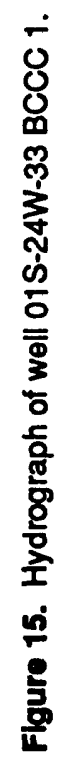


Table 3.--Daily mean water-Level measurements in recorder wells in southwestern Oklahoma--Continued

JACKSON COUNTY, OKLAHOMA--CONTInUEd

Index number on location map: 10

Station number: $\$ 43410099364401$ Local number: $01 \mathrm{~N}-23 W-10$ BBBA 1

Location: Lat $34^{\circ} 34^{\prime} 10^{\prime \prime}$, long $99^{\circ} 36^{\prime} 44^{\prime \prime}$, hydrologic unit 11130101

Aquifer: $313 V V C R$

Altitude of land-surface datum: $1,432 \mathrm{ft}$

Well depth: $49 \mathrm{ft}$

Water Year, October 1988 to September 1987

Day Dct. Nov. Dec. Jan. Fob. Mor. Apr. May Juno July Aug. Sept.

\begin{tabular}{|c|c|c|c|c|c|c|c|c|c|c|c|c|}
\hline 1 & - & 8.71 & 9.30 & 11.27 & 12.74 & 12.76 & 13.27 & 14.27 & 8.86 & 8.46 & 10.06 & 11.84 \\
\hline 2 & -- & 8.76 & 9.41 & 11.23 & 12.74 & 12.78 & 13.36 & 14.29 & 8.84 & 8.48 & 10.11 & 11.97 \\
\hline 3 & -- & 8.67 & 9.62 & 11.36 & 12.77 & 12.82 & 13.38 & 14.36 & 8.89 & 8.54 & 10.18 & 12.01 \\
\hline 4 & -- & 8.18 & 9.61 & 11.43 & 12.81 & 12.88 & 13.40 & 14.46 & 8.85 & 8.67 & 10.27 & 12.86 \\
\hline 6 & -- & 8.03 & 9.63 & 11.44 & 12.83 & 12.85 & 13.43 & 14.62 & 8.77 & 8.64 & 10.36 & 12.11 \\
\hline 6 & -- & 8.66 & 9.64 & 11.61 & 12.79 & 12.83 & 13.45 & 14.68 & 8.72 & 8.72 & 10.37 & 12.16 \\
\hline 7 & -- & 8.69 & 9.67 & 11.64 & 12.76 & 12.82 & 13.48 & 14.62 & 8.67 & 8.79 & 10.43 & 12.21 \\
\hline 8 & -- & 8.22 & 9.74 & 11.67 & 12.73 & 12.80 & 13.48 & 14.66 & 8.66 & 8.89 & 10.48 & 12.29 \\
\hline 9 & -- & 8.36 & 9.84 & 11.68 & 12.76 & 12.82 & 13.49 & 14.68 & 6.63 & 8.95 & 10.56 & 12.34 \\
\hline 10 & -- & 8.36 & 9.94 & 11.77 & 12.71 & 12.89 & 13.68 & 14.69 & 8.49 & 9.00 & 10.63 & 12.37 \\
\hline 11 & $-\infty$ & 8.42 & 9.94 & 11.86 & 12.72 & 12.94 & 13.60 & 14.73 & 8.37 & 9.06 & 10.86 & 12.43 \\
\hline 12 & -- & 8.48 & 10.05 & 11.88 & 12.76 & 12.95 & 13.60 & 14.78 & 8.36 & 9.12 & 10.70 & 12.49 \\
\hline 13 & -- & 8.60 & 10.11 & 11.90 & 12.76 & 12.93 & 13.67 & 14.82 & 8.26 & 9.20 & 10.77 & 12.64 \\
\hline 14 & -- & 8.46 & 10.13 & 11.92 & 12.71 & 12.86 & 13.62 & 14.87 & 8.23 & 9.24 & 10.83 & 12.68 \\
\hline 16 & -- & 8.42 & 10.19 & 12.03 & 12.72 & 12.88 & 13.67 & 14.92 & 8.23 & 9.26 & 10.90 & 12.61 \\
\hline 16 & -- & 8.43 & 10.27 & 12.11 & 12.76 & 12.87 & 13.69 & 14.96 & 8.24 & 9.30 & 10.96 & 12.85 \\
\hline 17 & 11.32 & 8.46 & 10.32 & 12.16 & 12.82 & 12.84 & 13.71 & 14.96 & 8.29 & 9.08 & 11.04 & 12.74 \\
\hline 18 & 11.31 & 8.62 & 10.41 & 12.19 & 12.86 & 12.89 & 13.72 & 14.99 & 8.37 & 9.69 & 11.11 & 12.82 \\
\hline 19 & 11.31 & 8.59 & 10.43 & 12.28 & 12.88 & 12.93 & 13.76 & 15.04 & 8.36 & 9.21 & 11.17 & 12.86 \\
\hline 20 & 11.27 & 8.81 & 10.61 & 12.39 & 12.91 & 12.94 & 13.86 & 16.07 & 8.38 & 9.32 & 11.23 & 12.90 \\
\hline 21 & 10.65 & 8.76 & 10.61 & 12.40 & 12.91 & 12.97 & 13.96 & 16.14 & 8.42 & 9.40 & 11.30 & 12.97 \\
\hline 22 & 8.61 & 8.74 & 10.66 & 12.48 & 12.91 & 12.96 & 13.96 & 16.22 & 8.46 & 9.46 & 11.37 & 13.00 \\
\hline 23 & 8.27 & 8.90 & 10.68 & 12.47 & 12.90 & 12.96 & 13.95 & 15.08 & 8.61 & 9.61 & 11.44 & 13.02 \\
\hline 24 & 8.40 & 8.98 & 10.69 & 12.61 & 12.91 & 13.01 & 13.99 & 14.92 & 8.42 & 9.69 & 11.49 & 13.02 \\
\hline 25 & 8.44 & 8.93 & 10.80 & 12.61 & 12.89 & 13.10 & 14.03 & 14.11 & 8.28 & 9.68 & 11.61 & 13.04 \\
\hline 28 & 8.48 & 9.07 & 10.89 & 12.69 & 12.61 & 13.14 & 14.08 & 13.27 & 8.36 & 9.73 & 11.67 & 13.06 \\
\hline 27 & 8.56 & 9.13 & 10.96 & 12.72 & 12.74 & 13.11 & 14.12 & 13.21 & 8.38 & 9.79 & 11.65 & 13.16 \\
\hline 28 & 8.62 & 9.16 & 11.02 & 12.70 & 12.69 & 13.17 & 14.18 & 11.48 & 8.41 & 9.86 & 11.73 & 13.26 \\
\hline 29 & 8.69 & 9.18 & 11.08 & 12.72 & $-\infty$ & 13.25 & 14.20 & 9.32 & 8.46 & 9.91 & 11.77 & 13.32 \\
\hline 30 & 8.66 & 9.18 & 11.12 & 12.81 & - & 13.29 & 14.22 & 8.90 & 8.41 & 9.98 & 11.81 & 13.40 \\
\hline 31 & 8.63 & -- & 11.16 & 12.77 & -- & 13.29 & -- & 8.88 & -- & 10.03 & 11.89 & - \\
\hline Mean & -- & 8.62 & 10.27 & 12.08 & 12.79 & 12.96 & 13.72 & 13.99 & 8.48 & 9.22 & 10.98 & 12.64 \\
\hline Max. & -- & 9.18 & 11.16 & 12.81 & 12.91 & 13.29 & 14.22 & 15.22 & 8.89 & 10.63 & 11.89 & 13.40 \\
\hline Min. & -- & 8.03 & 9.30 & 11.23 & 12.61 & 12.76 & 13.27 & 8.88 & 8.23 & 8.46 & 10.08 & 11.94 \\
\hline
\end{tabular}


Table 3.--Daily mean water-level measurements in recorder wells in southwestern Oklahoma--Continued

JACKSON COUNTY, OKLAHOMA--Continued

61N-23W-10 BBBA 1--Continued

\begin{tabular}{|c|c|c|c|c|c|c|c|c|c|c|c|c|}
\hline \multirow[b]{2}{*}{ Day } & \multirow[b]{2}{*}{ Oct. } & \multirow[b]{2}{*}{ Nov. } & \multirow[b]{2}{*}{ Dec. } & \multicolumn{5}{|c|}{ Water Year, October 1987 to September 1988} & \multirow[b]{2}{*}{ June } & \multirow[b]{2}{*}{ July } & \multirow[b]{2}{*}{ Aug. } & \multirow[b]{2}{*}{ Sept. } \\
\hline & & & & Jan. & Feb. & Mar. & Apr. & May & & & & \\
\hline 1 & 13.42 & 12.92 & 13.96 & 15.20 & 15.38 & 16.00 & 16.11 & 16.27 & 16.93 & 16.60 & 17.69 & 18.10 \\
\hline 2 & 13.47 & 13.00 & 13.98 & 16.22 & 16.45 & 15.80 & 16.13 & 16.26 & 16.93 & 16.61 & 17.20 & 18.11 \\
\hline 3 & 13.66 & 13.06 & 14.06 & 16.20 & 16.46 & 14.47 & 16.16 & 16.36 & 16.93 & 16.62 & 17.32 & 18.12 \\
\hline 4 & 13.67 & 13.13 & 14.11 & 16.28 & 16.63 & 14.33 & 16.16 & 16.36 & 16.94 & 16.64 & 17.34 & 18.12 \\
\hline 6 & 13.68 & 13.23 & 14.10 & 16.30 & 15.68 & 14.63 & 15.22 & 15.38 & 16.94 & 18.66 & 17.36 & 18.12 \\
\hline B & 13.66 & 13.24 & 14.15 & 16.29 & 16.60 & 14.82 & 16.33 & 15.36 & 16.94 & 16.71 & 17.37 & 18.12 \\
\hline 7 & 13.70 & 13.21 & 14.22 & 16.32 & 16.64 & 14.63 & 16.33 & 15.34 & 16.04 & 16.77 & 17.37 & 18.12 \\
\hline 8 & 13.71 & 13.26 & 14.27 & 16.33 & 16.54 & 14.72 & 16.33 & 16.34 & 16.11 & 16.78 & 17.38 & 18.12 \\
\hline 9 & 13.76 & 13.30 & 14.34 & 16.37 & 16.68 & 14.74 & 16.41 & 16.42 & 16.14 & 16.71 & 17.46 & 18.13 \\
\hline 10 & 13.87 & 13.38 & 14.36 & 15.41 & 16.67 & 14.71 & 15.60 & 15.46 & 16.19 & 16.69 & 17.61 & 18.13 \\
\hline 11 & 13.94 & 13.40 & 14.39 & 16.38 & 16.65 & 14.72 & 16.61 & 15.60 & 16.22 & 16.71 & 17.62 & 18.13 \\
\hline 12 & 13.96 & 13.39 & 14.47 & 16.43 & 16.63 & 14.86 & 16.52 & 16.62 & 16.24 & 16.72 & 17.64 & 18.13 \\
\hline 13 & 13.95 & 13.37 & 14.51 & 16.64 & 16.69 & 14.87 & 16.63 & 16.63 & 16.26 & 16.73 & 17.67 & 18.13 \\
\hline 14 & 13.98 & 13.36 & 14.65 & 16.61 & 15.60 & 14.89 & 16.65 & 15.63 & 16.29 & 16.76 & 17.68 & 18.14 \\
\hline 16 & 14.02 & 13.36 & 14.64 & 16.48 & 16.66 & 14.88 & 16.60 & 16.63 & 16.34 & 16.82 & 17.61 & 18.14 \\
\hline 16 & 14.10 & 13.39 & 14.71 & 16.43 & 15.65 & 14.90 & 16.63 & 15.67 & 16.36 & 16.89 & 17.64 & 18.16 \\
\hline 17 & 14.17 & 13.46 & 14.72 & 16.40 & 16.67 & 14.92 & 16.49 & 16.69 & 16.37 & 16.89 & 17.66 & 18.16 \\
\hline 18 & 14.17 & 13.56 & 14.72 & 16.30 & 15.68 & 14.98 & 16.10 & 16.60 & 16.37 & 16.89 & 17.69 & 18.26 \\
\hline 19 & 14.21 & 13.62 & 14.70 & 16.27 & 16.68 & 14.98 & 16.12 & 16.61 & 16.38 & 16.98 & 17.72 & 18.34 \\
\hline 20 & 14.33 & 13.66 & 14.78 & 16.30 & 16.69 & 14.95 & 16.16 & 16.64 & 16.46 & 17.05 & 17.76 & 18.32 \\
\hline 21 & 14.36 & 13.61 & 14.82 & 16.31 & 15.70 & 14.94 & 16.67 & 16.67 & 16.46 & 17.07 & 17.77 & 18.32 \\
\hline 22 & 14.38 & 13.69 & 14.84 & 16.33 & 16.70 & 14.94 & 16.08 & 15.69 & 16.51 & 17.07 & 17.79 & 18.32 \\
\hline 23 & 14.41 & 13.65 & 14.80 & 16.27 & 16.70 & 14.96 & 16.14 & 16.70 & 16.62 & 17.07 & 17.84 & 18.32 \\
\hline 24 & 14.44 & 13.69 & 14.87 & 15.32 & 15.70 & 14.98 & 16.16 & 16.72 & 16.64 & 17.67 & 17.88 & 18.30 \\
\hline 25 & 14.47 & 13.76 & 14.94 & 16.36 & 16.70 & 16.01 & 16.16 & 16.72 & 16.66 & 17.08 & 17.90 & 18.30 \\
\hline 28 & 14.48 & 13.79 & 14.98 & 15.36 & 16.70 & 16.65 & 15.18 & 16.72 & 16.67 & 17.68 & 17.91 & 18.30 \\
\hline 27 & 14.68 & 13.84 & 14.99 & 16.40 & 16.89 & 16.04 & 16.26 & 15.73 & 16.69 & 17.68 & 17.92 & 18.30 \\
\hline 28 & 14.60 & 13.86 & 16.08 & 16.38 & 16.94 & 16.00 & 15.26 & 16.73 & 16.59 & 17.68 & 17.97 & 18.29 \\
\hline 29 & 14.69 & 13.89 & 16.12 & 16.34 & 16.99 & 16.10 & 16.24 & 15.73 & 16.69 & 17.69 & 18.01 & 18.29 \\
\hline 30 & 14.58 & 13.92 & 15.68 & 16.31 & - & 16.12 & 16.27 & 16.73 & 16.59 & 17.69 & 18.03 & 18.29 \\
\hline 31 & 13.36 & $\ldots$ & 16.12 & 16.33 & -- & 16.11 & - & 16.79 & -- & 17.09 & 18.67 & -- \\
\hline Mean & 14.04 & 13.46 & 14.69 & 16.34 & 15.66 & 14.92 & 15.29 & 16.66 & 16.29 & 16.87 & 17.64 & 18.20 \\
\hline Max & 14.60 & 13.92 & 15.12 & 15.64 & 16.99 & 16.00 & 16.63 & 16.79 & 16.59 & 17.69 & 18.07 & 18.34 \\
\hline Min. & 13.36 & 12.92 & 13.96 & 15.20 & 16.38 & 14.33 & 16.67 & 16.26 & 16.93 & 16.60 & 17.69 & 18.10 \\
\hline
\end{tabular}


Table 3.--Daily mean water-level measurements in recorder wells in southwestern Oklahoma--Continued

JACKSON COUNTY, OKLAHOMA--COntinued

01N-23W-10 BBBA 1--Continued

\begin{tabular}{|c|c|c|c|c|c|c|c|c|c|c|c|c|}
\hline \multirow[b]{2}{*}{ Day } & \multirow[b]{2}{*}{ Oct. } & \multirow[b]{2}{*}{ Nov. } & \multirow[b]{2}{*}{ Dec. } & \multirow{2}{*}{$\begin{array}{l}\text { Water } \\
\text { Jan. }\end{array}$} & \multicolumn{4}{|c|}{ October 1988 to September 1989} & \multirow[b]{2}{*}{ June } & \multirow[b]{2}{*}{ July } & \multirow[b]{2}{*}{ Aug. } & \multirow[b]{2}{*}{ Sept. } \\
\hline & & & & & Feb. & Mar. & Apr. & May & & & & \\
\hline 1 & 18.29 & 18.51 & 19.39 & 19.95 & -- & -- & -- & -- & -- & -- & -- & -- \\
\hline 2 & 18.29 & 18.61 & 19.46 & 19.96 & -- & -- & -- & -- & -- & -- & -- & -- \\
\hline 3 & 18.29 & 18.61 & 19.46 & 20.06 & -- & -- & -- & -- & -- & -- & -- & -- \\
\hline 4 & 18.29 & 18.67 & 19.47 & 20.63 & -- & -- & -- & -- & -- & -- & -- & -- \\
\hline 5 & 18.29 & 18.64 & 19.48 & 20.02 & -- & -- & -- & -- & -- & -- & -- & -- \\
\hline 6 & 18.29 & 18.68 & 19.48 & 20.02 & -- & -- & -- & -- & -- & -- & -- & -- \\
\hline 7 & 18.29 & 18.71 & 19.49 & 20.03 & -- & -- & -- & -- & -- & -- & -- & -- \\
\hline 8 & 18.30 & 18.80 & 19.69 & 20.10 & -- & -- & -- & $-\infty$ & -- & -- & -- & -- \\
\hline 9 & 18.30 & 18.81 & 19.64 & 20.14 & -- & -- & -- & -- & -- & -- & -- & -- \\
\hline 10 & 18.30 & 18.88 & 19.64 & 20.14 & -- & -- & -- & -- & -- & -- & -- & -- \\
\hline 11 & 18.30 & 18.92 & 19.65 & 26.14 & -- & -- & -- & -- & -- & -- & -- & -- \\
\hline 12 & 18.31 & 18.94 & 19.67 & 20.16 & -- & -- & -- & -- & -- & -- & -- & -- \\
\hline 13 & 18.31 & 18.98 & 19.68 & 20.16 & -- & -- & -- & -- & -- & -- & -- & -- \\
\hline 14 & 18.31 & 18.99 & 19.68 & 20.16 & -- & -- & -- & -- & -- & -- & -- & -- \\
\hline 15 & 18.31 & 18.99 & 19.73 & 20.16 & -- & -- & -- & -- & -- & -- & -- & -- \\
\hline 16 & 18.31 & 19.06 & 19.79 & 20.17 & -- & -- & -- & -- & -- & -- & -- & -- \\
\hline 17 & 18.32 & 19.10 & 19.79 & 20.17 & -- & -- & -- & -- & -- & -- & -- & -- \\
\hline 18 & 18.34 & 19.10 & 19.79 & 20.23 & -- & -- & -- & -- & -- & -- & -- & -- \\
\hline 19 & 18.41 & 19.11 & 19.77 & -- & -- & -- & -- & -- & -- & -- & -- & -- \\
\hline 20 & 18.44 & 19.13 & 19.78 & -- & -- & -- & -- & -- & -- & -- & -- & -- \\
\hline 21 & 18.46 & 19.16 & 19.80 & -- & -- & -- & -- & -- & -- & -- & -- & -- \\
\hline 22 & 18.46 & 19.15 & 19.80 & -- & -- & -- & -- & -- & -- & -- & -- & -- \\
\hline 23 & 18.46 & 19.16 & 19.80 & -- & -- & -- & -- & -- & -- & -- & -- & -- \\
\hline 24 & 18.46 & 19.16 & 19.81 & -- & -- & -- & -- & -- & -- & -- & -- & -- \\
\hline 25 & 18.47 & 19.16 & 19.82 & -- & -- & -- & -- & -- & -- & -- & -- & -- \\
\hline 28 & 18.48 & 19.15 & 19.82 & -- & -- & -- & -- & -- & -- & -- & -- & -- \\
\hline 27 & 18.48 & 19.23 & 19.86 & -- & -- & -- & -- & -- & -- & -- & -- & -- \\
\hline 28 & 18.49 & 19.29 & 19.93 & -- & -- & -- & -- & -- & -- & -- & -- & -- \\
\hline 29 & 18.60 & 19.29 & 19.94 & -- & -- & -- & -- & -- & -- & -- & -- & -- \\
\hline 30 & 18.50 & 19.32 & 19.94 & -- & -- & -- & -- & -- & -- & -- & -- & -- \\
\hline 31 & 18.60 & -- & 19.94 & -- & -- & -- & -- & -- & -- & -- & -- & -- \\
\hline Mean & 18.37 & 18.97 & 19.71 & -- & -- & -- & -- & -- & -- & -- & -- & -- \\
\hline Max. & 18.60 & 19.32 & 19.94 & -- & -- & -- & -- & -- & -- & -- & -- & -- \\
\hline Min. & 18.29 & 18.61 & 19.39 & -- & -- & -- & -- & -- & -- & -- & -- & - \\
\hline
\end{tabular}




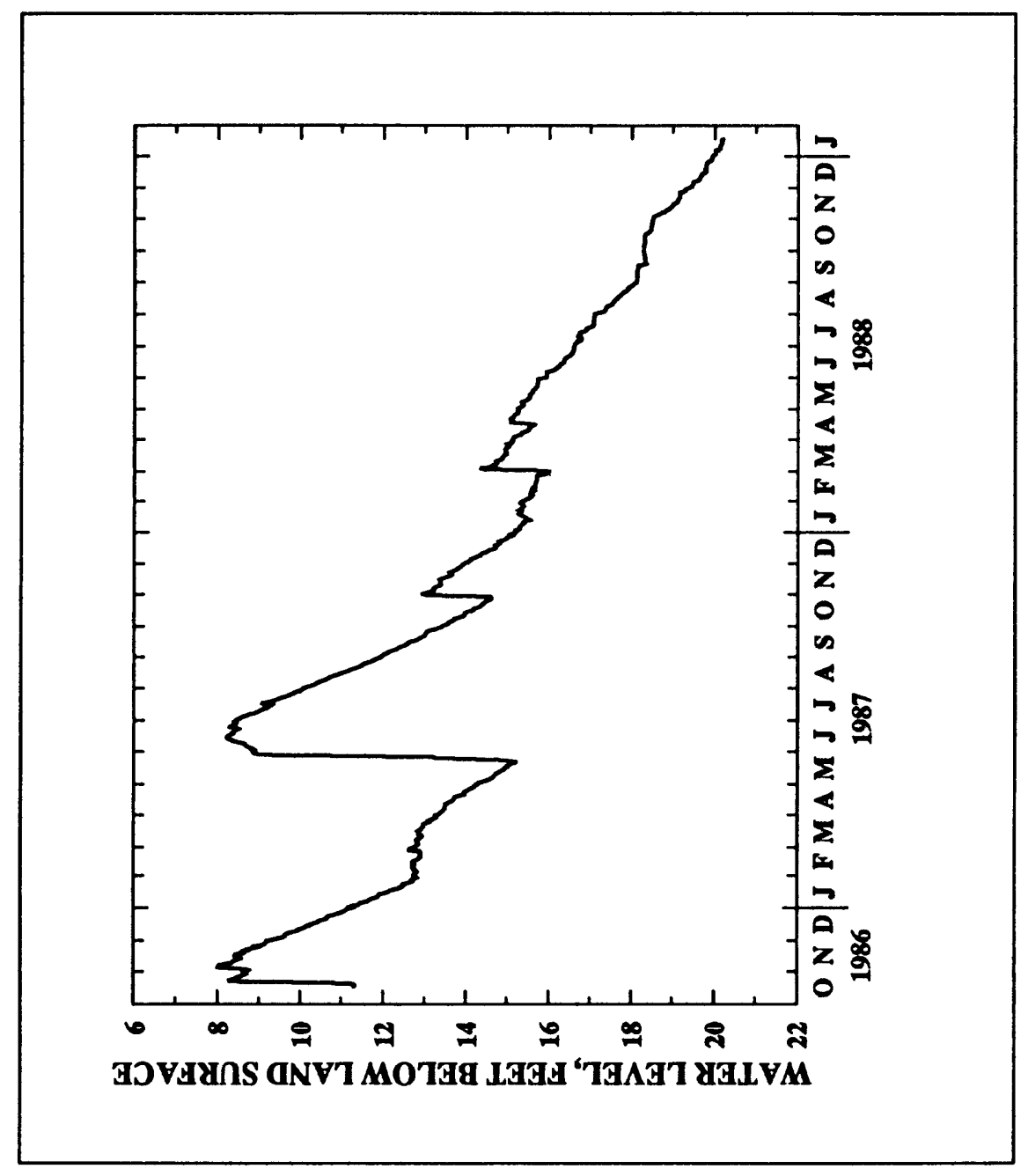


Table 3.--Daily mean water-level measurements in recorder vells in southwestern Oklahoma--Continued

JACKSON COUNTY, OKLAHOMA--CONtinuEd

Index number on location map: 11

Station number: 344204699365301 Local number: $03 N-23 W-28$ DADA 1

Location: Lat $34^{\circ} 42^{\prime} 64^{\prime \prime}$, long $99^{\circ} 36^{\prime} 63^{\prime \prime}$, hydrologic unit 11126202

Aquifer: $313 V V C R$

Altitude of land-surface datum: $1,434 \mathrm{ft}$

Well depth: $73.5 \mathrm{ft}$

Water Year, October 1986 to September 1986

Day Oct. Nov. Dec. Jan. Feb. Mer. Apr. May June July Aug. Sept.

\begin{tabular}{|c|c|c|c|c|c|c|c|c|c|c|c|c|}
\hline 1 & -- & -- & -- & -- & -- & -- & -- & -- & -- & 13.70 & 16.99 & 14.98 \\
\hline 2 & -- & -- & -- & -- & -- & -- & -- & -- & -- & 13.60 & 17.19 & 14.88 \\
\hline 3 & -- & -- & -- & $-\infty$ & -- & -- & -- & -- & -- & 13.69 & 17.46 & 14.02 \\
\hline 4 & -- & -- & -- & -- & -- & -- & -- & -- & -- & 13.54 & 17.59 & 12.99 \\
\hline 5 & -- & -- & -- & -- & -- & -- & -- & -- & -- & 13.68 & 17.58 & 12.02 \\
\hline 6 & -- & -- & -- & -- & -- & -- & -- & -- & -- & 13.68 & 17.64 & 11.94 \\
\hline 7 & -- & $-\infty$ & -- & -- & -- & -- & -- & -- & -- & 13.72 & 17.25 & 12.65 \\
\hline 8 & -- & -- & -- & -- & -- & -- & -- & -- & -- & 13.70 & 17.13 & 12.61 \\
\hline 9 & -- & -- & -- & -- & -- & -- & -- & -- & 14.11 & 13.68 & 17.01 & 11.98 \\
\hline 10 & -- & -- & -- & -- & -- & -- & -- & -- & 14.13 & 13.76 & 16.84 & 11.93 \\
\hline 11 & -- & -- & -- & -- & -- & -- & -- & -- & 14.12 & 13.88 & 16.68 & 12.04 \\
\hline 12 & -- & -- & -- & -- & -- & -- & -- & -- & 14.13 & 14.01 & 16.47 & 12.08 \\
\hline 13 & -- & -- & -- & -- & -- & -- & -- & -- & 14.17 & 14.22 & 16.32 & 12.09 \\
\hline 14 & -- & -- & -- & -- & -- & -- & -- & - & 14.17 & 14.32 & 16.33 & 11.65 \\
\hline 16 & -- & -- & -- & -- & -- & -- & -- & $\cdots$ & 14.24 & 14.28 & 16.34 & 11.30 \\
\hline 16 & -- & -- & -- & -- & -- & -- & -- & -- & 14.25 & 14.36 & 16.20 & 11.32 \\
\hline 17 & -- & - & - & -- & -- & -- & -- & -- & 14.28 & 14.49 & 16.12 & 11.31 \\
\hline 18 & -- & -- & -- & -- & -- & -- & -- & -- & 14.23 & 14.62 & 16.05 & 11.37 \\
\hline 19 & -- & -- & -- & -- & -- & -- & -- & -- & 14.20 & 14.90 & 16.03 & 11.43 \\
\hline 20 & -- & -- & -- & -- & -- & -- & -- & -- & 14.22 & 16.42 & 16.06 & 11.47 \\
\hline 21 & -- & -- & -- & - & -- & -- & -- & -- & 14.22 & 15.58 & 16.15 & 11.47 \\
\hline 22 & -- & -- & -- & -- & -- & -- & - & -- & 14.23 & 15.40 & 16.19 & 11.47 \\
\hline 23 & -- & -- & - & -- & -- & -- & -- & -- & 13.81 & 15.60 & 16.26 & 11.46 \\
\hline 24 & -- & - & -- & -- & -- & -- & -- & -- & 13.73 & 15.83 & 16.37 & 11.43 \\
\hline 26 & -- & -- & -- & -- & -- & -- & -- & -- & 13.64 & 16.90 & 16.46 & 11.65 \\
\hline 28 & -- & -- & -- & -- & -- & -- & -- & -- & 13.68 & 15.92 & 16.43 & 11.68 \\
\hline 27 & -- & -- & -- & -- & -- & -- & -- & -- & 13.72 & 16.05 & 16.34 & 11.63 \\
\hline 28 & -- & - & -- & -- & -- & -- & -- & -- & 13.71 & 16.22 & 18.04 & 11.63 \\
\hline 29 & -- & -- & -- & -- & -- & -- & -- & -- & 13.72 & 16.53 & 15.89 & 18.82 \\
\hline 30 & -- & -- & -- & -- & -- & -- & -- & -- & 13.72 & 16.80 & 15.78 & 10.69 \\
\hline 31 & -- & -- & -- & -- & -- & -- & -- & -- & -- & 16.91 & 15.37 & -- \\
\hline Mean & -- & -- & - & -- & -- & -- & -- & -- & -- & 14.77 & 16.63 & 11.95 \\
\hline Max. & -- & -- & -- & -- & -- & -- & -- & -- & -- & 16.91 & 17.59 & 14.96 \\
\hline Min. & -- & -- & -- & -- & -- & -- & -- & -- & -- & 13.54 & 16.37 & 10.69 \\
\hline
\end{tabular}


Table 3.--Daily mean water-level measurements in recorder wells in southwestern Oklahoma--Continued

JACKSON COUNTY, OKLAHOMA-CONtinued

$63 N-23 W-28$ DADA 1--Continued

Water Year, October 1986 to September 1987

\begin{tabular}{|c|c|c|c|c|c|c|c|c|c|c|c|c|}
\hline Day & Oct. & Nov. & Dec. & Jan. & Fob. & Mar. & Apr. & May & June & July & Aug. & Sept. \\
\hline 1 & 10.60 & 7.85 & 8.91 & 9.68 & 9.47 & 7.89 & 8.99 & 9.86 & 6.56 & 7.49 & 9.79 & 11.77 \\
\hline 2 & 10.13 & 7.93 & 9.00 & 9.51 & 9.40 & 7.82 & 9.21 & 9.94 & 6.81 & 7.61 & 10.20 & 11.69 \\
\hline 3 & 9.01 & 7.84 & 9.68 & 9.76 & 9.38 & 7.90 & 9.16 & 10.60 & 7.35 & 7.68 & 10.67 & 11.86 \\
\hline 4 & 9.69 & 7.73 & 9.14 & 9.71 & 9.39 & 7.98 & 9.26 & 10.89 & 7.51 & 7.68 & 10.71 & 11.76 \\
\hline 6 & 9.22 & 7.77 & 9.09 & 9.63 & 9.51 & 7.92 & 9.25 & 11.63 & 7.68 & 7.70 & 10.76 & 11.69 \\
\hline 6 & 9.26 & 7.76 & 9.02 & 9.68 & 9.46 & 7.96 & 9.21 & 11.17 & 7.68 & 7.79 & 10.51 & 11.68 \\
\hline 7 & 9.26 & 7.73 & 9.61 & 9.82 & 9.34 & 8.62 & 9.24 & 11.17 & 7.75 & 7.88 & 10.46 & 11.67 \\
\hline 8 & 9.29 & 7.91 & 9.65 & 9.76 & 9.36 & 8.63 & 9.24 & 11.19 & 7.84 & 7.98 & 10.60 & 11.76 \\
\hline 9 & 9.42 & 8.09 & 9.14 & 9.67 & 9.36 & 8.21 & 9.18 & 11.24 & 7.87 & 8.03 & 10.89 & 11.64 \\
\hline 10 & 9.37 & 8.05 & 9.19 & 9.78 & 9.26 & 8.31 & 9.26 & 11.29 & 6.70 & 8.08 & 10.88 & 11.69 \\
\hline 11 & 9.24 & 8.16 & 9.11 & 9.83 & 9.32 & 8.36 & 9.18 & 11.27 & 5.00 & 8.13 & 10.80 & 11.67 \\
\hline 12 & 9.15 & 8.28 & 9.23 & 9.77 & 9.40 & 8.41 & 9.22 & 11.22 & 5.21 & 8.21 & 10.80 & 11.47 \\
\hline 13 & 9.03 & 8.40 & 9.24 & 9.72 & 9.30 & 8.36 & 9.37 & 11.17 & 5.63 & 8.35 & 10.89 & 11.46 \\
\hline 14 & 9.06 & 8.17 & 9.21 & 9.69 & 9.21 & 8.26 & 9.43 & 11.20 & 6.86 & 8.37 & 10.94 & 11.41 \\
\hline 16 & 9.16 & 8.16 & 9.26 & 9.83 & 9.31 & 8.41 & 9.43 & 11.24 & 6.04 & 8.47 & 10.99 & 11.33 \\
\hline 16 & 9.16 & 8.21 & 9.28 & 9.90 & 9.32 & 8.38 & 9.42 & 11.30 & 6.22 & 8.60 & 11.06 & 11.32 \\
\hline 17 & 9.14 & 8.26 & 9.30 & 9.86 & 9.31 & 8.37 & 9.41 & 11.64 & 6.42 & 8.53 & 11.26 & 11.35 \\
\hline 18 & 9.20 & 8.47 & 9.36 & 9.84 & 9.29 & 8.53 & 9.43 & 11.69 & 6.63 & 8.67 & 11.33 & 11.38 \\
\hline 19 & 9.28 & 8.36 & 9.30 & 9.94 & 9.28 & 8.61 & 9.49 & 11.97 & 0.70 & 8.63 & 11.37 & 11.37 \\
\hline 20 & 9.28 & 8.60 & 9.37 & 10.11 & 9.29 & 8.62 & 9.64 & 11.79 & 6.83 & 8.71 & 11.38 & 11.36 \\
\hline 21 & 8.80 & 8.65 & 9.46 & 9.89 & 9.19 & 8.70 & 9.74 & 11.76 & 6.91 & 8.81 & 11.38 & 11.12 \\
\hline 22 & 7.39 & 8.50 & 9.44 & 10.62 & 9.20 & 8.62 & 9.64 & 11.77 & 6.94 & 8.86 & 11.47 & 11.16 \\
\hline 23 & 7.16 & 8.72 & 9.35 & 9.83 & 9.17 & 8.60 & 9.64 & 11.31 & 7.04 & 8.86 & 11.54 & 11.12 \\
\hline 24 & 7.13 & 8.74 & 9.36 & 9.85 & 9.19 & 8.77 & 9.73 & 11.03 & 7.14 & 8.92 & 11.51 & 11.06 \\
\hline 26 & 7.18 & 8.61 & 9.47 & 10.01 & 9.21 & 8.89 & 9.76 & 10.69 & 7.22 & 8.97 & 11.66 & 11.07 \\
\hline 28 & 7.34 & 8.80 & 9.54 & 10.10 & 9.12 & 8.88 & 9.79 & 10.56 & 7.32 & 8.98 & 11.80 & 11.06 \\
\hline 27 & 7.40 & 8.83 & 9.65 & 9.91 & 8.68 & 8.76 & 9.82 & 10.36 & 7.34 & 9.00 & 11.92 & 11.06 \\
\hline 28 & 7.45 & 8.83 & 9.57 & 9.74 & 8.00 & 8.97 & 9.86 & 8.96 & 7.39 & 9.02 & 11.93 & 11.11 \\
\hline 29 & 7.63 & 8.79 & 9.60 & 9.76 & -- & 9.09 & 9.81 & 6.30 & 7.46 & 9.12 & 11.81 & 11.16 \\
\hline 30 & 7.62 & 8.76 & 9.67 & 9.76 & -- & 9.07 & 9.84 & 5.99 & 7.46 & 9.25 & 11.77 & 11.14 \\
\hline 31 & 7.66 & -- & 9.56 & 9.58 & -- & 9.03 & -- & 6.29 & -- & 9.51 & 11.75 & -- \\
\hline $\begin{array}{l}\text { Mean } \\
\text { Max. } \\
\text { Mịn. }\end{array}$ & $\begin{array}{r}8.68 \\
10.66 \\
7.13\end{array}$ & $\begin{array}{l}8.29 \\
8.83 \\
7.70\end{array}$ & $\begin{array}{l}9.28 \\
9.60 \\
8.91\end{array}$ & $\begin{array}{r}9.80 \\
10.11 \\
9.51\end{array}$ & $\begin{array}{l}9.24 \\
9.61 \\
8.00\end{array}$ & $\begin{array}{l}8.44 \\
9.09 \\
7.82\end{array}$ & $\begin{array}{l}9.46 \\
9.85 \\
8.99\end{array}$ & $\begin{array}{r}10.57 \\
11.97 \\
5.99\end{array}$ & $\begin{array}{l}6.88 \\
7.87 \\
6.60\end{array}$ & $\begin{array}{l}8.44 \\
9.51 \\
7.49\end{array}$ & $\begin{array}{r}11.12 \\
11.93 \\
9.79\end{array}$ & $\begin{array}{l}11.46 \\
11.85 \\
11.05\end{array}$ \\
\hline
\end{tabular}


Table 3.--Daily mean water-level measurements in recorder wells in southwestern Oklahoma--Continued

JACKSON COUNTY, OKLAHOMA--Continued

63N-23W-28 DADA 1--Continued

Water Year, October 1987 to September 1988

Day Dct. Nov. Dec. Jan. Fob. Mar. Apr. May June July Aug. Sept.

\begin{tabular}{|c|c|c|c|c|c|c|c|c|c|c|c|c|}
\hline 1 & 11.08 & 10.68 & 10.88 & 11.02 & 10.68 & 10.84 & 10.48 & 10.39 & 11.18 & 11.67 & 12.72 & 14.24 \\
\hline 2 & 11.14 & 10.61 & 10.85 & 10.97 & 10.72 & 10.56 & 10.52 & 10.39 & 11.15 & 11.53 & 12.87 & 14.26 \\
\hline 3 & 11.21 & 10.66 & 10.93 & 10.85 & 10.68 & 10.06 & 10.68 & 10.65 & 11.21 & 11.48 & 13.09 & 14.12 \\
\hline 4 & 11.08 & 10.70 & 10.94 & 10.96 & 10.80 & 10.13 & 10.65 & 10.62 & 11.30 & 11.47 & 13.16 & 14.00 \\
\hline 5 & 11.06 & 10.81 & 10.82 & 10.93 & 10.84 & 10.19 & 10.68 & 10.60 & 11.57 & 11.46 & 13.14 & 13.88 \\
\hline 6 & 11.12 & 10.74 & 10.88 & 10.87 & 10.81 & 10.17 & 10.79 & 10.50 & 11.62 & 11.43 & 13.13 & 13.80 \\
\hline 7 & 11.11 & 10.66 & 10.90 & 10.91 & 10.67 & 10.10 & 10.67 & 10.62 & 11.44 & 11.41 & 13.16 & 13.69 \\
\hline 8 & 11.01 & 10.77 & 10.91 & 10.88 & 10.87 & 10.31 & 10.66 & 10.58 & 11.63 & 11.41 & 13.20 & 13.63 \\
\hline 9 & 11.09 & 10.86 & 10.98 & 10.94 & 10.73 & 10.25 & 10.81 & 10.73 & 11.73 & 11.10 & 13.25 & 13.65 \\
\hline 10 & 11.21 & 10.92 & 10.92 & 10.93 & 10.68 & 10.00 & 10.81 & 10.76 & 12.10 & 10.56 & 13.18 & 13.63 \\
\hline 11 & 11.22 & 10.85 & 10.89 & 10.79 & 10.84 & 10.21 & 10.74 & 10.80 & 12.24 & 10.63 & 13.32 & 13.60 \\
\hline 12 & 11.11 & 10.78 & 10.98 & 10.86 & 10.70 & 10.42 & 10.76 & 10.76 & 12.28 & 10.56 & 13.72 & 13.64 \\
\hline 13 & 11.05 & 10.71 & 10.97 & 10.97 & 10.69 & 10.51 & 10.73 & 10.76 & 12.31 & 10.67 & 14.06 & 13.64 \\
\hline 14 & 11.06 & 10.67 & 10.94 & 10.84 & 10.68 & 10.46 & 10.76 & 10.74 & 12.48 & 10.58 & 14.26 & 13.65 \\
\hline 15 & 11.87 & 10.68 & 11.05 & 10.74 & 10.75 & 10.41 & 16.87 & 10.75 & 12.63 & 10.59 & 14.22 & 13.47 \\
\hline 16 & 11.12 & 10.79 & 11.08 & 10.68 & 10.66 & 10.44 & 10.89 & 10.84 & 12.71 & 10.78 & 14.64 & 13.43 \\
\hline 17 & 11.18 & 10.84 & 11.02 & 10.50 & 10.79 & 10.47 & 10.43 & 10.82 & 12.78 & 10.92 & 14.76 & 13.46 \\
\hline 18 & -11.10 & 18.97 & 10.90 & 10.32 & 10.76 & 10.51 & 10.08 & 10.83 & 12.86 & 11.03 & 14.92 & 12.94 \\
\hline 19 & 11.18 & 10.97 & 10.70 & 10.27 & 10.77 & 10.45 & 10.13 & 10.85 & 12.91 & 11.17 & 15.00 & 11.78 \\
\hline 20 & 11.34 & 10.92 & 10.83 & 10.45 & 10.83 & 10.39 & 10.13 & 10.87 & 12.85 & 11.29 & 14.98 & 11.94 \\
\hline 21 & 11.36 & 10.79 & 10.79 & 10.49 & 10.82 & 10.41 & 10.13 & 10.90 & 12.89 & 11.33 & 14.76 & 11.97 \\
\hline 22 & 11.36 & 10.76 & 10.82 & 10.67 & 10.71 & 10.41 & 10.21 & 10.91 & 12.86 & 11.30 & 14.66 & 11.96 \\
\hline 23 & 11.43 & 10.84 & 10.71 & 10.43 & 10.96 & 10.44 & 10.38 & 10.93 & 12.83 & 11.42 & 14.60 & 11.92 \\
\hline 24 & 11.36 & 10.84 & 10.87 & 10.58 & 10.95 & 10.44 & 10.34 & 10.98 & 12.84 & 11.58 & 14.61 & 11.84 \\
\hline 26 & 11.27 & 10.91 & 10.87 & 10.64 & 10.91 & 10.56 & 10.39 & 11.03 & 12.77 & 11.68 & 14.43 & 11.84 \\
\hline 28 & 11.28 & 10.90 & 10.87 & 10.63 & 10.86 & 10.62 & 10.44 & 11.06 & 12.27 & 11.69 & 14.40 & 11.80 \\
\hline 27 & 11.37 & 10.88 & 10.86 & 10.68 & 10.89 & 10.61 & 10.62 & 11.06 & 11.99 & 11.72 & 14.38 & 11.76 \\
\hline 28 & 11.29 & 10.86 & 10.97 & 10.61 & 10.86 & 10.46 & 10.48 & 11.08 & 11.95 & 11.99 & 14.36 & 11.72 \\
\hline 29 & 11.22 & 10.86 & 11.02 & 10.66 & 10.95 & 10.66 & 10.41 & 11.09 & 11.87 & 12.11 & 14.22 & 11.81 \\
\hline 30 & 11.21 & 10.86 & 10.81 & 10.51 & -- & 10.61 & 10.47 & 11.13 & 11.83 & 12.48 & 14.18 & 11.78 \\
\hline 31 & 10.46 & - & 10.91 & 10.67 & -- & 10.64 & -- & 11.16 & -- & 12.60 & 14.20 & - \\
\hline Mean & 11.16 & 10.80 & 10.90 & 10.70 & 10.78 & 10.46 & 10.63 & 10.81 & 12.16 & 11.33 & 13.98 & 12.96 \\
\hline Max. & 11.43 & 10.97 & 11.08 & 11.82 & 10.95 & 10.84 & 10.89 & 11.16 & 12.91 & 12.60 & 15.00 & 14.26 \\
\hline Min. & 10.45 & 10.66 & 10.70 & 10.27 & 10.59 & 10.00 & 10.08 & 10.39 & 11.16 & 10.53 & 12.72 & 11.72 \\
\hline
\end{tabular}


Table 3.--Daily mean water-level measurements in recorder wells in southwestern oklahoma--Continued

JACKSON COUNTY, OKLAHOMA--CONtinued

O3N-23W-28 DADA 1--Continued

\begin{tabular}{|c|c|c|c|c|c|c|c|c|c|c|c|c|}
\hline \multirow[b]{2}{*}{ Day } & \multirow[b]{2}{*}{ oct. } & \multirow[b]{2}{*}{ Nov. } & \multirow[b]{2}{*}{ Dec. } & \multirow{2}{*}{$\begin{array}{l}\text { Water } \\
\text { Jan. }\end{array}$} & \multicolumn{2}{|c|}{ October 1988} & \multicolumn{2}{|c|}{ September 1989} & \multirow[b]{2}{*}{ June } & \multirow[b]{2}{*}{ July } & \multirow[b]{2}{*}{ Aug. } & \multirow[b]{2}{*}{ Sept. } \\
\hline & & & & & Feb. & Mar. & Apr. & May & & & & \\
\hline 1 & 11.72 & 11.90 & 12.22 & -- & -- & - & -- & -- & -- & -- & -- & - \\
\hline 2 & 11.80 & 11.81 & 12.19 & -- & -- & -- & $-\infty$ & -- & -- & -- & $-\infty$ & - \\
\hline 3 & 11.79 & 11.83 & 12.20 & -- & -- & -- & -- & -- & -- & -- & -- & - \\
\hline 4 & 11.79 & 11.94 & - & -- & $-\infty$ & -- & -- & -- & -- & -- & -- & - \\
\hline 6 & 11.79 & 12.06 & -- & -- & -- & -- & -- & -- & -- & -- & -- & - \\
\hline 6 & 11.76 & 12.61 & - & -- & $-\infty$ & - & -- & -- & -- & -- & -- & - \\
\hline 7 & 11.78 & 11.99 & -- & -- & -- & -- & -- & -- & -- & - & -- & - \\
\hline 8 & 11.70 & 12.07 & -- & -- & $-\infty$ & -- & - & -- & -- & -- & - & - \\
\hline 9 & 11.69 & 12.01 & -- & -- & -- & -- & -- & -- & -- & -- & -- & -- \\
\hline 10 & 11.70 & 12.17 & -- & -- & -- & -- & -- & -- & -- & -- & -- & -- \\
\hline 11 & 11.71 & 12.07 & -- & -- & -- & -- & -- & -- & -- & $-\infty$ & -- & - \\
\hline 12 & 11.73 & 12.09 & -- & -- & -- & -- & -- & -- & -- & -- & -- & -- \\
\hline 13 & 11.70 & 12.10 & -- & -- & -- & -- & -- & -- & -- & -- & -- & - \\
\hline 14 & 11.66 & 12.05 & $\rightarrow$ & -- & -- & -- & -- & - & -- & -- & -- & -- \\
\hline 16 & 11.66 & 12.04 & -- & -- & -- & -- & -- & -- & -- & -- & -- & $\rightarrow$ \\
\hline 16 & 11.70 & 12.26 & -- & - & $\rightarrow$ & - & $-\infty$ & $-\infty$ & - & -- & - & - \\
\hline 17 & 11.67 & 12.19 & -- & -- & -- & -- & -- & -- & -- & -- & -- & -- \\
\hline 18 & 11.80 & 12.12 & -- & -- & -- & -- & -- & -- & -- & -- & -- & -- \\
\hline 19 & 11.76 & 12.20 & -- & -- & -- & -- & -- & -- & -- & -- & -- & - \\
\hline 20 & 11.72 & 12.27 & -- & -- & -- & -- & -- & -- & -- & -- & -- & -- \\
\hline 21 & 11.79 & 12.24 & -- & -- & -- & -- & -- & -- & -- & -- & -- & -- \\
\hline 22 & 11.67 & 12.16 & -- & -- & -- & -- & -- & -- & -- & -- & -- & - \\
\hline 23 & 11.76 & 12.10 & -- & -- & -- & -- & -- & -- & -- & -- & -- & -- \\
\hline 24 & 11.76 & 12.04 & -- & -- & -- & -- & -- & -- & -- & -- & -- & -- \\
\hline 26 & 11.77 & 12.04 & -- & -- & -- & -- & -- & - & -- & -- & -- & - \\
\hline 28 & 11.81 & 12.04 & -- & -- & -- & - & -- & -- & -- & -- & -- & - \\
\hline 27 & 11.77 & 12.23 & -- & -- & -- & -- & -- & -- & -- & -- & -- & -- \\
\hline 28 & 11.96 & 12.19 & -- & -- & -- & -- & -- & -- & -- & -- & -- & -- \\
\hline 29 & 11.91 & 12.17 & -- & -- & -- & -- & -- & -- & -- & -- & -- & - \\
\hline 30 & 11.92 & 12.25 & $-\infty$ & $\rightarrow$ & -- & -- & $-\infty$ & -- & -- & -- & -- & -- \\
\hline 31 & 11.94 & -- & -- & -- & -- & -- & -- & -- & -- & -- & -- & -- \\
\hline Mean & 11.76 & 12.09 & -- & -- & -- & -- & -- & -- & -- & -- & -- & - \\
\hline Max. & 11.96 & 12.27 & -- & -- & -- & - & -- & -- & -- & -- & -- & -- \\
\hline Min. & 11.66 & 11.81 & -- & -- & $-\infty$ & $\rightarrow$ & -- & -- & -- & -- & $\rightarrow$ & - \\
\hline
\end{tabular}




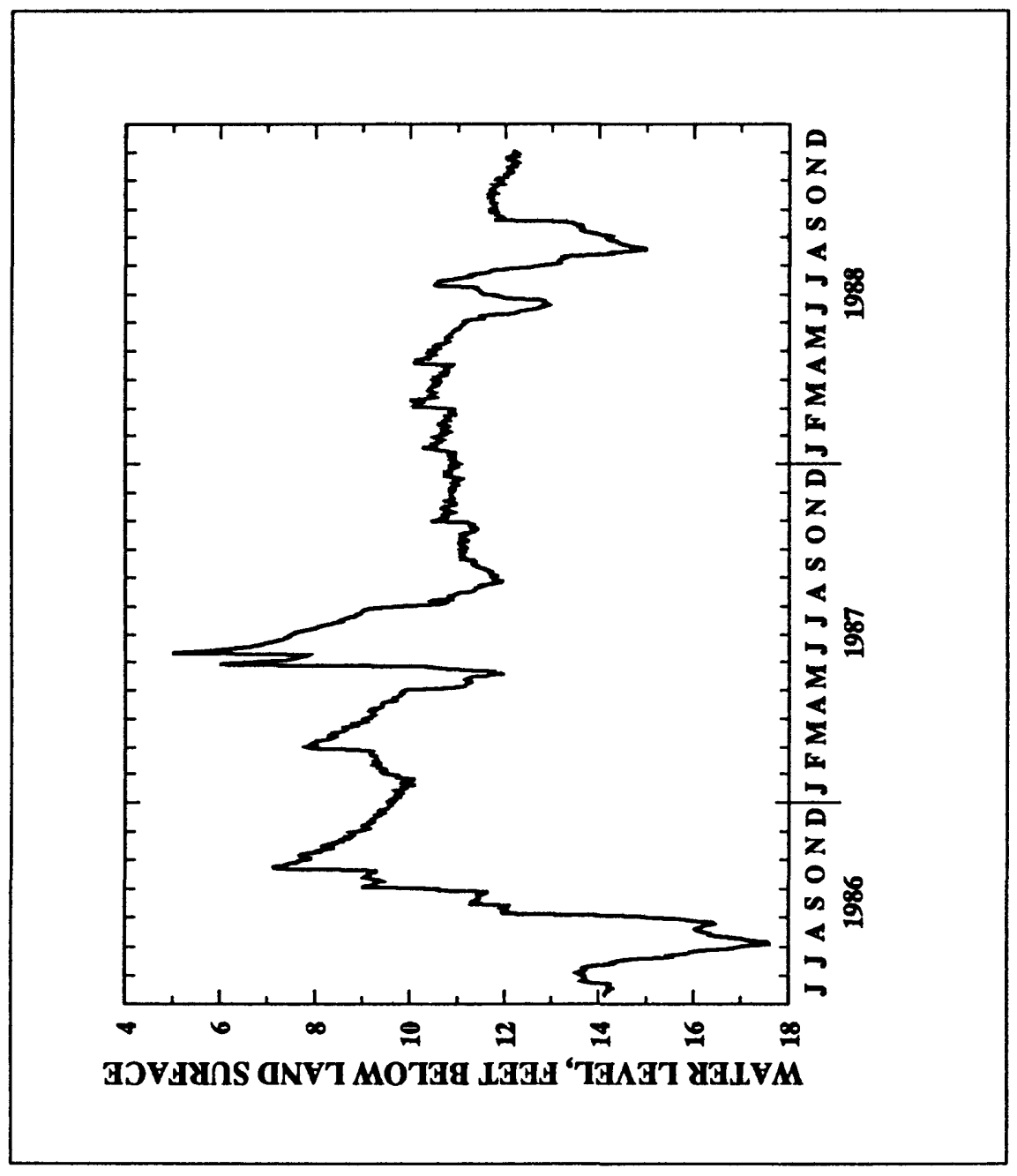

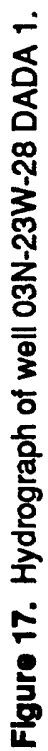



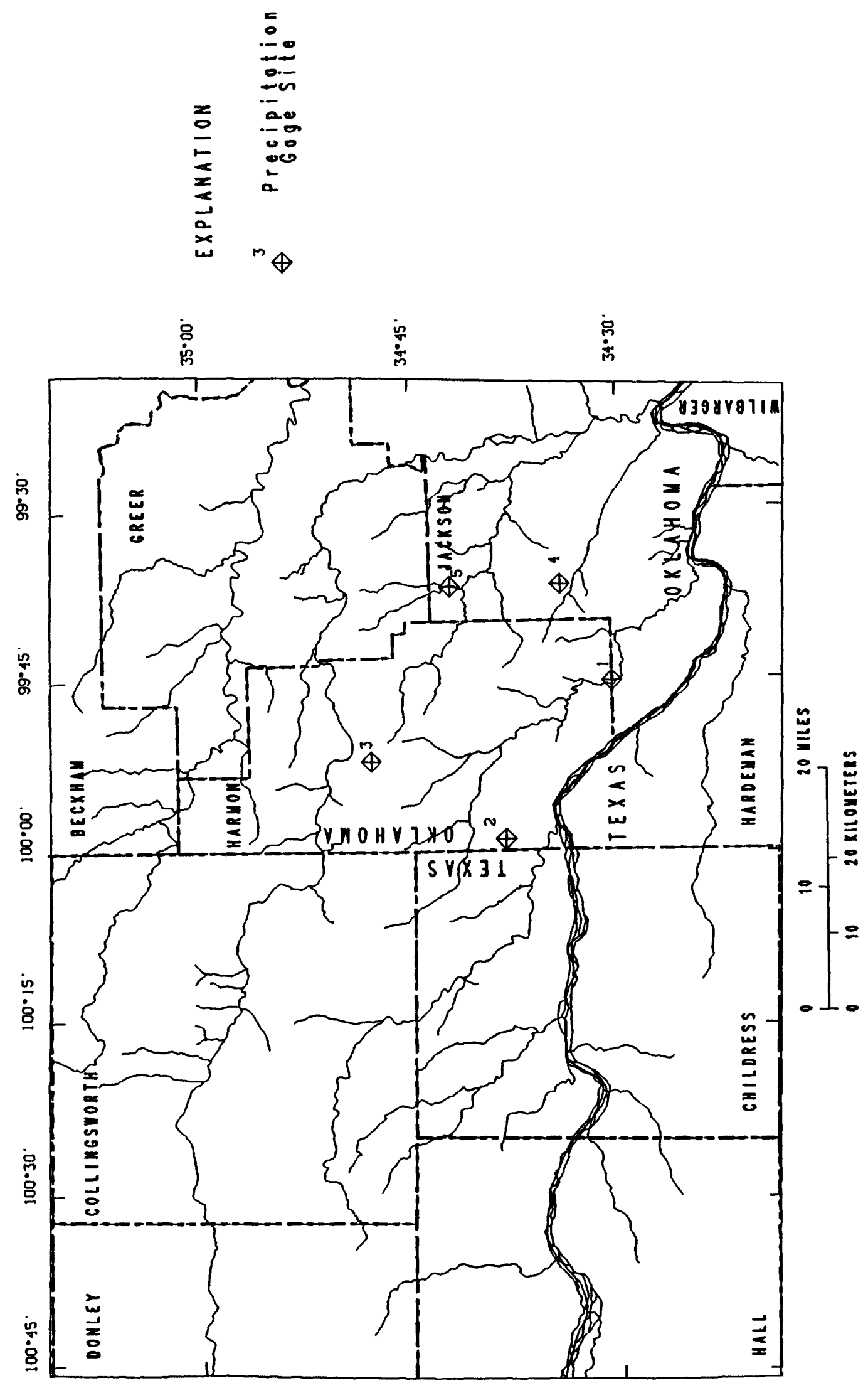

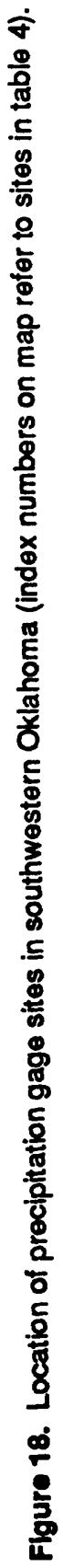


Table 4.--Daily precipitation measurements in southwestern Oklahoma

[Index number on location map refers to the number adjacent to the precipitation gage symbol in figure 20; $f t$, foot; - no data]

HARMON COUNTY, OKLAHOMA

Index number on location map: 1

Station number: 343041099451002 Local number: $01 \mathrm{~N}-24 \mathrm{~W}-32$ CBCC 2

Location: Lat $34^{\circ} 30^{\prime} 31^{\prime \prime}$, long $99^{\circ} 46^{\prime} 10^{\prime \prime}$, hydrologic unit 11130101

Altitude of land-surface datum: $1483 \mathrm{ft}$

Water Year, October 1985 to September 1986

Day Dct. Nov. Dec. Jen. Feb. Mar. Apr. May June July Aug. Sept.

\begin{tabular}{|c|c|c|c|c|c|c|c|c|c|c|c|c|c|}
\hline 1 & & -- & -- & -- & -- & -- & - & -- & $-\infty$ & -- & .00 & -- & - \\
\hline 2 & & -- & -- & $-\infty$ & $\cdots$ & -- & $-\infty$ & 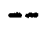 & $-\infty$ & $m$ & .10 & $\cdots$ & - \\
\hline 3 & & -- & -- & -- & -- & -- & -- & -- & -- & -- & .80 & -- & -- \\
\hline 4 & & -- & -- & -- & -- & -- & -- & -- & -- & -- & .00 & -- & -- \\
\hline 6 & & - & -- & -- & $-\infty$ & -- & -- & $-\infty$ & -- & -- & .60 & -- & -- \\
\hline 6 & & -- & -- & -- & -- & - & -- & -- & -- & -- & .00 & -- & - \\
\hline 7 & & -- & -- & -- & -- & -- & $-\infty$ & -- & -- & -- & $.0 B$ & -- & -- \\
\hline 8 & & -- & -- & -- & -- & -- & -- & -- & -- & -- & .00 & -- & - \\
\hline 9 & & - & -- & -- & - & - & -- & -- & -- & -- & .00 & - & - \\
\hline 10 & & -- & -- & -- & -- & -- & -- & -- & -- & -- & .00 & -- & -- \\
\hline 11 & & -- & -- & -- & -- & -- & $-\infty$ & -- & -- & -- & .80 & -- & - \\
\hline 12 & - & -- & -- & -- & -- & -- & -- & -- & -- & $-\infty$ &.$\varnothing \varnothing$ & -- & - \\
\hline 13 & & - & -- & -- & - & -- & -- & -- & -- & -- &.$\varnothing \varnothing$ & -- & - \\
\hline 14 & & -- & -- & -- & -- & -- & -- & -- & -- & -- & .00 & -- & -- \\
\hline 16 & & -- & -- & -- & -- & -- & -- & -- & -- & -- & .00 & -- & -- \\
\hline 16 & & -- & -- & -- & -- & -- & -- & -- & -- & -- & .00 & -- & - \\
\hline 17 & & -- & -- & -- & -- & -- & -- & -- & -- & - & .00 & -- & - \\
\hline 18 & & -- & -- & -- & - & -- & -- & -- & -- & -- & .06 & -- & .00 \\
\hline 19 & & -- & -- & -- & -- & -- & -- & -- & -- & -- & .060 & -- & .00 \\
\hline 20 & & -- & -- & -- & -- & -- & -- & -- & -- & -- & 1.10 & -- & .00 \\
\hline 21 & & -- & -- & -- & -- & -- & -- & - & -- & -- & .70 & -- & .00 \\
\hline 22 & & - & -- & -- & -- & -- & -- & -- & -- & -- & .06 & -- & .00 \\
\hline 23 & & -- & -- & -- & -- & -- & -- & -- & -- & -- &.$\varnothing 0$ & - & .00 \\
\hline 24 & & - & -- & -- & -- & -- & -- & -- & -- & -- & .80 & -- & .00 \\
\hline 25 & & -- & -- & -- & -- & -- & -- & -- & -- & -- &.$\varnothing 0$ & -- & .00 \\
\hline 26 & & -- & -- & -- & -- & -- & -- & -- & -- & -- & .00 & -- & .00 \\
\hline 27 & & -- & -- & -- & -- & -- & -- & -- & -- & -- &.$\varnothing \varnothing$ & -- & .00 \\
\hline 28 & & -- & -- & -- & -- & -- & -- & -- & -- & -- & .00 & -- & .50 \\
\hline 29 & & -- & -- & -- & -- & -- & -- & -- & -- & -- & .00 & -- & 3.90 \\
\hline 30 & & -- & -- & $\sim$ & -- & -- & -- & $\cdots$ & -- & -- & .00 & -- & .10 \\
\hline 31 & & -- & - & -- & -- & -- & - & -- & - & -- & -- & -- & -- \\
\hline
\end{tabular}

Total 
Table 4.--Daily precipitation measurements in southwestern Oklahoma--Continued

HARMON COUNTY, OKLAHOMA--CONTInUEd

D1N-24W-32 CBCC 2--Continued

Water Year, October 1986 to September 1987

Day Dct. Nov. Dec. Jan. Feb. Mar. Apr. May June July Mug. Sept.

\begin{tabular}{|c|c|c|c|c|c|c|c|c|c|c|c|c|}
\hline 1 & .00 & 1.20 & .00 &.$\varnothing \varnothing$ & .00 & .00 & .00 & .00 & .00 & -- & .00 & -- \\
\hline 2 & 3.40 & .00 & .00 &.$\varnothing \varnothing$ & .00 & $.0 B$ & .00 & .00 & .00 & -- & .00 & -- \\
\hline 3 & .30 & .00 & .00 &.$\emptyset 0$ & .00 & .00 & .00 & .00 & .00 & -- & $.0 \varnothing$ & -- \\
\hline 4 & .00 & .00 & .00 & .00 & .00 & .00 & .00 & .60 & .00 & -- & .00 & -- \\
\hline 5 & .10 & .00 & .00 & .00 & .70 & .00 & .00 & .00 & .10 & -- & 2.20 & -- \\
\hline 8 & .00 & .00 & .00 & .00 & .00 & .00 & .00 & .00 & .00 & -- & .00 & -- \\
\hline 7 & .00 & $.0 \varnothing$ & .10 & .00 & .00 & .00 & .00 & .10 & .00 & -- & .00 & -- \\
\hline 8 & .00 & .00 & .40 & .50 & .00 & .00 & .00 & .00 & .00 & -- & .00 & -- \\
\hline 9 & .10 & .00 & .00 & .10 & .00 & .00 & .00 & .00 & .20 & -- & .00 & -- \\
\hline 10 & .00 &.$\varnothing \varnothing$ & .86 & .00 & .00 & .00 & .00 & .00 & 5.80 & -- & .00 & -- \\
\hline 11 & 1.20 & .06 & .00 & .00 & .10 & .00 & .00 & .00 & $.0 \theta$ & -- & .00 & -- \\
\hline 12 & .00 & .00 & .00 & .00 & .10 & .00 & .00 & .00 & .00 & -- & .00 & -- \\
\hline 13 &.$\varnothing \varnothing$ & -- & .00 & -- & .10 & .00 & .00 & .00 & .00 & -- & .00 & -- \\
\hline 14 & .00 & -- & .00 & -- & .00 & .00 & .00 & .00 & .00 & -- & 1.50 & -- \\
\hline 16 & .00 & -- & .00 & -- & .90 & .00 & .00 &.$\varnothing \varnothing$ & .00 & -- & .00 & -- \\
\hline 16 & .00 & -- & .00 & -- & .00 & .10 & .00 & .00 & .00 & -- & .00 & -- \\
\hline 17 & .10 & -- & $.0 \varnothing$ & -- & .00 & .10 & .00 & .00 & .00 & -- & .00 &.$\theta \varnothing$ \\
\hline 18 & .00 & -- & .10 & -- & .06 & .00 & .00 & .00 & .00 & -- & .00 & .20 \\
\hline 19 & .00 & .00 & .00 & -- & .10 & .08 & $.0 \theta$ & .50 & .00 & -- & .00 & .00 \\
\hline 20 & 1.90 & .00 & .00 & -- & .10 & .00 & .00 & .10 & .20 & -- & .00 & .60 \\
\hline 21 & .90 & .00 & .06 & -- & .00 &.$\varnothing 0$ & .00 & .00 & .00 & -- & .00 & .00 \\
\hline 22 & .00 & .00 & $.0 \theta$ & -- & .10 & .36 & .00 & .50 & -- & -- & .60 & .60 \\
\hline 23 & .00 &.$\varnothing \varnothing$ &.$\varnothing \varnothing$ & -- & .10 & .40 &.$\varnothing \varnothing$ & 1.60 & -- & -- & .00 & .00 \\
\hline 24 & .00 & .00 & .00 & -- & .10 &.$\varnothing 0$ & .00 & .10 & -- & - & .00 & .00 \\
\hline 25 & .00 & .40 & .00 & -- & .00 & .00 &.$\varnothing \theta$ & .00 & -- & -- & -- & .080 \\
\hline 26 & .00 & .00 & .00 & -- & .20 & .00 & .00 & .00 & -- & -- & -- & .00 \\
\hline 27 & .00 & .00 & .00 & -- & .60 & .00 & .00 & 1.40 & -- & -- & -- & .00 \\
\hline 28 & .00 & .00 & .00 & -- & .00 & .00 & .00 & 2.70 & -- & -- & -- & .80 \\
\hline 29 & .00 & .00 & .00 & .00 & -- & .00 & .00 & .00 & -- & -- & -- & .00 \\
\hline 30 & .00 & .00 & .00 & $.0 B$ & -- & .00 & .00 & .00 & -- & .00 & -- & .00 \\
\hline 31 &.$\varnothing \varnothing$ & -- & .00 & .50 & -- & .00 & -- & .00 & -- & .00 & -- & -- \\
\hline
\end{tabular}


Table 4.--Daily precipitation masurements in southwestern Oklahoma--Continued

HARMON COUNTY, OKLAHOMA--CONtinued

D1N-24W-32 CBCC 2--Continued

Water Year, October 1987 to September 1988

Day Dct. Nov. Dec. Jan. Feb. Mar. Apr. May June July Aug. Sept.

\begin{tabular}{|c|c|c|c|c|c|c|c|c|c|c|c|c|}
\hline 1 &.$\varnothing \varnothing$ & .00 & .00 & -- & $.0 D$ & .00 & .20 & .00 & .00 & .10 & -- & - \\
\hline 2 & .06 & .00 & -- & -- & .00 & 2.00 & .00 & .00 &.$\varnothing 0$ & .10 & - & - \\
\hline 3 & .00 & .00 & -- & -- & .00 & .00 & .00 & .00 & .00 & .10 & -- & - \\
\hline 4 & .00 & .00 & -- & -- & .00 & .00 & .00 & .00 & .00 & .00 & -- & -- \\
\hline 5 & .00 & .00 & -- & -- & .00 & - & .00 & .60 & .00 & .00 & -- & -- \\
\hline 6 & .00 & .00 & - & -- &.$\varnothing \varnothing$ & -- & .00 & .000 & .00 & .00 & -- & -- \\
\hline 7 & .00 & .00 & -- & -- & .00 & -- & .00 & .00 &.$D D$ & .00 & -- & - \\
\hline 8 & .00 & .00 & -- & -- & .00 & .00 & .00 & .00 & .00 & .00 & - & - \\
\hline 9 &.$\varnothing 0$ & .00 & -- & -- & .00 & .00 & .20 & .00 & .00 & .10 & -- & - \\
\hline 10 & .00 & .00 & .00 & -- & .00 & .00 & .00 & .00 & .00 & .40 & -- & -- \\
\hline 11 & .00 & .00 & .00 & - & .00 & .00 & .00 & .00 &.$\infty 0$ &.$\infty$ & -- & -- \\
\hline 12 & .00 & .00 & .00 & -- & .06 & .00 & .00 & .00 & .00 & .60 & -- & -- \\
\hline 13 & .00 & .00 & .00 & -- & .00 & .00 & .00 & .00 & .00 & $.0 \varnothing$ & -- & -- \\
\hline 14 & .00 & .00 & .00 & -- & .00 & .00 & .00 & .60 & .00 & .00 & -- & -- \\
\hline 15 & .00 & .00 & .20 & -- & .00 & .00 & .00 & .00 & .00 & .00 & -- & -- \\
\hline 16 & .00 &.$\varnothing \varnothing$ & $.0 D$ & - &.$\varnothing \varnothing$ & .00 & .30 & .00 & .00 & .00 & - & - \\
\hline 17 &.$\varnothing 0$ & .00 & .00 & -- & .00 & .10 & 2.10 & .60 & .00 & .00 & -- & -- \\
\hline 18 & .00 &.$\varnothing 0$ & 1.50 & -- & .00 & .00 & .00 & .00 & .000 & .10 & -- & - \\
\hline 19 &.$\varnothing \varnothing$ & .00 & .40 & -- &.$\varnothing \varnothing$ & .08 & .00 & .10 & .00 & .10 & - & -- \\
\hline 20 & .00 & .06 & .00 & -- & .00 & .08 & .00 & .00 & .00 & -- & -- & -- \\
\hline 21 &.$\varnothing \theta$ &.$\varnothing \varnothing$ &.$\varnothing \varnothing$ & -- & .00 & .00 & .00 & .00 & .00 & - & - & - \\
\hline 22 & -- &.$\varnothing \sigma$ & .00 & -- & .00 & .068 & .00 & .06 & .00 & -- & -- & - \\
\hline 23 & -- & .80 &.$\varnothing 0$ & -- & .00 & $.0 D$ & .00 & .00 & .00 & -- & -- & -- \\
\hline 24 & -- & .80 & .00 & -- & .00 & .80 & .00 & .00 & .00 & -- & -- & - \\
\hline 25 & -- &.$\varnothing \varnothing$ & .30 & -- & .00 & .00 & .80 & .00 & .00 & - & -- & -- \\
\hline 28 & -- & .60 & .80 & -- & .00 & .00 & .00 & .00 & 1.68 & -- & - & - \\
\hline 27 & -- &.$\varnothing \varnothing$ & .10 & .00 & .00 & .00 & .00 & .00 & 1.64 & -- & -- & - \\
\hline 28 &.$\varnothing \varnothing$ &.$\varnothing \varnothing$ & .20 &.$\varnothing \varnothing$ & .06 & .00 & .00 & .00 & .20 & -- & -- & -- \\
\hline 29 & .00 &.$\varnothing \varnothing$ & .00 & .00 & .00 & .06 & .00 & .00 & .40 & -- & -- & - \\
\hline $3 \emptyset$ & .70 & .00 & -- & .00 & -- & .60 & .80 & .00 & .00 & -- & -- & -- \\
\hline 31 & .10 & - & - & $.0 \varnothing$ & -- & .10 & -- & .10 & -- & -- & -- & -- \\
\hline
\end{tabular}


Table 4.--Daily precipitation measurements in southwestern Oklahoma--Continued

HARMON COUNTY, OKLAHOMA--CONTInUEd

Index number on location map: 2

Station number: $\$ 43866099581802$ Local number: O2N-27W-24 AAAD 2

Location: Lat $34^{\circ} 38^{\prime} 12^{\prime \prime}$, long $99^{\circ} 68^{\prime} 58^{\prime \prime}$, hydrologic unit 11130101

Altitude of land-surface datum: $1716 \mathrm{ft}$

Water Year, October 1986 to September 1987

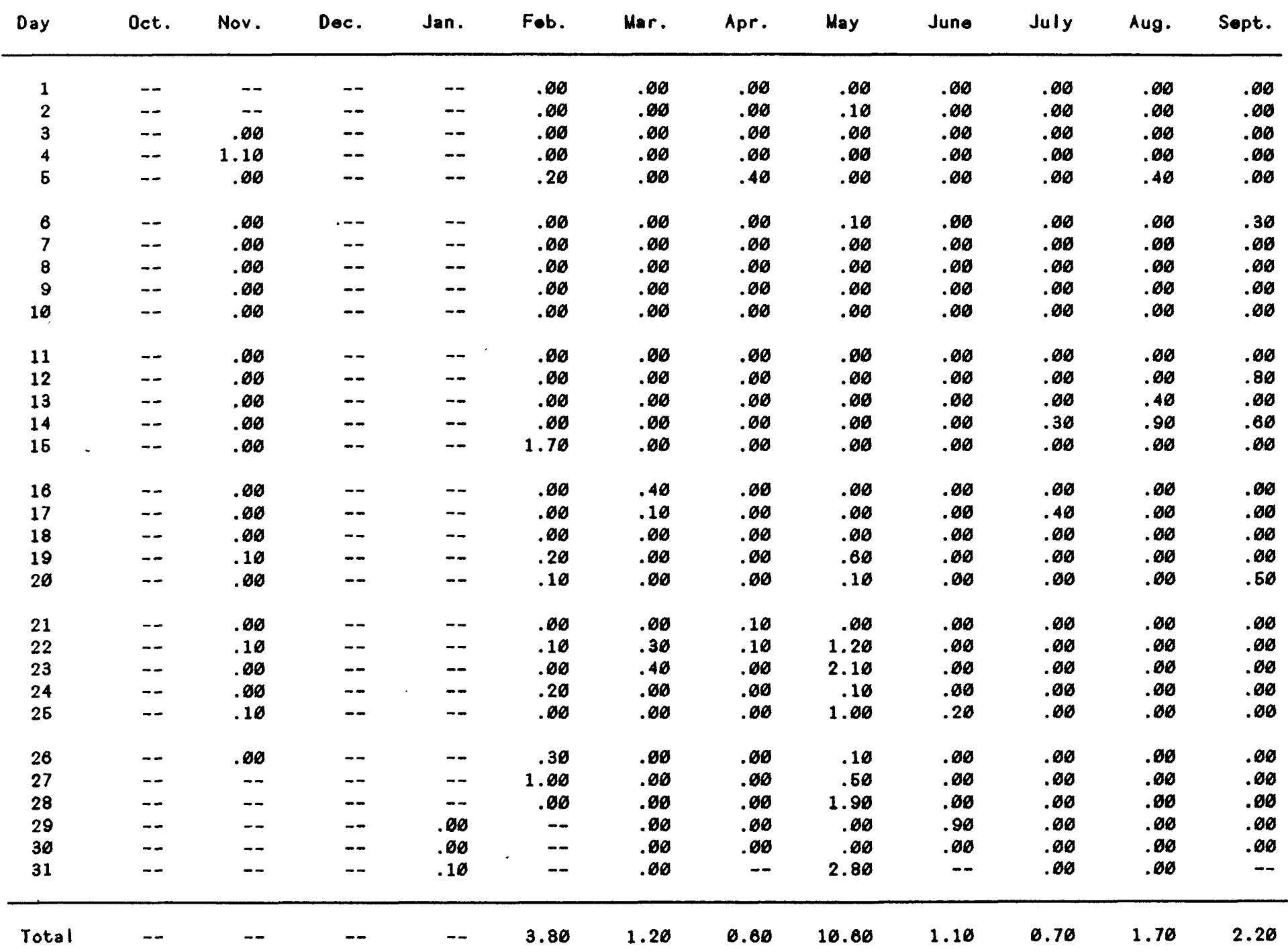


Table 4.--Daily precipitation measurements in southwestern Oklahoma--Continued

HARMON COUNTY, OKLAHOMA--Continued

62N-27W-24 AAAD 2--Continued

Water Year, October 1987 to September 1988

Day Dct. Nov Dec Jan Fob.

\begin{tabular}{|c|c|c|c|c|c|c|c|c|c|c|c|c|}
\hline 1 &.$\varnothing \varnothing$ &.$\varnothing \varnothing$ &.$\varnothing \theta$ & .00 & .00 & .00 & .10 & .00 & .00 & .30 & .00 & .80 \\
\hline 2 & .00 &.$\varnothing \varnothing$ &.$\varnothing \varnothing$ & .00 & .00 & 1.40 &.$\varnothing \varnothing$ & .00 & .00 & .80 & .10 & 1.00 \\
\hline 3 & .00 & .00 &.$\varnothing \varnothing$ & .00 & .00 & .00 & .00 & .00 &.$\varnothing \varnothing$ &.$\varnothing \varnothing$ & .00 & .00 \\
\hline 4 & .00 &.$\varnothing \varnothing$ &.$\varnothing 0$ &.$\not \varnothing \varnothing$ & .00 & .00 & .00 & .00 &.$\varnothing \varnothing$ & .00 & .000 & .00 \\
\hline 5 & .00 & .00 & .00 & .00 & .00 & .00 & .00 & .00 &.$\varnothing \varnothing$ & .00 & .00 & .00 \\
\hline 6 & .00 &.$\varnothing \varnothing$ & .00 & .00 & .00 & .00 & .60 & .00 &.$\varnothing \theta$ & .00 & .00 & .00 \\
\hline 7 &.$\varnothing \varnothing$ & .26 &.$\varnothing 0$ & .00 & .60 & .00 & .00 & .00 & .00 & .00 & .00 & .00 \\
\hline 8 & .00 & .00 & .60 & .30 & .060 & .00 & .26 & .60 & .00 & .20 & .00 & .00 \\
\hline 9 &.$\varnothing \sigma$ & .00 & .06 & .00 & .00 & .00 & .46 & .00 & .00 & .02 & .10 & .06 \\
\hline 10 & .00 & .00 & .00 & .00 & .00 & .10 & .00 & .06 & .00 & .48 & .00 & .00 \\
\hline 11 & .00 & .00 &.$\varnothing 0$ & .00 & .00 & .010 & .00 & .60 & .00 & .10 & .00 &.$\varnothing \varnothing$ \\
\hline 12 & .00 & .00 & .00 & .00 & .00 & .00 & .00 & .00 & .00 & .00 & .00 & .00 \\
\hline 13 & .00 & .00 & .00 & .00 & .00 & .00 & .10 & .00 & .00 & .60 & .00 & .40 \\
\hline 14 & .000 &.$\varnothing 0$ &.$\emptyset \emptyset$ & .00 & .06 & .00 & .00 & .00 & .30 & .00 & .00 & .00 \\
\hline 15 &.$\varnothing \varnothing$ & .00 & .26 & .00 & .00 & .00 & .00 & .00 & .00 &.$\varnothing \varnothing$ & .00 & .30 \\
\hline 16 & .00 &.$\varnothing 0$ &.$\varnothing \sigma$ & .00 & .00 & .10 & 2.70 & .00 & .60 & .00 & .00 &.$\varnothing \varnothing$ \\
\hline 17 & .00 & .00 & .60 & .00 & .00 & .00 &.$\varnothing \varnothing$ & .000 & .00 &.$\varnothing \theta$ & .00 & .20 \\
\hline 18 & .00 & .00 & .00 & .20 & .00 & .00 & .60 & .00 & .00 & .10 & .10 & 3.20 \\
\hline 19 & .00 & .00 & 1.10 & .00 &.$\varnothing \sigma$ & .00 & .00 & .00 & .00 & .10 & .00 & .10 \\
\hline 20 & .00 & .00 & .00 & .00 & .00 & .00 & .00 & .80 & .00 & .00 & .00 & .00 \\
\hline 21 &.$\varnothing \varnothing$ & .00 &.$\varnothing \varnothing$ & .00 & .00 & .00 &.$\varnothing 0$ & .00 & .00 &.$\theta \theta$ & .00 & .00 \\
\hline 22 & .00 &.$\varnothing 0$ &.$\varnothing 0$ & .60 & .00 &.$\varnothing 0$ &.$\varnothing \varnothing$ & .00 & .00 & .00 & .00 & 2.80 \\
\hline 23 & .20 &.$\varnothing \varnothing$ & .30 & .00 & .00 & .00 & .00 & .00 & .00 & .00 & .00 & .50 \\
\hline 24 & .10 & .00 & .00 & .00 & .00 & .00 & .00 & .00 & .00 & .30 & .00 & .00 \\
\hline 25 &.$\varnothing \varnothing$ &.$\varnothing \theta$ & .10 & .00 & .60 & .00 & .00 & .00 & .10 & .30 & .00 & .60 \\
\hline 26 &.$\varnothing \varnothing$ &.$\varnothing 0$ & .00 & $.0 B$ & $.0 D$ & .00 &.$\varnothing \varnothing$ & .20 & 1.000 & .00 & .00 & .000 \\
\hline 27 &.$\varnothing 0$ &.$\varnothing \varnothing$ &.$\varnothing \emptyset$ & .00 & .00 & .00 & .60 & .00 & .20 &.$\varnothing \theta$ & .60 & 2.10 \\
\hline 28 & .00 &.$\varnothing \varnothing$ & .46 & .00 & .00 & .00 & .60 & .10 & .60 & .00 & .20 & .00 \\
\hline 29 & .00 & .00 & .00 & .60 & .00 & .00 & .20 & .00 & .00 & .00 & .05 & .00 \\
\hline 30 & .40 & .00 & .00 & .00 & - & .00 & .00 & .60 & .00 & .00 & .15 & .10 \\
\hline 31 &.$\varnothing 0$ & -- & .60 & .00 & -- & .40 & -- & .78 & -- & .00 & .00 & -- \\
\hline
\end{tabular}

$\begin{array}{llllllllllllll}\text { Total } & 0.70 & 0.20 & 2.10 & 0.60 & 0.00 & 2.00 & 3.70 & 1.80 & 2.80 & 2.70 & 0.70 & 11.60\end{array}$


Table 4.--Daily precipitation measurements in southwestern Oklahoma--Continued

HARMON COUNTY, OKLAHOMA--COntinued

62N-27W-24 AAAD 2--Continued

Water Year, October 1988 to September 1989

Day Dct. Nov. Dec. Jan. Fob. Mar. Apr. May June July Aug. Sept.

\begin{tabular}{|c|c|c|c|c|c|c|c|c|c|c|c|c|}
\hline 1 & .00 & -- & -- & .00 & -- & -- & -- & -- & -- & -- & -- & -- \\
\hline 2 & .00 & -- & .00 & .00 & -- & -- & -- & -- & -- & -- & -- & -- \\
\hline 3 & .00 & - & .00 & .00 & -- & -- & -- & -- & -- & -- & -- & -- \\
\hline 4 & .00 & -- & .00 &.$\varnothing \varnothing$ & -- & -- & -- & -- & -- & -- & -- & -- \\
\hline 5 & 2.10 & -- & .00 & .00 & -- & -- & -- & -- & -- & -- & -- & -- \\
\hline 6 &.$\infty 0$ & -- & .00 & .00 & -- & -- & -- & -- & -- & -- & -- & -- \\
\hline 7 &.$\varnothing 0$ & -- & .70 & .00 & -- & -- & -- & -- & -- & -- & -- & -- \\
\hline 8 & 2.10 & -- & .10 & .00 & -- & -- & -- & -- & -- & -- & -- & -- \\
\hline 9 &.$\varnothing \varnothing$ & -- & .00 & .00 & -- & -- & -- & -- & -- & -- & -- & -- \\
\hline 10 & .00 & - & .20 & .00 & -- & -- & -- & -- & -- & -- & -- & -- \\
\hline 11 &.$\varnothing \theta$ & -- & .00 & .00 & -- & -- & -- & -- & -- & -- & -- & -- \\
\hline 12 & .00 & - &.$\varnothing \varnothing$ &.$\varnothing \varnothing$ & -- & -- & -- & -- & -- & -- & -- & -- \\
\hline 13 & .10 & -- & .30 & .00 & -- & -- & -- & -- & -- & -- & -- & -- \\
\hline 14 & .00 & -- & .00 & .00 & -- & -- & -- & -- & -- & -- & -- & -- \\
\hline 15 & .00 & -- & .00 & .00 & -- & -- & -- & -- & -- & - & -- & -- \\
\hline 16 & .00 & -- & .00 & .00 & -- & -- & -- & -- & -- & -- & -- & - \\
\hline 17 & .00 & -- & .00 & .00 & -- & -- & -- & -- & -- & -- & -- & -- \\
\hline 18 & .00 & -- & .00 & .00 & -- & -- & -- & -- & -- & -- & -- & -- \\
\hline 19 & -- & -- & .00 & -- & -- & -- & -- & -- & -- & -- & -- & -- \\
\hline 20 & -- & -- & .20 & - & -- & -- & -- & -- & -- & - & - & - \\
\hline 21 & -- & -- & .00 & -- & -- & -- & -- & -- & -- & -- & -- & -- \\
\hline 22 & -- & -- & .06 & -- & $\rightarrow$ & -- & -- & -- & -- & -- & -- & -- \\
\hline 23 & -- & -- & .00 & -- & -- & -- & -- & -- & -- & -- & -- & - \\
\hline 24 & -- & -- & .00 & -- & -- & -- & -- & -- & -- & -- & -- & - \\
\hline 25 & -- & -- & .06 & -- & -- & -- & -- & -- & -- & - & -- & - \\
\hline 26 & -- & -- &.$\varnothing \theta$ & -- & -- & -- & -- & -- & -- & -- & -- & -- \\
\hline 27 & -- & -- & .80 & -- & -- & -- & -- & -- & -- & -- & -- & - \\
\hline 28 & -- & -- & .00 & -- & -- & -- & -- & -- & -- & -- & -- & -- \\
\hline 29 & -- & -- & .00 & -- & -- & -- & -- & -- & -- & -- & -- & - \\
\hline 30 & -- & -- & .00 & -- & -- & -- & -- & -- & -- & -- & -- & -- \\
\hline 31 & -- & -- &.$\varnothing \theta$ & -- & -- & -- & -- & -- & -- & -- & -- & -- \\
\hline
\end{tabular}


Table 4.--Daily precipitation measurements in southwestern Oklahoma--Continued

HARMON COUNTY, OKLAHOMA--CONtinued

Index number on location map: 3

Station number: $\$ 44762099620601$ Local number: $64 \mathrm{~N}-25 \mathrm{~W}-19$ DCCC 1

Location: Lat $34^{\circ} 47^{\prime} 62^{\prime \prime}$, long $99^{\circ} 52^{\prime} 96^{\prime \prime}$, hydrologic unit 11130101

Altitude of land-surface datum: $1826 \mathrm{ft}$

Water Year, October 1986 to September 1987

Day Dct. Nov. Dec. Jan. Feb. Mar. Apr. May June July Aug. Sept.

\begin{tabular}{|c|c|c|c|c|c|c|c|c|c|c|c|c|}
\hline 1 & -- & -- & .00 & .00 & .00 &.$\varnothing 0$ & .00 & .000 & .00 & .00 & .00 &.$\varnothing 0$ \\
\hline 2 & -- & - & .00 & .00 & .80 &.$\varnothing \varnothing$ &.$\varnothing 0$ & .00 & .00 & .00 & .00 &.$\varnothing 0$ \\
\hline 3 & -- & .20 & .00 & .00 & .00 &.$\varnothing 0$ & .00 & .00 & .00 & .00 & .00 & .00 \\
\hline 4 & -- & 1.10 & .00 & .00 & .00 & .00 & .00 & .00 & .00 & .060 & .00 & .80 \\
\hline 5 & - & .00 & .00 & .00 & $.4 B$ & .60 & .10 & .00 & .00 & .00 & 1.40 & .00 \\
\hline 8 & -- & .00 &.$\varnothing \varnothing$ & .00 & .06 & .00 & .00 & .00 & .00 & .00 & .00 & .20 \\
\hline 7 & -- & .60 & .10 & .060 & .00 & .00 & .00 & .00 & .00 & .00 & .00 & .00 \\
\hline 8 & -- & .00 & .50 & .10 & .00 & .00 & .000 & .00 & .00 & .00 & .00 & .00 \\
\hline 9 & -- & .00 & .00 & .08 & .00 & .00 & .000 & .00 & .30 & .00 & .00 & .00 \\
\hline 10 & -- & .00 & .00 & .00 & .00 &.$\varnothing \theta$ & .00 &.$\varnothing \varnothing$ & .20 & .00 & .00 & $.0 \theta$ \\
\hline 11 & -- & .06 & .00 & .00 & .06 & .06 & .06 & .00 & .00 & .00 & .00 &.$\varnothing 0$ \\
\hline 12 & -- & .00 & .00 & .00 & .00 & .00 & .00 & .00 & .00 & .00 & .00 & .76 \\
\hline 13 & -- &.$\varnothing \varnothing$ & .00 & .00 & .00 & .00 & .00 & .00 & .00 & .10 & .40 & .00 \\
\hline 14 & -- & $\therefore \varnothing$ & .00 & .00 & .06 & .10 & .00 & .00 & .00 & .20 & .00 & .60 \\
\hline 16 & -- & .00 & .00 & .00 & 1.60 & .00 & .00 & .00 & .00 & .00 & .00 & - \\
\hline 16 & -- & .00 & .00 & .00 & .00 & 1.10 & .00 & .00 & .00 & .00 & .00 & - \\
\hline 17 & -- & .00 & .00 & .10 & .00 & .10 & .00 & .00 & .30 & .30 & .00 & -- \\
\hline 18 & -- & .00 & .10 & .00 & .80 & .00 & .00 & .00 & .00 & .00 & .00 & - \\
\hline 19 & -- & .10 & .00 & .20 & .00 & .60 & .00 & .80 & .00 & .00 & .00 & .00 \\
\hline 20 & -- & .00 & .00 & .00 & .10 & .00 & .00 & .20 &.$\varnothing \varnothing$ & .00 & .00 & .00 \\
\hline 21 & - &.$\varnothing \theta$ & .00 & .00 & .00 & .00 & .00 & .00 & .80 & .00 & .00 & .00 \\
\hline 22 & -- & .00 & .00 & .00 & .60 & .20 & .00 & .30 & .20 & .00 & .00 & .00 \\
\hline 23 & -- & .00 & .00 & .00 & .10 & .50 & .30 & 1.50 & .00 & .00 & .00 & .00 \\
\hline 24 & -- & .00 & .00 & .00 & .10 & .00 & .80 & .00 & .78 & .08 & .00 & -- \\
\hline 26 & -- & .20 & .00 & .00 & .00 & .00 & .00 & .50 & .00 & .00 & .00 & - \\
\hline 28 & -- & .00 & .00 & .00 & .40 & .00 & .00 & .10 & .00 & .00 & .60 & - \\
\hline 27 & -- &.$\varnothing 0$ & .00 & .00 & 1.10 &.$\varnothing 0$ & .00 & .40 & .00 & .00 & .00 & -- \\
\hline 28 & - & .00 & .00 & .00 & .00 & .20 & .00 & 2.00 & .00 & .00 & .00 & -- \\
\hline 29 & -- &.$\varnothing 0$ & .00 & .10 & -- & .00 & .00 & .00 & .00 & .00 & .00 & - \\
\hline 30 & -- &.$\varnothing 0$ & .00 & .00 & -- & .00 & .00 & .10 & .00 & .00 & .20 & -- \\
\hline 31 & -- & -- & .00 & .40 & - & .00 & -- & .00 & -- & .00 & .00 & -- \\
\hline
\end{tabular}

Total

0.70

0.90

3.70

2.20

0.40

5.70

2.60

0.60

2.50 
Table 4.--Daily precipitation measurements in southwestern Oklahoma--Continued

HARMON COUNTY, OKLAHOMA--CONtinued

64N-25W-19 DCCC 1--Continued

Water Yoar, October 1987 to September 1988

Day Oct. Nov. Dec. Jan. Feb. Mar. Apr. May June July Aug. Sept.

\begin{tabular}{|c|c|c|c|c|c|c|c|c|c|c|c|c|c|}
\hline 1 & & -- & .00 & .00 & .00 & .00 & .00 & .30 &.$\varnothing \varnothing$ &.$\varnothing \varnothing$ & .00 &.$\varnothing 0$ & .20 \\
\hline 2 & & -- & .00 & .00 &.$\varnothing 0$ & .00 & 2.80 & .00 & .00 & .80 & .00 & .00 & 1.10 \\
\hline 3 & & -- & .00 & .00 &.$\varnothing 0$ & .00 & .00 & .00 & .00 & .00 & .00 &.$\varnothing \varnothing$ & .00 \\
\hline 4 & & -- & .00 & .00 & .00 & .00 & .00 & .00 & .00 & .00 & .00 & .00 & .00 \\
\hline 5 & & -- & .00 & .00 & .00 & .00 & .00 &.$\varnothing 0$ & .00 & .00 &.$\varnothing \theta$ & .06 & .00 \\
\hline 6 & & -- & .00 & .00 & .00 & .00 & .00 & .80 & .00 & .00 & .80 & .00 & .80 \\
\hline 7 & & -- & .00 &.$\varnothing \varnothing$ & .00 & .00 & .00 & .00 & .00 & .00 & .00 &.$\varnothing 0$ & .00 \\
\hline 8 & & -- & .00 & .00 & .00 & .00 & .00 & .00 & .00 &.$\varnothing \varnothing$ & .00 &.$\varnothing \varnothing$ & .00 \\
\hline 9 & & -- & .00 & .00 & .08 & .00 & .00 & .20 & .00 & .00 & .82 & .08 & .06 \\
\hline 10 & & -- & .00 & .00 & .00 & .00 & .00 & .00 & .00 &.$\varnothing 0$ & .28 & .00 &.$\varnothing 0$ \\
\hline 11 & & -- & .00 & .80 & .20 &.$\varnothing 0$ & .00 & .00 & .00 &.$\varnothing 0$ & .00 & .50 & .00 \\
\hline 12 & & -- & .00 & .00 & .10 & .00 &.$\varnothing 0$ & .60 & .00 &.$\varnothing 0$ & .00 &.$\varnothing \varnothing$ & .00 \\
\hline 13 & & -- & .00 & .00 & .06 & .00 & .06 & .00 & .00 & .00 & .00 & .00 & .00 \\
\hline 14 & & -- & .00 & .00 & .08 & .00 & .00 & .00 & .00 & 1.50 & .00 & .80 & .10 \\
\hline 16 & & -- &.$\varnothing \varnothing$ & .00 & .00 & .60 & .00 & .00 & .00 & .10 & .00 & .00 & .30 \\
\hline 16 & & -- &.$\varnothing \varnothing$ &.$\varnothing \varnothing$ &.$\infty 0$ &.$\varnothing \varnothing$ &.$\varnothing 0$ & .00 & .00 &.$\varnothing 0$ & .00 &.$\varnothing \varnothing$ & .00 \\
\hline 17 & & -- &.$\infty 0$ & .20 & .00 &.$\varnothing 0$ & .30 & 1.90 & .00 & .00 & .00 & .00 & $.0 \theta$ \\
\hline 18 & - & -- & .00 & .60 & .00 & .00 & .00 & .00 & .00 & .00 & .06 & .00 & 7.46 \\
\hline 19 & & -- & .00 & .50 & .00 & .80 & .00 & .00 &.$\varnothing \varnothing$ & .00 & .00 & .00 & .80 \\
\hline 20 & & -- & .80 & .00 & .00 & .00 & .00 & .00 & .00 & .00 &.$\varnothing 0$ & .80 & .06 \\
\hline 21 & & -- &.$\varnothing 0$ & .00 & .00 &.$\varnothing \varnothing$ &.$\varnothing \varnothing$ & .00 & .00 & .00 &.$\varnothing \theta$ & .60 & .00 \\
\hline 22 & & -- & .00 &.$\varnothing 0$ & .60 & .00 & .00 & .00 & .00 & .00 & .00 & .00 & .00 \\
\hline 23 & & -- & .00 & .00 & .00 & .00 & .00 & .00 &.$\varnothing \theta$ & .00 & .00 & .00 & .80 \\
\hline 24 & & -- & .00 & .00 & .00 & .00 & .00 & .00 & .00 &.$\varnothing 0$ & .00 & .00 & .00 \\
\hline 25 & & -- & .00 & .00 & .00 & .00 & .06 & .00 & .00 & .10 & .00 & .00 & .00 \\
\hline 26 & & -- & $.0 \theta$ & .00 &.$\varnothing \theta$ & .00 &.$\varnothing 0$ &.$D D$ & .00 & .70 & .00 &.$\varnothing 0$ & .00 \\
\hline 27 & & -- & .10 & .00 & .00 & .00 & .08 & .60 & .00 & .10 & .00 & .00 & .000 \\
\hline 28 & & -- & .00 & .20 & .00 & .00 & .00 & .00 & .00 & .40 & .00 & .10 & .00 \\
\hline 29 & &.$\not \varnothing \varnothing$ & .00 & .080 & .00 & .08 & .00 & .00 &.$\varnothing \theta$ & $.8 D$ & .00 & .80 & .00 \\
\hline 30 & & .90 & .00 & .00 & .00 & -- & .00 & .00 &.$\varnothing 0$ & .00 & .00 & .00 & .00 \\
\hline 31 & & .00 & -- & .00 & .00 & -- & .00 & -- & .70 & -- & .00 & .20 & -- \\
\hline
\end{tabular}


Table 4.--Daily precipitation measurements in southwestern Oklahoma--Continued

HARMON COUNTY, OKLAHOMA--CONTinUEd

94N-25W-19 DCCC 1--Continued

Water Year, October 1988 to September 1989

Day Oct. Nov. Dec. Jan. Feb. Mar. Apr. May June July Aug. Sept.

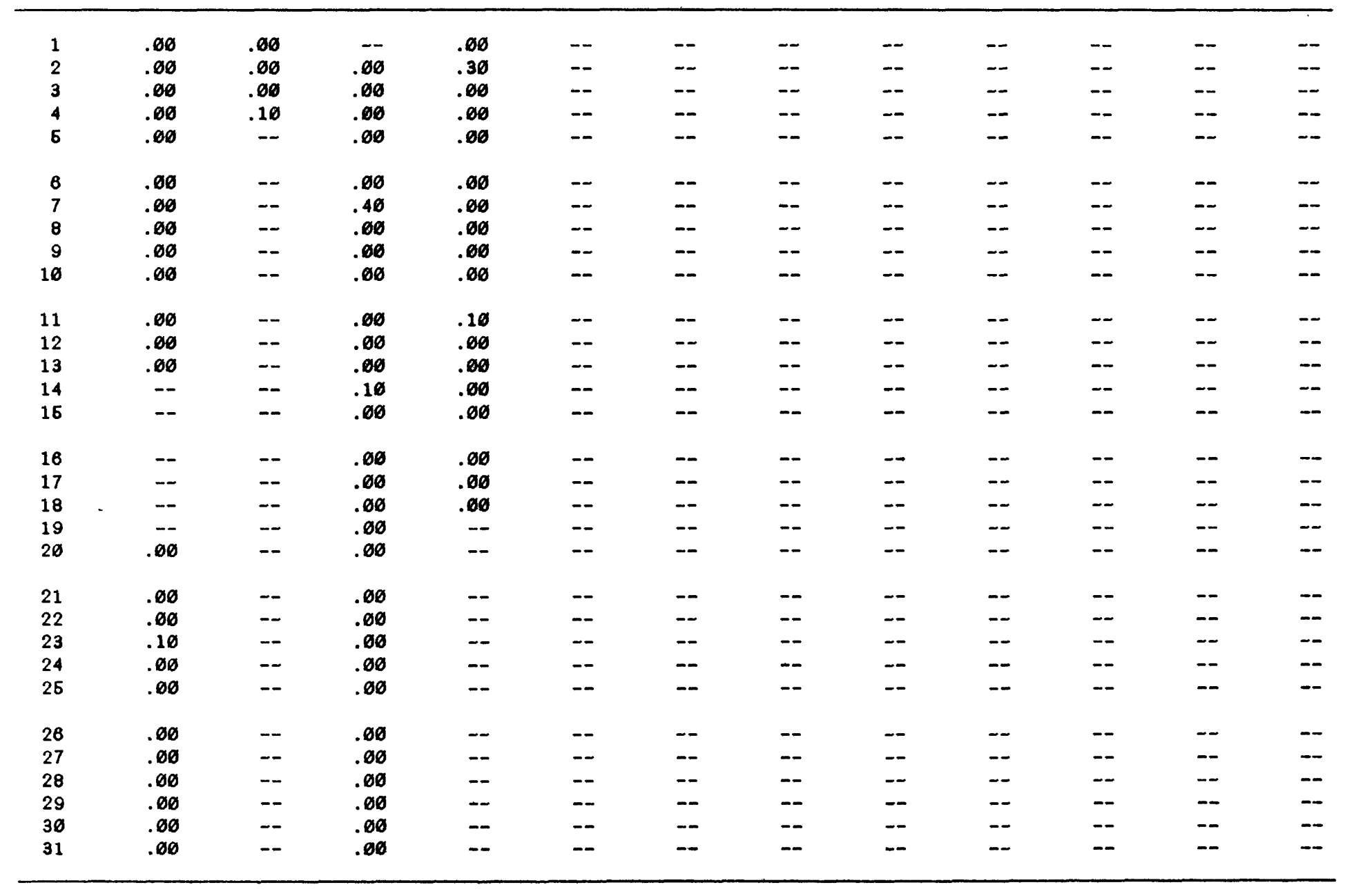

Total 
Table 4.--Daily precipitation measurements in southestern Oklahoma--Continued

JACKSON COUNTY, OKLAHOMA

Index number on location map: 4

Station number: $\$ 43410099364402$ Local number: $1 \mathrm{~N}-23 W-10$ BBBA 2

Location: Lat $34^{\circ} 34^{\prime} 10^{\prime \prime}$, long $99^{\circ} 36^{\prime} 44^{\prime \prime}$, hydrologic unit 11130101

Altitude of land-surface datum: $1432 \mathrm{ft}$

Water Year, October 1986 to September 1987

Day Oct. Nov. Dec. Jan. Feb. Mar. Apr. May June July Aug. Sept.

\begin{tabular}{|c|c|c|c|c|c|c|c|c|c|c|c|c|c|}
\hline 1 & & -- & .80 & .00 & .00 & .00 & .00 & .00 & .00 & .00 & .00 & .00 & .80 \\
\hline 2 & & -- & -- & .00 & .60 & .00 & .00 & .00 & .80 & .00 & .00 &.$\varnothing 0$ &.$\varnothing \varnothing$ \\
\hline 3 & & -- & .00 & .00 & .00 & .00 & .06 &.$\varnothing 0$ & .00 & .00 & .00 & .00 & .00 \\
\hline 4 & & -- & .90 & .00 & .00 & .60 & .10 & .00 & .00 & .00 & .00 & .00 & .60 \\
\hline 5 & & -- & .00 & .00 & .00 & .20 & .06 & .10 &.$\varnothing 0$ & .00 & .60 & .70 &.$\emptyset \emptyset$ \\
\hline 6 & & -- & .80 & .10 & .80 & .00 & .00 & .10 & .60 & .00 & .00 & .00 & .10 \\
\hline 7 & & -- & .00 & .00 & .10 & - & .00 & .00 & .00 & .00 & .00 & .00 & .00 \\
\hline 8 & & -- & .60 & .20 & .50 & -- & .00 & .00 & .00 & .00 & .00 & .00 & .10 \\
\hline 9 & & -- & .00 & .00 & .10 & -- & .00 & .00 & .00 & .40 & .00 & .00 & .00 \\
\hline 10 & & -- & .00 & .00 & .00 & -- & .00 & .00 & .00 & .60 & .00 & .00 & .00 \\
\hline 11 & & -- & .00 & .00 & .00 & -- & .00 & .00 & .80 & .80 & .00 & .00 & .00 \\
\hline 12 & & -- & .00 & .00 & .00 & -- & .10 & .00 & .00 & .50 & .60 & .00 & .50 \\
\hline 13 & & -- & .00 & .00 & .00 & -- & .60 & .86 & .60 & .00 & .06 & .00 & .00 \\
\hline 14 & & -- & .00 & .00 & .00 & -- & .00 & .00 & .00 & .00 & .10 & .20 & .00 \\
\hline 15 & - & -- & .00 & .00 & .00 & -- & .60 & .00 & .00 & .00 & .10 & .00 & $.0 \theta$ \\
\hline 16 & & -- & .00 &.$\varnothing \varnothing$ & .20 & -- & .20 & .00 & .00 & .00 & .10 &.$\varnothing \sigma$ & .00 \\
\hline 17 & & -- & .00 &.$\varnothing \varnothing$ & .00 & -- & .10 & .60 & .00 & .16 & 1.10 & .00 & .00 \\
\hline 18 & & -- &.$\varnothing 0$ & .10 & -- & .00 & .00 & .00 & .10 & .00 & .00 & .00 & .20 \\
\hline 19 & & -- & .00 & .00 & -- & .10 & .00 & .00 & .50 & .00 & .00 & .00 & .00 \\
\hline 20 & & -- & .00 & .00 & -- & .10 & .00 & .80 & .10 &.$\varnothing \varnothing$ & .80 &.$\varnothing \varnothing$ & .90 \\
\hline 21 & & -- & .00 &.$\varnothing 0$ & -- & .00 & .00 & .00 & .00 & .00 & .00 & .00 & .00 \\
\hline 22 & & -- & .00 & .00 & -- & .10 & .00 &.$\varnothing 0$ & .50 & .00 & .00 & .00 & .00 \\
\hline 23 & & -- & .10 &.$\varnothing \varnothing$ & -- & .00 & .70 & .00 & 2.90 & .80 & .00 & .00 & .08 \\
\hline 24 & & -- & .00 & .00 & -- & .20 & .00 & .00 & .10 & 1.20 & .00 & .00 & .10 \\
\hline 26 & & -- & .60 & .00 & -- & .00 & .00 & .00 & 1.30 & .20 & .00 & .10 & .00 \\
\hline 26 & & -- & .00 & .00 & -- & .30 & .00 & .00 & .00 & .00 & .00 & .30 & .80 \\
\hline 27 & & -- & .00 & .00 & - & .30 & .00 & .80 & 1.60 & .06 & .80 & $.0 \varnothing$ & .00 \\
\hline 28 & & -- & .00 & .00 &.$D D$ & .00 & .00 & .00 & 2.70 &.$D D$ &.$D D$ & .00 & .48 \\
\hline 29 & & -- & .00 & .00 & .00 & -- & .06 & .16 & .00 & .70 & .00 & .00 & .00 \\
\hline 30 & & -- & .00 & .00 & .00 & -- & .00 & .60 & .10 & .10 & .00 & .00 & .10 \\
\hline 31 & & -- & -- & .00 & .60 & -- & .00 & -- & .00 & -- & .60 & .00 & -- \\
\hline
\end{tabular}


Table 4.--Daily precipitation measurements in southwestern Oklahoma--Continued

JACKSON COUNTY, OKLAHOMA--CONtinued

61N-23W-10 BBBA 2--Continued

Water Year, October 1987 to September 1988

Day Dct. Nov. Dec. Jan. Fob. Mar. Apr. May Juno July Aug. Sopt.

\begin{tabular}{|c|c|c|c|c|c|c|c|c|c|c|c|c|}
\hline 1 & .06 & .08 & .00 & .06 & .00 & .20 & .30 &.$\varnothing 0$ & .00 & .00 & -- & .20 \\
\hline 2 & .00 & .00 & .80 & .30 & .00 & 2.50 & .00 & .00 & .00 & .40 & -- & 1.00 \\
\hline 3 &.$\varnothing 0$ & .00 & .00 & .20 & .00 & .00 & .00 & .00 & .00 & .10 & -- & .10 \\
\hline 4 & .80 & .00 & .00 & .00 & .00 & .00 & .00 & .00 & .000 & .060 & -- & .00 \\
\hline 5 & .00 & .00 & .00 & .00 & .00 & .00 & .00 & .60 & .00 & .00 & -- & .00 \\
\hline 6 & .000 & .00 & .00 & .00 & .00 & .00 & .00 &.$\varnothing 0$ & .00 & .00 & -- & .00 \\
\hline 7 & .00 & .00 & .00 & .00 & .00 & .00 & .00 & .00 & .00 & .800 & -- & .00 \\
\hline 8 & .00 & .00 & .00 & .00 & .00 & .00 & .00 & .00 & .00 & .87 & -- & .00 \\
\hline 9 & .00 & .80 & .60 & .00 & .00 & .00 & .40 & .00 & .00 & .43 & -- & .060 \\
\hline 10 & .00 & .000 & .00 & .00 & $.0 \varnothing$ & .00 & .00 & .00 & .00 & .20 & -- & .00 \\
\hline 11 &.$\varnothing \theta$ & .00 & .06 & .20 & .00 & .00 & .00 &.$\varnothing 0$ & .00 & .00 & -- & .00 \\
\hline 12 & .00 & .00 & .00 & .50 & .00 & .000 & .10 & .00 & .00 & .00 & -- & .60 \\
\hline 13 & .00 & .00 & .00 & .00 & .00 & .00 & .00 & .00 & .00 & .00 & -- & .10 \\
\hline 14 & .00 & .80 & .00 & .00 & .00 & .00 & .00 & .00 & .00 &.$\varnothing 0$ & - & .10 \\
\hline 16 & .80 &.$\varnothing \varnothing$ & .00 &.$\varnothing 0$ & .00 & .00 & .00 & .00 & .00 & .00 & -- & .30 \\
\hline 16 & .00 & .00 &.$\varnothing \varnothing$ & .00 & .00 & .00 & .00 & .00 & .00 & .00 & -- & .30 \\
\hline 17 & .06 & .00 & .00 &.$\varnothing \theta$ & .00 & .20 & 2.60 &.$\varnothing 0$ & .20 & .00 & -- & .30 \\
\hline 18 & .00 & .00 & .40 & .00 & .00 & .00 &.$\varnothing 0$ & .00 & .10 & .00 & -- & 3.10 \\
\hline 19 & .00 & .00 & .50 &.$\varnothing \varnothing$ & .00 & .00 & .00 &.$\varnothing 0$ & .00 & .00 & -- & .00 \\
\hline 20 & .00 & .00 & .00 & .00 & .00 & .00 &.$\varnothing 0$ &.$\varnothing 0$ & .00 & -- & -- & .00 \\
\hline 21 & .00 &.$\varnothing 0$ &.$\varnothing \varnothing$ & .00 & .00 & .00 & .00 &.$\varnothing 0$ & .00 & -- & -- & .00 \\
\hline 22 & .00 & .00 & .00 &.$\varnothing \varnothing$ & .00 & .00 & .00 & .00 & .00 & -- & -- & .00 \\
\hline 23 & 1.10 & .00 & .00 & .00 & .80 & .00 & .00 &.$\varnothing \varnothing$ & .00 & -- & -- & .80 \\
\hline 24 & .20 & .00 & .00 & .00 &.$\varnothing \varnothing$ & .00 & .00 & .00 & .00 & -- & -- & .00 \\
\hline 25 &.$\varnothing 0$ & .00 & .40 & .00 &.$\varnothing \theta$ & .00 & .00 &.$\varnothing \varnothing$ & .43 & -- & -- & .00 \\
\hline 26 & .00 & .30 & .40 & .00 &.$\varnothing \varnothing$ & .00 & .00 & .00 & 1.47 & -- & -- & .10 \\
\hline 27 & .00 & .00 & .10 & .00 &.$\varnothing \varnothing$ &.$\varnothing 0$ & .00 &.$\varnothing \varnothing$ & .10 & -- & -- & .00 \\
\hline 28 &.$\varnothing \varnothing$ &.$\varnothing \varnothing$ & .00 & .00 & .00 & .00 & .00 & .00 & .50 & -- & -- & .00 \\
\hline 29 & .00 & .60 & .10 & .00 &.$\varnothing 6$ & .00 & .30 & .00 & .00 & -- & -- & .00 \\
\hline 30 & 2.000 & .00 & .30 & .00 & -- & $.0 \sigma$ & .20 & .00 & .00 & -- & -- & .00 \\
\hline 31 & .10 & -- & .00 & .00 & - & .20 & -- & .20 & -- & - & -- & -- \\
\hline
\end{tabular}


Table 4.--Daily precipitation measurements in southwestern Oklahoma--Continued

JACKSON COUNTY, OKLAHOMA--Continued

$01 N-23 W-10$ BBBA 2--Continued

Water Year, October 1988 to September 1989

Day Det. Nov. Dec. Jan. Feb. Mar. Apr. May June July Aug. Sept.

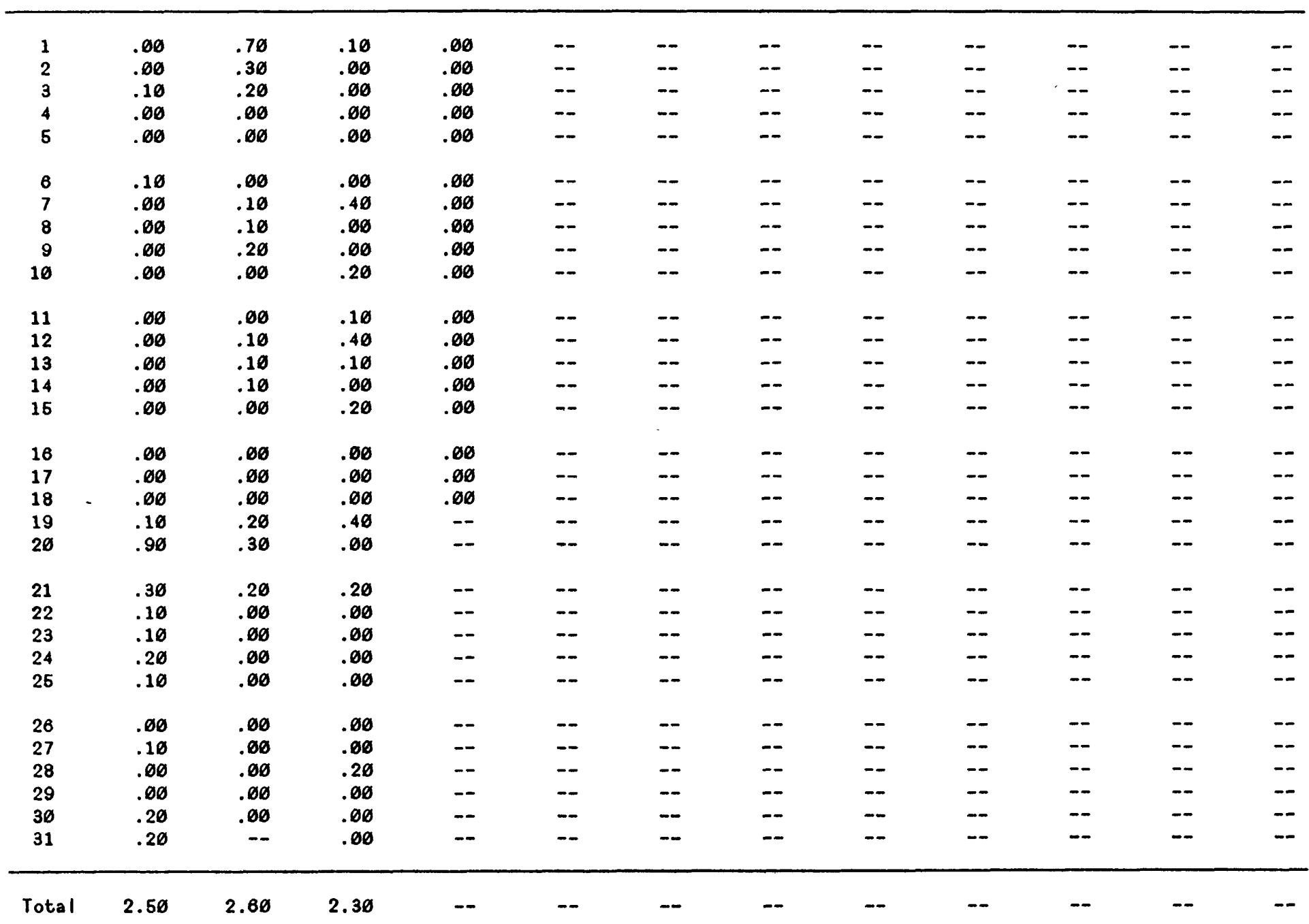


Index number on location map: 5 Station number: $3442 \varnothing 4 \varnothing 993663 \varnothing 2$ Local number: $\varnothing 3 N-23 W-28$ DADA 2 Location: Lat $34^{\prime} 42^{\prime} 04^{\prime \prime}$, long $99^{\circ} 36^{\prime} 63^{\prime \prime}$, hydrologic unit 11126262 Altitude of land-surface datum: $1434 \mathrm{ft}$

Water Year, October 1986 to September 1986

Day Oct. Nov. Dec. Jan. Feb. Mar. Apr. May June July Aug. Sept.

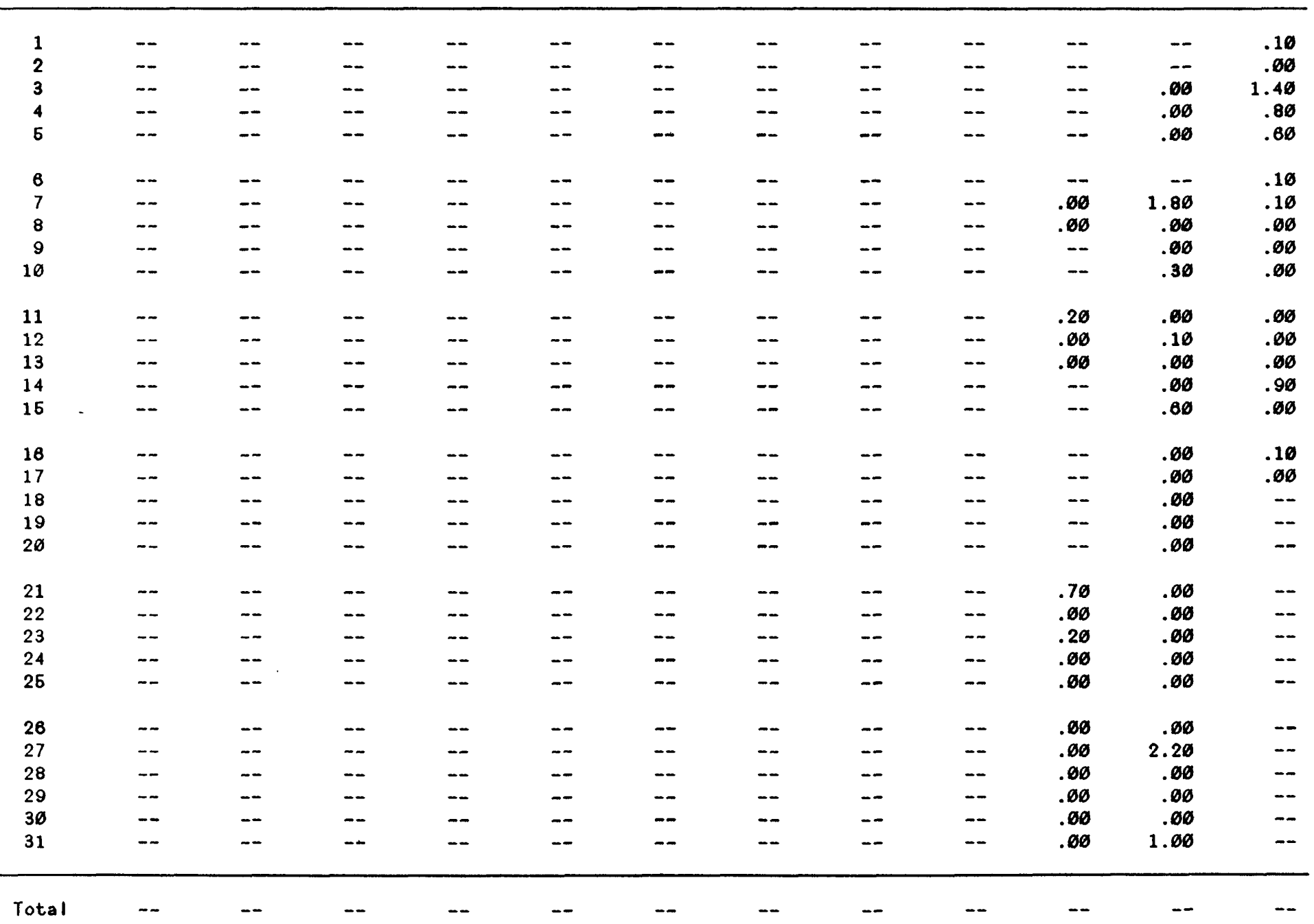


Table 4.--Daily precipitation measurements in southwestern Oklahoma--Continued

JACKSON COUNTY, OKLAHOMA--COntinued

63N-23W-28 DADA 2--Continued

Water Year, October 1988 to September 1989

Day Oct. Nov. Dec. Jan. Feb. Mar. Apr. May June July Aug. Sept.

\begin{tabular}{|c|c|c|c|c|c|c|c|c|c|c|c|c|}
\hline 1 &.$\varnothing \varnothing$ & .00 & -- & -- & -- & - & -- & -- & -- & -- & -- & -- \\
\hline 2 &.$\varnothing \varnothing$ &.$\varnothing 6$ & $\sim$ & $m$ & -- & -- & $m$ & - & - & -- & -- & -- \\
\hline 3 &.$\varnothing \varnothing$ & .10 & -- & -- & $\sim$ & -- & -- & -- & -- & -- & -- & -- \\
\hline 4 &.$\varnothing \varnothing$ &.$\varnothing \varnothing$ & -- & -- & -- & -- & -- & -- & $\cdots$ & - & -- & -- \\
\hline 5 &.$\varnothing \varnothing$ &.$\varnothing \varnothing$ & -- & -- & -- & -- & -- & -- & -- & -- & -- & -- \\
\hline 6 &.$\varnothing 0$ &.$\varnothing \varnothing$ & $\sim$ & $\sim$ & -- & -- & -- & -- & $\sim$ & -- & -- & -- \\
\hline 7 & .00 &.$\varnothing \varnothing$ & $\sim$ & -- & -- & -- & -- & -- & -- & -- & -- & -- \\
\hline 8 &.$\varnothing \varnothing$ &.$\varnothing \varnothing$ & $\sim$ & -- & -- & -- & -- & $-\infty$ & -- & $\sim$ & -- & - \\
\hline 9 &.$\varnothing \varnothing$ & .06 & -- & -- & -- & -- & -- & $-\infty$ & -- & $\sim$ & $\sim$ & -- \\
\hline 10 &.$\varnothing \varnothing$ & $.0 \theta$ & $\sim$ & $\sim$ & -- & - & $\sim$ & - & - & $\sim$ & $\sim$ & -- \\
\hline 11 &.$\varnothing \varnothing$ &.$\varnothing \varnothing$ & -- & -- & -- & -- & -- & -- & -- & $\sim$ & $\sim$ & -- \\
\hline 12 &.$\varnothing \varnothing$ & .00 & -- & -- & -- & -- & -- & -- & -- & -- & -- & -- \\
\hline 13 & .00 & .00 & -- & -- & -- & -- & -- & -- & -- & -- & -- & -- \\
\hline 14 & .00 &.$\varnothing \varnothing$ & -- & -- & -- & -- & -- & - & $\sim$ & -- & $-\sim$ & - \\
\hline 15 &.$\varnothing \varnothing$ & .00 & -- & - & $\sim$ & -- & -- & -- & -- & $\sim$ & $\sim$ & -- \\
\hline 16 & -- &.$\varnothing \varnothing$ & - & -- & -- & -- & -- & -- & $\sim$ & $\sim$ & -- & - \\
\hline 17 & -- &.$\varnothing \varnothing$ & $\sim$ & $m$ & $\sim-$ & -- & -- & -- & -- & -- & $\sim$ & -- \\
\hline 18 & $\sim-$ &.$\varnothing \varnothing$ & -- & -- & -- & -- & -- & - & - & - & - & -- \\
\hline 19 & $-\sim$ & .10 & -- & $\tilde{a}$ & -- & -- & -- & -- & - & -- & -- & - \\
\hline $2 \varnothing$ & .00 & $\sim$ & $\cdots$ & .00 & - & -- & -- & -- & $-\sim$ & $-\sim$ & $\sim$ & - \\
\hline 21 &.$\varnothing \varnothing$ & -- & -- &.$\varnothing \varnothing$ & -- & - & -- & -- & -- & -- & $\sim$ & -- \\
\hline 22 & .00 & -- & -- & .00 & -- & -- & -- & -- & -- & -- & -- & - \\
\hline 23 &.$\varnothing \varnothing$ & -- & -- & .00 & $\sim$ & - & -- & -- & -- & -- & -- & - \\
\hline 24 &.$\varnothing \varnothing$ & -- & -- &.$\varnothing \varnothing$ & -- & - & -- & -- & -- & $\sim$ & $\sim$ & - \\
\hline 26 &.$\varnothing \varnothing$ & -- & -- & .30 & -- & $\sim$ & -- & - & -- & $\sim$ & $\sim$ & - \\
\hline 26 &.$\varnothing \varnothing$ & $\sim$ & $\sim$ &.$\varnothing \varnothing$ & $\sim$ & -- & -- & -- & -- & $\sim$ & -- & - \\
\hline 27 &.$\varnothing \varnothing$ & -- & $-\sim$ & $.3 \varnothing$ & -- & -- & - & -- & -- & $\sim$ & -- & - \\
\hline 28 & .00 & -- & -- & .68 & -- & -- & -- & -- & -- & -- & -- & -- \\
\hline 29 & .00 & -- & -- & .00 & -- & -- & -- & -- & -- & -- & -- & -- \\
\hline $3 \varnothing$ & .10 & -- & -- & -- & -- & -- & -- & -- & -- & -- & -- & -- \\
\hline 31 &.$\varnothing \theta$ & - & $\sim$ & -- & - & - & -- & - & -- & - & $\sim$ & - \\
\hline Total & $\sim$ & $-\infty$ & -- & -- & -- & -- & -- & -- & -- & -- & -- & - \\
\hline
\end{tabular}



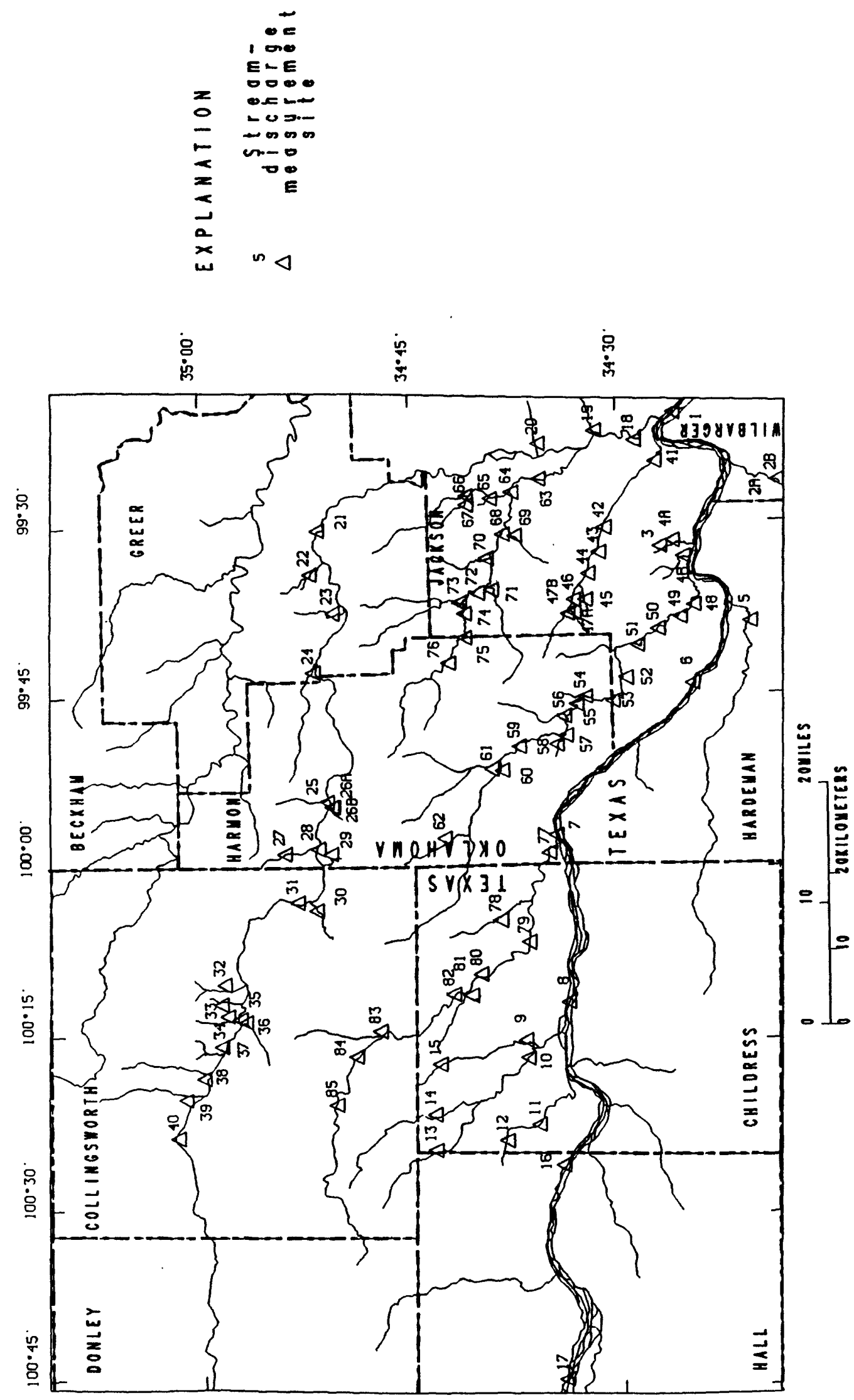

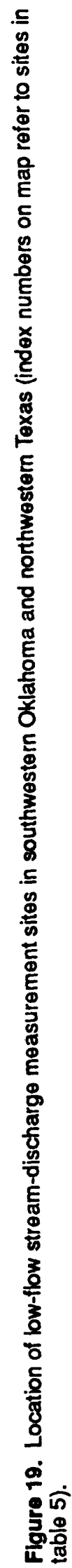




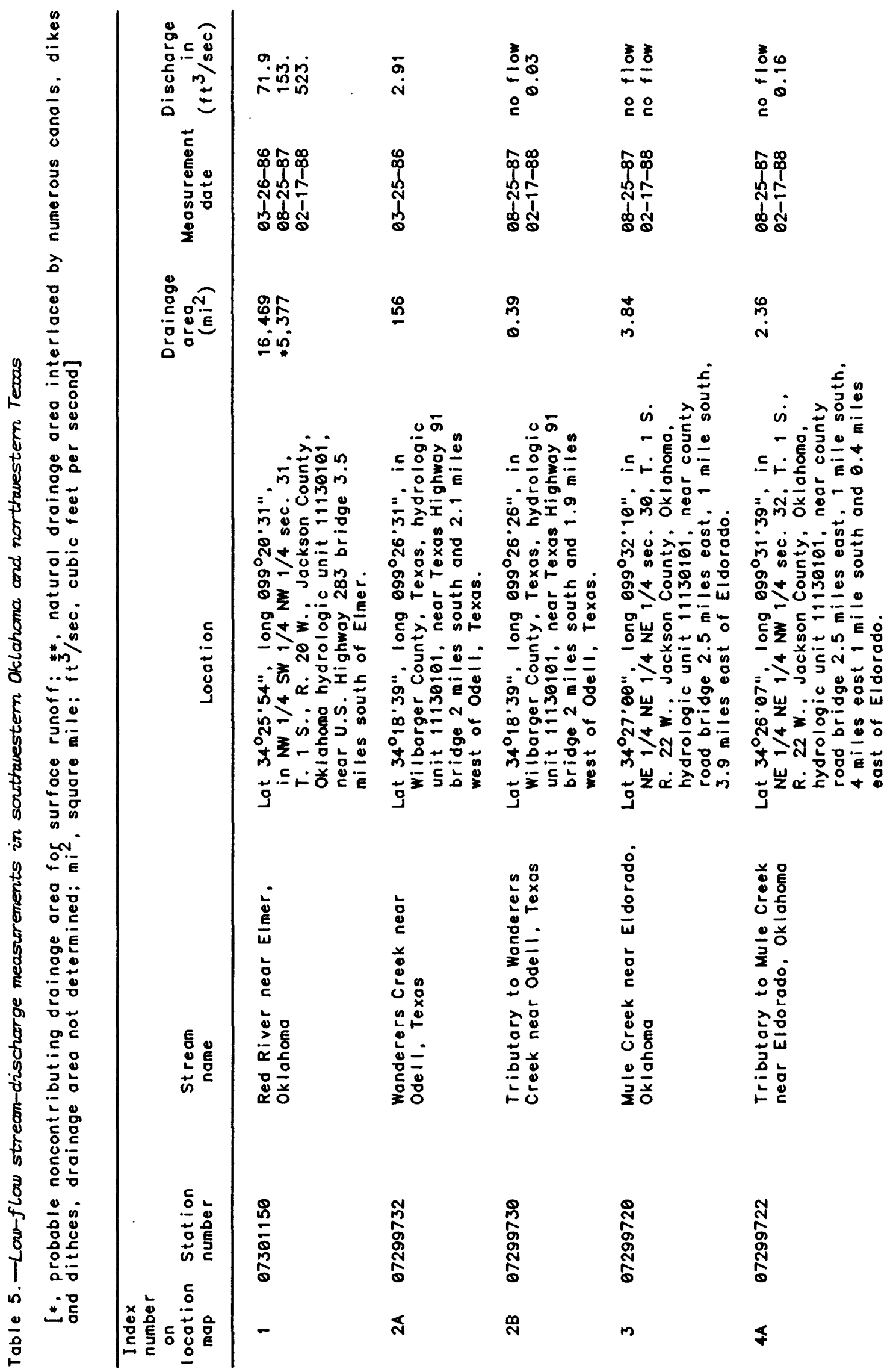




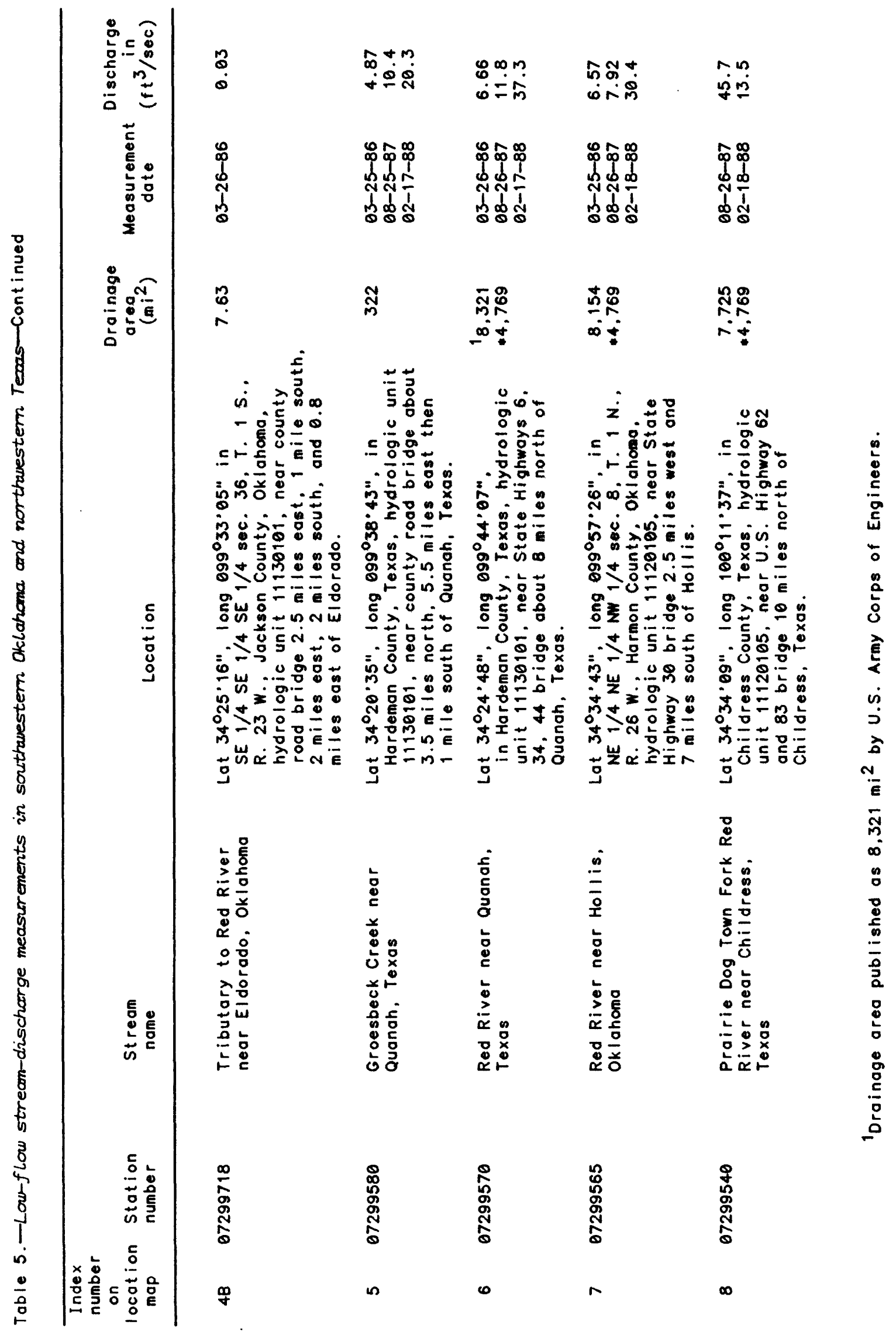




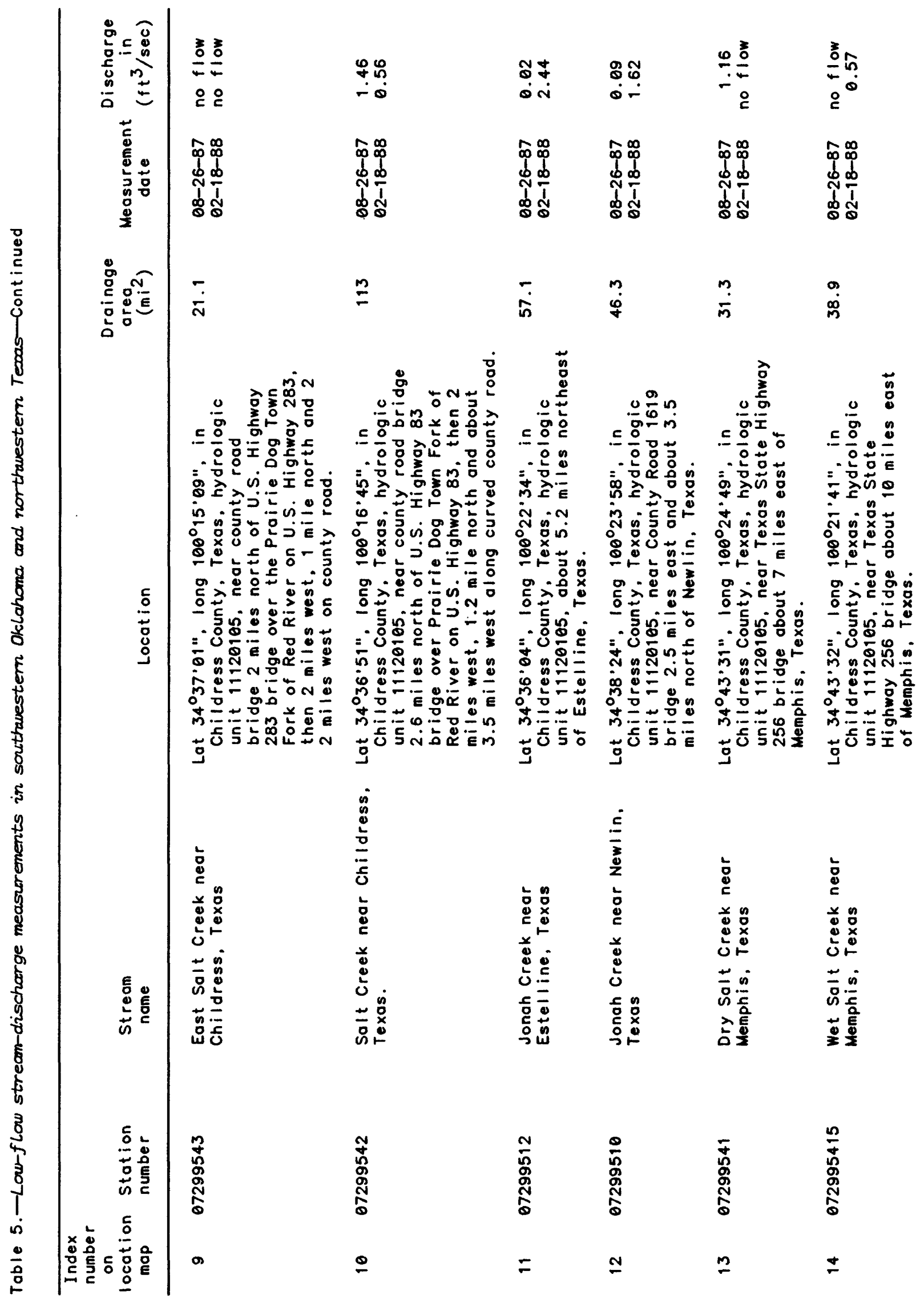




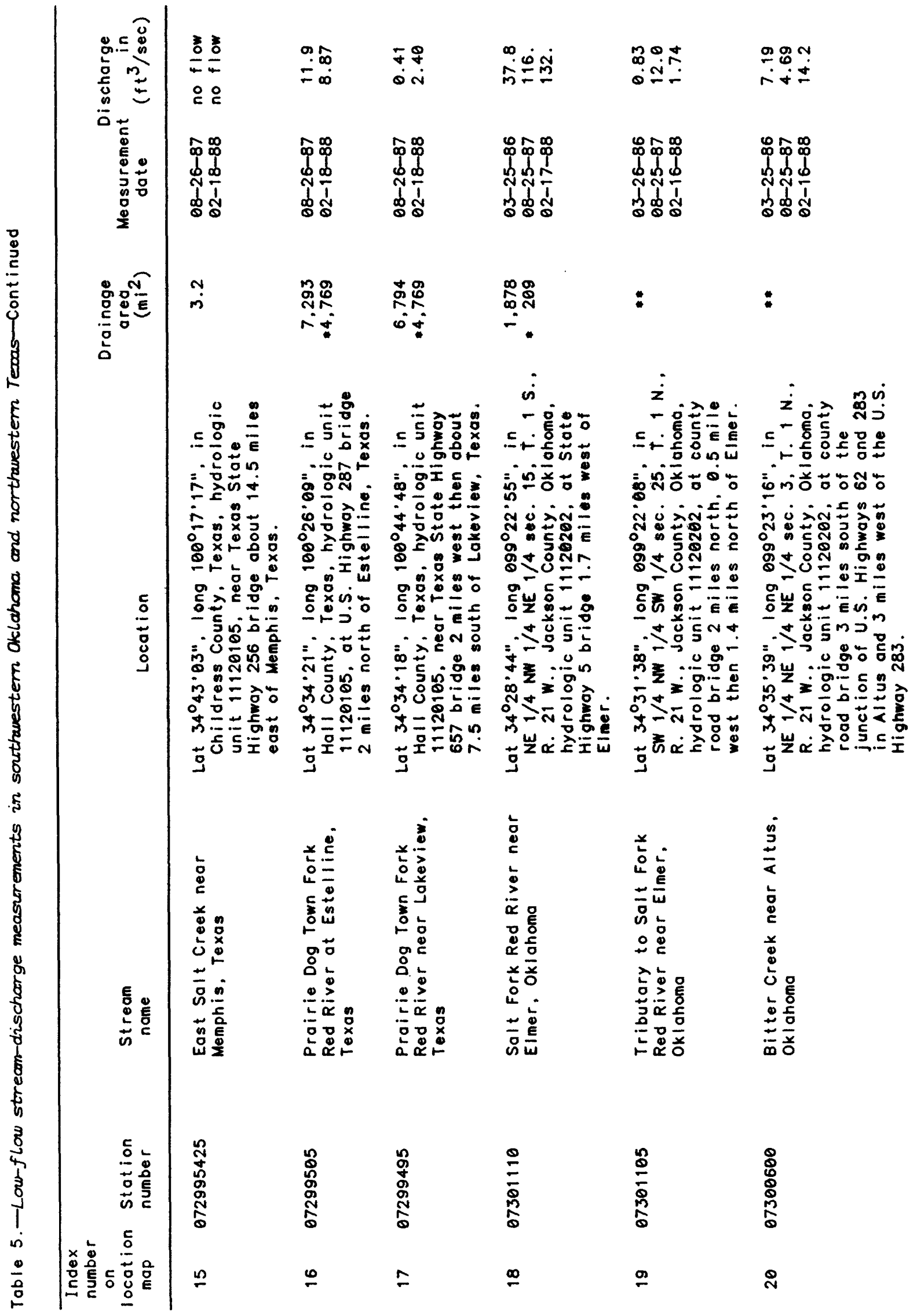




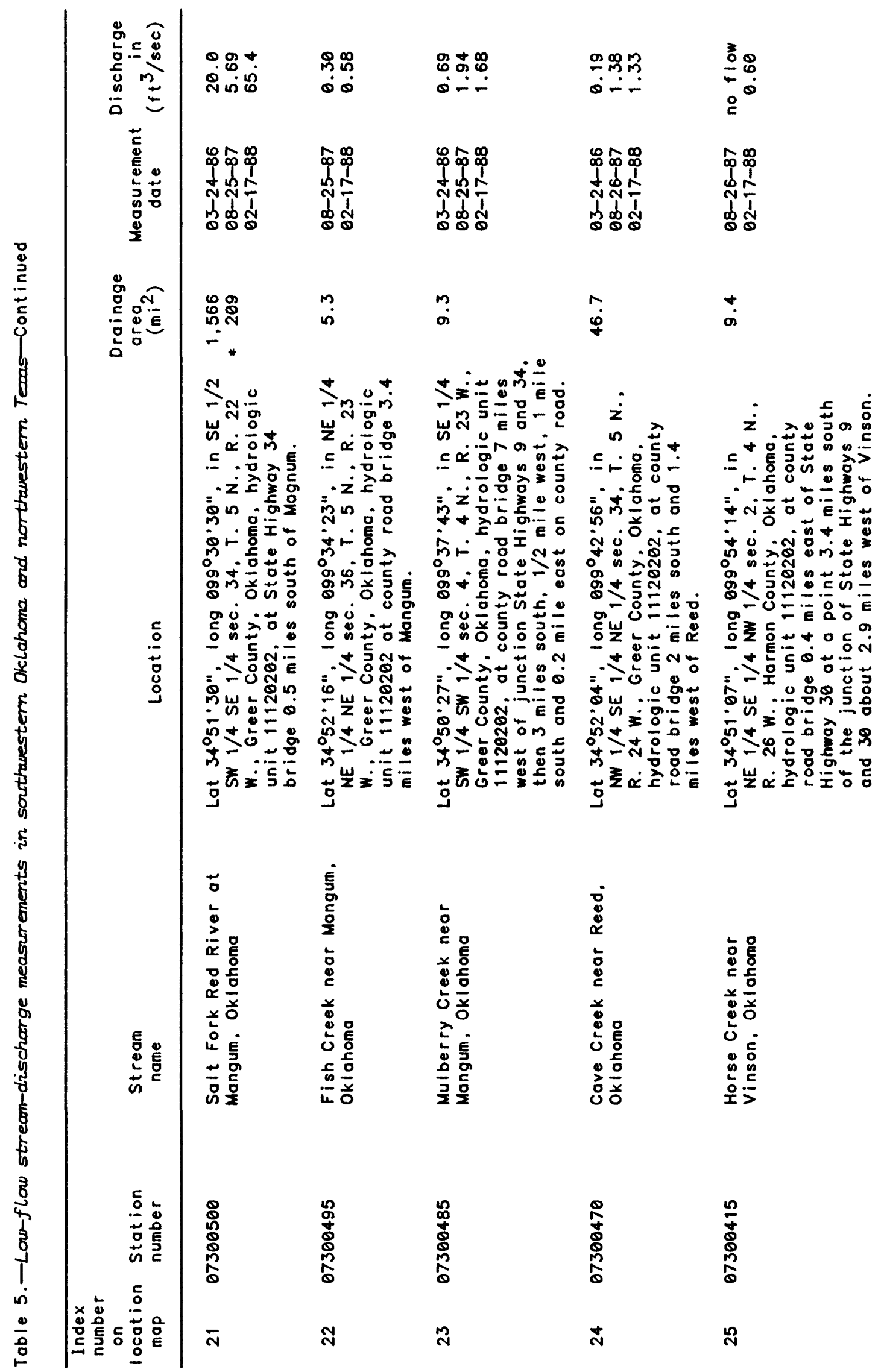




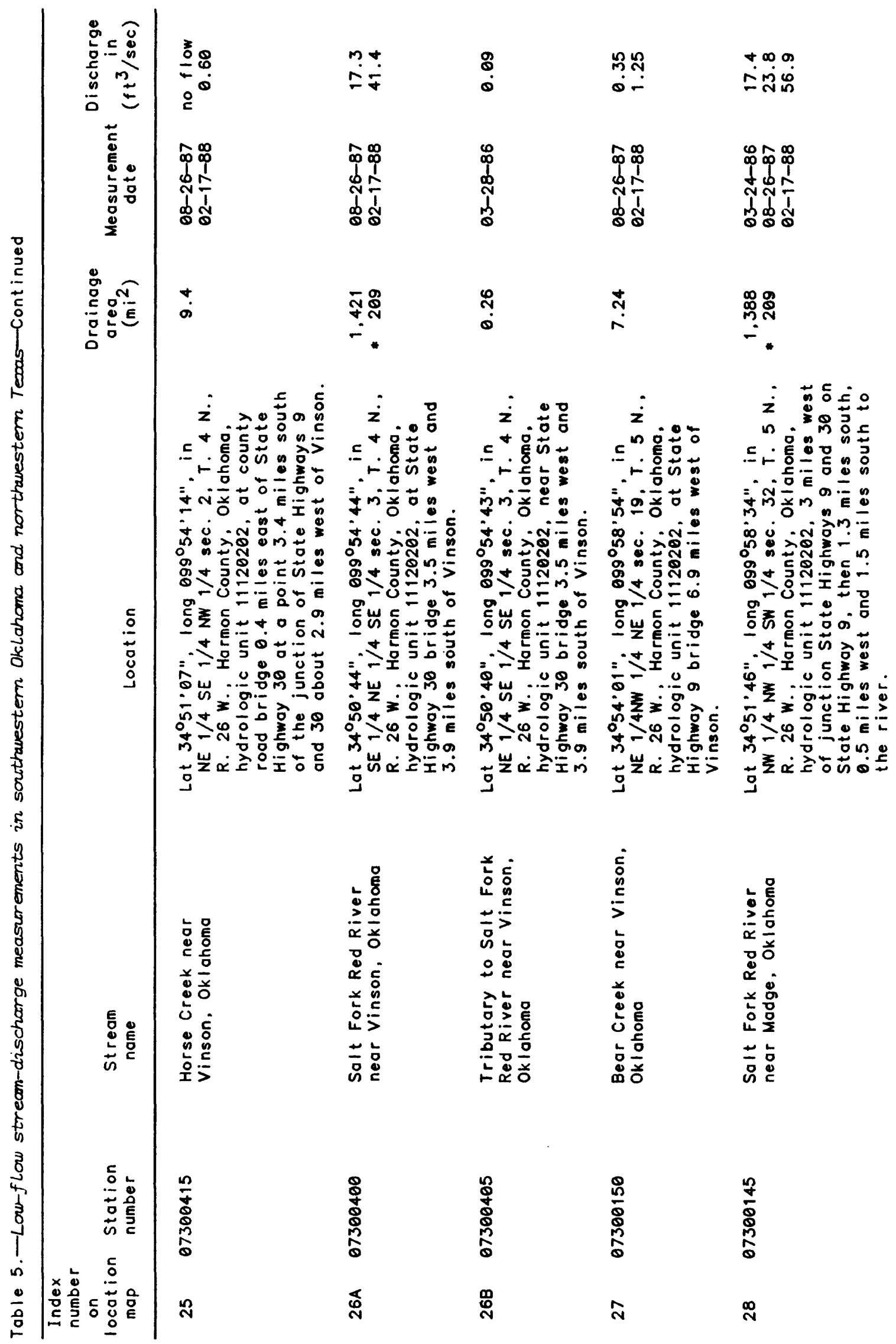




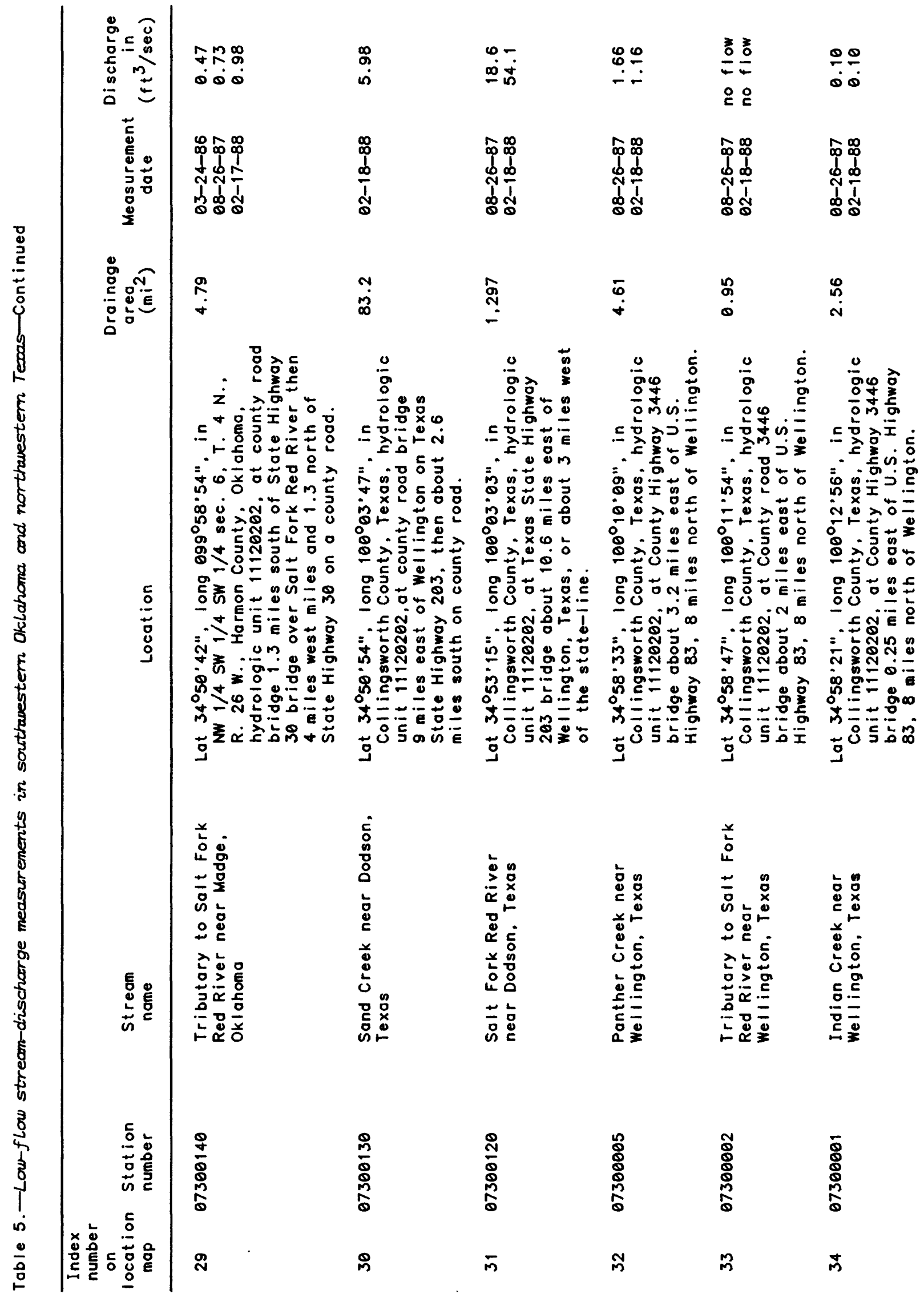




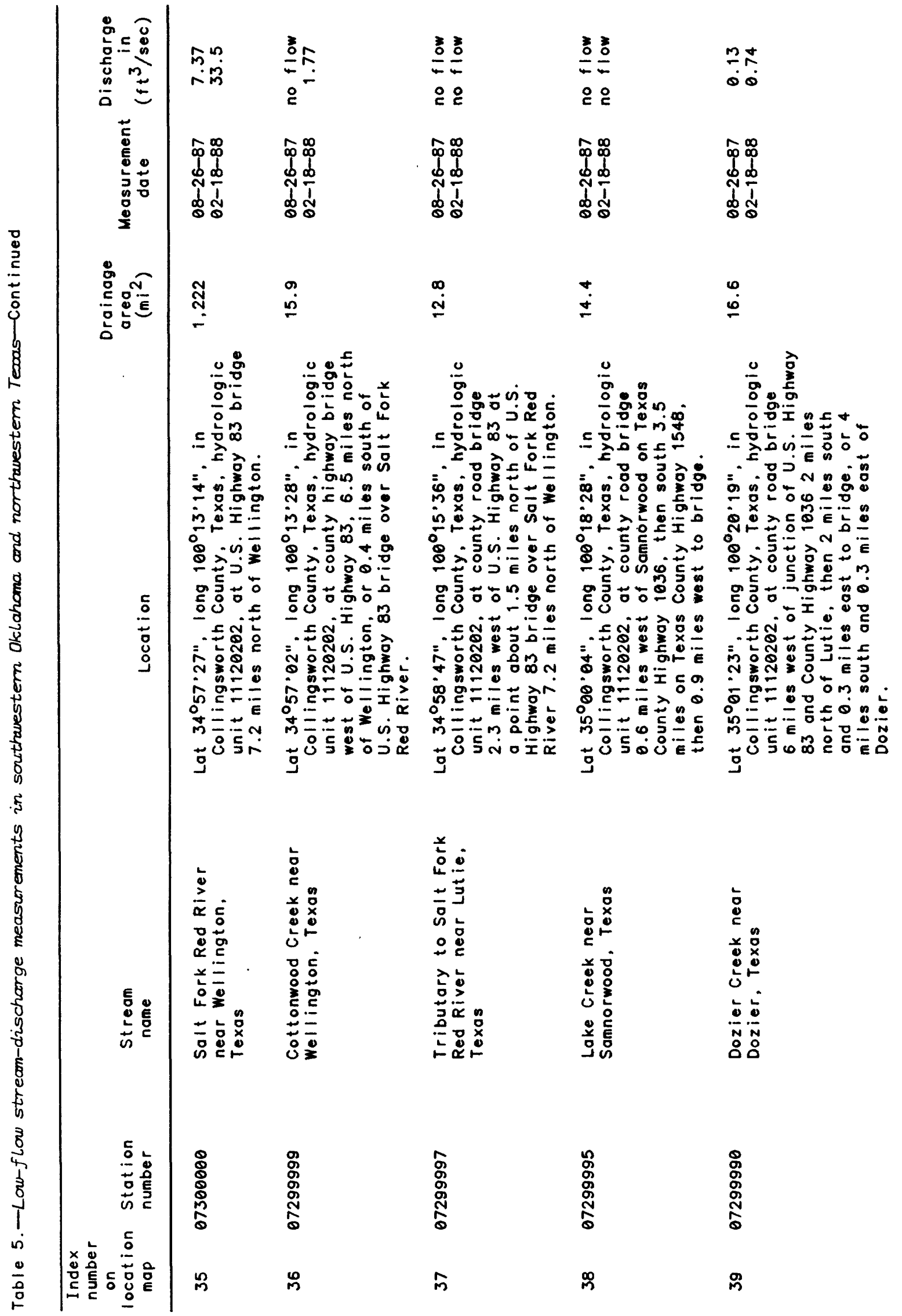




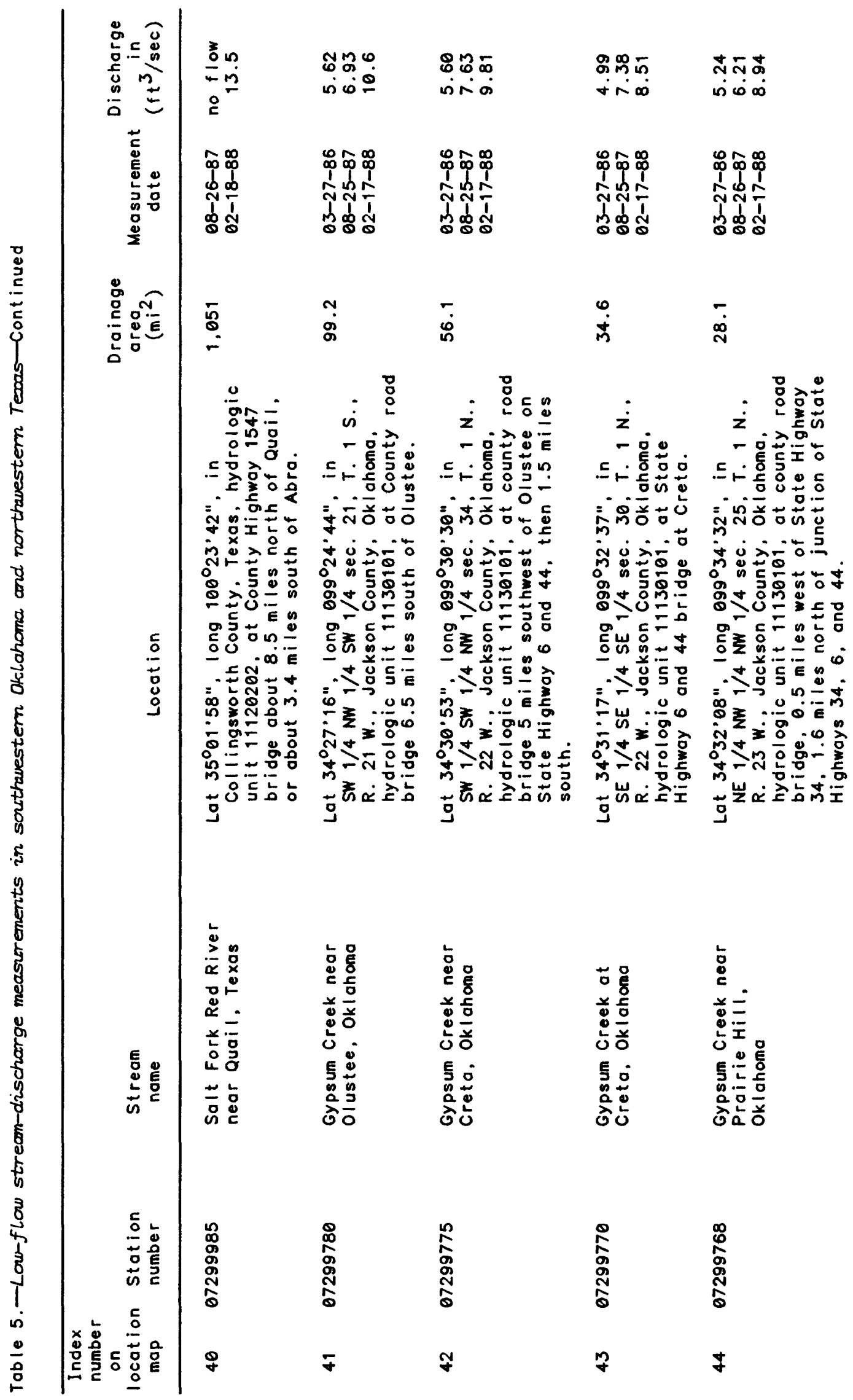




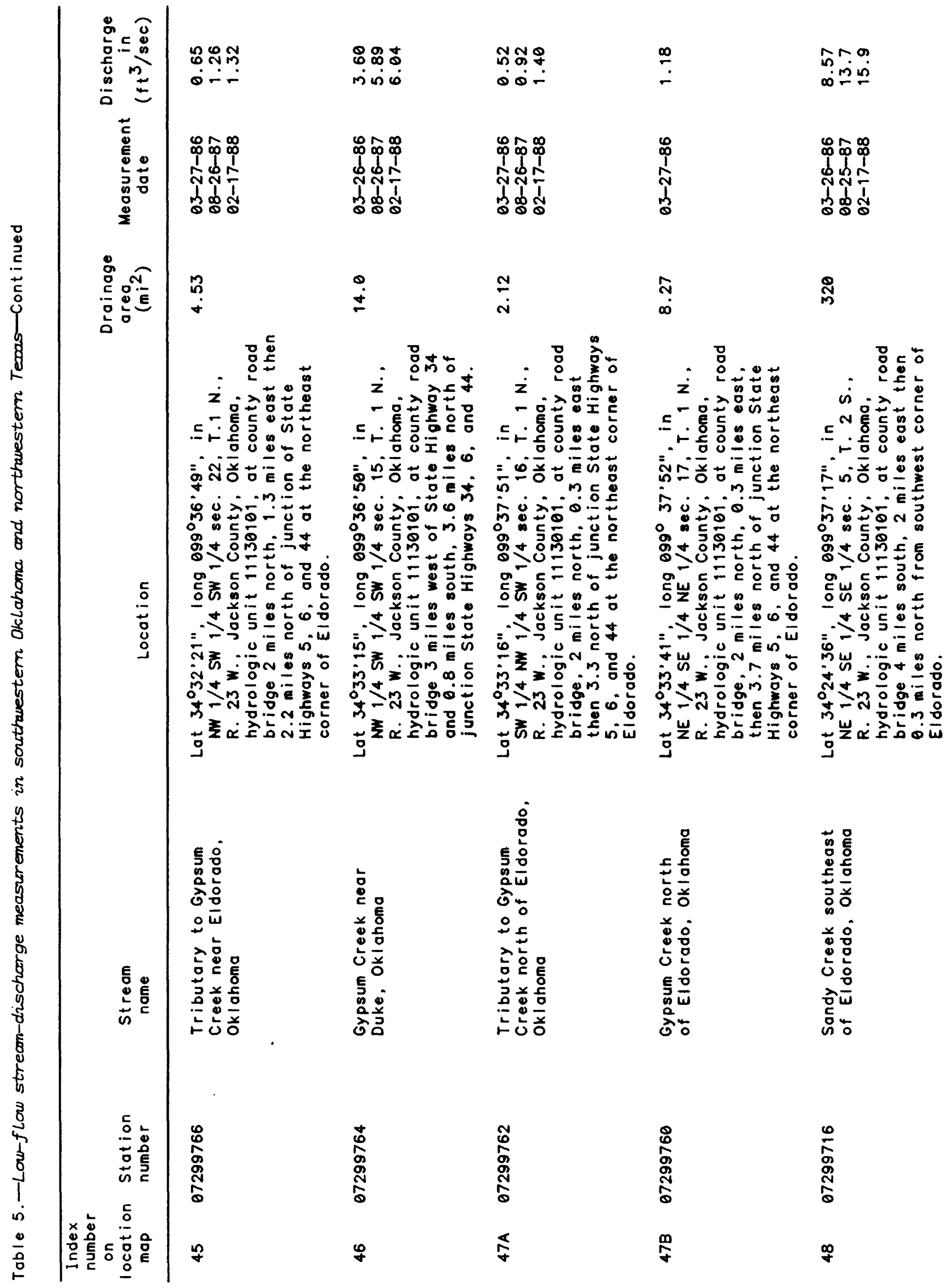




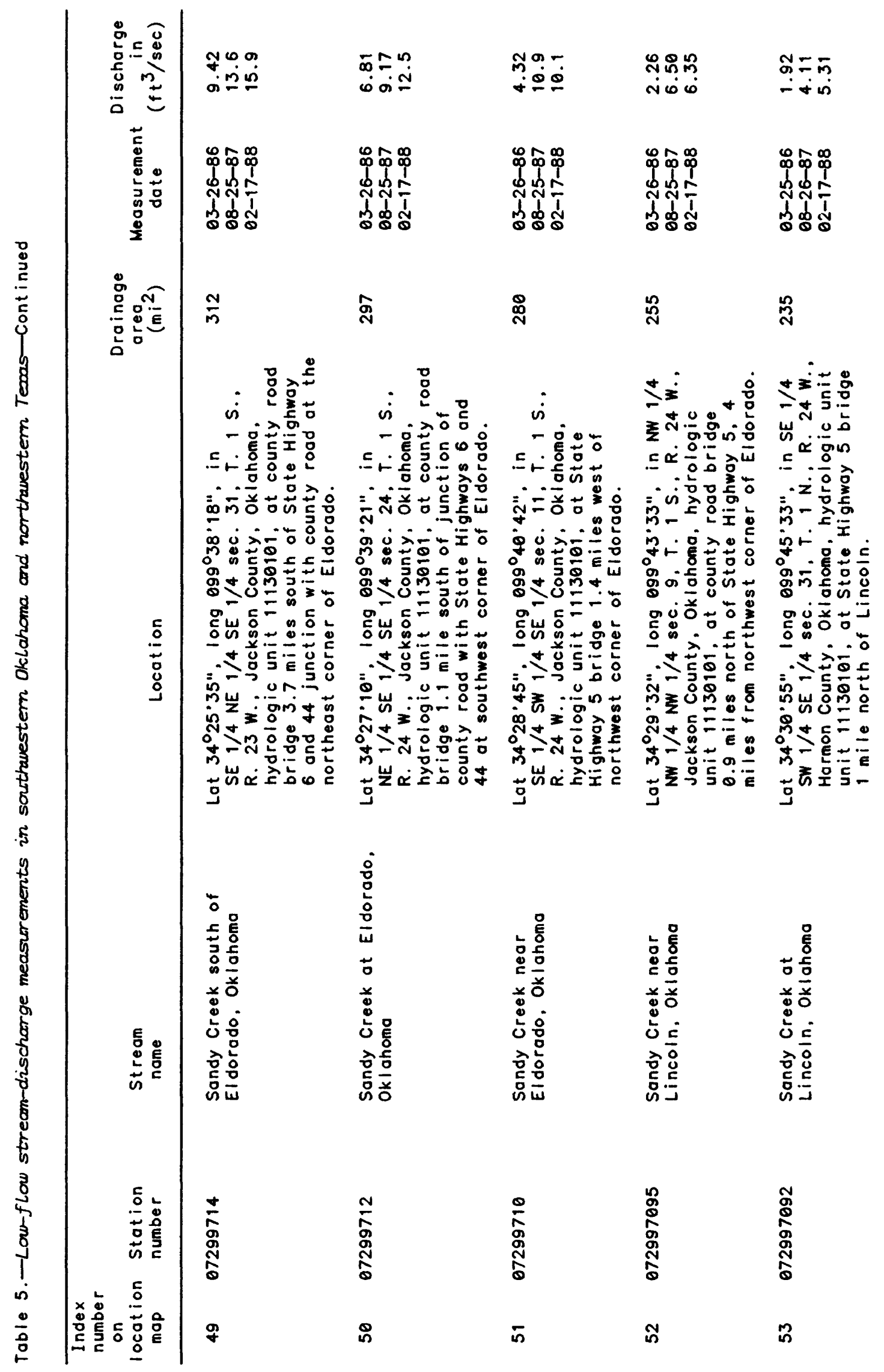




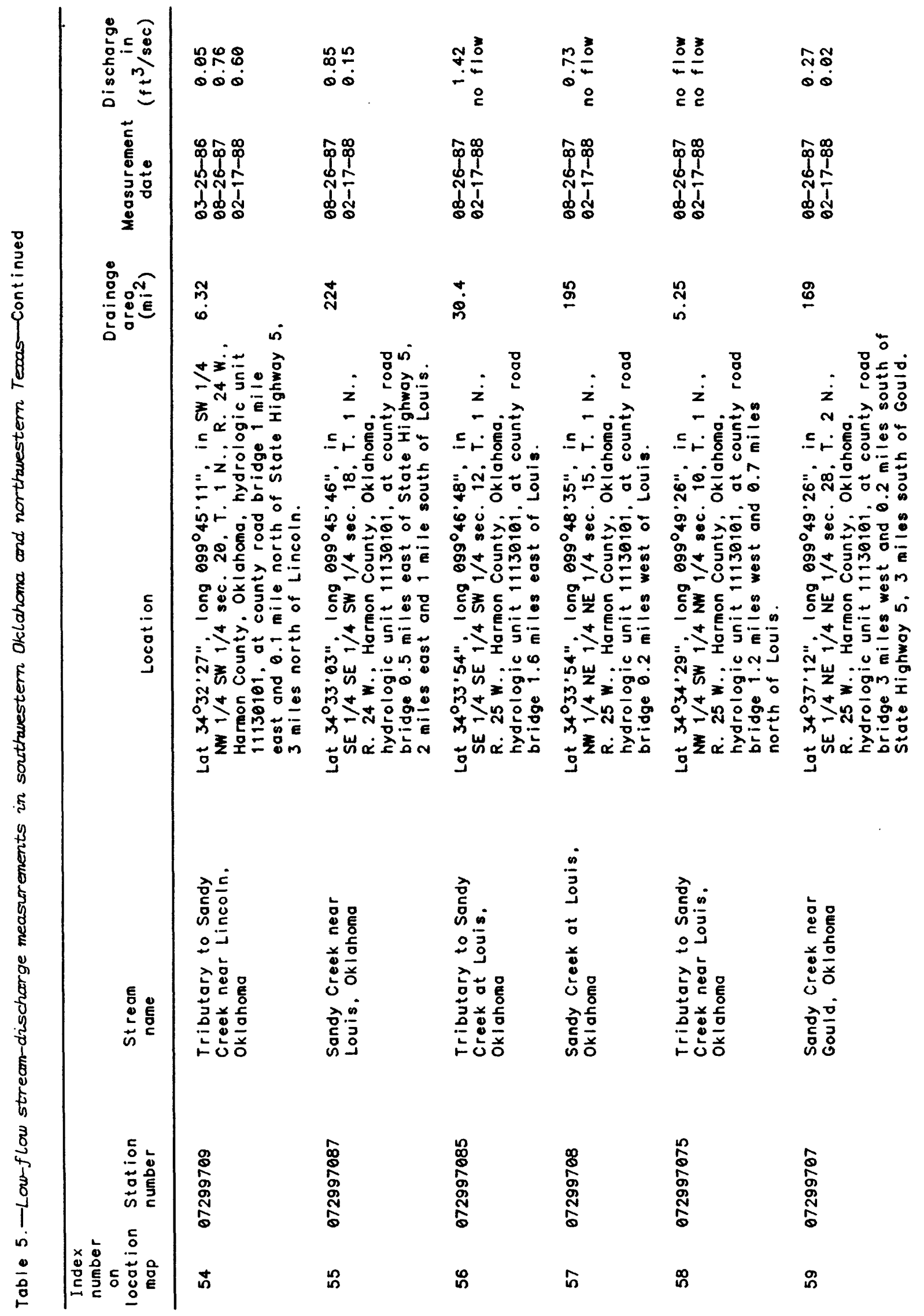




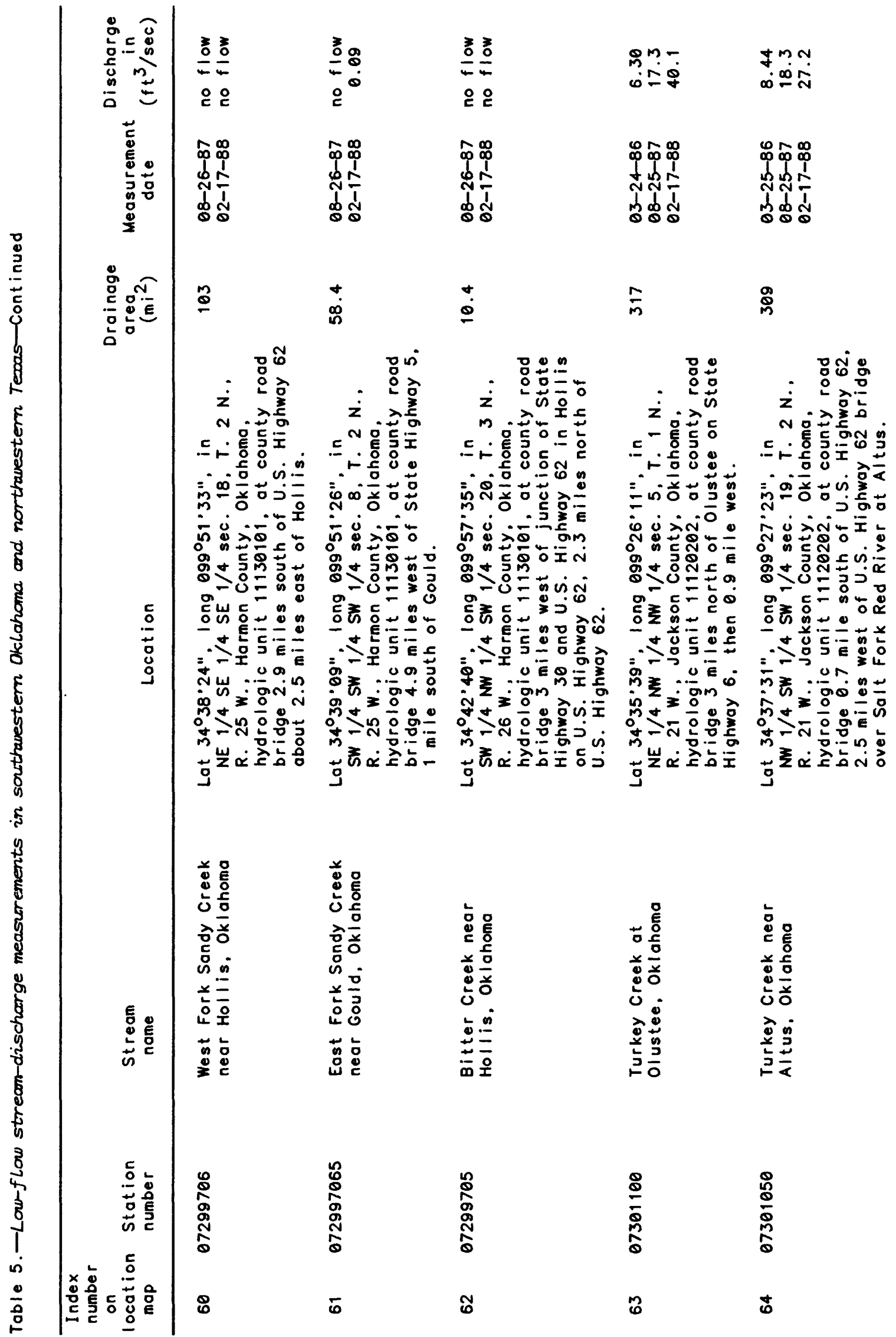




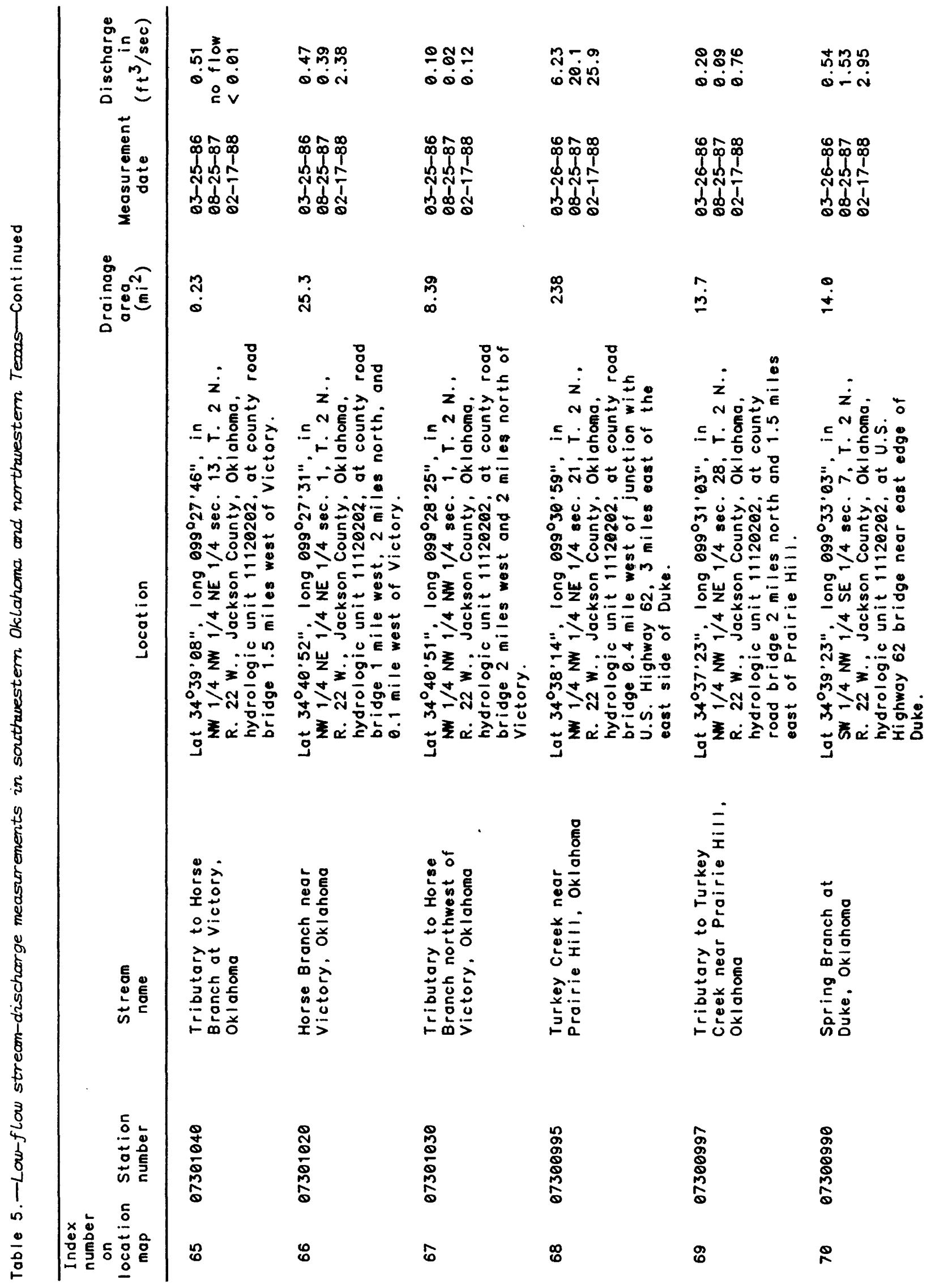




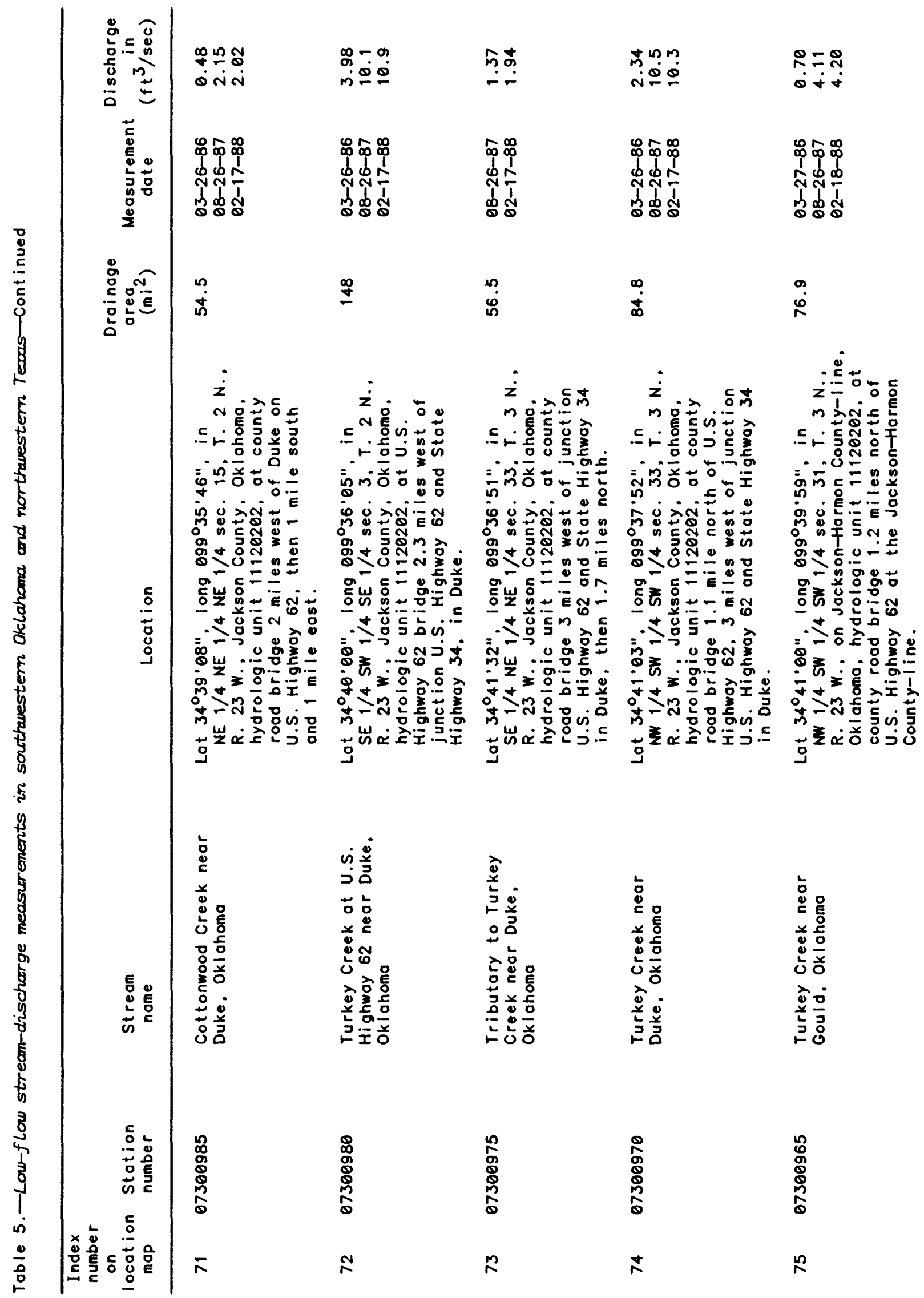




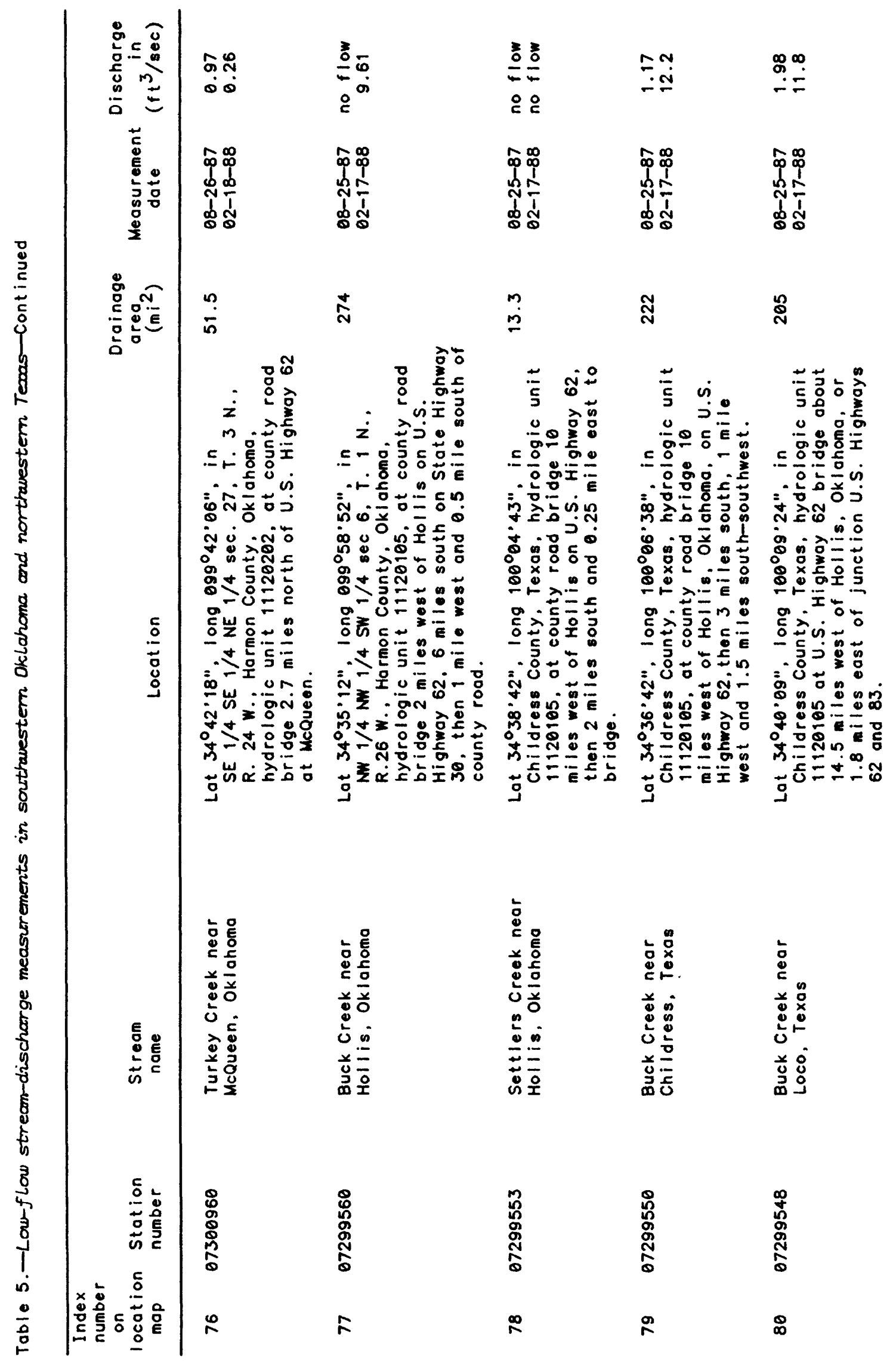




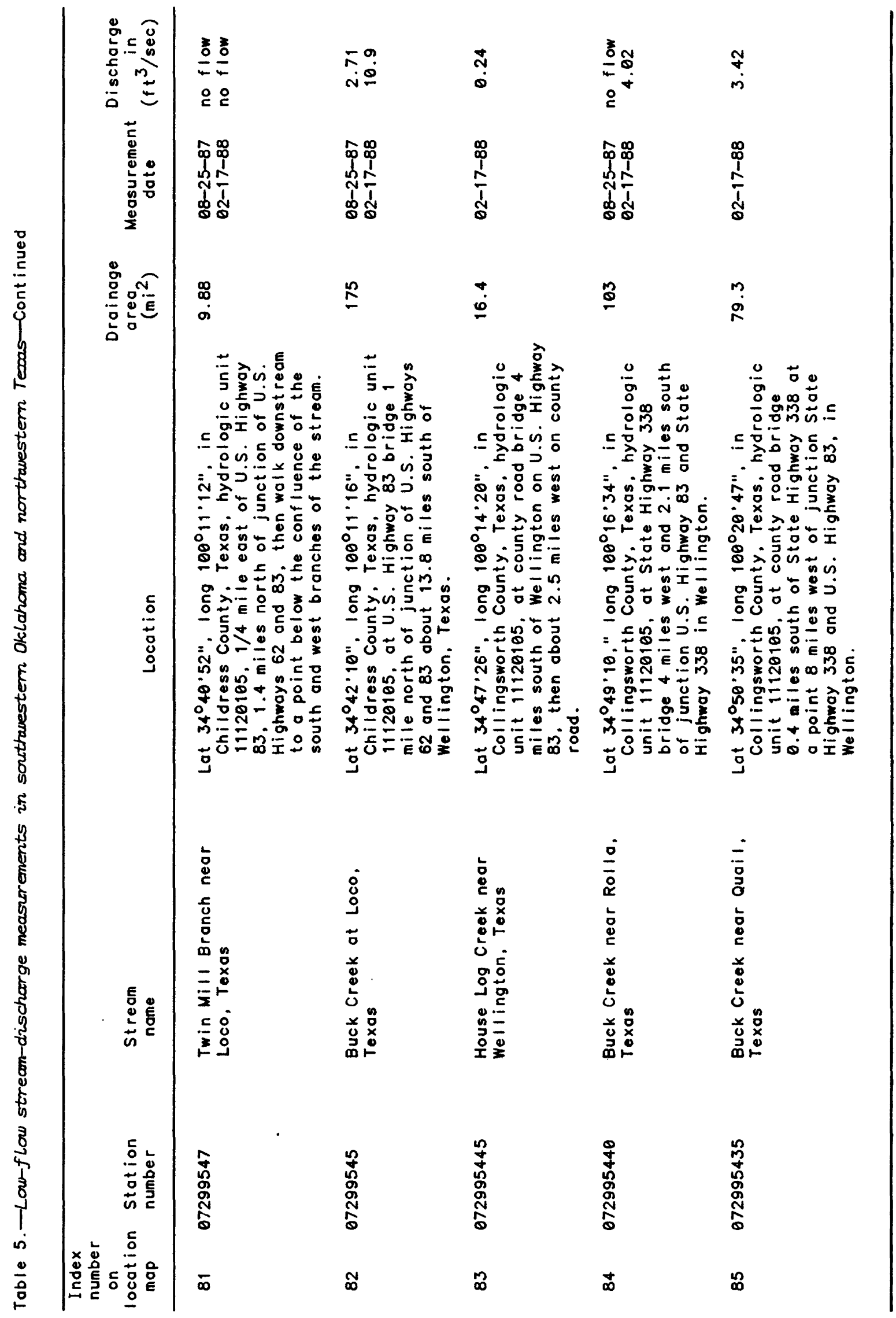



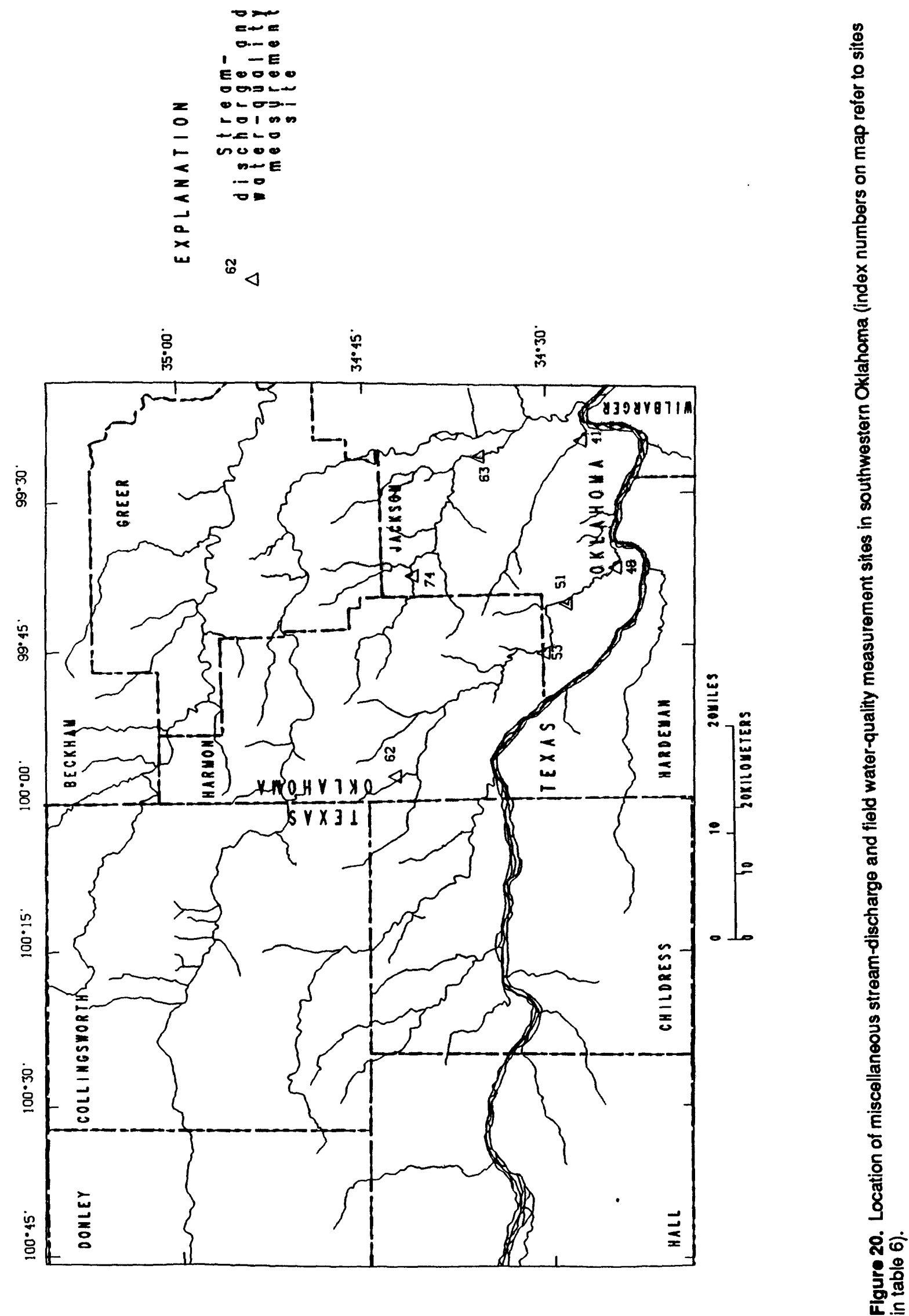


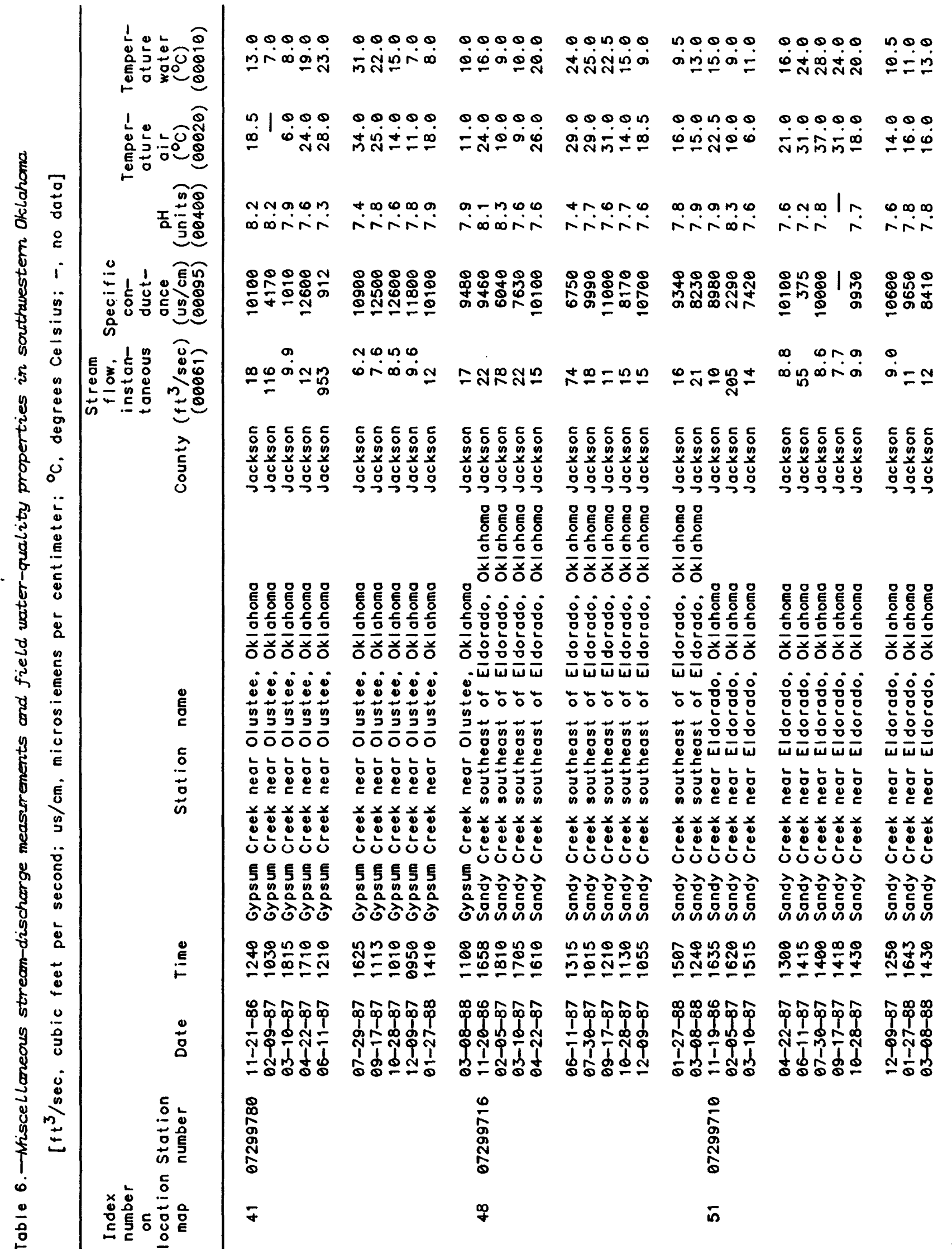




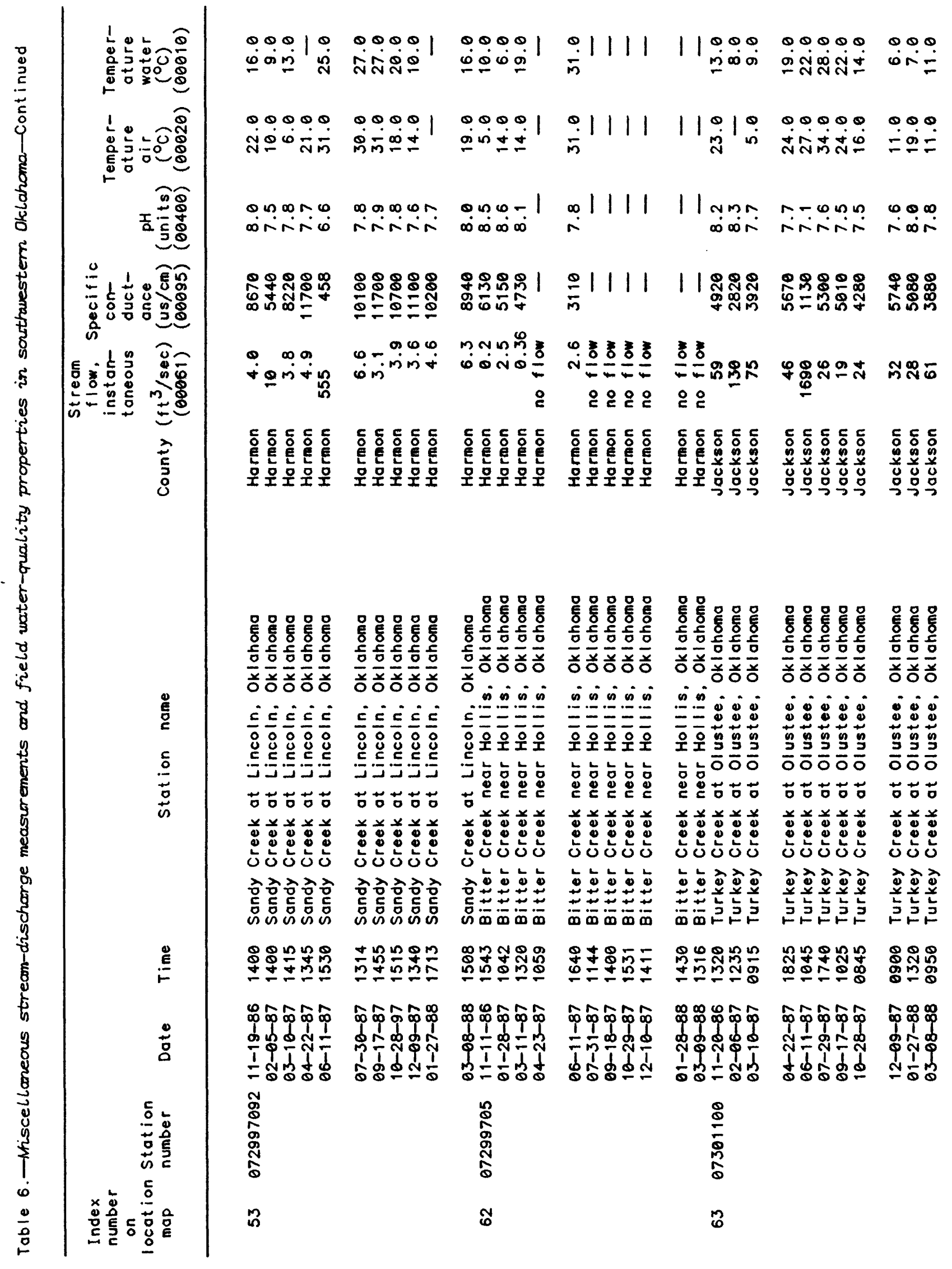




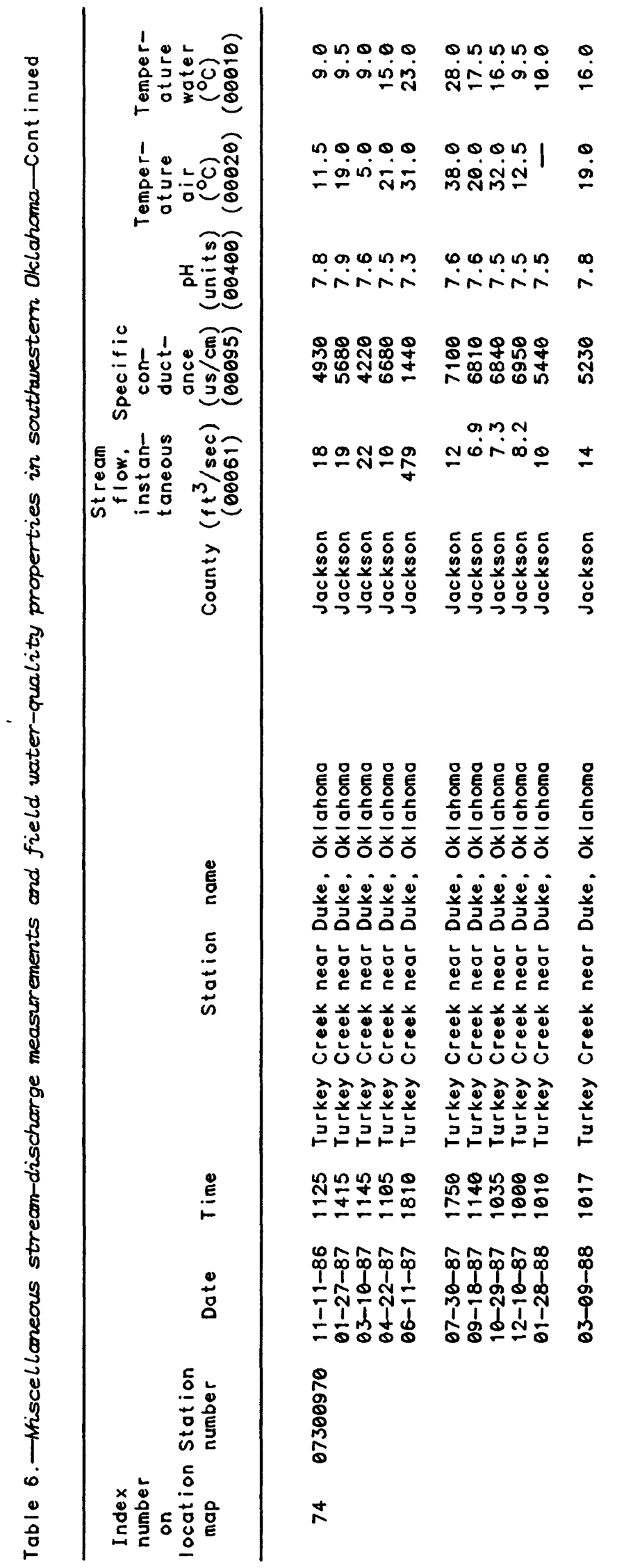



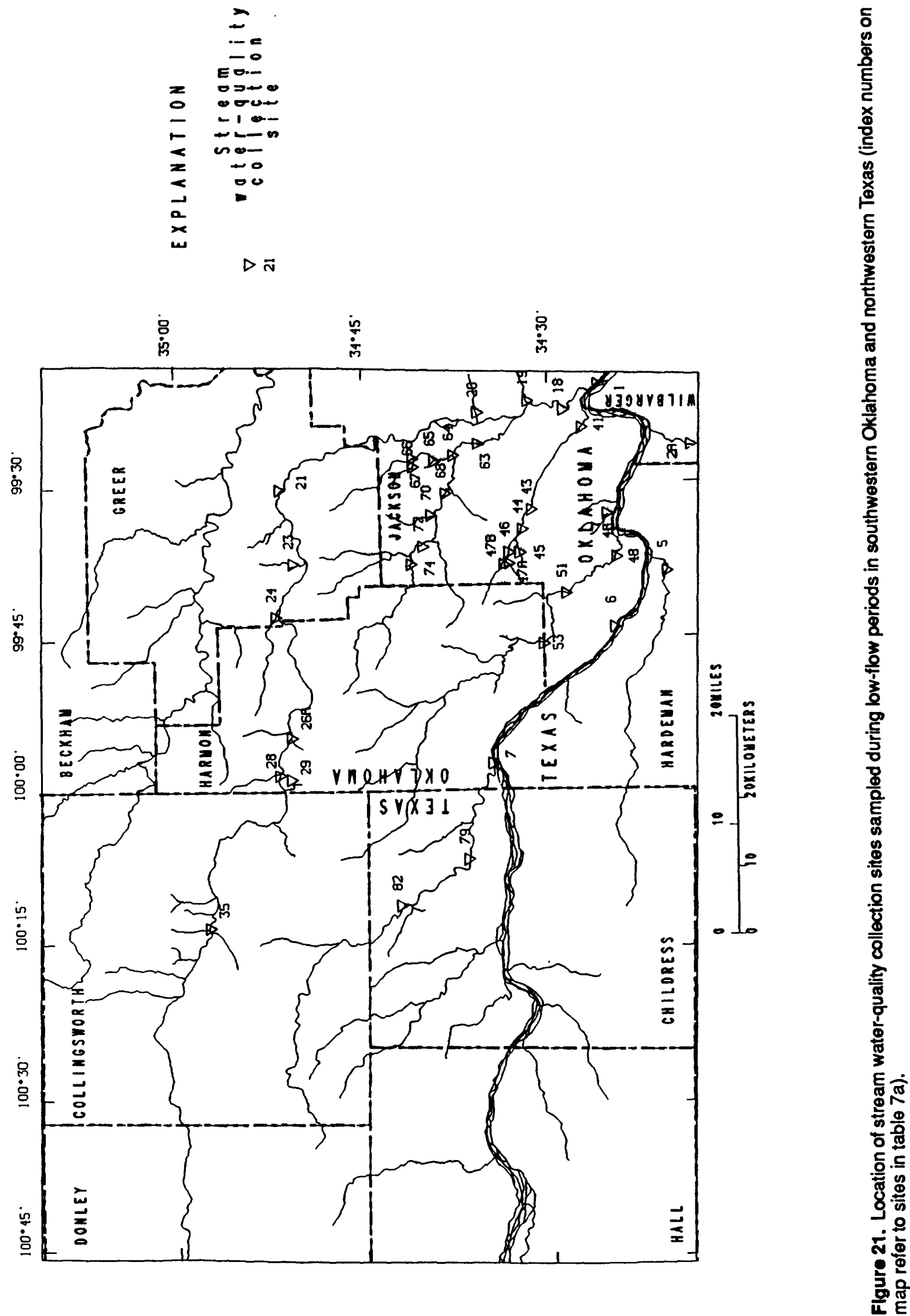


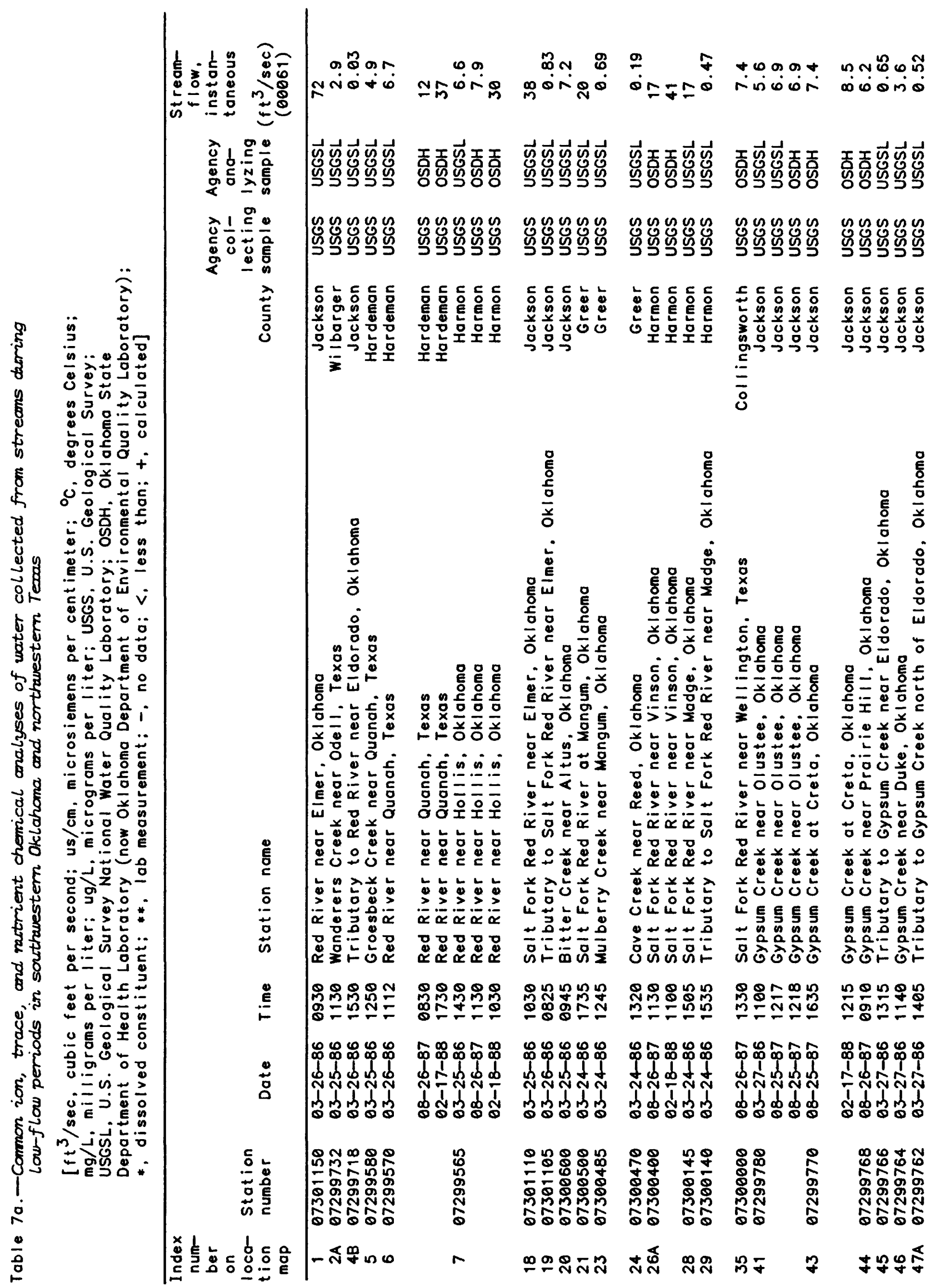




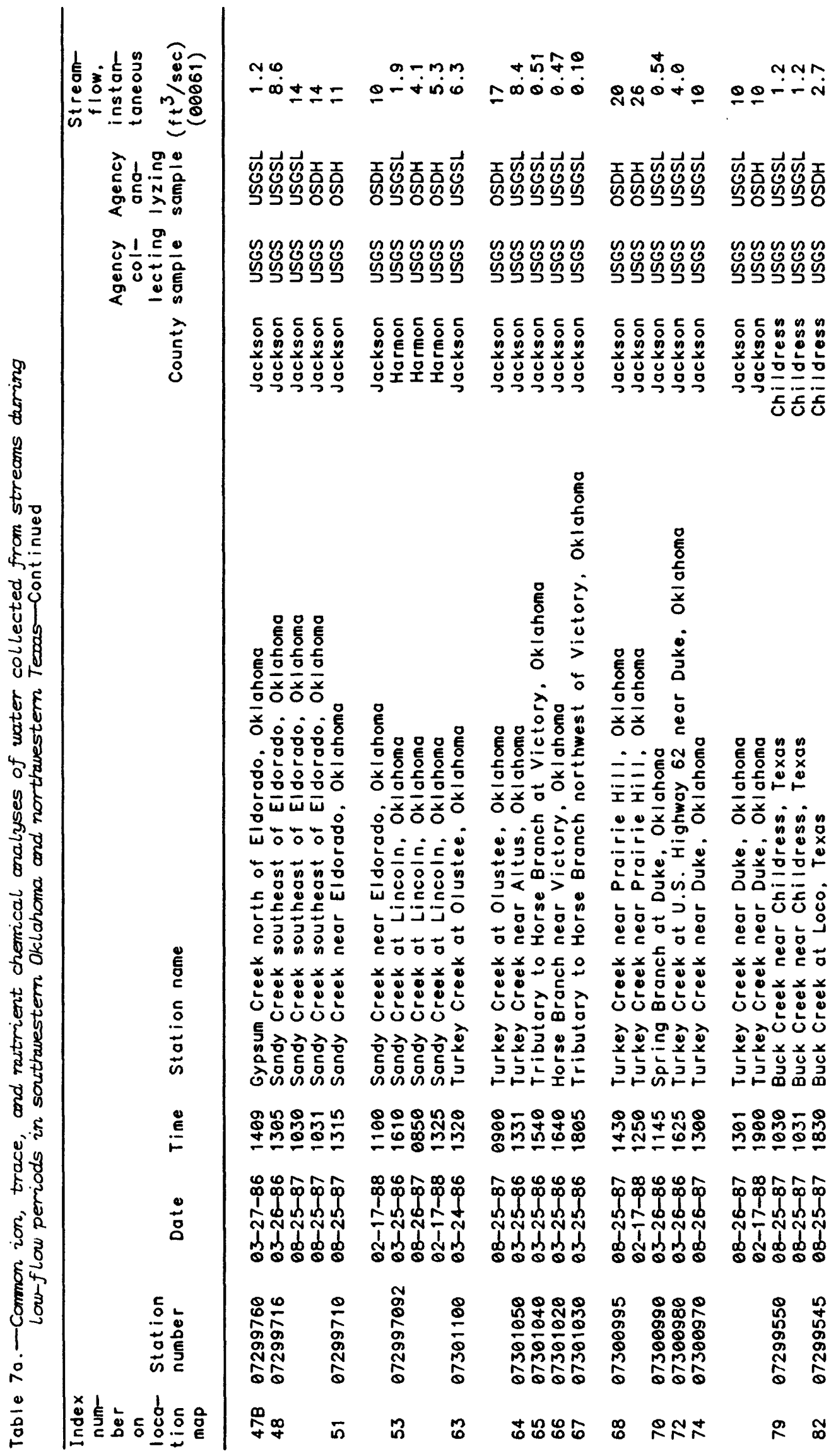




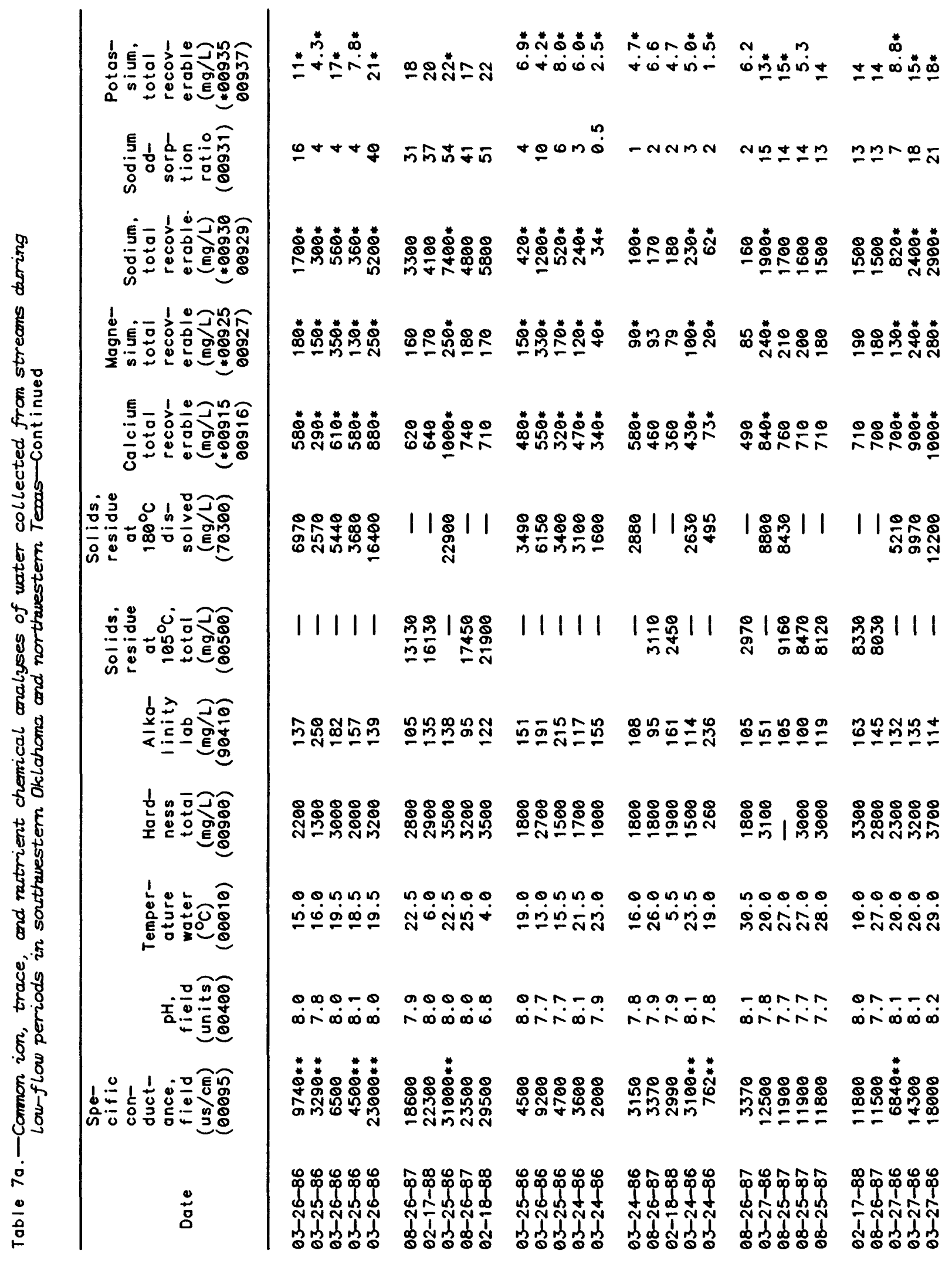




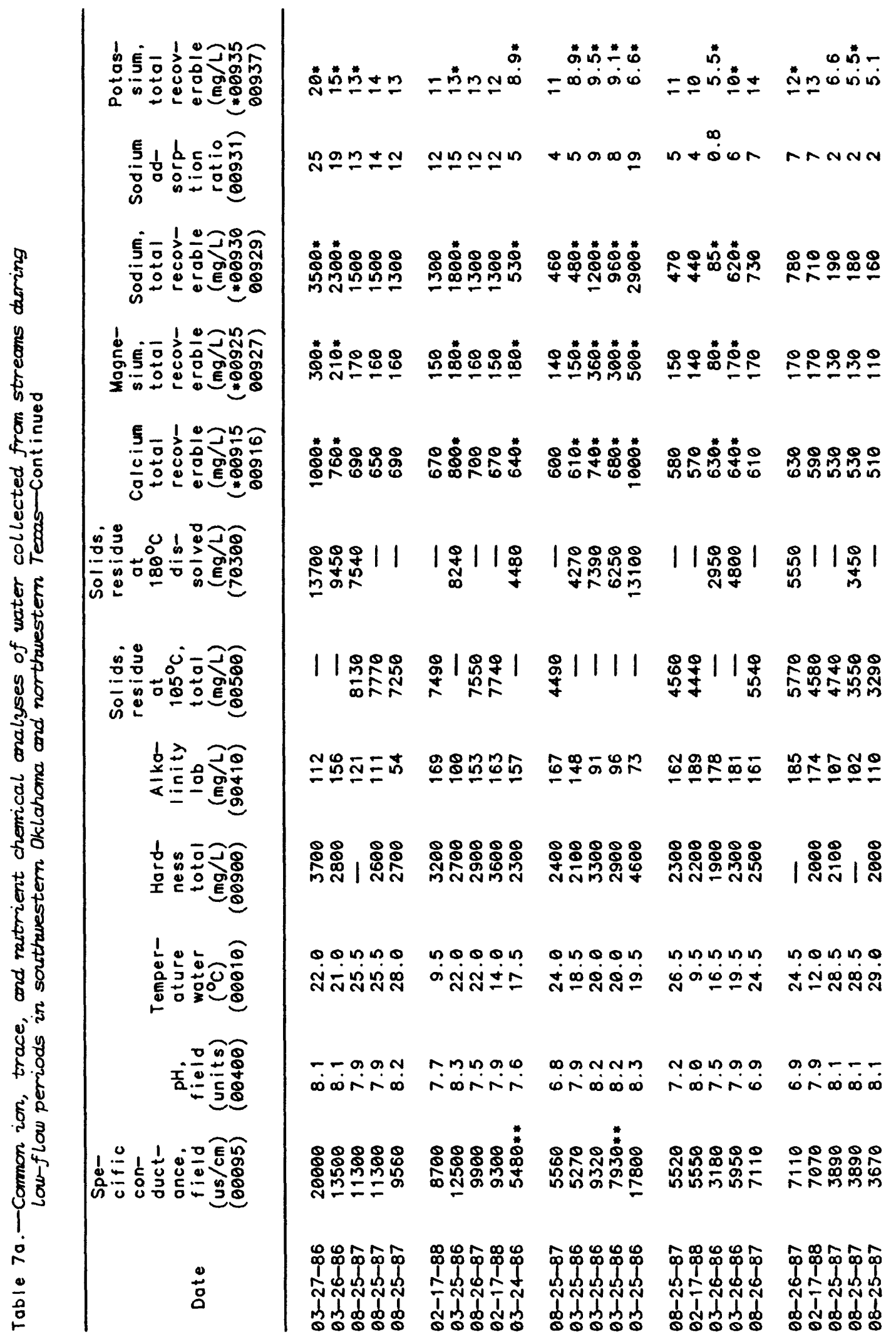




\begin{tabular}{|c|c|c|c|c|c|c|}
\hline 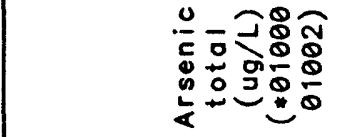 & $\ddot{*} \dot{*} \frac{*}{V} \cdot \dot{*}$ & : & ஸ் & $\stackrel{*}{*} \underset{V}{\mathbb{V}}$ & 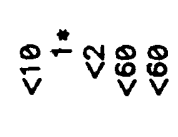 & 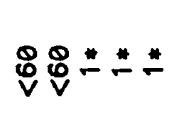 \\
\hline 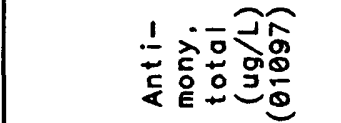 & 11111 & 号号 | 号号 & 11111 & | 亮员 | 1 & 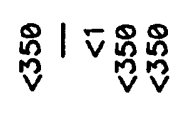 & 昜瓷 | | \\
\hline 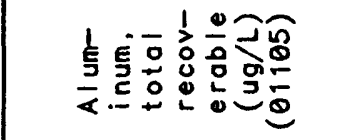 & 11111 & \$ $\$$ & 11111 & | 吕용 | & \& $18 \%$ & 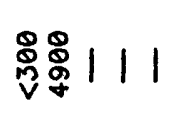 \\
\hline 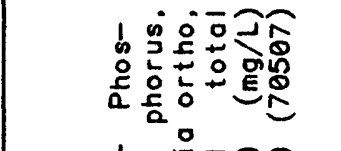 & 11111 & 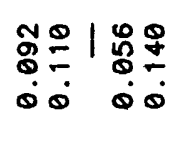 & 11111 & 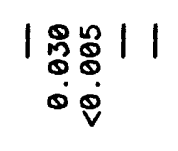 & 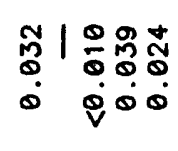 & 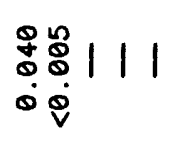 \\
\hline 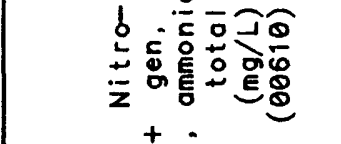 & 11111 & 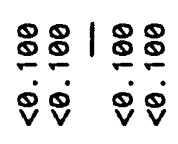 & 11111 & 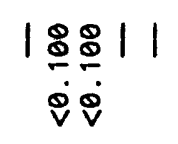 & 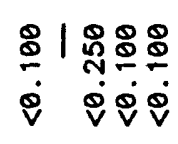 & 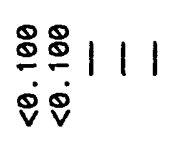 \\
\hline 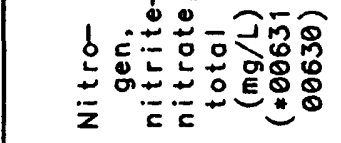 & 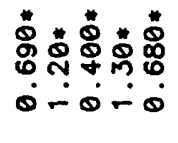 & 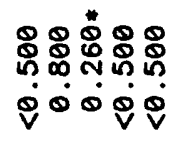 & 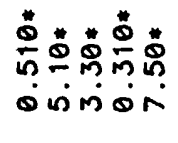 & 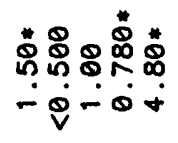 & 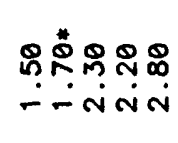 & 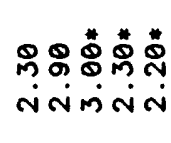 \\
\hline 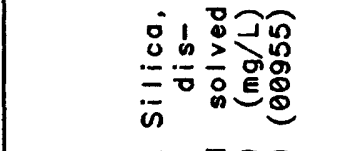 & MOळm & $11_{m}$ & 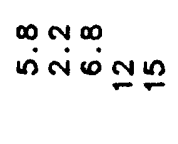 & $\stackrel{\sim}{*} \mid \stackrel{n}{\sim}$ & $\left.\right|_{0} ^{0}$ & $11=$ \\
\hline$\frac{0}{80} \frac{0}{0}$ & 11111 & 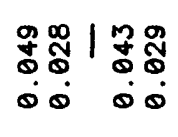 & 11111 & 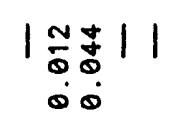 & 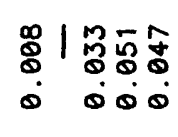 & 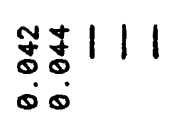 \\
\hline है & 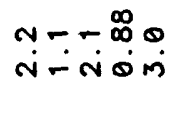 & $\ddot{N} \because \tilde{N} \dot{N}$ & 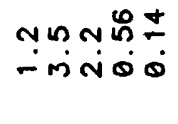 & 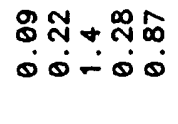 & 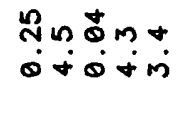 & NMOO \\
\hline 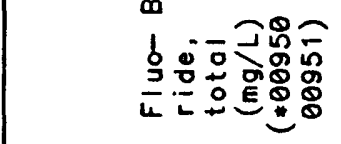 & 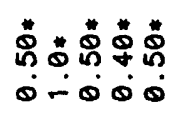 & 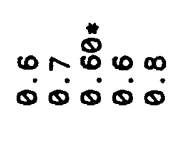 & 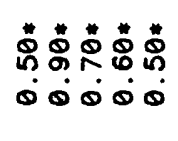 & 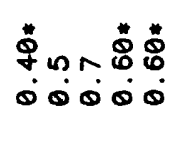 & 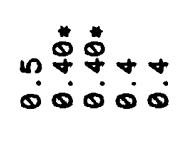 & 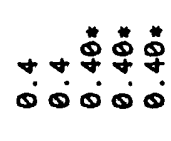 \\
\hline 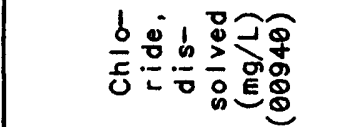 & 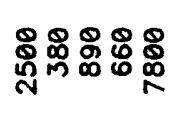 & 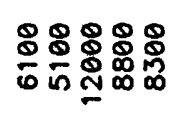 & 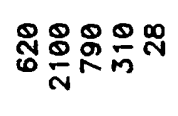 & 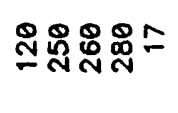 & స̊ํㅠ: & 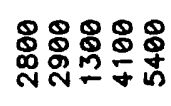 \\
\hline 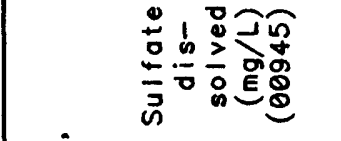 & ஓ̊: & 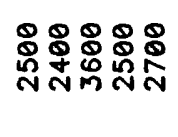 & ஃ:ஃஃ: & 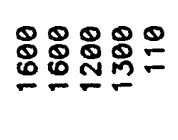 & 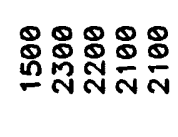 & : \\
\hline 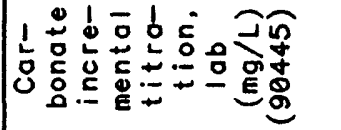 & むむむむま & 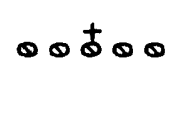 & むむむむむ & むののむむ & ○むむめ。 & ますま \\
\hline 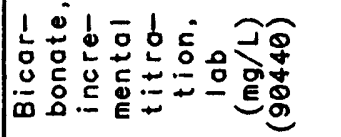 & 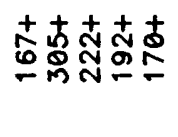 & 怘怘志㔾 & 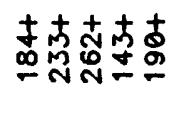 & 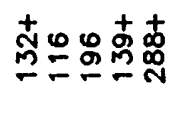 & : & 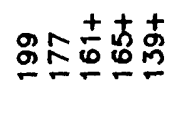 \\
\hline 范 & 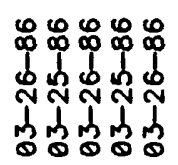 & 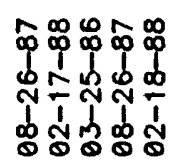 & 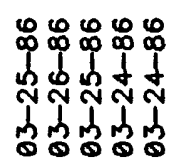 & 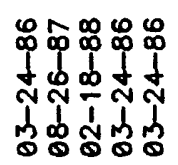 & 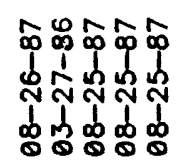 & 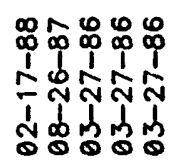 \\
\hline
\end{tabular}




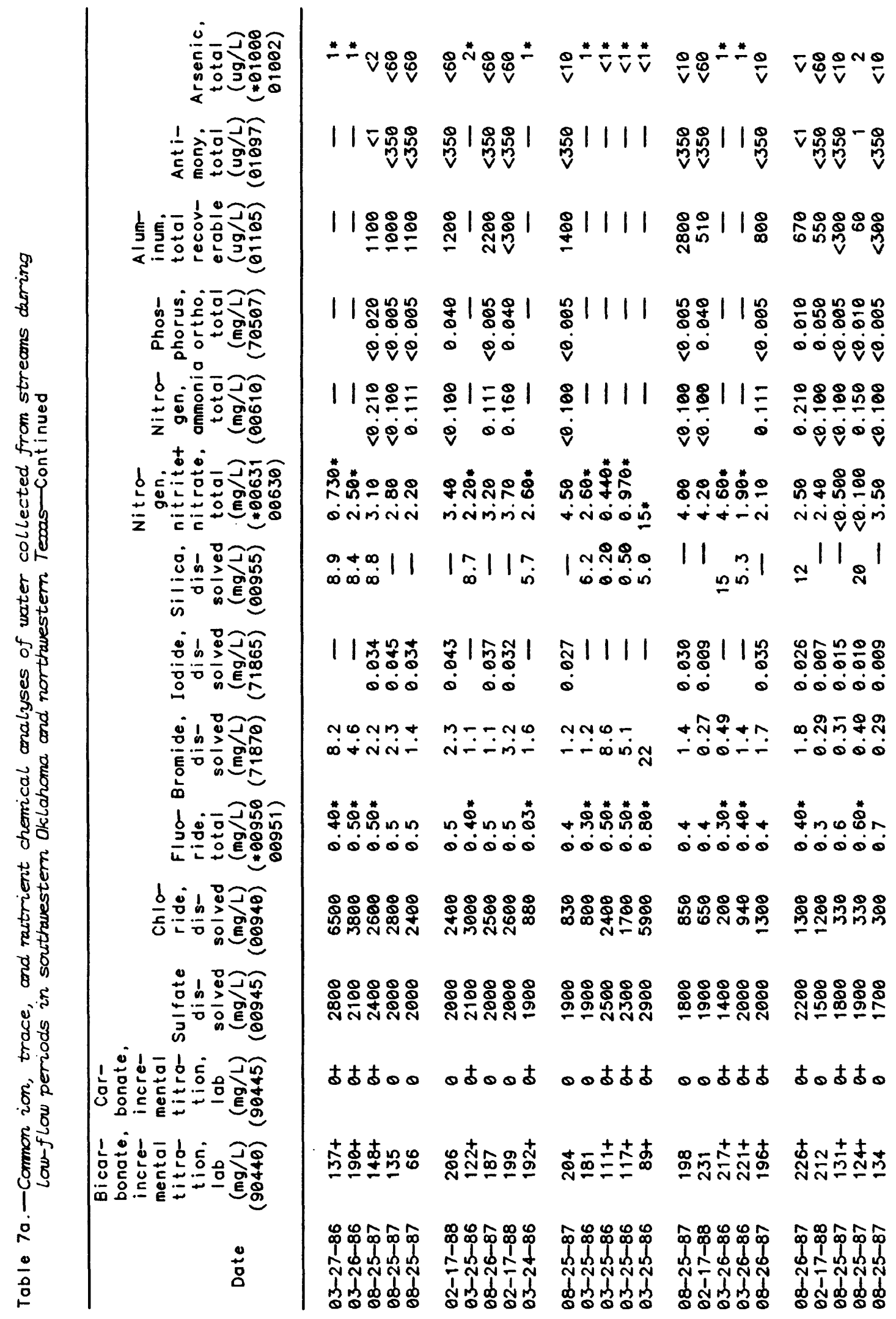




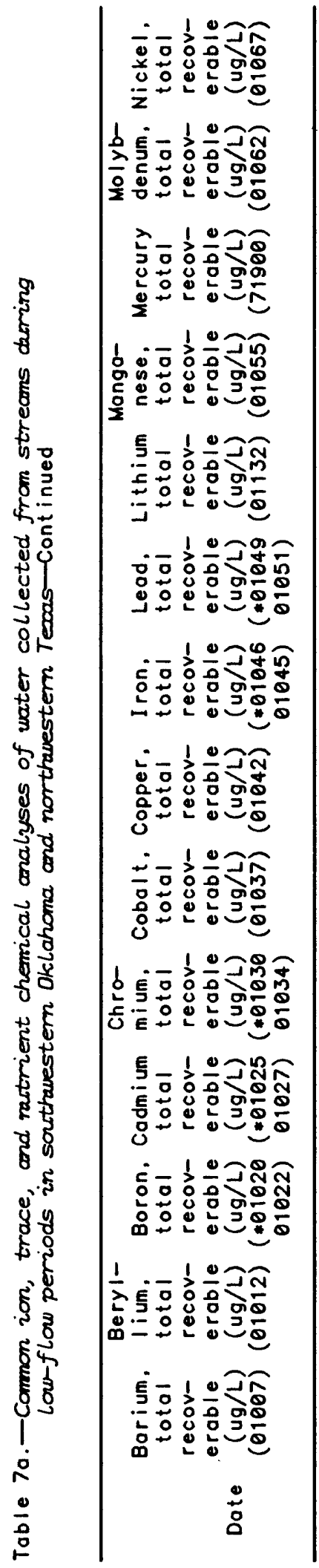

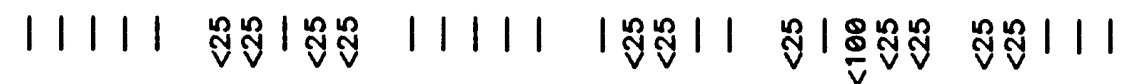

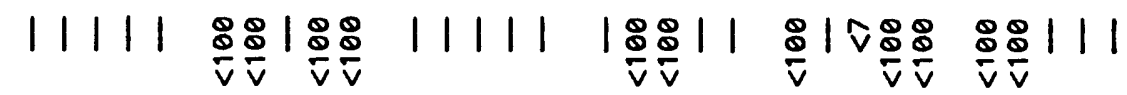

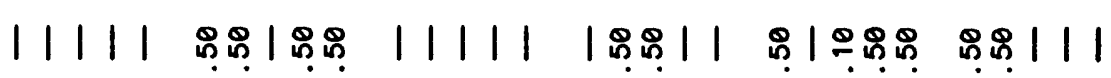

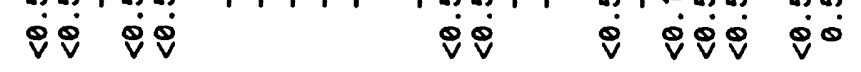

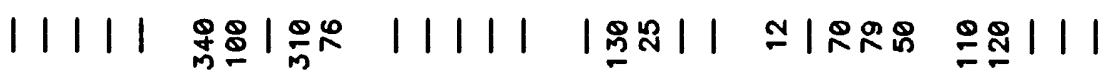
111111111111111111111111801111111

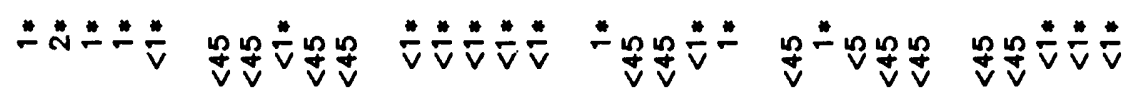

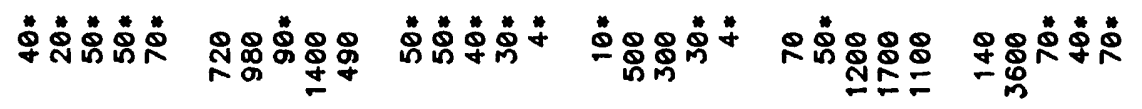

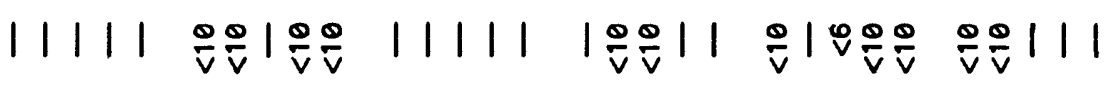
11111 每

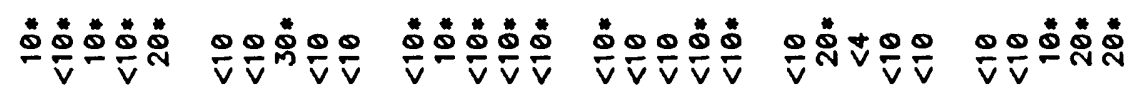

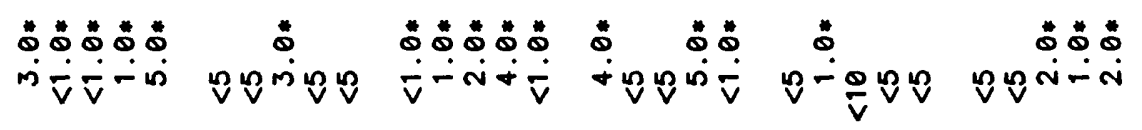

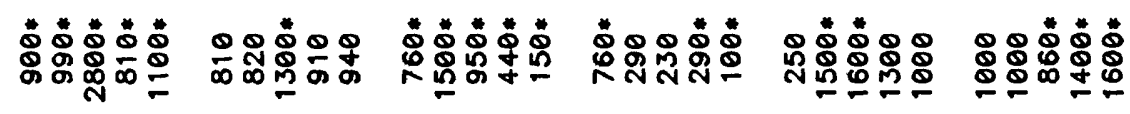

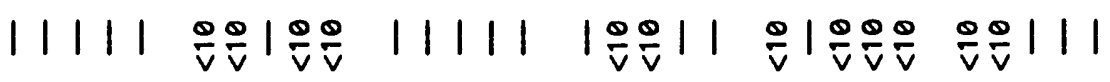

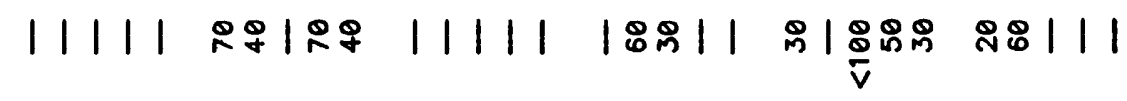

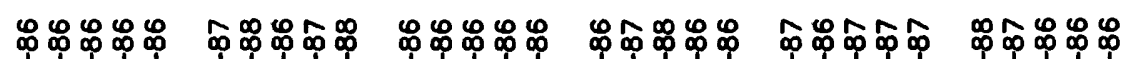

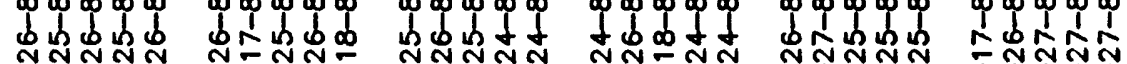

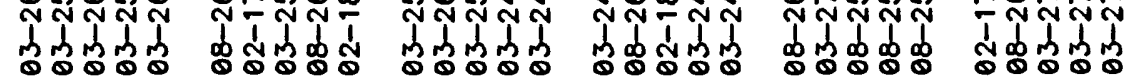




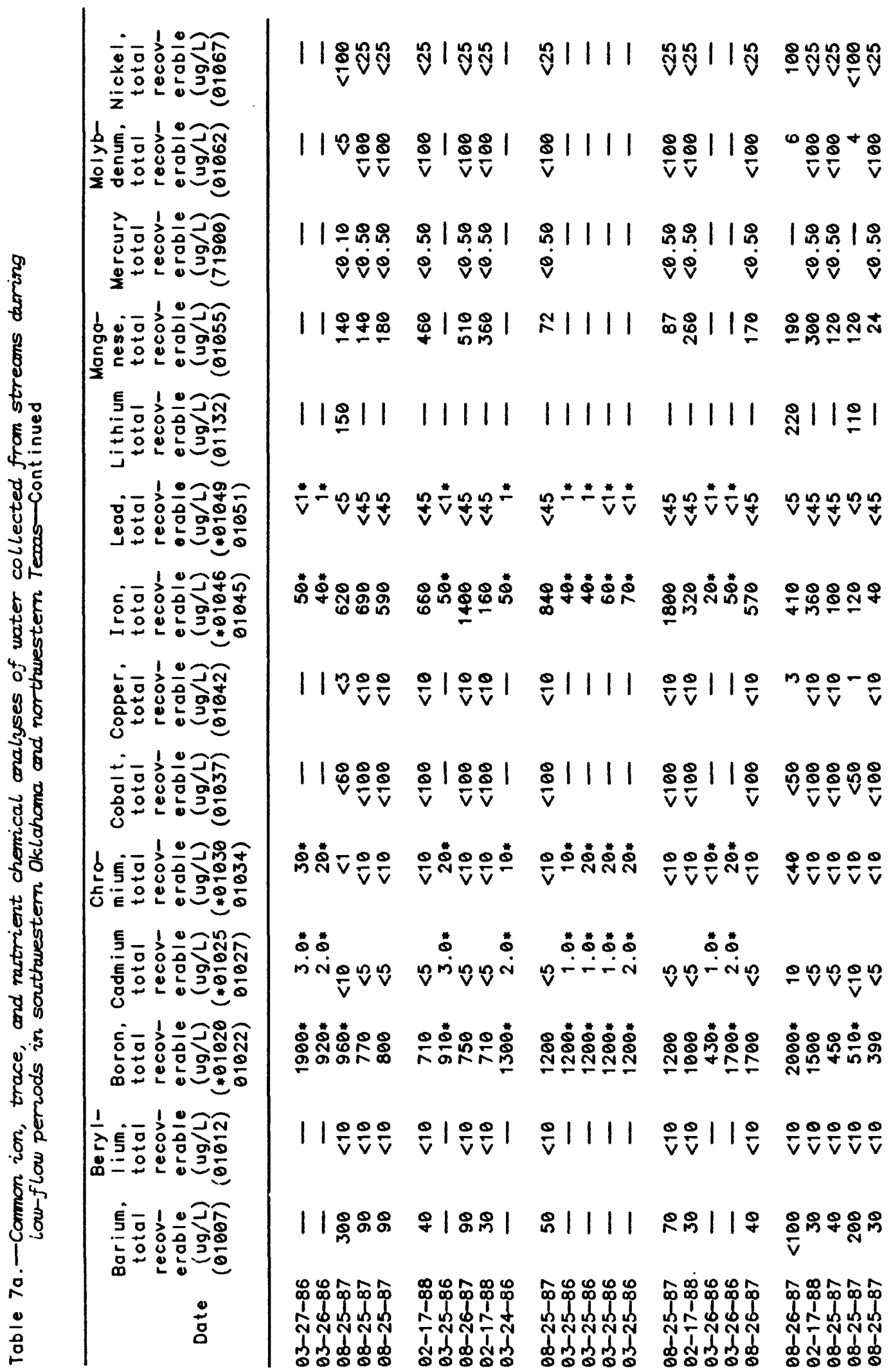




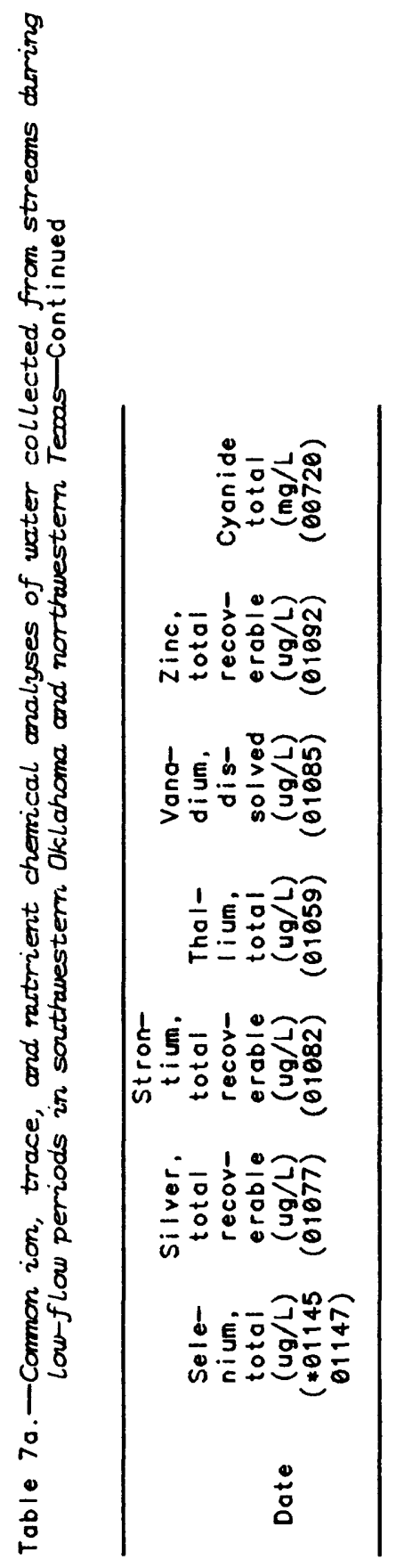

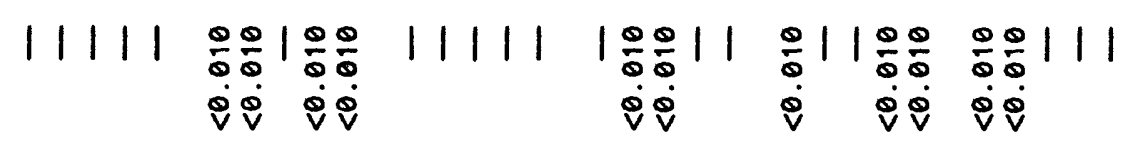

11111 ถูก

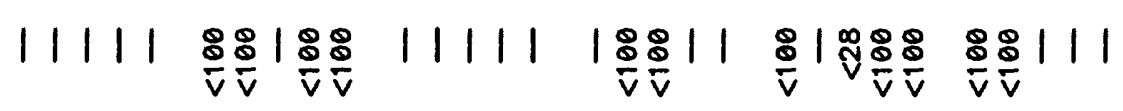

11111 ชูํํำ

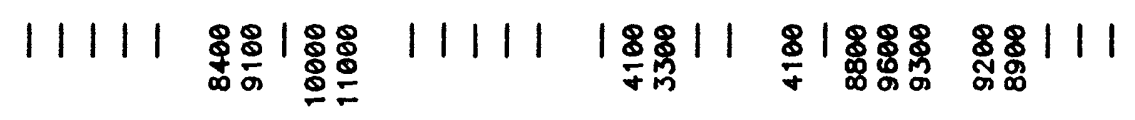

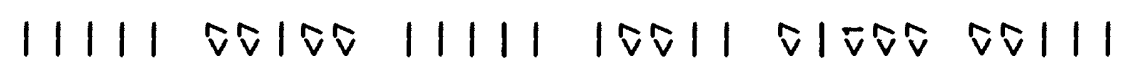

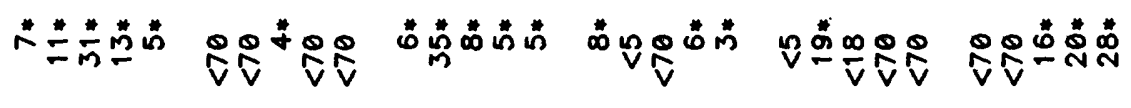

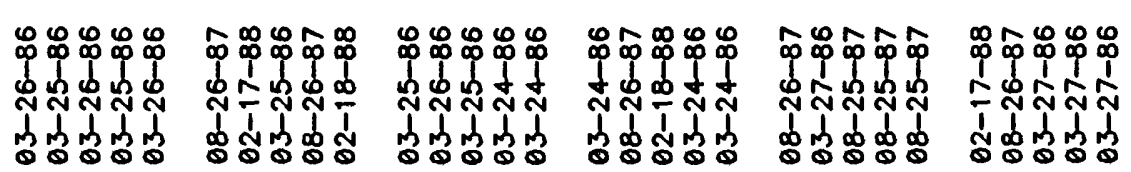




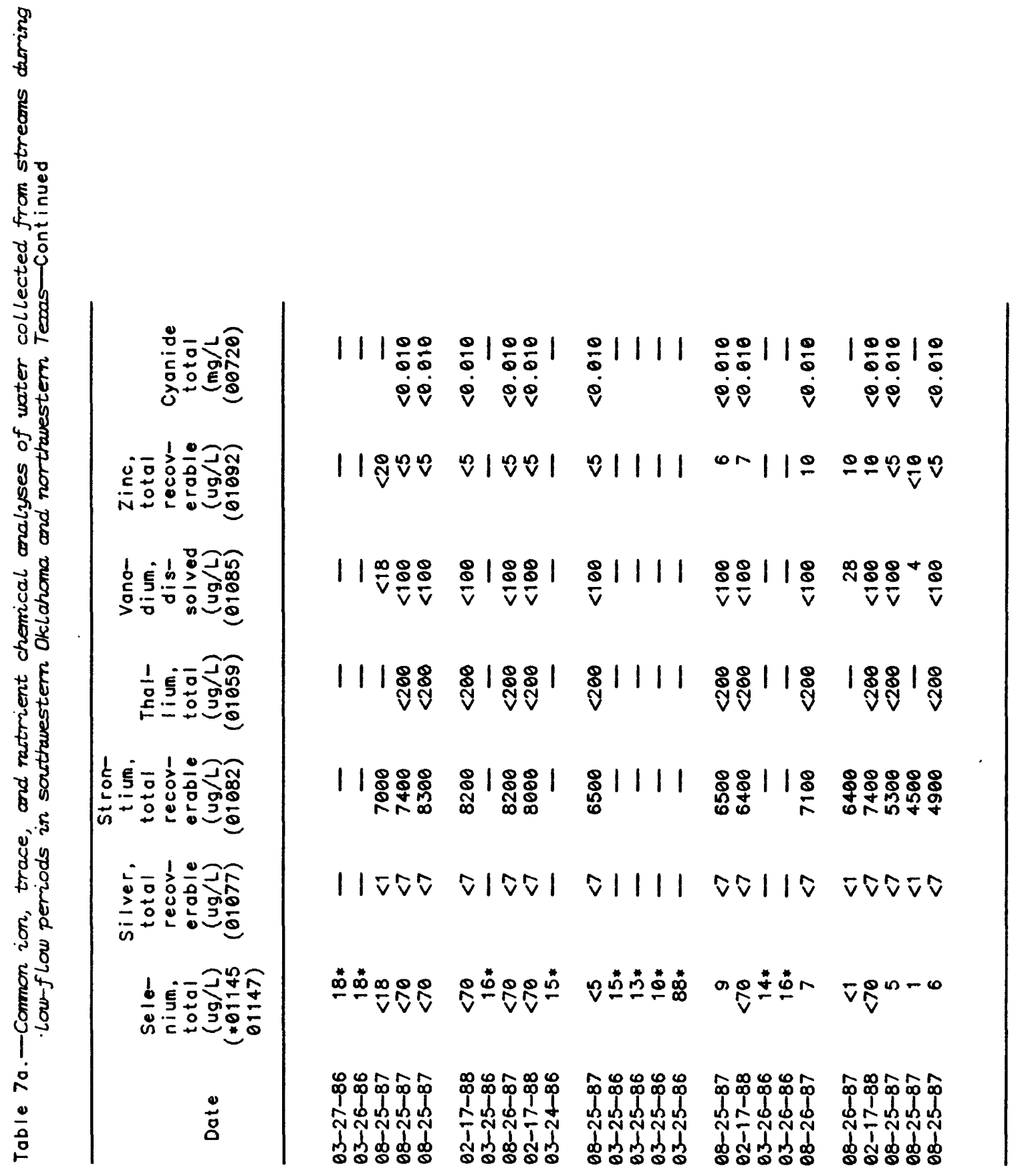



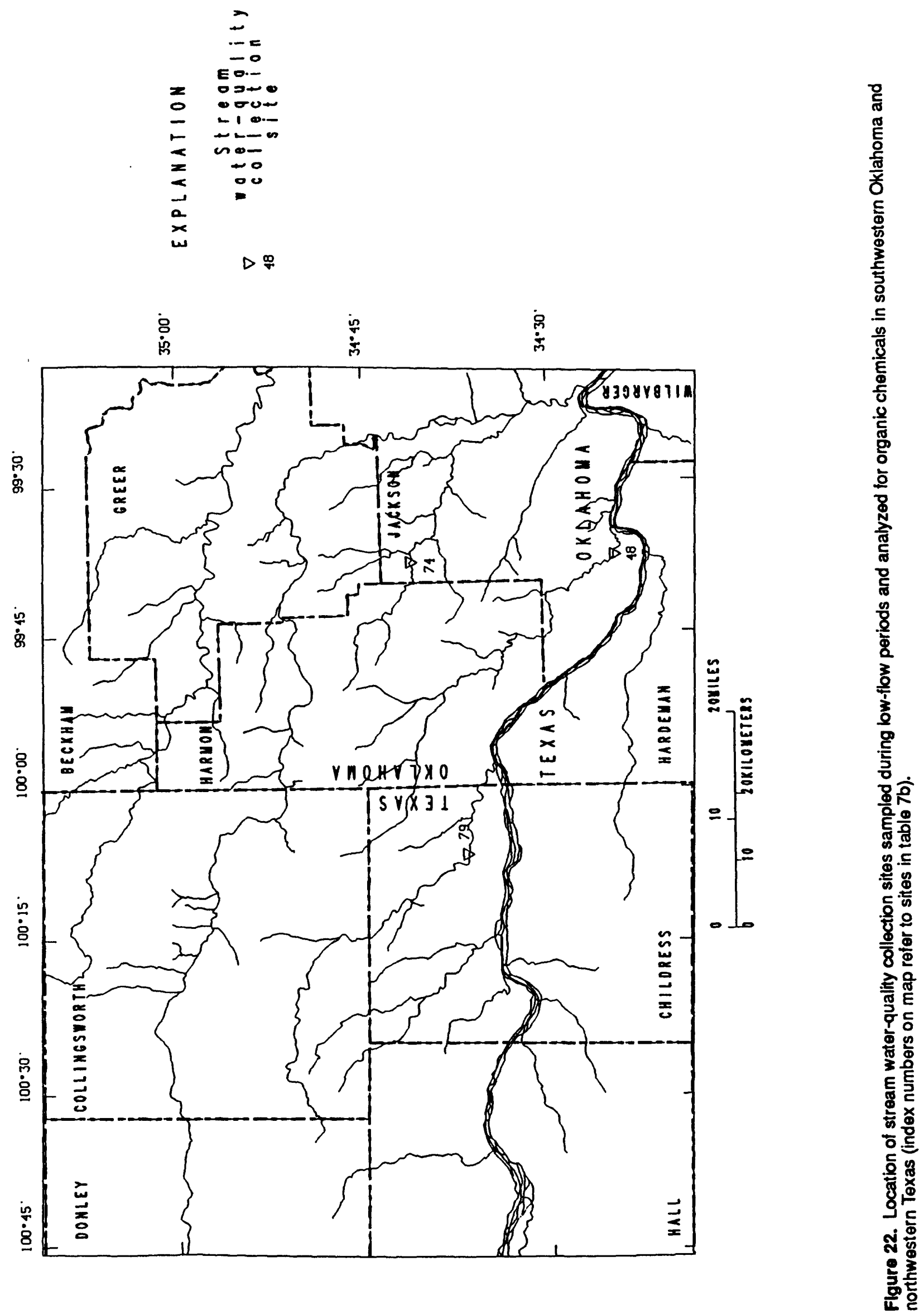


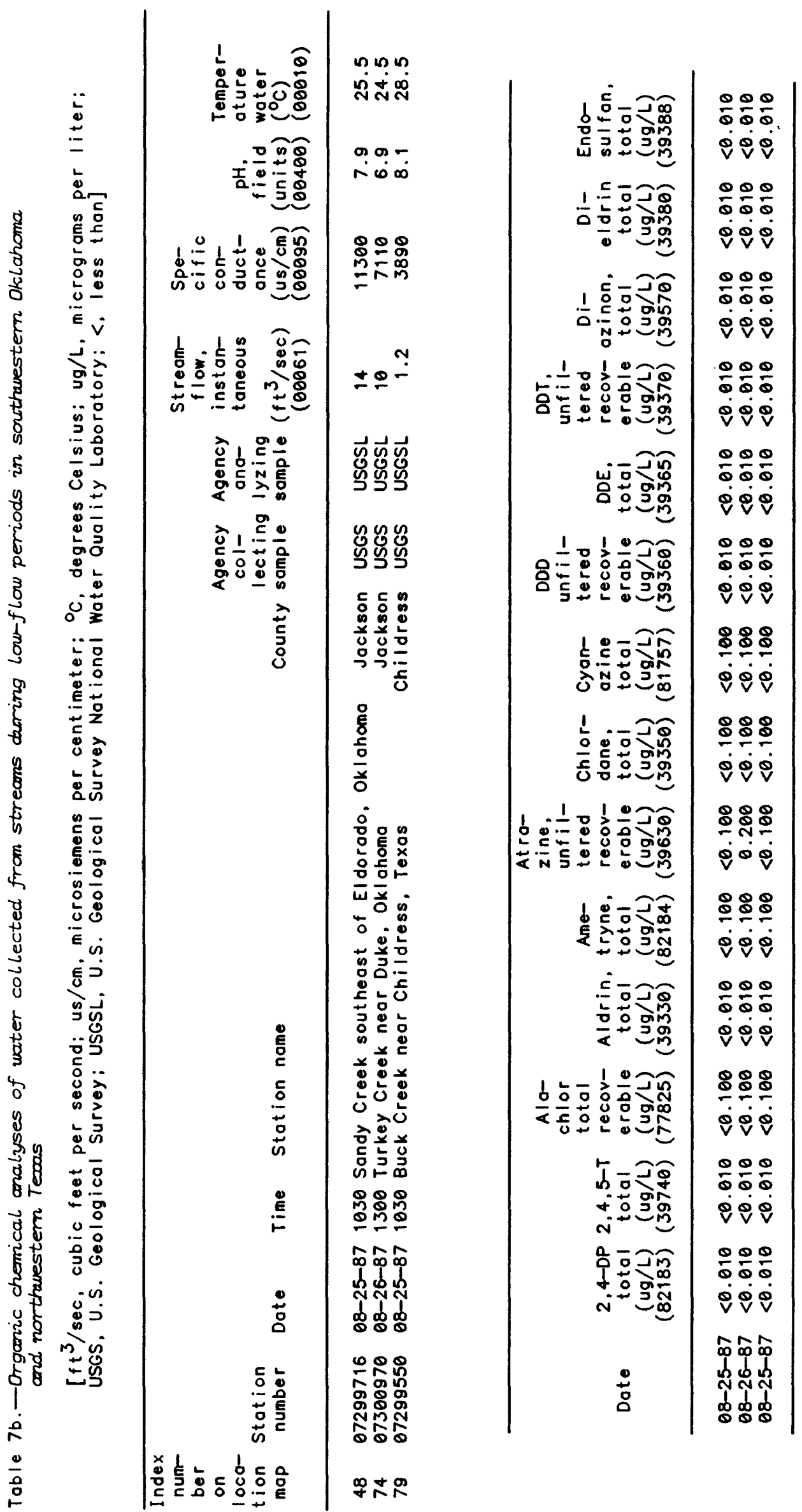



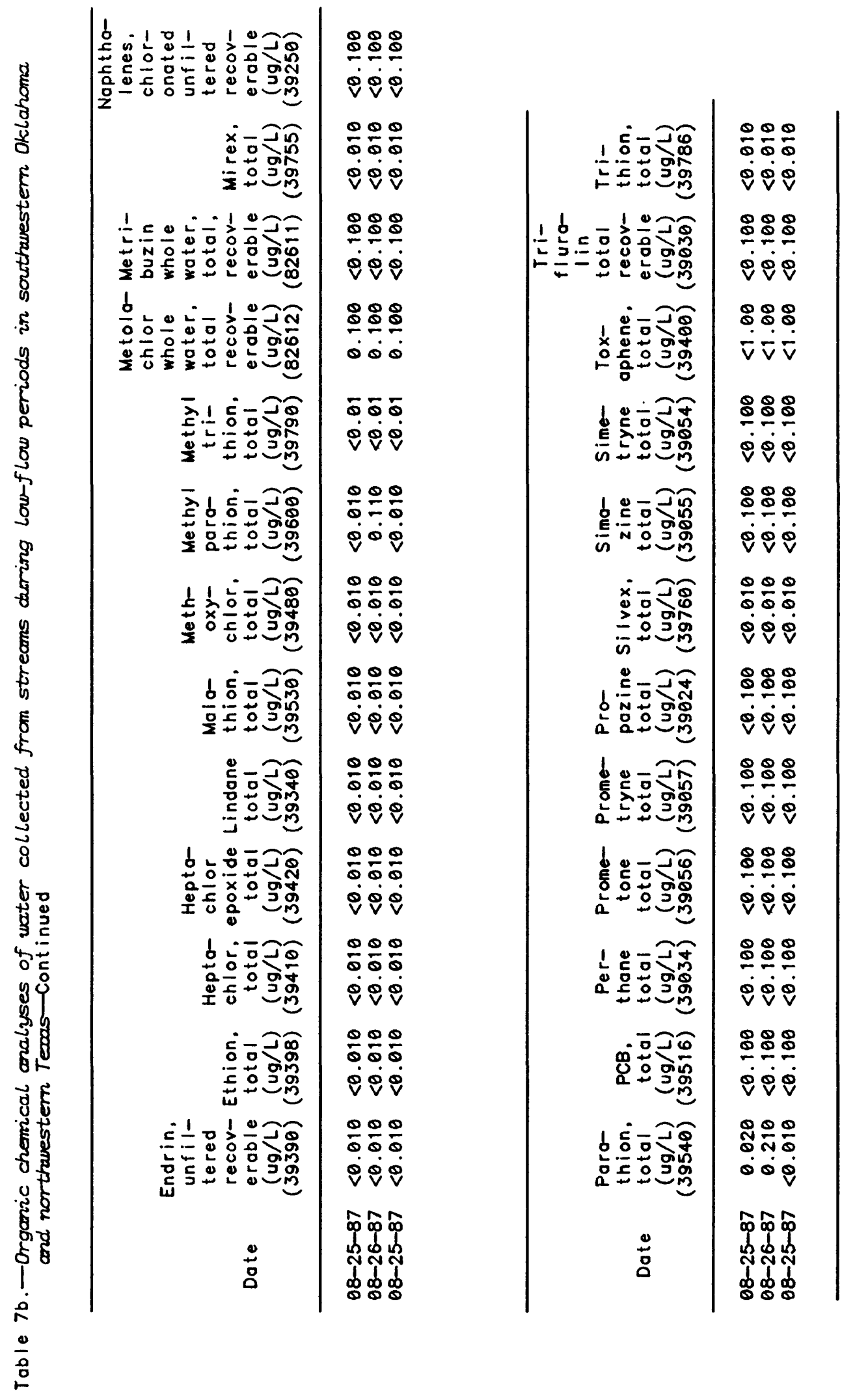


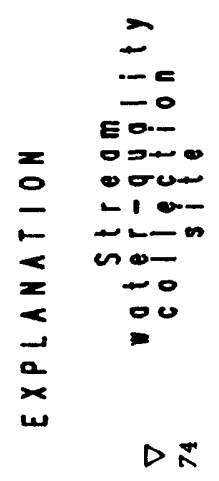
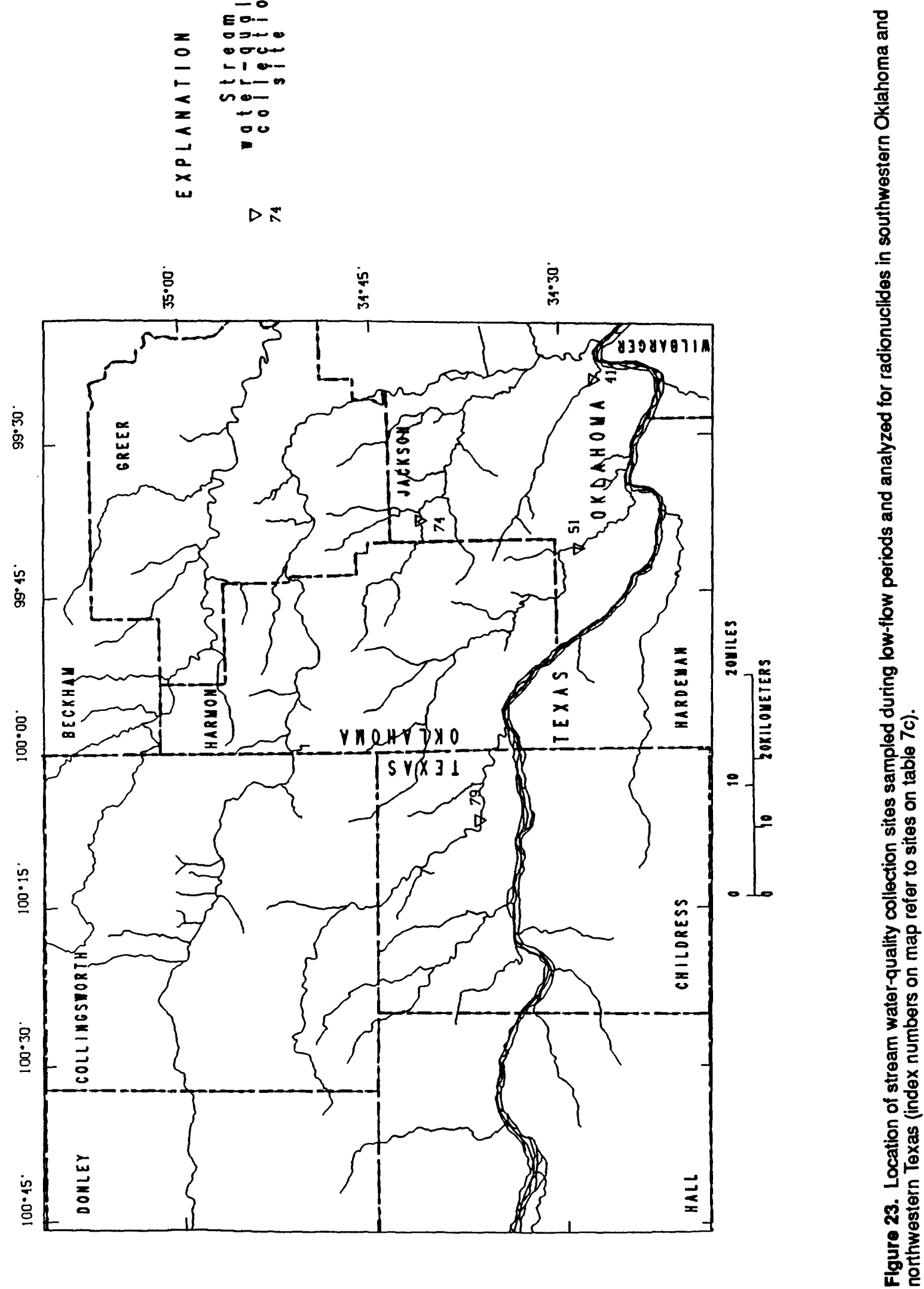


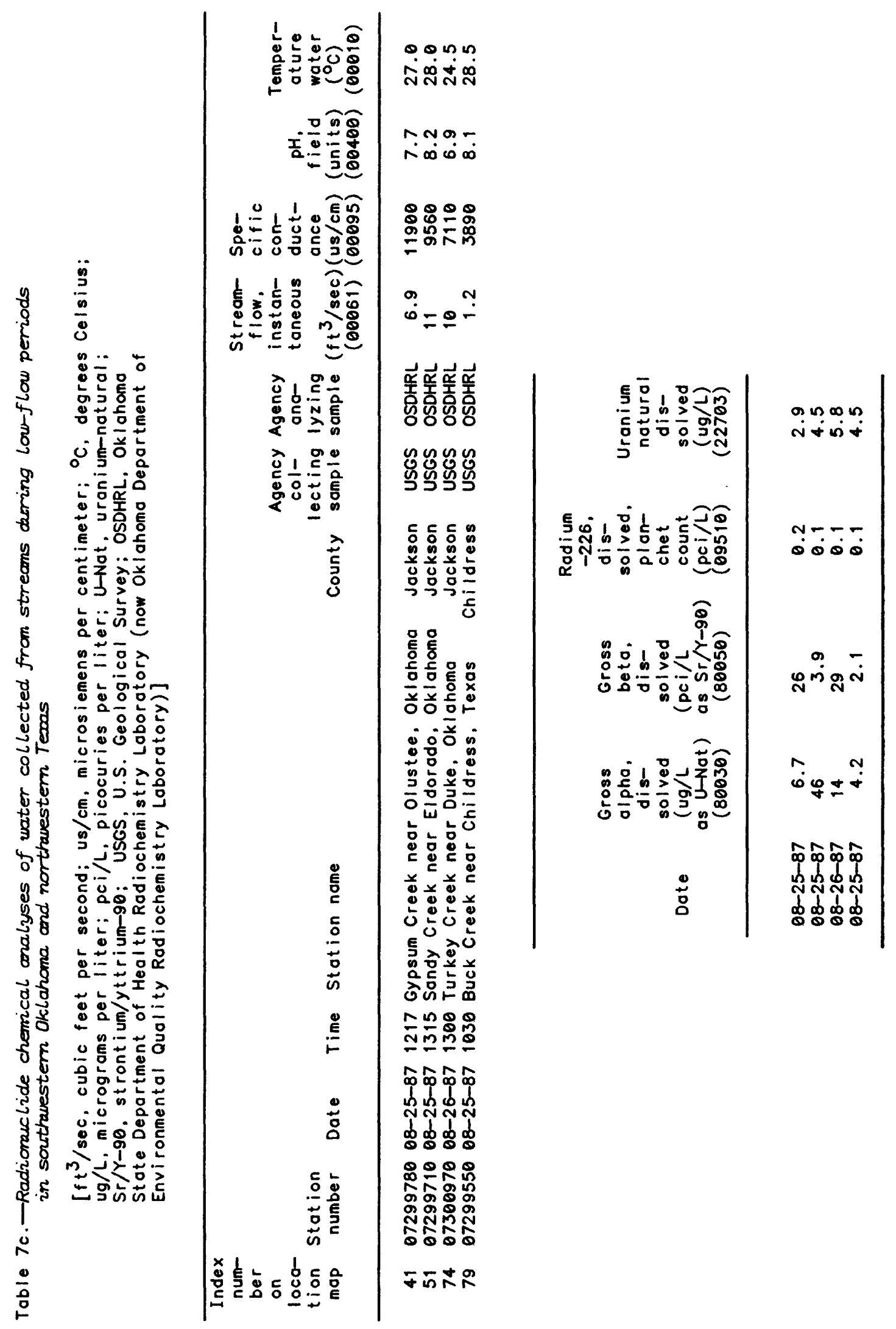




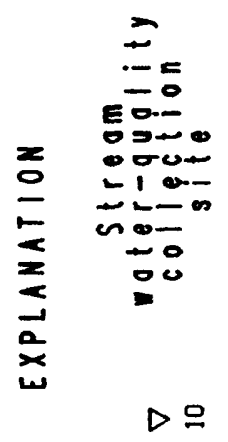

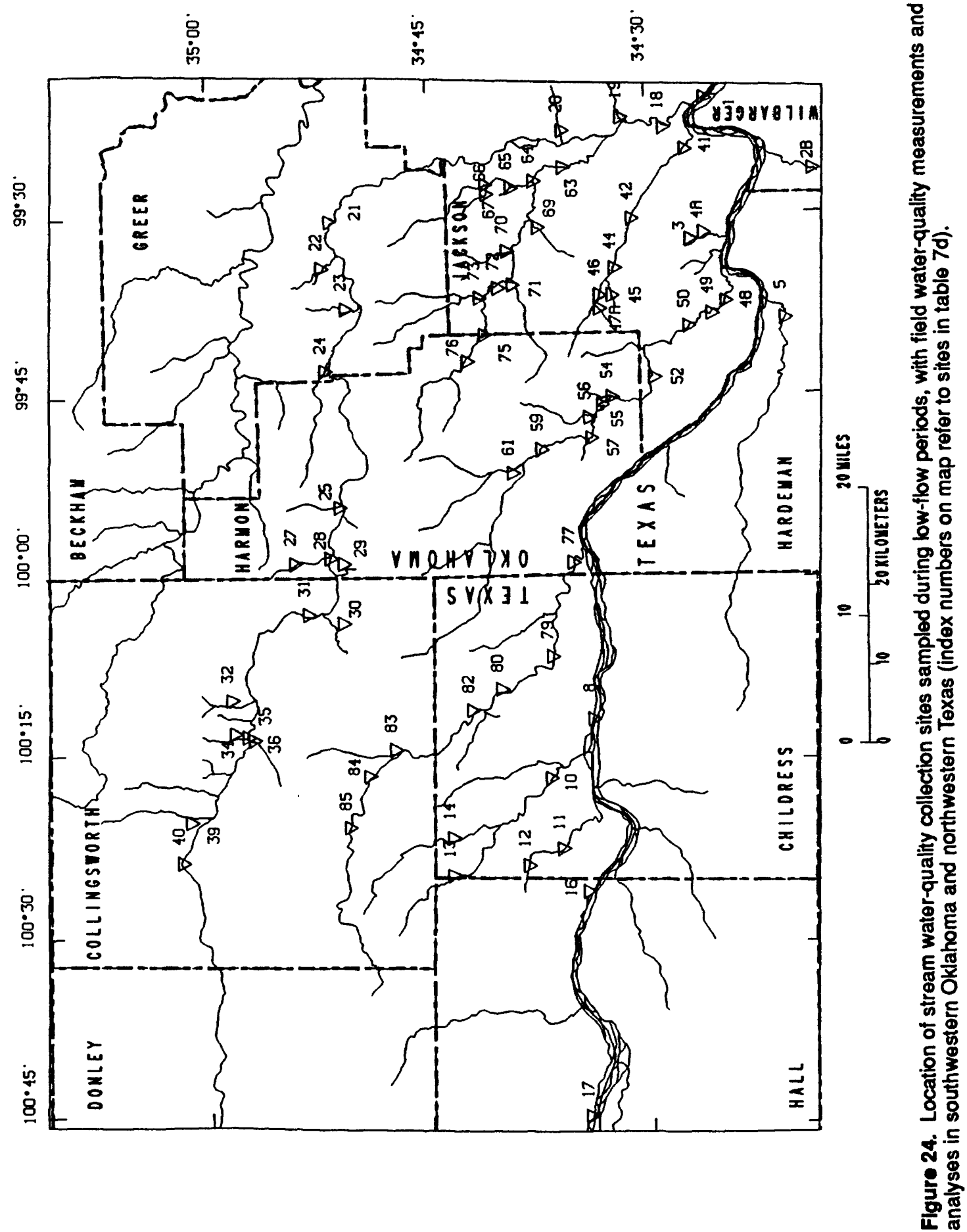




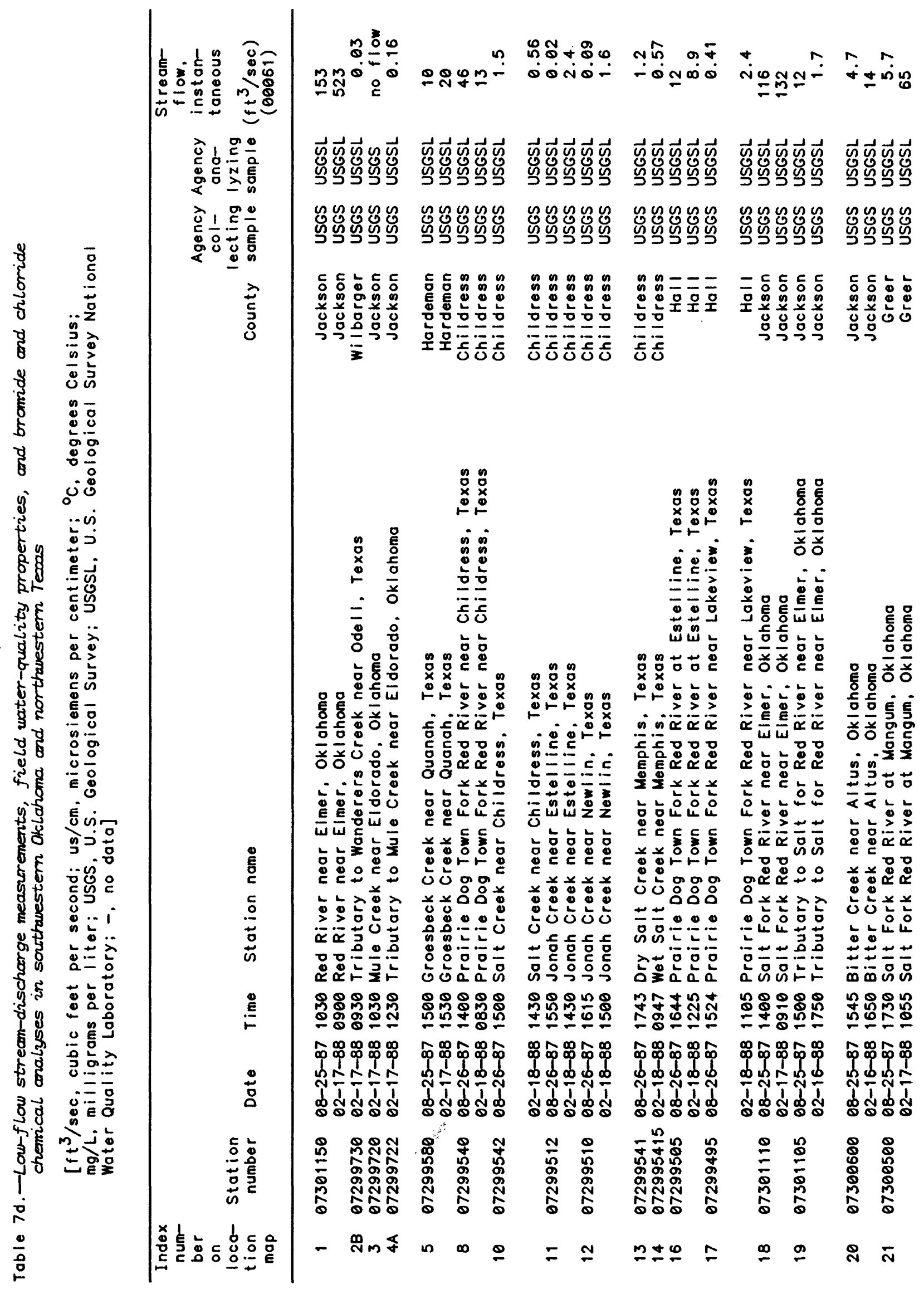




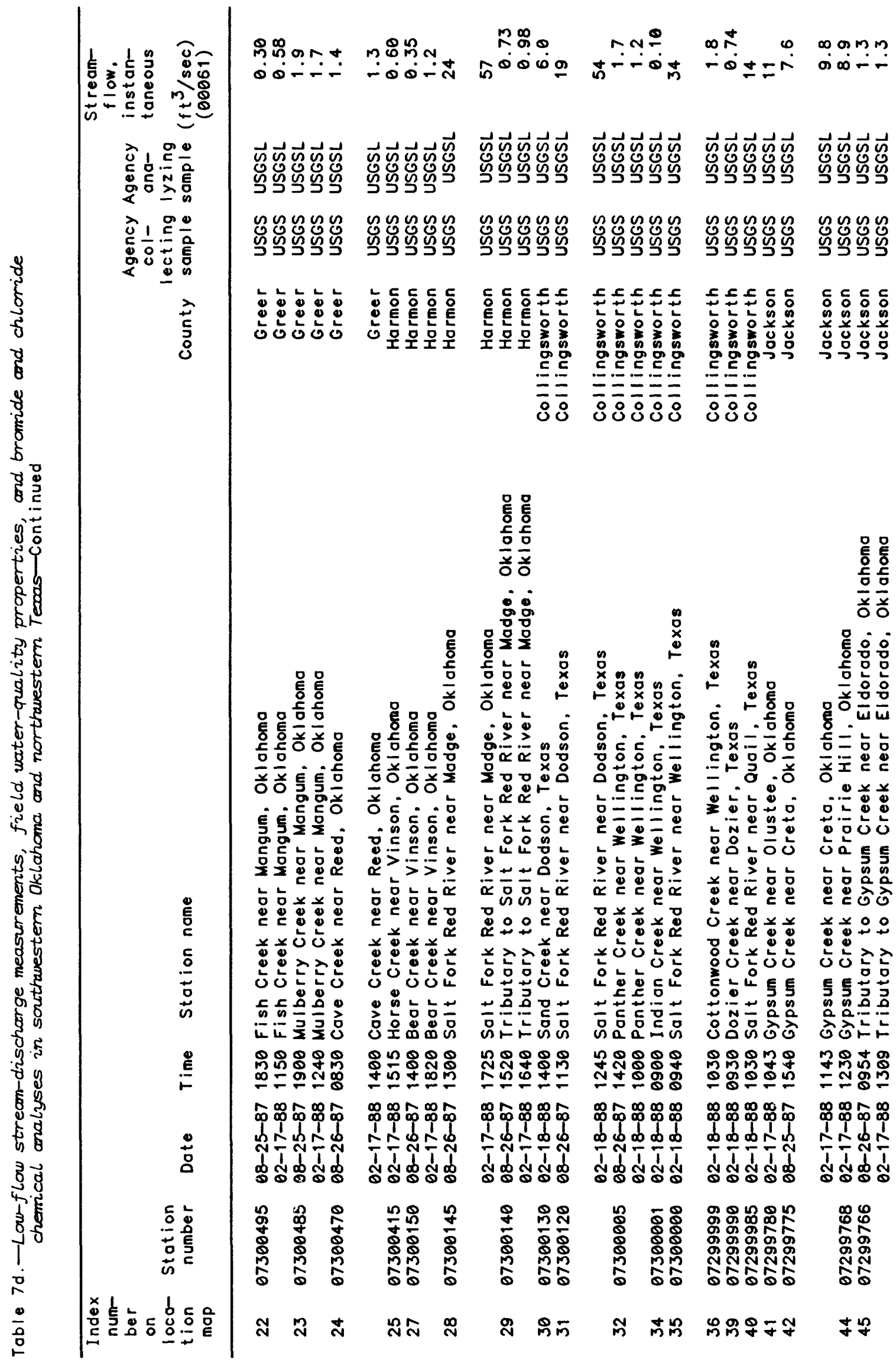




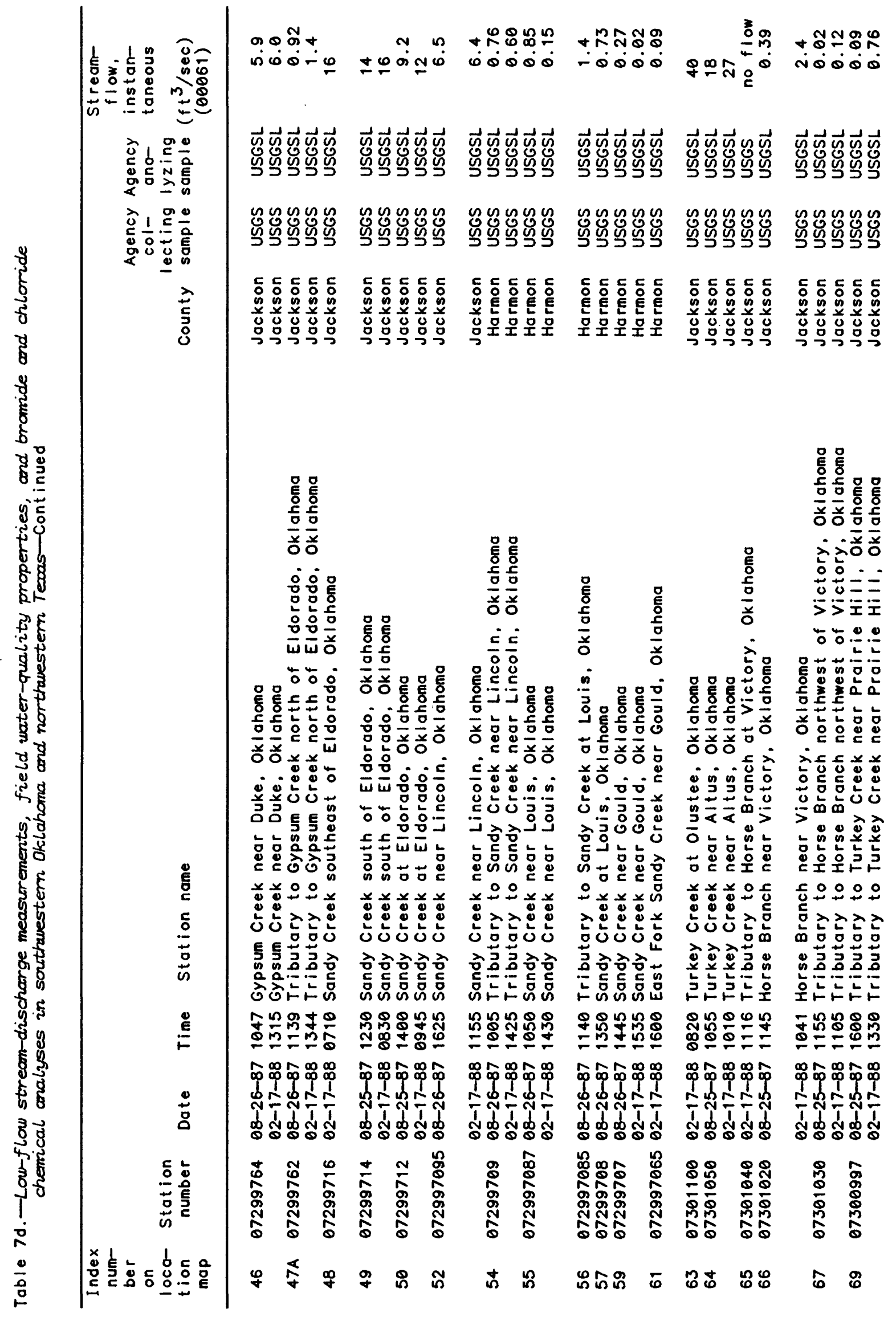




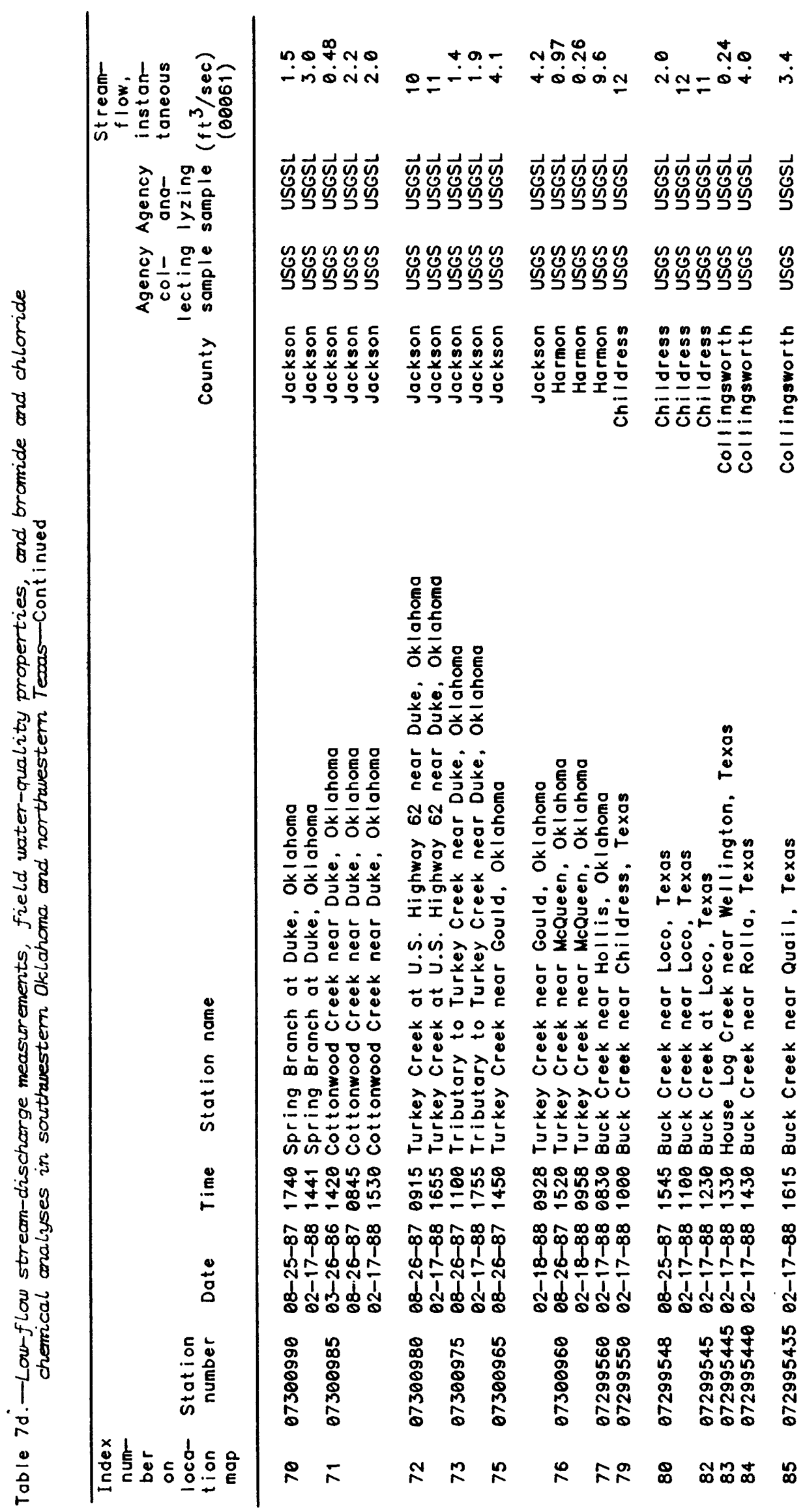




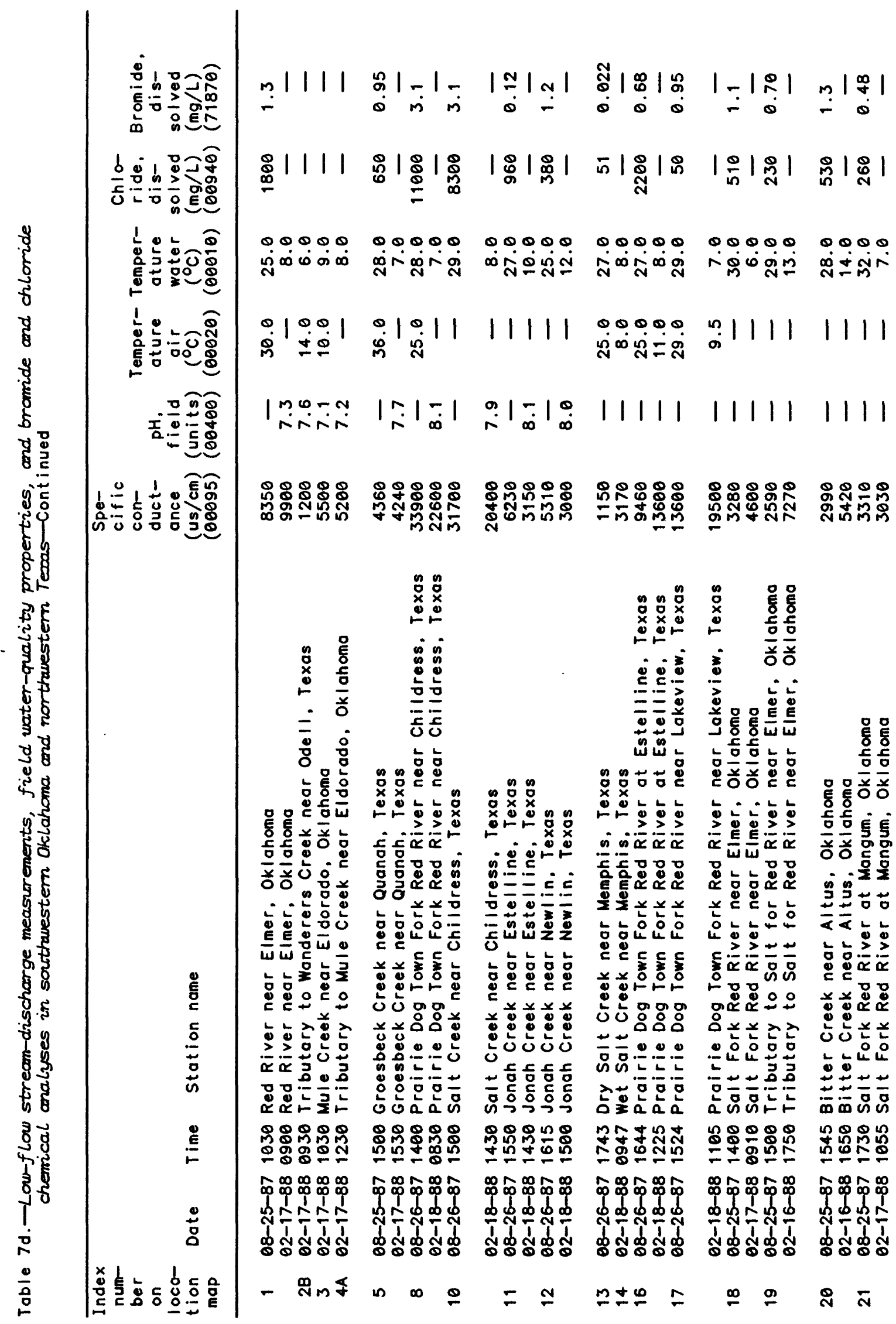




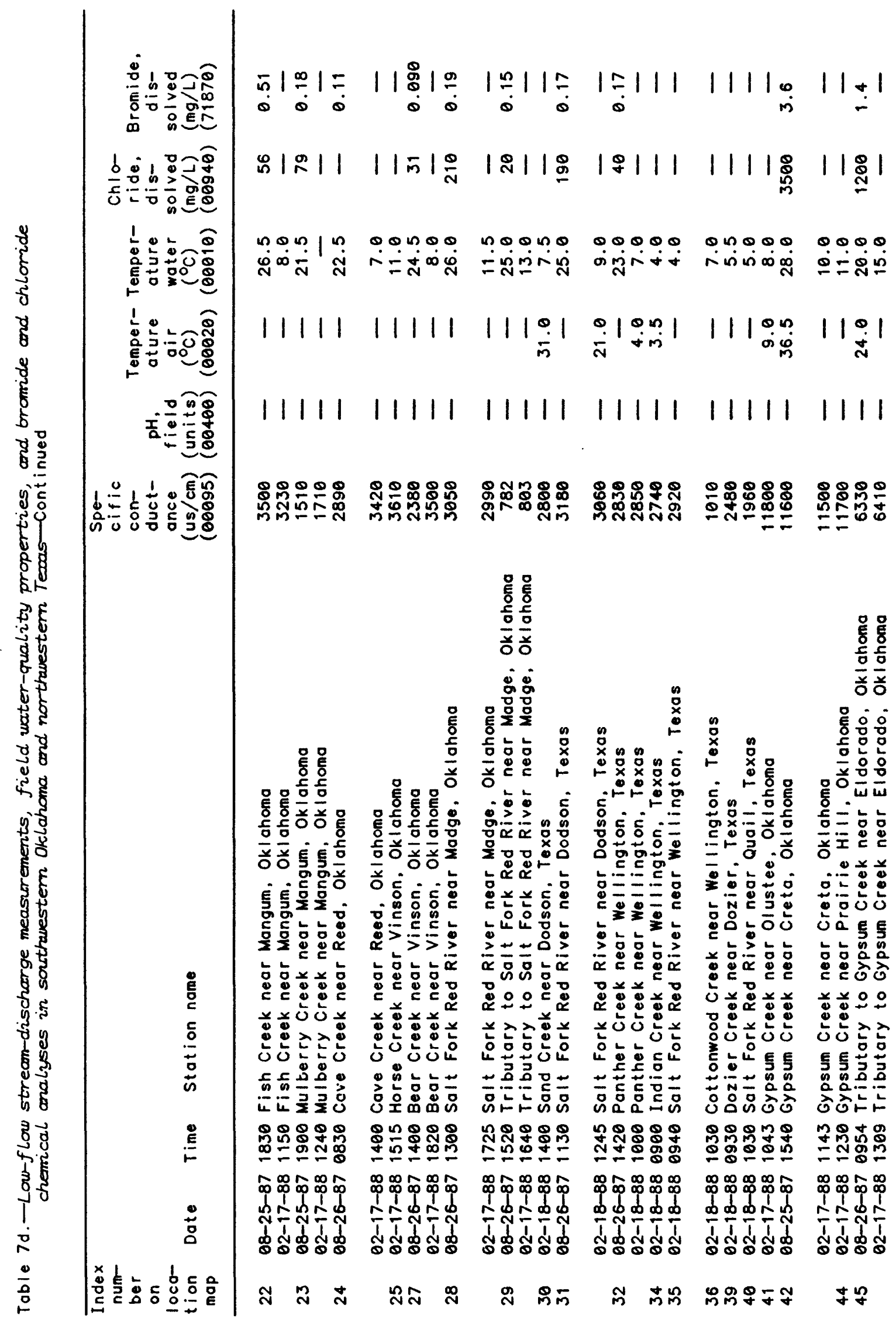




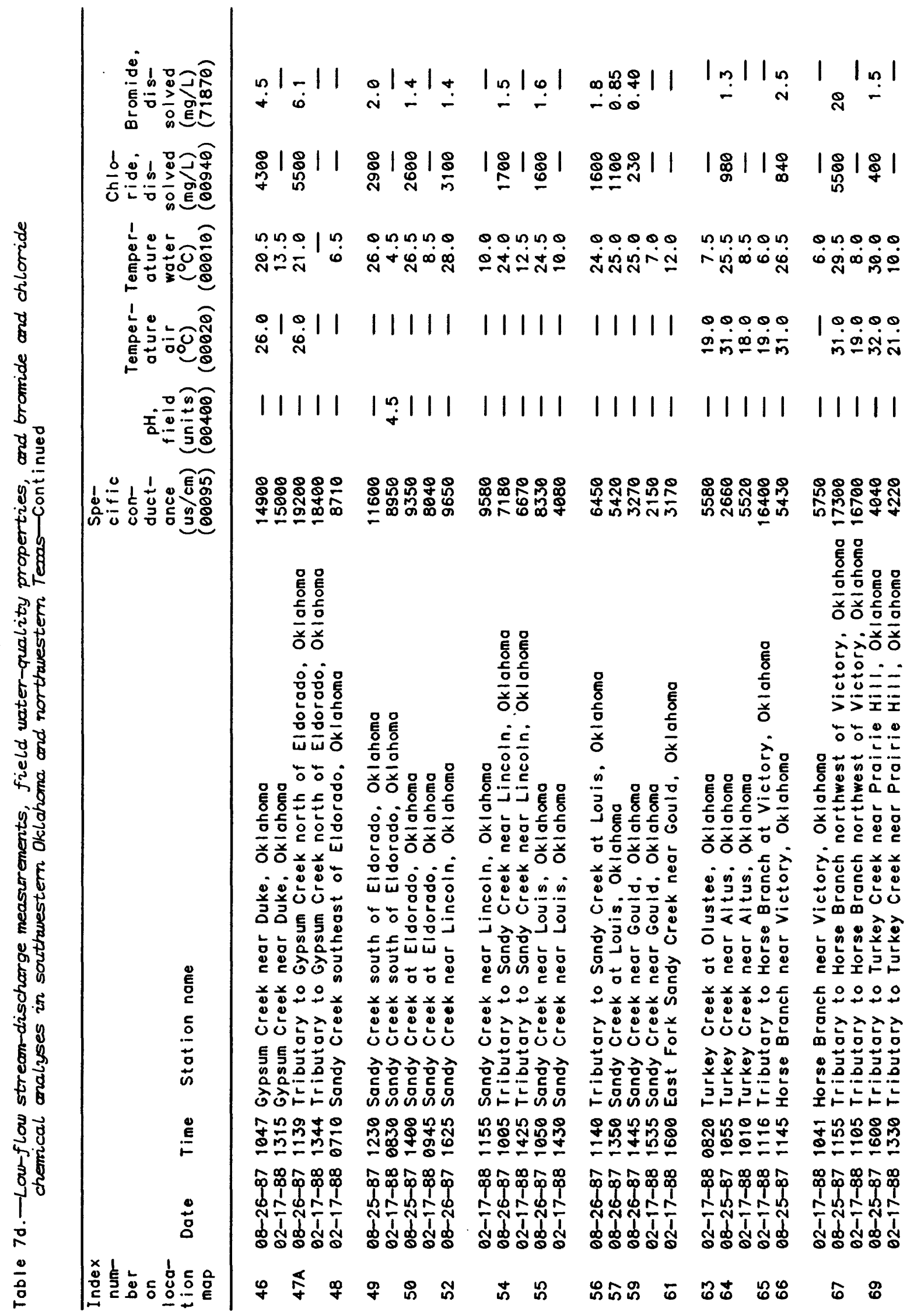




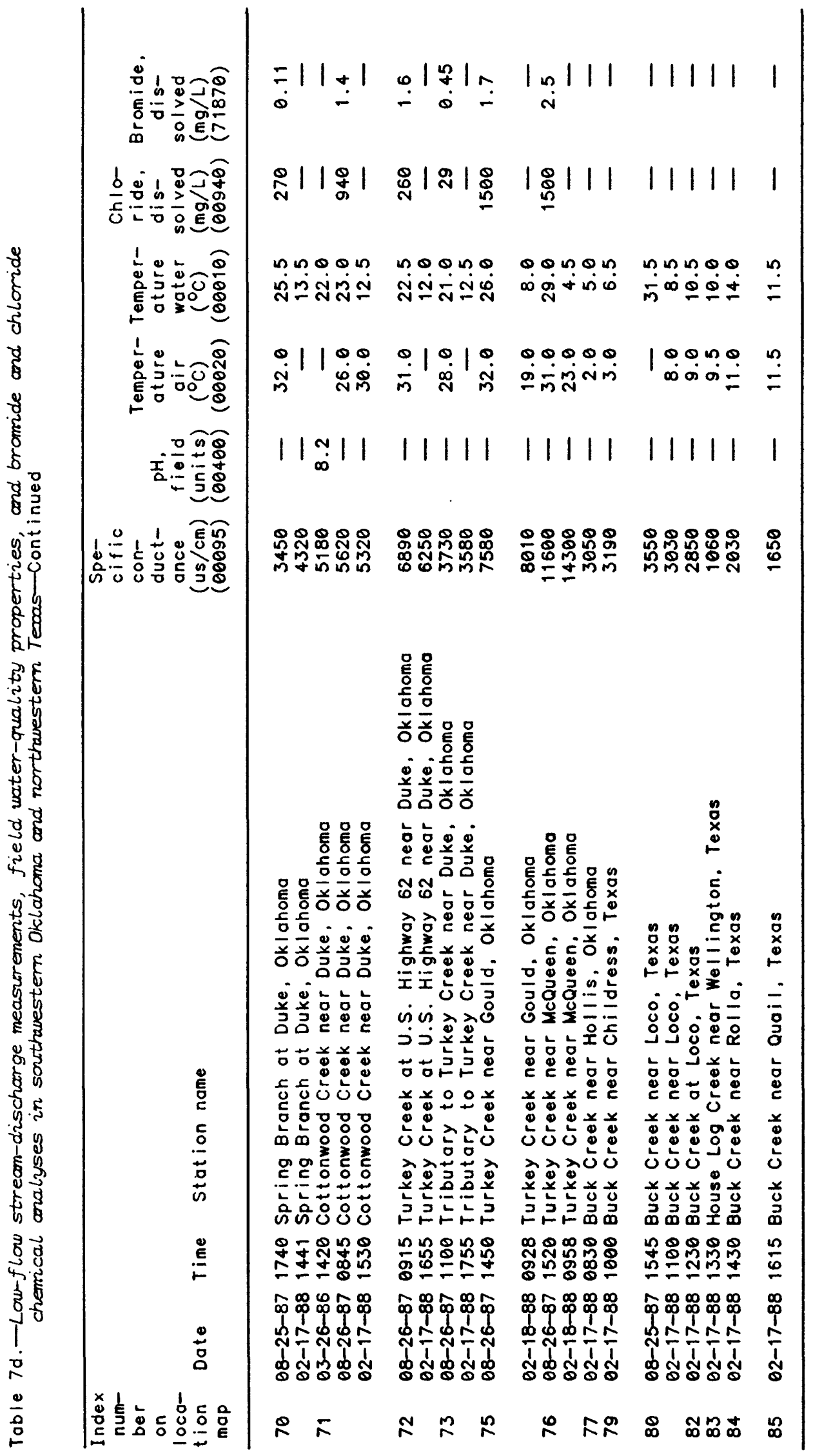



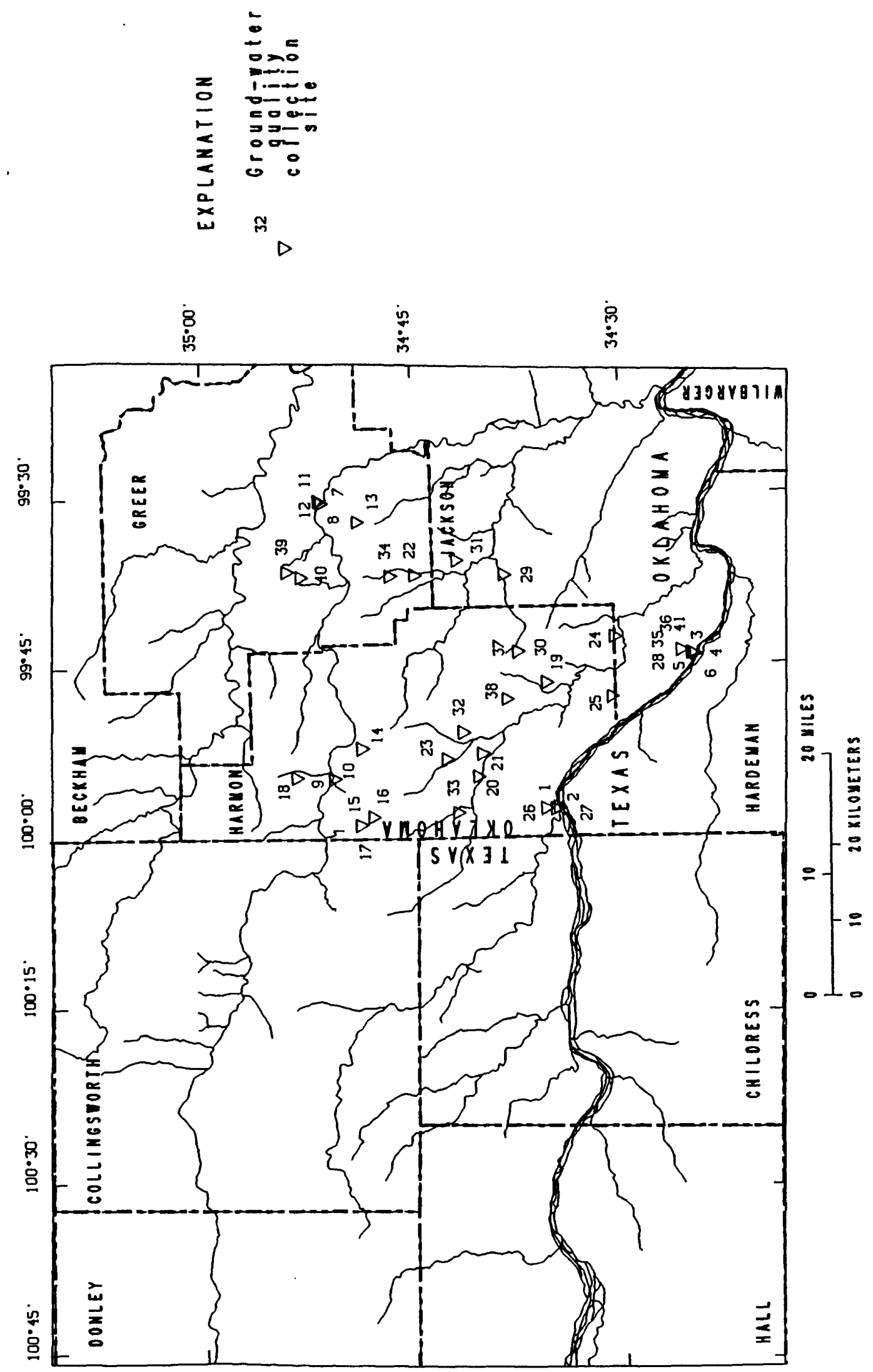


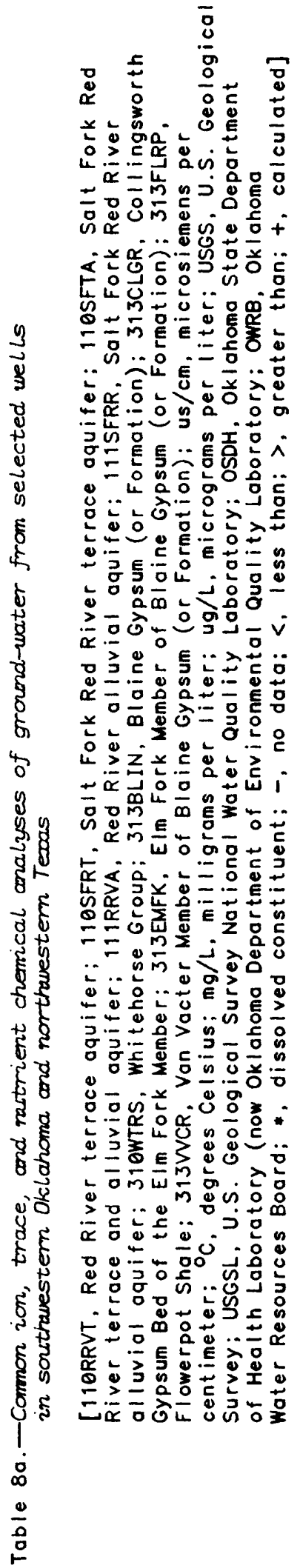

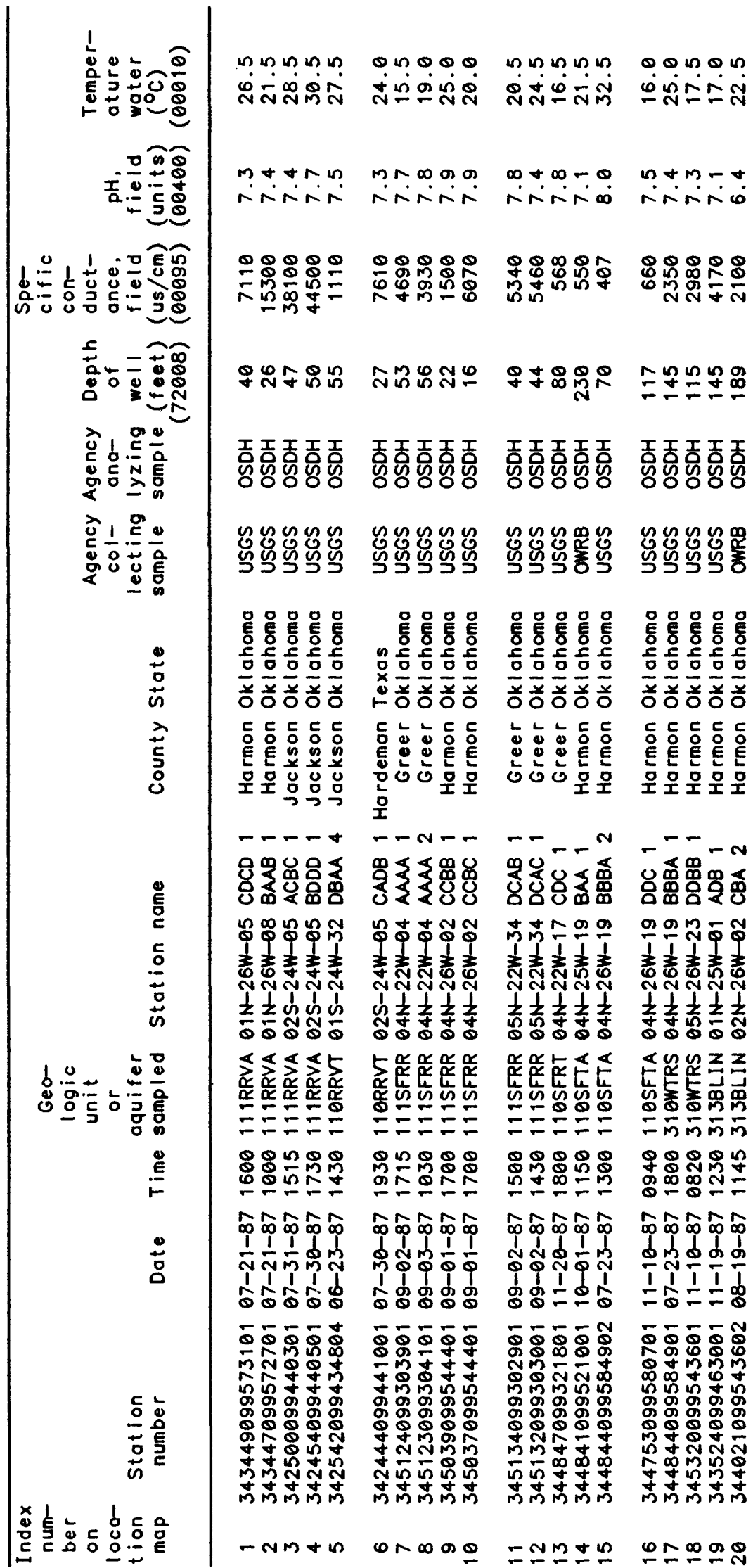




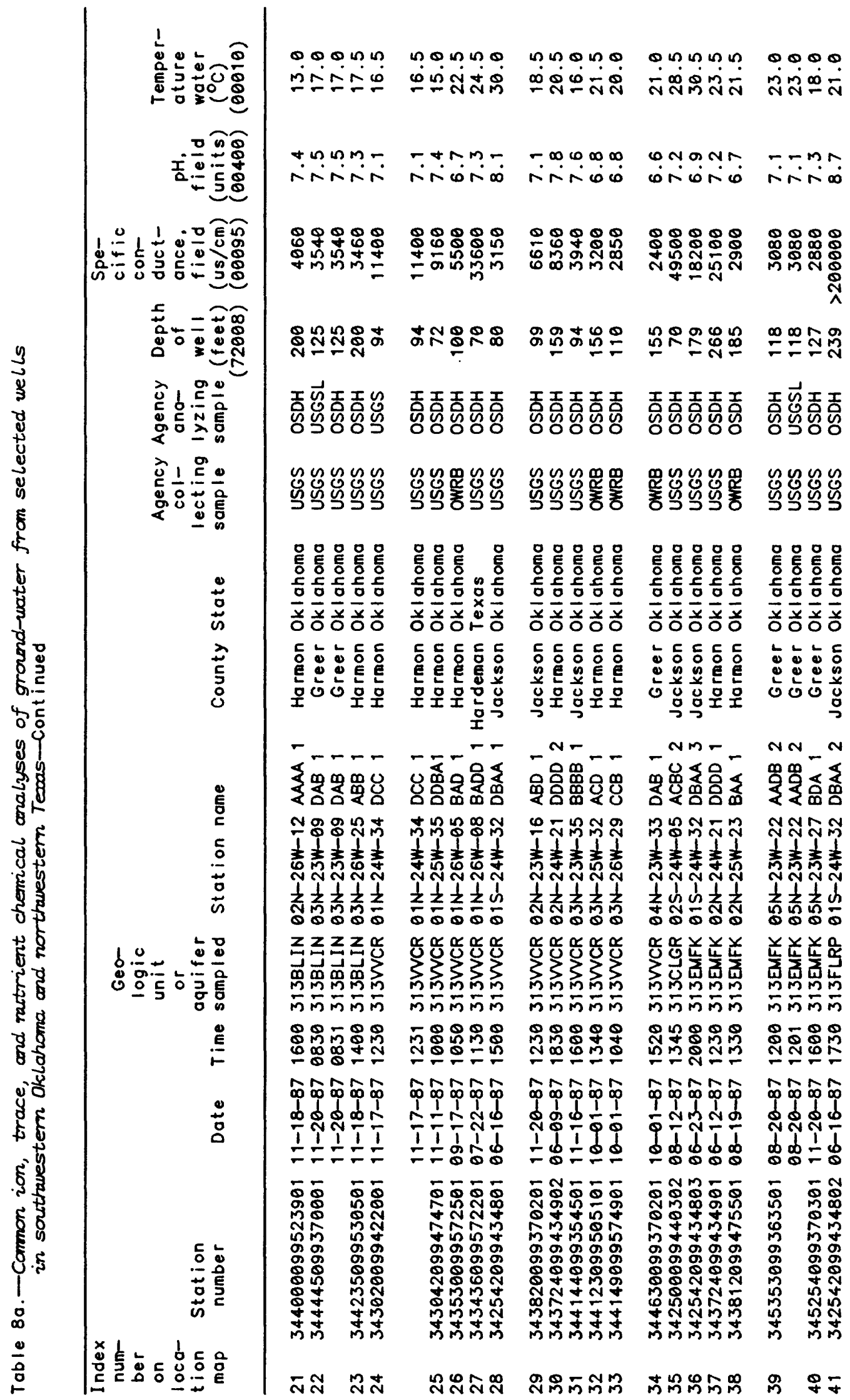




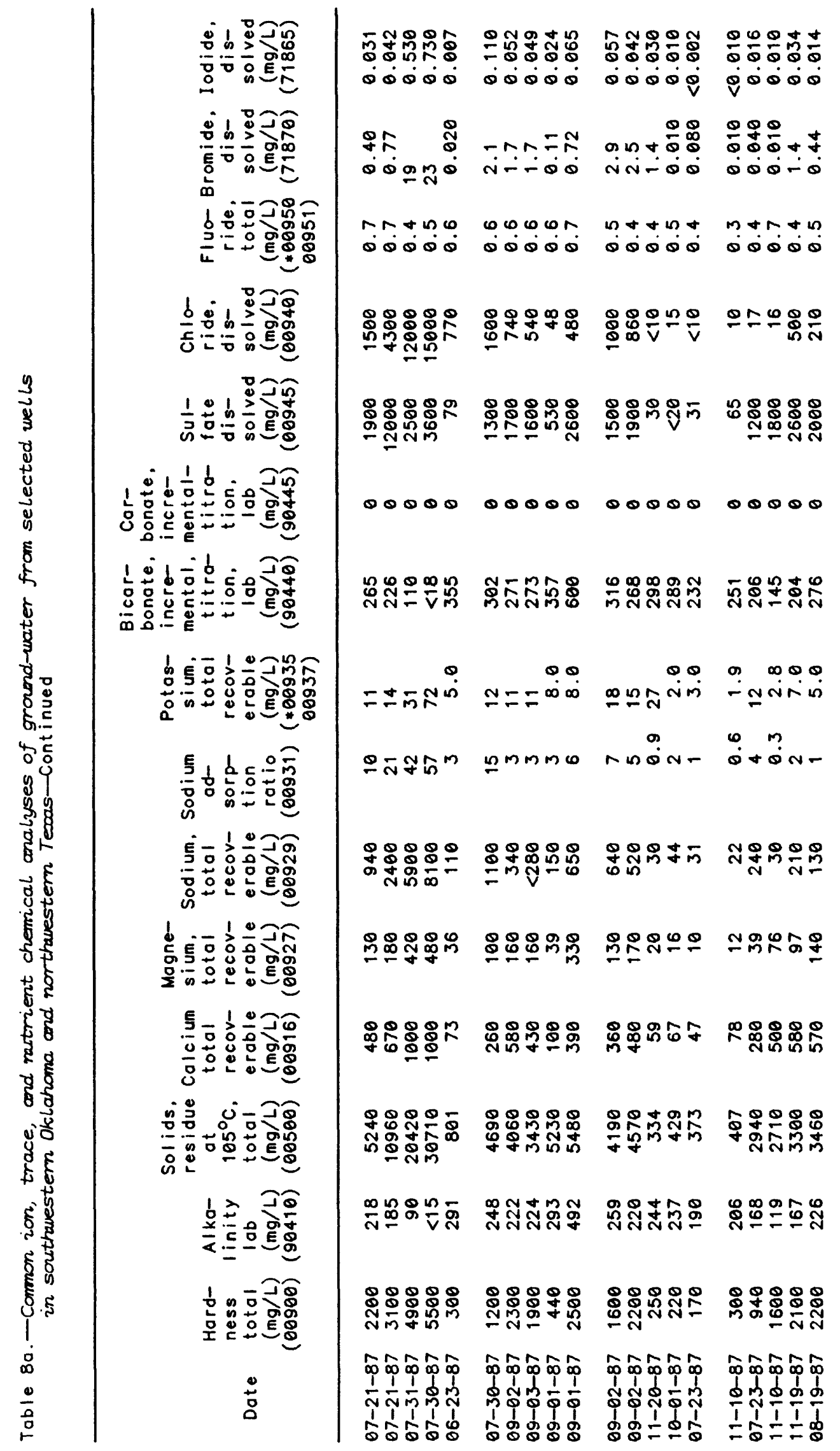




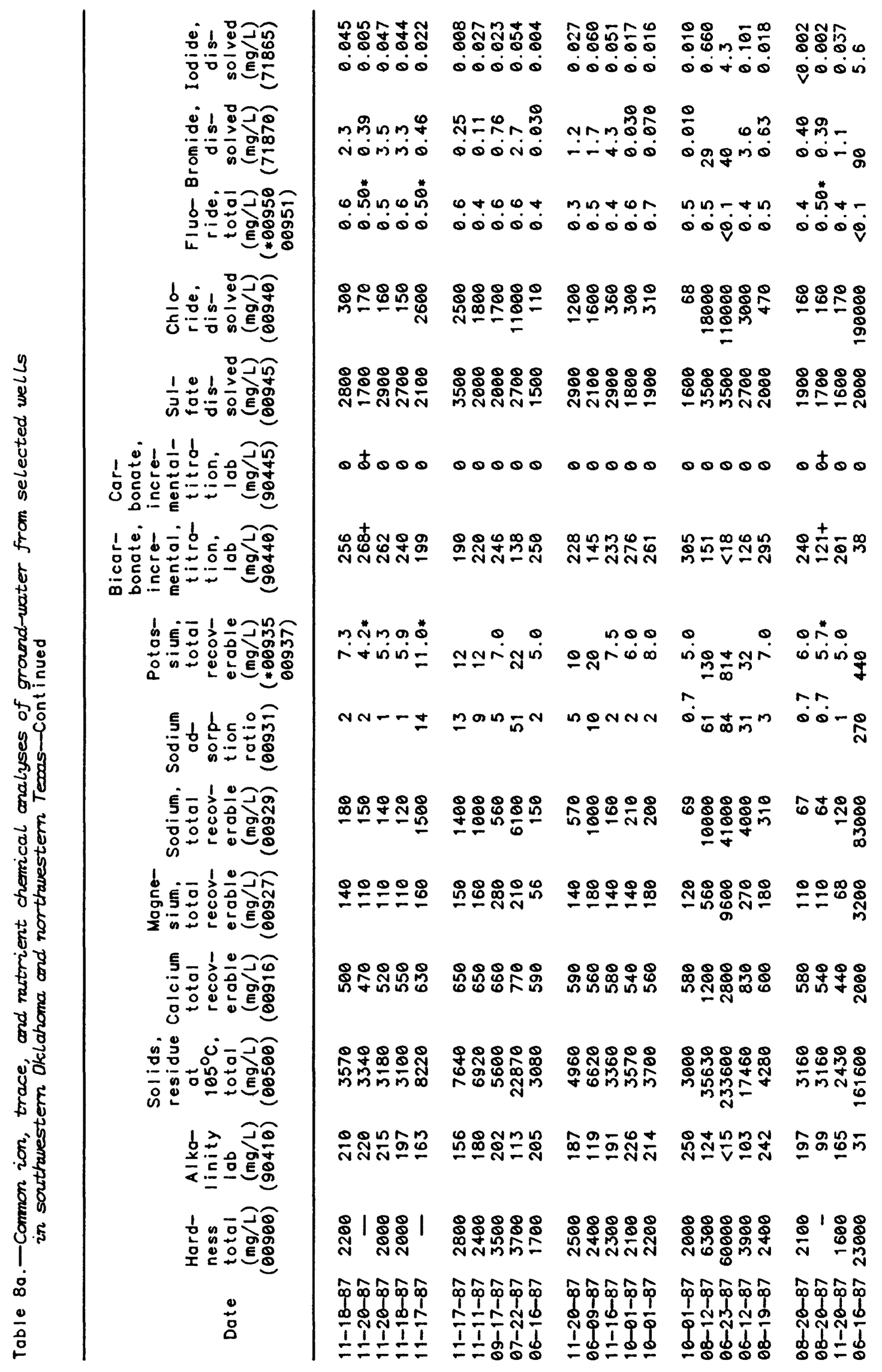




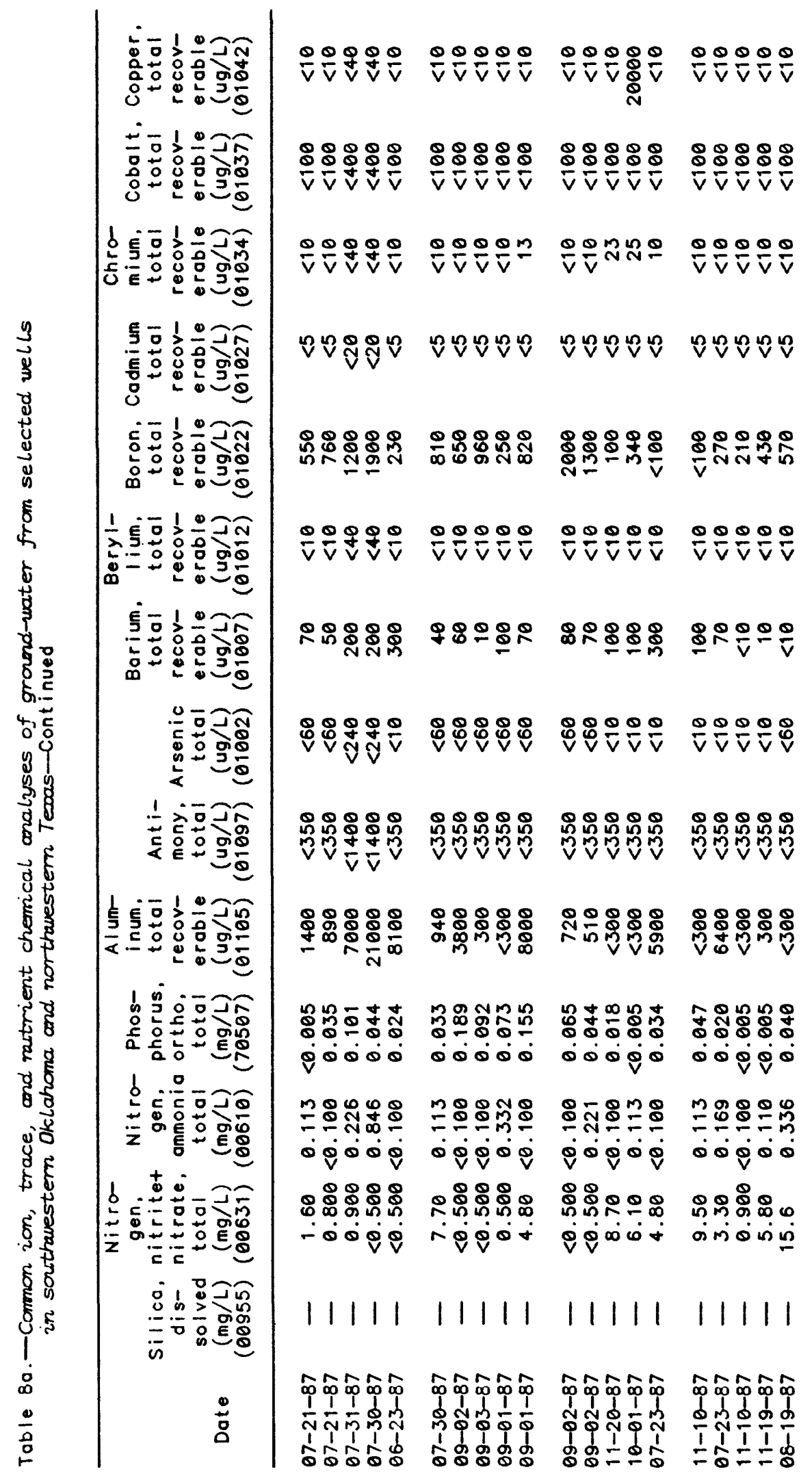




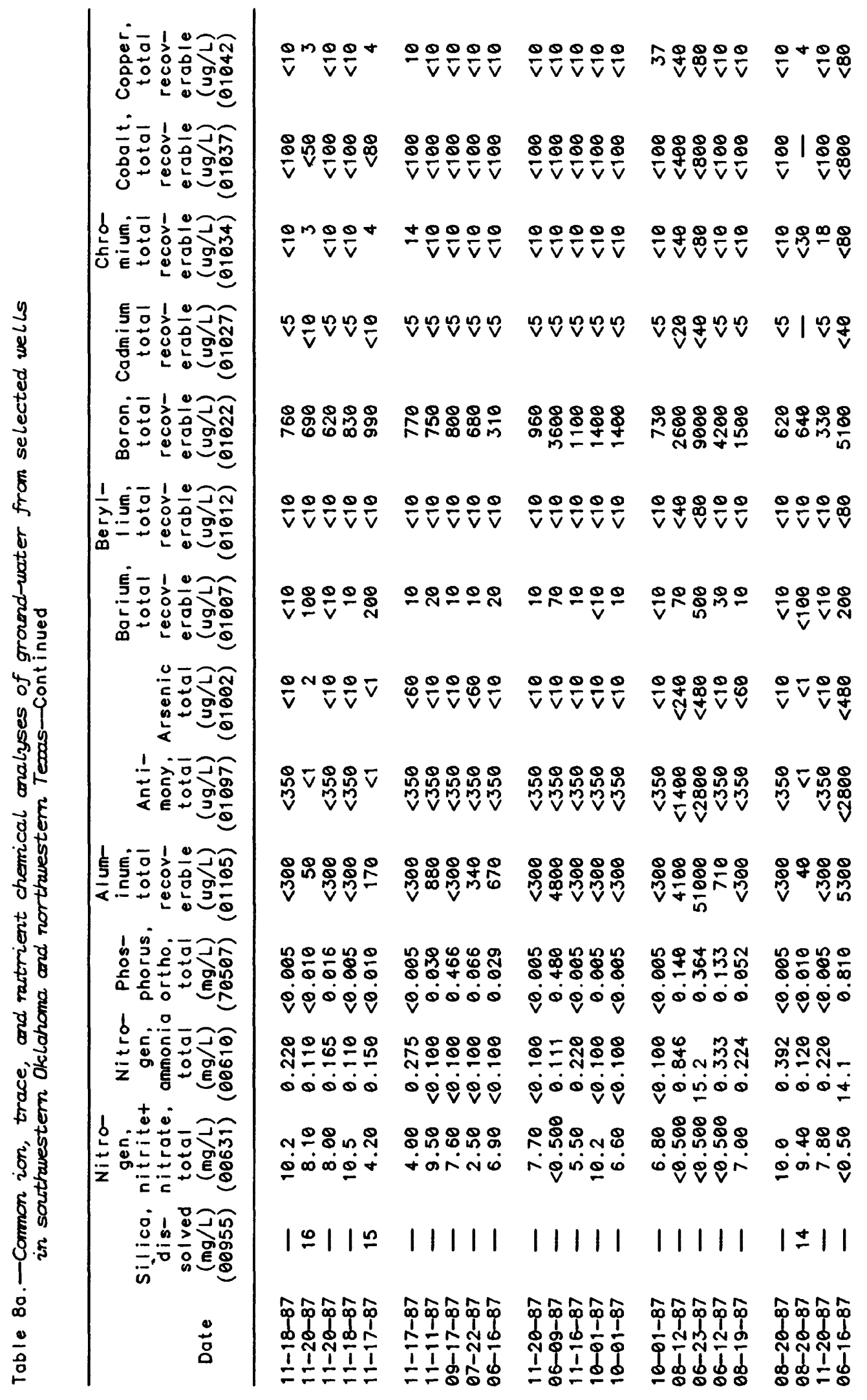




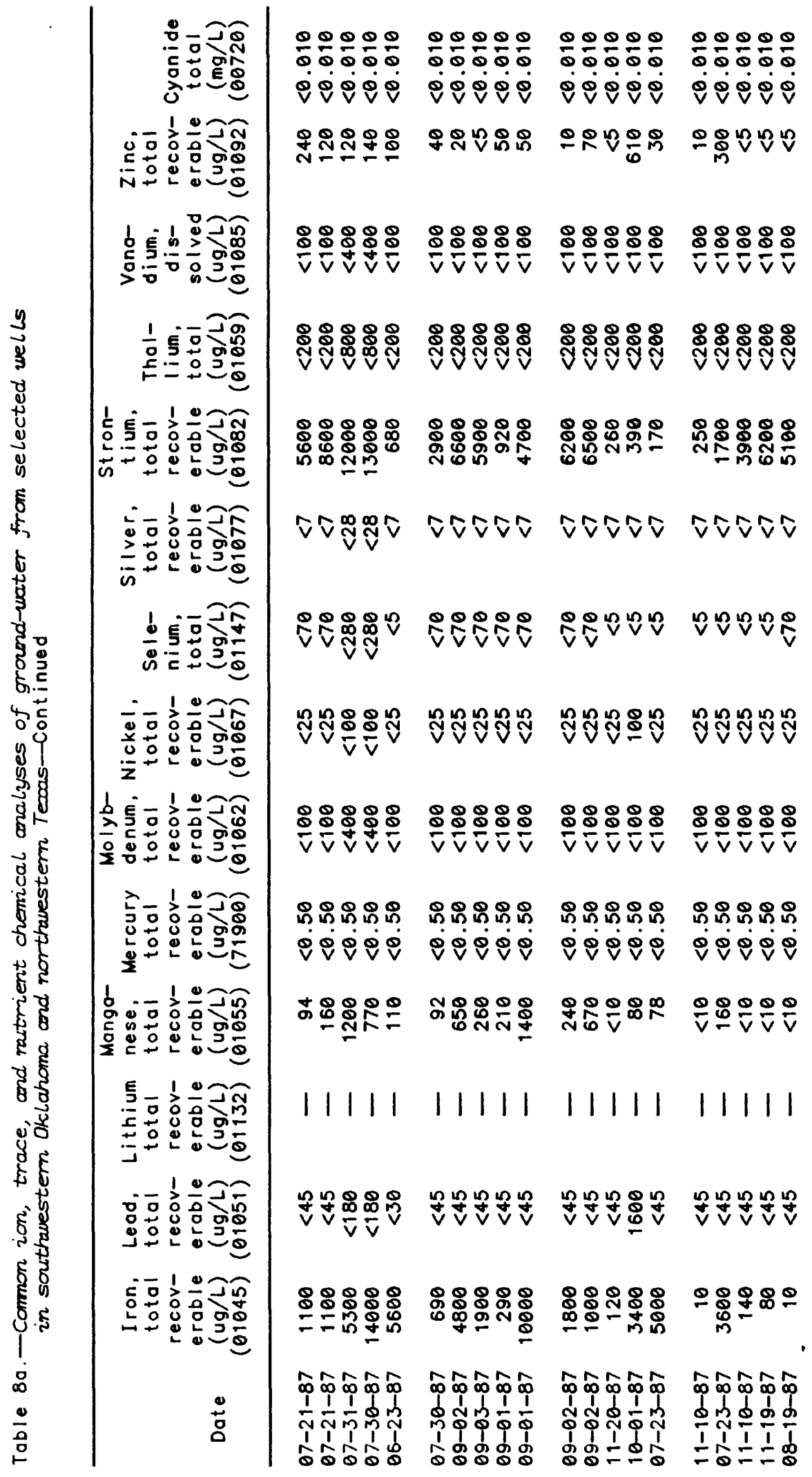




\begin{tabular}{|c|c|c|c|c|c|}
\hline 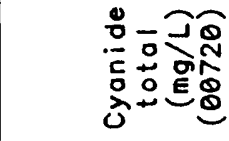 & 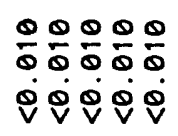 & 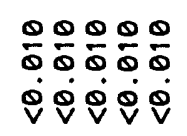 & 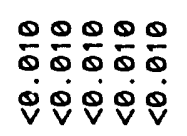 & 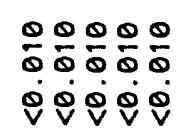 & 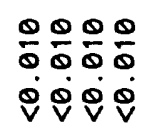 \\
\hline 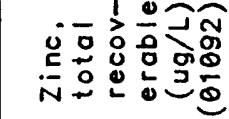 & ®ㄴำ & ロำกั & 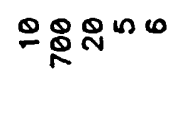 & 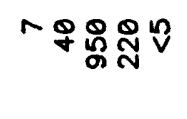 & 윤 \\
\hline 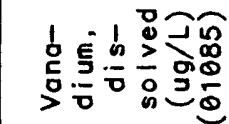 & $\frac{\otimes}{v} \frac{8}{v} \frac{\bar{v}}{\mathrm{v}}$ & 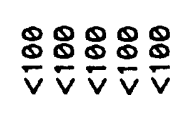 & 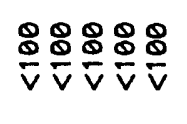 & 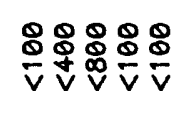 & \&N\& \\
\hline 高官可 & \& & \& & 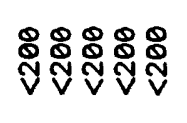 & \&: & 过 180 \\
\hline 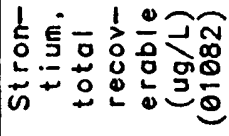 & இ8 & 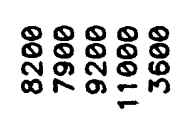 & 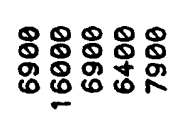 & 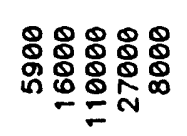 & 总: \\
\hline 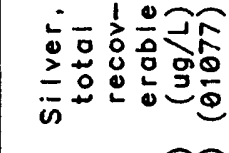 & $\hat{v} \bar{v} \hat{v} \hat{v} \bar{v}$ & nv̂ṽṽ & $\hat{v} \hat{v} \hat{v} \hat{v} \hat{v}$ & 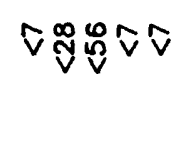 & $\hat{v} \bar{v} \hat{v} \stackrel{\leftrightarrow}{\mathscr{N}}$ \\
\hline 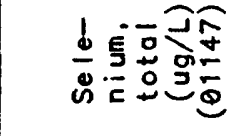 & $0 \bar{v} \vartheta \tilde{v} \bar{v}$ & 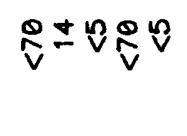 & ที่ง & 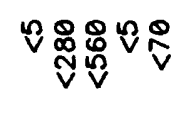 & 里 \\
\hline 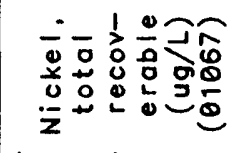 & 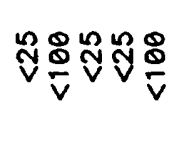 & 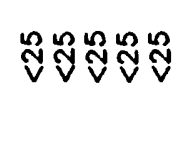 & 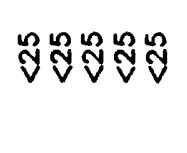 & 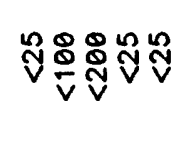 & $\stackrel{\sim}{\sim} \mid \stackrel{\sim}{v}$ \\
\hline 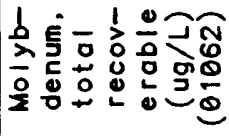 & 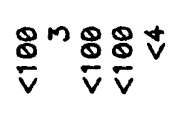 & 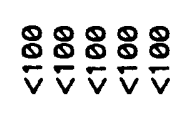 & 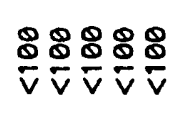 & 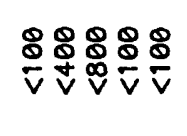 & :m\&: \\
\hline 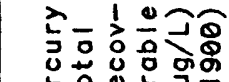 & ํำำำำำ & 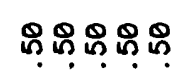 & 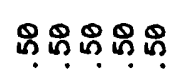 & ำ & คำกำ \\
\hline$\frac{5}{2}+$ & VQ8 & $8 \sigma$ & i & 8 & 8080 \\
\hline 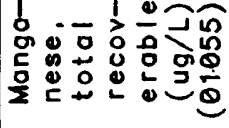 & 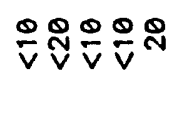 & ำกำํำ & $\frac{0}{v}=\frac{0}{v} \frac{0}{v} \pm$ & ะฌำ & ำำ \\
\hline 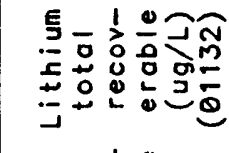 & 19119 & 11111 & 11111 & 11111 & 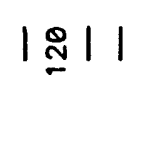 \\
\hline 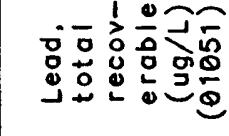 & 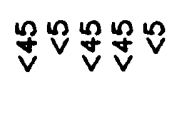 & 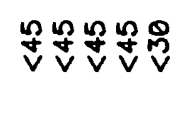 & 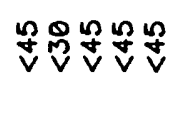 & 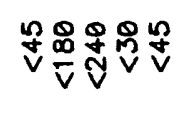 & ำำ \\
\hline 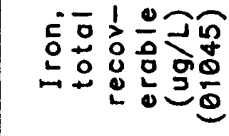 & ลำำํำ & 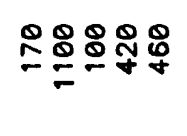 & 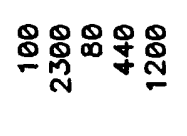 & 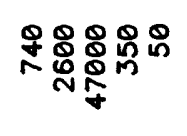 & 舟早早 \\
\hline & 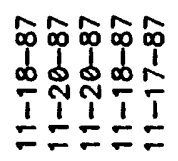 & 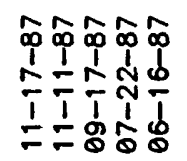 & 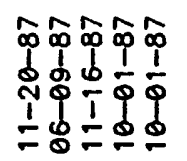 & 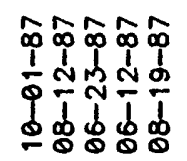 & 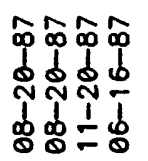 \\
\hline
\end{tabular}




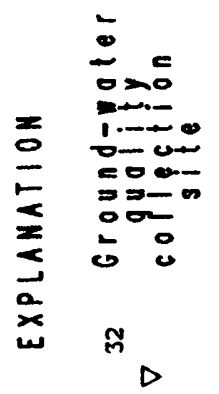

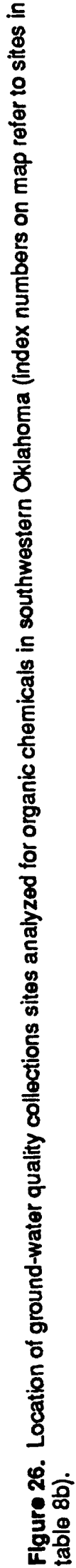




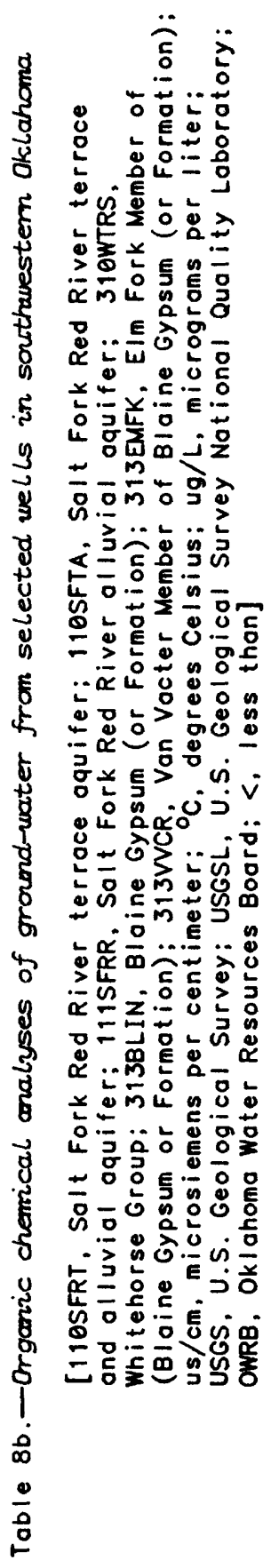

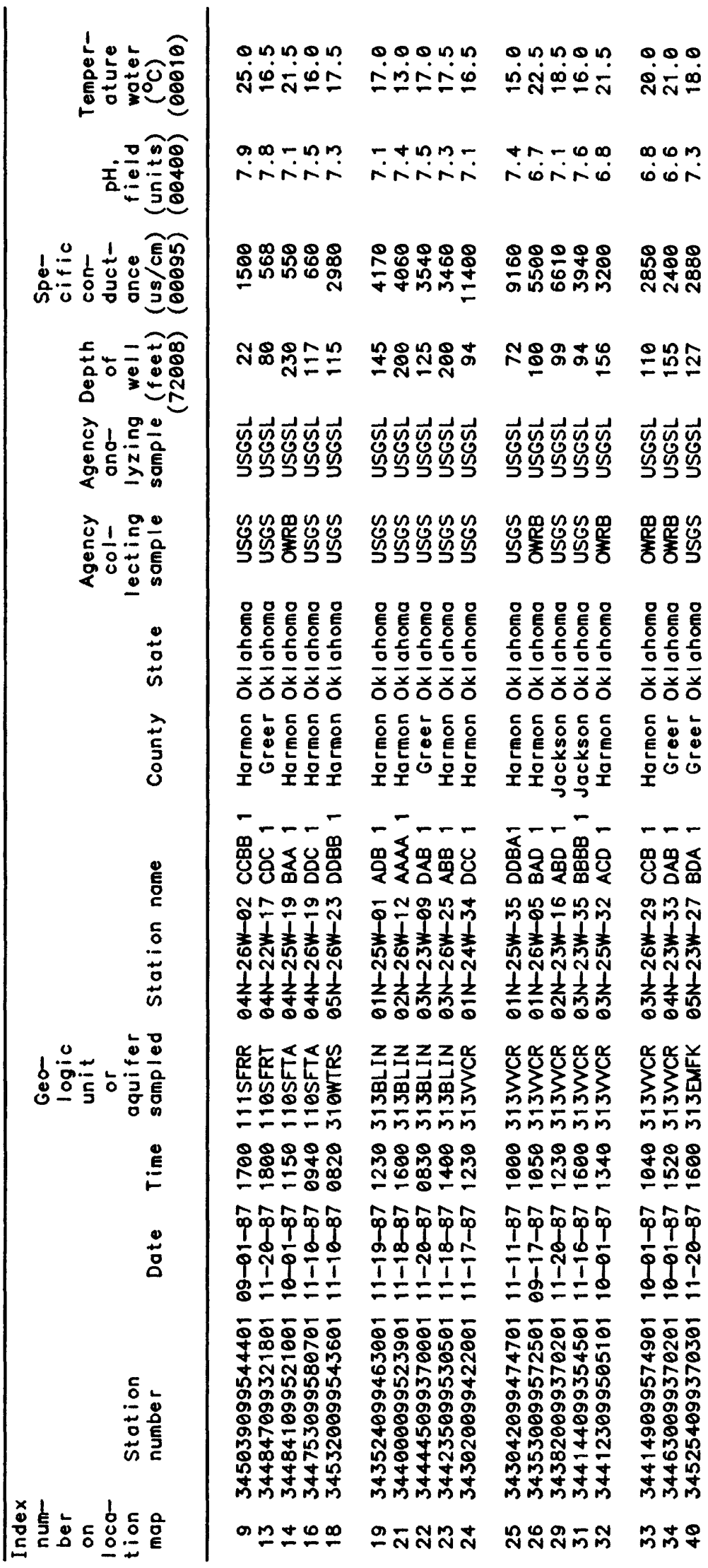




\begin{tabular}{|c|c|c|c|c|}
\hline$\therefore \dot{0} \overline{0}$ & $\frac{0}{6} \div \frac{0}{6} \frac{0}{6}$ & $\frac{0}{6} \div \frac{0}{0} \div \frac{0}{0} \div \frac{0}{0}$ & 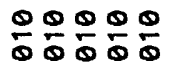 & $\frac{0}{6} \frac{0}{6}$ \\
\hline & $89888^{\circ}$ & $8 \% 8 \%$ & iq $8 \theta^{\circ}$ & 88 \\
\hline 10 & $\frac{9}{6} \frac{0}{0} \frac{0}{0} \frac{0}{0}$ & $\frac{0}{\phi} \frac{0}{0} \frac{0}{0} \frac{0}{0}$ & 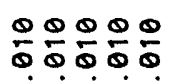 & $\frac{0}{0} \div \frac{0}{0}$ \\
\hline & 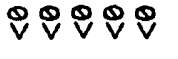 & அ $8: 8 R$ & அே $8 \% 8$ & $8 \%$ \\
\hline $5 \frac{1}{5}=$ & $\frac{0}{6} \div \frac{0}{0} \div \frac{0}{0} \div$ & $\frac{0}{6} \div \frac{0}{0} \div \frac{0}{0}$ & $\frac{0}{6} \div \frac{0}{0} \div \frac{0}{0} \div \frac{0}{0}$ & $\frac{0}{0} \frac{0}{0}$ \\
\hline 05 & $\dot{8} \nabla \dot{8} 8$ & Q $8 \dot{\nabla} 8$ & $8 \% 8 \% 8$ & $8 \% 8$ \\
\hline 送 & 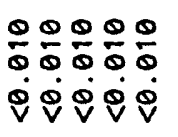 & 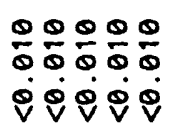 & 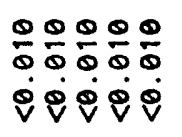 & $\begin{array}{l}\frac{0}{0} \frac{0}{0} \\
\dot{0} \dot{0}\end{array}$ \\
\hline 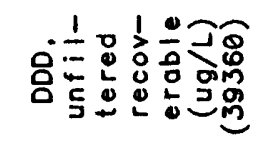 & 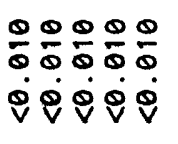 & 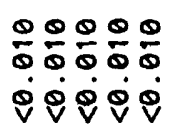 & 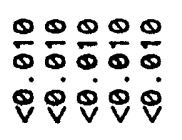 & 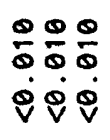 \\
\hline 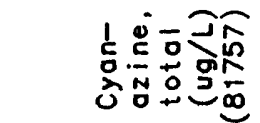 & $\begin{array}{l}\$ 8 \& \$ 8 \\
\dot{8} \dot{8} \dot{8}\end{array}$ & 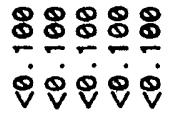 & 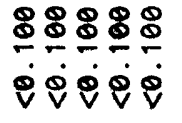 & $\begin{array}{l}\dot{8} 8 \\
\dot{8 \dot{8}}\end{array}$ \\
\hline 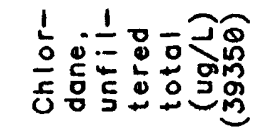 & $\frac{8: \$ 8 \%}{\dot{8} \dot{8} \dot{8}}$ & 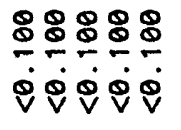 & 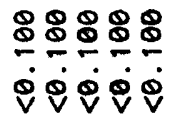 & $\frac{8 \% 8}{\dot{8} \dot{8}}$ \\
\hline 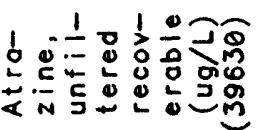 & 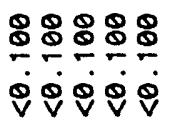 & 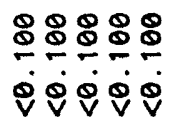 & $\frac{\$ \& \$ \$ 8}{\text { iष }}$ & $\frac{\$ 8 \%}{8 \%}$ \\
\hline b & 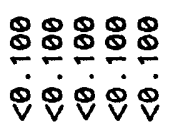 & 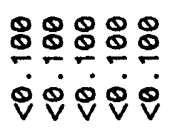 & 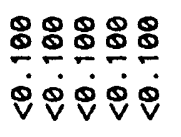 & $\begin{array}{l}8 \% 8 \\
\dot{8} \dot{8}\end{array}$ \\
\hline & $\frac{0}{6} \div \frac{0}{0} \div \frac{0}{0}$ & 웅웅웅유웅 & 웅융유융 & $\frac{0}{6} \div \frac{0}{0}$ \\
\hline & $8 \% 8 v^{\circ}$ & iq $8 \%$ & iq $88^{\circ}$ & $8 v^{\circ}$ \\
\hline 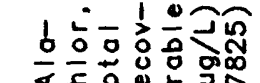 & \$8:8: & :\$ะ: & :8:8: & \&:8 \\
\hline <宅ㅇㅇㄴ & 운 & ㅇำ & ㅇvำ & $8 \otimes 8$ \\
\hline & 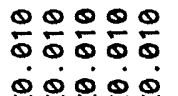 & $\begin{array}{l}\text { 웅웡ㅇㅇ } \\
00000\end{array}$ & 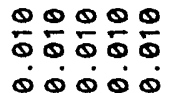 & 웅융 \\
\hline & $\bar{v} \bar{v}$ & $\vec{v}$ & & 8 \\
\hline$\therefore 0$ & 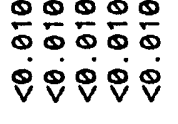 & 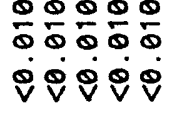 & 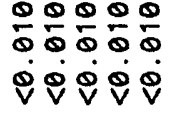 & 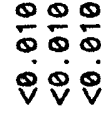 \\
\hline & 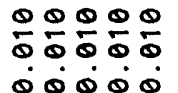 & 웅웡웅웅 & $\frac{0}{0} \div \frac{0}{6} \div \frac{0}{0}$ & $\frac{0}{6} \div \frac{0}{6}$ \\
\hline & $\sigma_{v}^{*} \sigma_{v}^{*}$ & $8 \& 8$ & ㅇvㅁㅇำ & 888 \\
\hline 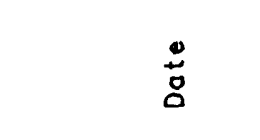 & 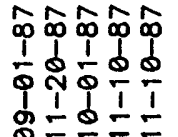 & 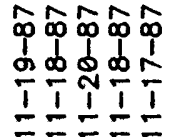 & 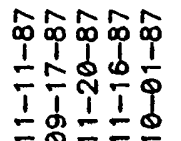 & 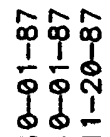 \\
\hline
\end{tabular}




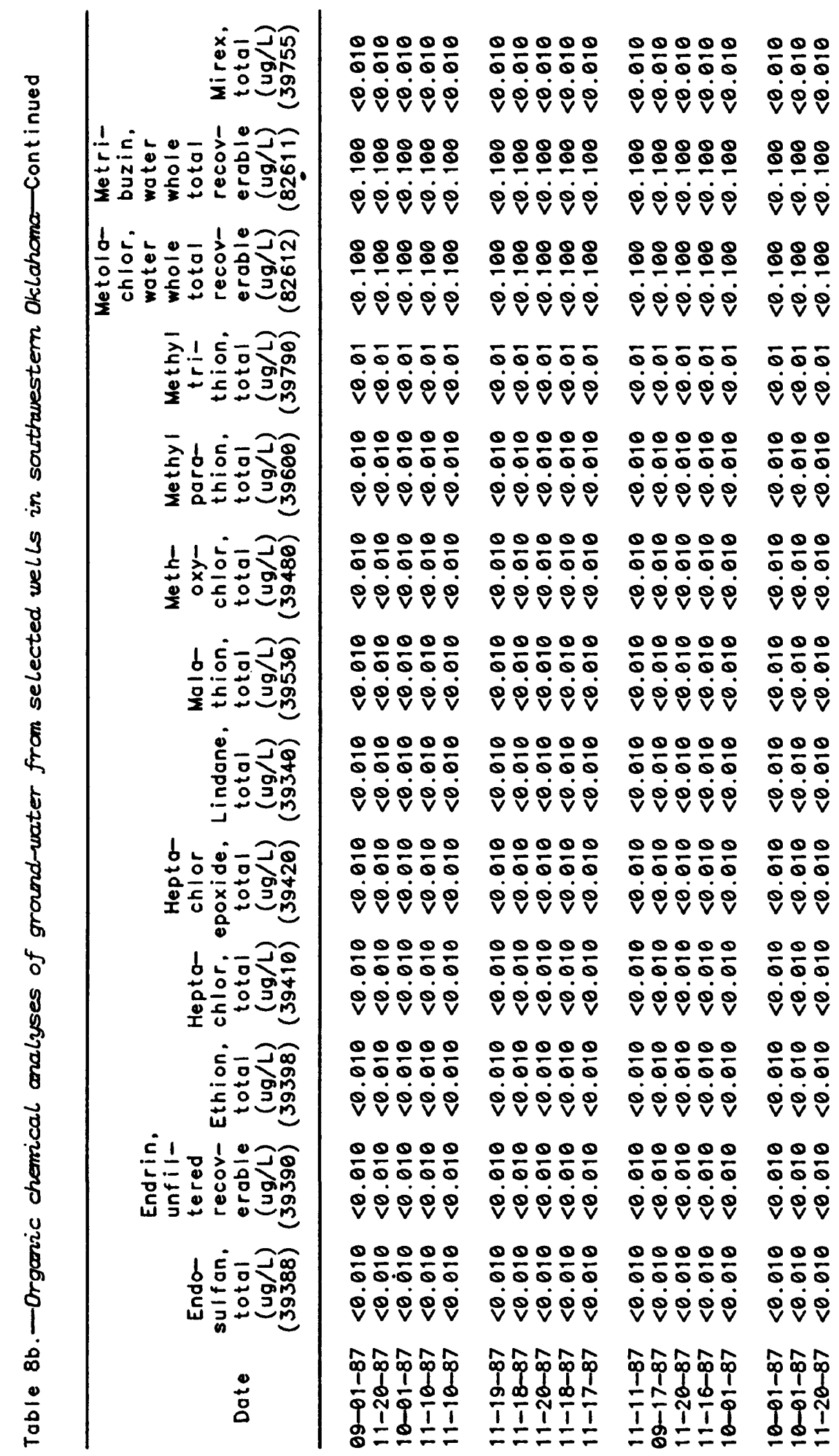




\begin{tabular}{|c|c|c|c|c|}
\hline 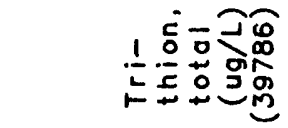 & 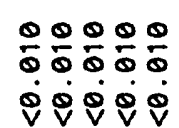 & 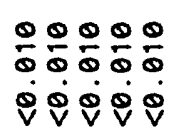 & 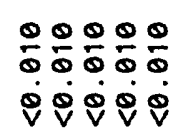 & 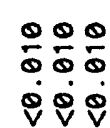 \\
\hline $1 \frac{1}{b}=\overline{0}$ & $\$ \& \& \&$ & \&\&\&\& & \&:\&: & 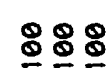 \\
\hline & Q $\dot{\nabla} \dot{\nabla} \dot{\nabla}$ & iq $\dot{\nabla} \dot{\nabla} \dot{\nabla}$ & iq $\dot{\nabla} \dot{\nabla}$ & $\dot{8} \dot{8}$ \\
\hline & ஃஃ\&\&\& & ஃஃ\&タ\& & ஃ:ஃஃ: & \&: \\
\hline & $\dot{\bar{v}} \bar{v} \dot{\bar{v}} \bar{v} \dot{v}$ & $\dot{\bar{v}} \dot{\bar{v}} \overline{\mathrm{v}} \dot{\mathrm{v}} \dot{\bar{v}}$ & $\dot{\bar{v}} \dot{\bar{v}} \dot{\bar{v}} \dot{\bar{v}} \bar{v}$ & $\dot{\bar{v}} \dot{\bar{v}} \dot{v}$ \\
\hline & இ\&\&\&\& & 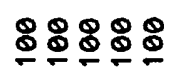 & ¿\&\&\& & \&\& \\
\hline & iqivi & $\dot{\theta} \dot{8} \dot{\nabla} \dot{8}$ & $\dot{\theta} \dot{8} \dot{8} \dot{8}$ & $\dot{\nabla} \dot{\nabla}$ \\
\hline & இ』‡』 & 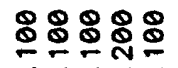 & ¿\&』』 & 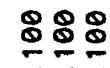 \\
\hline & Q $\dot{\nabla} \dot{\nabla} \dot{\nabla} \dot{\nabla}$ & $\dot{Q} \dot{Q} \dot{\theta} \dot{\theta}$ & iिi & $\dot{8} \dot{8}$ \\
\hline & 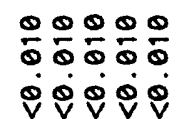 & 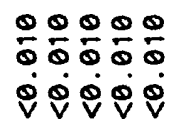 & 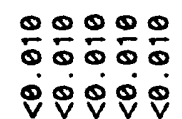 & 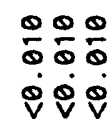 \\
\hline & ¿\&\&\&\& & ஓ\&\&\& & 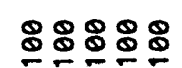 & $\$ \&$ \\
\hline & QPQPQP & 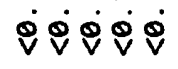 & Q & $88 \theta^{\circ}$ \\
\hline & ஓఖఖఖ & ¿\&ఖఖ & 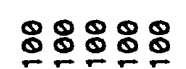 & $\$ \$ \$$ \\
\hline & Q & $\dot{\theta} \dot{8} \dot{\nabla} \dot{\nabla}$ & 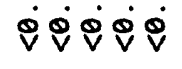 & $\dot{\nabla} \dot{8}$ \\
\hline & 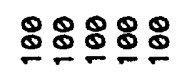 & \&\$\$\& & $\$ 8 \$ 8 \$$ & $\$ 8:$ \\
\hline & Q & 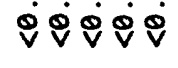 & 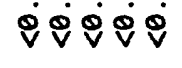 & $\dot{\theta} \dot{\nabla}$ \\
\hline & 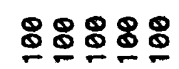 & $\$ \$ 8 \$ 8$ & \$\$\$\$ & $\$ \$ 8$ \\
\hline & $\dot{\theta} \dot{\theta} \dot{8} \dot{8}$ & Q $8 \dot{8} \dot{8}$ & Q $\dot{P} \dot{\nabla} \dot{\nabla}$ & $8 \dot{8}$ \\
\hline & ஓ\&\&\&\& & \&\&\&\& & ஓ\&\&\&\& & 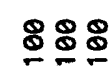 \\
\hline & Q & $\dot{\theta} \dot{\nabla} \dot{\nabla} \dot{\nabla} \dot{\nabla}$ & 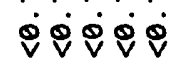 & Q $8 \dot{\nabla}$ \\
\hline b & $\frac{0}{0} \div \frac{0}{0} \div \frac{0}{0} \div \frac{0}{6}$ & 웅웅요웅 & $\frac{0}{6} \div \frac{0}{6} \div \frac{0}{0} \div \frac{0}{0}$ & $\frac{0}{6} \div \frac{0}{0}$ \\
\hline & 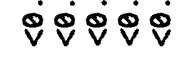 & VQVivi & iqivi & $8 \overline{8}$ \\
\hline$\frac{1}{0} \geq 201$ & இఖఖఖ & 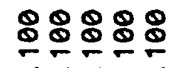 & இ\&\&\&\& & 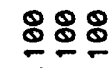 \\
\hline$=0-1$ & Q $\dot{\nabla} \dot{\nabla} \dot{\theta} \dot{8}$ & Q & 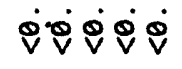 & i $\dot{\nabla}$ \\
\hline$\underset{\tau}{2}$ & 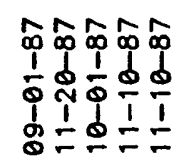 & 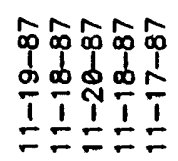 & 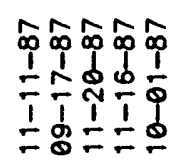 & 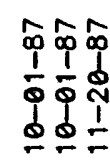 \\
\hline
\end{tabular}



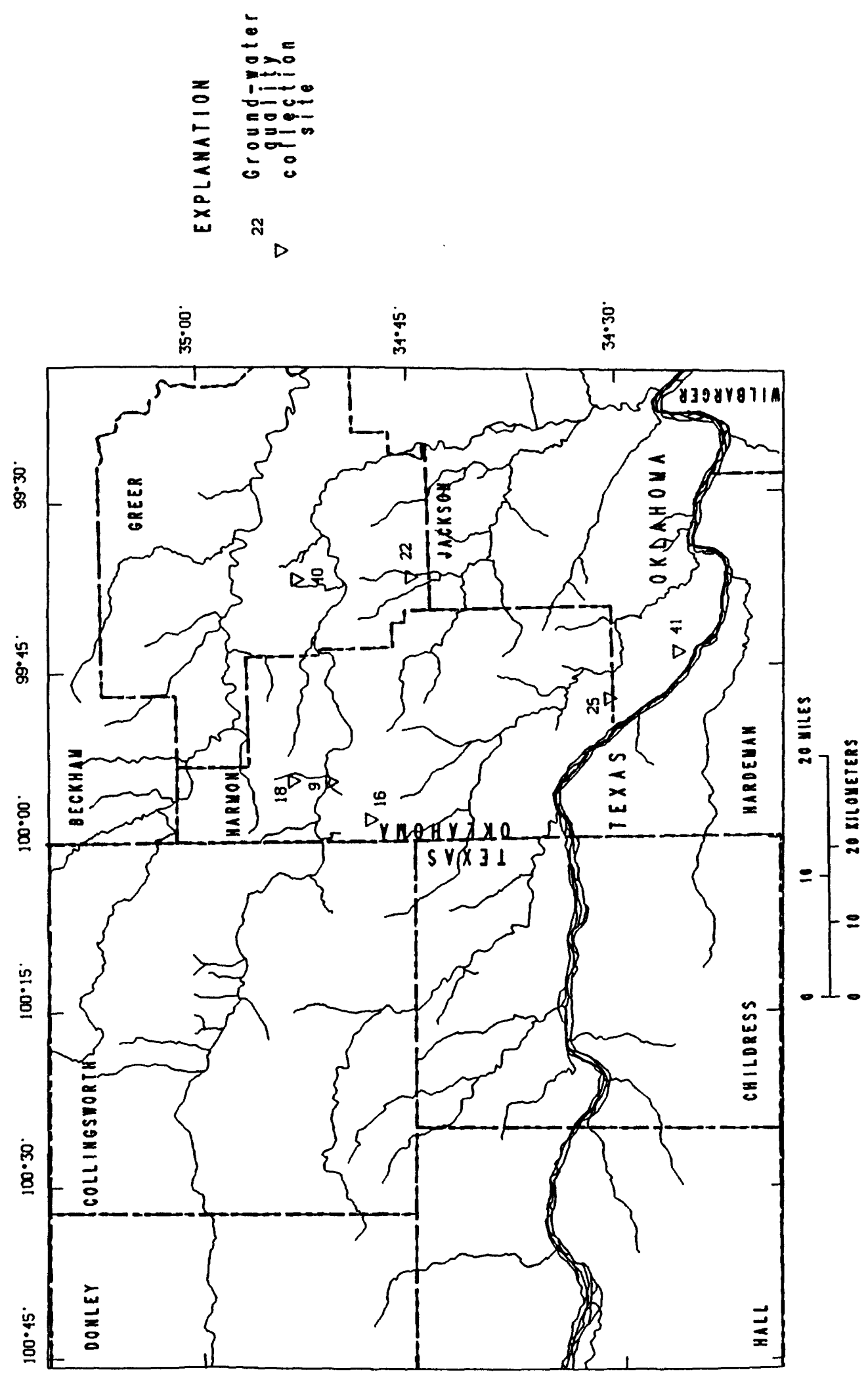


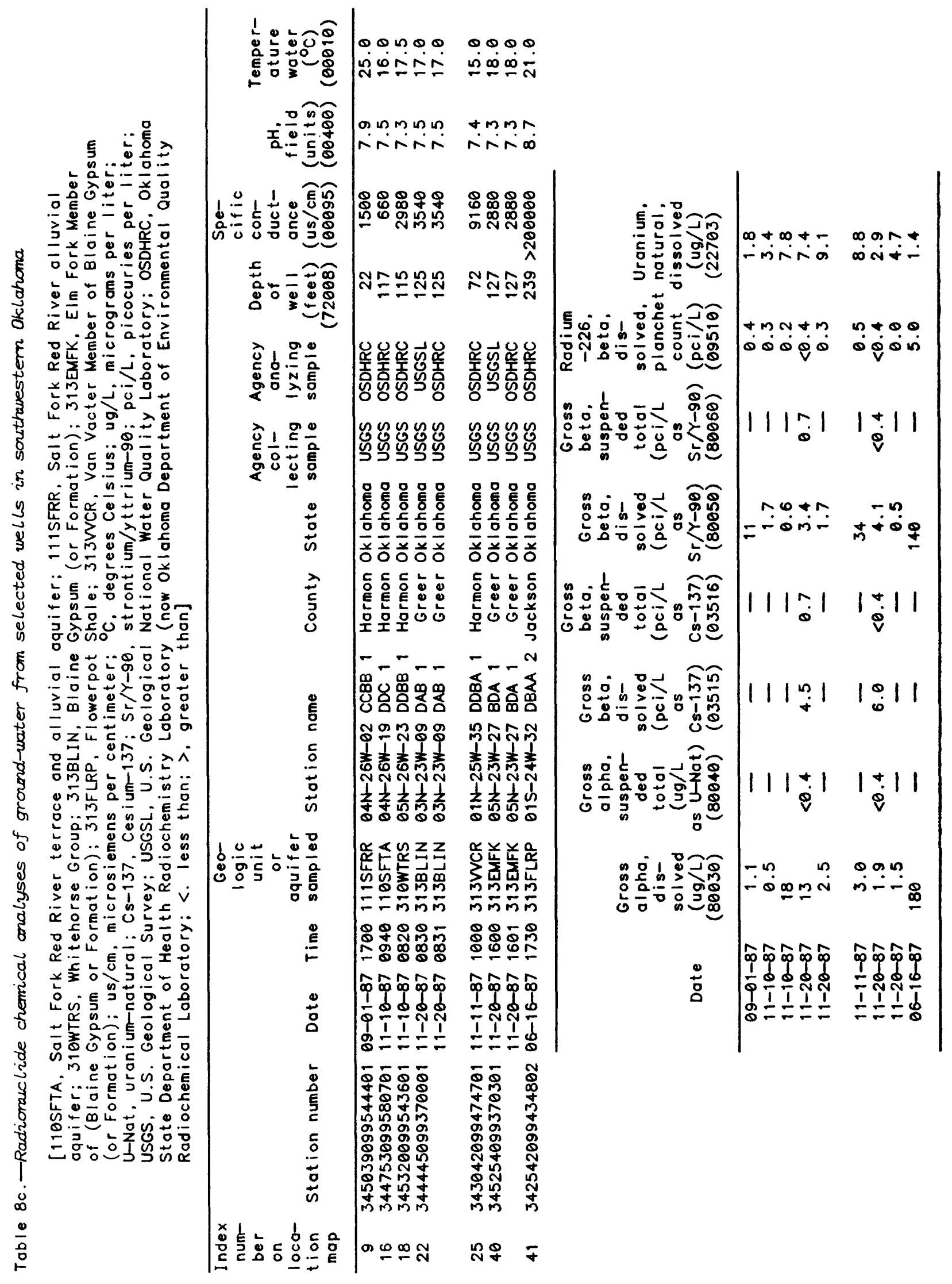



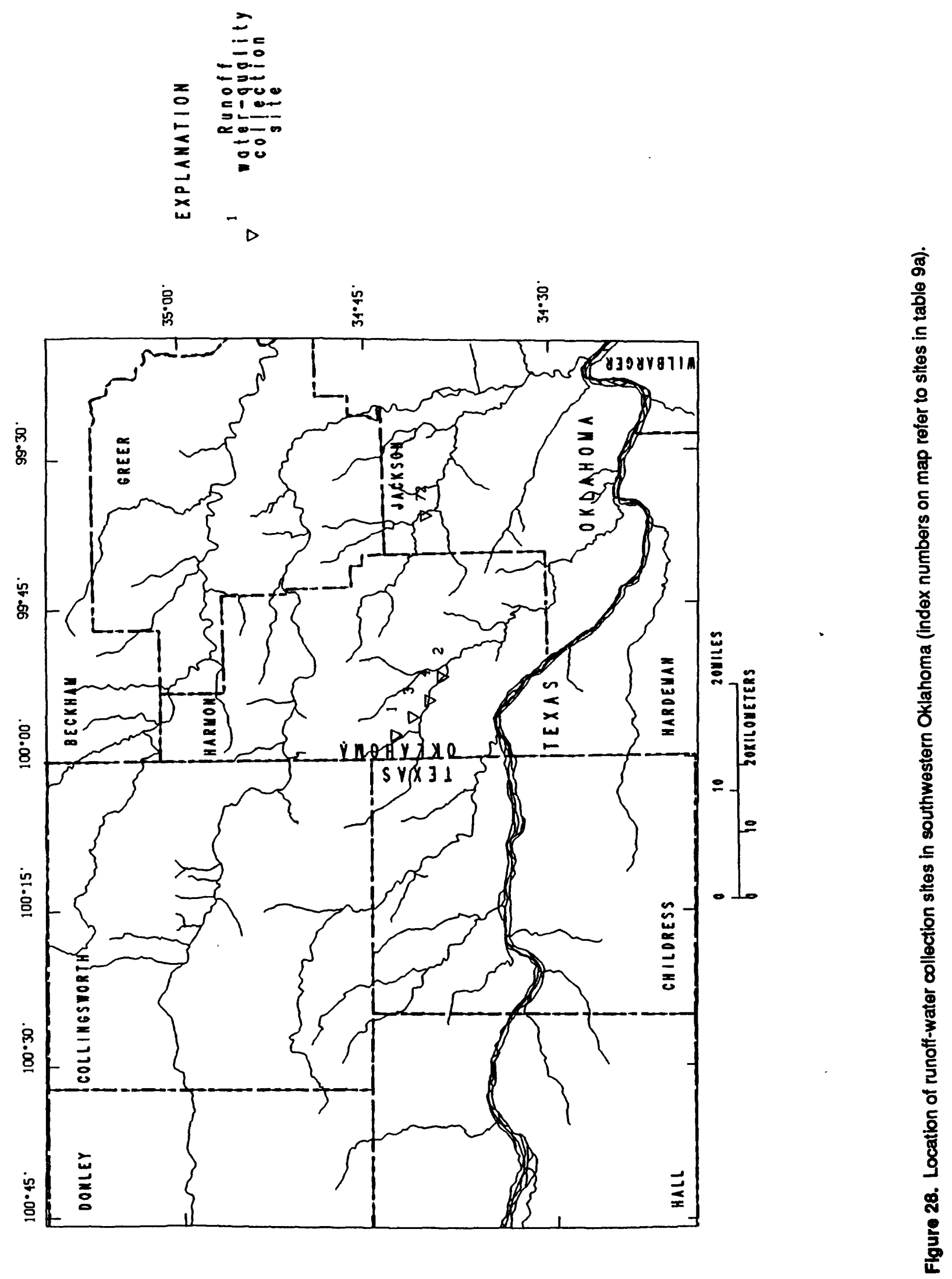


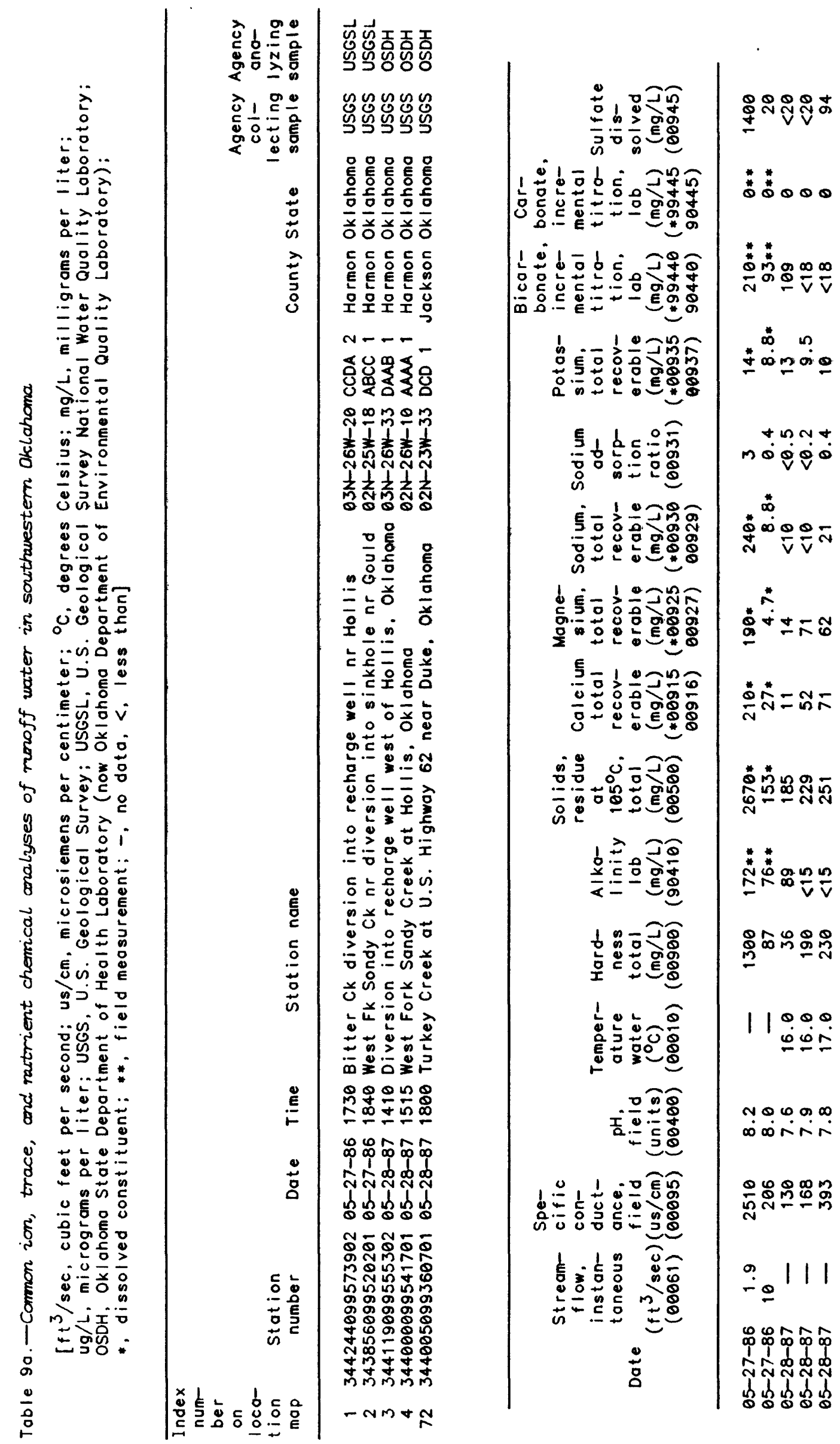




\begin{tabular}{|c|c|c|c|}
\hline 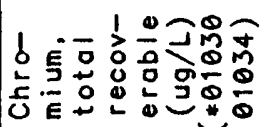 & ேㅗํำ & 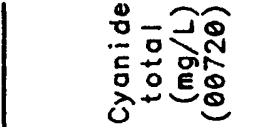 & 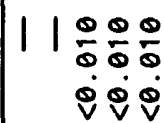 \\
\hline 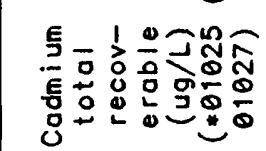 & 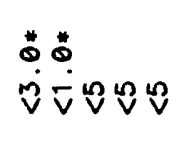 & 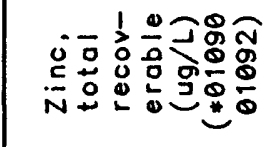 & 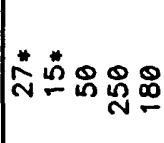 \\
\hline 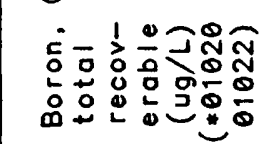 & 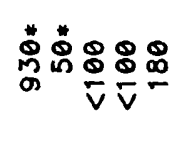 & 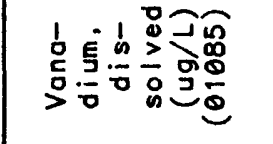 & $\bar{v}^{\infty} \frac{8}{6} \div$ \\
\hline 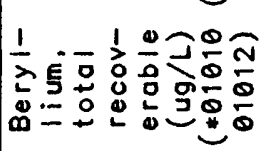 & 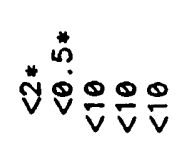 & 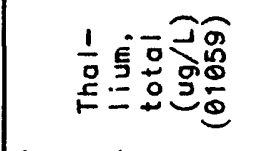 & 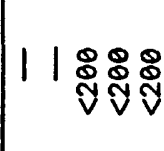 \\
\hline 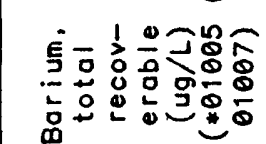 & 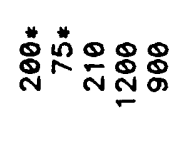 & 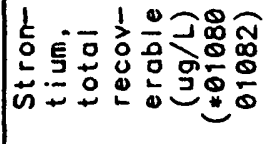 & 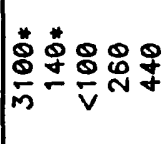 \\
\hline 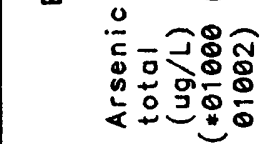 & 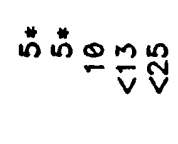 & 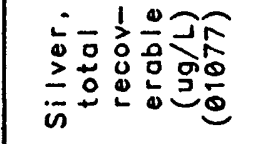 & $11 \hat{v} \hat{v}$ \\
\hline 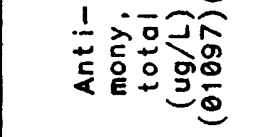 & 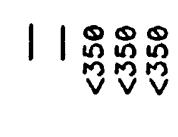 & 造 & * \\
\hline 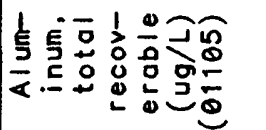 & 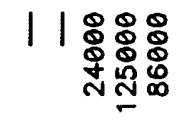 & 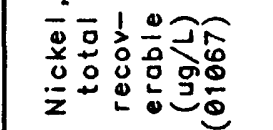 & 11 ลิ요 \\
\hline 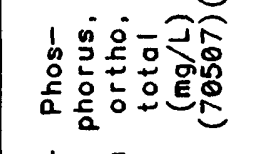 & 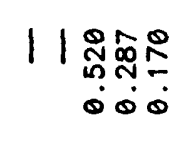 & 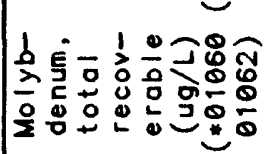 & மே. \\
\hline 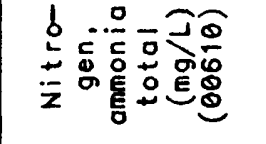 & 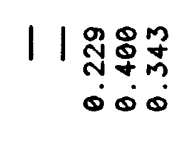 & 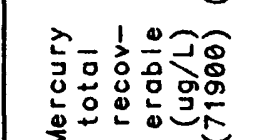 & 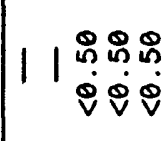 \\
\hline 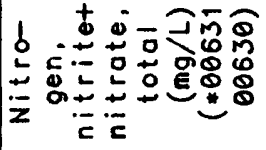 & 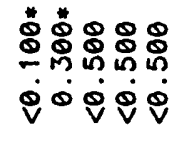 & 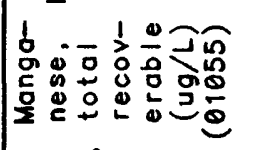 & | 1 | \\
\hline 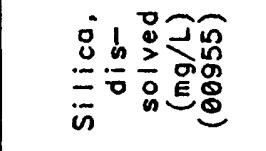 & $\stackrel{\infty}{\infty}|| \mid$ & 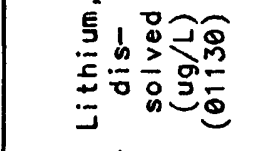 & $m^{n} 111$ \\
\hline 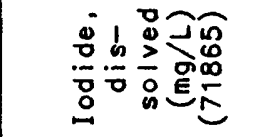 & $\begin{array}{r}118 \overline{8} \\
888 \\
8 \% 8\end{array}$ & 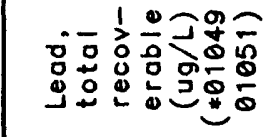 & 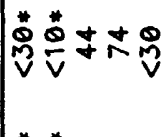 \\
\hline 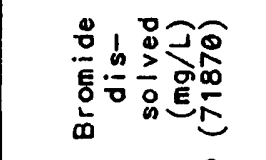 & 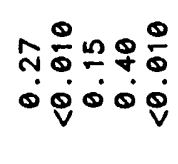 & 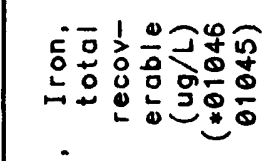 & v \\
\hline 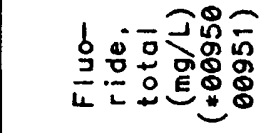 & 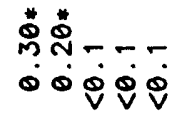 & 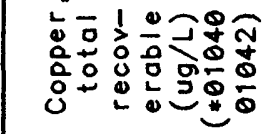 & 总 \\
\hline 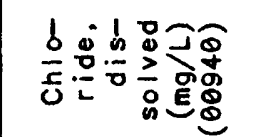 & 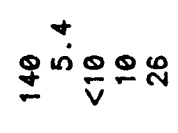 & 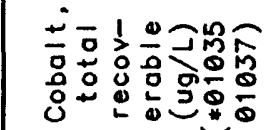 & 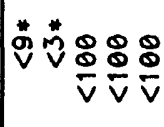 \\
\hline$c$ & 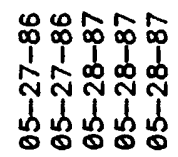 & $\stackrel{9}{0}$ & 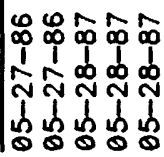 \\
\hline
\end{tabular}




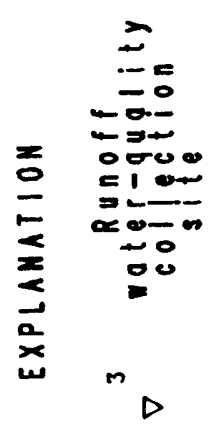

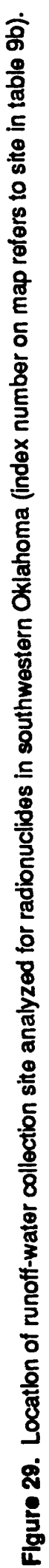




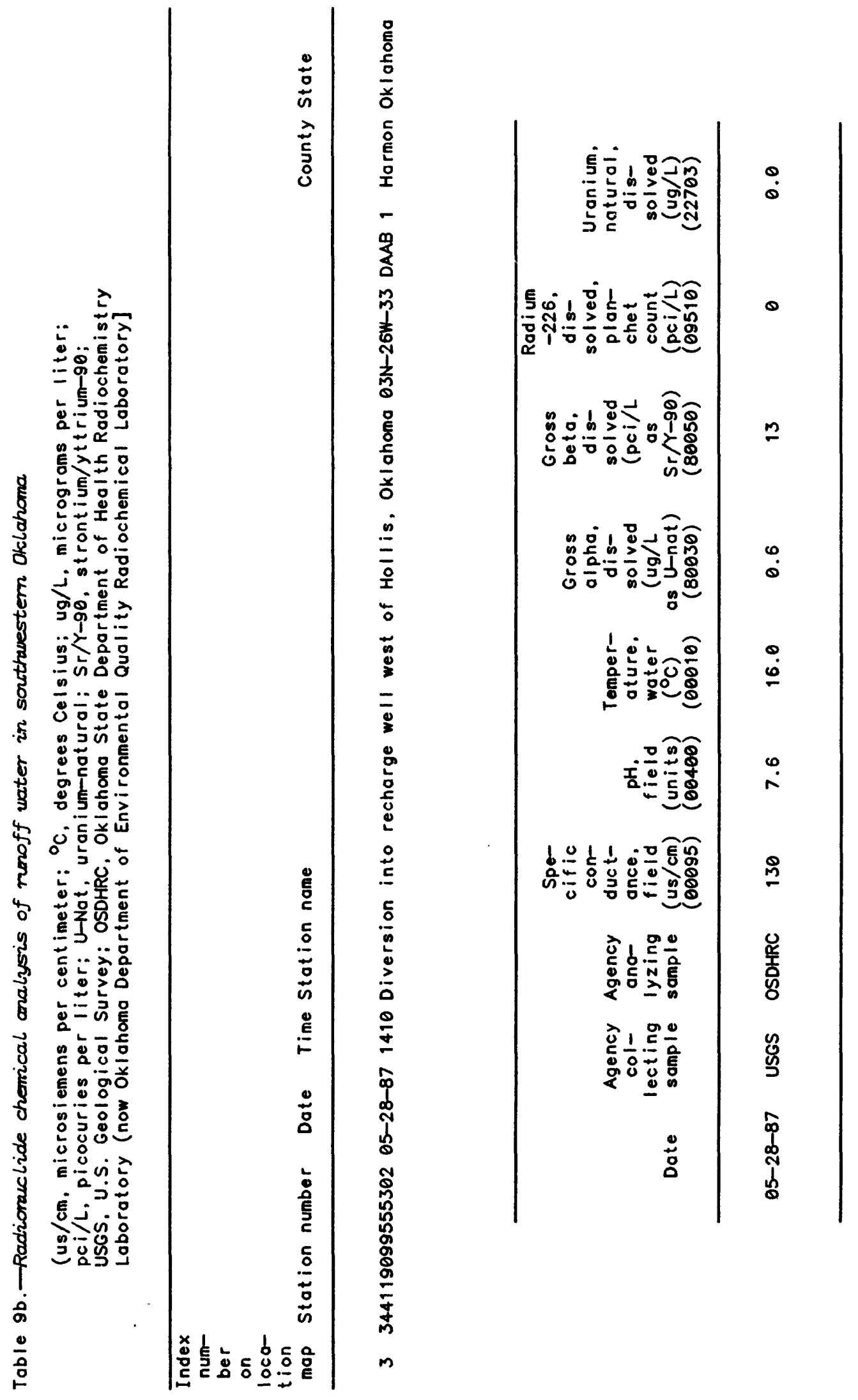

